\title{
Modified Gravity and Cosmology
}

\author{
Timothy Clifton ${ }^{\mathrm{a}}$, Pedro G. Ferreira ${ }^{\mathrm{a}}$, Antonio Padilla ${ }^{\mathrm{b}}$, Constantinos Skordis ${ }^{\mathrm{b}}$ \\ ${ }^{a}$ Department of Astrophysics, University of Oxford, UK. \\ ${ }^{b}$ School of Physics and Astronomy, University of Nottingham, UK.
}

\begin{abstract}
In this review we present a thoroughly comprehensive survey of recent work on modified theories of gravity and their cosmological consequences. Amongst other things, we cover General Relativity, Scalar-Tensor, Einstein-Aether, and Bimetric theories, as well as TeVeS, $f(R)$, general higher-order theories, Hořava-Lifschitz gravity, Galileons, Ghost Condensates, and models of extra dimensions including Kaluza-Klein, Randall-Sundrum, DGP, and higher co-dimension braneworlds. We also review attempts to construct a Parameterised Post-Friedmannian formalism, that can be used to constrain deviations from General Relativity in cosmology, and that is suitable for comparison with data on the largest scales. These subjects have been intensively studied over the past decade, largely motivated by rapid progress in the field of observational cosmology that now allows, for the first time, precision tests of fundamental physics on the scale of the observable Universe. The purpose of this review is to provide a reference tool for researchers and students in cosmology and gravitational physics, as well as a self-contained, comprehensive and up-to-date introduction to the subject as a whole.
\end{abstract}

Keywords: General Relativity, Gravitational Physics, Cosmology, Modified Gravity 


\section{Contents}

1 Introduction

2 General Relativity, and its Foundations 11

2.1 Requirements for Validity . . . . . . . . . . . . . . . . . . 11

2.1.1 The foundations of relativistic theories . . . . . . . . . 11

2.1.2 Observational tests of metric theories of gravity . . . . . . . . . 14

2.1.3 Theoretical considerations . . . . . . . . . . . . 20

2.2 Einstein's Theory . . . . . . . . . . . . . . . . . 22

2.2.1 The field equations . . . . . . . . . . . . . . 23

2.2 .2 The action ........................... 24

2.3 Alternative Formulations . . . . . . . . . . . . . . . . . . 25

2.3.1 The Palatini procedure . . . . . . . . . . . . . 25]

2.3.2 Metric-affine gravity and matter . . . . . . . . . . . . 27

2.3 .3 Other approaches . . . . . . . . . . . . . . . 27

2.4 Theorems . . . . . . . . . . . . . . . . . . . . . . . . . . . . . 29

2.4.1 Lovelock's theorem . . . . . . . . . . . . . . . 29

2.4 .2 Birkhoff's theorem . . . . . . . . . . . . . . . 30

2.4.3 The no-hair theorems ................... 31

2.5 The Parameterised Post-Newtonian Approach . . . . . . . . . . . . . . 32

2.5.1 Parameterised post-Newtonian formalism . . . . . . . . . . 32

2.5.2 Parameterised post-Newtonian constraints . . . . . . . . . . . 34

2.6 Cosmology . . . . . . . . . . . . . . . . . 37

2.6.1 The Friedmann-Lemaître-Robertson-Walker solutions . . . . . . 37

2.6.2 Cosmological distances . . . . . . . . . . . . . . . . . . 38

2.6.3 Perturbation theory .................... 40

2.6.4 Gravitational potentials and observations . . . . . . . . . 42

2.6.5 The evidence for the $\Lambda$ CDM model . . . . . . . . . . . . . . 44

2.6.6 Shortcomings of the $\Lambda$ CDM model . . . . . . . . . . . 46

3 Alternative Theories of Gravity with Extra Fields 49

3.1 Scalar-Tensor Theories . . . . . . . . . . . . . . 49

3.1.1 Action, field equations, and conformal transformations . . . . . . 49

3.1.2 Brans-Dicke theory . . . . . . . . . . . . . . 52 5

3.1.3 General scalar-tensor theories . . . . . . . . . . . . 59

3.1.4 The chameleon mechanism .............. 66 66

3.2 Einstein-Æther Theories . . . . . . . . . . . . . . . . . 68

3.2.1 Modified Newtonian dynamics . . . . . . . . . . . . 68 68

3.2 .2 Action and field equations . . . . . . . . . . . . . . . 69

3.2 .3 FLRW solutions . . . . . . . . . . . . . . . . . 70

3.2.4 Cosmological perturbations . . . . . . . . . . . . 71

3.2.5 Observations and constraints ................ 73

3.3 Bimetric Theories . . . . . . . . . . . . . . . 75

3.3.1 Rosen's theory, and non-dynamical metrics . . . . . . . . . 76

3.3.2 Drummond's theory . . . . . . . . . . . . . . 77

3.3.3 Massive gravity .................... 77 
3.3.4 Bigravity . . . . . . . . . . . . . . . . . 799

3.3.5 Bimetric MOND . . . . . . . . . . . . . . . . . . 80

3.4 Tensor-Vector-Scalar Theories . . . . . . . . . . . . . . . . . . . . . 81

3.4 .1 Actions and field equations . . . . . . . . . . 82

3.4 .2 Newtonian and MOND limits . . . . . . . . . . . . . . 84

3.4.3 Homogeneous and isotropic cosmology . . . . . . . . . . . 86

3.4.4 Cosmological perturbation theory . . . . . . . . . . . . . 91

3.4.5 Cosmological observations and constraints . . . . . . . . . 993

3.5 Other Theories . . . . . . . . . . . . . . . . . . . . 96

3.5.1 The Einstein-Cartan-Sciama-Kibble Theory . . . . . . . . . . . . 96

3.5.2 Scalar-Tensor-Vector Theory . . . . . . . . . . . . . . 99

4 Higher Derivative and Non-Local Theories of Gravity 101

$4.1 \quad f(R)$ Theories . . . . . . . . . . . . . . . . . . . . . . . . . . 101

4.1.1 Action, field equations and transformations . . . . . . . 102

4.1 .2 Weak-field limit . . . . . . . . . . . . . . . . . . 106

4.1.3 Exact solutions, and general behaviour . . . . . . . . . . . . . 111

4.1 .4 Cosmology . . . . . . . . . . . . . . . . . . . 114

4.1 .5 Stability issues . . . . . . . . . . . . . . . . 120

4.2 General combinations of Ricci and Riemann curvature. . . . . . . . . . 123

4.2 .1 Action and field equations . . . . . . . . . . . . . . . 123

4.2 .2 Weak-field limit . . . . . . . . . . . . . . . . . 125

4.2 .3 Exact solutions, and general behaviour . . . . . . . . . . . 126

4.2.4 Physical cosmology and dark energy . . . . . . . . . . . . 128

4.2 .5 Other topics . . . . . . . . . . . . . . . . . . . 131

4.3 Hořava-Lifschitz Gravity . . . . . . . . . . . . . . . . . . . . . . 137

4.3 .1 The projectable theory . . . . . . . . . . . . . . . . . . . 141

4.3 .2 The non-projectable theory . . . . . . . . . . . . . . . . . 144

4.3.3 Aspects of Hořava-Lifschitz cosmology . . . . . . . . . . . . . . 146

4.3.4 The $\Theta$ CDM model . . . . . . . . . . . . . . . . . . . . . . 148

4.3.5 HMT-da Silva theory . . . . . . . . . . . . . . . . . . . 148

4.4 Galileons . . . . . . . . . . . . . . . . . . . . . . . 150

4.4 .1 Galileon modification of gravity . . . . . . . . . . . . . 151

4.4 .2 Covariant galileon . . . . . . . . . . . . . . . 157

4.4 .3 DBI galileon . . . . . . . . . . . . . . . . . . . 158

4.4 .4 Galileon cosmology . . . . . . . . . . . . . . . . . . 160

4.4 .5 Multi-galileons . . . . . . . . . . . . . . . . . . 161

4.5 Other Theories . . . . . . . . . . . . . . . . . . . . . . . . . . 164

4.5.1 Ghost condensates . . . . . . . . . . . . . . . . . . . 164

4.5 .2 Non-metric gravity . . . . . . . . . . . . . . . . . 166

4.5.3 Dark energy from curvature corrections . . . . . . . . . . . 169

5 Higher Dimensional Theories of Gravity 172

5.1 Kaluza-Klein Theories of Gravity . . . . . . . . . . . . . . . . 172

5.1 .1 Kaluza-Klein compactifications . . . . . . . . . . . . . . 173

$5.1 .2 \quad$ Kaluza-Klein cosmology . . . . . . . . . . . . . . . . . . 174

5.2 The Braneworld Paradigm . . . . . . . . . . . . . . . . . . . . . 179 
5.2.1 The ADD model . . . . . . . . . . . . . . . . . . . 180

5.3 Randall-Sundrum Gravity . . . . . . . . . . . . . . . . . . . . . . . 181

5.3 .1 The RS1 model . . . . . . . . . . . . . . . . . . . . 182

5.3 .2 The RS2 model . . . . . . . . . . . . . . . . . . 184

5.3 .3 Other RS-like models . . . . . . . . . . . . . . . 185

5.3.4 Action and equations of motion . . . . . . . . . . . . 188

5.3.5 Linear perturbations in RS1 and RS2 . . . . . . . . . . . . . . . 189

5.4 Brane Cosmology . . . . . . . . . . . . . . . . . . . . . . 195

5.4.1 Brane based formalism - covariant formulation . . . . . . . . . 197

5.4.2 Bulk based formalism - moving branes in a static bulk . . . . . . . 199

5.4.3 Cosmological perturbations . . . . . . . . . . . . . . 200

5.5 Dvali-Gabadadze-Porrati Gravity . . . . . . . . . . . . . . . 207

5.5.1 Action, equations of motion, and vacua . . . . . . . . . . . 207

5.5.2 Linear perturbations on the normal branch . . . . . . . . . . . . 209

5.5.3 Linear perturbations (and ghosts) on the self-accelerating branch . 211

5.5.4 From strong coupling to the Vainshtein mechanism . . . . . . . . . 214

5.5.5 DGP cosmology . . . . . . . . . . . . . . . . . 221

5.6 Higher Co-Dimension Braneworlds . . . . . . . . . . . . . . . . . . 228

5.6 .1 Cascading gravity . . . . . . . . . . . . . . . . 230

5.6 .2 Degravitation . . . . . . . . . . . . . . . . . 235

5.7 Einstein Gauss-Bonnet Gravity . . . . . . . . . . . . . . . . . . . . 236

5.7.1 Action, equations of motion, and vacua . . . . . . . . . . 237

5.7.2 Kaluza-Klein reduction of EGB gravity . . . . . . . . . . . . . . . 240

5.7.3 Co-dimension one branes in EGB gravity . . . . . . . . . . . . . . 240

5.7.4 Co-dimension two branes in EGB gravity . . . . . . . . . . . . . 244

6 Parameterised Post-Friedmannian Approaches and Observational Constraints

6.1 The Formalism . . . . . . . . . . . . . . . . . . . . . 247

6.1.1 Evolution of perturbations on super-horizon scales . . . . . . . . 248

6.1.2 The simplified PPF approach, and its extensions . . . . . . . . . 249

6.1.3 The Hu-Sawicki frame-work . . . . . . . . . . . . . . . . . . 253

6.2 Models for $\mu$ and $\zeta$ on Sub-Horizon Scales . . . . . . . . . . . . . . 254

6.2.1 The importance of shear . . . . . . . . . . . . . . . . 254

6.2 .2 The growth function . . . . . . . . . . . . . . 255

6.2.3 Current constraints on the PPF parameters . . . . . . . . . 257

6.2.4 Constraining the growth rate . . . . . . . . . . . . . . . 258

6.2 .5 The $E_{G}$ diagnostic . . . . . . . . . . . . . . . . . . . . 259

6.3 Forecasting Constraints from Future Surveys . . . . . . . . . . . . . 260

7 Discussion $\quad 262$ 


\section{Introduction}

The General Theory of Relativity is an astounding accomplishment: Together with quantum field theory, it is now widely considered to be one of the two pillars of modern physics. The theory itself is couched in the language of differential geometry, and was a pioneer for the use of modern mathematics in physical theories, leading the way for the gauge theories and string theories that have followed. It is no exaggeration to say that General Relativity set a new tone for what a physical theory can be, and has truly revolutionised our understanding of the Universe.

One of the most striking facts about General Relativity is that, after almost an entire century, it remains completely unchanged: The field equations that Einstein communication to the Prussian Academy of Sciences in November 1915 are still our best description of how space-time behaves on macroscopic scales. These are

$$
G_{\mu \nu}=\frac{8 \pi G}{c^{4}} T_{\mu \nu}
$$

where $G_{\mu \nu}$ is the Einstein tensor, $T_{\mu \nu}$ is the energy momentum tensor, $G$ is Newton's constant, and $c$ is the speed of light. It is these equations that are thought to govern the expansion of the Universe, the behaviour of black holes, the propagation of gravitational waves, and the formation of all structures in the Universe from planets and stars all the way up to the clusters and super-clusters of galaxies that we are discovering today. It is only in the microscopic world of particles and high energies that General Relativity is thought to be inadequate. On all other scales it remains the gold standard.

The great success of General Relativity, however, has not stopped alternatives being proposed. Even during the very early days after Einstein's publication of his theory there were proposals being made on how to extend it, and incorporate it in a larger, more unified theory. Notable examples of this are Eddington's theory of connections, Weyl's scale independent theory, and the higher dimensional theories of Kaluza and Klein. To some extent, these early papers were known to have been influential on Einstein himself. They certainly influenced the physicists who came after him.

The ideas developed by Eddington during this period were later picked up by Dirac, who pointed out the apparent coincidence between the magnitude of Newton's constant and the ratio of the mass and scale of the Universe. This relationship between a fundamental constant and the dynamical state of a particular solution led Dirac to conjecture that Newton's constant may, in fact, be varying with time. The possibility of a varying Newton's constant was picked up again in the 1960s by Brans and Dicke who developed the prototypical version of what are now known as scalar-tensor theories of gravity. These theories are still the subject of research today, and make up Section 3.1 of our report.

Building on the work of Hermann Weyl, the Soviet physicist Andrei Sakharov proposed in 1967 what would prove to be one of the most enduring theories of modified gravity. In Sakharov's approach, the Einstein-Hilbert action, from which the Einstein field equations can be derived, is simply a first approximation to a much more complicated action: Fluctuations in space-time itself lead to higher powers corrections to Einstein's theory. In 1977 Kellogg Stelle showed formally that these theories are renormalizable in the presence of matter fields at the one loop level. This discovery was followed by a surge of interest, that was boosted again later on by the discovery of the potential cosmological consequences of these theories, as found by Starobinsky and others. In Section 4 we review this work. 
The idea of constructing a quantum field theory of gravity started to take a front seat in physics research during the 1970s and 80s, with the rise of super-gravity and superstring theories. Both of these proposals rely on the introduction of super-symmetry, and signalled a resurgence in the ideas of Kaluza and Klein involving higher dimensional spaces. Boosted further by the discovery of D-branes as fundamental objects in string theories, this avenue of research led to a vastly richer set of structures that one could consider, and a plethora of proposals were made for how to modify the effective field equations in four dimensions. In Section 5 we review the literature on this subject.

By the early 1970s, and following the 'golden age' of general relativity that took place in the 1960s, there was a wide array of candidate theories of gravity in existence that could rival Einstein's. A formalism was needed to deal with this great abundance of possibilities, and this was provided in the form of the Parameterised Post-Newtonian (PPN) formalism by Kenneth Nordtvedt, Kip Thorne and Clifford Will. The PPN formalism was built on the earlier work of Eddington and Dicke, and allowed for the numerous theories available at the time to be compared to cutting edge astrophysical observations such as lunar laser ranging, radio echo, and, in 1974, the Hulse-Taylor binary pulsar. The PPN formalism provided a clear structure within which one could compare and assess various theories, and has been the benchmark for how theories of gravity should be evaluated ever since. We will give an outline of the PPN formalism, and the constraints available within it today, in Section 2

The limits of General Relativity have again come into focus with the emergence of the 'dark universe' scenario. For almost thirty years there has existed evidence that, if gravity is governed by Einstein's field equations, there should be a substantial amount of 'dark matter' in galaxies and clusters. More recently, 'dark energy' has also been found to be required in order to explain the apparent accelerating expansion of the Universe. Indeed, if General Relativity is correct, it now seems that around $96 \%$ of the Universe should be in the form of energy densities that do not interact electromagnetically. Such an odd composition, favoured at such high confidence, has led some to speculate on the possibility that General Relativity may not, in fact, be the correct theory of gravity to describe the Universe on the largest scales. The dark universe may be just another signal that we need to go beyond Einstein's theory.

The idea of modifying gravity on cosmological scales has really taken off over the past decade. This has been triggered, in part, by theoretical developments involving higher dimensional theories, as well as new developments in constructing renormalizable theories of gravity. More phenomenologically, Bekenstein's relativistic formulation of Milgrom's Modified Newtonian Dynamics (MoND) has provided a fresh impetus for new study: What was previously a rule of thumb for how weak gravitational fields might behave in regions of low acceleration, was suddenly elevated to a theory that could be used to study cosmology. Insights such as Bertschinger's realisation that large-scale perturbations in the Universe can be directly related to the overall expansion rate have also made it possible to characterise large classes of theories simply in terms of how they make the Universe evolve. Finally, and just as importantly, there has been tremendous progress observationally. A key step here has been the measurement of the growth of structure at redshifts of $z \simeq 0.8$, by Guzzo and his collaborators. With these measurements one can test, and reject, a large number of proposals for modified gravity. This work is complemented by many others that carefully consider the impact of modifications to gravity on the cosmic microwave background, weak lensing and a variety of other 
cosmological probes. As a result, testing gravity has become one of the core tasks of many current, and future, cosmological missions and surveys.

In this report we aim to provide a comprehensive exposition of the many developments that have occurred in the field of modified gravity over the past few decades. We will focus on how these theories differ from General Relativity, and how they can be distinguished from it, as well as from each other. A vast range of modified theories now exist in the literature. Some of these have extra scalar, vector or tensor fields in their gravitational sector; some take Sakharov's idea in an altogether new direction, modifying gravity in regions of low, rather than high, curvature; others expand on the ideas first put forward by Kaluza and Klein, and take them into new realms by invoking new structures. Indeed, as the reader will see from our table of contents, there are now a great many possible ways of modifying gravity that can, in principle, be tested against the real Universe. We will attempt to be as comprehensive in this report as we consider it reasonably possible to be. That is, we will attempt to cover as many aspects of as many different theories as we can.

To be able to efficiently assess the different candidate theories of gravity we have opted to first lay down the foundations of modern gravitational physics and General Relativity in Section 2. We have aimed to make this a self-contained section that focuses, to some extent, on why general relativity should be considered 'special' among the larger class of possibilities that we might consider. In this section we also survey the current evidence for the 'dark universe', and explain why it has become the standard paradigm. From here we move on to discuss and compare alternative theories of gravity and their observational consequences. While the primary focus of this report is to elucidate particular theories, we will also briefly delve into the recent attempts that have been made to construct a formalism, analogous to the PPN formalism, for the cosmological arena. We dub these approaches 'Parameterised Post Friedmannian'.

Let us now spell out the conventions and definitions that we will use throughout this review. We will employ the 'space-like convention' for the metric, such that when it is diagonalised it has the signature $(-+++)$. We will choose to write space-time indices using the Greek alphabet, and space indices using the Latin alphabet. Where convenient, we will also choose to use units such that speed of light is equal to 1 . Under these conventions the line-element for Minkowski space, for example, can then be written

$$
d s^{2}=\eta_{\mu \nu} d x^{\mu} d x^{\nu}=-d t^{2}+d x^{2}+d y^{2}+d z^{2} .
$$

For the Riemann and Einstein curvature tensors we will adopt the conventions of Misner, Thorne and Wheeler 902:

$$
\begin{aligned}
R_{\nu \alpha \beta}^{\mu} & =\partial_{\alpha} \Gamma_{\nu \beta}^{\mu}-\partial_{\beta} \Gamma_{\nu \alpha}^{\mu}+\Gamma_{\sigma \alpha}^{\mu} \Gamma_{\nu \beta}^{\sigma}-\Gamma_{\sigma \beta}^{\mu} \Gamma_{\nu \alpha}^{\sigma} \\
G_{\mu \nu} & =R_{\mu \nu}-\frac{1}{2} g_{\mu \nu} R,
\end{aligned}
$$

where $R_{\mu \nu}=R^{\alpha}{ }_{\mu \alpha \nu}$ and $R=R_{\alpha}^{\alpha}$. The energy-momentum tensor will be defined with respect to the Lagrangian density for the matter fields as

$$
T^{\mu \nu}=\frac{2}{\sqrt{-g}} \frac{\delta \mathcal{L}_{m}}{\delta g_{\mu \nu}},
$$


where the derivative here is a functional one. Throughout this review we will refer to the energy density of a fluid as $\rho$, and its isotropic pressure as $P$. The equation of state, $w$, is then defined by

$$
P=w \rho .
$$

When writing the Friedmann-Lemaitre-Robertson-Walker (FLRW) line-element we will use $t$ to denote the 'physical time' (proper time of observers comoving with the fluid), and $\tau=\int d t / a(t)$ to denote the 'conformal time' coordinate. Unless otherwise stated, when working with linear perturbations about an FLRW background we will work in the conformal Newtonian gauge in which

$$
d s^{2}=a^{2}(\tau)\left[-(1+2 \Psi) d \tau^{2}+(1-2 \Phi) q_{i j} d x^{i} d x^{j}\right],
$$

where $q_{i j}$ is the metric of a maximally symmetric 3 -space with Gaussian curvature $\kappa$ :

$$
d s_{(3)}^{2}=q_{i j} d x^{i} d x^{j}=\frac{d r^{2}}{1-\kappa r^{2}}+r^{2} d \theta^{2}+r^{2} \sin ^{2} \theta d \phi^{2} .
$$

When dealing with time derivatives in cosmology we will use the dot and prime operators to refer to derivatives with respect to physical and conformal time, respectively, such that

$$
\begin{aligned}
& \equiv \frac{d}{d t} \\
& \equiv \frac{d}{d \tau} .
\end{aligned}
$$

In four dimensional space-time we will denote covariant derivatives with either a semicolon or a $\nabla_{\mu}$. The four dimensional d'Alembertian will then be defined as

$$
\square \equiv g^{\mu \nu} \nabla_{\mu} \nabla_{\nu}
$$

On the conformally static three-dimensional space-like hyper-surfaces the grad operator will be denoted with an arrow, as $\vec{\nabla}_{i}$, while the Laplacian will be given by

$$
\Delta \equiv q^{i j} \vec{\nabla}_{i} \vec{\nabla}_{j}
$$

As is usual, we will often make use of the definition of the Hubble parameter defined with respect to both physical and conformal time as

$$
\begin{aligned}
H & \equiv \frac{\dot{a}}{a} \\
\mathcal{H} & \equiv \frac{a^{\prime}}{a} .
\end{aligned}
$$

The definitions we have made here will be restated at various points in the review, so that each section remains self-contained to a reasonable degree. The exception to this will be Section 5 , on higher dimensional theories, which will require the introduction of new notation in order to describe quantities in the bulk.

Let us now move onto the definitions of particular terms. We choose to define the equivalence principles in the following way: 
- Weak Equivalence Principle (WEP): All uncharged, freely falling test particles follow the same trajectories, once an initial position and velocity have been prescribed.

- Einstein Equivalence Principle (EEP): The WEP is valid, and furthermore in all freely falling frames one recovers (locally, and up to tidal gravitational forces) the same laws of special relativistic physics, independent of position or velocity.

- Strong Equivalence Principle (SEP): The WEP is valid for massive gravitating objects as well as test particles, and in all freely falling frames one recovers (locally, and up to tidal gravitational forces) the same special relativistic physics, independent of position or velocity.

Of these, the EEP in particular is known to have been very influential in the conception of General Relativity. One may note that some authors refer to what we have called the EEP as the 'strong equivalence principle'.

Let us now define what we mean by 'General Relativity'. This term is often used by cosmologists to refer simply to Einstein's equations. Particle physicists, on the other hand, refer to any dynamical theory of spin-2 fields that incorporates general covariance as 'general relativity', even if it has field equations that are different from Einstein's? In this report when we write about 'General Relativity' we refer to a theory that simultaneously exhibits general covariance, and universal couplings to all matter fields, as well as satisfying Einstein's field equations. When we then discuss 'modified gravity' this will refer to any modification of any of these properties. However, it will be clear from reading through this report that almost all the proposals we report on preserve general covariance, and the universality of free fall. Let us now clarify further what exactly we mean by 'modified' theories of gravity.

As we will discuss in the next section, the effect of gravity on matter is tightly constrained to be mediated by interactions of the matter fields with a single rank-2 tensor field. This does not mean that this field is the only degree of freedom in the theory, but that whatever other interactions may occur, the effect of gravity on the matter fields can only be through interactions with the rank-2 tensor (up to additional weak interactions that are consistent with the available constraints). The term 'gravitational theory' can then be functionally defined by the set of field equations obeyed by the rank- 2 tensor, and any other non-matter fields it interacts with. If these equations are anything other than Einstein's equations, then we consider it to be a 'modified theory of gravity'. We will not appeal to the action or Lagrangian of the theory itself here; our definition is an entirely functional one, in terms of the field equations alone.

While we have constructed the definition above to be as simple as possible, there are of course a number of ambiguities involved. Firstly, exactly what one should consider as a 'matter field' can be somewhat subjective. This is especially true in terms of the exotic fields that are sometimes introduced into cosmology in order to try and understand the apparent late-time accelerating expansion of the Universe. Secondly, we have not defined exactly what we mean by 'Einstein's equations'. In four dimensions it is usually clear what this term refers to, but if we allow for the possibility of extra dimensions

\footnotetext{
${ }^{1}$ Note that under this definition the Einstein-Hilbert and Brans-Dicke Lagrangians, for example, represent different models of the same theory, which is called General Relativity.
} 
then we may choose for it to refer either to the equations derived from an EinsteinHilbert action in the higher dimensional space-time, or to the effective set of equations in four dimensional space-time. Clearly these two possible definitions are not necessarily consistent with each other. Even in four dimensions it is not always clear if 'Einstein's equations' include the existence of a non-zero cosmological constant, or not.

To a large extent, the ambiguities just mentioned are a matter of taste, and have no baring on the physics of the situation. For example, whether one chooses to refer to the cosmological constant as a modification of gravity, as an additional matter field, or as part of Einstein's equations themselves makes no difference to its effect on the expansion of the Universe. In this case it is only convention that states that the Einstein equations with $\Lambda$ is not a modified theory of gravity. Although less established than the case of the cosmological constant, similar conventions have started to develop around other modifications to the standard theory. For example, quintessence fields that are minimally coupled to the metric are usually thought of as additional matter fields, whereas scalar fields that non-minimally couple to the Einstein-Hilbert term in the action are usually thought of as being 'gravitational' fields (this distinction existing despite what numerous studies call non-minimally coupled quintessence fields). Although not always clear, we try to follow what we perceive to be the conventions that exist in the literature in this regard. We therefore include in this review a section on non-minimally coupled scalartensor theories, but not a section on minimally coupled quintessence fields. 


\section{General Relativity, and its Foundations}

General Relativity is the standard theory of gravity. Here we will briefly recap some of its essential features, and foundations. We will outline the observational tests of gravity that have been performed on Earth, in the solar system, and in other astrophysical systems, and we will then explain how and why it is that General Relativity satisfies them. We will outline why General Relativity should be considered a special theory in the more general class of theories that one could consider, and will present some of the theorems it obeys as well as the apparatus that is most frequently used to parameterise deviations away from it. This will be followed by a discussion of the cosmological solutions and predictions of the concordance general relativistic $\Lambda$ CDM model of the Universe.

\subsection{Requirements for Validity}

In order to construct a relativistic theory of gravity it is of primary importance to establish the properties it must satisfy in order for it to be considered viable. These include foundational requirements, such as the universality of free fall and the isotropy of space, as well as compatibility with a variety of different observations involving the propagation of light and the orbits of massive bodies. Today, radio and laser signals can be sent back and forth from the Earth to spacecraft, planets and the moon, and detailed observations of the orbits of a variety of different astrophysical bodies allow us to look for ever smaller deviations from Newtonian gravity, as well as entirely new gravitational effects. It is in this section that we will discuss the gravitational experiments and observations that have so far been performed in these environments. We will discuss what they can tell us about relativity theory, and the principles that a theory must obey in order for it to stand a chance of being considered observationally viable.

\subsubsection{The foundations of relativistic theories}

First of all let us consider the equivalence principles. We will not insist immediately that any or all of these principles are valid, but will rather reflect on what can be said about them experimentally. This will allow us to separate out observations that test equivalence principles, from observations that test the different gravitational theories that obey these principles - an approach pioneered by Dicke [423.

The least stringent of the equivalence principles is the WEP. The best evidence in support of the WEP still comes from Eötvös type experiments that use a torsion balance to determine the relative acceleration of two different materials towards distant astrophysical bodies. In reality these materials are self-gravitating, but their mass is usually small enough that they can effectively be considered to be non-gravitating test particles in the gravitational field of the astrophysical body. Using beryllium and titanium the tightest constraint on the relative difference in accelerations of the two bodies, $a_{1}$ and $a_{2}$, is currently 1110 .

$$
\eta=2 \frac{\left|a_{1}-a_{2}\right|}{\left|a_{1}+a_{2}\right|}=(0.3 \pm 1.8) \times 10^{-13}
$$

This is an improvement of around 4 orders of magnitude on the original results of Eötvös from 1922 [472. It is expected that this can be improved upon by up to a further 5 orders of magnitude when space based tests of the equivalence principle are performed 
[1282. These null results are generally considered to be a very tight constraint on the foundations of any relativistic gravitational theory if it is to be thought of as viable: The WEP must be satisfied, at least up to the accuracy specified in Eq. 15.

Let us now briefly consider the gravitational redshifting of light. This is one of the three "classic tests" of General Relativity, suggested by Einstein himself in 1916 [465. It is not, however, a particularly stringent test of relativity theory. If we accept energymomentum conservation in a closed system then it is only really a test of the WEP, and is superseded in its accuracy by the Eötvös experiment we have just discussed. The argument for this is the following [423, 594]: Consider an atom that initially has an inertial mass $M_{i}$, and a gravitational mass $M_{g}$. The atom starts near the ceiling of a lab of height $h$, in a static gravitational field of strength $g$, and with an energy reservoir on the lab floor beneath it. The atom emits a photon of energy $E$ that then travels down to the lab floor, such that its energy has been blue-shifted by the gravitational field to $E^{\prime}$ when it is collected in the reservoir. This process changes the inertial and gravitational masses of the atom to $M_{i}^{\prime}$ and $M_{g}^{\prime}$, respectively. The atom is then lowered to the floor, a process which lowers its total energy by $M_{g}^{\prime} g h$. At this point, the atom re-absorbs a photon from the reservoir with energy $E^{\prime}=\left(M_{i}^{\prime \prime}-M_{i}^{\prime}\right) c^{2}$ and is then raised to its initial position at the ceiling. This last process raises its energy by $M_{g}^{\prime \prime} g h$, where here $M_{i}^{\prime \prime}$ and $M_{g}^{\prime \prime}$ are the inertial and gravitational masses of the atom after reabsorbing the photon. The work done in lowering and raising the atom in this way is then $w=\left(M_{g}^{\prime \prime}-M_{g}^{\prime}\right) g h$. Recalling that the energy gained by the photon in travelling from the lab ceiling to the lab floor is $E^{\prime}-E$, the principle of energy conservation then tells us that $\left(E^{\prime}-E\right)=w=\left(M_{g}^{\prime \prime}-M_{g}^{\prime}\right) g h$. Now, if the WEP is obeyed then $M_{i}=M_{g}$, and this equation simply becomes $\left(E^{\prime}-E\right)=E^{\prime} g h$. This, however, is just the usual expression for gravitational redshift. Crucial here is the assumption that local position invariance is valid, so that both $M_{i}$ and $M_{g}$ are independent of where they are in the lab. If the laws of physics are position independent, and energy is conserved, gravitational redshift then simply tests the equivalence of gravitational and inertial masses, which is what the Eötvös experiment does to higher accuracy. Alternatively, if we take the WEP to be tightly constrained by the Eötvös experiment, then gravitational redshift experiments can be used to gain high precision constraints on the position dependence of the laws of physics [118. The gravitational redshift effect by itself, however, does not appear to be able to distinguish between different theories that obey the WEP and local position invariance. In Dicke's approach it should therefore be considered as a test of the foundations of relativistic gravitational theories, rather than a test of the theories themselves.

The next most stringent equivalence principle is the EEP. Testing this is a considerably more demanding task than was the case for the WEP, as one now not only has to show that different test particles follow the same trajectories, but also that a whole set of special relativistic laws are valid in the rest frames of these particles. Despite the difficulties involved with this, there is still very compelling evidence that the EEP should also be considered valid to high accuracy. The most accurate and direct of this evidence is due to the Hughes-Drever experiments 633, 433, which test for local spatial anisotropies by carefully observing the shape and spacing of atomic spectral lines. The basic idea here is to determine if any gravitational fields beyond a single rank-2 tensor are allowed to couple directly to matter fields. To see why this is of importance, let us first consider a number of point-like particles coupled to a single rank-2 tensor, $g_{\mu \nu}$. The 
Lagrangian density for such a set of particles is given by

$$
\mathcal{L}=\sum_{I} \int m_{I} \sqrt{-g_{\mu \nu} u^{\mu} u^{\nu}} d \lambda
$$

where $m_{I}$ are the masses of the particles, and $u^{\mu}=d x^{\mu} / d \lambda$ is their 4-velocity measured with respect to some parameter $\lambda$. The Euler-Lagrange equations derived from $\delta \mathcal{L}=$ 0 then tell us that the particles in Eq. 16. follow geodesics of the metric $g_{\mu \nu}$, and Riemannian geometry tells us that at any point we can choose coordinates such that $g_{\mu \nu}=\eta_{\mu \nu}$ locally. We therefore recover special relativity at every point, and the EEP is valid. Now, if the matter fields couple to two rank-2 tensors then the argument used above falls apart. In this case the Lagrangian density of our particles reads

$$
\mathcal{L}=\sum_{I} \int\left[m_{I} \sqrt{-g_{\mu \nu} u^{\mu} u^{\nu}}+n_{I} \sqrt{-h_{\mu \nu} u^{\mu} u^{\nu}}\right] d \lambda,
$$

where $h_{\mu \nu}$ is the new tensor, and $n_{I}$ is the coupling of each particle to that field. The particles above can now no longer be thought of as following the geodesics of any one metric, as the Euler-Lagrange Equations (17) are not in the form of geodesic equations. We therefore have no Riemannian geometry with which we can locally transform to Minkowski space, and the EEP is violated. The relevance of this discussion for the Hughes-Drever experiments is that EEP violating couplings, such as those in Eq. (17), cause just the type of spatial anisotropies that these experiments constrain. In this case the 4-momentum of the test particle in these experiments becomes

$$
p_{\mu}=\frac{m g_{\mu \nu} u^{\nu}}{\sqrt{-g_{\alpha \beta} u^{\alpha} u^{\beta}}}+\frac{n h_{\mu \nu} u^{\nu}}{\sqrt{-h_{\alpha \beta} u^{\alpha} u^{\beta}}},
$$

and as $g_{\mu \nu}$ and $h_{\mu \nu}$ cannot in general be made to be simultaneously spatially isotropic, we then have that $p_{\mu}$ is spatially anisotropic, and should cause the type of shifts and broadening of spectral lines that Hughes-Drever-type experiments are designed to detect. The current tightest constraints are around 5 orders of magnitude tighter than the original experiments of Hughes and Drever [765, 301, and yield constraints of the order

$$
n \lesssim 10^{-27} \mathrm{~m}
$$

so that couplings to the second metric must be very weak in order to be observationally viable. This result strongly supports the conclusion that matter fields must be coupled to a single rank-2 tensor only. It then follows that particles follow geodesics of this metric, that we can recover special relativity at any point, and hence that the EEP is valid. It should be noted that these constraints do not apply to gravitational theories with multiple rank-2 tensor fields that couple to matter in a linear combination, so that they can be written as in Eq. 16 with $g_{\mu \nu}=\sum_{I} c_{I} h_{\mu \nu}^{(I)}$, where $c_{I}$ are a set of $I$ constants. Local spatially isotropy, and the EEP, is always recovered in this case.

Beyond direct experimental tests, such as Hughes-Drever-type experiments, there are also theoretical reasons to think that the EEP is valid to high accuracy. This is a conjecture attributed to Schiff, that states 'any complete and self-consistent gravitational theory that obeys the WEP must also satisfy the EEP'. It has been shown using conservation of energy that preferred frame and preferred location effects can cause violations 
of the WEP [594]. This goes some way towards demonstrating Schiff's conjecture, but there is as yet still no incontrovertible proof of its veracity. We will not consider the issue further here.

The experiments we have just described provide very tight constraints on the WEP, the EEP, and local position invariance. It is, of course, possible to test various other aspects of relativistic gravitational theory that one may consider as 'foundational' (for example, the constancy of a constant of nature [1240]). For our present purposes, however, we are mostly interested in the EEP. Theories that obey the EEP are often described as being 'metric' theories of gravity, as any theory of gravity based on a differentiable manifold and a metric tensor that couples to matter, as in Eq. (16), can be shown to have test particles that follow geodesics of the resulting metric space. The basics of Riemannian geometry then tells us that at every point in the manifold there exists a tangent plane, which in cases with Lorentzian signature is taken to be Minkowski space. This allows us to recover special relativity at every point, up to the effects of second derivatives in the metric (i.e. tidal forces), so that the EEP is satisfied. Validity of the EEP can then be thought of as implying that the underlying gravitational theory should be a metric one 1273 .

\subsubsection{Observational tests of metric theories of gravity}

In what follows we will consider gravitational experiments and observations that can potentially be used to distinguish between different metric theories of gravity.

\section{Solar system tests}

As well as the gravitational redshifting of light that we have already mentioned, the other two 'classic tests' of General Relativity are the bending of light rays by the Sun, and the anomalous perihelion precession of Mercury. These can both be considered tests of gravitational theories beyond the foundational issues discussed in the previous section. That is, each of these tests is (potentially) able to distinguish between different metric theories of gravity. As well as these two tests, there are also a variety of other gravitational observations that can be performed in the solar system in order to investigate relativistic gravitational phenomena. A viable theory of gravity must be compatible with all of them. For convenience we will split these into tests involving null trajectories (such as light bending) and tests involving time-like trajectories (such as the perihelion precession of planets).

First of all let us consider tests involving null geodesics. As already mentioned, the most famous of these is the spatial deflection of star light by the Sun. In General Relativity the deflection angle, $\theta$, of a photon's trajectory due to a mass, $M$, with impact parameter $d$, is given by

$$
\theta=\frac{2 M}{d}(1+\cos \varphi) \simeq 1.75^{\prime \prime}
$$

where $\varphi$ is the angle made at the observer between the direction of the incoming photon and the direction of the mass. The $1.75^{\prime \prime}$ is for a null trajectory that grazes the limb of the Sun. This result is famously twice the size of the effect that one might naively estimate using the equivalence principle alone 464. The tightest observational constraint to date on $\theta$ is due to Shapiro, David, Lebach and Gregory who use around 2500 days worth of observations taken over a period of 20 years. The data in this study was taken using 87 
VLBI sites and 541 radio sources, yielding more than $1.7 \times 10^{6}$ measurements that use standard correction and delay rate estimation procedures. The result of this is [1131]

$$
\theta=(0.99992 \pm 0.00023) \times 1.75^{\prime \prime},
$$

which is around 3 orders of magnitude better than the observations of Eddington in 1919.

A further, and currently more constraining, test of metric theories of gravity using null trajectories involves the Shapiro time-delay effect [1130. Here the deflection in time is taken into account when a photon passes through the gravitational field of a massive object, as well as the deflection in space that is familiar from the lensing effects discussed above. The effect of this in General Relativity is to cause a time delay, $\Delta t$, for a light-like signal reflected off a distant test object given by

$$
\Delta t=4 M \ln \left[\frac{4 r_{1} r_{2}}{d^{2}}\right] \simeq 20\left(12-\ln \left[\left(\frac{d}{R_{\odot}}\right)^{2}\left(\frac{a u}{r_{2}}\right)\right]\right) \mu s,
$$

where $r_{1}$ and $r_{2}$ (both assumed $\gg d$ ) are the distances of the observer and test object from an object of mass $M$, respectively. The second equality here is the approximate magnitude of this effect when the photons pass close by the Sun, and the observer is on Earth. Here we have written $R_{\odot}$ as the radius of the Sun, and au as the astronomical unit. The best constraint on gravity using this effect is currently due to Bertotti, Iess and Tortora using radio links with the Cassini spacecraft between the 6th of June and the 7th of July 2002 [147]. These observations result in the constraint

$$
\Delta t=(1.00001 \pm 0.00001) \Delta t_{G R},
$$

where $\Delta t_{G R}$ is the expected time-delay due to general relativity. The Shapiro time-delay effect in fact constrains the same aspect of relativistic gravity as the spatial deflection of light (this will become clear when we introduce the parameterised post-Newtonian formalism later on). This aspect is sometimes called the 'unit curvature' of space.

Let us now consider tests involving time-like trajectories. The 'classical' test of General Relativity that falls into this category is the anomalous perihelion precession of Mercury (this is called a test, despite the fact that it was discovered long before General Relativity [777). In Newtonian physics the perihelion of a test particle orbiting an isolated point-like mass stays in a fixed position, relative to the fixed stars. Adding other massive objects into the system perturbs this orbit, as does allowing the central mass to have a non-zero quadrupole moment, so that the perihelion of the test particle's orbit slowly starts to precess. In the solar system the precession of the equinoxes of the coordinate system contribute about $5025^{\prime \prime}$ per century to Mercury's perihelion precession, while the other planets contribute about $531^{\prime \prime}$ per century. The Sun also has a non-zero quadrupole moment, which contributes a further $0.025^{\prime \prime}$ per century. Taking all of these effects into account, it still appears that the orbit of Mercury in the solar system has an anomalous perihelion precession that cannot be explained by the available visible matter, and Newtonian gravity alone. Calculating this anomalous shift exactly is a complicated matter, and depends on the exact values of the quantities described above. In Table 1 we display the observed anomalous perihelion precession of Mercury, $\Delta \omega$, as calculated by various different groups. For a more detailed overview of the issues involved, and a number of other results, the reader is referred to [1039]. In relativistic theories of gravity 


\begin{tabular}{|c|c|}
\hline Source & $\Delta \omega /\left({ }^{\prime \prime}\right.$ per century $)$ \\
\hline Anderson et al. [48] & $42.94 \pm 0.20$ \\
Anderson et al. [49] & $43.13 \pm 0.14$ \\
Krasinsky et al. [746]: EPM1988 & $42.984 \pm 0.061$ \\
DE200 & $42.977 \pm 0.061$ \\
Pitjeva [1040]: EPM1988 & $42.963 \pm 0.052$ \\
DE200 & $42.969 \pm 0.052$ \\
\hline
\end{tabular}

Table 1: The value of the perihelion precession of Mercury obtained from observations by various authors. The acronyms EPM1988 and DE200 refer to different numerical ephemerides, which are reviewed in 1041.

the additional post-Newtonian gravitational potentials mean that the perihelion of a test particle orbiting an isolated mass is no longer fixed, as these potentials do not drop off as $\sim 1 / r^{2}$. There is therefore an additional contribution to the perihelion precession, which is sensitive to the relative magnitude and form of the gravitational potentials, and hence the underlying relativistic theory. For General Relativity, the predicted anomalous precession of a two body system is given by

$$
\Delta \omega=\frac{6 \pi M}{p} \simeq 42.98^{\prime \prime}
$$

where $m$ is the total mass of the two bodies, and $p$ is the semi-latus rectum of the orbit. The last equality is for the Sun-Mercury system, and is compatible with the observations shown in Table 1. Each relativistic theory predicts its own value of $\Delta \omega$, and by comparing to observations such as those in Table 1 we can therefore constrain them. This test is an additional one beyond those based on null geodesics alone as it tests not only the 'unit curvature' of space, but also the non-linear terms in the space-time geometry, as well as preferred frame effects.

Another very useful test involving time-like geodesics involves looking for the 'Nordtvedt effect' 986. This effect is the name given to violations of the SEP. In the previous section we only considered tests of the WEP and EEP, which provide strong evidence that viable gravitational theories should be 'metric' ones. Now, it is entirely possible to satisfy the WEP and EEP, with a metric theory of gravity, while violating the SEP. Such violations do not occur in General Relativity, but do in most other theories. Every test of the Nordtvedt effect is therefore a potential killing test of general relativity, if it delivers a non-null result. To date, the most successful approach in searching for SEP violations is to use the Earth-Moon system in the gravitational field of the Sun as a giant Eötvös experiment. The difference between this and the laboratory experiments described in the previous section is that while the gravitational fields of the masses in WEP Eötvös experiments are entirely negligible, this is no longer the case with the Earth and Moon. By tracking the separation of the Earth and Moon to high precision, using lasers reflected off reflectors left on the Moon by the Apollo 11 mission in 1969, it is then possible to gain the constraint 1277

$$
\eta=(-1.0 \pm 1.4) \times 10^{-13},
$$

where $\eta$ is defined as in Eq. (15). This is indeed a null result, consistent with General Relativity, and is tighter even than the current best laboratory constraint on the WEP. It 
can therefore be used to constrain possible deviations from General Relativity, and in fact constrains a similar (but not identical) set of gravitational potentials to the perihelion precession described previously.

A third solar system test involving time-like geodesics is the observation of spinning objects in orbit. While currently less constraining than the other tests discussed so far, these observations allow insight into an entirely relativistic type of gravitational behaviour: gravitomagnetism. This is the generation of gravitational fields by the rotation of massive objects, and was discovered in the very early days of General Relativity by Lense and Thirring [1204, 785. The basic idea here is that massive objects should 'drag' space around with them as they rotate, a concept that is in good keeping with Mach's principle. Although one can convincingly argue that the same aspects of the gravitational field that cause frame-dragging are also being tested by perihelion precession and the Nordtvedt effect, it is not true that in these cases the gravitational fields in question are being communicated through the rotation of matter. Now, in the case of General Relativity it can be shown that the precession of a spin vector $\mathbf{S}$ along the trajectory of a freely-falling gyroscope in orbit around an isolated rotating massive body at rest is given by

$$
\frac{d \mathbf{S}}{d \tau}=\mathbf{\Omega} \times \mathbf{S},
$$

where

$$
\boldsymbol{\Omega}=\frac{3}{2} \mathbf{v} \times \nabla U-\frac{1}{2} \nabla \times \mathbf{g} .
$$

Here we have written the vector $\mathbf{g}=g_{0 i}$, and have taken $\mathbf{v}$ and $U$ to be the velocity of the gyroscope and the Newtonian potential at the gyroscope, respectively. The first term in (27) is called 'geodetic precession', and is caused by the 'unit curvature' of the space. This effect exists independent of the massive bodies rotation. The second term in (27) is the Lense-Thirring term, and causes the frame-dragging discussed above. The most accurate measurement of this effect claimed so far is at the level of $5-10 \%$ accuracy, and has been made using the LAser GEOdynamics Satellites (LAGEOS) 302] (there has, however, been some dispute of this result [641, 642]). The Gravity Probe B mission is a more tailor made experiment which was put in orbit around the Earth between April 2004 and September 2005. The current accuracy of results from this mission are at the level of $\sim 15 \%$ [476], although this could improve further after additional analysis is performed.

All of the tests discussed so far in this section have been for long-ranged modifications to Newtonian gravity. As well as these, however, there are a host of alternatives to General Relativity that also predict short-ranged deviations from $1 / r^{2}$ gravity. These range from extra-dimensional theories 673, 702, to fourth-order theories [307] and bimetric theories [308, all of which predict 'Yukawa' potentials of the form

$$
U=\alpha \int \frac{\rho\left(x^{\prime}\right) e^{-\left|x-x^{\prime}\right| / \lambda}}{\left|x-x^{\prime}\right|} d^{3} x^{\prime 3},
$$

where $\alpha$ parameterises the 'strength' of the interaction, and $\lambda$ parameterises its range. The genericity of these potentials, often referred to as 'fifth-forces', provides strong motivation for experimental attempts to detect them. Unfortunately, due to the their scale dependence, one can no longer simply look for the extra force on one particular scale, and 
then extrapolate the result to all scales. Instead, observations must be made on a whole range of different scales, so that we end up with constraints on $\alpha$ at various different values of $\lambda$. These observations are taken from a variety of different sources, with the scale of the phenomenon being observed typically constraining $\lambda$ of similar size. So, for example, on the larger end of the observationally probed scale we have planetary orbits [1197] and lunar laser ranging [1277] constraining $\alpha \lesssim 10^{-8}$ between $10^{8} \mathrm{~m} \lesssim \lambda \lesssim 10^{12} \mathrm{~m}$. On intermediate scales the LAGEOS satellite, and observations of gravitational accelerations at the top of towers and under the oceans provide constraints of $\alpha$ that range from $\alpha \lesssim 10^{-8}$ at $\lambda \sim 10^{7} m$ [1053] to $\alpha \lesssim 10^{-3}$ at $10^{-1} m \lesssim \lambda \lesssim 10^{4} m$ [459, 1312]. At smaller scales laboratory searches must be performed, and current constraints in this regime range from $\alpha \lesssim 10^{-2}$ at $\lambda \sim 10^{-2} m$, to $\alpha \lesssim 10^{6}$ at $\lambda \sim 10^{-5} m$ [624, 819, 291]. Weaker constraints at still smaller scales are available using the Casimir effect. For a fuller discussion of these searches, and the experiments and observations involved, the reader is referred to the reviews by Fischbach and Talmadge [507, and Adelberger, Heckel and Nelson [1].

\section{Gravitational waves, and binary pulsars}

A generic prediction of all known relativistic theories of gravity is the existence of gravitational waves: Propagating gravitational disturbances in the metric itself. However, while all known relativistic gravitational theories predict gravitational radiation, they do not all predict the same type of radiation as the quadrupolar, null radiation that we are familiar with from General Relativity. It is therefore the case that while the mere existence of gravitational radiation is not itself enough to effectively discriminate between different gravitational theories, the type of gravitational radiation that is observed is. The potential differences between different types of gravitational radiation can take a number of different forms, which we will now discuss.

Firstly, one could attempt to determine the propagation speed of gravitational waves. In General Relativity it is the case that gravitational waves have a velocity that is strictly equal to that of the speed of light in vacuum. Generically, however, this is not true: Some theories predict null gravitational radiation, and others do not. So, for example, if one were able to detect gravitational waves from nearby supernovae, then comparing the arrival time of this radiation with the arrival time of the electromagnetic radiation would provide a potentially killing test of General Relativity. There are, however, a number of different theories that predict null gravitational radiation. Tests of the velocity of gravitational waves therefore have the potential to rule out a number of theories, but by themselves are not sufficient to distinguish any one in particular.

A second, more discriminating test, is of the polarity of gravitational radiation. General Relativity predicts radiation with helicity modes \pm 2 only, and so far is the only proposed theory of gravity that does so. In general, there are six different polarisation states - one for each of the six 'electric' components of the Riemann tensor, $R_{0 i 0 j}$. These correspond to the two modes familiar from General Relativity, as well as two modes with helicity \pm 1 , and two further modes with helicity 0 . One of these helicity- 0 modes corresponds to an additional oscillation in the plane orthogonal to the wave vector $k^{\mu}$, while the remaining 3 modes all correspond to oscillations in a plane containing $k^{\mu}$. The extent to which observations of these modes can constrain gravitational theory depends on whether or not the source of the radiation can be reliably identified. If the source can be identified, then the vector $k^{\mu}$ is known, and one should then be able to uniquely 
identify the individual polarisation modes discussed above. We then have 6 different tests of relativistic gravitational theory - one for each of the modes. In the absence of any knowledge of $k^{\mu}$, however, one cannot necessarily uniquely identify all of the modes that are present in a gravitational wave, although it may still be possible to constrain the modes being observed to a limited number of possibilities.

Direct observations of gravitational waves, of the kind discussed above, provide an excellent opportunity to further constrain gravity. Indeed, some theories can be shown to be indistinguishable from General Relativity using post-Newtonian gravitational phenomena in the solar system alone, while being easily distinguishable when one also considers gravitational radiation. This is the case with Rosen's bimetric theory of gravity [1068, 779, 1275. To date, however, the direct detection of gravitational radiation has yet to be performed. At present the highest accuracy null-observations of gravitational radiation are those of the Laser Interferometer Gravitational-wave Observatory (LIGO). This experiment consists of two sites in the USA (one in Livingston, Louisiana and one in Richland, Washington). Each site is an independent interferometer constructed from two $4 \mathrm{~km}$ arms, along which laser beams are shone. The experiment has an accuracy capable of detecting oscillations in space at the level of $\sim 1$ part in $10^{21}$, but has yet to make a positive detection. Further experiments are planned for the future, including Advanced LIGO, which is scheduled to start in 2014, and the Laser Interferometer Space Antenna (LISA). Both Advanced LIGO and LISA are expected to make positive detections of gravitational waves.

Another way to search for gravitational waves is to look for their influence on the systems that emitted them. In this regard binary pulsar systems are of particular interest. Pulsars are rapidly rotating neutron stars that emit a beam of electromagnetic radiation, and were first observed in 1967 604. When these beams pass over the Earth, as the star rotates, we observe regular pulses of radiation. The first pulsar observed in a binary system was PSR B1913+16 in 1974, by Russell Hulse and Joseph Taylor 634. This is a particularly 'clean' binary system of a pulsar with rotational period $\sim 59 \mathrm{~ms}$ in orbit around another neutron star. Binary pulsars are of particular significance for gravitational physics for a number of reasons. Firstly, they can be highly relativistic. The Hulse-Taylor binary system, for example, exhibits a relativistic periastron advance that is more than 30000 times that of the Mercury-Sun system. In this regard they provide an important compliment to the observations of post-Newtonian gravity that we observe in the solar system. Secondly, they are a source of gravitational waves. Given the high degree of accuracy to which the orbits of these systems are known, the change in angular momentum due to gravitational radiation can be determined and observed. In the Hulse-Taylor system the observed decrease in orbital period over the past 30 years is $0.997 \pm 0.002$ of the rate predicted by General Relativity [1267]. Finally, neutron stars are composed of a type of compact matter that is of particular interest for the study of self-gravitational effects. For a review of pulsars in this context the reader is referred to 1177.

There are large number of relativistic parameters that can be probed by observations of binary pulsar systems [359. To date, however, the most constrained are the 5 'postKeplerian' effects, which are the rate of periastron advance, the rate of change of orbital period, the gravitational redshift, and two Shapiro time-delay effects. These effects are familiar from the solar system tests discussed above, apart from the change in orbital period that is negligible in the solar system. One further effect that has been measured 
only relatively recently is the 'geodetic' precession of the pulsar spin vector about its angular momentum vector [744. This is a purely relativistic effect that is observed via changes in the observed pulse profile over a period of time that can be attributed to our line of sight to the pulsar crossing the emitting region at varying positions due to the precession. The determination of the precession rate using these observations is, however, complicated somewhat by a degeneracy between the a priori unknown shape of the emitting region and the geometry of the system as a whole [1268, 314].

Not all of the post-Keplerian effects are always apparent in any given binary system, and not all provide independent tests of gravity. For example, in the Hulse-Taylor binary only three of these effects can be observed (the inclination angle of the system on the sky is too large to observe any significant Shapiro delay), and there are two unknown quantities in the system (the masses of the pulsar, and that of its companion). The HulseTaylor binary therefore provides only $3-2=1$ test of relativistic gravity. The recently discovered 'Double Pulsar' PSR J0737-3039A/B [840, however, does significantly better [745. All five post-Keplerian effects are visible in this system, and because both neutron stars are observable as pulsars the ratio of their masses can be directly inferred from their orbits. This leaves only one unknown quantity, and hence gives $5-1=4$ independent tests of relativistic gravity. So far, all binary pulsar tests of gravity, including those of the double pulsar, are consistent with General Relativity.

Finally, let us return to constraining gravitational theory through the emission of gravitational waves. The effect of emitting gravitational radiation from a binary system is to change its orbital period. In General Relativity we know that only quadrupole radiation with positive energy should be emitted from a system. For most relativistic theories, however, dipole gravitational radiation is also expected, and sometimes this can carry away negative energy. The existence of dipole radiation is sometimes attributed to violations of the SEP, whereby the centre of the mass responsible for gravitational radiation is no longer the same as the centre of inertial mass. If the centre of inertial mass is what stays fixed, then the centre of mass responsible for the gravitational radiation can move and generate dipole radiation. Dipole radiation is expected to be most dominant in binary systems with high eccentricity, and where the companion mass is a white dwarf. No evidence for dipolar radiation yet exists [154, 1776. Null observations that attest to this result therefore allow for experimental limits to be set on theories that predict positive energy dipolar radiation. The lack of any observation of dipolar radiation can also be used to rule out with high confidence theories that allow negative energy dipolar radiation, such as Rosen's theory [1275].

\subsubsection{Theoretical considerations}

As he developed the Special Theory of Relativity, it is often assumed that Einstein's inspiration came from experiments pointing towards the constancy of the speed of light. It is true that he was certainly aware of these experiments, but he was also inspired by theory, specifically his faith in the principle of relativity and the validity of Maxwell's equations in any inertial frame. So too, in developing models of modified gravity, we should not only take our lead from observation but also from theory. Indeed, theoretical considerations are a very powerful tool in testing new models. Typically these involve the study of classical and quantum fluctuations about classical solutions. Do the classical fluctuations propagate super-luminally? Can we excite a ghost? Do the quantum fluctuations become strongly coupled at some unacceptably low energy scale? 
Ghosts

Ghosts are a common feature of many modified gravity models that hope to explain dark energy. Intuitively it is easy to see why this might be the case. To get cosmic acceleration we need an additional repulsive force to act between massive objects at large distances. If this force is to be mediated by a particle of even spin, such as a scalar (spin 0) or a tensor (spin 2), then the kinetic term describing this must have the "wrong" sign ${ }^{2}$ that is, it must be a ghost.

We should be clear about the distinction between the kind of ghost that arises in certain modified gravity models and the Faddeev-Popov ghost used in the quantisation of non-abelian gauge theories. The latter is introduced in the path integral to absorb unphysical gauge degrees of freedom. It does not describe a physical particle and can only appear as an internal line in Feynman diagrams. In contrast, the ghosts that haunt modified gravity describe physical excitations and can appear as external lines in Feynman diagrams.

When a physical ghost is present one has a choice: Accept the existence of negative norm states and abandon unitarity, or else accept that the energy eigenvalues of the ghost are negative 317. Since the former renders the entire quantum description completely non-sensical, one usually accepts the latter. However, it now follows that the ghost will generate instabilities if it couples to other, more conventional, fields. When these fields are already excited, the ghost can and will continually dump its energy into the "conventional" sector through classical processes, since its energy is unbounded from below. Even in vacuum, one will get the spontaneous (quantum) production of ghostnon-ghost pairs, and in a Lorentz invariant theory, the production rate is divergent 317 .

There are a few ways to try to exorcise the ghost. One is to isolate it somehow, such that it completely decouples from other fields. Another option is to make it heavy, so much so that its mass exceeds the cut-off for the effective theory describing the relevant fluctuations, and one can happily integrate it out. A third option is to break Lorentz invariance, perhaps spontaneously, so that one can introduce an explicit Lorentz non-invariant cut-off to regulate the production rate of ghost-non-ghost pairs (see, for example, 653]). However, perhaps the safest way to deal with a ghost is to dismiss as unphysical those solutions of a theory upon which the ghost can fluctuate. This school of thought is exploited to good effect in the ghost condensate model [590].

\section{Strong coupling}

Some modified gravity models are said to suffer from "strong coupling" problems. Given a classical solution to the field equations, this refers to quantum fluctuations on that solution becoming strongly coupled at an unacceptably low scale. For example, in DGP gravity, quantum fluctuations on the Minkowski vacuum becomes strongly coupled at around $\Lambda \sim 10^{-13} \mathrm{eV} \sim 1 /(1000 \mathrm{~km})$. In other words, for scattering processes above $\Lambda$, perturbative quantum field theory on the vacuum is no longer well defined, and one

\footnotetext{
${ }^{2}$ In our conventions, the Lagrangian for a canonical scalar is $\mathcal{L}=-\frac{1}{2}(\partial \psi)^{2}$, whereas a ghost has $\mathcal{L}=+\frac{1}{2}(\partial \psi)^{2}$.
} 
must sum up the contribution from all the multi-loop diagrams. One then has complete loss of predictivity. Furthermore, the classical solution itself is meaningless at distances below $\Lambda^{-1}$ since it would require a scattering process involving energies above the cut-off to probe its structure.

The strong coupling scale is, of course, dependent on the background classical solution, and may even depend on position in space-time. Whether the inferred strong coupling scale is acceptable, or not, again depends on the background. For example, strong coupling at $1000 \mathrm{~km}$ on the Minkowski vacuum of DGP gravity is not really an issue as Minkowski space does not represent a good approximation to the classical solution in the vicinity of the Earth. Indeed, for the classical solutions sourced by the Earth to leading order, quantum fluctuations will become strongly coupled at some scale that depends on the radial distance from the Earth's centre. Computed at the Earth's surface one should require that this lies below an meV since quantum gravity effects have yet to show up in any lab based experiments up to this scale.

It has actually been argued that strong coupling on the vacuum can be a virtue in modified gravity models [447. This is because it can be linked to a breakdown of classical perturbation theory, which is necessary for the successful implementation of the Vainshtein mechanism [1241, 399]. We discuss the Vainshtein mechanism and strong coupling in some detail in the context of DGP gravity in Section 5.5.4. Here we will make some generic statements. Consider a model of gravity that deviates from GR at large distances. To be significant in terms of understanding dark energy, this deviation must be at least $\mathcal{O}(1)$ on cosmological scales, but be suppressed down to $\lesssim \mathcal{O}\left(10^{-5}\right)$ on Solar System scales. Therefore, the field or fields that are responsible for the modification must be screened within the Solar System. How can this screening occur? One way is for the fields to interact so strongly that they are frozen together, so much so that they are unable to propagate freely. This is the idea behind the Vainshtein mechanism - higher order derivative interactions help to suppress the extra modes near the source (the Sun).

Alternative ways to screen the extra fields have been suggested in the form of the chameleon 689, 688, and the symmetron 608 mechanisms. Both methods exploit the dependence of the effective potential on the environment. For the chameleon, the mass of the field is environmentally dependent, getting heavy in the Solar System. For the symmetron, the strength of the matter coupling is (indirectly) environmentally dependent, tending to zero near a heavy source.

\subsection{Einstein's Theory}

Having considered the requirements that must be satisfied by a viable relativistic theory of gravity, let us now consider Einstein's theory of General Relativity in particular. General Relativity satisfies all of the requirements described in the previous section, either by construction (for the foundational requirements) or by trial (in the case of tests of metric theories of gravity).

General Relativity is a gravitational theory that treats space-time as a 4-dimensional manifold. The connection associated with covariant differentiation, $\Gamma_{\alpha \beta}^{\mu}$, should be viewed as an additional structure on this manifold, which, in general, can be decomposed into parts that are symmetric or antisymmetric in its last two indices:

$$
\Gamma_{\alpha \beta}^{\mu}=\Gamma_{(\alpha \beta)}^{\mu}+\Gamma_{[\alpha \beta]}^{\mu} .
$$


In General Relativity we take $\Gamma_{[\alpha \beta]}^{\mu}=0$, or, in the language of differential geometry, we assume that torsion vanishes. We are then left with only the symmetric part of the connection, which describes the curvature of the manifold.

Now, to define distances on the manifold one also requires a metric tensor, $g_{\mu \nu}$. Along the curve $\gamma$ this gives the measure of distance

$$
s=\int_{\gamma} d \lambda \sqrt{g_{\mu \nu} \dot{x}^{\mu} \dot{x}^{\nu}},
$$

where $\lambda$ is a parameter along the curve, $x^{\mu}=x^{\mu}(\lambda)$, and over-dots here mean differentiation with respect to $\lambda$. The metric should also be considered as an additional structure on the manifold, which is in general independent from the connection. The relationship between the connection and the metric is defined via the non-metricity tensor, $Q_{\mu \alpha \beta} \equiv \nabla_{\mu} g_{\alpha \beta}$. In General Relativity it is assumed that the non-metricity tensor vanishes. We can now use the metric to define the Levi-Civita connection, which has components given by the Christoffel symbols:

$$
\Gamma_{\alpha \beta}^{\mu}=\left\{\begin{array}{c}
\mu \\
\alpha \beta
\end{array}\right\} \equiv \frac{1}{2} g^{\mu \nu}\left(g_{\alpha \nu, \beta}+g_{\beta \nu, \alpha}-g_{\alpha \beta, \nu}\right) .
$$

To summarise, as a consequence of the two assumptions $Q_{\mu \alpha \beta}=0$ and $\Gamma_{[\alpha \beta]}^{\mu}=0$, the components of the connection are uniquely given by the Christoffel symbols via (31), and so the connection, and all geometric quantities derived from it, are defined entirely in terms of the metric. In General Relativity, therefore, the metric tells us everything there is to know about both distances and parallel transport in the space-time manifold.

The resulting set of structures is known as a Riemannian manifold (or, more accurately, pseudo-Riemannian in the case where the metric is not positive definite, as is required to recover special relativity in the tangent space to a point in space-time). Riemannian manifolds have a number of useful properties including tangent vectors being parallel to themselves along geodesics, the geodesic completeness of space-time implying the metric completeness of space-time, and a particularly simple form for the contracted Bianchi identities:

$$
\nabla_{\mu}\left(R^{\mu \nu}-\frac{1}{2} g^{\mu \nu} R\right)=0,
$$

where $R_{\mu \nu}$ and $R=g^{\mu \nu} R_{\mu \nu}$ are the Ricci tensor and scalar curvature, respectively . This last equation is of great significance for Einstein's equations.

\subsubsection{The field equations}

Having briefly discussed the geometric assumptions implicit in General Relativity, let us now display the field equations of this theory:

$$
R_{\mu \nu}-\frac{1}{2} g_{\mu \nu} R=8 \pi G T_{\mu \nu}-g_{\mu \nu} \Lambda .
$$

Here $T_{\mu \nu}$ is the energy-momentum tensor of matter fields in the space-time, and $\Lambda$ is the cosmological constant. These equations are formulated such that energy-momentum is a conserved quantity (due to the contracted Bianchi identity and metric-compatibility of the connection), so that special relativity can be recovered in the neighbourhood of 
every point in space-time (up to tidal forces), and so that the usual Newtonian Poisson equation for weak gravitational fields is recovered in non-inertial frames kept at a fixed space-like distances from massive objects (up to small corrections).

The Field Equations (33) are a set of 10 generally covariant, quasi-linear second-order PDEs in 4 variables, for the 10 independent components of the metric tensor. They constitute 4 constraint equations and 6 evolution equations, with the contracted Bianchi identities ensuring that the constraint equations are always satisfied. Furthermore, the conserved nature of $T_{\mu \nu}$ and the Riemannian nature of the manifold ensure that the WEP and EEP are always satisfied: Massless test particles follow geodesics, and in any freely falling frame one can always choose 'normal coordinates' so that local space-time is well described as Minkowski space.

\subsubsection{The action}

As with most field theories, the Field Equations (33) can be derived from the variation of an action. In the case of General Relativity this is the Einstein-Hilbert action:

$$
S=\frac{1}{16 \pi G} \int \sqrt{-g}(R-2 \Lambda) d^{4} x+\int \mathcal{L}_{m}\left(g_{\mu \nu}, \psi\right) d^{4} x,
$$

where $\mathcal{L}_{m}$ is the Lagrangian density of the matter fields, $\psi$, and the gravitational Lagrangian density has been taken to be $\mathcal{L}_{g}=\sqrt{-g}(R-2 \Lambda) / 16 \pi G$. Let us now assume the Ricci scalar to be a function of the metric only, so that $R=R(g)$. Variation of Eq. (34) with respect to the metric tensor then gives the Field Equations (33), where

$$
T^{\mu \nu} \equiv \frac{2}{\sqrt{-g}} \frac{\delta \mathcal{L}_{m}}{\delta g_{\mu \nu}} .
$$

The factors of $\sqrt{-g}$ are included in Eq. (34) to ensure that the $\mathcal{L} \mathrm{s}$ transform as scalar densities under coordinate transformations, i.e. as

$$
\overline{\mathcal{L}}=\operatorname{det}\left(\frac{\partial x^{\mu}}{\partial \bar{x}^{\nu}}\right) \mathcal{L},
$$

under coordinate transformations $\bar{x}^{\mu}=\bar{x}^{\mu}\left(x^{\nu}\right)$. This property ensures $S$ is invariant under general coordinate transformation, and that the resulting tensor field equations are divergence free (i.e. the contracted Bianchi identities and energy-momentum conservation equations are automatically satisfied).

We have outlined here how Einstein's equations can be obtained from the variation of an invariant action with respect to the metric, once it has been assumed that the spacetime manifold is Riemannian. The vanishing of torsion and non-metricity then tell us that the metric is the only independent structure on the manifold, and the invariant action principle ensures that we end up with a set of tensor field equations in which energymomentum is conserved. Because of this formulation the WEP and EEP are satisfied identically. Now, when considering alternative theories of gravity one often wants to modify the field equations while conserving these basic properties. Modified theories of gravity are therefore often formulated in a similar way; from the metric variation of an invariant action principle under the assumption of Riemannian geometry, with a universal coupling of all matter fields to the same metric. 


\subsection{Alternative Formulations}

The discussion in the previous section involved deriving Einstein's equations under the a priori assumption of Riemannian geometry (i.e. assuming to begin with that the torsion vanishes and that the connection is metric compatible). In this case the metric is the only remaining geometric structure, and a simple metric variation of the action is the only option. We can, however, be less restrictive in specifying the type of geometry we wish to consider. For the case of the Einstein-Hilbert action, Eq. (34), this usually still leads to the Einstein Equations (33). For alternative theories of gravity, however, this is often not the case: Different variational procedures, and different assumptions about the geometric structures on the manifold, can lead to different field equations. It is for this reason that we now outline some alternative formulations of General Relativity. A large collection of many such formulations can be found in [1032.

\subsubsection{The Palatini procedure}

The most well known deviation from the metric variation approach is the 'Palatini procedure' 1020. Here the connection is no longer immediately assumed to be metric compatible, but is still assumed to be symmetric and thus torsionless. In addition, all matter fields are still taken to couple universally to the metric only ${ }^{3}$ The action to be varied is then

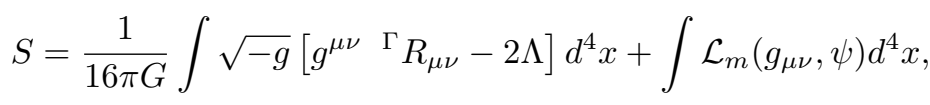

where ${ }^{\Gamma} R_{\mu \nu}$ indicates that the Ricci tensor here is defined with respect to the connection and not the metric (at this stage the metric and connection are still independent variables), and is given by

$$
{ }^{\Gamma} R_{\mu \nu}=\partial_{\alpha} \Gamma_{\mu \nu}^{\alpha}-\partial_{\mu} \Gamma_{\alpha \nu}^{\alpha}+\Gamma_{\beta \alpha}^{\beta} \Gamma_{\mu \nu}^{\alpha}-\Gamma_{\mu \beta}^{\alpha} \Gamma_{\alpha \nu}^{\beta} .
$$

The Ricci tensor defined above, as well as the Einstein tensor derived from it, are in general asymmetric. However, they become symmetric as soon as we assume the connection is symmetric. Variation of Eq. (37) with respect to the connection gives the condition that the connection is in fact the Levi-Civita connection. Variation with respect to the metric then recovers the Einstein equations.

If the torsionless condition on the connection is dropped then complications arise, as has been shown by Hehl and Kerlick 600. The general form of the connection can be shown to be given by

$$
\Gamma_{\alpha \beta}^{\mu}=\left\{\begin{array}{c}
\mu \\
\alpha \beta
\end{array}\right\}+J_{\alpha \beta}^{\mu}=\left\{\begin{array}{c}
\mu \\
\alpha \beta
\end{array}\right\}+K_{\alpha \beta}^{\mu}+L_{\alpha \beta}^{\mu} .
$$

The tensor field $K^{\mu \beta}$ is the contorsion tensor, that can be defined in terms of the antisymmetric components of the connection, known as the torsion, as

$$
K_{\alpha \beta}^{\mu} \equiv S_{\alpha \beta}^{\mu}-S_{\alpha \beta}{ }^{\mu}-S_{\beta \alpha}{ }^{\mu},
$$

\footnotetext{
${ }^{3}$ This assumption has limited validity, however, as it cannot be applied to tensor fields without using a covariant derivative.
} 
where $S^{\mu}{ }_{\alpha \beta}=\Gamma_{[\alpha \beta]}^{\mu}$ is the torsion tensor. The tensor field $L_{\alpha \beta}^{\mu}$ is defined in terms of the non-metricity tensor as

$$
L_{\alpha \beta}^{\mu} \equiv \frac{1}{2}\left(Q_{\alpha \beta}^{\mu}-Q_{\alpha \beta}{ }^{\mu}-Q_{\beta \alpha}{ }^{\mu}\right) .
$$

To avoid confusion, we continue to denote the covariant derivative associated with the Levi-Civita connection as $\nabla_{\mu}$, while we use ${ }^{\Gamma} \nabla_{\mu}$ to denote the covariant derivative associated with $\Gamma_{\alpha \beta}^{\mu}$.

Varying the action with respect to the metric $g_{\mu \nu}$ we find the analogue of the Einstein equations:

$$
G_{(\mu \nu)}+\Lambda g_{\mu \nu}=8 \pi G \tilde{T}_{\mu \nu}
$$

One should note that only the symmetric part of the Einstein tensor appears here, and that we have used $\tilde{T}_{\mu \nu}$ rather than $T_{\mu \nu}$ to emphasise the fact that $\tilde{T}_{\mu \nu}$ is defined at constant $\Gamma_{\alpha \beta}^{\mu}$ in the variation, i.e. $\tilde{T}_{\mu \nu}=-\left.\frac{2}{\sqrt{-g}} \frac{\delta \mathcal{L}_{m}}{\delta g^{\mu \nu}}\right|_{\Gamma}$. On the other hand $T_{\mu \nu}=$ $-\left.\frac{2}{\sqrt{-g}} \frac{\delta \mathcal{L}_{m}}{\delta g^{\mu \nu}}\right|_{J}$. This is not an important distinction at this stage, as we have assumed that matter field do not couple to the connection, and hence $\tilde{T}_{\mu \nu}=T_{\mu \nu}$. It will, however, be important in the following subsection and in Section 3.5.1.

Varying with respect to the connection defines the Palatini tensor as $P_{\mu}{ }^{\alpha \beta}=\frac{8 \pi G}{\sqrt{-g}} \frac{\delta(\sqrt{-g} R)}{\delta \Gamma^{\mu}{ }_{\alpha \beta}}$, that can be written as

$$
P_{\alpha \mu \beta}=S_{\mu \alpha \beta}+2 g_{\mu[\alpha} S_{\beta]}+g_{\mu[\alpha} Q_{\beta]}-g_{\mu[\alpha} \bar{Q}_{\nu] \beta}^{\nu},
$$

where $S_{\mu}=S^{\alpha}{ }_{\mu \alpha}$, and where we have split the non-metricity tensor into trace and traceless parts as $Q_{\mu \alpha \beta}=Q_{\mu} g_{\alpha \beta}+\bar{Q}_{\mu \alpha \beta}$, with $g^{\alpha \beta} \bar{Q}_{\mu \alpha \beta}=0$. The Palatini tensor has only 60 independent components because it is identically traceless: $P_{\alpha}{ }^{\mu \alpha}=0$. Now, the second field equation is the vanishing of the Palatini tensor,

$$
P_{\mu}^{\alpha \beta}=0,
$$

but this provides only 60 constraints among the 64 independent components of the connection. In fact it may be shown that the equation $P_{\mu}^{\alpha \beta}=0$ is equivalent to the connection taking the following form $[600$ :

$$
\Gamma_{\alpha \beta}^{\mu}=\left\{\begin{array}{c}
\mu \\
\alpha \beta
\end{array}\right\}-\frac{1}{2} Q_{\alpha} \delta_{\beta}^{\mu}=\left\{\begin{array}{c}
\mu \\
\alpha \beta
\end{array}\right\}+\frac{2}{3} S_{\alpha} \delta_{\beta}^{\mu} .
$$

Clearly then, there are 4 degrees of freedom left undetermined by the field equations. Thus the Palatini approach in its most general form does not lead to a unique set of field equation: 4

The constraint $Q_{\mu}=0$ is sufficient to produce a consistent theory. This, however, has to be imposed as a Lagrange multiplier in the action via a term $\int d^{4} x \sqrt{-g} \lambda^{\alpha} Q_{\alpha}$. Once this is done, one recovers General Relativity uniquely. For theories of gravity other than General Relativity the difference between the metric variation and the Palatini procedure is even more significant: The resulting field equations are, in general, different. This will be spelt out explicitly for some specific theories in the sections that follow.

\footnotetext{
${ }^{4}$ It is often said that the Palatini procedure uniquely recovers GR. As we have seen, however, this is a myth. It does so only after further assumptions, for instance that the torsion vanishes, or that the connection is metric compatible, or that $Q_{\alpha}=0$. To make the Palatini variation well defined one has to impose such conditions in the action by means of Lagrange multipliers.
} 


\subsubsection{Metric-affine gravity and matter}

A further generalisation of the metric variation approach is to keep the metric and connection completely independent, as discussed above, and further allow matter to couple not only to the metric, but also the connection [600. In this case the action takes the form

$$
S=\frac{1}{16 \pi G} \int \sqrt{-g}\left(g^{\mu \nu} \Gamma_{\mu \nu}-2 \Lambda\right) d^{4} x+\int \mathcal{L}_{m}\left(g_{\mu \nu}, \Gamma_{\alpha \beta}^{\mu}, \psi\right) d^{4} x,
$$

where $\Gamma_{\alpha \beta}^{\mu}$ and $g_{\mu \nu}$ are once again independent. Performing the variations we recover Eq. 42, as before, and

$$
P_{\mu}^{\alpha \beta}=8 \pi G \Delta_{\mu}{ }^{\alpha \beta},
$$

where $\Delta_{\mu}{ }^{\alpha \beta}=-\frac{1}{\sqrt{-g}} \frac{\delta \mathcal{L}_{m}}{\delta \Gamma^{\mu^{\prime} \beta}}$ is called the hypermomentum tensor [600, 601].

In this case $T_{\mu \nu} \neq \tilde{T}_{\mu \nu}$, but it is straightforward to find that

$$
T_{\mu \nu}=\tilde{T}_{\mu \nu}+\nabla_{\rho}\left[\Delta_{(\mu \nu)}^{\rho}-\Delta_{(\mu \nu)}^{\rho}-\Delta_{(\mu \nu)}^{\rho}\right],
$$

where $\nabla_{\mu}$ is the covariant derivative associated with the Levi-Civita connection.

Equation (47) can be shown to be self-inconsistent for reasonable forms of matter, as the Palatini tensor is invariant under projective transformations of the form $\Gamma_{\alpha \beta}^{\mu} \rightarrow$ $\Gamma_{\alpha \beta}^{\mu}+\lambda_{\alpha} \delta^{\mu}{ }_{\beta}$, while there is no reason to suspect this invariance is exhibited by the matter fields and hence the hypermomentum. Equivalently, the Palatini tensor obeys the identity $P_{\alpha \mu}{ }^{\alpha}=0$, while there is no reason that this should identically hold for the hypermomentum 5 . One way to impose self-consistency is to demand that both torsion and non-metricity must vanish (by using Lagrange multipliers in the action), leading again to General Relativity. This type of self-consistency is, however, very strong, and weaker constraints have been found in 600. One such weaker constraint leads to the Einstein-Cartan-Sciama-Kibble theory [601, that we shall briefly describe in Section 3.5 .1

\subsubsection{Other approaches}

There are a variety of other formalisms that one can use to derive Einstein's equations. We will not go into the full details of all of these here, but merely mention some of the approaches that exist in the literature. For brevity we will only consider vacuum general relativity here.

In the 'vierbein' formalism the Einstein-Hilbert action can be written

$$
S=\int d^{4} x e e_{\hat{\alpha}}^{\mu} e_{\hat{\beta}}^{\nu} R_{\mu \nu}^{\hat{\alpha} \hat{\beta}}
$$

\footnotetext{
${ }^{5}$ Consider for example a simple Einstein-Æther model for which the matter action is $S_{M}=$ $\int d^{4} x \sqrt{-g}\left[\alpha \nabla_{\mu} A^{\nu} \nabla_{\nu} A^{\mu}+\lambda\left(A_{\mu} A^{\mu}+1\right)\right]$. The hypermomentum is $\Delta_{\mu}{ }^{\alpha \beta}=-2 A^{\beta} \nabla_{\mu} A^{\alpha}$ which clearly does not obey $\Delta_{\alpha \mu}{ }^{\alpha}=0$. The variation done this way is inconsistent. On the other hand using the Lagrange constraint $\int d^{4} x \sqrt{-g} \beta_{\mu}{ }^{\alpha \beta} J_{\alpha \beta}^{\mu}$ in the action imposes $J_{\alpha \beta}^{\mu}=0$, and hence the vanishing of the Palatini tensor. This leads to a modified Eq. 47 , as $P_{\mu}{ }^{\alpha \beta}=0=8 \pi G\left(\Delta_{\mu}{ }^{\alpha \beta}-\beta_{\mu}{ }^{\alpha \beta}\right)$, and to a modified Eq. 42, which now includes derivatives of $\beta_{\mu}{ }^{\alpha \beta}$. After using Eq. 48, however, the resulting equations are completely equivalent to the metric variation.
} 
where indices with hats correspond to a basis in the tangent space defined by the set of contravariant vectors, $e_{\hat{\mu}}{ }^{\mu}$, with determinant $e=\operatorname{det}\left[e_{\hat{\mu}}{ }^{\mu}\right]$. The inverse of $e_{\hat{\mu}}{ }^{\mu}$ is $e_{\mu}{ }^{\hat{\mu}}$, such that $e_{\rho}{ }^{\hat{}} e_{\hat{\nu}}{ }^{\rho}=\delta^{\hat{\mu}}{ }_{\hat{\nu}}$, and $e_{\hat{\rho}}{ }^{\mu} e_{\nu}{ }^{\hat{\rho}}=\delta^{\mu}{ }_{\nu}$. The metric tensor is constructed as $g_{\mu \nu}=$ $\eta_{\hat{\mu} \hat{\nu}} e_{\mu}{ }^{\hat{\mu}} e_{\nu}^{\hat{\nu}}$. The spin connection $\omega_{\mu}^{\hat{\alpha} \hat{\beta}}$ then defines a space-time and Lorentz covariant derivative, $\mathcal{D}_{\mu}$, as $\mathcal{D}_{\mu} v_{\nu}^{\hat{\rho}}=\nabla_{\mu} v_{\nu}^{\hat{\rho}}+\omega_{\mu}^{\hat{\rho}} v_{\nu}^{\hat{\lambda}}$, where $\nabla_{\mu}$ is the Levi-Civita connection ${ }^{6}$. The curvature tensor $R_{\mu \nu}{ }^{\hat{\alpha} \hat{\beta}}$ is defined in terms of the spin connection as

$$
R_{\mu \nu}^{\hat{\mu} \hat{\nu}} \equiv \partial_{\mu} \omega_{\nu}^{\hat{\mu} \hat{\nu}}-\partial_{\nu} \omega_{\mu}^{\hat{\mu} \hat{\nu}}+\omega_{\mu}^{\hat{\mu} \hat{\rho}} \omega_{\nu \hat{\rho}}^{\hat{\nu}}-\omega_{\nu}^{\hat{\mu} \hat{\rho}} \omega_{\mu \hat{\rho}}^{\hat{\nu}} .
$$

Variation now proceeds as in the Palatini formalism by assuming that the spin connection and vierbein are independent fields, from which one obtains the two field equations

$$
\mathcal{D}_{[\mu} e^{\hat{\alpha}}{ }_{\nu]}=0,
$$

and

$$
G_{\hat{\rho}}^{\alpha} \equiv e_{\hat{\alpha}}^{\alpha} e_{\hat{\rho}}^{\mu} e_{\hat{\beta}}^{\nu} R_{\mu \nu}^{\hat{\alpha} \hat{\beta}}-\frac{1}{2}\left(e_{\hat{\alpha}}^{\mu} e_{\hat{\beta}}^{\nu} R_{\mu \nu}^{\hat{\alpha} \hat{\beta}}\right) e_{\hat{\rho}}^{\alpha}=0,
$$

where $G_{\hat{\nu}}^{\mu}$ is the Einstein tensor. Equation (51) can be used to obtain the spin connection in terms of the partial derivatives of the vierbein, and the resulting relation implies that $\omega_{\mu}^{\hat{\alpha} \hat{\beta}}$ is torsion-less, i.e. one recovers Cartan's first structure equation, $d e^{\hat{\mu}}+\omega^{\hat{\mu}} \wedge e^{\hat{\nu}}=0$. The second equation says that the vacuum Einstein equations are recovered.

Another interesting alternative formulation of General Relativity is given by the Plebanski formalism [1042]. It is derived from the action

$$
S=\int \Sigma^{A B} \wedge R_{A B}-\frac{1}{2} \Psi_{A B C D} \Sigma^{A B} \wedge \Sigma^{C D}
$$

where upper case indices denote two component spinor indices to be raised and lowered with $\epsilon^{A B}$ and its inverse, and where the exterior product $\wedge$ acts on space-time indices, which have been suppressed. The curvature 2-form $R_{A B} \equiv d \omega_{A B}+\omega_{A}{ }^{C} \wedge \omega_{C B}$ is defined with respect to a spin connection 1-form $\omega_{A}{ }^{B}$. Variation of this action with respect to $\Psi_{A B C D}$ and $\omega_{A B}$ then tells us that the 2 -form $\Sigma^{A B}$ is the exterior product of some set of 1 -forms that we can identify with the tetrad $\theta^{A A^{\prime}}$, and that the connection $\omega_{A B}$ is torsion-free with respect to $\Sigma^{A B}$. Using this together with the variation of the action with respect to $\Sigma^{A B}$ then gives the vacuum Einstein equations, where the metric is given by $g=\theta^{A A^{\prime}} \otimes \theta_{A A^{\prime}}$.

One further alternative formulation of General Relativity is the purely affine 'Eddington formalism' 460. In previous subsections we outlined how one can either treat the metric as the only independent structure on the manifold, or treat the metric and connection as being two independent structures. Another approach is to take the connection as the only structure on the manifold. In this case, the simplest way of constructing a Lagrangian density with the correct weight (and without a metric) is to simply take the square root of the determinant of the Ricci tensor itself:

$$
S=\int \sqrt{-\operatorname{det}\left[R_{\mu \nu}(\Gamma)\right]} d^{4} x .
$$

\footnotetext{
${ }^{6}$ Given a metric, $g_{\mu \nu}$, the Levi-Civita connection can always be defined. The question is whether that is the connection that is used to define parallel transport.
} 
Varying this action with respect to the connection then gives the field equations

$$
\nabla_{\rho}\left(\sqrt{-\operatorname{det}\left[R_{\alpha \beta}(\Gamma)\right]} R^{\mu \nu}\right)=0,
$$

which can be shown to be equivalent to Einstein's equations in vacuum with a cosmological constant, if we take the connection to be the Levi-Civita connection. Due to the lack of a metric in the action for this theory, however, it is not a trivial matter to introduce matter fields into the theory [69.

Finally, let us mention that approaches exist that treat gravity as simply a spin-2 field on flat space [114, 115]. It has been conjectured that one could reconstruct the Einstein-Hilbert action in such an approach by considering consistency conditions order by order in perturbation theory. This will, of course, be an invalid treatment when gravity is strong, and in cosmology.

\subsection{Theorems}

There a number of theorems in General Relativity that are of great importance for the structure of the theory itself, as well as for the solutions to the field equations. These theorems underpin a lot of the acquired intuition on how gravity should function in different environments, and what the resulting phenomenology should be. In alternative theories of gravity, however, the theorems of General Relativity often fail, allowing new behaviours that would otherwise be impossible.

Here we briefly recap what we consider to be some of the most important theorems of General Relativity. In later sections we will show how these theorems are violated in alternative theories, and discuss the consequences of this.

\subsubsection{Lovelock's theorem}

Lovelock's theorem [831, 832 limits the theories that one can construct from the metric tensor alone. To enunciate this theorem, let us begin by assuming that the metric tensor is the only field involved in the gravitational action. If the action can be written in terms of the metric tensor $g_{\mu \nu}$ alone, then we can write

$$
S=\int d^{4} x \mathcal{L}\left(g_{\mu \nu}\right) .
$$

If this action contains up to second derivatives of $g_{\mu \nu}$, then extremising it with respect to the metric gives the Euler-Lagrange expression

$$
E^{\mu \nu}[\mathcal{L}]=\frac{d}{d x^{\rho}}\left[\frac{\partial \mathcal{L}}{\partial g_{\mu \nu, \rho}}-\frac{d}{d x^{\lambda}}\left(\frac{\partial \mathcal{L}}{\partial g_{\mu \nu, \rho \lambda}}\right)\right]-\frac{\partial \mathcal{L}}{\partial g_{\mu \nu}},
$$

and the Euler-Lagrange equation is $E^{\mu \nu}(\mathcal{L})=0$. Lovelock's theorem can then be stated as the following:

Theorem 2.1. (Lovelock's Theorem)

The only possible second-order Euler-Lagrange expression obtainable in a four dimensional space from a scalar density of the form $\mathcal{L}=\mathcal{L}\left(g_{\mu \nu}\right)$ is

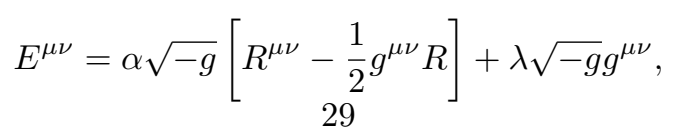


where $\alpha$ and $\lambda$ are constants, and $R_{\mu \nu}$ and $R$ are the Ricci tensor and scalar curvature, respectively.

This powerful theorem means that if we try to create any gravitational theory in a four-dimensional Riemannian space from an action principle involving the metric tensor and its derivatives only, then the only field equations that are second order or less are Einstein's equations and/or a cosmological constant. This does not, however, imply that the Einstein-Hilbert action is the only action constructed from $g_{\mu \nu}$ that results in the Einstein equations. In fact, in four dimensions or less one finds that the most general such action is

$\mathcal{L}=\alpha \sqrt{-g} R-2 \lambda \sqrt{-g}+\beta \epsilon^{\mu \nu \rho \lambda} R_{\mu \nu}^{\alpha \beta} R_{\alpha \beta \rho \lambda}+\gamma \sqrt{-g}\left(R^{2}-4 R^{\mu}{ }_{\nu} R_{\mu}^{\nu}+R_{\rho \lambda}^{\mu \nu} R_{\mu \nu}^{\rho \lambda}\right)$,

where $\beta$ and $\gamma$ are also constants. The third and fourth terms in this expression do not, however, contribute to the Euler-Lagrange equations as

$$
\begin{aligned}
E^{\mu \nu}\left[\epsilon^{\alpha \beta \rho \lambda} R^{\gamma \delta}{ }_{\alpha \beta} R_{\gamma \delta \rho \lambda}\right] & =0 \\
E^{\mu \nu}\left[\sqrt{-g}\left(R^{2}-4 R^{\alpha}{ }_{\beta} R_{\alpha}^{\beta}+R_{\rho \lambda}^{\alpha \beta} R_{\alpha \beta}^{\rho \lambda}\right)\right] & =0,
\end{aligned}
$$

where the action of $E^{\mu \nu}$ on any function $X$ is defined as in Eq. (57). The first of these equations is valid in any number of dimensions, and the second is valid in four dimensions only.

Lovelock's theorem means that to construct metric theories of gravity with field equations that differ from those of General Relativity we must do one (or more) of the following:

- Consider other fields, beyond (or rather than) the metric tensor.

- Accept higher than second derivatives of the metric in the field equations.

- Work in a space with dimensionality different from four.

- Give up on either rank $(2,0)$ tensor field equations, symmetry of the field equations under exchange of indices, or divergence-free field equations.

- Give up locality.

The first three of these will be the subject of the next three sections of this report. The fourth option requires giving up on deriving field equations from the metric variation of an action principle, and will not be considered further here.

\subsubsection{Birkhoff's theorem}

Birkhoff's theorem ${ }^{7}$ is of great significance for the weak-field limit of General Relativity. The theorem states [160]

\footnotetext{
${ }^{7}$ This theorem is commonly attributed to Birkhoff, although it was already published two years earlier by Jebsen [659. It is not to be confused with Birkhoff's pointwise ergodic theorem.
} 
Theorem 2.2. (Birkhoff's Theorem)

All spherically symmetric solutions of Einstein's equations in vacuum must be static and asymptotically flat (in the absence of $\Lambda$ ).

Strictly speaking, there are very few situations in the real Universe in which Birkhoff's theorem is of direct applicability: Exact spherical symmetry and true vacuums are rarely, if ever, observed. Nevertheless, Birkhoff's theorem is very influential in how we understand the gravitational field around (approximately) isolated masses. It provides strong support for the relativistic extension of our Newtonian intuition that far from such objects their gravitational influence should become negligible, or, equivalently, space-time should be asymptotically flat ${ }^{8}$. We can therefore proceed with some confidence in treating the weak-field limit of General Relativity as a perturbation about Minkowski space. Birkhoff's theorem also tells us that certain types of gravitational radiation (from a star that pulsates in a spherically symmetric fashion, for example) are not possible.

As we will show below, Birkhoff's theorem does not hold in many alternative theories of gravity. We therefore have less justification, aside from our own intuition, in treating the weak field limit of these theories as perturbations about Minkowski space. We must instead be more careful, as the space-time we perform our expansion around can have asymptotic curvature, leading to either time or space-dependence of the background (or some combination of the two). What is more, the perturbations themselves may be timedependent, and their form can be sensitive to the type of asymptotic curvature that the background exhibits. Behaviours such as these are not expected in General Relativity 837.

\subsubsection{The no-hair theorems}

These theorems are named after the phrase coined by Wheeler that "black holes have no hair". The first of these theorems was given by Israel and showed that the only static uncharged asymptotically flat black hole solution to Einstein's equations is the Schwarzschild solution 650. He later extended this theorem to include charged objects 651], and Carter extended it to black holes with angular momentum [262]. The theorem is therefore often stated today as "the generic final state of gravitational collapse is a Kerr-Newman black hole, fully specified by its mass, angular momentum, and charge" 1254.

Complementary to the black hole no-hair theorems is the no-hair 'theorem' of de Sitter space. The claim here is that in the context of General Relativity with a cosmological constant all expanding universe solutions should evolve towards de Sitter space. This has been shown explicitly by Wald for all Bianchi type models $9^{9} 1255$.

These theorems play an important role in General Relativity and cosmology. Some progress has been made in extending them to alternative theories of gravity, but there have also been explicit examples of them being violated in particular theories. This will be discussed further in subsequent sections.

\footnotetext{
${ }^{8}$ Of course, in a cosmological setting asymptotic regions are never realised as we will eventually come across the other masses in the Universe.

${ }^{9}$ Except type-IX universes with large amounts of spatial curvature.
} 


\subsection{The Parameterised Post-Newtonian Approach}

This section is a recap of the Parameterised Post-Newtonian (PPN) formalism that is widely used by both theoretical and observational gravitational physicists. The idea here is to create a construction that encompasses a wide array of different gravitational theories, and that contains parameters that can be constrained by observations in a reasonably straightforward fashion. In this way labour can be saved on both the theoretical and observational ends of the spectrum: Observers can apply their results to constrain a wide array of theories without having to trawl through the details of the individual theories themselves, and theorists can straightforwardly constrain their new theories by comparing to the already established bounds on the PPN parameters without having to re-calculate individual gravitational phenomena. To date, this approach has been highly successful, and in the following sections of this report we will often refer to it. We will therefore outline here how the PPN formalism proceeds. For a more detailed explanation of the principles and consequences of this formalism the reader is referred to [1274].

\subsubsection{Parameterised post-Newtonian formalism}

The PPN formalism is a perturbative treatment of weak-field gravity, and therefore requires a small parameter to expand in. For this purpose an "order of smallness" is defined by

$$
U \sim v^{2} \sim \frac{P}{\rho} \sim \Pi \sim O(2),
$$

where $U$ is the Newtonian potential, $v$ is the 3 -velocity of a fluid element, $P$ is the pressure of the fluid, $\rho$ is its rest-mass density and $\Pi$ is the ratio of energy density to rest-mass density. Time derivatives are also taken to have an order of smallness associated with them, relative to spatial derivatives:

$$
\frac{|\partial / \partial t|}{|\partial / \partial x|} \sim O(1) .
$$

Here we have chosen to set $c=1$. The PPN formalism now proceeds as an expansion in this order of smallness.

For time-like particles coupled to the metric only the equations of motion show that the level of approximation required to recover the Newtonian limit is $g_{00}$ to $O(2)$, with no other knowledge of other metric components beyond the background level being necessary. The post-Newtonian limit for time-like particles, however, requires a knowledge of

$$
\begin{array}{lll}
g_{00} & \text { to } & O(4) \\
g_{0 i} & \text { to } & O(3) \\
g_{i j} & \text { to } & O(2) .
\end{array}
$$

Latin letters here are used to denote spatial indices. To obtain the Newtonian limit of null particles we only need to know the metric to background order: Light follows straight lines, to Newtonian accuracy. The post-Newtonian limit of null particles requires a knowledge of $g_{00}$ and $g_{i j}$ both to $O(2)$.

The way in which the PPN formalism then proceeds is as follows. First one identifies the different fields in the theory. All dynamical fields should then be perturbed from 
their expected background values, and the perturbations assigned an appropriate order of smallness each. For theories containing a metric the appropriate expansion is usually

$$
\begin{aligned}
& g_{00}=-1+h_{00}^{(2)}+h_{00}^{(4)}+O(6) \\
& g_{0 i}=h_{0 i}^{(3)}+O(5) \\
& g_{i j}=\delta_{i j}+h_{i j}^{(2)}+O(4),
\end{aligned}
$$

where superscripts in brackets denote the order of smallness of the term. If, for example, the theory contains an additional scalar field, then the usual expansion for this quantity is

$$
\phi=\phi_{0}+\varphi^{(2)}+\varphi^{(4)}+O(6),
$$

where $\phi_{0}$ is the constant background value of $\phi$. Additional vector and tensor gravitational fields can be specified in a corresponding way.

The energy-momentum tensor in the PPN formalism is then taken to be that of a perfect fluid. To the relevant order, the components of this tensor are given by

$$
\begin{aligned}
& T_{00}=\rho\left(1+\Pi+v^{2}-h_{00}\right)+O(6) \\
& T_{0 i}=-\rho v_{i}+O(5) \\
& T_{i j}=\rho v_{i} v_{j}+P \delta_{i j}+O(6) .
\end{aligned}
$$

Taking these expressions, the field equations for the theory in question, and substituting in the perturbed expressions for the dynamical fields in the theory, as prescribed above, the field equations can then be solved for order by order in the smallness parameter.

The first step in such calculations is usually to solve for $h_{00}^{(2)}$. With this solution in hand, one then proceeds to solve for $h_{i j}^{(2)}$ and $h_{0 i}^{(3)}$ simultaneously, and finally $h_{00}^{(4)}$ can be solved for. If additional fields exist, beyond the metric, then these quantities must also be solved for to increasing order of smallness as the calculation proceeds. In finding $h_{i j}^{(2)}$, $h_{0 i}^{(3)}$ and $h_{00}^{(4)}$ one needs to specify a gauge. After such a specification one still, of course, has the freedom to make additional gauge transformations of the form $x^{\mu} \rightarrow x^{\mu}+\xi^{\mu}$, where $\xi^{\mu}$ is $O(2)$ or smaller. This freedom should be used at the end of the process to transform the metric that has been obtained into the "standard post-Newtonian gauge". This is a gauge in which the spatial part of the metric is diagonal, and terms containing time derivatives are removed. Once this has been done then one is in possession of the PPN limit of the theory in question.

We have so far outlined the procedure that one needs to follow in order to gain the appropriate form of the metric that couples to matter fields in the weak-field limit. Once done, the result can then be compared to the 'PPN test metric' below:

$$
\begin{aligned}
g_{00}= & -1+2 G U-2 \beta G^{2} U^{2}-2 \xi G^{2} \Phi_{W}+\left(2 \gamma+2+\alpha_{3}+\beta_{1}-2 \xi\right) G \Phi_{1} \\
& +2\left(1+3 \gamma-2 \beta+\beta_{2}+\xi\right) G^{2} \Phi_{2}+2\left(1+\beta_{3}\right) G \Phi_{3}-\left(\beta_{1}-2 \xi\right) G \mathcal{A} \\
& +2\left(3 \gamma+3 \beta_{4}-2 \xi\right) G \Phi_{4} \\
g_{0 i}= & -\frac{1}{2}\left(3+4 \gamma+\alpha_{1}-\alpha_{2}+\beta_{1}-2 \xi\right) G V_{i}-\frac{1}{2}\left(1+\alpha_{2}-\beta_{1}+2 \xi\right) G W_{i} \\
g_{i j}= & (1+2 \gamma G U) \delta_{i j} .
\end{aligned}
$$


Here $\beta, \gamma, \xi, \beta_{1}, \beta_{2}, \beta_{3}, \beta_{4}, \alpha_{1}, \alpha_{2}$ and $\alpha_{3}$ are the 'post-Newtonian parameters', $U$ is the Newtonian gravitational potential that solves the Newtonian Poisson equation, and $\Phi_{W}, \Phi_{1}, \Phi_{2}, \Phi_{3}, \Phi_{4}, \mathcal{A}, V_{i}$ and $W_{i}$ are the 'post-Newtonian gravitational potentials' (the precise form of these potentials is given in [1274]). The particular combination of parameters before each of these potentials is chosen here so that they have particular physical significance, once gravitational phenomena have been computed.

\subsubsection{Parameterised post-Newtonian constraints}

Comparison of the weak field metric of a particular theory with the PPN test metric above allows one to read off values for the PPN parameters $\beta, \gamma, \xi, \beta_{1}, \beta_{2}, \beta_{3}, \beta_{4}, \alpha_{1}, \alpha_{2}$ and $\alpha_{3}$ for the theory in question. The test metric has been constructed to include the type of potentials that often appear when one modifies gravity 10 . The great utility of the PPN formalism is that observers can take the PPN test metric above and constrain the parameters without having a particular theory in mind. These constraints can then be applied directly to a large number of gravitational theories, without having to work out how complicated gravitational phenomena work in each theory individually.

In General Relativity we have that $\beta=\gamma=1$ and $\xi=\beta_{1}=\beta_{2}=\beta_{3}=\beta_{4}=\alpha_{1}=$ $\alpha_{2}=\alpha_{3}=0$. Other theories will predict other values for these parameters, and we will discuss these on a case by case basis in the sections that follow. Observationally, one can use the gravitational phenomena discussed in Section 1 to impose the constraints that follow.

As already discussed, observations that involve only null geodesics are sensitive to the Newtonian part of the metric, $g_{00}^{(2)}$, and the term $g_{i j}^{(2)}$ only. These two terms involve the PPN parameter $\gamma$ only. We can now use constraints on the bending of light by the Sun to get a constraint on $\gamma$. Using the PPN test metric the predicted bending of light that one should observe is 1274

$$
\theta=2(1+\gamma) \frac{m}{r}=\frac{(1+\gamma)}{2} \theta_{G R},
$$

where $m$ is the mass of the Sun, $r$ is its radius, and $\theta_{G R}$ is the general relativistic prediction. Using the observed value of $\theta$ given in Section 1 then gives [1131]

$$
\gamma-1=(-1.7 \pm 4.5) \times 10^{-4}
$$

which is consistent with the general relativistic value of $\gamma=1$. Similarly, we can use the PPN test metric to find that the Shapiro time delay effect is given by 1274 .

$$
\Delta t=\frac{(1+\gamma)}{2} \Delta t_{G R}
$$

where subscript $G R$ again means the value of this quantity as predicted by General Relativity. Taking the observed value of $\Delta t$ given in Section 1 then gives the even tighter constraint 147

$$
\gamma-1=(2.1 \pm 2.3) \times 10^{-5}
$$

\footnotetext{
${ }^{10}$ It is not, however, an exhaustive collection of all possible potentials, and in some theories it is occasionally necessary to include additional terms.
} 
again consistent with $\gamma=1$. It can now be clearly seen that the bending of light by the Sun, and the Shapiro time delay effect do, in fact, constrain the same aspect of space-time geometry. They can therefore be considered as complimentary to each other.

If we now consider observations of gravitational phenomena that involve time-like geodesics then we are able to observe, potentially, all of the post-Newtonian potentials in the PPN test metric. This becomes clear from the expression for perihelion precession, which now becomes

$$
\Delta \omega=\frac{6 \pi M}{p}\left[\frac{1}{3}(2+2 \gamma-\beta)+\frac{1}{6}\left(2 \alpha_{1}-\alpha_{2}+\alpha_{3}+2 \beta_{2}\right) \frac{\mu}{M}+J_{2}\left(\frac{r^{2}}{2 M p}\right)\right],
$$

where $M$ is the total mass of the two bodies involved, $\mu$ is their reduced mass, and $p$ is the semi-latus rectum of the orbit. The affect of modifying the geometry can be seen here to be degenerate with the effect due to the solar quadrupole moment, $J_{2}$. Once the value of this quantity is known, however, then one is able to gain constraints on the above combination of $\beta, \gamma, \alpha_{1}, \alpha_{2}, \alpha_{3}$ and $\beta_{2}$. This can be done for any or all of the observations of the perihelion precession of Mercury given in Section 1 and if we take the value of $\gamma$ to be that given by Eq. (71), as well a: ${ }^{11} \alpha_{1} \sim \alpha_{2} \sim \alpha_{3} \sim \beta_{2} \sim 0$ and a reasonable value of $J_{2} \sim 10^{-7}$, then this gives constraints on $\beta$ of the order $\beta-1 \sim O\left(10^{-3}\right)$ or $O\left(10^{-4}\right)$. However, as already noted, these constraints are somewhat sensitive to a number of assumptions about the orbits of the other planets, as well as the solar quadrupole moment.

The Nordtvedt effect is similarly an observation of time-like geodesics. In this case it is convenient to define the 'Nordtvedt parameter'

$$
\eta_{N} \equiv 4 \beta-\gamma-3-\frac{10}{3} \xi-\alpha_{1}+\frac{2}{3} \alpha_{2}-\frac{2}{3} \beta_{1}-\frac{1}{3} \beta_{2},
$$

which is not to be confused with the equivalence principle violation parameter $\eta$ defined in Equation (15). The observations of Williams, Turyshev and Boggs [1277] then give the constraint $\eta_{N}=(4.4 \pm 4.5) \times 10^{-4}$, which, if we again take $\gamma$ to be given by observations of the Shapiro time delay effect with all other PPN parameters being zero, gives us

$$
\beta-1=(1.2 \pm 1.1) \times 10^{-4},
$$

which is a much cleaner constraint on $\beta$ than those which can be derived from observations of the perihelion precession of Mercury.

In 'conservative' theories of gravity it is usually only the PPN parameters $\beta$ and $\gamma$ (and sometimes $\xi$ ) that vary from their general relativistic values. These quantities are often interpreted as the degree of non-linearity in the gravitational theory, and the amount of spatial curvature per unit mass that is produced, respectively. The other parameters $\xi, \alpha_{i}$ and $\beta_{i}$ are usually interpreted as corresponding to preferred location effects, preferred frame effect and the violation of conserved quantities. When considering theories in which such effects are expected to be absent it is therefore usual to assume that these parameters are all zero, and to search instead for constraints on $\beta$ and $\gamma$.

Of course, one can subject the $\xi, \alpha_{i}$ and $\beta_{i}$ parameters to observational scrutiny in a number of ways. The table below gives a selection of the tightest constraints currently available:

\footnotetext{
${ }^{11}$ These values will be given some justification shortly.
} 


\begin{tabular}{|c|c|l|}
\hline Parameter & Limit & Source \\
\hline$\xi$ & $10^{-3}$ & Ocean tides [1274] \\
$\alpha_{1}$ & $10^{-3}$ & Lunar laser ranging 928] \\
$\alpha_{2}$ & $4 \times 10^{-7}$ & Alignment of Sun's spin axis with ecliptic [987] \\
$\alpha_{3}$ & $4 \times 10^{-20}$ & Pulsar acceleration [1176] \\
\hline
\end{tabular}

Further constraints and discussion on the $\beta_{i}$ parameters can be found in 1274. For more details of the observations leading to these constraints on $\xi$ and $\alpha_{i}$ the reader is referred to the source material cited above and [1274].

The constraints on the PPN parameters that we have discussed above are all, to date, in reasonably good agreement with General Relativity, and it is likely that future observations of, for example, the 'double pulsar' 840, 745, will tighten these constraints even further in coming years. This excellent concordance of numerous different physical phenomena means that one must reconcile any alterations to General Relativity with observations in weak field systems that appear to be narrowing down on a general relativistic description. As we will describe in the sections that follow, this places tight constraints on a variety of different modified theories of gravity: It must be the case that any alternative theories that we consider should reproduce General Relativity in the appropriate weak field limit, or at least something very close to it.

There are a number of mechanisms that have been considered in the literature that allow for a general relativistic weak field limit even in theories that are, in general, very different from General Relativity. These include the Vainshtein mechanism [1241] which occurs when large derivative interactions are present, the Chameleon mechanism of theories with non-minimal coupling to scalar fields $\underline{689}$, as well as the attractor mechanism of Damour and Nordtvedt [356. These different approaches allow, potentially, for theories that deviate considerably from General Relativity to exist without disturbing gravitational physics in the solar system to a large extent. They are thought to be successful in a number of different environments, and have sometimes been applied to situations that are quite different to the ones in which they were originally conceived.

As well as successful reproductions of general relativistic behaviour, however, there have also been a number cases found in the literature of theories that produce weak field gravity that is surprisingly inconsistent with the predictions of General Relativity. Perhaps the most famous of these is the van Dam-Veltman-Zakharov (vDVZ) discontinuity that was originally found in the context of Pauli-Fierz gravity [1243, 1296) (a theory with one dynamical metric, and one non-dynamical a priori specified metric). Here the graviton acquires a mass through the introduction of terms into the gravitational Lagrangian that, in the weak field limit, look like mass terms for the perturbations $h_{\mu \nu}$ around Minkowski space, i.e. like $m^{2} h^{\mu \nu} h_{\mu \nu}$. Naively one might then expect in the limit $m \rightarrow 0$, when the graviton becomes massless, that the zero mass theory of General Relativity should be recovered. This is, however, not the case. Instead one finds from the study of linear perturbations around Minkowski space that $\gamma \rightarrow 1 / 2$, which can be seen from the constraints above to be in strong disagreement with a number of different observations, including light bending and time delay effects. The general relativistic limit in this case is therefore a singular one, and any finite but non-zero graviton mass, no matter how small, appears to give results that are incompatible with observations. Similar results 
have also been found in some theories of gravity constructed from more general functions of the Ricci curvature than the Einstein-Hilbert action [294, and are expected in other theories as well. In these cases one must either abandon the theory as being incompatible with observations, or show that the treatment being applied is unsatisfactory because, for example, one of the mechanisms discussed previously should be applied.

Issues such as those just discussed can make the study of weak field gravity in modified theories a more complicated subject than it is in General Relativity. One must be careful to make sure that the treatments being applied are justifiable, that the limits of the theory take the expected form (rather than being singular), and that non-linear mechanisms and non-perturbative effects are being fully taken into account. How this should be done for specific modified theories of gravity will be the subject of subsequent sections. In some cases it is still an active area of research.

\subsection{Cosmology}

We now turn to cosmology, which forms a major part of this review. In this section we first describe cosmology from the point of view of General Relativity, including Friedmann-Lemaittre-Robertson-Walker (FLRW) solutions, cosmic distance measures and cosmological perturbation theory. We then consider the observational evidence that has led to the rise of the "Dark Sector", thus arriving at the so-called $\Lambda \mathrm{CDM}$ "concordance model'. We end this section with a short discussion of the successes of $\Lambda \mathrm{CDM}$, its predictions and potential shortcomings.

\subsubsection{The Friedmann-Lemaître-Robertson-Walker solutions}

The Robertson-Walker metric in the synchronous coordinate system is

$$
d s^{2}=-d t^{2}+a^{2}(t) q_{i j} d x^{i} d x^{j},
$$

where $q_{i j}$ is a maximally symmetric 3 -metric of Gaussian curvature $\kappa$. In a spherically symmetric coordinate system this can be written

$$
d s^{2}=-d t^{2}+a^{2}\left[\frac{d r^{2}}{1-\kappa r^{2}}+r^{2} d \Omega\right]
$$

where $\kappa$ is a real constant. If $\kappa=0$, the hyper-surfaces of constant $t$ are flat, if $\kappa>0$ they are positively curved, and if $\kappa<0$ they are negatively curved. The function $a(t)$ is called the scale factor, and we assume it to be normalised to unity today.

The Hubble parameter is defined as

$$
H=\frac{1}{a} \frac{d a}{d t}
$$

with $H_{0}=H(a=1)$ being the Hubble constant, i.e. the value of the Hubble parameter at the present time.

The dynamics of the scale factor is given by the Friedmann equation

$$
3 H^{2}=8 \pi G \sum_{i} \rho_{i}
$$


where $\rho_{i}$ are the energy densities of all possible fluids, including photons $\rho_{\gamma}$, neutrinos $\rho_{\nu}$ (possibly with mass $m_{\nu}$ ), pressureless matter $\rho_{m}$, and spatial curvature $\rho_{\kappa}$. We may also define the relative densities

$$
\Omega_{i}=\frac{\rho_{i}}{\rho_{T}}
$$

where $\rho_{T}$ is the total energy density $\rho_{T}=\frac{3 H^{2}}{8 \pi G}=\sum_{i} \rho_{i}$. The Friedmann equation then becomes the constraint $\sum_{i} \Omega_{i}=1$.

If each fluid is uncoupled then energy-momentum conservation gives

$$
\dot{\rho}_{i}+3 H\left(1+w_{i}\right) \rho_{i}=0
$$

where $w$ is the equation of state, defined by $P=w \rho$. For the known forms of matter $w_{\gamma}=\frac{1}{3}, w_{m}=0, w_{\kappa}=-\frac{1}{3}$, and $w_{\nu}$ is in the range $\left[0, \frac{1}{3}\right]$. We may solve Eq. (78) for a few cases of interest, and then determine the dynamics of the scale factor. For radiation we obtain $\rho_{r}=\rho_{0 r} a^{-4}$, for pressureless matter $\rho_{m}=\rho_{0 m} a^{-3}$, for curvature $\rho_{\kappa}=-\frac{3 \kappa}{8 \pi G} a^{-2}$, and for a cosmological constant $\rho_{\Lambda}=\frac{1}{8 \pi G} \Lambda$.

A general analytic solution in the case where all the above fluids are present is impossible. However, analytic solutions can be found in certain special cases. If a single fluid is present and $w$ is constant then $a^{3(1+w) / 2}=\frac{3(1+w)}{2} H_{0} t$, provided $w \neq-1$. For the case of radiation we get $a=\sqrt{2 H_{0} t}$, and for pressureless matter $a=\left(\frac{3 H_{0} t}{2}\right)^{2 / 3}$. The case of a cosmological constant is special: One obtains $a=e^{H_{0} t}$, the de Sitter solution, in which space (in this coordinate system) is exponentially expanding.

In many cases of interest it is convenient to use a different time coordinate, the conformal time, $\tau$, defined by $d t=a d \tau$. In a radiation dominated universe we then have $a(\tau)=H_{0} \tau$, in a matter dominated universe $a=\frac{1}{4}\left(H_{0} \tau\right)^{2}$, and for the de Sitter universe $a=\frac{1}{H_{0}\left(\tau_{\infty}-\tau\right)}$, where $\tau_{\infty}$ is the value of the conformal time at $a \rightarrow \infty$. In a universe filled with both radiation and matter we get $a=\sqrt{\Omega_{0 r}} H_{0} \tau+\frac{\Omega_{0 m}}{4}\left(H_{0} \tau\right)^{2}$. A summary of these solutions is shown in Table 2 .

\begin{tabular}{|l|l|l|}
\hline Matter type & $a(t)$ & $a(\tau)$ \\
\hline radiation & $a=\left(2 H_{0} t\right)^{1 / 2}$ & $a=H_{0} \tau$ \\
dust & $a=\left(\frac{3}{2} H_{0} t\right)^{2 / 3}$ & $a=\frac{1}{4}\left(H_{0} \tau\right)^{2}$ \\
radiation \& dust & complicated & $a=\sqrt{\Omega_{0 r}} H_{0} \tau+\frac{\Omega_{0 m}}{4}\left(H_{0} \tau\right)^{2}$ \\
$\Lambda$ & $a=e^{H_{0} t}$ & $a=\frac{1}{H_{0}\left(\tau_{\infty}-\tau\right)}$ \\
\hline
\end{tabular}

Table 2: A summary of particular solutions to the Friedmann equation.

\subsubsection{Cosmological distances}

Given a Friedmann universe obeying Einstein's field equations, it is useful to define observables that characterise the background evolution. Distances play an important role if we are to map out its behaviour (see 612 for a more detailed explanation). Hubble's law $v=H_{0} d$ allows us to define a Hubble time, $t_{H}=\frac{1}{H_{0}}=9.78 \times 10^{9} h^{-1}$ yr and the Hubble distance, $D_{H}=\frac{c}{H_{0}}=3000 h^{-1} \mathrm{Mpc}$. We can also integrate along a light ray to 
get the comoving distance:

$$
D_{C}=c \int_{t}^{t_{0}} \frac{c d t^{\prime}}{a\left(t^{\prime}\right)}
$$

From $-\kappa=\Omega_{\kappa} / D_{H}^{2}$, and performing the radial integral (assuming the observer is at $r=0$ ), we have

$$
D_{C}=\int_{0}^{D_{M}} \frac{d r}{\sqrt{1-\kappa r^{2}}}= \begin{cases}\frac{D_{H}}{\sqrt{\Omega_{\kappa}}} \sinh ^{-1}\left[\sqrt{\Omega_{\kappa}} D_{M} / D_{H}\right] & \text { for } \Omega_{\kappa}>0 \\ D_{M} & \text { for } \Omega_{\kappa}=0 \\ \frac{D_{H}}{\sqrt{\left|\Omega_{\kappa}\right|}} \sin ^{-1}\left[\sqrt{\left|\Omega_{\kappa}\right|} D_{M} / D_{H}\right] & \text { for } \Omega_{\kappa}<0\end{cases}
$$

where the proper motion distance (also known as the transverse comoving distance) is $D_{M}$. This can be rewritten as

$$
D_{M}= \begin{cases}\frac{D_{H}}{\sqrt{\Omega_{\kappa}}} \sinh \left[\sqrt{\Omega_{\kappa}} D_{C} / D_{H}\right] & \text { for } \Omega_{\kappa}>0 \\ D_{C} & \text { for } \Omega_{\kappa}=0 \\ \frac{D_{H}}{\sqrt{\left|\Omega_{\kappa}\right|}} \sin \left[\sqrt{\left|\Omega_{\kappa}\right|} D_{C} / D_{H}\right] & \text { for } \Omega_{\kappa}<0\end{cases}
$$

It is then possible to find an expression for the angular diameter distance:

$$
D_{A}=\frac{D_{M}}{1+z} .
$$

Hence, if we know the proper size of an object and its redshift we can work out, for a given universe, the angular diameter distance, $D_{A}$. If we measure the brightness or luminosity of an object, we know that the flux of that object at a distance $D_{L}$ is given by $F=\frac{L}{4 \pi D_{L}^{2}}$, where $D_{L}$ is aptly known as the luminosity distance and is related to other distances through:

$$
D_{L}=(1+z) D_{M}=(1+z)^{2} D_{A} .
$$

This relation is a consequence of Etherington's theorem [474, and holds in any metric theory of gravity, irrespective of the field equations. It is however violated if the photon number is not conserved (e.g. due to photon-axion mixing), or if photons are extinguished due to the presence of dust. It turns out that in astronomy one often works with a logarithmic scale, i.e. with magnitudes. One can then define the distance modulus:

$$
D M \equiv 5 \log \left(\frac{D_{L}}{10 \mathrm{pc}}\right)
$$

which can be measured from the apparent magnitude, $m$, (related to the flux at the observer), and the absolute magnitude, $M$, (what the magnitude would be if the observer was at $10 \mathrm{pc}$ from the source) through $m=M+D M$.

Finally, let us consider Hubble's law. Take two objects that are a distance $d$ apart, and Taylor expand the scale factor today to find

$$
a(t)=a\left(t_{0}\right)+\dot{a}\left(t_{0}\right)\left[t-t_{0}\right]+\frac{1}{2} \ddot{a}\left(t_{0}\right)\left[t-t_{0}\right]^{2}+\cdots .
$$


On small scales the distance to the emitter is roughly related to the time of emission, $t$, by $d \simeq c\left(t_{0}-t\right)$. We can then rewrite the above expression as

$$
(1+z)^{-1}=1-H_{0} \frac{d}{c}-\frac{q_{0} H_{0}^{2}}{2}\left(\frac{d}{c}\right)^{2}+\cdots,
$$

where $q_{0}=-\ddot{a} a /\left.\dot{a}^{2}\right|_{t=t_{0}}$ is the deceleration parameter. On small scales and at small redshifts we then have Hubble's law, $c z=H_{0} d$.

To constrain the background evolution it is necessary to have good distance measurements. So, for example, with measurement of supernovae light curves at different redshifts it is, in principle, possible to measure $D_{L}(z)$. Alternatively one might try to measure $D_{A}(z)$ by observing known length scales in the universe. This has been done spectacularly well with the sound horizon of the cosmic microwave background at redshift $z \simeq 1100$. More tentatively, there is a constraint on a combination of $D_{A}(z)$ and $H(z)$ using the imprint of acoustic oscillations of baryons on galaxy clustering at moderate to low redshifts, $z \simeq 0.1-0.3$.

\subsubsection{Perturbation theory}

We now turn to perturbation theory, which is an indispensable tool for making predictions for a variety of cosmological observations. For extensive treatments of cosmological perturbation theory the reader is referred to [90, 717, 469, 917. Here we shall only consider scalar fluctuations, for which the perturbed FLRW metric can be written

$$
d s^{2}=a^{2}\left\{-(1-2 \Xi) d \tau^{2}-2\left(\vec{\nabla}_{i} \beta\right) d \tau d x^{i}+\left[\left(1+\frac{1}{3} \chi\right) q_{i j}+D_{i j} \nu\right] d x^{i} d x^{j}\right\},
$$

where $D_{i j} \equiv \vec{\nabla}_{i} \vec{\nabla}_{j}-\frac{1}{3} q_{i j} \Delta$ is a trace-less spatial derivative operator. We note that $\vec{\nabla}_{i}$ is the covariant derivative compatible with the 3-metric $q_{i j}$. Perfect fluids with shear have energy-momentum tensors that can be written as

$$
T_{\mu \nu}=(\rho+P) u_{\mu} u_{\nu}+P g_{\mu \nu}+\Sigma_{\mu \nu}
$$

where $\rho$ is the energy density, $P$ is the pressure, $u_{\mu}$ the 4-velocity of the fluid (normalised to $u^{\mu} u_{\mu}=-1$ ), and $\Sigma_{\mu \nu}$ is the anisotropic stress tensor which obeys $u^{\mu} \Sigma_{\mu \nu}=\Sigma^{\mu}{ }_{\mu}=0$. In a homogeneous and isotropic space $\Sigma_{\mu \nu}=0$, and $u_{\mu}$ is aligned with the time direction such that in the coordinate system used above it has components $u_{\mu}=(a, \overrightarrow{0})$. For first order scalar perturbations we can parameterise $T^{\mu}{ }_{\nu}$ as

$$
\begin{aligned}
T_{0}^{0} & =-\rho \delta \\
T^{0}{ }_{i} & =-(\rho+P) \vec{\nabla}_{i} \theta \\
T_{0}^{i} & =(\rho+P) \vec{\nabla}^{i}(\theta-\beta) \\
T_{j}^{i} & =\delta P \delta^{i}{ }_{j}+(\rho+P) D^{i}{ }_{j} \Sigma,
\end{aligned}
$$

while the fluid velocity is $u_{\mu}=a\left(1-\Xi, \vec{\nabla}_{i} \theta\right)$. Here $\delta P$ is the pressure perturbation, and $\Sigma$ the scalar anisotropic stress.

For any variable $\mathbf{X}$, its perturbation $\delta \mathbf{X}$ is not necessarily an observable quantity, and may depend on a gauge. In particular, one can always define a new perturbation 
$\delta \mathbf{X}^{\prime}=\delta \mathbf{X}+\mathcal{L}_{\xi} \overline{\mathbf{X}}$ through the Lie derivative acting on the background tensor $\overline{\mathbf{X}}$ through a vector field $\xi^{\mu}$. The perturbations $\delta \mathbf{X}$ are thus in general gauge-dependent122, For scalar perturbations we can write $\xi_{\mu}=a\left(-\xi, \vec{\nabla}_{i} \psi\right)$, and then find how our variables transform under gauge transformations using the Lie derivative. All of them, apart from $\Sigma$, are gauge-dependent, with transformations given by

$$
\begin{array}{ll}
\Xi \rightarrow \Xi-\frac{\xi^{\prime}}{a} & \beta \rightarrow \beta+\frac{1}{a}\left[\xi+\mathcal{H} \psi-\psi^{\prime}\right] \\
\chi \rightarrow \chi+\frac{1}{a}[6 \mathcal{H} \xi+2 \Delta \psi] & \nu \rightarrow \nu+\frac{2}{a} \psi \\
\delta \rightarrow \delta-\frac{3}{a}(1+w) \mathcal{H} \xi & \theta \rightarrow \theta+\frac{1}{a} \xi \\
\frac{\delta P}{\rho} \rightarrow \frac{\delta P}{\rho}+\frac{1}{a}\left[w^{\prime}-3 w(1+w) \mathcal{H}\right] \xi & \Sigma \rightarrow \Sigma .
\end{array}
$$

where $\mathcal{H}=\frac{a^{\prime}}{a}$.

Given our set of perturbation variables, two linear combinations of them can be removed ${ }^{13}$ (set to zero). Popular gauges are

- Newtonian gauge: $\nu=\beta=0$. The remaining metric perturbations give rise to the Newtonian potentials $\Phi=-\frac{1}{6} \chi$ and $\Psi=-\Xi$.

- Synchronous gauge: $\Xi=\beta=0$ (this does not completely fix the gauge). The remaining metric perturbations are related to the Ma-Bertschinger 841 variables as $\chi=h$ and $-k^{2} \nu=h+6 \eta$.

- Comoving gauge: $\theta=\nu=0$. Strictly speaking there is a multitude of comoving gauges depending on which velocity $\theta$ is set to zero. Thus we may speak of a "baryon comoving gauge" if $\theta_{b}=0$, a "photon comoving gauge" if $\theta_{\gamma}=0$, the total matter comoving gauge if $\theta_{T}=\frac{\sum_{X}\left(\rho_{X}+P_{X}\right) \theta_{X}}{\sum_{X}\left(\rho_{X}+P_{X}\right)}=0$, etc.

- Uniform density gauge: $\delta=\nu=0$. Once again there is a multitude of uniform density gauges depending on which density fluctuation is set to zero, as in the comoving gauges above.

- Spatially flat gauge: $\chi=\nu=0$.

It is possible to find combinations of perturbation variables that are gauge invariant, but note that there are an infinite number of them as any linear combination of gaugeinvariant variables is also gauge-invariant. Two popular gauge-invariant metric variables are the Bardeen potentials $\hat{\Phi}$ and $\hat{\Psi}$ :

$$
\hat{\Phi} \equiv-\frac{1}{6}(\chi-\Delta \nu)+\frac{1}{2} \mathcal{H}\left(\nu^{\prime}+2 \beta\right),
$$

and

$$
\hat{\Psi} \equiv-\Xi-\frac{1}{2}\left(\nu^{\prime \prime}+2 \beta^{\prime}\right)-\frac{1}{2} \mathcal{H}\left(\nu^{\prime}+2 \beta\right) .
$$

\footnotetext{
${ }^{12}$ The Stewart-Walker lemma 1188 states that the only gauge-invariant perturbed tensors are those that have background values that are either zero or a constant multiple of the identity matrix.

${ }^{13}$ One has to be careful and not over constrain the gauge by removing two combinations that transform with the same gauge variable, e.g. $\delta$ and $\theta$ both transform with $\xi$ and therefore cannot be set to zero simultaneously.
} 
The Newtonian gauge is special in this case as $\hat{\Phi}=\Phi$ and $\hat{\Psi}=\Psi$. From now on we will refer to $\Phi$ and $\Psi$ without a "hat" as the Newtonian gauge potentials. The Einstein equations in the Newtonian gauge give

$$
\begin{aligned}
& 2(\Delta+3 \kappa) \Phi-6 \mathcal{H}\left(\Phi^{\prime}+\mathcal{H} \Psi\right)=8 \pi G a^{2} \sum_{i} \rho_{i} \delta_{i} \\
& 2\left(\Phi^{\prime}+\mathcal{H} \Psi\right)=8 \pi G a^{2} \sum_{i}\left(\rho_{i}+P_{i}\right) \theta_{i} \\
& \Phi^{\prime \prime}+\mathcal{H} \Psi^{\prime}+2 \mathcal{H} \Phi^{\prime}+\left(2 \mathcal{H}^{\prime}+\mathcal{H}^{2}+\frac{1}{3} \Delta\right) \Psi-\left(\frac{1}{3} \Delta+\kappa\right) \Phi=4 \pi G a^{2} \sum_{i} \delta P_{i}
\end{aligned}
$$

and

$$
\Phi-\Psi=8 \pi G a^{2} \sum_{i}\left(\rho_{i}+P_{i}\right) \Sigma_{i}
$$

Combining Eqs. 87) and (88) we can find $\Phi$ in terms of the matter variables as

$$
2(\Delta+3 \kappa) \Phi=3 \mathcal{H}^{2} \sum_{i} \Omega_{i}\left[\delta_{i}+3 \mathcal{H}\left(1+w_{i}\right) \theta_{i}\right]
$$

while $\Psi$ is then obtained using Eq. 900.

Finally, all scalar modes can be decomposed in terms of a complete set of eigen-modes of the Laplace-Beltrami operator. For example, a variable $A$ can be decomposed as

$$
A\left(x^{i}, t\right)=\int d^{3} k Y\left(x^{j}, k_{k}\right) \tilde{A}\left(k_{i}, t\right),
$$

where the eigen-modes, $Y\left(x^{j}, k_{k}\right)$, obey $\left(\Delta+k^{2}\right) Y=0$. In the special case of topologically trivial and spatially flat hyper-surfaces of constant $t$, we simply have $Y=e^{i k_{j} x^{j}}$. The integral transform above is then a Fourier transform. The value of $k$ depends on the geometry and topology of the spatial hyper-surfaces: In the case of trivial topology $k$ takes values $k=\sqrt{k_{*}^{2}-\kappa}$, where $k_{*}$ is continuous and obeys $k_{*} \geq 0$ for zero or negative spatial curvature, while $k_{*}=N \sqrt{\kappa}$ for positive spatial curvature, where $N \geq 3$ is an integer.

\subsubsection{Gravitational potentials and observations}

One of the main sources of information in cosmology is through the observation of perturbations about a Friedmann background. Such perturbations can be probed through their effects on the dynamics of particles and light, which we will now describe (see [1028] for further details):

- Density fluctuations: Fluctuations in the matter density field, $\delta(\mathbf{x})$, will reflect various properties of the cosmological model. The simplest approach is to assume that $\delta(\mathbf{x})$ is a multivariate Gaussian random field that is entirely described by the power spectrum, $P(k)$, defined by

$$
\left\langle\left|\delta_{\mathbf{k}}\right|^{2}\right\rangle \equiv P(k),
$$


where $\delta_{\mathbf{k}}$ is the Fourier transform of $\delta(\mathbf{x})$. The shape of the power spectrum contains a wealth of information: The amplitude of clustering as a function of scale, its redshift dependence, how its shape on small scales is distorted by small scale velocities (known as redshift space distortions), and acoustic features imprinted by the baryons from pre-recombination (known as baryon acoustic oscillations) can all be used as distance indicators. The power spectrum can be estimated from surveys of galaxies or clusters of galaxies, the clustering properties of which can be directly related to the amplitude of fluctuations in the density field (under certain assumptions of how galaxies (or clusters) trace the density field (known as bias)).

- Peculiar velocities: The motion of galaxies relative to the Hubble flow, $v^{i}$, is described by the non-relativistic geodesic equation given above. In the linear regime, the peculiar velocity can be related directly to the density field via the gravitational potential:

$$
v_{\mathbf{k}}^{i}=-i a f H_{0} \frac{k^{i} \delta_{\mathbf{k}}}{k^{2}},
$$

where $f \equiv d \ln \delta / d \ln a$ and we have assumed the general relativistic result $\Phi=$ $\Psi$. Peculiar velocities will be observable through their effects on the redshift of objects, either in redshift galaxy surveys (through their distortion of $P(k)$ ), or when supplemented with independent distance measurements of each object (using the Tully-Fisher relation or supernova light curves) in peculiar velocity surveys.

- Anisotropies in the Cosmic Microwave Background (CMB): The CMB will be sensitive to density fluctuations, peculiar velocities, and the gravitational potentials. It is usual to characterise anisotropies in the CMB in terms of $\frac{\delta T(\hat{n})}{T}$, the dimensionless deviations of the black-body temperature of the Universe in a direction given by the unit vector, $\hat{n}$. We can expand

$$
\frac{\delta T(\hat{n})}{T}=\sum_{\ell m} a_{\ell m} Y_{\ell m}(\hat{n}),
$$

where we have spherical harmonics, $Y_{\ell m}(\hat{n})$, and define the angular power spectrum $C_{\ell}=\frac{1}{2 \ell+1} \sum_{m}\left\langle\left|a_{\ell m}\right|^{2}\right\rangle$. Like $P(k)$, the $C_{\ell}$ s contain a wealth of information about the cosmological model. It is now instructive to delve slightly further into the form of $\frac{\delta T(\hat{n})}{T}$. We can schematically split CMB anisotropies into three cosmological contributions,

$$
\frac{\delta T(\hat{n})}{T}=\left.\frac{\delta T(\hat{n})}{T}\right|_{\mathrm{LS}}+\left.\frac{\delta T(\hat{n})}{T}\right|_{\mathrm{ISW}}+\left.\frac{\delta T(\hat{n})}{T}\right|_{\mathrm{SEC}}
$$

where the first term encompasses all effects from the surface of last scattering, the second term (the Integrated Sachs Wolfe effect) is due to integrated effects along the line of sight, and the last term encompasses secondary effects such as weak lensing of the CMB, the Sunyaev-Zel'dovich effect and other such contributions. Let us focus on the effect of the gravitational potentials, the consequence of which we can see through the geodesic equation for light rays given above [150]. The 
accumulated redshift of a beam of light along the line of sight is given by $(1+z)=\frac{E_{\mathrm{obs}}}{E_{\mathrm{em}}}=1+\Psi_{\mathrm{em}}-\Psi_{\mathrm{obs}}-\int_{0}^{z}(\dot{\Phi}+\dot{\Psi})(d \tau / d z) d z+$ higher order terms.

where factors such as the integrated visibility function have been ignored for simplicity. The first term is the Sachs-Wolfe effect and, in the case of the CMB, will give a redshift to the photons as they climb out of potential wells at the surface of last scattering. The second term is the ISW effect, and depends on the time dependence of the gravitational potentials along the line of sight, as advertised above.

- Weak lensing: Lensing arise when photon light rays are deflected due to the gravitational potentials along the line of sight. The deflection angle is given by $\delta \vec{\theta}=-\vec{\nabla}_{\perp}(\Phi+\Psi) d \tau$, and allows us to relate the true position, $\vec{\theta}_{\text {true }}$, to the deflected position, $\vec{\theta}_{\text {def }}$, via $\vec{\theta}_{\text {true }}=\vec{\theta}_{\text {def }}-\frac{r_{L S}}{r_{L}} \delta \vec{\theta}$, where $r_{L}\left(r_{L S}\right)$ is the distance to the lens (between the lens and the source). In practise we probe the gradient of the deflection through the inverse magnification matrix:

$$
\mathbf{M}^{-1}=\frac{\partial \vec{\theta}_{\text {true }}}{\partial \vec{\theta}_{\text {def }}}=\mathbf{I}+\int_{0}^{z_{S}} \frac{r_{L} r_{L S}}{r_{S}} \vec{\nabla}_{\perp} \vec{\nabla}_{\perp}(\Phi+\Psi) d \tau .
$$

This two by two matrix is parameterised by the convergence, $K$, and shear parameters $\gamma_{1}$ and $\gamma_{2}$. In the case of small deflections this gives

$$
\mathbf{M}=\left(\begin{array}{cc}
1+K+\gamma_{1} & \gamma_{2} \\
\gamma_{2} & 1+K-\gamma_{1}
\end{array}\right)
$$

This information can be extracted from imaging surveys of distant galaxies. The galaxy shapes (or ellipticities) will be distorted by the intervening gravitational potentials. These distortions will induce correlations between the galaxy shapes that will reflect the underlying cosmology. Lensing will, of course, also affect the CMB photons as they pass through potential wells.

\subsubsection{The evidence for the $\Lambda C D M$ model}

There is currently a consensus that in an FLRW Universe that is governed by Einstein's field equations, roughly $95 \%$ of the overall energy density must be 'dark' in order to be compatible with observations. The current best fit model claims that about $25 \%$ of this dark material is in the form of a non-relativistic, non-interacting form of matter called dark matter, and that the remaining $70 \%$ is in the form of a non-clustering form of energy density with a negative equation of state known as dark energy.

The broad case for Cold Dark Matter (CDM) is as follows [1028]:

- The rotation curves of galaxies tend to flatten out at large radii. This flattening can be explained if the baryonic part of the galaxy resides in a halo of dark matter with a density profile that falls of as $1 / r^{2}$. 
- Clusters of galaxies appear to have deeper potential wells than would be inferred from baryonic matter. This is manifest in the motions of galaxies, as well as the X-ray temperature of gas, and weak lensing measurements of the integrated gravitational potentials. Dark matter halos surrounding clusters explain all these observations.

- Diffusion damping during recombination is expected to wipe out all small-scale structure in baryons, preventing the formation of galaxies at late times. Dark matter, however, can sustain structure during the damping regime, and will seed the formation of galaxies.

The case for dark energy has been around since the early 1980s. After the proposal of the original models of inflation, the idea that the Universe should have Euclidean spatial geometry became ever more entrenched in the standard lore. Given that baryons made up a small fraction of the total energy budget, and that dark matter makes up about $25 \%$, there was clearly a shortfall of pressureless matter at late times. Furthermore, estimates of the ages of globular clusters of around 12-14 billion years were incompatible with a flat, matter dominated universe [1228.

There was also tentative evidence from large-scale clustering that a flat, cold dark matter dominated Universe could not explain some of the observations. Most notably, an analysis of the APM galaxy catalogue in [463] seemed to show that a Universe with a cosmological constant might explain the amount of galaxy clustering on a wide range of scales. Now, with the advent of what has been dubbed "precision cosmology" in the late 1990s, the evidence for dark energy has become even more compelling. In particular, the following results make a strong case for presence of an energy density with negative equation of state:

- Measurements of the luminosity distance of type Ia supernovae are consistent with a universe with a cosmological constant, and inconsistent with a flat, matter dominated universe or an open universe 1034, 1063. The latest results seem to constrain the equation of state, $w=P / \rho \simeq-1.068_{-0.082}^{+0.080}$ [330, 1190].

- Measurements of the CMB anisotropies from large to small scales [732, 442, combined with measurements of galaxy clustering from the Sloan Digital Sky Survey (SDSS) [1058, greatly favour a model with $\Omega_{\Lambda}=0.725 \pm 0.016$ and $w=$ $-1.10 \pm 0.14$.

- The cross correlation between the ISW effect from the CMB and a variety of surveys of large-scale structure favour $w=-1.01_{-0.40}^{+0.30}$, at around $4 \sigma$ [534, 611].

- The number density of clusters of galaxies as a function of redshift disfavour a flat, matter dominated universe. The presence of massive clusters at high redshift point accelerating expansion out to redshift $z \simeq 232$.

Although each individual observation may be subject to a variety of interpretations, and different systematic effects, the overall concordance is remarkable. Indeed, the model that best fits these observations is now known as the concordance model, or $\Lambda$ CDM. 


\subsubsection{Shortcomings of the $\Lambda C D M$ model}

Perhaps the most serious problem with $\Lambda \mathrm{CDM}$ is the cosmological constant problem: That the observed value of $\Lambda$ is around 120 orders of magnitude smaller than the naive expectation that it should be of the Planck Mass, $M_{\mathrm{Pl}}^{4}$. Super-Symmetric (SUSY) theories can lower this expectation to that of the SUSY breaking scale, but this still required a bare $\Lambda_{0}$ to cancel the vacuum energy coming from the SUSY symmetry breaking scale to about 60 decimal places. One could consider arguing that some unknown physics at high energies may provide a mechanism for achieving this level of fine-tuning, but this seems unlikely as the problem already manifests itself at low energies.

Now, suppose that we want to describe all physics up to scales just above the electron mass. Then the contribution to the vacuum energy $\Lambda$ will include a bare term $\Lambda_{1}$, a term coming from the electron and a term coming from the neutrino. This is schematically given by

$$
\Lambda=\Lambda_{1}+c_{e} m_{e}^{4}+c_{\nu} m_{\nu}^{4} \ldots,
$$

where $c_{e}$ and $c_{\nu}$ are coefficients. If we now lower the energy below the electron mass, and integrate out the electron, we instead have

$$
\Lambda=\Lambda_{0}+c_{\nu} m_{\nu}^{4} \ldots
$$

for a new bare term $\Lambda_{0}$. To get the same observable vacuum energy, $\Lambda$, we must now have that $\Lambda_{1}$ and $\Lambda_{0}$ cancel to 32 decimal places.

It may be thought that there could exist some mechanism that relaxes the effective cosmological constant ${ }^{14}$ to zero dynamically, but Weinberg 1265 has shown that this is impossible. Suppose that there is a set of $N$ scalars, $\phi^{A}$, that are responsible for driving the effective $\Lambda$ to zero. These scalars will contribute an effective potential, $V\left(\phi^{A}\right)$, to the cosmological constant. If we are to approach a global Minkowski metric at these energy levels, then $V\left(\phi^{A}\right)$ must cancel the other contributions to $\Lambda$ to high accuracy as the fields settle to the minimum. However, this is hardly a readjustment mechanism: If the cosmological constant changes slightly, then the mechanism fails. This proof assumes Poincaré invariance in the scalar sector, which could, however, be considered an unnecessary assumption (see Horndeski's theory in Section 3.1.3.

The present value of $\Lambda$, as implied by cosmological observations, has another potential problem associated with it: It has an energy density of the same order of magnitude as the average matter density in the Universe today,

$$
\left.\left.\rho_{\Lambda}\right|_{a=1} \sim \rho_{m}\right|_{a=1}
$$

These two quantities scale with the size of the Universe in very different ways, and so their similarity at the present time appears naively to be somewhat of a coincidence. Hence, this problem is sometimes referred to as the coincidence problem.

Aside from the problems of the cosmological constant, there are some problems that plague dark matter as well. The first is another coincidence problem: Why is the dark matter energy density so close to the baryon energy density? This is actually worse than it might seem. Baryons are produced non-thermally, out of equilibrium. CDM is

\footnotetext{
${ }^{14}$ By effective cosmological constant we mean the Ricci curvature of the vacuum.
} 
usually thought to be produced thermally, as weak interaction cross-sections naturally give rise to the right dark matter abundance via thermal production. But how can two components that have very different production mechanisms have very similar energy densities ${ }^{15}$ ? Solutions to this puzzle have been proposed [91, 679, 1205, 699, 680, 365, [210, 587, 586, 876, 27. but they typically require additional particles to those that form the dark matter, and there is as yet no well accepted mechanism.

Other problems with dark matter are observational, and we will discuss them only briefly. The density profile of CDM, as determined from N-body simulations, is inferred to be cuspy. For example the Navarro-Frenk-White (NFW) profile [946] gives $\rho_{C D M} \propto \frac{1}{r}$ close to the centre of a halo. Other simulations give similar results: $\rho_{C D M} \propto r^{-\alpha}$ with $\alpha \sim 0.7-1.5$. Galaxies, however, are observed to have cores such that $\rho$ flattens out at the centre. This is the cusp problem [366] and proposed solutions within the CDM paradigm include self-interacting dark matter [1174], fuzzy dark matter [628], or various feedback processes that expel dark matter. Note that simulations do not have enough resolution to probe the small scales where the problem manifests itself, but rely instead on extrapolations. However, simulations with increasingly smaller resolutions (although still above the probed scales) have not indicated any kind of alleviation to the cusp problem.

Another problem is that of missing satellites 867, 211. The CDM paradigm predicts a rich sub-structure within the main galactic halo that should lead to numerous dwarf galaxies orbiting the main galaxy. Indeed, simulations indicate that about 500 satellite galaxies should be orbiting the Milky way [909]. On the contrary, however, only about 30 such dwarfs have been observed. A possible resolution within the CDM paradigm is that most of these galaxies are dark galaxies, i.e. have very little or no stars in them, and are instead completely dominated by dark matter [1146.

A third problem is the tight correlation between dark matter and baryons in galaxies that manifests itself in a universal acceleration scale, $a_{0} \sim 1.2 \times 10^{-10} \mathrm{~m} \mathrm{~s}^{-2}$ [895, 1094, the Tully-Fisher relation [879, 878], and the Faber-Jackson relation [1092]. Within the CDM paradigm, such correlations are not expected to be present, as baryons should not know how the dark matter behaves. For further apparent discrepancies between $\Lambda$ CDM and small scale observations the reader is referred to [756.

On cluster scales and larger, the $\Lambda \mathrm{CDM}$ model can boast of success coming from a host of observations: Strong and weak lensing of clusters, X-ray observations of clusters, the CMB angular power spectrum, the matter power spectrum, $P(k)$, and supernova data. Yet there are a few cases of interesting discrepancies. The collision velocity of the bullet cluster 318 may be so large that the probability of it occurring in a $\Lambda$ CDM scenario is at best $\sim 10^{-9}[780$. In [510, however, the opposite conclusion is reached, so this appears far from settled. Cosmological voids seem to be more empty of galaxies than expected, as has been championed by Peebles [1030. The CMB angular power spectrum has a lack of large-scale power above $60^{\circ}$ [1172] (although the statistical significance of this is debatable, due to cosmic variance). Certain violations of statistical isotropy or other anomalies on large scales in the CMB have also been reported [591, 337, 1294]. It remains to be seen whether these are really problems with $\Lambda \mathrm{CDM}$, if they are due to

\footnotetext{
${ }^{15}$ There are also non-thermal candidates for dark matter, e.g. axions, but this does not change the argument.
} 
systematic effects, or if they are statistical flukes. These difficulties do, however, provide some motivation for looking at alternatives to $\Lambda$ CDM. 


\section{Alternative Theories of Gravity with Extra Fields}

In General Relativity the gravitational force is mediated by a single rank-2 tensor field, or a massless spin-2 particle in the quantum field theory picture. While there is good reason to couple matter fields to gravity in this way, there is less reason to think that the field equations of gravity should not contain other fields, and one is in general free to speculate on the existence of such additional fields in the gravitational sector. The simplest scenario that one could consider in this context is the addition of an extra scalar field, but one might also choose to consider extra vectors, tensors, or even higher rank fields [511, 1247. Of course, the effect of such additional fields needs to be suppressed at scales where General Relativity has been well tested, such as in the lab or solar system. This is usually achieved making couplings very weak, although novel screening mechanisms such as the chameleon mechanism [689, 688] and Vainshtein mechanism [1241] have also been explored.

This section represents an overview of four-dimensional gravity theories with extra fields, focusing on additional scalars, vectors and tensors. We note that some theories in other sections of this review can also be considered as theories with extra fields (e.g. $f(R)$ gravity, galileons, and ghost condensates). The reader is referred to later sections for details of this.

\subsection{Scalar-Tensor Theories}

The scalar-tensor theories of gravity are some of the most established and well studied alternative theories of gravity that exist in the literature. They are often used as the prototypical way in which deviations from General Relativity are modelled, and are of particular interest as the relatively simple structure of their field equations allow exact analytic solutions to be found in a number of physically interesting situations. Scalartensor theories arise naturally as the dimensionally reduced effective theories of higher dimensional theories, such as Kaluza-Klein and string models. They are also often used as simple ways to self-consistently model possible variations in Newton's constant, $G$.

\subsubsection{Action, field equations, and conformal transformations}

A general form of the scalar-tensor theory can be derived from the Lagrangian density $142,988,1253$.

$$
\mathcal{L}=\frac{1}{16 \pi} \sqrt{-g}\left[f(\phi) R-g(\phi) \nabla_{\mu} \phi \nabla^{\mu} \phi-2 \Lambda(\phi)\right]+\mathcal{L}_{m}\left(\Psi, h(\phi) g_{\mu \nu}\right),
$$

where $f, g, h$ and $\Lambda$ are arbitrary functions of the scalar field $\phi$ and $\mathcal{L}_{m}$ is the Lagrangian density of the matter fields $\Psi$. The function $h(\phi)$ can be absorbed into the metric by a conformal transformation of the form [422]

$$
h(\phi) g_{\mu \nu} \rightarrow g_{\mu \nu} .
$$

The conformal frame picked out by this choice is one in which there is no direct interaction between the scalar field and matter fields, and is usually referred to as the Jordan frame. As discussed in previous sections, test-particles in this conformal frame follow geodesics of the metric to which they are coupled, and the weak equivalence principle is satisfied for massless test particles. The effect of this transformation on the remainder of the 
Lagrangian can then be absorbed into redefinitions of the as yet unspecified functions $f$, $g$ and $\Lambda$.

By a redefinition of the scalar field $\phi$ we can now set $f(\phi) \rightarrow \phi$, without loss of generality. The Lagrangian density (95) can then be written as

$$
\mathcal{L}=\frac{1}{16 \pi} \sqrt{-g}\left[\phi R-\frac{\omega(\phi)}{\phi} \nabla_{\mu} \phi \nabla^{\mu} \phi-2 \Lambda(\phi)\right]+\mathcal{L}_{m}\left(\Psi, g_{\mu \nu}\right),
$$

where $\omega(\phi)$ is an arbitrary function, often referred to as the 'coupling parameter', and $\Lambda$ is a $\phi$-dependent generalisation of the cosmological constant. This theory reduces to the well known Brans-Dicke theory [184 in the limit $\omega \rightarrow$ constant and $\Lambda \rightarrow 0$, and approaches General Relativity with a cosmological constant in the limit $\omega \rightarrow \infty$, $\omega^{\prime} / \omega^{2} \rightarrow 0$ and $\Lambda \rightarrow$ constant.

The variation of the action derived from integrating (97) over all space, with respect to $g^{\mu \nu}$, gives the field equations

$$
\phi G_{\mu \nu}+\left[\square \phi+\frac{1}{2} \frac{\omega}{\phi}(\nabla \phi)^{2}+\Lambda\right] g_{\mu \nu}-\nabla_{\mu} \nabla_{\nu} \phi-\frac{\omega}{\phi} \nabla_{\mu} \phi \nabla_{\nu} \phi=8 \pi T_{\mu \nu} .
$$

Now, as well as the metric tensor $g_{\mu \nu}$, these theories also contain the dynamical scalar field $\phi$, and so we must vary the action derived from Eq. (97) with respect to this additional degree of freedom. After eliminating $R$ with the trace of $(98)$, this yields

$$
(2 \omega+3) \square \phi+\omega^{\prime}(\nabla \phi)^{2}+4 \Lambda-2 \phi \Lambda^{\prime}=8 \pi T .
$$

where primes here denote differentiation with respect to $\phi$. These are the field equations of the scalar-tensor theories of gravity.

It is well known that these theories admit the very useful property of being 'conformally equivalent' to General Relativity. By this we mean that under a transformation of the metric that alters scales, but not angles, one can find a new metric that obeys the Einstein equation, with the scalar contributing as an ordinary matter field. This does not, however, mean that scalar-tensor theories are the same as General Relativity, as the metric that couples to matter fields must also transform. The theory that is recovered after conformally transforming is one in which the metric obeys a set of fields equations similar to Einstein's, but with an unusual matter content that does not follow geodesics of the new metric (with the exception of radiation fields, or null geodesics, which are themselves conformally invariant). This property of scalar-tensor theories can sometimes allow their field equations to be manipulated into more familiar forms, that allow solutions to be found more readily.

To be explicit, a conformal transformation of the metric $g_{\mu \nu}$ into $\bar{g}_{\mu \nu}$ can be written

$$
g_{\mu \nu}=e^{2 \Gamma(x)} \bar{g}_{\mu \nu}
$$

where $\Gamma(x)$ is an arbitrary function of the space-time coordinates $x^{\mu}$. The line-element is then correspondingly transformed as $d s^{2}=e^{2 \Gamma(x)} d \bar{s}^{2}$, and the square root of the determinant of the metric as $\sqrt{-g}=e^{4 \Gamma} \sqrt{-\bar{g}}$ (in four dimensions). After performing such a transformation we can use the term 'conformal frame' to distinguish the new, rescaled metric from the original. 
Among the infinite possible conformal frames we can then identify two which have particular significance: The Jordan frame and the Einstein frame. The Jordan frame is the one in which the energy-momentum tensor is covariantly conserved and in which testparticles follow geodesics of the metric. This is the frame picked out by the transformation (96), and is the one in which scalar-tensor theories are most usually formulated. The Einstein frame is the conformal frame in which the field equations of the theory take the form of the Einstein equations with the scalar contributing as an ordinary scalar field, as discussed above.

Under the transformation 100 it can be shown that the Ricci tensor and Ricci scalar transform as

$$
\begin{aligned}
R_{\mu \nu} & =\bar{R}_{\mu \nu}-2 \bar{\nabla}_{\mu} \bar{\nabla}_{\nu} \Gamma+2 \bar{\nabla}_{\mu} \Gamma \bar{\nabla}_{\nu} \Gamma-\left(2 \bar{\nabla}_{\alpha} \Gamma \bar{\nabla}^{\alpha} \Gamma+\bar{\square} \Gamma\right) \bar{g}_{\mu \nu} \\
e^{2 \Gamma} R & =\bar{R}-6 \bar{\nabla}_{\mu} \Gamma \bar{\nabla}^{\mu} \Gamma-6 \bar{\square} \Gamma
\end{aligned}
$$

while the d'Alembertian transforms as $e^{2 \Gamma} \square \phi=\bar{\square} \phi+2 \bar{\nabla}_{\mu} \Gamma \bar{\nabla}^{\mu} \phi$. Here, over-bars on operators or indices denote that they are defined using the metric $\bar{g}_{\mu \nu}$. Under these transformations we will now show how the scalar-tensor theories defined by the Lagrangian (97), in the Jordan frame, can all be transformed into the Einstein frame.

First, consider the term in Eq. (97) containing the Ricci scalar, which under the conformal transformation 100 becomes

$$
\mathcal{L}_{1}=\frac{1}{16 \pi} \sqrt{-\bar{g}} \phi e^{2 \Gamma}\left(\bar{R}-6 \bar{g}^{\mu \nu} \Gamma_{, \mu} \Gamma_{, \nu}-6 \bar{\square} \Gamma\right) .
$$

The non-minimal coupling to the Ricci scalar can now be removed by making the choice of conformal factor $e^{2 \Gamma}=\phi^{-1}$ such that $g_{\mu \nu}=\bar{g}_{\mu \nu} / \phi$. This defines the conformal transformation between Jordan and Einstein frames in the scalar-tensor theories. Applying the transformation (100) to the rest of Eq. (97) then gives

$$
\mathcal{L}=\frac{1}{16 \pi} \sqrt{-\bar{g}}\left(\bar{R}-2(3+2 \omega) \bar{\nabla}_{\mu} \Gamma \bar{\nabla}^{\mu} \Gamma-2 e^{4 \Gamma} \Lambda\right)+\mathcal{L}_{m}\left(\Psi, e^{2 \Gamma} \bar{g}_{\mu \nu}\right) .
$$

Now, by making the definitions $\sqrt{4 \pi /(3+2 \omega)} \equiv \partial \Gamma / \partial \psi$ and $8 \pi V(\psi) \equiv e^{4 \Gamma} \Lambda$, for the scalar $\psi$ and the function $V(\psi)$, we can write the transformed Lagrangian (104) as

$$
\mathcal{L}=\frac{1}{16 \pi} \sqrt{-\bar{g}} \bar{R}-\sqrt{-\bar{g}}\left(\frac{1}{2} \bar{\nabla}_{\mu} \psi \bar{\nabla}^{\mu} \psi+V(\psi)\right)+\mathcal{L}_{m}\left(\Psi, e^{2 \Gamma} \bar{g}_{\mu \nu}\right) .
$$

In the absence of any matter fields the scalar-tensor theories can now be clearly seen to be conformally related to Einstein's theory in the presence of a scalar field in a potential. This potential disappears when $\Lambda=0$

In the Brans-Dicke theory 184 the coupling parameter $\omega$ is constant, and the scalar fields $\phi$ and $\psi$ are therefore related by

$$
\ln \phi=\sqrt{\frac{16 \pi}{(3+2 \omega)}} \psi
$$

For more general theories with $\omega=\omega(\phi)$ the definition of $\psi$ must be integrated to obtain a relation between $\phi$ and $\psi$. By extremising the action 105 with respect to $\bar{g}_{\mu \nu}$ and $\psi$ 
we get the Einstein frame field equations

$$
\bar{G}_{\mu \nu}=8 \pi\left[\bar{T}_{\mu \nu}+\bar{\nabla}_{\mu} \psi \bar{\nabla}_{\nu} \psi-\left(\frac{1}{2} \bar{\nabla}_{\alpha} \psi \bar{\nabla}^{\alpha} \psi+V\right) \bar{g}_{\mu \nu}\right]
$$

and

$$
\tilde{\square} \psi-\frac{d V}{d \psi}=-\sqrt{4 \pi} \alpha \tilde{T}
$$

where $\alpha^{-2}=3+2 \omega$ and where we have defined the energy-momentum tensor $\bar{T}_{\mu \nu}$ with respect to $\bar{g}_{\mu \nu}$ so that $\bar{T}^{\mu \nu}=e^{6 \Gamma} T^{\mu \nu}$. It can now be explicitly seen that while the Jordan frame energy-momentum tensor is covariantly conserved, $\nabla_{\mu} T^{\mu \nu}=0$, its counterpart in the Einstein frame is not, $\bar{\nabla}_{\mu} \bar{T}^{\mu \nu}=\sqrt{4 \pi} \alpha \bar{T} \bar{\nabla}^{\nu} \psi$.

\subsubsection{Brans-Dicke theory}

The Brans-Dicke theory is given by the Lagrangian density (97) with $\omega=$ constant, and $\Lambda=0$ [184]. The behaviour of this theory in the vicinity of isolated masses is well understood, and in the case of static and spherical symmetry can be solved exactly by the line-element 185 .

$$
d s^{2}=-e^{2 \alpha} d t^{2}+e^{2 \beta}\left(d r^{2}+r^{2} d \Omega^{2}\right)
$$

where $\alpha=\alpha(r)$ and $\beta=\beta(r)$ are given by one of the following four solutions:

I

$$
\begin{aligned}
e^{\alpha} & =e^{\alpha_{0}}\left[\frac{1-\frac{B}{r}}{1+\frac{B}{r}}\right]^{\frac{1}{\lambda}} \\
e^{\beta} & =e^{\beta_{0}}\left(1+\frac{B}{r}\right)^{2}\left[\frac{1-\frac{B}{r}}{1+\frac{B}{r}}\right]^{\frac{(\lambda-C-1)}{\lambda}} \\
\phi & =\phi_{0}\left[\frac{1-\frac{B}{r}}{1+\frac{B}{r}}\right]^{\frac{C}{\lambda}}
\end{aligned}
$$

II

$$
\begin{aligned}
& \alpha=\alpha_{0}+\frac{2}{\Lambda} \tan ^{-1}\left(\frac{r}{B}\right) \\
& \beta=\beta_{0}-\frac{2(C+1)}{\Lambda} \tan ^{-1}\left(\frac{r}{B}\right)-\ln \left[\frac{r^{2}}{\left(r^{2}+B^{2}\right)}\right] \\
& \phi=\phi_{0} e^{\frac{2 C}{\Lambda} \tan ^{-1}\left(\frac{r}{B}\right)}
\end{aligned}
$$

III

$$
\alpha=\alpha_{0}-\frac{r}{B}
$$$$
\beta=\beta_{0}-2 \ln \left(\frac{r}{B}\right)+\frac{(C+1) r}{B}
$$

IV

$$
\begin{aligned}
\phi & =\phi_{0} e^{-\frac{C r}{B}} \\
\alpha & =\alpha_{0}-\frac{1}{B r} \\
\beta & =\beta_{0}+\frac{(C+1)}{B r} \\
\phi & =\phi_{0} e^{-\frac{C}{B r}} .
\end{aligned}
$$


Here we have defined $\lambda^{2} \equiv(C+1)^{2}-C(1-\omega C / 2)>0$ in solution I, and $\Lambda^{2} \equiv$ $C(1-\omega C / 2)-(C+1)^{2}>0$ in solution II. The constant $C$ is arbitrary in I and II, and given by $C=(-1 \pm \sqrt{-2 \omega-3}) /(\omega+2)$ in III and IV. The constants $B, \alpha_{0}, \beta_{0}$ and $\phi_{0}$ are arbitrary throughout.

Now, while solution $I$ is valid for all values of $\omega$, solutions $I I, I I I$ and $I V$ are only valid for $\omega<-3 / 2$. Solution $I$ is also known to be conformally related to the minimally coupled massless scalar field solution of Buchdahl 207. It can be seen that these solutions are not all independent of each other. By a transformation of the form $r \rightarrow 1 / r$ and some redefinition of constants, solution $I I$ can be transformed into the $\omega<-3 / 2$ range of solution $I$ [152 and solution $I I I$ can be transformed into solution $I V$ [153]. It was also shown in [152] that the independent solutions $I$ and $I V$ are both conformally related to the general solution of the static, spherically symmetric case in the Einstein frame, as found by Wyman 1289 .

These solutions are very useful for understanding the gravitational fields around an isolated body in Brans-Dicke theory, but are not the only spherically symmetric vacuum solutions of the Brans-Dicke field equations. A non-static spherically symmetric vacuum exact solution is also known [313:

$$
\begin{aligned}
d \bar{s}^{2}=-A(r)^{\alpha\left(1-\frac{1}{\sqrt{3} \beta}\right)} d t^{2} & \\
+ & A(r)^{-\alpha\left(1+\frac{1}{\sqrt{3} \beta}\right)} t^{\frac{2(\beta-\sqrt{3})}{3 \beta-\sqrt{3}}}\left[d r^{2}+A(r) r^{2}\left(d \theta^{2}+\sin ^{2} \theta d \phi^{2}\right)\right],
\end{aligned}
$$

with

$$
\phi(r, t)=\left(1-\frac{2 C}{r}\right)^{ \pm \frac{1}{2 \beta}} t^{2 /(\sqrt{3} \beta-1)}
$$

where we have $A(r)=1-\frac{2 C}{r}, \alpha= \pm \frac{\sqrt{3}}{2}, \beta=\sqrt{2 \omega+3}$, and $C=$ constant. This solution reduces to a flat vacuum FLRW metric in the limit $C \rightarrow 0$ (an inhomogeneous solution requires $C \neq 0$ ). The metric $(108)$ is spatially homogeneous at large $r$ and has singularities at $t=0$ and $r=2 C$; the coordinates $r$ and $t$ therefore cover the ranges $0 \leq t<\infty$ and $2 C \leq r<\infty$. This solution is known to be conformally related to 635, and shows explicitly the lack of validity of Birkhoff's theorem in Brans-Dicke theory. It also reduces to the Schwarzschild solution when $\omega \rightarrow \infty$. Black hole solutions in Brans-Dicke theory with a power-law potential have been investigated in 850.

Let us now consider the weak field limit of this theory. Following the PPN prescription outlined in previous sections one can straightforwardly find that the relevant values for the PPN parameters are:

$$
\beta_{P P N}=1 \quad \text { and } \quad \gamma_{P P N}=\frac{1+\omega}{2+\omega},
$$

with all other parameters equalling zero. The value of Newton's constant can also be shown to be given by

$$
G=\left(\frac{4+2 \omega}{3+2 \omega}\right) \frac{1}{\phi_{0}}
$$

where $\phi_{0}$ is the background (unperturbed) value of the scalar field. It can be seen that in the general relativistic limit $\omega \rightarrow \infty$ we then recover the usual values of the 
PPN parameters, and that for finite $\omega$ the only parameter that deviates from its general relativistic value is $\gamma$.

This value of $\gamma$ is valid for both the static and non-static exact solutions shown above. It is interesting to note, however, that it is not the value of $\gamma$ that one should expect to measure outside of a black hole that has formed from gravitational collapse in this theory. Such an object can be shown to have an external gravitational field with $\gamma=1$ [1107], as predicted by Hawking [595]. This does not, however, mean that gravitational collapse to a black hole proceeds in the same way in Brans-Dicke theory as it does in General Relativity. In the Brans-Dicke case apparent horizons are allowed to pass outside of the event horizon, scalar gravitational waves are emitted during the collapse, and the surface area of the event horizon can decrease with time. Such behaviour does not occur in General Relativity, and is allowed here because Brans-Dicke theory can violate the condition $R_{\mu \nu} k^{a} k^{\nu} \geq 0$, where $k^{a} k_{a}=0$. The problem of understanding black hole thermodynamics in Brans-Dicke theory has been addressed in 674]. Here it was found that the expression for the entropy of a black hole with an horizon $\Sigma$ of area $A$ is given by

$$
S_{B H}=\frac{1}{4} \int_{\Sigma} d^{2} x \sqrt{g^{(2)}} \phi=\frac{\phi A}{4},
$$

such that $S_{B H}$ is always non-decreasing, even if the area decreases. This shows that the second law of black hole thermodynamics can indeed be extended to Brans-Dicke theories, with the effective gravitational constant being replaced by $1 / \phi$. For an intuitive interpretation of this result in the Einstein frame, and for further discussion on this topic, the reader is referred to [489].

Having discussed the gravitational fields of point-like objects in Brans-Dicke theory, let us now proceed to use observations of weak field phenomena to constrain the theory. This can be done most effectively using the constraint on $\gamma$ given in Equation (71), derived from observations of the time delay of radio signals from the Cassini spacecraft as it passed behind the Sun. Together with the expression (110), shown above, this gives the $2 \sigma$ constraint on the coupling parameter

$$
\omega \gtrsim 40000 .
$$

This is a very restricting constraint on the theory, and shows that deviations of this kind from General Relativity must be very small indeed (see the following subsection, however, for a discussion of scalar-tensor theories that can evade this bound while still exhibiting significantly different behaviour to General Relativity in the early universe).

Let us now proceed to discuss the cosmology of Brans-Dicke theory. Using the usual FLRW line-element, and assuming a perfect fluid matter content, the field equations reduce to:

$$
\begin{aligned}
H^{2} & =\frac{8 \pi \rho}{3 \phi}-\frac{\kappa}{a^{2}}-H \frac{\dot{\phi}}{\phi}+\frac{\omega}{6} \frac{\dot{\phi}^{2}}{\phi^{2}} \\
\frac{\ddot{\phi}}{\phi} & =\frac{8 \pi}{\phi} \frac{(\rho-3 P)}{(2 \omega+3)}-3 H \frac{\dot{\phi}}{\phi}
\end{aligned}
$$

where over-dots denote differentiation with respect to the proper time of a comoving observer, $H=\dot{a} / a$, and $\dot{\rho}+3 H(\rho+P)=0$. The general solutions to Eq. 114 and 1115 
are now fully understood [576, 95]. At early times the vacuum solutions of O'Hanlon and Tupper 993] are recovered, while at late-times one approaches the power-law solutions of Nariai 938] (when $\kappa=0$ ):

$$
\begin{aligned}
& a(t)=t^{2[1+\omega(1-W)] /\left[4+3 \omega\left(1-W^{2}\right)\right]} \\
& \phi(t)=\phi_{0} t^{[2(1-3 W)] /\left[4+3 \omega\left(1-W^{2}\right)\right]},
\end{aligned}
$$

wher $\varepsilon^{16} p=W \rho$. These solutions can be considered "Machian" in the sense that the matter fields are driving the expansion of the Universe, rather than $\phi$.

Let us now consider the general FLRW solutions in terms of a transformed time coordinate $\eta=\eta(t)$. Such solutions can be found any equations of state $W$ [576], but here let us consider only the radiation dominated solutions with $W=1 / 3$. In this case the new time coordinate $\eta$ is simply the conformal time $\tau$ given by $a d \tau \equiv d t$, and the general solution for $\omega>-3 / 2$ is

$$
\begin{aligned}
& a(\tau)=a_{1}\left(\tau+\tau_{+}\right)^{\frac{1}{2}+\frac{1}{2 \sqrt{1+\frac{2}{3} \omega}}}\left(\tau+\tau_{-}\right)^{\frac{1}{2}-\frac{1}{2 \sqrt{1+\frac{2}{3} \omega}}} \\
& \phi(\tau)=\phi_{1}\left(\tau+\tau_{+}\right)^{-\frac{1}{2 \sqrt{1+\frac{2}{3} \omega}}}\left(\tau+\tau_{-}\right)^{+\frac{1}{2 \sqrt{1+\frac{2}{3} \omega}}}
\end{aligned}
$$

where $\tau_{ \pm}, a_{1}$ and $\phi_{1}$ are integration constants, and where $8 \pi \rho_{r 0} / 3 \phi_{1} a_{1}^{2}=1$. For $\omega<$ $-3 / 2$, however, we instead find

$$
\begin{aligned}
& a(\tau)=a_{1} \sqrt{\left(\tau+\tau_{-}\right)^{2}+\tau_{+}^{2}} \exp \left(\frac{-1}{\sqrt{\frac{2}{3}|\omega|-1}} \tan ^{-1} \frac{\tau+\tau_{-}}{\tau_{+}}\right), \\
& \phi(\tau)=\phi_{1}\left(\frac{2}{\sqrt{\frac{2}{3}|\omega|-1}} \tan ^{-1} \frac{\tau+\tau_{-}}{\tau_{+}}\right) .
\end{aligned}
$$

For $\omega>-3 / 2(\omega<-3 / 2)$ we see that the scale factor here undergoes an initial period of rapid (slow) expansion and at late times is attracted towards the solution $a(\tau) \propto \tau$, or, equivalently, $a(t) \propto t^{\frac{1}{2}}$. Similarly, $\phi$ can be seen to be changing rapidly at early times and slowly at late times. These two different behaviours, at early and late times, can be attributed to periods of free scalar-field domination and radiation domination, respectively. If $\rho_{r 0}=0$ is chosen then these solutions become vacuum ones that are driven by the $\phi$ field alone, and for $\omega<-3 / 2$ the initial singularity can be seen to be avoided. Corresponding behaviour can also be shown to exist for other equations of state, $W$. For a more detailed discussion of this phenomenon we refer the reader to [105].

Unlike in General Relativity, in the Brans-Dicke theory it is also possible to have spatially flat and positively curved exact vacuum solutions. Spatially flat solutions can

\footnotetext{
${ }^{16}$ Note that in this section we use an upper case $W$ to denote the equation of state of the fluid, rather than the usual lower case $w$ used in the rest of the review. This is to avoid confusion with the coupling parameter $\omega$.
} 
be found by assuming $\phi \propto t^{x}$ and $a \propto t^{y}$, and by setting $a(0)=0$. When $\kappa=0$ the vacuum Brans-Dicke equations are then solved by 993 .

$$
\begin{aligned}
& a(t) \propto t^{\frac{1}{3}\left(1+2(1-\sqrt{3(3+2 \omega)})^{-1}\right)}, \\
& \phi(t) \propto\left(\frac{t}{t_{0}}\right)^{-2(1-\sqrt{3(3+2 \omega)})^{-1}} .
\end{aligned}
$$

For spatially closed solutions one can follow the method prescribed in 95]. Here one defines a new quantity $y \equiv \phi a^{2}$, and uses the conformal time coordinate $\tau$, to write the field equations as

$$
(\ln \phi),_{\tau}=\sqrt{3} A y^{-1}(2 \omega+3)^{-1 / 2} \quad \text { and } \quad y_{\tau}^{2}=-4 \kappa y^{2}+A^{2},
$$

where $A$ is a constant. For $\kappa>0$ these equations can be integrated to find $y=$ $(A / 2 \sqrt{\kappa}) \sin \left(2 \sqrt{\kappa}\left(\tau-\tau_{0}\right)\right)$, which then gives the solutions

$$
\begin{aligned}
& \phi(\tau) \propto \tan \sqrt{\frac{3}{(2 \omega+3)}}\left(\sqrt{\kappa}\left(\tau-\tau_{0}\right)\right), \\
& a(\tau) \propto \frac{\sin ^{1 / 2}\left(2 \sqrt{\kappa}\left(\tau-\tau_{0}\right)\right)}{\tan \sqrt{\frac{3}{4(2 \omega+3)}}\left(\sqrt{\kappa}\left(\tau-\tau_{0}\right)\right)} .
\end{aligned}
$$

Spatially flat and closed vacuum FLRW solutions such as those shown here do not exist in General Relativity, and show the potential for interesting new behaviour at early times in scalar-tensor theories of gravity. Phase plane analyses of perfect fluid FLRW solutions to the Brans-Dicke field equations have been performed in [731, 1096, 613.

A number of anisotropic cosmological solutions of the Brans-Dicke field equations are also known. Bianchi type- $I$ solutions have been found in 1075, 133, type- $I I$ in 822 , [580, 825, type- $I I I$ solutions in [826], type- $V$ solutions in [821, 823, 827, 828, 581], type$V I_{0}$ and $V I_{h}$ solutions in $829,830,131$, type- $V I I_{h}$ solutions in 824, 579, type-VIII solutions in 825, 820, type- $I X$ solutions in 825, 820, and Kantowski-Sachs solutions in 826. Inhomogeneous cosmological solutions have also been found 313, and braneworld cosmologies have been considered in [75, 1054]. We will not reproduce any of these solutions here, but rather refer the reader to the citations above, and references therein. For a discussion of the cosmic no-hair theorems in Brans-Dicke theory the reader is referred to [582, where it is shown that these theorems are valid without imposing any strong constraints on the coupling constant, $\omega$, so that initially anisotropic universes can evolve towards an isotropic final state.

Now let us consider perturbed FLRW space-times, within which cosmological observations are usually interpreted. For the Brans-Dicke theory these equations have been studied many times before, starting with [939. Here we will present these equations in the synchronous gauge and with $\kappa=0$, as found in 290. In this case the equations take on a simpler form. For the more general case the reader is referred to [934, or to the $\omega=$ constant limit of Eqs. 145- 149 in Section 3.1 .3 for the corresponding equations in the conformal Newtonian gauge. Now, the perturbed metric can be written as

$$
g_{\mu \nu}=\bar{g}_{\mu \nu}+a^{2}(\tau) h_{\mu \nu}
$$

where $a(\tau)$ is the FLRW scale factor, $\bar{g}_{\mu \nu}$ is the unperturbed FLRW metric with $\kappa=0$, and $h_{\mu \nu}$ is the perturbation that satisfies $h_{00}=h_{0 \nu}=0$ in the synchronous gauge. We 
can then proceed as normal, and decompose the remaining non-zero $h_{i j}$ perturbations into harmonic modes, and decouple the scalar, vector and tensor components. The scalar part of the perturbations can be written as in Section 2

$$
h_{i j}=\frac{1}{3} h q_{i j}+D_{i j} \nu,
$$

where the synchronous gauge has been adopted, while $\delta \phi$ is the perturbation to the BransDicke scalar. The $D_{i j}$ operator, as in Section 2 is defined by $D_{i j}=\vec{\nabla}_{i} \vec{\nabla}_{j}-\frac{1}{3} q_{i j} \Delta$. As usual we define $\eta=-\left(h+k^{2} \nu\right) / 6$ (see section 2 ). The perturbed equations are17

$$
\begin{aligned}
-2 k^{2} \eta+\left(\mathcal{H}+\frac{1}{2} \frac{\phi^{\prime}}{\phi}\right) h^{\prime} & =\frac{8 \pi a^{2}}{\phi} \sum_{f} \rho_{f} \delta_{f}+\left(\omega \frac{\phi^{\prime}}{\phi}-3 \mathcal{H}\right) \frac{\delta \phi^{\prime}}{\phi} \\
& -\left[k^{2}+3 \mathcal{H}^{2}+\frac{\omega}{2} \frac{\phi^{\prime 2}}{\phi^{2}}\right] \frac{\delta \phi}{\phi} \\
2 \eta^{\prime} & =\frac{8 \pi a^{2}}{\phi} \sum_{f}\left(\rho_{f}+P_{f}\right) \theta_{f}+\frac{1}{\phi} \delta \phi^{\prime}-\frac{1}{\phi}\left(\mathcal{H}-\omega \frac{\phi^{\prime}}{\phi}\right) \delta \phi \\
\frac{1}{2} \nu^{\prime \prime}+\left(\mathcal{H}+\frac{\phi^{\prime}}{2 \phi}\right) \nu^{\prime}+\eta & =\frac{8 \pi a^{2}}{\phi}(\rho+P) \Sigma_{f}+\frac{\delta \phi}{\phi}
\end{aligned}
$$

and

$$
\delta \phi^{\prime \prime}+2 \mathcal{H} \delta \phi^{\prime}+k^{2} \delta \phi+\frac{1}{2} \phi^{\prime} h^{\prime}=\frac{8 \pi a^{2}}{2 \omega+3} \sum_{f}\left(\delta \rho_{f}-3 \delta P_{f}\right) .
$$

Here the perturbations to the energy density and pressure of the non-interacting fluids $f$ are written as $\delta \rho_{f}$ and $\delta P_{f}$, with the peculiar velocity potentials and anisotropic stress written as $\theta_{f}$ and $\Sigma_{f}$, respectively. Primes denote differentiation with respect to conformal time, $\tau$.

For the tensor modes we can write the metric perturbations as $h_{i j}=\tilde{h}_{T} Q_{i j}$, where $Q_{i j}$ is a harmonic function, and with no tensor component involved in $\delta \phi$. The evolution equation for $\tilde{h}_{T}$ is then given by

$$
\tilde{h}_{T}^{\prime \prime}+2 \mathcal{H} \tilde{h}_{T}^{\prime}+k^{2} \tilde{h}_{T}=\frac{8 \pi a^{2}}{\phi} \sum_{f}\left(\rho_{f}+P_{f}\right) \tilde{\Sigma}_{f}
$$

where $\tilde{\Sigma}_{f}$ is the tensor contribution to the anisotropic stress of the fluid $f$. We will not write the vector perturbation equations here, which are not expected to be significant for most cosmological applications. For perturbation equations written in terms of gauge invariant variables the reader is referred to $[1285$ for the covariant approach, or 934 for the Bardeen variable approach (for the Brans-Dicke theory one should take $\omega=$ constant in this last reference).

The background cosmological evolution and perturbations can be used to place constraints on Brans-Dicke theory from a number of different sources. The CMB is one

\footnotetext{
${ }^{17}$ Various typos in the corresponding equations in 290 have been corrected.
} 
such source, and can be used to place constraints on the coupling parameter, $\omega$. This has been done a number of times in the literature [935, 7, 1284, with the latest results based on constraints given by the WMAP 5 year data, the ACBAR 2007 data, the CBI polarisation data, and the BOOMERanG 2003 flight, together with large-scale structure data from the SDSS data release 4, giving $\omega>97.8$ or $\omega<-120.0$ to $2 \sigma$ [1284. This is in keeping with the results of [7, but significantly weaker than those claimed by [935] of $\omega>1000$ to $2 \sigma$ based on the WMAP first year data. Among the detailed processes that lead to these constraints one can see that the change in the horizon size at matterradiation equality is altered in Brans-Dicke theory due to the different expansion rates 799. This length scale is imprinted on the spectrum of perturbations as during the radiation era perturbations inside the horizon are effectively frozen, while during matter domination perturbations grow on all length scales. Different expansion rates also affect the horizon size at recombination, which affects the level of 'Silk damping' that occurs on small scales due to viscosity and heat conduction. What is more, the thickness of the last scattering surface is also changed, which affects anisotropy on small scales through the exponential damping which has its cutoff determined by this quantity. The upcoming data from the Planck satellite is, of course, expected to tighten the constraints given above still further.

Another cosmological probe that has been extensively applied to Brans-Dicke theory is that of the primordial nucleosynthesis of light elements [1292, 59, 354, 265, 266, 1126, 312. In the Brans-Dicke theory the scalar field $\phi$ is approximately constant during the epoch of radiation domination. Nucleosynthesis therefore proceeds largely as in a general relativistic cosmology (up to the effect of 'kicks' on the scalar field due to the annihilation of electron-positron pairs [358]), but with a different value of $G$ during this process, and hence a different expansion rate. Of course, the time at which weak interactions freeze out in the early universe is determined by equality between the rate of the relevant weak interactions and the Hubble rate. When the weak interaction rate is the greater then the ratio of neutrons to protons it tracks its equilibrium value, while if the Hubble rate is greater than the weak-interaction rate then the ratio of neutrons to protons is effectively 'frozen-in', and $\beta$-decay is the only weak process that still operates with any efficiency. This is the case until the onset of deuterium formation, at which time the neutrons become bound and $\beta$-decay ceases. Now, the onset of deuterium formation is primarily determined by the photon to baryon ratio, $\eta_{\gamma}$, which inhibits the formation of deuterium nuclei until the critical temperature for photodissociation is past. As the vast majority of neutrons finally end up in ${ }^{4} \mathrm{He}$ the primordial abundance of this element is influenced most significantly by the number of neutrons at the onset of deuterium formation, which is sensitive to the temperature of weak-interaction freeze-out, and hence the Hubble rate, and so $G$, at this time. Conversely, the primordial abundances of the other light elements are mostly sensitive to the temperature at deuterium formation, and hence $\eta_{\gamma}$, when nuclear reactions occur and the light elements form. The reader is referred to 83 for further discussion of these points. The typical bounds that can be achieved on the coupling parameter from observations of element abundances are then given by $\omega \gtrsim 300$ or $\omega \lesssim-30$, assuming the power-law solutions (116) and (117). By using the general solutions (118)-(121), however, these bounds can be somewhat relaxed or tightened, depending on the behaviour of $\phi$ in the early universe [312.

While the cosmological bounds discussed above are weaker than those derived in the solar system, and in binary pulsars, they probe a very different physical environment and 
scale. They are therefore usually considered complimentary to the constraints imposed from observations of weak field gravity, and a useful consistency check. After all, one may wish to consider theories in which the coupling parameter $\omega$ varies throughout cosmic history. Theories in which such behaviour can occur explicitly are the subject of Section 3.1 .3

\subsubsection{General scalar-tensor theories}

The Brans-Dicke theory that has so far been considered is a very special scalar-tensor theory, with only a single constant parameter. The more general class of scalar-tensor theories contains two free functions, given by $\omega(\phi)$ and $\Lambda(\phi)$ in Eq. 97). Let us now consider these more general theories.

First of all let us consider the case in which $\Lambda(\phi)=0$. Such theories have been well studied in the literature, and are often used to model the possibility of having a coupling parameter $\omega$ in the early universe that is small enough to have interesting effects, while being large enough in the late universe to be compatible with the stringent bounds imposed upon such couplings by observations of gravitational phenomena in the solar system, and other nearby astrophysical systems. This interest is bolstered by the presence of an attractor mechanism that ensures General Relativity is recovered as a stable asymptote at late times in FLRW cosmology [356]. We will explain this attractor in more detail below.

Of course, in generalising the Brans-Dicke theory we want to know what the consequences are for constraints imposed in the weak field limit. The extra complication caused by allowing $\omega$ to be a function of $\phi$ means that exact solutions are hard to find. Perturbative analyses can still be readily performed, however, leading to the PPN parameters

$$
\beta_{P P N}=1+\frac{d \omega / d \phi}{(4+2 \omega)(3+2 \omega)^{2}} \quad \text { and } \quad \gamma_{P P N}=\frac{1+\omega}{2+\omega},
$$

with all other parameters equalling zero. The value of $\gamma$ here can be seen to be the same as in the Brans-Dicke theory, while the value of $\beta$ reduces to the Brans-Dicke (and General Relativity) value of unity when $\omega=$ constant. Observations from the Cassini satellite therefore place upon $\omega$ the same tight constraint as in Brans-Dicke theory $(\omega \gtrsim 40000$ to $2 \sigma$ ). This constraint, however, now only applies to the local value of $\omega$ (i.e. with the present day value of $\phi$ in the solar system). The variation of $\omega$ with $\phi$ can then be constrained by observations of post-Newtonian phenomena that constrain $\beta$, such as the lunar laser ranging experiments described in previous sections. To constrain $\omega$ for other values of $\phi$, however, requires making observations in other physical environments, such as in the early universe, or near black holes.

Let us now consider the cosmological solutions of these theories. It has been shown by Clarkson, Coley and O'Neill in [303, that the Ehlers-Geren-Sachs theorem can be extended to cover scalar-tensor theories of gravity. Taking the FLRW line-element, and assuming a perfect fluid matter content, the field equations in this case reduce to

$$
\begin{aligned}
H^{2} & =\frac{8 \pi \rho}{3 \phi}-\frac{\kappa}{a^{2}}-H \frac{\dot{\phi}}{\phi}+\frac{\omega}{6} \frac{\dot{\phi}^{2}}{\phi^{2}} \\
\frac{\ddot{\phi}}{\phi} & =\frac{8 \pi}{\phi} \frac{(\rho-3 P)}{(2 \omega+3)}-3 H \frac{\dot{\phi}}{\phi}-\frac{(d \omega / d \phi) \dot{\phi}^{2}}{(2 \omega+3) \phi}
\end{aligned}
$$


where over-dots again denote differentiation with respect to the proper time of comoving observers. These equations are similar to those of the Brans-Dicke theory, Eqs. (114 and (115), except for the extra term on the RHS of Eq. (135). Exact solutions with $\kappa=0$ have been found to Eqs. (134) and 135) in 94, 1004, 108, 942, and vacuum and radiation dominated solutions for arbitrary spatial curvature have been found in [95, 899, 111. Some of the methods used in these papers are extended to anisotropic cosmologies in 898, and the asymptotics of FLRW cosmologies in scalar-tensor theories have been studied in [112, 1125]. Exact homogeneous and anisotropic solutions are found in [327, 156] that act as past and future attractors for the general solution. Exact homogeneous self-similar solutions are found in [132, and inhomogeneous self-similar solutions are found in [157. We will not reproduce these solutions here, some of which can be quite complicated, but will instead return to the attractor mechanism expounded in [356.

This mechanism is most easily seen in the Einstein conformal frame, given by the Lagrangian 105, such that for a spatially flat FLRW geometry the evolution equation for the scalar field can be written as

$$
\frac{8 \pi}{\left(3-4 \pi \psi^{\prime 2}\right)} \psi^{\prime \prime}+4 \pi(1-w) \psi^{\prime}+\sqrt{4 \pi}(1-3 w) \alpha=0
$$

where here primes denote differentiation with respect to the natural log of the Einstein frame scale factor, $\bar{a}$, and $w$ is the equation of state $P=w \rho$. The reader will recall that $\psi=\sqrt{(3+2 \omega) / 16 \pi} \ln \phi$ is the scalar field in the Einstein frame, and $\alpha^{-2}=3+2 \omega$ denotes the strength of coupling between the scalar and tensor degrees of freedom. Equation 136 is clearly the equation for a simple harmonic oscillator with a dynamical mass, a damping force given by $-4 \pi(1-w)$, and a driving force given by the gradient of a potential $(1-3 w) \Gamma$, where the reader will recall $\Gamma=\sqrt{4 \pi} \int \alpha d \psi$. This interpretation of $\Gamma$ as an effective potential is often used to justify an expansion of the form

$$
\Gamma=\alpha_{0}\left(\psi-\psi_{0}\right)+\frac{\beta_{0}}{2}\left(\psi-\psi_{0}\right)^{2}+O\left(\left(\psi-\psi_{0}\right)^{3}\right),
$$

where $\psi_{0}$ is an assumed local minimum of $\Gamma(\psi)$, and $\alpha_{0}$ and $\beta_{0}$ are constants. In terms of this parameterisation the PPN parameters $\beta_{P P N}$ and $\gamma_{P P N}$ then become

$$
\begin{aligned}
1-\beta_{P P N} & =-\frac{\alpha_{0}^{2} \beta_{0}}{2\left(1+\alpha_{0}^{2}\right)^{2}} \\
1-\gamma_{P P N} & =\frac{2 \alpha_{0}^{2}}{1+\alpha_{0}^{2}} .
\end{aligned}
$$

The requirement of positive mass in (136) can also be seen to be equivalent to the requirement of positive energy density, $\bar{\rho}$, in the Einstein frame.

The cosmological dynamics that result from Eq. 136 are that $\psi$, and hence $\phi$, approach a constant value during the radiation dominated epoch. This is due to the vanishing of the 'potential' in (136) when $w=1 / 3$, and the negativity of the effective 'damping force'. Once radiation domination ends, however, and matter domination begins, then the scalar field rolls down to the minimum, $\psi_{0}$, of the now non-zero potential $\Gamma(\psi)$ (assuming such a minimum exists). Once this minimum is reached, after some possible oscillations in the case of an under-damped system, then we are left with $\alpha=0$, 
which is the general relativistic limit of these theories. This is a very useful general property of any scalar-tensor theory which has a local minimum in its parameter $\Gamma(\psi)$, and means that interesting new behaviour is possible at early times, while still being (potentially) compatible with observations that appear to point towards General Relativity at late-time.

Using order-of-magnitude approximations, the authors of [356] claim that this attractor mechanism is powerful enough to drive the value of the PPN parameter $1-\gamma$ down to values of as low as $\sim 10^{-7}$. This is a couple of order of magnitudes below the level that is probed by even the observations of the Cassini spacecraft, but is not inconceivably small. In particular, it may be that upcoming observations of binary pulsar systems could achieve such levels. Further predictions of this scenario are a possible oscillation in the effective value of Newton's constant near the beginning of the matter dominated epoch of the Universe's history, as well as a prediction for the locally measured value of $\beta_{P P N}$ given by

$$
\beta_{P P N}-1=\frac{\beta_{0}}{32 \pi}\left(1-\gamma^{2}\right),
$$

where $\beta_{0}$ is defined in Eq. (137). The validity and limitations of these results are extended, and are further studied in [357, 1095.

Let us now consider perturbations around a general FLRW background, in these generalised theories. We will work in the conformal Newtonian gauge, which has the usual correspondence with Bardeen's gauge invariant variables. Tensor perturbations on cosmological backgrounds have been studied in [109], while the scalar part of the perturbed line-element takes the form

$$
d s^{2}=a^{2}\left[-(1+2 \Psi) d \tau^{2}+(1-2 \Phi) q_{i j} d x^{i} d x^{j}\right],
$$

where we have used conformal time, $\tau$, and $q_{i j}$ is now the metric of a static 3 -space with constant curvature. Perturbations to the scalar field and energy momentum tensor are given by $\delta \phi$ and

$$
\begin{aligned}
\delta T_{0}^{0} & =-\delta \rho \\
\delta T^{0}{ }_{i} & =-(\rho+P) \vec{\nabla}_{i} \theta \\
\delta T^{i}{ }_{j} & =\delta P \delta^{i}{ }_{j}+(\rho+P) D^{i}{ }_{j} \Sigma,
\end{aligned}
$$

where $\rho, P$ and $\theta$ are the total energy density, pressure and peculiar velocity of the matter fields. The first-order perturbation equations are then given by 934 ]

$$
\begin{aligned}
\frac{2}{a^{2}}\left[3\left(\frac{a^{\prime}}{a}\right)^{2} \Psi+3 \frac{a^{\prime}}{a} \Phi^{\prime}+\left(k^{2}-3 \kappa\right) \Phi\right]+\frac{3 \delta \phi}{a^{2} \phi}\left[\left(\frac{a^{\prime}}{a}\right)^{2}+\kappa\right] \\
=-\frac{8 \pi}{\phi} \delta \rho-\frac{1}{a^{2} \phi}\left[\left[6\left(\frac{a^{\prime}}{a}\right) \Psi+3 \Phi^{\prime}\right] \phi^{\prime}-3\left(\frac{a^{\prime}}{a}\right) \delta \phi^{\prime}-k^{2} \delta \phi\right] \\
-\frac{\delta \phi}{2 a^{2}}\left(\frac{\phi^{\prime}}{\phi}\right)^{2} \frac{d \omega}{d \phi}+\frac{\omega}{a^{2} \phi}\left[\frac{\delta \phi}{2}\left(\frac{\phi^{\prime}}{\phi}\right)^{2}-\left(\frac{\phi^{\prime}}{\phi}\right) \delta \phi^{\prime}+\frac{\phi^{\prime 2}}{\phi} \Psi\right]
\end{aligned}
$$




$$
\begin{aligned}
\frac{2}{a^{2}}\left[\frac{a^{\prime}}{a} \Psi^{\prime}+\right. & {\left.\left[2\left(\frac{a^{\prime}}{a}\right)^{\prime}+\left(\frac{a^{\prime}}{a}\right)^{2}-\frac{k^{2}}{3}\right] \Psi+\Phi^{\prime \prime}+2 \frac{a^{\prime}}{a} \Phi^{\prime}+\frac{k^{2}}{3} \Phi-\kappa \Phi\right] } \\
= & \frac{8 \pi \delta P}{\phi}+\frac{\delta \phi}{a^{2} \phi}\left[2\left(\frac{a^{\prime}}{a}\right)^{\prime}+\left(\frac{a^{\prime}}{a}\right)^{2}+\kappa\right] \\
& -\frac{1}{a^{2} \phi}\left[2 \phi^{\prime \prime} \Psi+\phi^{\prime}\left[\Psi^{\prime}+2 \frac{a^{\prime}}{a} \Psi+2 \Phi\right]-\delta \phi^{\prime \prime}-\frac{a^{\prime}}{a} \delta \phi^{\prime}-\frac{2 k^{2} \delta \phi}{3}\right] \\
& +\frac{\delta \phi}{2 a^{2}}\left(\frac{\phi^{\prime}}{\phi}\right)^{2} \frac{d \omega}{d \phi}-\frac{\omega}{a^{2} \phi}\left[\frac{\delta \phi}{2}\left(\frac{\phi^{\prime}}{\phi}\right)^{2}-\left(\frac{\phi^{\prime}}{\phi}\right) \delta \phi^{\prime}+\frac{\phi^{\prime 2}}{\phi} \Psi\right]
\end{aligned}
$$

and

$$
\frac{2}{a^{2}}\left[\frac{a^{\prime}}{a} \Psi+\Phi^{\prime}\right]=\frac{8 \pi}{\phi}(\rho+P) \theta-\frac{1}{a^{2} \phi}\left[\left(\frac{a^{\prime}}{a}\right) \delta \phi+\phi^{\prime} \Psi-\delta \phi^{\prime}\right]+\frac{\omega \phi^{\prime} \delta \phi}{a^{2} \phi^{2}} .
$$

We also have the perturbed scalar field equation

$$
\begin{aligned}
& \delta \phi^{\prime \prime}+2 \frac{a^{\prime}}{a} \delta \phi^{\prime}+k^{2} \delta \phi-2 \phi^{\prime \prime} \Psi-\phi^{\prime}\left(\Psi^{\prime}+4 \frac{a^{\prime}}{a} \Psi+3 \Phi^{\prime}\right)-\frac{8 \pi a^{2}(\delta \rho-3 \delta P)}{(2 \omega+3)} \\
& =-\frac{a^{2}}{(2 \omega+3)}\left[\frac{d^{2} \omega}{d \phi^{2}} \frac{\phi^{\prime 2} \delta \phi}{a^{2}}+\frac{2}{a^{2}} \frac{d \omega}{d \phi}\left(\phi^{\prime} \delta \phi^{\prime}-\phi^{\prime 2} \Psi\right)+\frac{2}{a^{2}} \frac{d \omega}{d \phi}\left(\phi^{\prime \prime}+2 \frac{a^{\prime}}{a} \phi^{\prime}\right) \delta \phi\right],
\end{aligned}
$$

as well as the condition

$$
\Phi-\Psi=\frac{8 \pi a^{2}}{\phi}(\rho+P) \Sigma+\frac{\delta \phi}{\phi} .
$$

This last equation shows that, unlike in General Relativity, $\Phi \neq \Psi$ when anisotropic stresses vanishes (unless the perturbations to the scalar field also vanish). Primes here denote differentiation with respect to the conformal time, $\tau$, and $k$ is the wave-number of the perturbation.

Using the equations given above with $\kappa=0$, an analysis of the first year WMAP data has been performed and used to constrain the parameters $\alpha_{0}$ and $\beta_{0}$ of the attractor model in [935. The authors of this study find that the following constraint can be imposed on these parameters at the $2 \sigma$ level of significance:

$$
\alpha_{0}<5 \times 10^{-4-7 \beta_{0}} \text {. }
$$

One should bear in mind here that as $\beta_{0} \rightarrow 0$ Brans-Dicke theory is recovered, and as $\alpha_{0} \rightarrow 0$ General Relativity is recovered. As a corollary of this result these authors also constrain the value of Newton's constant at recombination to be no more than $5 \%$ different from the value measured in the solar system today, at the $2 \sigma$ confidence level. The effect of allowing a non-zero spatial curvature should be expected to weaken these bounds.

Big bang nucleosynthesis has also been explored in the context of general scalar-tensor theories [1210, 358, 319, 773. In 358 it is found that the inferred upper bound on the baryon density in the Universe is relatively insensitive to the presence of a gravitational scalar field, and that the parameters of the attractor model must satisfy the constraint

$$
\alpha_{0}^{2} \lesssim 10^{-6.5} \beta_{0}^{-1}\left(\frac{\Omega_{m} h^{2}}{0.15}\right)^{-1.5}
$$


when $\beta_{0} \gtrsim 0.5$. For $\beta_{0} \lesssim 0.5$ these bounds are weakened by a few orders of magnitude. These results are extended and refined in 319, who also allow for a non-zero self-interaction potential for the scalar field. The apparent tension between observed and theoretically predicted abundances of Lithium- 7 is addressed in the context of scalartensor theories in 773. Here the authors point out that a period of expansion slower than in General Relativity before primordial nucleosynthesis, together with a period of more rapid expansion during nucleosynthesis, can resolve this conflict. They find such behaviour in numerous scalar-tensor gravity theories, both with and without self-interaction potentials.

Inflation in scalar-tensor theories of gravity has been extensively studied, often under the name 'extended inflation', as coined by La and Steinhardt for the case of Brans-Dicke theory [762. The motivation behind this is the possibility of producing a successful inflationary phase transition from a false vacuum state, thus avoiding the fine tuning problems associated with 'new inflation'. Unfortunately, it was soon found that bubble collisions at the end of inflation produce unacceptable fluctuations in the CMB [1264, 761, 804. Suggestions to improve this situation were to include a self-interaction potential for the scalar field 763, generalise the couplings of the Brans-Dicke scalar to other fields 617] (see also [614, 1260]), include quantum effects [616, add additional couplings between the inflaton and the space-time curvature [775, or to consider more general scalar-tensor theories of gravity [1185, 526]. The latter of these approaches was dubbed 'hyper-extended inflation'. The inflationary solutions of general scalar-tensor theories have been studied in detail in [106, 212, 86], and specific models that could be compatible with observations were proposed in 554. Density perturbations in inflationary scalartensor scenarios have been investigated extensively in [861, 578, 1127, 937, 908, 798, 410 , 412, 345, 411, 760, 1181, 1193, 863, 1262. Studies of topological defects 333, 1089, black holes [625], gravitational waves [1229, 145], baryogenesis [99, 100, baryon asymmetry [1295], dark matter [874, 875, the formation of voids [802, 803, bubble nucleation rates and dynamics [1283, 1088, reheating [328, stochastic inflation [523, 525, 524, 1191, 1192, slow roll inflation [96, 527, 1212, non-Gaussianity [1047, isotropisation of the Universe [583, 584, and quantum cosmology [533] have all also been performed in the context of inflation in scalar-tensor theories. The initial conditions for inflation in scalar-tensor theories have been considered in [407, 441, 406. For further details the reader is referred to 1993 review of extended inflation by Steinhardt [1184.

Theories of gravity with non-minimally coupled scalar fields and non-zero self-interaction potentials have been studied by a number of authors under the name 'extended quintessence' [1035, 74, 35, 1061, 1109. Such theories can act as dark energy as well as model possible deviations from General Relativity at early times. These papers include studies of small angle CMB temperature and polarisation power spectra, the integrated Sachs-Wolfe effect, the matter power spectrum, supernovae observations and the affects that should be expected on weak lensing observations. The FLRW solutions of theories with power-law self-interaction potentials have been studied in further detail in 252, where the attractor mechanism to general relativity is investigated, as well the presence of periods of accelerating expansion at late and early times. Late-time acceleration in models without a potential for the scalar field is studied in 483 .

Another interesting possibility in scalar-tensor theories of gravity is the idea of 'gravitational memory', proposed by Barrow in 93. The idea here is that when a black hole forms one of two things can happen (or some combination of them). Firstly, the 
Schwarzschild radius of a black hole, which is given by $r_{S}=2 G(t) m$, could vary as the value of the scalar field controlling the value of $G$ varies in the background universe. In this case there is no such thing as a static black hole solution to the gravitational field equations, unless the black hole exists in a static universe. Secondly, the Schwarzschild radius of a black hole could be frozen in at its value when the black hole formed, so that $r_{S}=2 G\left(t_{f}\right) m$, where $t_{f}$ is the time when the black hole formed. In this case black holes that formed early on in the Universe's history would remember, in some sense, the conditions of the early universe, this being reflected in the value of $G\left(t_{f}\right)$. As Barrow points out, these two possibilities have consequences for the evaporation, and explosion, of black holes in the late universe. This idea has motivated a number of studies on the gravitational field of collapsed objects in scalar-tensor theories of gravity [97, 1211, 1213, 654, 593, 860, 948, 947]. One particularly interesting approach is that of matched asymptotic expansions, which suggests that the first option is followed, and black holes do not have any gravitational memory [1135, 1136, 1134].

\section{Horndeski's theory}

The most general four dimensional scalar-tensor theory with second-order field equations was worked out by Horndeski in 623 . It has the following Lagrangian

$$
\begin{aligned}
\mathcal{L}_{H}= & \delta_{\mu \nu \sigma}^{\alpha \beta \gamma}\left[\kappa_{1} \nabla^{\mu} \nabla_{\alpha} \phi R_{\beta \gamma}{ }^{\nu \sigma}-\frac{4}{3} \kappa_{1, X} \nabla^{\mu} \nabla_{\alpha} \phi \nabla^{\nu} \nabla_{\beta} \phi \nabla^{\sigma} \nabla_{\gamma} \phi\right. \\
& \left.+\kappa_{3} \nabla_{\alpha} \phi \nabla^{\mu} \phi R_{\beta \gamma}{ }^{\nu \sigma}-4 \kappa_{3, X} \nabla_{\alpha} \phi \nabla^{\mu} \phi \nabla^{\nu} \nabla_{\beta} \phi \nabla^{\sigma} \nabla_{\gamma} \phi\right] \\
& +\delta_{\mu \nu}^{\alpha \beta}\left[(F+2 W) R_{\alpha \beta}{ }^{\mu \nu}-4 F_{, X} \nabla^{\mu} \nabla_{\alpha} \phi \nabla^{\nu} \nabla_{\beta} \phi+2 \kappa_{8} \nabla_{\alpha} \phi \nabla^{\mu} \phi \nabla^{\nu} \nabla_{\beta} \phi\right] \\
& -3\left[2(F+2 W)_{, \phi}+X \kappa_{8}\right] \nabla_{\mu} \nabla^{\mu} \phi+\kappa_{9}(\phi, X),
\end{aligned}
$$

where $X=\nabla_{\mu} \phi \nabla^{\mu} \phi$, and $\delta_{\mu_{1} \mu_{2} \ldots \mu_{n}}^{\nu_{1} \nu_{2} \ldots \nu_{n}}=n ! \delta_{\mu_{1}}^{\left[\nu_{1}\right.} \delta_{\mu_{2}}^{\nu_{2}} \ldots \delta_{\mu_{n}}^{\left.\nu_{n}\right]}$. The theory depends on four arbitrary functions of $\phi$ and $X, \kappa_{i}=\kappa_{i}(\phi, X)$ as well as $F=F(\phi, X)$, which is constrained so that $F_{, X}=\kappa_{1, \phi}-\kappa_{3}-2 X \kappa_{3, X}$. Note that $W=W(\phi)$, which means that it can be absorbed into a redefinition of $F(\phi, X)$. This paper is not very well known, and as a result Horndeski's theory has not been well explored. It has, however, been recently resurrected in [276, where aspects of the theory on FLRW backgrounds were studied. The effective Lagrangian describing the cosmology in the minisuperspace approximation is given by

$$
L_{H}^{\mathrm{eff}}(a, \dot{a}, \phi, \dot{\phi})=a^{3} \sum_{n=0}^{3}\left(A_{n}-B_{n} \frac{\kappa}{a^{2}}\right) H^{n},
$$

where $H=\dot{a} / a$ is the Hubble parameter, and where we have

$$
\begin{aligned}
& A_{0}=-\tilde{Q}_{7, \phi} \dot{\phi}+\kappa_{9} \\
& B_{0}=\tilde{Q}_{1, \phi} \dot{\phi}+12 \kappa_{3} \dot{\phi}^{2}-12 F \\
& A_{1}=-12 F_{, \phi} \dot{\phi}+3\left(Q_{7} \dot{\phi}-\tilde{Q}_{7}\right)+6 \kappa_{8} \dot{\phi}^{3} \\
& B_{1}=-Q_{1} \dot{\phi}+\tilde{Q}_{1} \\
& A_{2}=-12 F-12 F_{, A} \dot{\phi}^{2} \\
& A_{3}=8 \kappa_{1, A} \dot{\phi}^{3},
\end{aligned}
$$


where

$$
Q_{1}=\frac{\partial \tilde{Q}_{1}}{\partial \dot{\phi}}=-12 \kappa_{1}, \quad \text { and } \quad Q_{7}=\frac{\partial \tilde{Q}_{7}}{\partial \dot{\phi}}=6 F_{, \phi}-3 \dot{\phi}^{2} \kappa_{8}
$$

It is assumed that matter is minimally coupled to the metric $g_{\mu \nu}$, and not to the scalar. Indeed, it is argued that if the equivalence principle is to hold then this can be assumed without further loss of generality. The cosmological field equations are presented implicitly as a generalised Friedmann equation:

$$
\frac{1}{a^{3}}\left[\dot{a} \frac{\partial L_{H}^{\mathrm{eff}}}{\partial \dot{a}}+\dot{\phi} \frac{\partial L_{H}^{\mathrm{eff}}}{\partial \dot{\phi}}-L_{H}^{\mathrm{eff}}\right]=-\rho,
$$

and the scalar equation of motion

$$
\frac{\partial L_{H}^{\mathrm{eff}}}{\partial \phi}-\frac{d}{d t}\left[\frac{\partial L_{H}^{\mathrm{eff}}}{\partial \dot{\phi}}\right]=0
$$

where $\rho$ is the energy density of the cosmological fluid.

In 276] the authors look for those corners of Horndeski's theory that admit a selftuning mechanism. They demand that the vacuum space-time is Minkowski, irrespective of the value of the cosmological constant, and that this should remain true even after a phase transition in which the cosmological constant changes by some amount. This is not in violation of Weinberg's theorem since Poincaré invariance is explicitly broken by the scalar. These considerations reduce Horndeski's theory to four base Lagrangians known as the Fab Four:

$$
\begin{aligned}
\mathcal{L}_{\text {john }} & =\sqrt{-g} V_{\text {john }}(\phi) G^{\mu \nu} \nabla_{\mu} \phi \nabla_{\nu} \phi \\
\mathcal{L}_{\text {paul }} & =\sqrt{-g} V_{\text {paul }}(\phi) P^{\mu \nu \alpha \beta} \nabla_{\mu} \phi \nabla_{\alpha} \phi \nabla_{\nu} \nabla_{\beta} \phi \\
\mathcal{L}_{\text {george }} & =\sqrt{-g} V_{\text {george }}(\phi) R \\
\mathcal{L}_{\text {ringo }} & =\sqrt{-g} V_{\text {ringo }}(\phi) \hat{G},
\end{aligned}
$$

where $\hat{G}=R_{\mu \nu \alpha \beta} R^{\mu \nu \alpha \beta}-4 R_{\mu \nu} R^{\mu \nu}+R^{2}$ is the Gauss-Bonnet combination, and

$$
P_{\alpha \beta}^{\mu \nu}=-R_{\alpha \beta}^{\mu \nu}+2 R_{[\alpha}^{\mu} \delta_{\beta]}^{\nu}-2 R_{[\alpha}^{\nu} \delta_{\beta]}^{\mu}-R \delta_{[\alpha}^{\mu} \delta_{\beta]}^{\nu}
$$

is the double dual of the Riemann tensor. These terms give rise to self-tuning cosmologies for $\kappa<0$. The relevant cosmological field equations are given by

$$
\mathcal{H}_{\text {john }}+\mathcal{H}_{\text {paul }}+\mathcal{H}_{\text {george }}+\mathcal{H}_{\text {ringo }}=-\left[\rho_{\Lambda}+\rho_{\text {matter }}\right]
$$

where we have separated the net cosmological constant contribution, $\rho_{\Lambda}$, and the matter contribution, $\rho_{\text {matter }}$, and where

$$
\begin{aligned}
& \mathcal{H}_{\text {john }}=3 V_{\text {john }}(\phi) \dot{\phi}^{2}\left(3 H^{2}+\frac{\kappa}{a^{2}}\right) \\
& \mathcal{H}_{\text {paul }}=-3 V_{\text {paul }}(\phi) \dot{\phi}^{3} H\left(5 H^{2}+3 \frac{\kappa}{a^{2}}\right) \\
& \mathcal{H}_{\text {george }}=-6 V_{\text {george }}(\phi)\left[\left(H^{2}+\frac{\kappa}{a^{2}}\right)+H \dot{\phi} \frac{V_{\text {george }}^{\prime}}{V_{\text {george }}}\right] \\
& \mathcal{H}_{\text {ringo }}=-24 V_{\text {ringo }}^{\prime}(\phi) \dot{\phi} H\left(H^{2}+\frac{\kappa}{a^{2}}\right) . \\
& 65
\end{aligned}
$$


The scalar equations of motion are $\mathcal{E}_{\text {john }}+\mathcal{E}_{\text {paul }}+\mathcal{E}_{\text {george }}+\mathcal{E}_{\text {ringo }}=0$ where

$$
\begin{aligned}
& \mathcal{E}_{\text {john }}=6 \frac{d}{d t}\left[a^{3} V_{\text {john }}(\phi) \dot{\phi} \Delta_{2}\right]-3 a^{3} V_{\text {john }}^{\prime}(\phi) \dot{\phi}^{2} \Delta_{2} \\
& \mathcal{E}_{\text {paul }}=-9 \frac{d}{d t}\left[a^{3} V_{\text {paul }}(\phi) \dot{\phi}^{2} H \Delta_{2}\right]+3 a^{3} V_{\text {paul }}^{\prime}(\phi) \dot{\phi}^{3} H \Delta_{2} \\
& \mathcal{E}_{\text {george }}=-6 \frac{d}{d t}\left[a^{3} V_{\text {george }}^{\prime}(\phi) \Delta_{1}\right]+6 a^{3} V_{\text {george }}^{\prime \prime}(\phi) \dot{\phi} \Delta_{1} \\
& +6 a^{3} V_{\text {george }}^{\prime}(\phi) \Delta_{1}^{2} \\
& \mathcal{E}_{\text {ringo }}=-24 V_{\text {ringo }}^{\prime}(\phi) \frac{d}{d t}\left[a^{3}\left(\frac{\kappa}{a^{2}} \Delta_{1}+\frac{2}{3} \Delta_{3}\right)\right],
\end{aligned}
$$

and we define $\Delta_{n}=H^{n}-\left(\frac{\sqrt{-\kappa}}{a}\right)^{n}$. We see that the self-tuning is achieved at the level of the scalar equation of motion, since on a Minkowski solution one has $H^{2}=-\frac{\kappa}{a^{2}} \Longrightarrow$ $\Delta_{n}=0$ for $n \geq 1$. In vacuum, the cosmological constant controls the value of the scalar via the generalised Friedmann equation. A detailed study of the phenomenology of the fab four has yet to be carried out, but the authors of [276] argue that the 'john' and 'paul' terms are expected to play a crucial role, as their derivative interactions could give rise to Vainshtein effects that could help pass solar system constraints. The Vainshtein mechanism is discussed in detail in Section 5.5.4.

Note that it has been shown that Horndeski's general theory is equivalent to [402] in four dimensions [710]. Aspects of cosmological perturbations are studied in [710] that may be applied to the Fab Four in the appropriate special case.

\subsubsection{The chameleon mechanism}

The 'chameleon mechanism' was introduced as a concept in gravitational physics by Khoury and Weltman in 689,688 . The basic concept here is that if we consider theories with a non-minimally coupled scalar field, then in the presence of other matter fields these scalars can acquire an effective mass parameter that is environmentally dependent. One can then potentially satisfy the tight constraints on non-minimally coupled scalar degrees of freedom that are imposed in relatively dense environments, such as exist in the solar system, while still having interesting new behaviour in less dense environments, such as those that can exist in cosmology.

This mechanism is usually formulated in the Einstein conformal frame, where the coupling between the scalar curvature and scalar field is minimal, but where the scalar field couples non-minimally to matter fields. The relevant action is then

$$
\mathcal{L}=\sqrt{-\bar{g}}\left\{\frac{1}{16 \pi} \bar{R}-\frac{1}{2} \bar{g}^{\mu \nu} \psi_{, \mu} \psi_{, \nu}-V(\psi)\right\}+\mathcal{L}_{m}\left(\Psi_{i}, e^{2 \sqrt{8 \pi} \beta_{i} \psi} \bar{g}_{\mu \nu}\right),
$$

where, in the notation used in Eq. 105 , we have taken $\Gamma=\sqrt{8 \pi} \beta_{i} \psi$, and where the $\beta_{i}$ are a set of constants denoting the coupling of $\psi$ to each of the $i$ matter fields $\Psi_{i}$. In the scalar-tensor theories so far discussed the scalar field should be considered coupled to each of the matter fields with the same universal coupling, which in the Brans-Dicke theory is given by $\beta^{-2}=2(3+2 \omega)$. Assuming such a coupling, the non-relativistic limit of the scalar field equation can then be written as

$$
\nabla^{2} \psi=\frac{d V_{\mathrm{eff}}}{d \psi},
$$


where $V_{\text {eff }}(\psi) \equiv V(\psi)+\rho e^{\sqrt{8 \pi} \beta \psi}$. This new 'effective potential' can be seen to be dependent on the ambient energy density, and if $\psi$ and $\beta$ are both positive then any runaway potential with $d V / d \psi<0$ will result in an effective potential with a local minimum whose position depends on $\rho$. What is more, for couplings of the type specified in Eq. 168 the local effective mass of the scalar field $\psi$, given by $m_{\psi}=d^{2} V_{\text {eff }} / d \psi^{2}$, can be seen to generically increase with increasing $\rho$. Hence, the name 'chameleon'.

The behaviour of scalar fields outside of massive objects, when the chameleon mechanism is present, can be shown to be crucially dependent on the ratio of $\Delta \psi$ to $\Phi_{c}$. Here $\Delta \psi$ denotes the difference in the value of the scalar field inside the object, $\psi_{c}$, and asymptotically, $\psi_{\infty}$, while $\Phi_{c}$ is the value of the Newtonian potential at the surface of the object, where $r=R_{c}$. More precisely, when one satisfies the condition

$$
\frac{\sqrt{8 \pi}\left(\psi_{\infty}-\phi_{c}\right)}{6 \beta \Phi_{c}} \ll 1
$$

then the resulting configuration of gravitational fields is found to be one in which $\psi$ occupies the minimal of the effective potential inside the bulk of the massive object, except for a thin region of depth $\Delta R_{c}$ just below its surface where the value of $\psi$ rises. Outside of the object $\psi$ increases further, and approaches its asymptotic value $\psi_{\infty}$ as

$$
\psi \simeq \psi_{\infty}-\frac{2 \beta}{\sqrt{8 \pi}}\left(\frac{3 \Delta R_{c}}{R_{c}}\right) \frac{M_{c} e^{-m_{\infty}\left(r-R_{c}\right)}}{r},
$$

where $m_{\infty}$ is the effective mass of the field at asymptotically large distances from the object of mass $M_{c}$. Now, the ratio of the thickness of the shell just below the object's surface to the object's overall radius, $\Delta R_{c} / R_{c}$, can be shown to be well approximated by the LHS of Eq. (170). The condition given in 170 is then equivalent to the condition that a 'thin shell' should be present, with $\Delta R_{c} / R_{c} \ll 1$.

If the 'thin shell' condition is not met then one instead has $\psi \sim \psi_{\infty}$ everywhere, and the exterior solution is given by

$$
\psi \simeq \psi_{\infty}-\frac{2 \beta}{\sqrt{8 \pi}} \frac{M_{c} e^{-m_{\infty}\left(r-R_{c}\right)}}{r} .
$$

A comparison of Eq. 172 with Eq. (171) immediately shows that without a thin shell variations in $\psi$ are no longer suppressed by the small factor of $3 \Delta R_{c} / R_{c}$, and that we should therefore expect in this case more obvious consequences to the existence of $\psi$ within the vicinity of massive objects. Khoury and Weltman proceed to argue that in order to avoid violations of the weak equivalence principle, and unacceptable deviations from the predictions of General Relativity in the solar system, we should require that the Earth, and other astrophysical bodies, should satisfy the thin shell condition 668.

This idea of a scalar field with an environmentally dependent mass has sparked widespread interest since it was proposed. In particular, it allows for the possibility of measuring fifth forces, or violations of the weak equivalence principle, that are different in space than they are on Earth, 689, 688, 499, 912, 1236, 190, 913, 1199, 1091, 1225, 198. It can act as dark energy [191, 197, and has been studied in the context of structure formation [196, 188, as well as a number of other cosmological scenarios 1912, 190, 913, 191, 484, 239, 362. The effect of 'chameleon particles' on searches for axion-like particles 
and experiments involving magnetic fields have been studied in 192, 14, 537, and their effect on the propagation of light in astrophysics in 219, 220. Experimental searches for chameleons have now been performed by GammeV [298, 1182, 1238, 1183, and ADMX [1079, which have started to constrain the viable parameter space of these theories. Other tests of this scenario are also proposed in [187, 789, 788, 1108, 199, 186.

\subsection{Einstein-Ether Theories}

Vector-tensor theories, in the form of Einstein-æther theories, have had a revival over the past decade, and are now often used as a counterfoil to test General Relativity. They have the particular property that they single out a preferred reference frame and have become somewhat of a theoretical workhorse for studying violations of Lorentz symmetry in gravitation. In the Einstein-æther theory [655] violations of Lorentz invariance arise within the framework of a diffeomorphism-invariant theory, and their modern incarnations are a refinement of the gravitationally coupled vector field theories first proposed by Will and Nordtvedt in the 1970s [1276, 989]. The presence of a Lorentz-violating vector field, henceforth called the ather, can dramatically affect cosmology: It can lead to a renormalisation of the Newton constant [257, leave an imprint on perturbations in the early universe [809, 677, and in more elaborate actions it can even affect the growth rate of structure in the Universe [1311, 1314, 589.

\subsubsection{Modified Newtonian dynamics}

Some of the theories that we will discuss in this subsection and the next have been constructed to give modifications to Newtonian gravity on galactic scales. To be more specific, they should lead to Milgrom's Modified Newtonian Dynamics [895, also known as MOND, in regimes of low acceleration. Given its relevance for Einstein-æther theories, we will now briefly describe the motivation for MOND, and how it works. We also briefly mention some of its successes and failures.

MOND was first proposed as a possible explanation of the need for dark matter in galaxies, based on observations of their rotational velocities. With Newtonian gravity and the visible baryonic matter in galaxies only one expects that the rotational velocity, $v_{r}$, should depend on the distance from the centre of the galaxy, $r$, as $v_{r} \propto r^{-1 / 2}$. What is in fact found in observations of spiral galaxies is that $v_{r}$ is approximately constant at large radii. The conventional answer to this problem is to posit that galaxies sit in halos of dark matter, with energy density profiles that vary as $\rho \sim r^{-2}$ for large $r$. Milgrom's proposal was that, alternatively, Newton's inverse square law of gravity could be modified in the low-acceleration regime of galactic dynamics. Such a modification, it was ventured, may be able to account for the anomalously high rotational velocities in spiral galaxies without invoking any new matter fields.

In MOND the spherically symmetric gravitational potential has two regimes: High acceleration and low acceleration. In regions of high acceleration (where $|\vec{a}| \gg a_{0}$, for constant $a_{0}$ ), it simply satisfies Newton's second law: $\vec{a}=-\nabla \Phi$ where $\Phi$ is the gravitational potential. On the other hand, in the low acceleration regime (where $|\vec{a}| \ll a_{0}$ ), Newton's second law is modified to $\left(\mid \vec{a} / a_{0}\right) \vec{a}=-\nabla \Phi$. Albeit a simple rule of thumb, Milgrom's proposal is remarkably successful at fitting a large range of spiral galaxy observations. Furthermore, it can be used to explain the Tully-Fisher relation that relates the velocity of rotation of a spiral galaxy with its intrinsic luminosity. Unfortunately 
MOND is unable to explain the dynamics of clusters of galaxies without recourse to additional dark matter (possibly in the form of neutrinos), and the behaviour of dwarf spheroidals in different environments is also problematic. Nevertheless, it is an interesting proposal that has had a renewed surge of interest in the past decade.

The non-relativistic Poisson equation in MOND can be written as

$$
\vec{\nabla} \cdot\left[\mu\left(\frac{|\vec{\nabla} \Phi|}{a_{0}}\right) \vec{\nabla} \Phi\right]=4 \pi G \rho,
$$

where $\rho$ is the energy density in baryons, and the function $\mu(x) \rightarrow 1$ as $x \rightarrow \infty$ and $\mu(x) \rightarrow x$ as $x \rightarrow 0$. There are a variety of proposals for the precise form of $\mu(x)$ that fit observations of galaxies to a greater or lesser degree. As a theory of modified gravity, however, MOND's greatest limitation is that it is restricted to non-relativistic regimes. It therefore cannot be used to make prediction on cosmological scales, nor can it be used to calculate fundamentally relativistic observables, such as lensing. Many of the theories that follow in this section have been constructed to address this deficiency: They are relativistic gravitational theories that have MOND as a non-relativistic limit.

\subsubsection{Action and field equations}

As the name suggests, vector-tensor theories involve the introduction of a space-time 4 -vector field, $A^{\mu}$. A general action for such theories is given by

$$
S=\int d^{4} x \sqrt{-g}\left[\frac{1}{16 \pi G} R+\mathcal{L}\left(g^{\mu \nu}, A^{\nu}\right)\right]+S_{M}\left(g^{\mu \nu}, \Psi\right),
$$

where $S_{m}$ is the matter action. Note that the matter fields $\Psi$ in $S_{M}$ couple only to the metric $g_{\mu \nu}$, and not to $A^{\nu}$.

Let us now focus on Einstein-æther theories, and hence forth consider $A^{\mu}$ to have a time-like direction. The simplest (and most thoroughly studied) version of the Einsteinæther theory is quadratic in derivatives of $A^{\nu}$, and has the form

$$
\mathcal{L}_{E A}\left(g^{\mu \nu}, A^{\nu}\right) \equiv \frac{1}{16 \pi G}\left[K_{\alpha \beta}^{\mu \nu} \nabla_{\mu} A^{\alpha} \nabla_{\nu} A^{\beta}+\lambda\left(A^{\nu} A_{\nu}+1\right)\right],
$$

where $K^{\mu \nu}{ }_{\alpha \beta} \equiv c_{1} g^{\mu \nu} g_{\alpha \beta}+c_{2} \delta^{\mu}{ }_{\alpha} \delta^{\nu}{ }_{\beta}+c_{3} \delta^{\mu}{ }_{\beta} \delta^{\nu}{ }_{\alpha}-c_{4} A^{\mu} A^{\nu} g_{\alpha \beta}$ and $\lambda$ is a Lagrange multiplier. In what follows we will use the notation $c_{12 \ldots} \equiv c_{1}+c_{2}+\ldots$. We call the theory derived from Eqs. (174) and (175) the linear Einstein-æther theory.

A more general, non-linear Lagrangian for the æther field can be written in the form

$$
\mathcal{L}_{G E A}\left(g^{\mu \nu}, A^{\mu}\right)=\frac{M^{2}}{16 \pi G} F(K)+\frac{1}{16 \pi G} \lambda\left(A^{\mu} A_{\mu}+1\right),
$$

where $K=K_{\alpha \beta}^{\mu \nu} \nabla_{\mu} A^{\alpha} \nabla_{\nu} A^{\beta}$, and $M$ has the dimension of mass. We shall call this a generalised Einstein-æther theory.

Such actions arise from Lorentz violating physics in quantum gravity. Indeed, the linear Einstein-æther theory can be constructed using the rules of effective field theory, and has been shown to be stable with regard to quantum effects [1279]. Such theories, however, can suffer from instabilities at the classical level, with the onset of caustics in a finite time 331. This raises the question of whether the vector field in such theories are 
merely an effective (possibly composite) degrees of freedom, or whether they are genuine fundamental fields.

The gravitational field equations for this theory, obtained by varying the action for the Generalised Einstein-æther theory with respect to $g^{\mu \nu}$ are given by

$$
\begin{aligned}
G_{\mu \nu}= & \tilde{T}_{\mu \nu}+8 \pi G T_{\mu \nu}^{\text {matter }}, \\
\tilde{T}_{\mu \nu}= & \frac{1}{2} \nabla_{\alpha}\left(F_{K}\left(J_{(\mu}{ }^{\alpha} A_{\nu)}-J^{\alpha}{ }_{(\mu} A_{\nu)}-J_{(\mu \nu)} A^{\alpha}\right)\right) \\
& -F_{K} Y_{(\mu \nu)}+\frac{1}{2} g_{\mu \nu} M^{2} F+\lambda A_{\mu} A_{\nu},
\end{aligned}
$$

where $F_{K} \equiv \frac{d F}{d K}$ and $J_{\alpha}^{\mu} \equiv\left(K_{\alpha \beta}^{\mu \nu}+K_{\beta \alpha}^{\nu \mu}\right) \nabla_{\nu} A^{\beta}$. Brackets around indices denote symmetrisation, and $Y_{\mu \nu}$ is defined by the functional derivative $Y_{\mu \nu}=\nabla_{\alpha} A^{\rho} \nabla_{\beta} A^{\sigma} \frac{\partial\left(K^{\alpha \beta} \rho\right)}{\left.\partial g^{\mu \nu}\right)}$. The equations of motion for the vector field, obtained by varying with respect to $A^{\nu}$, are

$$
\nabla_{\mu}\left(F_{K} J_{\nu}^{\mu}\right)+F_{K} y_{\nu}=2 \lambda A_{\nu}
$$

where we have defined $y_{\nu}=\nabla_{\alpha} A^{\rho} \nabla_{\beta} A^{\sigma} \frac{\partial\left(K^{\alpha \beta}{ }_{\rho \sigma}\right)}{\partial A^{\nu}}$. Finally, variations of the action with respect to $\lambda$ fix $A^{\nu} A_{\nu}=-1$.

\subsubsection{FLRW solutions}

In a homogeneous and isotropic universe with perfect fluid matter content, the vector field will only have a non-vanishing ' $t$ ' component, so that $A^{\mu}=(1,0,0,0)$. The equations of motion then simplify dramatically, so that $\nabla_{\mu} A^{\mu}=3 H$ and $K=3 \frac{\alpha H^{2}}{M^{2}}$, where $\alpha \equiv$ $c_{1}+3 c_{2}+c_{3}$. Note that the $\alpha$ we have defined here has the same sign as $K$. The field equations then reduce to

$$
\begin{aligned}
{\left[1-\alpha K^{1 / 2} \frac{d}{d K}\left(\frac{F}{K^{1 / 2}}\right)\right] H^{2} } & =\frac{8 \pi G}{3} \rho, \\
\frac{d}{d t}\left(-2 H+F_{K} \alpha H\right) & =8 \pi G(\rho+P) .
\end{aligned}
$$

If we now take $F(x)=\gamma x^{n}$, the modified Friedmann equations become

$$
\left[1+\epsilon\left(\frac{H}{M}\right)^{2(n-1)}\right] H^{2}=\frac{8 \pi G}{3} \rho,
$$

where $\epsilon \equiv(1-2 n) \gamma(-3 \alpha)^{n} / 6$. We also get the relationship

$$
\gamma=\frac{6\left(\Omega_{m}-1\right)}{(1-2 n)(-3 \alpha)^{n}}\left(\frac{M}{H_{0}}\right)^{2(n-1)}
$$

where $\Omega_{m} \equiv 8 \pi G \rho_{0} / 3 H_{0}^{2}$, and $H_{0}$ is the Hubble constant today. Let us now consider a few special cases: If $n=1 / 2$, the Friedmann equations are unchanged $(\epsilon=0)$ and there is no effect on the background cosmology; with $n=1$ we have that $\epsilon=\gamma \alpha / 2$ and Newton's constant is rescaled by a factor of $1 /(1+\epsilon)$ [257]; if $n=0$ we recover a cosmological constant, $\Lambda \simeq \operatorname{sign}(-\gamma) M^{2}$. More generally, we will obtain different regimes depending on the relative size of each term in the modified Friedmann equation. We can summarise these behaviours in Figure 3.2 .3 . 


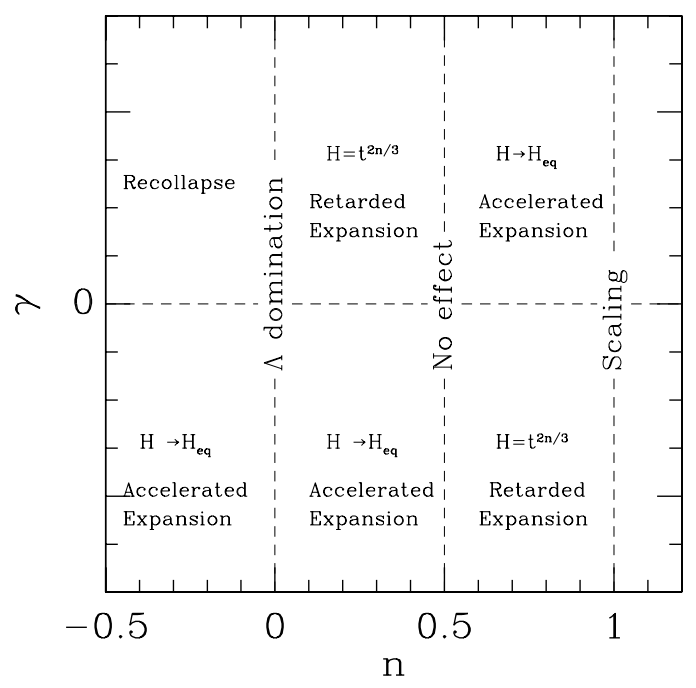

Figure 1: A schematic representation of the late-time evolution of FLRW solutions as a function of $n$ and $\gamma$, for $n<1$.

\subsubsection{Cosmological perturbations}

The four-vector $A^{\mu}$ can be perturbed as $A^{\mu}=\left(1-\Psi, \frac{1}{a} \vec{\nabla}^{i} V\right)$, where $V$ is a small quantity. Perturbing $K$ to linear order then gives $K=K_{0}+K_{1}$, where $K_{0}=3 \frac{\alpha H^{2}}{M^{2}}$ and $K_{1}=-2 \frac{\alpha H}{M^{2}}\left(k^{2} \frac{V}{a}+3 H \Psi+3 \dot{\Phi}\right)$. The gravitational potentials $\Psi$ and $\Phi$ come from the perturbed metric:

$$
d s^{2}=a^{2}(\tau)\left[-(1+2 \Psi) d \tau^{2}+(1-2 \Phi) q_{i j} d x^{i} d x^{j}\right]
$$

were $q_{i j}$ is the unperturbed conformal metric of the hyper-surfaces of constant $\tau$.

The evolution equation for the perturbations in the vector field are

$$
\begin{aligned}
0= & c_{1}\left[V^{\prime \prime}+k^{2} V+2 \mathcal{H} V^{\prime}+2 \mathcal{H}^{2} V+\Psi^{\prime}+\Phi^{\prime}+2 \mathcal{H} \Psi\right] \\
& +c_{2}\left[k^{2} V+6 \mathcal{H}^{2} V-3 \frac{a^{\prime \prime}}{a} V+3 \Phi^{\prime}+3 \mathcal{H} \Psi\right] \\
& +c_{3}\left[k^{2} V+2 \mathcal{H}^{2} V-\frac{a^{\prime \prime}}{a} V+\Phi^{\prime}+\mathcal{H} \Psi\right] \\
& +\frac{F_{K K}}{F_{K}}\left[-K_{1} \alpha \mathcal{H}-K_{0}^{\prime}\left(-c_{1}\left(V^{\prime}+\Psi\right)+3 c_{2} \mathcal{H} V+c_{3} \mathcal{H} V\right)\right] .
\end{aligned}
$$

The perturbation in the vector field is sourced by the two gravitational potentials $\Phi$ and 
$\Psi$. The first-order perturbations to the vector field's stress-energy tensor are

$$
\begin{aligned}
& a^{2} \delta \tilde{T}_{0}^{0}= F_{K} c_{1}\left[-\mathcal{H} k^{2} V-k^{2} V^{\prime}-k^{2} \Psi\right] \\
&+F_{K} \alpha\left[\mathcal{H} k^{2} V+3 \mathcal{H} \Phi^{\prime}+3 \mathcal{H}^{2} \Psi\right]-3 F_{K K} \alpha \mathcal{H}^{2} K_{1} \\
&= F_{K} c_{1}\left[-\mathcal{H} k^{2} V-k^{2} V^{\prime}-k^{2} \Psi\right]+F_{K} \alpha(2 n-1)\left[\mathcal{H} k^{2} V+3 \mathcal{H} \Phi^{\prime}+3 \mathcal{H}^{2} \Psi\right] \\
& a^{2} \delta \tilde{T}_{i}^{0}= i k_{i} F_{K} c_{1}\left[V^{\prime \prime}+2 \mathcal{H} V^{\prime}+\frac{a^{\prime \prime}}{a} V+\Psi^{\prime}+\mathcal{H} \Psi\right] \\
&+i k_{i} F_{K} \alpha\left[2 \mathcal{H}^{2} V-\frac{a^{\prime \prime}}{a} V\right]+i k_{i} F_{K K} K_{0}^{\prime}\left[c_{1}\left(\mathcal{H} V+V^{\prime}+\Psi\right)-\alpha \mathcal{H} V\right] \\
& a^{2} \delta \tilde{T}_{j}^{i}= F_{K} c_{2} k^{2}\left[2 \mathcal{H} V+V^{\prime}\right] \delta^{i}{ }_{j}+F_{K}\left(c_{1}+c_{3}\right)\left[2 \mathcal{H} V+V^{\prime}\right] k^{i} k_{j} \\
&+F_{K} \alpha\left[2 \mathcal{H} \Phi^{\prime}+\Phi^{\prime \prime}+2 \frac{a^{\prime \prime}}{a} \Psi-\mathcal{H}^{2} \Psi+\mathcal{H} \Psi^{\prime}\right] \delta^{i}{ }_{j}+F_{K K}\left(c_{1}+c_{3}\right) K_{0}^{\prime} V k^{i} k_{j} \\
&-F_{K K}\left[\alpha K_{1} \frac{a^{\prime \prime}}{a}+\left(c_{1}+c_{2}+c_{3}\right) K_{1} \mathcal{H}^{2}+\alpha \mathcal{H} K_{1}^{\prime}\right. \\
&\left.-\alpha K_{0}^{\prime} \Phi^{\prime}-2 \alpha K_{0}^{\prime} \mathcal{H} \Psi+\alpha \ln \left(F_{K K}\right)^{\prime} K_{1} \mathcal{H}-c_{2} K_{0}^{\prime} k^{2} V\right] \delta^{i}{ }_{j},
\end{aligned}
$$

where the second expression for $a^{2} \delta \tilde{T}_{0}^{0}$ assumes the monomial form for $F(K)$. In the absence of anisotropic stresses in the matter fields, we may obtain an algebraic relation between the metric potentials $\Phi$ and $\Psi$ by computing the transverse, traceless part of the perturbed Einstein equations. This gives

$$
\begin{aligned}
k^{2}(\Psi-\Phi) & =\frac{3}{2} a^{2}\left(\hat{k}_{i} \hat{k}_{j}-\frac{1}{3} \delta_{i j}\right)\left(\delta \tilde{T}_{j}^{i}\right) \\
& =\left(c_{1}+c_{3}\right) k^{2}\left[F_{K}\left(2 \mathcal{H} V+V^{\prime}\right)+F_{K K} K_{0}^{\prime} V\right] .
\end{aligned}
$$

We then find the following expression for the perturbed field equations:

$$
\begin{aligned}
k^{2} \Phi= & -\frac{1}{2} F_{K} c_{1} k^{2}\left[V^{\prime}+\Psi+\left(3+2 \tilde{c}_{3}\right) \mathcal{H} V\right] \\
& -4 \pi G a^{2} \sum_{a}\left(\bar{\rho}_{a} \delta_{a}+3\left(\bar{\rho}_{a}+\bar{P}_{a}\right) \mathcal{H} \theta_{a}\right) .
\end{aligned}
$$

Before we look at the cosmological consequences of these theories, and constraints that can be imposed on them, it is instructive to study the effect of the vector field during matter domination. This should allow us some insight into how the growth of structure proceeds in the generalised Einstein-æether case. First let us consider the simplest case in which the dominant contribution to the energy density is baryonic, so that we can treat it as a pressureless perfect fluid. Let us also introduce the new variable $V^{\prime} \equiv E$. For illustrative ease we will initially consider only the case where $V$ is described by a growing monomial, such that $V=V_{0}\left(\frac{\tau}{\tau_{0}}\right)^{p}$, During the matter dominated era we then have $a^{2} \delta T_{0}^{0} \simeq-l_{E} \xi(k) k^{2} \tau^{5+p-6 n}$ and $k^{2}(\Psi-\Phi) \simeq-l_{S} \xi(k) k^{2} \tau^{5+p-6 n}$, where $l_{E} \equiv-\left(c_{1}(2+p) n+2 \alpha(1-2 n) n\right), l_{S} \equiv-\left(c_{1}+c_{3}\right) n(6 n-p-10)$, and

$$
\xi(k) \sim \gamma V_{0}(k)\left(\frac{1}{\tau_{0}}\right)^{p} k_{h u b}^{6-6 n}\left(3 \alpha \Omega_{m}\left(\frac{H_{0}}{M}\right)^{2}\right)^{n-1},
$$


where $k_{\text {hub }} \equiv 1 / \tau_{\text {today }}$. Hence, the vector field affects the evolution equations for the matter and metric perturbations only through its contribution to the energy density and anisotropic stress. On large scales, $k \tau \ll 1$, and assuming adiabatic initial conditions for the fields $\delta, \Phi, \theta$, this leads to $\delta=C_{1}(k)+\frac{6 l_{S} \xi(k)}{(10+p-6 n)} \tau^{5+p-6 n}$, where $C_{1}$ is a constant of integration and we have omitted the decaying mode. Therefore, even before horizon crossing, the anisotropic stress term due to the vector field can influence time evolution of the baryon density contrast. On small scales, $k \tau \gg 1$, we find $\delta(k, \tau)=C_{2}(k) \tau^{2}+\frac{\left(\frac{1}{2} l_{E}+l_{S}\right)}{(5+p-6 n)(10+p-6 n)} \xi(k)(k \tau)^{2} \tau^{5+p-6 n}$, where $C_{2}(k)$ is another constant of integration. Hence, for sub-horizon modes, the influence of the vector field on the evolution of $\delta$ is a combination of its affect on the energy density and anisotropic stress contributions, though both, in this limit, result in the same contributions to the scale dependence and time evolution of the density contrast.

\subsubsection{Observations and constraints}

Let us now consider the constraints that can be imposed on these theories. First of all we will consider the linear Einstein-æther theory, and then we will consider the generalised Einstein-æther models.

In the case of the linear Einstein-æther theory, a number of non-cosmological constraints on the $c_{i}$ have been derived: Most notably, a Parameterised Post-Newtonian (PPN) analysis of the theory leads to a reduction in the dimensionality of parameter space. This is occurs due to the requirement that $c_{2}$ and $c_{4}$ must be expressed in terms of the other two parameters in the theory as $c_{2}=\left(-2 c_{1}^{2}-c_{1} c_{3}+c_{3}^{2}\right) / 3 c_{1}$ and $c_{4}=-c_{3}^{2} / c_{1}$. Additionally, the squared speeds of the gravitational and æther waves with respect to the preferred frame must be greater than unity, so as to prevent the generation of vacuum Cerenkov radiation by cosmic rays. A final constraint arises from considering the effects of the æther on the damping rate of binary pulsars. The rate of energy loss in such systems by gravitational radiation agrees with the prediction of General Relativity to one part in $10^{3}$. In the case of the Einstein-æther theory it has been shown that to agree with General Relativity in these systems we must require that $c_{+} \equiv c_{1}+c_{3}$ and $c_{-} \equiv c_{1}-c_{3}$ are related by an algebraic constraint. A more exotic, but viable, subset of the parameter space can be considered if we set $c_{1}=c_{3}=0$. The PPN and pulsar constraints are then no longer applicable, and a cosmological analysis is potentially the only way of constraining the values of the coupling constants.

Using a combination of CMB and large-scale structure data [1315] it is possible to impose constraints on the coefficients of the theory, $c_{i}$, as well as the overall energy density in the æther field. The main effect on the evolution of perturbations is through the change in the background evolution, and not necessarily through the presence of perturbations in the vector field. Indeed, artificially switching off the perturbations in the æther field has essentially no effect on the power spectrum of large-scale structure, and a small effect (of approximately 10\%) on the angular power spectrum of the CMB. In Figure 2 we plot the join constraints on $c_{+}$and $c_{-}$that can be imposed from these observables.

If we consider the generalised Einstein-æther theory, we find that the effect on the $\mathrm{CMB}$ is much more pronounced. Let us first consider a Universe with no dark matter, and in which the perturbations in the æther field simultaneously mimic a perturbed pressureless fluid in the formation of large scale structure, whilst behaving entirely differently 


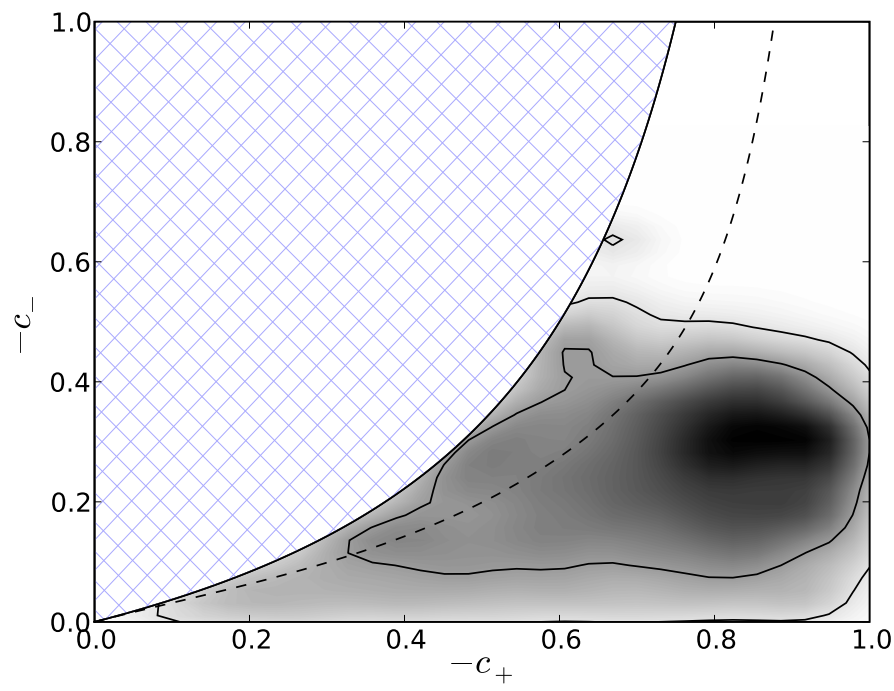

Figure 2: Likelihood plot in the parameter space of $-c_{+}$and $-c_{-}$from observations of the CMB and large-scale structure. The black lines are the 1 and $2 \sigma$ contours, for which we have marginalised over the values of the other parameters. The hatched region is excluded by Cerenkov constraints. The dashed line indicates the constraints available from binary pulsars.

in the cosmological background 1314. The first requirement for successful perturbation evolution is that structure can form at all. One necessary condition for this is that the sound speed of the structure seed is not too large, since this would wash out structure. It is therefore required that the sound horizon in the models we are considering should be less than the smallest scales where linear structure can form: $C_{S} k_{\max } \tau \lesssim 1$, where $k_{\max } \sim 0.2 h / M$ pc. For matter power observations at $\tau \sim 3 \times 10^{4}$, which is the present epoch, this yields $C_{S} \lesssim 10^{-4}$.

There are two underlying physical processes that can constrain these models. The first is a change in the rate of growth of the amplitude of perturbations. This can cause discrepancies between the amplitudes we expect in the matter power spectrum and the $\mathrm{CMB}$, since the evolution between the two is different. It can also lead to an integrated Sachs-Wolfe effect during the matter era, as $\Phi$ becomes time dependent. The second process is due to the increased magnitude $\Phi-\Psi$. This also leads directly to a nonnegligible integrated Sachs-Wolfe effect in the matter dominated era. The details of each of these processes depends on the functional form of $F$, the time-dependence of the $\xi$ growing mode, and the choice of the parameters $c_{i}$. It is extremely challenging to find combinations of the parameters that allows for a realistic growth of structure, while simultaneously ensuring the integrated Sachs-Wolfe effect is acceptably small.

A consequence of these two effects is that it is impossible to find models where the æether field replaces the dark matter that fit the available cosmological data. This is not due to the matter power spectra, which can be reasonably fitted to the SDSS data, but from the CMB. In the low- $\ell$ regime a large ISW effect is clearly present, destroying any 
chance of fitting the CMB data at large scales. The positions of the peaks are also poorly fit by the model. Finally, to fit the matter power spectrum to the data requires rescaled by a factor 0.02 , which corresponds to a galaxy bias of 0.14 . Such a scaling is considered to be improbably small, on physical grounds. All these effects cause severe problems when attempting to simultaneously fit the $\mathrm{CMB}$ and large-scale structure.

Finally, let us consider the possibility of late-time accelerating expansion. A detailed comparison with the data seems to allow a range of values for the index $n$, and the three coupling terms of the theory, which can produce this behaviour. In the limit $n_{\mathrm{ae}} \rightarrow 0$, however, the æther field behaves exactly as a cosmological constant term.

\subsection{Bimetric Theories}

In this section we will consider theories that involve two rank-2 tensors. These are often referred to as "bimetric", or "tensor-tensor", theories of gravity. The first formulation of a bimetric theory appears to be due to Rosen [1067, 1068], and involves the addition of an extra non-dynamical rank-2 tensor into the theory. Rosen's theory, however, is now known to lead to the existence of states that are unbounded from below in their energy. As a result Rosen's theory predicts the spin up of pulsars, as gravitational waves with negative energy are emitted. This severely violates the constraints on these systems that have been imposed by observations of millisecond pulsars [779.

Following in Rosen's footsteps, there were a number of proposals over the years of how one could formulate a viable bimetric theory of gravity. Here we highlight what we consider to be some of the most interesting cases. These include Drummond's bimetric (or "bi-vierbein") theory, which is claimed to mimic the dark matter in spiral galaxies [434, as well as arguments by Magueijo that bimetric theories could exhibit a variable speed of light, thus providing a way to model time-varying fundamental constants. More recently, Bañados and collaborators have shown that a general form of bigravity, which includes specific forms previously proposed in 647, 355] might allow one to account for some aspects of the dark sector [85, 70. Finally, Milgrom has recently proposed a bimetric theory that reduces to MOND in the appropriate limits. In what we follows, we will briefly outline each of these theories.

The basic idea behind bimetric, or tensor-tensor, theories is the introduction of a second 'metric' tensor into the theory ${ }^{18}$ a dynamical metric, $g_{\mu \nu}$, and a second metric, $\tilde{g}_{\alpha \beta}$. The first of these is usually universally coupled to the matter fields, and is used to construct the energy-momentum tensor of the non-gravitational fields. It is this field is used to define the geodesic equations of test particles. The equations that govern $g_{\mu \nu}$, however, are not the Einstein field equations: They invariably involve $\tilde{g}_{\alpha \beta}$ as well.

If the $\tilde{g}_{\alpha \beta}$ is not dynamical, then it is usually taken to be highly symmetric (i.e. exhibiting the maximal 10 Killing vectors $X$, such that $\mathcal{L}_{X} \tilde{g}_{\alpha \beta}=0$ ). An obvious choice for $\tilde{g}_{\alpha \beta}$ is the Minkowski metric, $\eta_{\mu \nu}$, so that all components of the Riemann tensor constructed from $\tilde{g}_{\alpha \beta}$ vanish. Rosen's bimetric theory is a particular example of such a construction, as are some attempts to construct a massive theory of gravity. If $\tilde{g}_{\alpha \beta}$ is to be dynamical, then a kinetic term of the Einstein-Hilbert form is required in the

\footnotetext{
${ }^{18} \mathrm{~A}$ second rank-2 tensor would probably be a more accurate description of what is actually being added here, as the term 'metric' implies a particular geometric function. Nevertheless, the term 'metric' for this additional field is commonly used, and so we follow this convention here.
} 
gravitational action. Coupling terms are then also required between $\tilde{g}_{\alpha \beta}$ and $g_{\mu \nu}$, with the matter fields usually coupling to either one, or a combination of both, metrics.

\subsubsection{Rosen's theory, and non-dynamical metrics}

As advertised, Rosen's bimetric theory is constructed with an extra flat metric, $\tilde{g}_{\alpha \beta}=$ $\eta_{\alpha \beta}$, such that ${ }^{19}$

$$
\tilde{R}_{\nu \rho \sigma}^{\mu}\left(\tilde{g}_{\alpha \beta}\right)=0 .
$$

We can now define a covariant derivative in terms of $\tilde{g}_{\alpha \beta}$, which we will call $\tilde{\nabla}_{\mu}$, such that the field equations for the dynamical metric can be written

$$
\frac{1}{2} \tilde{g}^{\alpha \beta} \tilde{\nabla}_{\alpha} \tilde{\nabla}_{\beta} g_{\mu \nu}-\frac{1}{2} \tilde{g}^{\alpha \beta} g^{\delta \epsilon} \tilde{\nabla}_{\alpha} g_{\delta \mu} \tilde{\nabla}_{\beta} g_{\epsilon \nu}=-8 \pi G \frac{\sqrt{-g}}{\sqrt{-\tilde{g}}}\left(T_{\mu \nu}-\frac{1}{2} g_{\mu \nu} g^{\alpha \beta} T_{\alpha \beta}\right)
$$

The energy-momentum tensor satisfies the conventional conservation equation $\nabla^{\mu} T_{\mu \nu}=$ 0 .

Rosen's theory has been the subject of a number studies over the years. It has been found to be extremely successful when subjected to a PPN analysis, and compared to Solar System observations 779 . In fact, almost all the PPN parameters in Rosen's theory are indistinguishable from those of General Relativity. The only exception is $\alpha_{2}=v_{g}^{2} / c^{2}-1$, where $v_{g}$ is the speed of gravitational waves in Rosen's theorem, and $c$ is the speed of light. One should note here, however, that $v_{g}$ is not uniquely determined by the theory, but rather by the cosmological solutions to the theory. One can then adjust the initial conditions of the Universe in order to tune $\alpha_{2}$. If this is done then the theory is observationally indistinguishable from General Relativity in the weak field, low velocity regime of post-Newtonian gravitational physics.

Rosen's theory fails, however, when its predictions for the emission of gravitational waves are compared to observations of binary pulsars. Will and Eardley found that unless the binary system under consideration obeys very specific properties, in terms of masses and mass differences, then Rosen's theory leads to the emission of a large amount of dipole gravitational radiation [1275. This in turn results in a sizeable increase in the orbital period of the system, which is not observed. Binary pulsar observations are therefore incompatible with this theory. Rosen later proposed replacing flat space metric by an a priori specified, but time-varying, cosmological background [1069]. Unfortunately this does not circumvent the pulsar problem.

Other bimetric theories that also have been proposed with an additional a priori specified, non-dynamical metric field. These include Rastall's theory 1055, 1056 and Lightman and Lee's theory [808, for which the PPN limits of both theories are known. It has been conjectured by Will, however, that all such theories that incorporate prior specified geometry could suffer the same deficiency as Rosen's, when it comes to calculating the emission of gravitational radiation from binary systems [1274.

\footnotetext{
${ }^{19}$ Note that this equation can be derived from an action principle by including a space-time dependent rank-2 tensor as a Lagrange multiplier. We will not go into the details of how to do this here.
} 


\subsubsection{Drummond's theory}

Let us now turn to a more recent formulation of the bimetric theory. In 434 it was proposed to work in the vierbein formulation with $g_{\alpha \beta}=\eta_{A B} e_{\alpha}^{A} e_{\beta}^{B}$ and $\tilde{g}_{\alpha \beta}=$ $\eta_{\tilde{A}} \tilde{B}_{\alpha}^{\tilde{A}} \tilde{e}_{\beta}^{\tilde{B}}$. In this case both sets of vierbein are dynamical. While $\tilde{e}_{\alpha}^{\tilde{A}}$ is used to construct the Einstein-Hilbert action, $e_{\beta}^{B}$ is used to construct the action from which the energymomentum tensor is derived. The missing pieces of the theory are then a transformation tensor, $M_{B}^{\tilde{A}}$, and a scalar, $\phi$, which relate $\tilde{e}_{\mu}^{\tilde{A}}$ and $e_{\mu}^{B}$ by

$$
\tilde{e}_{\mu}^{\tilde{A}}=e^{\phi} M_{B}^{\tilde{A}} e_{\mu}^{B} .
$$

Finally, we then need to define a "linking action". This is given by Drummond as

$$
\begin{aligned}
S_{L}= & \frac{1}{16 \pi G_{1}} \int d^{4} x \sqrt{-\tilde{g}} \tilde{g}^{\mu \nu} \operatorname{Tr}\left(j_{\mu} j_{\nu}\right) \\
& +\frac{1}{16 \pi G_{2}} \int d^{4} x \sqrt{-\tilde{g}} \tilde{g}^{\mu \nu}\left(\partial_{\mu} \phi \partial_{\nu} \phi\right) \\
& +\frac{1}{16 \pi G_{1}} \int d^{4} x \sqrt{-\tilde{g}} \frac{m^{2}}{4}\left(M_{A}^{\tilde{A}} M_{\tilde{A}}^{A}+M_{\tilde{A}}^{A} M_{A}^{\tilde{A}}-\gamma\right) \\
& -\frac{1}{16 \pi G_{2}} \int d^{4} x \sqrt{-\tilde{g}} m^{2} \phi^{2},
\end{aligned}
$$

where the current $j_{\mu}$ is defined as

$$
j_{\mu}^{\tilde{A} \tilde{B}} \equiv \tilde{g}^{\tilde{B} \tilde{C}}\left[\tilde{\nabla}_{\mu} M_{B}^{\tilde{A}}\right] M_{\tilde{C}}^{B}
$$

and where $G_{1}$ and $G_{2}$ are new gravitational constants, $m$ is a mass parameter, and $\gamma$ is a free parameter which corresponds to the cosmological constant. Note that the action for $M$ is similar to that of the non-linear sigma model found in meson physics.

Drummond has shown that his bimetric theory has a well defined Newtonian limit and so, in principle, can satisfy the time delay measurements from radio signals. He also claims that the higher order correction is exactly what is needed to satisfy Solar System constraints from the precession of the orbit of Mercury, and that in the weak field limit the dynamical metric $g_{\alpha \beta} \simeq \eta_{\alpha \beta}+h_{\alpha \beta}$ gives rise to a potential of the form

$$
h_{00}=-\frac{G M}{r}\left(1+\frac{G_{1}}{G} e^{-m r}\right) \text {. }
$$

Hence, for $m r \ll 1$ the effective Newton's constant is $G_{N}=G+G_{1}$, while for large scales $G_{N} \simeq G$. Such a correction can alleviate the problem of flat galactic rotation curves that arises in standard Newtonian gravity with no dark matter, but does not completely resolve it. Albeit an intriguing proposal for a theory of modified gravity, there has been little progress in studying the various astrophysical and cosmological consequences of Drummond's theory.

\subsubsection{Massive gravity}

The theory of a single massive spin-2 field can also be considered as a bimetric theory, with a non-dynamical background metric $\tilde{g}_{\alpha \beta}$ and a dynamical fluctuation given by $g_{\alpha \beta}=$ 
$\tilde{g}_{\alpha \beta}+h_{\alpha \beta}$. Taking the background to be Minkowski space, for simplicity, we can then generate a mass for the spin-2 field $h_{\alpha \beta}$ by adding the Pauli-Fierz (PF) term to the Einstein-Hilbert action [504, resulting in

$$
S_{P F}=\frac{1}{16 \pi G} \int d^{4} x \sqrt{-g} R+\frac{m^{2}}{4} \sqrt{-g}\left[g^{\mu \nu} g^{\alpha \beta}-g^{\mu \alpha} g^{\mu \beta}\right] h_{\mu \nu} h_{\alpha \beta},
$$

where $m$ is a constant mass parameter. It is well known that, in four dimensions, a massive spin-2 field ought to have five propagating degrees of freedom: Two of helicity 2 , two of helicity 1 , and one of helicity 0 . However, a generic mass term with arbitrary coefficients will result in higher derivative terms for the helicity- 0 mode, giving rise to an additional ghost-like degree of freedom. The form of the PF mass term is specifically chosen so that this is not the case to linear order. Massive gravity exhibits some interesting phenomenology, not least the so-called vDVZ discontinuity, and its possible resolution via the Vainshtein mechanism. These will be discussed in more detail in the context of DGP gravity in Section 5.5 .

Unfortunately, the PF Lagrangian by itself cannot describe a consistent theory because the ghost-like mode reappears at non-linear order [175. This mode is often referred to as the Boulware-Deser ghost, and it was believed that one could not find generalisations of the theory that succeeded in eliminating it to all orders 342. There has, however, been some recent progress on this issue by de Rham and Gabadadze and collaborators [517, 380, 383, 382, 385] who have proposed the following action [382, 385]:

$$
S_{G P F}=\frac{1}{16 \pi G} \int d^{4} x \sqrt{-g} R+m^{2} \sqrt{-g} U(g, h),
$$

where $U(g, h)=\sum_{n=2}^{4} a_{m} \delta_{\left[\nu_{1}\right.}^{\mu_{1}} \ldots \delta_{\left.\nu_{m}\right]}^{\mu_{m}} K_{\nu_{1}}^{\mu_{1}} \cdots K_{\nu_{m}}^{\mu_{m}}$ and where the $a_{m}$ are constants, and

$$
K_{\nu}^{\mu}=\sqrt{\delta_{\nu}^{\mu}+g^{\mu \alpha} h_{\alpha \nu}}-\delta_{\nu}^{\mu}=\frac{1}{2} g^{\mu \alpha} h_{\alpha \nu}-\frac{1}{8} g^{\mu \alpha} h_{\alpha \beta} g^{\beta \gamma} h_{\gamma \nu}+\ldots
$$

It is now clear that the leading order part of the potential gives the PF mass term upon choosing $a_{2}=2$. To study the behaviour of the theory beyond linear order it is convenient to restore general coordinate invariance by means of the Stuckelberg trick. To this end one can perform the following field redefinition [382, 64,

$$
\begin{aligned}
h_{\mu \nu} & =\frac{\hat{h}_{\mu \nu}}{M_{p l}}+\eta_{\mu \nu}-\eta_{\alpha \beta} \frac{\partial \phi^{\alpha}}{\partial x^{\mu}} \frac{\partial \phi^{\beta}}{\partial x^{\nu}} \\
& =\frac{\hat{h}_{\mu \nu}}{M_{p l}}+2 \frac{\partial_{\mu} \partial_{\nu} \pi}{\Lambda_{3}^{3}}-\frac{\eta^{\alpha \beta} \partial_{\alpha} \partial_{\mu} \pi \partial_{\beta} \partial_{\nu} \pi}{\Lambda_{3}^{6}},
\end{aligned}
$$

where in going from Eq. 201 to Eq. 202 we have set $\phi^{\alpha}=x^{\alpha}-\eta^{\alpha \beta} \partial_{\beta} \pi / \Lambda_{3}$, in order to focus on the dynamics of the helicity zero mode. Note that $\Lambda_{3}=\left(M_{p l} m^{2}\right)^{1 / 3}$ and $M_{p l}=1 / \sqrt{8 \pi G}$. For the original PF action, Eq. 198), the Boulware-Deser ghost reveals itself by expanding the action in terms of $\hat{h}_{\mu \nu}$. At zeroth order one finds higher derivative terms for $\pi$ that contribute to the equations of motion, indicating the presence of the ghostly extra mode. In contrast, the generalised PF action is chosen so that the resulting higher derivative terms contribute a total derivative at zeroth order in $\hat{h}_{\mu \nu}$. 
This is a crucial first step in avoiding the extra mode. One can go further and study the theory in the so-called decoupling limit, $m \rightarrow 0, M_{p l} \rightarrow \infty$, and $\Lambda_{3}=$ constant. After some suitable field redefinitions one finds that the theory contains the quintic galileon Lagrangian [382. Note that it does not reproduce the galileon theory discussed in Section 4.4 exactly, since generically there is mixing with a graviton of the form $\hat{h}^{\mu \nu} X_{\mu \nu}^{3}$, where $X_{\mu \nu}^{3}$ is cubic in $\pi$. This mixing cannot be eliminated by a local field redefinition and may have important phenomenological consequences. In particular, when this coupling comes in with a particular sign it can prevent the recovery of GR inside the Vainshtein radius around a heavy source [738, 739, 297]. In any event, one can confidently say that the Boulware-Deser ghost does not appear in the decoupling limit. Of course, it is possible that this limit corresponds to taking its mass to infinity, and that it will reemerge in the full theory. Whether or not this is the case has yet to be established.

Self-accelerating and self-tuning cosmologies were studied de Rham and Gabadadze's theory in 384, whilst spherically symmetric solutions have also been considered recently [738, 739, 297].

\subsubsection{Bigravity}

A class of theories that were first proposed in the 1970s by Isham, Salam, Stradthee 647, and revisited a few years ago by Kogan 355 and collaborators, have recently been resurrected by Bañados and collaborators [84, 85, 70, (see for [1150] a short overview). Further studies of bi-gravity include weak-field solutions and gravitational waves [140], exact spherically symmetric solutions [141] and the energy of black holes [329. The starting point [84 is an extension of Eddington's affine theory (see section 20) so that the dynamical fields are a metric $g_{\mu \nu}$ (with curvature scalar $R$ ) and a connection $C_{\mu \nu}^{\alpha}$ with Ricci tensor $K_{\mu \nu}[C]$. The action is

$$
S[g, C]=\frac{1}{16 \pi G} \int d^{4} x \sqrt{-g}(R-2 \Lambda)+\frac{2}{\alpha \ell^{2}} \sqrt{-\operatorname{det}\left[g_{\mu \nu}-\ell^{2} K_{\mu \nu}\right]}
$$

where $\Lambda$ is a cosmological constant, $\alpha$ is a dimensionless parameter and $\ell$ is a length scale. It may be shown [85 by introducing a 2 nd metric $\tilde{g}_{\mu \nu}$ corresponding to the connection $C_{\mu \nu}^{\alpha}$ that the above theory is a special case of bigravity with action given by

$$
S=\frac{1}{16 \pi G} \int d^{4} x\left[\sqrt{-g}(R-2 \Lambda)+\sqrt{-\tilde{g}}(\tilde{R}-2 \tilde{\Lambda})-\sqrt{-\tilde{g}} \frac{1}{\ell^{2}}\left(\tilde{g}^{-1}\right)^{\alpha \beta} g_{\alpha \beta}\right],
$$

where $\tilde{\Lambda}=\frac{\alpha}{\ell^{2}}$ is a cosmological constant term. In these theories, both metrics are used to build Einstein-Hilbert actions even though only one of them couples to the matter content.

Such bigravity theories lead to interesting behaviour on cosmological scales [85, 70]. The homogeneous and isotropic FLRW metrics can be written as $g_{\alpha \beta}=\operatorname{diag}\left(-1, a^{2}, a^{2}, a^{2}\right)$ and $\tilde{g}_{\alpha \beta}=\operatorname{diag}\left(-X^{2}, Y^{2}, Y^{2}, Y^{2}\right)$, where $X$ and $Y$ are functions of $t$ alone. The corresponding Friedmann equations are then of the form

$$
H^{2}=\frac{8 \pi G}{3}(\tilde{\rho}+\rho)
$$

where $\tilde{\rho}=Y^{3} /\left(8 \pi G \ell^{2} X a^{3}\right)$. This fluid satisfies a conventional conservation equation of the form

$$
\frac{d \tilde{\rho}}{d t}=-3(1+\tilde{w}) \tilde{\rho},
$$


where $\tilde{w}$, satisfies a somewhat intricate evolution equation, given by

$$
\frac{d \tilde{w}}{d t}=2 \tilde{w}\left[1+3 \tilde{w}+\sqrt{4(-\tilde{w})^{3 / 2} \tilde{\Omega} \alpha-2 \frac{(1+3 \tilde{w}) \rho_{\ell}}{\rho_{c}}}\right],
$$

where $\rho_{c}=\rho+\tilde{\rho}, \rho_{\ell}=\left(8 \pi G \ell^{2}\right)^{-1}$ and $\tilde{\Omega}=\tilde{\rho} / \rho_{c}$. The extra metric here can lead to a range of interesting behaviours and, in particular, can drive the expansion to a de Sitter phase, or mimic the effects of dark matter. Anisotropic universes in these models were studied in [1066.

The cosmological evolution of perturbations in these theories has been worked out in some detail, and it turns out that the perturbations in the auxiliary field can be rewritten in the form of a generalised dark matter fluid 626 with density, momentum, pressure and shear that obey evolution equations. As a result, it is possible to work out cosmological observables such as CMB anisotropies and large-scale structure. In [70] it was found that distinctive signatures emerge during periods of accelerated expansion. If the $\tilde{\rho}$ field dominates, and is responsible for cosmic acceleration, there is a clear instability in the gravitational potentials; they not only grow but diverge leading very rapidly to an overwhelming integrated Sachs-Wolfe effect on large scales. It is difficult to reconcile the angular power spectrum of fluctuations and the power spectrum of the galaxy distribution predicted by a bimetric theory that unifies the dark sector with current data. If we restrict ourselves to a regime in which $\tilde{\rho}$ simply behaves as dark matter, however, then the best-fit bimetric model is entirely indistinguishable from the standard CDM scenario.

Bigravity theory can also be extended to consider more complicated actions, such as

$$
S_{L}=-\frac{1}{16 \pi G} \int d^{4} x \sqrt{-\tilde{g}}\left[\kappa_{0}\left(\tilde{g}^{-1}\right)^{\alpha \beta} g_{\alpha \beta}+\kappa_{1}\left(\left(\tilde{g}^{-1}\right)^{\alpha \beta} g_{\alpha \beta}\right)^{2}+\kappa_{2}\left(\tilde{g}^{-1}\right)^{\alpha \beta}\left(\tilde{g}^{-1}\right)_{\alpha \beta}\right],
$$

and, although a full analysis of its PPN parameters has been undertaken [308, its cosmology remains to be explored. Black holes, and their thermodynamics, have been studied in bimetric gravity in [71].

\subsubsection{Bimetric MOND}

A bimetric theory of MOND, somewhat akin to bigravity, has recently been proposed by Milgrom [896. The action for bimetric MOND, or BIMOND, is of the form

$$
\begin{aligned}
S= & \frac{1}{16 \pi G} \int d^{4} x\left[\beta \sqrt{-g} R+\alpha \sqrt{-\tilde{g}} \tilde{R}-2(\tilde{g} g)^{1 / 4} a_{0}^{2} \mathcal{M}\right] \\
& -\tilde{S}_{M}\left(\tilde{g}_{\mu \nu}, \tilde{\psi}\right)-S_{M}\left(g_{\mu \nu}, \psi\right)
\end{aligned}
$$

where $\mathcal{M}$ is the interaction term that connects the two metrics, and $\psi$ and $\tilde{\psi}$ are the matter fields that couple to $g_{\mu \nu}$ and $\tilde{g}_{\mu \nu}$, respectively. The factor $\mathcal{M}$ is a non-linear function of the tensor $\Upsilon_{\mu \nu}$, given by

$$
\Upsilon_{\mu \nu}=C^{\alpha}{ }_{\mu \beta} C^{\beta}{ }_{\nu \alpha}-C^{\alpha}{ }_{\mu \nu} C^{\alpha}{ }_{\alpha \alpha},
$$

where

$$
C_{\mu \nu}^{\alpha}=\tilde{\Gamma}^{\alpha}{ }_{\mu \nu}-\Gamma_{\mu \nu}^{\alpha}
$$


and $\Gamma_{\mu \nu}^{\alpha}$ and $\tilde{\Gamma}_{\mu \nu}^{\alpha}$ are the Christoffel symbols constructed from $g_{\mu \nu}$ and $\tilde{g}_{\mu \nu}$, respectively. Note that the even though the $\Gamma$ s are not tensors, $C$, constructed in this way, is a tensor. The constants $\alpha$ and $\beta$ can be kept unrelated, leading to different gravitational couplings in the two sectors. If we set $\alpha=\beta$, however, but leave $\beta$ arbitrary, then we get the field equations

and

$$
\beta G_{\mu \nu}+S_{\mu \nu}=-8 \pi G T_{\mu \nu}
$$

$$
\beta \tilde{G}_{\mu \nu}+\tilde{S}_{\mu \nu}=-8 \pi G \tilde{T}_{\mu \nu}
$$

which look like the conventional Einstein equations, except for the contributions from $S_{\mu \nu}$ and $\tilde{S}_{\mu \nu}$ which contain the interaction terms between the two metrics. These tensors are quadratic in $C^{\alpha}{ }_{\mu \nu}$, and are non-linear functions of $g, \tilde{g}$ and $g^{\mu \nu} \tilde{g}_{\mu \nu}$.

This theory has been constructed to reproduce MOND phenomenology on small scales, in the weak field and low acceleration regime. Its cosmological implications have been studied in [315, 897, where it was shown that in the high acceleration regime BIMOND reproduces conventional FLRW behaviour. In low acceleration regime, however, we have that the scale factor $a(\tau)$ (where $\tau$ is conformal time) can take the form the form $a \simeq \tau^{p}$, where 315

$$
p=\frac{1-3 \tilde{w}}{(1+2 w+8 n w-3 \tilde{w}-8 n \tilde{w}-6 w \tilde{w})} .
$$

and where $w$ and $\tilde{w}$ are the equations of state for the matter coupled to $g_{\mu \nu}$ and $\tilde{g}_{\mu \nu}$, and $n$ is one of the parameters in $\mathcal{M}$. This leads to an interesting range of behaviours. For example, it is possible to have a dust filled universe that is static, if the matter coupled to the second metric is radiation. It is shown in 897] that to calculate fluctuations about an FLRW background in either metric requires a knowledge of the matter coupled to both metrics. It is also shown that the growth of fluctuations does not proceed in a purely Newtonian way, but has a MOND contribution as well.

\subsection{Tensor-Vector-Scalar Theories}

In General Relativity, the space-time metric $g_{\mu \nu}$ is the sole dynamical agent of gravity. We have seen above that scalar-tensor theories extend this by adding a scalar field that mediates a spin- 0 gravitational interaction, while in Einstein-æther theories one makes use of a vector field. TeVeS has both of these types of fields as extra degrees of freedom: A scalar field, $\phi$, and a (dual) vector field, $A_{\mu}$, both of which participate in the gravitational sector. Like GR, it obeys the Einstein equivalence principle, but unlike GR it violates the strong equivalence principle.

$\mathrm{TeVeS}$ is a product of past antecedent theories, namely the Aquadratic Lagrangian theory of gravity (AQUAL) and its relativistic version [125, the phase-coupling gravitation [126], the disformal relativistic scalar field theory [130], and the Sanders' stratified vector field theory 1093. Since its inception 128, TeVeS has been intensively researched, including studies of cosmology [128, [592, 1153, 419, 427, 1147, 177, 1305, 1148, 502, spherically symmetric solutions [128, 535, 660, 1082, 774, 1154, gravitational collapse and stability [1124, 331, solar system tests [128, 535, 124, 1198, 1081, gravitational lensing [128, 295, 288, 1307, 1306, 287, 498, 1291, 1128, 296, 870, issues of superluminality [205], and the travel time of gravitational waves [664, 663, 414. A thorough and up-to-date review of TeVeS can be found in [1152. Here we will concentrate mostly on cosmological features of the theory. 


\subsubsection{Actions and field equations}

The original and most common way to specify $\mathrm{TeVeS}$ is to write the action in a mixed frame. That is, we write the action in the "Bekenstein frame" for the gravitational fields, and in the 'physical frame', for the matter fields. In this way we ensure that the Einstein equivalence principle is obeyed. The three gravitational fields are the metric, $\tilde{g}_{\mu \nu}$ (with connection $\tilde{\nabla}_{a}$ ), that we refer to as the Bekenstein metric, the Sanders vector field, $A_{\mu}$, and the scalar field, $\phi$. To ensure that the Einstein equivalence principle is obeyed, we write the action for all matter fields using a single 'physical metric', $g_{\mu \nu}$ (with connection $\nabla_{\mu}$ ), that we call the 'universally coupled metric' ${ }^{20}$. The universally coupled metric is algebraically defined via a disformal relation [127] as

$$
g_{\mu \nu}=e^{-2 \phi} \tilde{g}_{\mu \nu}-2 \sinh (2 \phi) A_{\mu} A_{\nu} .
$$

The vector field is further enforced to be unit-time-like with respect to the Bekenstein metric, i.e.

$$
\tilde{g}^{\mu \nu} A_{\mu} A_{\nu}=-1 .
$$

The unit-time-like constraint is a phenomenological requirement for the theory to give an acceptable amount of light bending. Using the unit-time-like constraint, Eq. 216, it can be shown that the disformal transformation for the inverse metric is

$$
g^{\mu \nu}=e^{2 \phi} \tilde{g}^{\mu \nu}+2 \sinh (2 \phi) A^{\mu} A^{\nu},
$$

where $A^{\mu}=\tilde{g}^{\mu \nu} A_{\nu}$. The existence of a scalar and a vector field may seem odd at first, but they are both the product of a series of extensions from older theories, based on theoretical and phenomenological constraints.

\section{Actions}

TeVeS is based on an action, $S$, which is split as

$$
S=S_{\tilde{g}}+S_{A}+S_{\phi}+S_{m},
$$

where $S_{\tilde{g}}, S_{A}, S_{\phi}$ and $S_{m}$ are the actions for $\tilde{g}_{\mu \nu}$, the vector field, $A_{\mu}$, the scalar field, $\phi$, and matter fields, respectively.

As already discussed, the action for $\tilde{g}_{\mu \nu}, A_{\mu}$, and $\phi$ is written using only the Bekenstein metric, $\tilde{g}_{\mu \nu}$, and not $g_{\mu \nu}$, and is such that $S_{\tilde{g}}$ is of Einstein-Hilbert form

$$
S_{\tilde{g}}=\frac{1}{16 \pi G} \int d^{4} x \sqrt{-\tilde{g}} \tilde{R},
$$

where $\tilde{g}$ and $\tilde{R}$ are the determinant and scalar curvature of $\tilde{g}_{\mu \nu}$, respectively, while $G$ is the bare gravitational constant. The relation between $G$ and the measured value of Newton's constant, $G_{N}$, will be elaborated on below, in Section 3.4.2.

\footnotetext{
${ }^{20}$ Some work on TeVeS, including the original articles by Sanders [1093] and Bekenstein [128], refer to the Bekenstein frame metric as the "geometric metric", and denote it as $g_{\mu \nu}$, while the universally coupled metric is referred to as the "physical metric", and is denoted by $\tilde{g}_{\mu \nu}$. Since it is more common to denote the metric which universally couples to matter as $g_{\mu \nu}$, in this review we interchange the tilde.
} 
The action for the vector field, $A_{\mu}$, is given by

$$
S_{A}=-\frac{1}{32 \pi G} \int d^{4} x \sqrt{-\tilde{g}}\left[K F^{\mu \nu} F_{\mu \nu}-2 \lambda\left(A_{\mu} A^{\mu}+1\right)\right],
$$

where $F_{\mu \nu}=\nabla_{\mu} A_{\nu}-\nabla_{\nu} A_{\mu}$ leads to a Maxwellian kinetic term, $\lambda$ is a Lagrange multiplier that ensures the unit-timelike constraint on $A_{\mu}$, and $K$ is a dimensionless constant. Indices on $F_{\mu \nu}$ are moved using the Bekenstein metric, i.e. $F^{\mu}{ }_{\nu}=\tilde{g}^{\mu \alpha} F_{\alpha \nu}$. This form of a vector field action has been considered by Dirac as a way of incorporating electrons into the electromagnetic potential [424, 425, 426. More recently it has been considered as a natural generalisation of GR, in the Einstein-æther theories discussed in Section 3.2 656, 655.

The action for the scalar field, $\phi$, is given by

$$
S_{\phi}=-\frac{1}{16 \pi G} \int d^{4} x \sqrt{-\tilde{g}}\left[\mu \hat{g}^{\mu \nu} \tilde{\nabla}_{\mu} \phi \tilde{\nabla}_{\nu} \phi+V(\mu)\right],
$$

where $\mu$ is a non-dynamical dimensionless scalar field, $\hat{g}^{\mu \nu}$ is a new metric defined by

$$
\hat{g}^{\mu \nu}=\tilde{g}^{\mu \nu}-A^{\mu} A^{\nu},
$$

and $V(\mu)$ is an arbitrary function which typically depends on a scale, $\ell_{B}$. Not all choices of $V(\mu)$ give the correct Newtonian or MONDian limits in a quasi-static situation. The allowed choices are presented in Section 3.4.2. The metric $\hat{g}^{\mu \nu}$ is used in the scalar field action, rather than $\tilde{g}^{\mu \nu}$, to avoid the superluminal propagation of perturbations. Note that it is possible to write the TeVeS action using $\hat{g}^{\mu \nu}$, with the consequence of having more general vector field kinetic terms (see the appendix of [1152]).

It is also easier in some cases to work with an alternative form for the scalar field action that does not have the non-dynamical field, $\mu$, but rather has the action written directly in terms of a non-canonical kinetic term for $\phi$ given by a free function $f(X)$, with $X$ defined by

$$
X=\ell_{B}^{2} \hat{g}^{\mu \nu} \nabla_{\mu} \phi \nabla_{\nu} \phi .
$$

The field $\mu$ is then given in terms of $f(X)$ by $\mu=\frac{d f}{d X}$, while $f(X)$ can be related to $V$ by $f=\mu X+\ell_{B}^{2} V$.

The matter fields in the action are coupled only to the 'universally coupled metric', $g_{\mu \nu}$, and thus their action is of the form

$$
S_{m}\left[g, \chi^{A}, \nabla \chi^{A}\right]=\int d^{4} x \sqrt{-g} L\left[g, \chi^{A}, \nabla \chi^{A}\right],
$$

for some generic collection of matter fields, $\chi^{A}$. The matter stress-energy tensor is then defined with respect to $\delta S_{m}$ in the usual way.

It should be stressed that the action for the scalar field has been constructed such that the theory has a MONDian non-relativistic limit, under the right conditions, for specific choices of functions $V(\mu)$ (or equivalently $F(X)$ ). The action for the vector field has no particular significance other than the fact that it is simple. More general actions can be considered without destroying the MOND limit, but that in addition provide new features or improved phenomenology. 
The field equations

The field equations of TeVeS are found using a variational principle. This gives two constraint equations, namely the unit-timelike constraint, given in Eq. 216), and the $\mu$-constraint:

$$
\hat{g}^{\mu \nu} \nabla_{\mu} \phi \nabla_{\nu} \phi=-\frac{d V}{d \mu},
$$

that is used to find $\mu$ as a function of $\nabla_{\mu} \phi$. The field equations for $\tilde{g}_{\mu \nu}$ are given by

$$
\begin{aligned}
\tilde{G}_{\mu \nu}= & 8 \pi G\left[T_{\mu \nu}+2\left(1-e^{-4 \phi}\right) A^{\alpha} T_{\alpha(\mu} A_{\nu)}\right] \\
& +\mu\left[\tilde{\nabla}_{\mu} \phi \tilde{\nabla}_{\nu} \phi-2 A^{\alpha} \tilde{\nabla}_{\alpha} \phi A_{(\mu} \tilde{\nabla}_{\nu)} \phi\right]+\frac{1}{2}\left(\mu V^{\prime}-V\right) \tilde{g}_{\mu \nu} \\
& +K\left[F_{\mu}^{\alpha} F_{\alpha \nu}-\frac{1}{4} F^{\alpha \beta} F_{\alpha \beta} \tilde{g}_{\mu \nu}\right]-\lambda A_{\mu} A_{\nu},
\end{aligned}
$$

where $\tilde{G}_{\mu \nu}$ is the Einstein tensor constructed from $\tilde{g}_{\mu \nu}$. The field equations for the vector field, $A_{\mu}$, are

$$
K \tilde{\nabla}_{\alpha} F_{\mu}^{\alpha}=-\lambda A_{\mu}-\mu A^{\nu} \tilde{\nabla}_{\nu} \phi \tilde{\nabla}_{\mu} \phi+8 \pi G\left(1-e^{-4 \phi}\right) A^{\nu} T_{\nu \mu},
$$

and the field equation for the scalar field, $\phi$, is

$$
\tilde{\nabla}_{\mu}\left[\mu \hat{g}^{\mu \nu} \tilde{\nabla}_{\nu} \phi\right]=8 \pi G e^{-2 \phi}\left[g^{\mu \nu}+2 e^{-2 \phi} A^{\mu} A^{\nu}\right] T_{\mu \nu} .
$$

The Lagrange multiplier can be solved for by contracting Eq. (227) with $A^{\mu}$.

\subsubsection{Newtonian and MOND limits}

We now describe the quasi-static limit, as relevant for establishing the existence of the Newtonian and MONDian limits. The details of the derivation we consider here can be found in [128, while an alternative shorter derivation in the spirit of the PPN formalism is given in [1152.

It can be shown that the PPN parameter $\gamma$ is unity in TeVeS, hence the universally coupled metric can be written as

$$
d s^{2}=-(1+2 \Phi) d t^{2}+(1-2 \Phi) \delta_{i j} d x^{i} d x^{j},
$$

where $\Phi$ is related to the acceleration of particles, $\vec{a}$, by $\vec{a}=-\vec{\nabla} \Phi$. The scalar field is perturbed as $\phi=\phi_{c}+\varphi$, where $\phi_{c}$ is the cosmological value of $\phi$. The Bekenstein metric takes a similar form to $g_{\mu \nu}$ :

$$
d \tilde{s}^{2}=-e^{-2 \phi_{c}}(1+2 \tilde{\Phi}) d t^{2}+e^{2 \phi_{c}}(1-2 \tilde{\Phi}) \delta_{i j} d x^{i} d x^{j} .
$$

The vector field does not play a role at this order of perturbations, and is simply given by

$$
A_{\mu}=e^{-\phi_{c}}(-1-\tilde{\Phi}, 0,0,0) \text {. }
$$


The field equations to $O\left(v^{2}\right)$ are then

$$
\Delta \tilde{\Phi}=\frac{8 \pi G}{2-K} \rho,
$$

and

$$
\vec{\nabla} \cdot[\mu \vec{\nabla} \varphi]=8 \pi G \rho
$$

while the potential $\Phi$ is given via the disformal transformation $\Phi=\tilde{\Phi}+\varphi$. Eqs. 232 and 2233 can be solved for any quasi-static situation, regardless of the boundary conditions or the symmetry of the system in question, provided a function $\mu(|\vec{\nabla} \varphi|)$ is supplied.

To find the Newtonian and MONDian limits we can consider, for simplicity, spherically symmetric situations. In this case we can combine Eqs. 232 and 233 into a single equation for $\Phi$, called the AQUAL equation:

$$
\vec{\nabla} \cdot\left[\mu_{m} \vec{\nabla} \Phi\right]=4 \pi G_{N} \rho,
$$

where

$$
\mu_{m}=\frac{G_{N}}{2 G} \frac{\mu}{\left(1+\frac{\mu}{2-K}\right)} .
$$

The ratio $G_{N} / G$ is not free, but is found by taking the limit $\mu_{m} \rightarrow 1$, i.e. the Newtonian limit. Consistency requires that $\mu \rightarrow \mu_{0}$ which is then a constant ${ }^{21}$ contained in the function $f$ (or $V$ ). This gives the relation

$$
\frac{G_{N}}{G}=\frac{2}{\mu_{0}}+\frac{2}{(2-K)}
$$

The MOND limit is now clearly recovered as $\mu_{m} \rightarrow \frac{\left|\vec{\nabla} \Phi_{N}\right|}{a_{0}}$, and we get

$$
\mu \rightarrow \frac{2 G}{G_{N}} \frac{|\vec{\nabla} \varphi|}{a_{0}}=\frac{2 G}{G_{N}} \frac{1}{\ell_{B} a_{0}} e^{\phi_{c}} \sqrt{X},
$$

where $X$ is given in Eq. 223). Since $\mu=\frac{d f}{d X}$, we may integrate the above equation to find the function $f(X)$, which in the MOND limit should be given by

$$
f \rightarrow \frac{2}{3 \ell_{B} a_{0}} \frac{1}{\left(\frac{1}{\mu_{0}}+\frac{1}{2-K}\right)} e^{\phi_{c}} X^{3 / 2},
$$

where the integration constant has been absorbed into the cosmological constant associated with the metric $\tilde{g}_{\mu \nu}$. Since both $X$ and $f$ are dimensionless we may define a new constant $\beta_{0}$, such that $a_{0}$ is a derived quantity given by

$$
a_{0}=\frac{2}{3 \beta_{0} \ell_{B}} \frac{1}{\left(\frac{1}{\mu_{0}}+\frac{1}{2-K}\right)} e^{\phi_{c}},
$$

\footnotetext{
${ }^{21}$ The constant $\mu_{0}$ is related to the constant $k$ introduced by Bekenstein as $\mu_{0}=\frac{8 \pi}{k}$.
} 
and the function $f$ has the MONDian limit $f \rightarrow \beta_{0} X^{3 / 2}$. Since in the Newtonian limit we have $f \rightarrow \mu_{0} X$, there are at least three constants that can appear in $f(X)$, namely $\mu_{0}, \beta_{0}$ and $\ell_{B}$.

In terms of the function $\frac{d V}{d \mu}$ the MONDian limiting case implies that $\frac{d V}{d \mu} \rightarrow-\frac{4}{9 \beta_{0}^{2} \ell_{B}^{2}} \mu^{2}$ as $\mu \rightarrow 0$, while it diverging as $\mu \rightarrow \mu_{0}$ in the Newtonian limit. This second limit is imposed if $\frac{d V}{d \mu} \rightarrow\left(\mu_{0}-\mu\right)^{-m}$, for some constant $m$. Bekenstein chooses this to be $m=1$, although other choices are equally valid, even functions that have essential singularities.

It is clear from Eq. 239 that $a_{0}$ depends on the cosmological boundary condition, $\phi_{c}$, which can differ for each system, depending on when it was formed. It could thus be considered as a slowly varying function of time. This possibility has been investigated by Bekenstein and Sagi [129, and by Limbach et al. [810.

The two limiting cases for $f(X)$ are somewhat strange. In particular we require that $f(X) \rightarrow X$ for $X \gg 1$ to recover the Newtonian limit, and that $f(X) \rightarrow X^{3 / 2}$ for $X \ll 1$ (i.e. a higher power) to recover the MONDian limit. This signifies that in this kind of formulation of relativistic MOND (i.e. in terms of a scalar field) the function $f(X)$ should be non-analytic. It further signifies that $f(X)$ can be expanded in positive powers of $\sqrt{X}$ for small $X$, and in positive powers of $\frac{1}{X}$ for large $X$, but that these two expansions cannot be connected. In other words, it is impossible to perturbatively connect the Newtonian regime with the MONDian regime via a perturbation series in $|\vec{\nabla} \varphi|$.

The Bekenstein free function in 128 is given in the notation used in this review by

$$
\frac{d V}{d \mu}=-\frac{3}{32 \pi \ell_{B}^{2} \mu_{0}^{2}} \frac{\mu^{2}\left(\mu-2 \mu_{0}\right)^{2}}{\left(\mu_{0}-\mu\right)}
$$

which means that $\beta_{0}=\frac{4}{3} \sqrt{\frac{2 \pi \mu_{0}}{3}}$, and thus

$$
a_{0}=\frac{\sqrt{3}}{2 \sqrt{2 \pi \mu_{0}} \ell_{B}} \frac{1}{\left(\frac{1}{\mu_{0}}+\frac{1}{2-K}\right)} e^{\phi_{c}} .
$$

This is in agreement with [129] (the authors of [177] erroneously inverted a fraction in their definition of $a_{0}$ ).

\subsubsection{Homogeneous and isotropic cosmology}

Homogeneous and isotropic Friedmann-Lemaitre-Robertson-Walker (FLRW) solutions to the field equations of TeVeS have been extensively studied [128, 592, 1153, 419, 427, 1147, 177, 1305, 1148, 502. In this case the universally coupled metric can be written in the conventional synchronous form as

$$
d s^{2}=-d t^{2}+a^{2}(t) q_{i j} d x^{i} d x^{j},
$$

where $a(t)$ is the 'physical scale factor'. Here we assume for simplicity that the hypersurfaces of constant $t$ are spatially flat (see [1147] for the curved case). The Bekenstein metric then has a similar form, and can be written as

$$
d \tilde{s}^{2}=-d \tilde{t}^{2}+b^{2}(\tilde{t}) q_{i j} d x^{i} d x^{j}
$$


for a second scale factor $b(\tilde{t})$. The disformal transformation relates the two scale factors by $a=b e^{-\phi}$, while the two time coordinates $t$ and $\tilde{t}$ are related by $d t=e^{\phi} d \tilde{t}$. The physical Hubble parameter is defined as usual as by $H=\frac{\dot{a}}{a}$, while the Bekenstein frame Hubble parameter is $\tilde{H}=\frac{d \ln b}{d \tilde{t}} e^{\phi} H+\frac{d \phi}{d \tilde{t}}$. Cosmological evolution is governed by the analogue of the Friedmann equation:

$$
3 \tilde{H}^{2}=8 \pi G e^{-2 \phi}\left(\rho_{\phi}+\rho\right),
$$

where $\rho$ is the energy density of physical matter, which obeys the energy conservation equation with respect to the universally coupled metric, and where the scalar field energy density, $\rho_{\phi}$, is given by

$$
\rho_{\phi}=\frac{e^{2 \phi}}{16 \pi G}\left(\mu \frac{d V}{d \mu}+V\right) .
$$

Similarly, one can define a scalar field pressure by

$$
P_{\phi}=\frac{e^{2 \phi}}{16 \pi G}\left(\mu \frac{d V}{d \mu}-V\right)
$$

The scalar field evolves according to the two differential equations:

$$
\frac{d \phi}{d \tilde{t}}=-\frac{1}{2 \mu} \Gamma
$$

and

$$
\frac{d \Gamma}{d \tilde{t}}+3 \tilde{H} \Gamma=8 \pi G e^{-2 \bar{\phi}}(\rho+3 P),
$$

where $\mu$ is found by inverting ${\phi^{\prime}}^{2}=\frac{1}{2} \frac{d V}{d \mu}$.

It is important to note that the vector field must point to the time direction, so that it can be written as $A_{\mu}=\left(\sqrt{\tilde{g}_{00}}, \overrightarrow{0}\right)$. In this case it does not contain any independent dynamical information, and it does not explicitly contribute to the energy density. Its only effect is on the disformal transformation which relates the Bekenstein-frame Friedmann equation, Eq. 244, with the physical Friedmann equation. This is also true in cases where the vector field action is generalised, and where the only effect is a constant rescaling of the left-hand-side of the Bekenstein-frame Friedmann equation, as discussed in [1148].

\section{FLRW solutions with the Bekenstein function}

In the original TeVeS paper [128] Bekenstein studied the cosmological evolution of an FLRW universe by assuming that the free function is given by Eq. 240. He showed that the scalar field contribution to the Friedmann equation is very small, and that $\phi$ evolves very little from the early universe until today. He noted that with this choice of function, a cosmological constant term has to be added in order to have an accelerating expansion today, as appears to be required by cosmological observations.

Many other studies on cosmology in TeVeS have also used the Bekenstein function, see for example [592, 1153, 427, 1148. In particular, Hao and Akhoury noted that the integration constant obtained by integrating Eq. 240 can be used to get a period 
of accelerating expansion, and that TeVeS therefore has the potential to act as dark energy [592. However, such an integration constant cannot be distinguished from a bare cosmological constant term in the Bekenstein frame, and so it is somewhat dubious as to whether this can really be interpreted as dark energy arising from TeVeS. Nevertheless, it would not be a surprising result if some other TeVeS functions could, in fact account for dark energy, as the scalar field action in TeVeS close resemblances that of k-essence 65, 66]. Zhao has investigated this issue further [1305](see below).

Exact analytical and numerical solutions with the Bekenstein free function, Eq. 240, have been found by Skordis et al.in [1153, and by Dodelson and Liguori in [427. It turns out that not only, is the scalar field is subdominant, as Bekenstein noted, but its energy density also tracks the matter fluid energy density. The ratio of the energy density in the scalar field to that of ordinary matter then remains approximately constant, so that the scalar field tracks the matter dynamics. One then gets that

$$
\Omega_{\phi}=\frac{(1+3 w)^{2}}{6(1-w)^{2} \mu_{0}}
$$

where $w$ is the equation of state of the matter fields ${ }^{22}$. Since $\mu_{0}$ is required to be very large, the energy density in the scalar field is always small, with values typically less than $\Omega_{\phi} \sim 10^{-3}$ in a realistic situation. Tracker solutions are also present for this choice of function in versions of TeVeS with more general vector field actions [1148].

In realistic situations, tracking in the radiation era is almost never realised, as has been noted by Dodelson and Liguori [27. Rather, during the radiation era, the scalar field energy density is subdominant but slowly growing, such that $\phi \propto a^{4 / 5}$. However, upon entering the matter era $\phi$ settles into the tracker solution. This transient solution in the radiation era has been generalised by Skordis to arbitrary initial conditions for $\phi$, more general free functions (see below), and a general vector field action [1148]. It should be stressed that the solution in the radiation era is important for setting up initial conditions for the perturbations about FLRW solutions that are relevant for studying the CMB radiation and Large-Scale Structure (LSS).

From Eq. (239) we see that $a_{0}$ for a quasi-static system depends on the cosmological value of the scalar field at the time the system broke off from the expansion, and collapsed to form a bound structure. It is then possible that different systems could exhibit different values of $a_{0}$ depending on when they formed. The impact of evolving $a_{0}$ on observations has been investigated in [129, 810.

Finally, note that the sign of $\dot{\phi}$ changes between the matter and cosmological constant eras. In doing so, the energy density of the scalar field goes momentarily through zero, since it is purely kinetic and vanishes for zero $\dot{\phi}[1153$.

\section{FLRW solutions by generalising the Bekenstein function}

Bourliot et al. [177] studied more general free functions, that have the Bekenstein function as a special case. In particular they introduced two new parameters, a constant,

\footnotetext{
${ }^{22}$ Note that this excludes the case of a stiff fluid with $w=1$.
} 
$\mu_{a}$, and a power index, $n$, such that the free function is generalised to

$$
\frac{d V^{(n)}}{d \mu}=-\frac{3}{32 \pi \ell_{B}^{2} \mu_{0}^{2}} \frac{\mu^{2}\left(\mu-\mu_{a} \mu_{0}\right)^{n}}{\mu_{0}-\mu} .
$$

This function ${ }^{23}$ reduces to the Bekenstein function when $n=2$ and $\mu_{a}=2$. It retains the property of having a Newtonian limit as $\mu \rightarrow \mu_{0}$ and a MOND limit as $\mu \rightarrow 0$. The cosmological evolution depends on the power index, $n$. More general functions can also be constructed by considering the sum of the above prototypical function with arbitrary coefficients, i.e. by taking $\frac{d V}{d \mu}=\sum_{n} c_{n} \frac{d V^{(n)}}{d \mu}[177$.

Clearly $\frac{d V}{d \mu}\left(\mu_{a} \mu_{0}\right)=0$, and at this point $\dot{\phi} \rightarrow 0$. Now suppose that the integration constant is chosen such that $V\left(\mu_{a} \mu_{0}\right)=0$ as well. Then, just like the case of the Bekenstein function, one finds tracker solutions: The function $\mu$ is driven to $\mu=\mu_{a} \mu_{0}$, at which point $\dot{\phi}=0$. There are no oscillations around that point, but it is approached slowly so that it is exactly reached only in the infinite future. The scalar field relative density is now given by

$$
\Omega_{\phi}=\frac{(1+3 w)^{2}}{3 \mu_{a}(1-w)^{2} \mu_{0}}
$$

independent of the value of $n$. It should also be pointed out that the evolution of the physical Hubble parameter, $H$, can be different than the case of $G R$ even in the tracking phase [177. For example in the case $w=0$ we have $H \propto a^{-n_{h}}$, where $n_{h}=\frac{1+3 \mu_{a} \mu_{0}}{2\left(\mu_{a} \mu_{0}-1\right)}$.

Furthermore, just like the Bekenstein case, the radiation era tracker is untenable for realistic cosmological evolutions, for which $\mu_{0}$ must be large so that $\Omega_{\phi}$ is small $\left(\lesssim 10^{-3}\right)$. In this case we once again get a transient solution where the scalar field evolves as $\phi \propto a^{4 /(3+n)}$ [1148. In the case that the integration constant is chosen such that $V\left(\mu_{a} \mu_{0}\right) \neq 0$ one has an effective cosmological constant present. Thus, once again, we get tracker solutions until the energy density of the Universe drops to values comparable with this cosmological constant, at which time tracking comes to an end, and the Universe enters a de Sitter phase.

The cases $-2<n<0$ turn out to be pathological as they lead to singularities in the cosmological evolution [177. The case $n=-3$ is well behaved when the matter fluid is a cosmological constant, but is also pathological when $w=-1$ [177]. The cases for which $n \leq-4$ are well behaved in the sense that no singularities occur in the cosmological evolution. Contrary to the $n \geq 1$ cases, the cosmological evolution drives the function $\mu$ to infinity. Moreover, these cases do not display the tracker solutions of $n \geq 1$, but rather the evolution of $\rho_{\phi}$ is such that it evolves more rapidly than the matter density, $\rho$, and so quickly becomes subdominant. The general relativistic Friedmann equation is thus recovered, such that $3 H^{2}=8 \pi G \rho$. This also results in $\tilde{H}=H$, which means that the scalar field is slowly rolling.

The evolution of the scalar field variables $\Gamma, \phi$ and $\mu$ then depends on the equation of state of the matter fields. If the background fluid is a cosmological constant, then we get de Sitter solutions for both metrics, and it can be shown that $\Gamma=2 H\left(e^{-3 H t}-1\right)$.

\footnotetext{
${ }^{23}$ Note that [177] uses a different normalisation for $V$, and their results can be recovered by rescaling the $\ell_{B}$ used in this report by $\ell_{B} \rightarrow \ell_{B} \sqrt{\frac{3}{2} \mu_{0}^{n-3}}$.
} 
For the case of a stiff fluid, with $w=1$, we get that $\Gamma$ has power-law solutions that are inverse powers of $t$, so that $\Gamma=\frac{6}{t}+\frac{\Gamma_{0}}{t^{3}}$. A similar situation arises when $-1<w<1$, for which we get $\Gamma=\frac{2(1+3 w)}{1-w} H$, and the Hubble parameter evolves as $H=\frac{2}{3(1+w)} \frac{1}{t}$. Notice that the limit $w \rightarrow 1$, for the $-1<w<1$ case, does not smoothly approach the $w=1$ case.

Mixing different powers of $n \geq 1$ leads once again to tracker solutions. One may have to add an integration constant in order to keep $V\left(\mu_{a} \mu_{0}\right)=0$, although for certain combinations of powers $n$ and coefficients $c_{i}$ this is not necessary. Mixing $n=0$ with some other $n \geq 1$ cannot remove the pathological situation associated with the $n=0$ case. Mixing $n=0$ with both positive and negative powers could however lead to acceptable cosmological evolution since the effect of the negative power is to drive $\mu$ away from the $\mu=\mu_{a} \mu_{0}$ point. In general, if we mix an arbitrary number of positive and negative powers we get tracker solutions provided we can expand the new function in positive definite powers of $\left(\mu-\mu_{a}^{\prime} \mu_{0}\right)$, where $\mu_{a}^{\prime}$ is some number different from the old $\mu_{a}$.

The observational consequences for the CMB and LSS have not been investigated for this class of function, unlike the case of the Bekenstein function.

\section{Inflationary/accelerating expansion for general functions}

Diaz-Rivera, Samushia and Ratra 419] have studied cases where TeVeS leads to inflationary, or self-accelerating, solutions. They first consider the vacuum case, in which they find that de Sitter solutions exist with $b \sim e^{\tilde{H}_{0} \tilde{t}}$, where the Bekenstein frame Hubble constant $\tilde{H}_{0}$ is given by the free function as $\tilde{H}_{0}=\sqrt{\frac{\mu_{0}^{2} V}{6}}$, and where $\frac{d V}{d \mu}=0$ (i.e. the scalar field is constant, $\phi=\phi_{i}$ ). Such a solution will always exist in vacuum provided that the free function satisfies $\frac{d V}{d \mu}\left(\mu_{v}\right)=0$ and $V\left(\mu_{v}\right) \neq 0$, for some constant $\mu_{v}$. In that case, the general solution is not de Sitter since both $\phi$ and $\mu$ will be time-varying, but will tend to de Sitter as $\mu \rightarrow \mu_{v}$. Indeed, the $n \geq 1$ case of Bourliot et al. [177] with an integration constant is precisely this kind of situation.

In the non-vacuum case, for a fluid with equation of state $P=w \rho$, they make the ansatz $b^{3(1+w)}=e^{(1+3 w) \phi}$. This brings the Friedmann equation into the form $3 \tilde{H}^{2}=8 \pi G \rho_{0}+\frac{1}{2}\left(\mu \frac{d V}{d \mu}+V\right)$, where $\rho_{0}$ is the matter density today. Once again, they assume that the free-function-dependent general solution drives $\mu$ to a constant $\mu_{v}$, but $\phi$ is evolving. Thus, we must have that $\phi=\phi_{1} \tilde{t}+\phi_{2}$, such that $\dot{\phi}=\phi_{1}$ is a constant. In order for $\phi_{1}$ to be non-zero we must have $\frac{d V}{d \mu}\left(\mu_{v}\right) \neq 0$. However, there is a drawback to this approach. As they point out, consistency with the scalar field equation requires that $w<-1$. Furthermore, although this solution is a de Sitter solution in the Bekensteinframe, it corresponds to a power-law solution for the universally coupled metric. In order for this power-law solution to lead to acceleration, they find that $-5 / 3<w<-1$. This range of $w$ corresponds to a phantom fluid.

\section{Accelerated expansion in TeVeS}

The simplest case of accelerated expansion in TeVeS is provided by a cosmological constant term. This is equivalent to adding an integration constant to $V(\mu)$ [592, 177, and it corresponds to the accelerated expansion considered by Diaz-Rivera, Samushia and Ratra [419] in both the vacuum and non-vacuum cases (see above). These solutions 
therefore suffer from the usual fine-tuning and coincidence problems, and so it is of interest to look for accelerating solutions without such a constant, simply by employing the scalar field (these need not be de Sitter solutions).

Zhao used a function $\frac{d V}{d \mu} \propto \mu^{2}$ to obtain solutions which provide acceleration, and compared his solution with the SN1a data 1305, finding good agreement. However, it is not clear whether other observables, such as the CMB angular power spectrum, or observations of LSS are compatible with this function. Furthermore, this function is not realistic, as it does not have a Newtonian limit (it is always MONDian). Although no further studies of accelerated expansion in TeVeS have been performed, it is plausible to think that certain choices of function could lead to acceleration. This is because the scalar field action has the same form as a k-essence/k-inflation action 65, 66, which has been considered as a candidate theory for acceleration. More precisely, the system of cosmological equations corresponds to k-essence coupled to matter. It is not known in general whether this type of model has similar features as the uncoupled k-essence models, although Zhao's study indicates that this a possibility.

\section{Realistic FLRW cosmology}

In TeVeS, cold dark matter is absent. Therefore in order to get acceptable values for the physical Hubble constant today (i.e. around $H_{0} \sim 70 \mathrm{~km} \mathrm{~s}^{-1} \mathrm{Mpc}^{-1}$ ), we have to supplement the absence of CDM with something else. The reason for this is simply that if all the energy density in the Universe today was in the form of baryons, then the Hubble constant would be lower than what is observed by a factor of $\sim 5$. Possibilities of what this supplementary material could be include the scalar field itself, massive neutrinos [1153, 502], and a cosmological constant. At the same time, one has to get the right angular diameter distance to recombination [502. These two requirements can place severe constraints on the allowed form of the free functions.

\subsubsection{Cosmological perturbation theory}

Cosmological perturbation theory in TeVeS has been formulated to linear order in [147, and in variants of TeVeS with more general vector field actions in [1148. The scalar modes of the linearly perturbed universally coupled metric are given in the conformal Newtonian gauge, as usual, by

$$
d s^{2}=-a^{2}(1+2 \Psi) d \tau^{2}+a^{2}(1-2 \Phi) q_{i j} d x^{i} d x^{j},
$$

where $\tau$ is conformal time, defined by $d t=a d \tau$. Here, we will assume, for simplicity, that the spatial curvature is zero. The reader is referred to [1147, 1148, for the curved cases, as well as for an enunciation of vector and tensor perturbations. The scalar field is perturbed as $\phi=\bar{\phi}+\varphi$, where $\bar{\phi}$ is the FLRW background scalar field, and $\varphi$ is the perturbation. The vector field is perturbed as $A_{\mu}=a e^{-\bar{\phi}}\left(1+\Psi-\varphi, \vec{\nabla}_{i} \alpha\right)$, such that the unit-timelike constraint is satisfied. This removes the time component of $A_{\mu}$ as an independent dynamical degree of freedom. Thus, there are two additional dynamical degrees of freedom, when comparing to cosmological perturbation theory in GR: The scalar field perturbation, $\varphi$, and the vector field scalar mode, $\alpha$.

The perturbed field equations for the scalar modes can be found in the conformal Newtonian gauge in [1153], and in more form (including in the synchronous gauge) 
in [1147. Perturbation equations for more general TeVeS actions are given in [1148. Here we only present the Newtonian gauge equations of the original TeVeS formulation. We define the following variables: $\tilde{\Phi}=\Phi-\varphi, \tilde{\Psi}=\Psi-\varphi, \tilde{\zeta}=-\left(1-e^{-4 \bar{\phi}}\right) \alpha$ and $\tilde{\mathcal{H}}=\frac{b^{\prime}}{b}$. The scalar field obeys the two first order equations

$$
\begin{aligned}
\gamma^{\prime}= & -3 \tilde{\mathcal{H}} \gamma+\frac{\bar{\mu}}{a} e^{-3 \bar{\phi}} k^{2}\left(\varphi+\bar{\phi}^{\prime} \alpha\right)-\frac{e^{\bar{\phi}}}{a} \bar{\mu} \bar{\phi}^{\prime}\left[6 \tilde{\Phi}^{\prime}+2 k^{2} \tilde{\zeta}\right] \\
& +8 \pi G a e^{-3 \bar{\phi}}\left[\sum_{f} \delta \rho_{f}+3 \delta P_{f}+\sum_{f}\left(\bar{\rho}_{f}+3 \bar{P}_{f}\right)(\tilde{\Psi}-2 \varphi)\right]
\end{aligned}
$$

and

$$
\varphi^{\prime}=-\frac{1}{2 U} a e^{-\bar{\phi}} \gamma+\bar{\phi}^{\prime} \tilde{\Psi}
$$

The vector field equations are given by

$$
K\left(E^{\prime}+\tilde{\mathcal{H}} E\right)=-\bar{\mu} \bar{\phi}^{\prime}\left(\varphi-\bar{\phi}^{\prime} \alpha\right)+8 \pi G a^{2}\left(1-e^{-4 \bar{\phi}}\right) \sum_{f}\left(\bar{\rho}_{f}+\bar{P}_{f}\right)\left(\theta_{f}-\alpha\right)
$$

and

$$
\alpha^{\prime}=E+\tilde{\Psi}+\left(\bar{\phi}^{\prime}-\mathcal{H}\right) \alpha
$$

and finally the Einstein equations are given by the Hamiltonian constraint

$$
\begin{aligned}
& -2\left(k^{2}-3 \kappa\right) \tilde{\Phi}-2 e^{4 \bar{\phi}} \tilde{\mathcal{H}}\left[3 \tilde{\Phi}^{\prime}+k^{2} \tilde{\zeta}+3 \tilde{\mathcal{H}} \tilde{\Psi}\right]+a e^{3 \bar{\phi}} \bar{\phi}^{\prime} \gamma \\
& -K k^{2} E=8 \pi G a^{2} \sum_{f} \bar{\rho}_{f}\left[\delta_{f}-2 \varphi\right]
\end{aligned}
$$

the momentum constraint equation

$$
\tilde{\Phi}^{\prime}+\kappa \tilde{\zeta}+\tilde{\mathcal{H}} \tilde{\Psi}-\bar{\mu} \bar{\phi}^{\prime} \varphi=4 \pi G a^{2} e^{-4 \bar{\phi}} \sum_{f}\left(\bar{\rho}_{f}+\bar{P}_{f}\right) \theta_{f}
$$

and the two propagation equations

$$
\begin{aligned}
& 6 \tilde{\Phi}^{\prime \prime}+2 k^{2}\left(\tilde{\zeta}^{\prime}-e^{-4 \bar{\phi}} \tilde{\Psi}\right)+2 e^{-4 \bar{\phi}}\left(k^{2}-3 \kappa\right) \tilde{\Phi}+2 \tilde{\mathcal{H}}\left[6 \tilde{\Phi}^{\prime}+3 \tilde{\Psi}^{\prime}+2 k^{2} \tilde{\zeta}\right] \\
& +4 \bar{\phi}^{\prime}\left[3 \tilde{\Phi}^{\prime}+k^{2} \tilde{\zeta}\right]+3 \frac{\mu}{U} a e^{-\bar{\phi}} \bar{\phi}^{\prime} \gamma+6\left[2 \tilde{\mathcal{H}}^{\prime}+\tilde{\mathcal{H}}^{2}+4 \bar{\phi}^{\prime} \tilde{\mathcal{H}}\right] \tilde{\Psi} \\
& =24 \pi G a^{2} e^{-4 \bar{\phi}}(\delta P-2 P \varphi)
\end{aligned}
$$

and

$$
\tilde{\Phi}-\tilde{\Psi}+e^{4 \bar{\phi}}\left[\tilde{\zeta}^{\prime}+2\left(\tilde{\mathcal{H}}+\bar{\phi}^{\prime}\right) \tilde{\zeta}\right]=8 \pi G a^{2} \sum_{f}\left(\bar{\rho}_{f}+\bar{P}_{f}\right) \Sigma_{f}
$$

Let us now turn to the problem of specifying initial conditions for the scalar modes, which in general should depend on the chosen form of the free function. The exact adiabatic growing mode in TeVeS, and generalised variants, have been found by Skordis in [1148, but only for the case of the generalised Bekenstein function. If the free function 
is such so that the scalar field contribution to the background expansion during the radiation era is very small, however, then the adiabatic modes for other free functions should be only marginally different from the ones found in 1148. In particular, the only effect should be a difference in the initial conditions of $\varphi$, which is not expected to make any difference to observations.

The only study that has been performed of the observational signatures of TeVeS in the CMB radiation and in LSS is due to Skordis, Mota, Ferreira and Boehm [1153. Here the initial conditions were chosen such that both $\varphi$ and $\alpha$, as well as their derivatives, are initially zero. While this is not a purely adiabatic initial condition, it turns out that it is close enough to ensure that no observable difference can be seen from isocurvature contamination. Detailed studies of isocurvature modes in TeVeS have not yet been conducted. In the light of the problems that TeVeS has with observations of the CMB radiation [1153, however, it may be important to investigate what effects isocurvature modes are likely to have. Preliminary studies by Mota, Ferreira and Skordis have shown that setting the vector field perturbations to be large initially can have a significant impact 910.

In addition to the four regular isocurvature modes that exist in GR, there could in principle exist four further isocurvature modes in TeVeS: Two associated with the scalar field, and two associated with the vector field. Preliminary studies by Skordis have shown that none of the scalar field isocurvature modes are regular in either the synchronous or conformal Newtonian gauges. Conversely, under certain conditions of the vector field parameters one of the vector field isocurvature modes can be regular, while the other one is never regular. Thus, it may be possible to have one regular isocurvature mode in the TeVeS sector. The observational consequences of this mode are still unknown, as is its generation method from early universe inflation. Studies of the observable spectra based on vector or tensor modes are also yet to be conducted although the necessary equations can be found in [1147, 1148].

\subsubsection{Cosmological observations and constraints}

Let us now consider the observational signatures of the perturbation theory discussed above, and how they can be used to constrain TeVeS.

\section{Large-scale structure observations}

A traditional criticism of MOND-type theories was their lack of a dark matter component, and therefore their presumed inability to form large-scale structure compatible with current observational data. This criticism was based on intuition formed from a general relativistic universe filled with baryons only. In that case it is well known that, since baryons are coupled to photons before recombination, they do not have enough time to grow into structures on their own. Furthermore, their oscillatory behaviour at recombination is preserved, and is visible as large oscillation in the observed galaxy power spectrum $P_{g g}(k)$. Finally, on scales smaller than the diffusion damping scale they are exponentially suppressed due to Silk damping. Cold dark matter solves all of these problems because it does not couple to photons, and therefore can start creating potential wells early on in the Universe's history, into which the baryons can fall. This is enough to generate the right amount of structure, erase most of the oscillations, and overcome the Silk damping. 
TeVeS contains two additional fields, not present in GR, that change the structure of the equations significantly. The first study of large-scale structure observations in TeVeS was conducted by Skordis, Mota, Ferreira and Bøhm in 1153. Here the perturbed TeVeS equations were solved numerically for the case of the Bekenstein function, and the effects on the matter power spectrum, $P(k)$, were determined. It was found that TeVeS can indeed form large-scale structure compatible with observations, depending on the choice of TeVeS parameters in the free function. In fact, the form of the matter power spectrum, $P(k)$, in TeVeS looks quite similar to the corresponding spectrum in $\Lambda \mathrm{CDM}$. Thus, one has to turn to other observables to distinguish the TeVeS from General Relativity.

Dodelson and Liguori [427] provided an analytical explanation of the growth of structure that was found numerically in [1153]. They concluded the growth in TeVeS cannot be due to the scalar field, as the scalar field perturbations are Bessel functions that decaying in an oscillatory fashion. Instead, they reasoned, the growth of large-scale structure in $\mathrm{TeVeS}$ is due to the vector field perturbation.

Let us see how the vector field leads to growth. Using the tracker solutions in the matter era, from Bourliot et al. [177, we can find the behaviour of the background functions $a, b$ and $\bar{\phi}$. Using these in the perturbed field equations, after setting the scalar field perturbations to zero, it can be shown that in the matter era the vector field perturbation $\alpha$ obeys the equation

$$
\alpha^{\prime \prime}+\frac{b_{1}}{\tau} \alpha^{\prime}+\frac{b_{2}}{\tau^{2}} \alpha=S\left(\Psi, \Psi^{\prime}, \theta\right)
$$

in the conformal Newtonian gauge, where

$$
\begin{aligned}
& b_{1}=\frac{4\left(\mu_{0} \mu_{a}-1\right)}{\mu_{0} \mu_{a}+3}, \\
& b_{2}=\frac{2}{\left(\mu_{0} \mu_{a}+3\right)^{2}}\left[\mu_{0}^{2} \mu_{a}^{2}-\left(5+\frac{4}{K}\right) \mu_{0} \mu_{a}+6\right],
\end{aligned}
$$

and where $S$ is a source term which does not explicitly depend on $\alpha$. If we simultaneously take the limits $\mu_{0} \rightarrow \infty$ and $K \rightarrow 0$, for which $\Omega_{\phi} \rightarrow 0$, meaning that the TeVeS contribution is absent, then we get $b_{1} \rightarrow 4$ and $b_{2} \rightarrow 2$. In this case the two homogeneous solutions to Eq. 261) are $\tau^{-2}$ and $\tau^{-1}$, which are decaying.

Dodelson and Liguori show that the source term $S(\Psi, \dot{\Psi}, \theta)$ is not sufficient to create a growing mode in the general solution to Eq. (261), and that in the general relativistic limit TeVeS does not, therefore, provide enough growth for structure formation. Now let us consider the general case. Assuming that the homogeneous solutions to 261) can be written as $\tau^{n}$, it can be shown that for the generalised Bekenstein function of Bourliot et al. [177] we can get

$$
n \approx-\frac{3}{2}+\frac{1}{2} \sqrt{1+\frac{32}{K \mu_{0} \mu_{a}}} .
$$

Thus, we can have $n>0$, provided that for fixed $\mu_{0} \mu_{a}$ we also have ${ }^{24}$

$$
K \lesssim 0.01
$$

\footnotetext{
${ }^{24}$ Smaller values of $\mu_{0} \mu_{a}$ can also raise this threshold.
} 

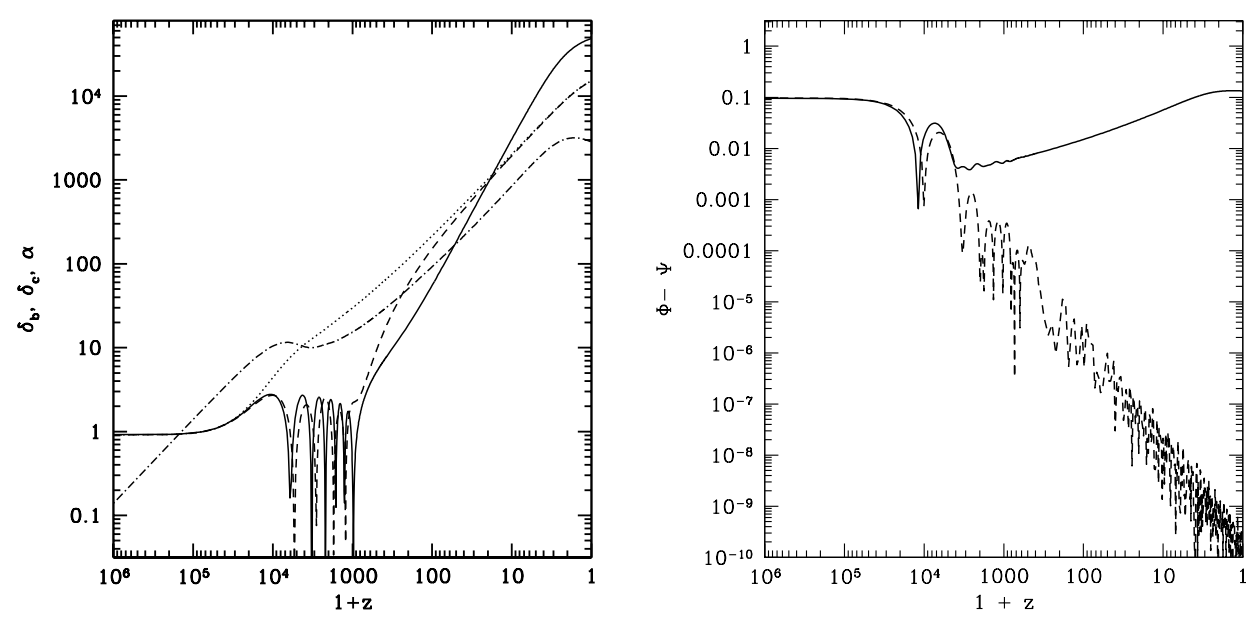

Figure 3: LEFT: The evolution in redshift of baryon density fluctuations in TeVeS (solid line), and in $\Lambda \mathrm{CDM}$ (dashed line) for a wavenumber $k=10^{-3} \mathrm{Mpc}^{-1}$. In both cases, the baryon density fluctuates before recombination, and grows afterwards. In the case of $\Lambda$ CDM, the baryon density eventually follows the CDM density fluctuation (dotted line), which starts growing before recombination. In the case of TeVeS, the baryons grow due to the potential wells formed by the growing mode in the vector field, $\alpha$ (dot-dashed line). RIGHT: The difference of the two gravitational potentials, $\Phi-\Psi$, for a wavenumber $k=10^{-3} \mathrm{Mpc}^{-1}$ as a function of redshift for both TeVeS (solid line), and $\Lambda$ CDM (dotted line).

If this condition is met then there can exist a growing mode in $\alpha$, which in turn feeds back into the perturbed Einstein equation and sources a non-decaying mode in $\Phi$ that can drive structure formation. This is displayed graphically in the left panel of Figure 3 . It is a striking result that even if the contribution of the TeVeS fields to the background FLRW equations is negligible $\left(\sim 10^{-3}\right.$ or less), one can still get a growing mode that drives structure formation.

\section{$C M B$ observations}

A general relativistic universe dominated by baryons cannot fit the most up to date observations of anisotropies in the CMB [984]. This is true even if a cosmological constant and/or three massive neutrinos are incorporated into the matter budget, so that the first peak of the CMB angular power spectrum is at the right position 25 . This, however, is not proof that only a theory with CDM can fit CMB observations (as claimed in 1155, 1173). A prime example to the contrary is the Eddington-Born-Infeld theory [70]. However, the linear Boltzmann equation, and the resulting CMB angular power spectrum, have been calculated in TeVeS, using initial conditions that are close to adiabatic 1153. The resulting fits to the data were poor, at least for the Bekenstein free function, showing that CMB observations are, nevertheless, problematic for TeVeS. It may be that the new isocurvature modes discussed above can provide a richer phenomenology but it remains to be seen whether this can save this theory.

\footnotetext{
${ }^{25}$ In this case the third peak is unacceptably lower than the second.
} 
The difference in the gravitational potentials: $\Phi-\Psi$

The result of Dodelson and Liguori 427] have a further direct consequence: The perturbation equations in $\mathrm{TeVeS}$ that relate the difference of the two gravitational potentials, $\Phi-\Psi$, to the shear of matter, have additional contributions coming from the perturbed vector field, $\alpha$. This is not due to the existence of the vector field per se, but comes from the disformal transformation in which the vector field plays an important part. Indeed, in a single metric theory where the vector field action is Maxwellian, as in TeVeS, there is no contribution from the vector field to $\Phi-\Psi$. Now, as the vector field is required to grow in order to drive structure formation, it will also inevitably lead to growth in $\Phi-\Psi$. This is precisely what we see numerically in the right panel of Figure 3 . If $\Phi-\Psi$ can be measured observationally, then it will provide an excellent test of TeVeS. This possibility is discussed in more detail in section 6 .

\subsection{Other Theories}

\subsubsection{The Einstein-Cartan-Sciama-Kibble Theory}

In this subsection we will briefly describe the Einstein-Cartan-Sciama-Kibble (ECSK) theory [259, 260, 261, 1104, 1105, 691. The ECSK theory is in many cases equivalent to General Relativity and departs from GR only when at least one matter field has intrinsic spin. The ECSK theory has been reviewed by Hehl et al. 601, and more recently by Trautman [1215].

\section{The ECSK theory as a theory with torsion}

The ECSK theory is basically General Relativity with the addition of torsion. The connection is assumed to be metric compatible, but has non-zero torsion, and is thus given by

$$
\Gamma_{\alpha \beta}^{\mu}=\left\{\begin{array}{c}
\mu \\
\alpha \beta
\end{array}\right\}+K_{\alpha \beta}^{\mu},
$$

where $K^{\mu \beta}$ is the contorsion tensor given in terms of the torsion $S^{\mu \beta}$ by Eq. 40 . The Riemann tensor is antisymmetric in both the first and the last two indices, and hence the Ricci tensor is its only unique non-vanishing contraction. It is, however, asymmetric, and is given by Eq. (38).

The addition of torsion to the connection has a direct consequence on the geometry of curves. In this case, autoparallels (straightest lines) are not necessarily extremals (shortest or longest lines) as they are in GR. The former are given by

$$
\frac{d^{2} x^{\mu}}{d s^{2}}+\Gamma_{\alpha \beta}^{\mu} \frac{d x^{\alpha}}{d s} \frac{d x^{\beta}}{d s}=0
$$

while the latter are found by minimising the proper length $\int_{\gamma} \sqrt{-g_{\mu \nu} d x^{\mu} d x^{\nu}}$, and are given by

$$
\frac{d^{2} x^{\mu}}{d s^{2}}+\left\{\begin{array}{c}
\mu \\
\alpha \beta
\end{array}\right\} \frac{d x^{\alpha}}{d s} \frac{d x^{\beta}}{d s}=0 .
$$

Spin-less test particles and gauge-fields (e.g. photons) do not feel the torsion and follow the extremals. However, spinning test particles do feel the torsion and obey analogues 
of the Mathisson-Papapetrou equations [868, 1024. The notions of autoparallel and extremal curves coincide if and only if the torsion is totally antisymmetric. The reader is referred to [599, 1214, 8] for further discussion.

\section{The action and field equations}

The action for the ECSK theory is the same as the one for the metric-affine gravity described in Eq. (46), with the additional assumption that the connection is metric compatible. To make sure that no inconsistency arises one has to impose $Q_{\mu \alpha \beta}=0$ with a Lagrange multiplier. Variation with respect to $\Gamma_{\alpha \beta}^{\mu}$ then proceeds in the usual way.

We can follow a different approach, however, and assume from the start that $Q_{\mu \alpha \beta}=$ 0 , and that the connection is given by Eq. 266. We can then vary the action, Eq. 46, without the Lagrange constraint, but instead take as independent variables the metric and the contorsion. Variation with respect to the inverse metric at constant contorsion then gives

$$
{ }^{\Gamma} G_{(\mu \nu)}+\nabla_{\alpha}\left[P_{(\mu \nu)}{ }^{\alpha}-P_{(\mu \nu)}^{\alpha}\right]=8 \pi G T_{\mu \nu}
$$

where the matter stress-energy tensor is given by $T_{\mu \nu}=-\left.\frac{1}{2 \sqrt{-g}} \frac{\delta \mathcal{L}_{m}}{\delta g^{\mu \nu}}\right|_{K}$. Variation with respect to the contorsion gives

$$
P_{\mu}{ }^{\alpha \beta}=8 \pi G \tau_{\mu}{ }^{\alpha \beta}
$$

where $\tau_{\mu}{ }^{\alpha \beta}=-\frac{1}{\sqrt{-g}} \frac{\delta \mathcal{L}_{m}}{\delta K^{\mu}{ }_{\alpha \beta}}$ is the spin angular momentum tensor of matter. Due to the anti-symmetry of the contorsion in the first and third indices we also have that $\tau_{\alpha \mu \beta}=-\tau_{\beta \mu \alpha}$. Note, however, that the metricity assumption also means that the Palatini tensor simplifies to

$$
P_{\alpha \mu \beta}=S_{\mu \alpha \beta}+2 g_{\mu[\alpha} S_{\beta]}
$$

which is also antisymmetric in the first and third indices. Hence no inconsistency arises from Eq. 270, as it does in the general metric-affine case. Now, Eq. 269 only determines the symmetric part of the Einstein tensor, but we also need the anti-symmetric part. Using Eqs. 271) and (38) gives the anti-symmetric part of the Einstein tensor as

$$
G_{[\mu \nu]}=R_{[\mu \nu]}=\stackrel{*}{\nabla}_{\alpha} P_{\mu \nu}^{\alpha}
$$

where $\stackrel{*}{*}_{\mu}={ }^{\Gamma} \nabla_{\mu}+2 S_{\mu}$. Equations 269, 270 and 272 form a consistent set of field equations for the ECSK theory.

We can proceed one further step, however, and consider the torsion rather than the contorsion as the 2 nd independent variable. This leads to the definition of the spin-energy potential, $\mu_{\nu}{ }^{\alpha \beta} \equiv-\frac{1}{\sqrt{-g}} \frac{\delta \mathcal{L}_{m}}{\delta S^{\mu}{ }_{\alpha \beta}}$. Due to the antisymmetry of the torsion in the last two indices, the spin-energy potential also obeys $\mu_{\nu \alpha \beta}=-\mu_{\nu \beta \alpha}$. It is straightforward to show that the spin-energy potential and the spin angular momentum tensors are related to each other by

$$
\tau_{\alpha \mu \beta}=-\mu_{[\alpha \beta] \mu}
$$

and

$$
\mu_{\mu \alpha \beta}=\tau_{\mu \alpha \beta}-\tau_{\beta \mu \alpha}+\tau_{\alpha \beta \mu}
$$


Carrying out the variation this way defines a new stress-energy tensor, $\sigma_{\mu \nu}$, at constant $S^{\mu}{ }_{\alpha \beta}$ by $\sigma_{\mu \nu} \equiv-\left.\frac{1}{2 \sqrt{-g}} \frac{\delta \mathcal{L}_{m}}{\delta g^{\mu \nu}}\right|_{S}$. This is related to $T_{\mu \nu}$ by

$$
\sigma_{\mu \nu}=T_{\mu \nu}-2\left[S_{\beta \alpha(\nu} \tau_{\mu)}^{\alpha \beta}+S_{\alpha \beta(\nu} \tau_{\mu)}^{\alpha \beta}-\tau_{(\mu}^{\beta \alpha} S_{\nu) \alpha \beta}-\tau_{(\mu}^{\alpha \beta} S_{\nu) \beta \alpha}\right] .
$$

The field equations obtained from varying the action with respect to the metric are

$$
\begin{aligned}
& { }^{\Gamma} G_{(\mu \nu)}+\nabla_{\alpha}\left[P_{\mu \nu}{ }^{\alpha}+P_{\nu \mu}{ }^{\alpha}\right] \\
& +2\left[P_{(\mu}^{\beta \alpha} S_{\nu) \alpha \beta}+P_{(\mu}^{\alpha{ }^{\beta}} S_{\nu) \beta \alpha}-S_{\beta \alpha(\nu} P_{\mu)}{ }^{\alpha \beta}-S_{\alpha \beta(\nu} P_{\mu)}{ }^{\alpha \beta}\right]=8 \pi G \sigma_{\mu \nu} .
\end{aligned}
$$

After some algebra, and using Eq. (272) to form the full asymmetric Einstein tensor, we find that the Einstein equations simplify to

$$
{ }^{\Gamma} G_{\mu \nu}=8 \pi G \Sigma_{\mu \nu}
$$

where

$$
\Sigma_{\mu \nu}=\sigma_{\mu \nu}+\stackrel{*}{\nabla}_{\alpha} \mu_{\mu \nu}^{\alpha}
$$

is a new stress-energy tensor. This new stress-energy tensor has a very important interpretation 601]: It is none other than the canonical stress-energy tensor. Thus, in the ECSK theory the usual symmetrisation procedure of the canonical stress-energy tensor to obtain the stress-energy tensor that enters the Einstein equations is not necessary.

Equations 277) and 270) form a consistent set of field equations that determine the geometry of the space-time from the matter stress-energy and spin distribution. They are supplemented by the conservation laws

$$
\stackrel{*}{\nabla}_{\nu} \Sigma_{\mu}{ }^{\nu}=2 \Sigma_{\alpha}{ }^{\beta} S^{\alpha}{ }_{\mu \beta}-\tau_{\alpha}{ }^{\beta \lambda} R^{\alpha}{ }_{\mu \beta \lambda}
$$

and

$$
\stackrel{*}{\nabla}_{\alpha} \tau_{\mu \nu}^{\alpha}=\Sigma_{[\mu \nu]} .
$$

Let us now discuss a further property of the ECSK theory. By inspecting of the field equations (277) and (270) we notice that there are no derivatives of the torsion appearing anywhere. Thus, the torsion in the ECSK theory is non-dynamical. Its presence is directly given in terms of the spin-angular momentum of matter by Eq. (270). This means that it completely vanishes in vacuum, or in cases where matter does not couple to contorsion (e.g. scalar and gauge fields). Since $S^{\alpha}{ }_{\mu \nu}$ is algebraically determined one can eliminate it from all of the field equations. The final form of the field equations after eliminating torsion is then found to be

$$
\begin{aligned}
G_{\mu \nu}= & 8 \pi G T_{\mu \nu}+(8 \pi G)^{2}\left\{2 \tau_{\beta \alpha(\mu} \tau_{\nu)}^{\alpha}{ }_{\nu}{ }^{\beta}-2 \tau_{\alpha} \tau_{(\mu \nu)}^{\alpha}-\tau_{\alpha \mu \beta} \tau_{\nu}^{\alpha \beta}\right. \\
& \left.+\frac{1}{2}\left[2 \tau^{\mu \alpha \beta} \tau_{\alpha \mu \beta}+\tau^{\alpha \mu \beta} \tau_{\alpha \mu \beta}-2 \tau_{\alpha} \tau^{\alpha}\right] g_{\mu \nu}\right\},
\end{aligned}
$$

where $G_{\mu \nu}$ is the Einstein tensor of $g_{\mu \nu}$, obtained in the usual way from the Levi-Civita connection, and $\tau_{\mu}=\tau_{\beta \mu}^{\beta}$. 


\section{Consequences of the ECSK theory}

We introduced the ECSK theory as a minimal modification of GR through the introduction of torsion and the application of the Palatini procedure. As was shown by Kibble 691, and then later by Hehl et al. 601, the ECSK theory emerges as the minimal description of space-time when one constructs a local gauge theory of the Poincaré group. There are, however, a number of unsatisfactory features of this interpretation. Quantisation of the theory fares no better than in GR. It is still non-renormalizable (the spin contact interactions are reminiscent of 4-fermion interaction terms in quantum field theory), and the torsion has vanishing canonical momentum, which makes it hard to construct a quantum description. The reader is referred to 601 for more details.

One may then ask when is the ECSK theory different from GR? By inspection, Eq. (281) tells us that the spin-potential modifications enter with an additional power of the Planck mass. Thus, we only expect the ECSK theory to deviate from GR at very high spin-densities of matter. For electrons the critical mass density such that spin effects are important is $\sim 10^{38} \mathrm{~kg} \mathrm{~m}^{-3}$ while for neutrons it is $\sim 10^{45} \mathrm{~kg} \mathrm{~m}^{-3} 602$. Conditions for such high densities can exist in the early universe.

Cosmology with spin and torsion has been studied in the hope that the cosmological singularity may be avoided. However, it appears that only under very unrealistic circumstances can one avoid the cosmological singularity in the ECSK theory (see [601, 686, 1195, 757]).

\subsubsection{Scalar-Tensor-Vector Theory}

As a matter of completeness, we will briefly present the Scalar-Tensor-Vector theory of gravity (STVG) proposed in [905. STVG is a theory that contains a vector field, $\phi_{\mu}$, three scalar fields, $G, \omega$ and $\mu$, and a metric, $g_{\alpha \beta}$. A key characteristic of this theory is that the modified acceleration law for weak gravitational fields has a repulsive Yukawa force, as well as the normal Newtonian force. The action for STVG takes the form

$$
S=S_{G r a v}+S_{\phi}+S_{S}+S_{M}
$$

where

$$
\begin{aligned}
S_{\text {Grav }}= & \frac{1}{16 \pi} \int d^{4} x \sqrt{-g}\left[\frac{1}{G}(R+2 \Lambda)\right], \\
S_{\phi}= & \int d^{4} x \sqrt{-g}\left[\omega\left(\frac{1}{4} B^{\mu \nu} B_{\mu \nu}+V(\phi)\right)\right], \\
S_{S}= & \int d^{4} x \sqrt{-g}\left[\frac{1}{G^{3}}\left(\frac{1}{2} g^{\mu \nu} \vec{\nabla}_{\mu} G \vec{\nabla}_{\nu} G-V(G)\right)\right] \\
& \int d^{4} x \sqrt{-g}\left[\frac{1}{G}\left(\frac{1}{2} g^{\mu \nu} \vec{\nabla}_{\mu} \omega \vec{\nabla}_{\nu} \omega-V(\omega)\right)\right] \\
& \int d^{4} x \sqrt{-g}\left[\frac{1}{\mu^{2} G}\left(\frac{1}{2} g^{\mu \nu} \vec{\nabla}_{\mu} \mu \vec{\nabla}_{\nu} \mu-V(\mu)\right)\right],
\end{aligned}
$$

and where $B_{\mu \nu}=\partial_{\mu} \phi_{\nu}-\partial_{\nu} \phi_{\mu}$. 
This action has been studied in [905] where the full field equations, the weak field limit, and cosmological solutions are presented. As would be expected from the complexity of the action, it is difficult to completely solve this system in the detail required to make accurate predictions. Hence, the author of 905 , has posited a certain number of scaling relations that translate into spatial dependences for $G, \omega$ and $\mu$. It is argued in [905] that the classical action for this theory could be considered as an effective field theory for a renormalisation-group-flow-quantum-gravity scenario. The reader is referred to 905 ] for further details. 


\section{Higher Derivative and Non-Local Theories of Gravity}

Recall from Section 2.4.1 that General Relativity represents the most general theory describing a single metric that in four dimensions has field equations with at most second-order derivatives [831, 832. One way to extend GR is therefore to permit the field equations to be higher than second order. Indeed, such a generalisation might be considered desirable as it will cause the graviton propagator to fall off more quickly in the UV, thereby improving the renormalisability properties. Modifying gravity in this way, however, also has a number of drawbacks. In particular, it can introduce instabilities into the theory, such as ghost-like degrees of freedom (see Sections 2.1.3, 4.1.5, and 4.2.5. and [1281] for an overview).

In this section we consider those gravity theories that are higher than second-order in derivatives. Such theories can have interesting phenomenology, and in many cases can be shown (or, at least, argued to be) less susceptible to instabilities than one may have initially suspected. For example, if the higher derivatives act only on what would otherwise be non-dynamical modes, then they may simply render them dynamical, rather than automatically generating a ghost. This is what happens in $f(R)$ gravity, where the higher order derivatives act on the conformal mode that does not propagate in GR. In HořavaLifschitz gravity, as another example, one allows for higher-order spatial derivatives, as opposed to higher time derivatives, in order to guard against ghost-like instabilities. In both of these examples the theory can deviate considerably from GR, while still maintaining some basic stability properties.

This section also includes galileon and ghost condensate theories. Strictly speaking these are not higher-derivative theories since their field equations are at most second order in derivatives. In fact, the galileon theory in particular is constructed with this in mind. Nevertheless we include them in this section as both theories contain nontrivial derivative interaction terms. We will not discuss theories with infinite derivatives, as occur in string field theory, or p-adic string theory (see 904 for discussion of such theories).

\section{1. $f(R)$ Theories}

Fourth-order theories of gravity have a long history, dating back to as early as 1918 [1269, only a few years after the first papers on General Relativity by Einstein. These theories generalise the Einstein-Hilbert action by adding additional scalar curvature invariants to the action, or by making the action a more general function of the Ricci scalar then the simple linear one that leads to Einstein's equations. Here we consider the latter of these options, a choice that leads by Lovelock's theorem to fourth-order field equations for anything except the addition of a constant term to the gravitational Lagrangian. Such theories, generically referred to as $f(R)$ theories of gravity, have been intensively studied, and have a number of reviews dedicated to them [969, 1118, 1167, 374, 978. This interest was stimulated in the 1960s, 70s and 80 s by the revelations that the quantisation of matter fields in an unquantised space-time can lead to such theories [1239], that $f(R)$ theories of gravity can have improved renormalisation properties [1186, and that they can lead to a period of accelerating expansion early in the Universe's history [1179. More recently they have been of considerable interest as a possible explanation for the observed late-time accelerating expansion of the Universe. 


\subsubsection{Action, field equations and transformations}

The $f(R)$ generalisations of Einstein's equations are derived from a Lagrangian density of the form

$$
\mathcal{L}=\sqrt{-g} f(R),
$$

where the factor of $\sqrt{-g}$ is included, as usual, in order to have the proper weight. This is clearly about as simple a generalisation of the Einstein-Hilbert density as one could possibly conceive of. The field equations derived from such an action are automatically generally covariant and Lorentz invariant for exactly the same reasons that Einstein's equations are. Unlike the Einstein-Hilbert term, however, the field equations that one obtains from the least action principle associated with Eq. (286) depend on the variational principle that one adopts. Different possibilities are the 'metric variation' where the connection is assumed to be the Levi-Civita one, the 'Palatini approach' in which Eq. 286 is varied with respect to the metric and connection independently, and the 'metric-affine' approach in which the same process occurs but the matter action is now taken to be a functional of the connection as well as the metric. In this section we will mostly be concerned with the metric variational approach, although we will also outline how the other procedures work below.

\section{Metric variational approach}

Let us now derive the field equations in the metric variational approach. Integrating Eq. 286 over a 4-volume, including a matter term and varying with respect to $g_{\mu \nu}$ yields

$$
\delta S=\int d \Omega \sqrt{-g}\left[\frac{1}{2} f g^{\mu \nu} \delta g_{\mu \nu}+f_{R} \delta R+\frac{\chi}{2} T^{\mu \nu} \delta g_{\mu \nu}\right],
$$

where $f_{R}$ means the functional derivative of $f$ with respect to $R, \chi$ is a constant, and $T_{\mu \nu}$ is the energy-momentum tensor defined by a variation of the matter action with respect to $g_{\mu \nu}$ in the usual way. Assuming the connection is the Levi-Civita one we can then write

$$
f_{R} \delta R \simeq-\left[f_{R} R^{\mu \nu}+f_{R ; \rho \sigma}\left(g^{\mu \nu} g^{\rho \sigma}-g^{\mu \rho} g^{\nu \sigma}\right)\right] \delta g_{\mu \nu},
$$

where $\simeq$ is used here to mean equal up to surface terms [568. Looking for a stationary point of the action, by setting the first variation to zero, then gives

$$
f_{R} R_{\mu \nu}-\frac{1}{2} f g_{\mu \nu}-f_{R ; \mu \nu}+g_{\mu \nu} \square f_{R}=\frac{\chi}{2} T_{\mu \nu} .
$$

These are the $f(R)$ field equations with which we will be primarily concerned in this section. It can be seen that for the special case $f=R$ the LHS of Eq. 289p reduces to the Einstein tensor, and the field equations are second-order in derivatives of the metric. For all other cases, except an additional constant, the equations in 289 are fourth-order in derivatives.

\section{Conformal transformation in the metric variational approach}

As with scalar-tensor theories, the $f(R)$ theories of gravity derived from the metric variational approach can be conformally transformed into a frame in which the field 
equations become those of General Relativity, with a minimally coupled scalar field. This is sometimes referred to as 'Bicknell's theorem' in the case of $f(R)$ theories, particularly when the minimally coupled scalar field is in a quadratic potential [155]. In the general case we consider conformal transformations of the form [101, 853.

$$
\bar{g}_{\mu \nu}=f_{R} g_{\mu \nu}
$$

together with the definition

$$
\phi \equiv \sqrt{\frac{3}{\chi}} \ln f_{R},
$$

which allows the field equations from the metric variational principle, Eqs. 289, to be transformed into

$$
\bar{R}_{\mu \nu}-\frac{1}{2} \bar{g}_{\mu \nu} \bar{R}=\frac{\chi}{2}\left(\phi_{, \mu} \phi_{, \nu}-\frac{1}{2} \bar{g}_{\mu \nu} \bar{g}^{\rho \sigma} \phi_{, \rho} \phi_{, \sigma}-\bar{g}_{\mu \nu} V\right)+\frac{\chi}{2} \bar{T}_{\mu \nu} .
$$

Here $\bar{T}_{\mu \nu}$ is a non-conserved energy-momentum tensor, and we have defined

$$
V=V(\phi) \equiv \frac{\left(R f_{R}-f\right)}{\chi f_{R}^{2}} .
$$

Theories derived from an action of the form (286) can therefore always be conformally transformed into General Relativity with a massless scalar field (as long as $f_{R} \neq 0$ ), and a non-metric coupling to the matter fields.

Legendre transformation in the metric variational approach

As well as conformal transformations, one can also perform Legendre transformations on $f(R)$ theories in the metric variational approach. Such transformations allow the field equations of $f(R)$ to take the form of a scalar-tensor theory (albeit it a slightly strange one). These transformed theories maintain the universal metric coupling of the matter fields, unlike the case of conformal transformations. form

The first step here is to notice that the Eq. 286 can be written in the equivalent

$$
\mathcal{L}=\sqrt{-g}\left[f(\chi)+f^{\prime}(\chi)(R-\chi)\right],
$$

where $\chi$ is a new field, and the prime denotes differentiation. Variation with respect to $\chi$ then gives

$$
f^{\prime \prime}(\chi)(R-\chi)=0
$$

so that $\chi=R$ for all $f^{\prime \prime}(\chi) \neq 0$. Substitution of this result back into Eq. 294 then immediately recovers Eq. 286, showing that the two Lagrangian densities are indeed equivalent. What is more, the special case $f^{\prime \prime}(\chi)=0$ can be seen to correspond to the Einstein-Hilbert action.

Now, if we make the definition

$$
\phi \equiv f^{\prime}(\chi)
$$

and assume that $\phi(\chi)$ is an invertible function, then we can define a potential

$$
\Lambda(\phi) \equiv \frac{1}{2}\left[\begin{array}{c}
\chi(\phi) \phi-f(\chi(\phi))] \\
103
\end{array}\right.
$$


In terms of this new scalar field we can then write the density (294) as

$$
\mathcal{L}=\sqrt{-g}[\phi R-2 \Lambda(\phi)],
$$

which is clearly just a scalar-tensor theory, as specified in Eq. (97), with vanishing coupling constant, $\omega=0$. As we have not transformed the metric, the coupling of this field to any matter fields that are present remains unchanged.

\section{The Palatini procedure}

Starting again from the density (286) we can also proceed in a entirely different way to the metric variational approach just described. Instead of assuming the connection from which the Ricci scalar is constructed is the Levi-Civita one, we could instead treat the metric and connection as independent fields. For the case of General Relativity a variation with respect to the connection then simply results in the connection being shown to be the Levi-Civita one, so that the difference between the metric variational approach and the Palatini approach is a semantic one. For the case of $f(R)$ theories, however, the Palatini approach leads to an entirely different set of field equations.

Starting with an integral of Eq. 286 over some 4-volume, and extremising with respect to $g_{\mu \nu}$ now gives

$$
f_{R} R_{\mu \nu}-\frac{1}{2} g_{\mu \nu} f=\frac{\chi}{2} T_{\mu \nu},
$$

where $T_{\mu \nu}$ is once again the usual energy-momentum tensor. In this expression $R_{\mu \nu}$ is now defined independently from the metric, and $R$ should be taken to be given by $g^{\mu \nu} R_{\mu \nu}$. The next step is the variation of Eq. 286) with respect to $\Gamma_{\nu \sigma}^{\mu}$, which results in

$$
\left(\sqrt{-g} g^{\mu \nu} f_{R}\right)_{; \sigma}=0,
$$

where the covariant derivative here should be understood to be taken with respect to $\Gamma_{\nu \sigma}^{\mu}$, which is not the Levi-Civita connection unless $f_{R}=$ constant (as is the case in GR). It is remarkable that the field equations (299) do not involve any derivatives of the metric, and only first derivatives of the connection. These are a different set of field equations to Eq. 289, and should be considered a different set of theories to the $f(R)$ in which $R$ is a priori taken to be constructed from the Levi-Civita connection.

It can be noted from Eq. (300) that even if the connection is not compatible with the metric $g_{\mu \nu}$, we can still define a new metric, $\bar{g}_{\mu \nu}=f_{R} g_{\mu \nu}$, with which it is compatible. Rewriting the full field equations under this conformal transformation we see that we recover General Relativity with a minimally coupled scalar field in a potential, but no kinetic term:

$$
\mathcal{L}=\sqrt{-\bar{g}}[\bar{R}-2 V(\phi)],
$$

where $\phi \equiv f_{R}$ and $V(\phi)=(R(\phi) \phi-f(\phi)) / 2 \phi^{2}$. Here $R(\phi)$ and $f(\phi)$ are given by inverting the definition of $\phi$, and $\bar{R}$ should be understood to be constructed from the metric connection compatible with $\bar{g}_{\mu \nu}$. Transforming back to the original conformal frame this theory then can be shown to be equivalent to a scalar-tensor theory with $\omega=-3 / 2$ and $\Lambda=\left(R f_{R}-f\right) / 2$ [999].

While being an interesting variant on the metric variational incarnation of the $f(R)$ theories, there are a number of very severe problems in proceeding with the Palatini 
procedure in this way. Not least of these is the apparent ill-posed nature of the Cauchy problem in the presence of most matter fields, which is discussed in [766. Without a well-posed initial value problem $f(R)$ gravity in the Palatini formalism lacks predictive power, and hence is not a good candidate for a viable theory of gravity. Furthermore, the Palatini approach to $f(R)$ gravity also appears to introduce problematic strong couplings between gravity and matter fields at low energies [509, 640, and singularities in systems that are usually well described by weak fields $[87,88,89]$. For these reasons we will focus on $f(R)$ theories in the metric variational approach in the sections that follow.

For further details of the Palatini approach to $f(R)$ gravity, and the results that follow from it, the reader is referred to [1167]. For studies of weak field gravity in the Palatini formalism the reader is referred to 92,429, 881, 999, 30, 1000, 1163, 1001, 1078, 224, 665, 31, 1077, and for cosmology to [1251, 509, 883, 28, 882, 884, 29, 1162, 1164, 724, 34, 727, 258, 793, 1232, 725, 781, 782, 497. An interesting class of theories that interpolate between the Palatini approach to $f(R)$ theories and the metric variational approach to $f(R)$ theories is investigated in [39, 726].

\section{The metric-affine approach}

One further approach to the $f(R)$ theories of gravity is the 'metric-affine' formulation. Here one again considers the metric and connection to be independent, as in the Palatini procedure, but now allows the matter action to be a function of both metric and connection (rather than metric alone, as is the case in Palatini and metric variational formalisms). The relevant action for the theory then becomes [1168]

$$
S=\int d \Omega \sqrt{-g} f(R)+S_{m}\left(g_{\mu \nu}, \Gamma_{\nu \sigma}^{\mu}, \Psi\right),
$$

where $R=g^{\mu \nu} R_{\mu \nu}$, and $R_{\mu \nu}$ is taken to be a function of the connection only, as in the Palatini procedure. One can therefore think of the action (302) as a generalisation of the Palatini action, which is recovered when the dependence of the matter action, $S_{m}$, on $\Gamma^{\mu}{ }_{\nu \sigma}$ vanishes.

As is the case in General Relativity, the invariance of the Ricci scalar under the projective transformation $\Gamma^{\mu}{ }_{\nu \sigma} \rightarrow \Gamma^{\mu}{ }_{\nu \sigma}+\lambda_{\nu} \delta^{\mu}{ }_{\sigma}$ can lead to inconsistency of the field equations, as matter fields do not generically exhibit an invariance of this type. This invariance can be cured by adding to the action an additional Lagrange multiplier term of the form $S=\int d \Omega \sqrt{-g} B^{\mu} \Lambda_{[\nu \sigma]}^{\nu}$, and results in the field equations

$$
f_{R} R_{\mu \nu}-\frac{1}{2} g_{\mu \nu} f=\frac{\chi}{2} T_{\mu \nu}
$$

together with $\Gamma_{[\mu \nu]}^{\mu}=0$, and

$$
\begin{array}{r}
\frac{1}{\sqrt{-g}}\left[\left(\sqrt{-g} f_{R} g^{\mu \sigma}\right)_{; \sigma} \delta_{\rho}^{\nu}-\left(\sqrt{-g} f_{R} g^{\mu \nu}\right)_{; \rho}\right]+2 f_{R} g^{\mu \sigma} \Gamma_{[\sigma \rho]}^{\nu} \\
=\frac{\chi}{2}\left[\Delta_{\rho}^{\mu \nu}-\frac{2}{3} \Delta_{\sigma}^{\sigma[\nu} \delta_{\rho}^{\mu]}\right],
\end{array}
$$

where $\Delta_{\mu}{ }^{\nu \rho} \equiv-(2 / \sqrt{-g}) \delta S_{m} / \delta \Gamma^{\mu}{ }_{\nu \rho}$. It can then be shown that $\Delta_{\mu}{ }^{[\nu \rho]}=0$ corresponds to a vanishing of the torsion, and $\Delta_{\mu}{ }^{(\nu \rho)} \neq 0$ introduces non-zero non-metricity [1168]. 
The metric-affine approach has not been studied as intensively as the other approaches to $f(R)$ gravity that we have already discussed, and will not feature heavily in the sections that follow.

\subsubsection{Weak-field limit}

The weak field limit of $f(R)$ theories of gravity has been extensively studied in the literature. Here we will first consider perturbations about Minkowski space, and then perturbations around de Sitter space, time dependent backgrounds, and inhomogeneous backgrounds.

\section{Perturbations about Minkowski space}

The first attempt at finding the Newtonian limit of an $f(R)$ theory appears to have been performed by Pechlaner and Sexl for the case of $f=R+\alpha R^{2}$ [1029]. The first step here is to write down the perturbed line-element as

$$
d s^{2}=-(1+2 \psi) d t^{2}+(1-2 \phi)\left(d x^{2}+d y^{2}+d z^{2}\right)
$$

neglecting time derivatives and second order terms in $\phi$ and $\psi$ one then finds that the Ricci scalar, $R=-2 \Delta \psi+4 \Delta \phi$, obeys

$$
6 \alpha \delta R-R=-\frac{\chi}{2} \rho
$$

and that the potential $\psi$ obeys

$$
(1+6 \alpha \Delta) \Delta \psi=\frac{\chi}{4}(1+8 \alpha \Delta) \rho
$$

where $\Delta$ is the Laplacian on Euclidean 3-space. The derivatives of $\rho$ in this last equation occur due to replacing terms containing $\phi$ with those obtained from taking derivatives of Eq. (306), and do not occur in the actual field equations themselves.

Inserting a delta function source, $\rho=m \delta$, and integrating Eq. (307), using the solution to Eq. 306 to find $\phi$, one then gets the solutions [1029]

$$
\begin{aligned}
16 \pi \psi & =-\frac{\chi m}{r}\left(1+\frac{e^{-m_{0} r}}{3}\right), \\
16 \pi \phi & =-\frac{\chi m}{r}\left(1-\frac{e^{-m_{0} r}}{3}\right),
\end{aligned}
$$

where boundary conditions at infinity have been imposed to eliminate exponentially increasing modes, and where we have defined the mass

$$
m_{0}^{2} \equiv \frac{1}{6 \alpha}
$$

Mass terms similar to this continue to exist for more general theories, and more general situations, as we will outline shortly. One can see straight away that for large masses, with $m_{0} \gg r^{-1}$, the Yukawa potentials in Eqs. 308 and 309 are exponentially suppressed, and we recover $\psi=\phi \propto-m / r$. In the limit of small masses, however, when $m_{0} \ll r^{-1}$, 
we instead find that $\psi=2 \phi \propto-m / r$. For the case of $f=R+\alpha R^{2}$ gravity we therefore already expect the PPN parameter $\gamma$ to be 1 when the mass of the scalar degree of freedom is large, and $1 / 2$ when it is small.

One is, of course, also interested in other functions of $f(R)$, and can consider the case of analytic $f(R)$ theories that can be expanded as

$$
f(R)=\sum_{i=1}^{\infty} c_{i} R^{i},
$$

where the $c_{i}$ are constants. To full post-Newtonian order the weak field solution in the presence of a perfect fluid is then given in full generality as [307.

$$
\begin{aligned}
g_{00}= & -1+\frac{2}{c_{1}}\left(U+c_{2} R\right)-\frac{2}{c_{1}^{2}} U^{2}+2 \frac{c_{2}^{2}}{c_{1}^{2}} R^{2}-\frac{16 c_{2}}{3 c_{1}^{2}} U R-\frac{7}{18 \pi c_{1}} \mathcal{V}(U R) \\
& +\frac{3 c_{2}}{4 \pi c_{1}} \mathcal{V}\left(R^{2}\right)+\frac{64}{9 c_{1}^{2}} \mathcal{V}(\rho U)-\frac{44 c_{2}}{3 c_{1}^{2}} \mathcal{V}(\rho R)-\frac{40 c_{2}}{3 c_{1}^{3}} \mathcal{V}(\vec{\nabla} \rho \cdot \vec{\nabla} U) \\
& +\frac{40 c_{2}^{2}}{c_{1}^{3}} \mathcal{V}(\vec{\nabla} \rho \cdot \vec{\nabla} R)+\frac{2}{c_{1}} \mathcal{V}(\rho \Pi)+\frac{4}{c_{1}} \mathcal{V}\left(\rho v^{2}\right)+\frac{6}{c_{1}} \mathcal{V}(P)+\frac{1}{6 \pi c_{1}} X(U R) \\
& -\frac{1}{4 \pi}\left(\frac{c_{2}}{c_{1}}-\frac{c_{3}}{2 c_{2}}\right) X\left(R^{2}\right)-\frac{4}{3 c_{1}^{2}} X(\rho U)+\frac{8 c_{2}}{3 c_{1}^{2}} X(\rho R) \\
& +\frac{8 c_{2}}{3 c_{1}^{3}} X(\vec{\nabla} \rho \cdot \vec{\nabla} U)-\frac{8 c_{2}^{2}}{c_{1}^{3}} X(\vec{\nabla} \rho \cdot \vec{\nabla} R)-\frac{2}{c_{1}} X(P)+\frac{2}{3 c_{1}} X(\rho \Pi) \\
g_{0 i}= & -\frac{7 V_{i}}{2 c_{1}}-\frac{W_{i}}{2 c_{1}}+\frac{X\left(\rho v_{i}\right)}{6 c_{1}}-\frac{Y_{i}}{6 c_{1}}-\frac{Z_{i}}{6 \sqrt{6 c_{1} c_{2}}} \\
g_{i j}= & \left(1+\frac{2}{c_{1}}\left(U-c_{2} R\right)\right) \delta_{i j}
\end{aligned}
$$

where $U$ is the usual Newtonian potential, and the other potentials are defined as

$$
\begin{array}{ll}
V_{i} \equiv \int \frac{\rho\left(\mathbf{x}^{\prime}\right) v_{i}\left(\mathbf{x}^{\prime}\right)}{\left|\mathbf{x}-\mathbf{x}^{\prime}\right|} d^{3} x^{\prime} & W_{i} \equiv \int \frac{\rho\left(\mathbf{x}^{\prime}\right)\left(\mathbf{v}\left(\mathbf{x}^{\prime}\right) \cdot\left(\mathbf{x}-\mathbf{x}^{\prime}\right)\right)\left(x-x^{\prime}\right)_{i}}{\left|\mathbf{x}-\mathbf{x}^{\prime}\right|^{3}} d^{3} x^{\prime} \\
X(Q) \equiv \int \frac{Q^{\prime} e^{-} \sqrt{\frac{c_{1}}{6 c_{2}}}\left|\mathbf{x}-\mathbf{x}^{\prime}\right|}{\left|\mathbf{x}-\mathbf{x}^{\prime}\right|} d^{3} x^{\prime} & Y_{i} \equiv \int \frac{\rho^{\prime} \mathbf{v}^{\prime} \cdot\left(\mathbf{x}-\mathbf{x}^{\prime}\right)\left(x-x^{\prime}\right)_{i}}{\left.\mid \mathbf{x}-\mathbf{x}^{\prime}\right)^{3}} e^{-\sqrt{\frac{c_{1}}{6 c_{2}}}\left|\mathbf{x}-\mathbf{x}^{\prime}\right|} d^{3} x^{\prime} \\
\mathcal{V}(Q) \equiv \int \frac{Q^{\prime}}{\left|\mathbf{x}-\mathbf{x}^{\prime}\right|} d x^{\prime 3} & Z_{i} \equiv \int \frac{\rho^{\prime} \mathbf{v}^{\prime} \cdot\left(\mathbf{x}-\mathbf{x}^{\prime}\right)\left(x-x^{\prime}\right)_{i}}{\left|\mathbf{x}-\mathbf{x}^{\prime}\right|^{2}} e^{-\sqrt{\frac{c_{1}}{6 c_{2}}}\left|\mathbf{x}-\mathbf{x}^{\prime}\right|} d^{3} x^{\prime} \\
\hat{\chi} \equiv \int \rho^{\prime} e^{-\sqrt{\frac{c_{1}}{6 c_{2}}\left|\mathbf{x}-\mathbf{x}^{\prime}\right|} d^{3} x^{\prime}} & R=\frac{1}{3 c_{2}} \int \frac{\rho\left(\mathbf{x}^{\prime}\right)}{\left|\mathbf{x}-\mathbf{x}^{\prime}\right|} e^{-\sqrt{\frac{c_{1}}{6 c_{2}}}\left|\mathbf{x}-\mathbf{x}^{\prime}\right|} d^{3} x^{\prime},
\end{array}
$$

where $R$ is the Ricci scalar, $P$ is pressure, $\rho$ is the rest-mass energy density, and $\Pi$ is the specific energy density (as defined in 1274). The terms in Eqs. (313)-(314) that are functionals of derivatives of $\rho, U$ and $R$ can be recast into a form where such derivatives do not appear by further manipulations [307.

From the above it can be seen that the results of General Relativity are recovered, to the appropriate order, when $c_{2} \rightarrow c_{3} \rightarrow 0$. For non-vanishing values of the these constants a large number of extra Yukawa potentials are present, and for large enough values of $c_{2}$ we can again see that $\gamma \rightarrow 1 / 2$, as the scalar degree of freedom becomes massless. 
The study of gravitational waves about a Minkowski space background in $f(R)$ theories has been undertaken by Berry and Gair in [146]. Here the authors consider analytic functions of the type prescribed by Eq. (311), and find that an extra mode of oscillation is possible. The gravitational waves emitted by extreme-mass-ratio inspirals are then calculated, and the authors conclude that current laboratory bounds (that result in $\left|c_{2} / c_{1}\right|<10^{-9} m^{2}$ ) mean that the extra oscillatory mode they find cannot be excited by astrophysical sources.

\section{Perturbations about de Sitter backgrounds}

As well as the usual expansions about Minkowski space, in order to determine the post-Newtonian behaviour of a theory, one is also interested in perturbations about other backgrounds. Here we will consider de Sitter space as a background. This is not meant to be an elucidation of cosmological perturbation theory, but rather a consideration of weak field expansions as applicable to systems such as the solar system and binary pulsars. Minkowski space is not always a stable solution of $f(R)$ theories of gravity that attempt to produce self-accelerating cosmologies at late-times, and in these cases time-dependent backgrounds, and de Sitter space in particular, become of increasing interest for weak field studies.

Much work has been performed on establishing the weak field limit of $f(R)$ theories about a de Sitter background, as relevant for theories that try and account for latetime accelerating expansion without dark energy, see e.g. [1050, 421, 1171, 943, 998, 1129, 1300, 930. The majority of these studies conclude that, in the absence of extra mechanisms to mask such behaviour, one should expect to find $\gamma=1 / 2$. This was shown in an early paper on the subject in [292, and is the familiar limit of theories in which a scalar degree of freedom has low mass. Here we will briefly sketch out the method by which such a result is found for more general $f(R)$, following the approach of 294.

The first step here is to identify a de Sitter solution with constant Ricci curvature $R=R_{0}=12 H_{0}^{2}$. The line-element is then perturbed as

$$
d s^{2}=-\left(1+2 \psi-H_{0}^{2} r^{2}\right) d t^{2}+\left(1-2 \phi+H_{0}^{2} r^{2}\right) d r^{2}+r^{2} d \Omega^{2},
$$

where we have chosen to present the de Sitter background using a static coordinate patch. We then proceed by perturbing the Ricci scalar as

$$
R=R_{0}+R_{1}
$$

where $R_{1} \ll R_{0}$. The perturbative expansion then linearises all field equations with respect to $\phi, \psi, R_{1}$ and $H_{0}^{2} r^{2}$, and their derivatives, while neglecting all time derivatives. The case of spherical symmetry is considered for simplicity.

To lowest order the trace of the field equations is now

$$
\Delta R_{1}-\left(\frac{f_{R}-f_{R R} R_{0}}{3 f_{R R}}\right) R_{1}=-\frac{\chi \rho}{6 f_{R R}}
$$

where $f_{R}$ and $f_{R R}$ should be understood to be the value of these quantities at $R=R_{0}$ (implicit here is an assumption that these quantities are all of the same order of magnitude as $R_{0}$, and that $R_{0}$ in the weak-field systems under consideration takes the same value as 
in the cosmological background solution). From cosmological considerations the second term on the left-hand side of Eq. (317) is then neglected, as the factor in brackets corresponds to the mass squared of the scalar degree of freedom, which must be small compared to $r^{-1}$ in order to have late-time accelerating expansion. The resulting form of $R_{1}$ is therefore found to be

$$
R_{1} \simeq \frac{\chi m}{24 \pi f_{R R} r},
$$

where $m$ is the mass of the object at the centre of symmetry. Applying the same approximations to the $(t, t)$ component of the field equations results in

$$
\Delta \psi=\frac{\chi \rho}{2 f_{R}}-\frac{R_{1}}{2}+\frac{f_{R R}}{f_{R}} \nabla^{2} R_{1},
$$

which on substitution of the expression for $R_{1}$ gives to lowest order

$$
\psi \simeq-\frac{\chi m}{12 \pi f_{R} r} .
$$

The remaining field equations then give $\phi$, to the same order of approximation, as

$$
\phi \simeq-\frac{\chi m}{24 \pi f_{R} r} \simeq \frac{\psi}{2} .
$$

This calculation has not been performed in the PPN gauge, which uses an isotropic spatial coordinate system, but nevertheless one can verify that when interpreted within the standard PPN framework it does indeed give [1001]

$$
\gamma=\frac{1}{2}
$$

In this section we have discussed de Sitter space as a background to perturb around. However, establishing whether de Sitter space is, in fact, a stable asymptotic solution of $f(R)$ theories, and establishing the genericity of initial conditions that lead to de Sitter space at late times, has not yet been discussed. We will consider this subject in following sections.

Perturbations about other backgrounds

Having considered the maximally symmetric Minkowski space and de Sitter space backgrounds, we can now also consider less symmetric spaces to perturb around. This enterprise is hindered by our ability to find less symmetric solutions to the field equations (289). We can, however, make progress with some simple cases.

If we consider $f(R)$ theories in which $f=R^{1+\delta}$ then one can find exact non-static, homogeneous and isotropic vacuum solutions 241. Such solutions can be shown to be, under certain conditions, stable asymptotic attractors for the general class of spatially flat, vacuum FLRW solutions [248]. They will be discussed further in the cosmology section that follows. For this same class of $f(R)$ theories exact static, spherically symmetric vacuum solutions are also known [310, which can also be seen to be generic asymptotes of the general solution, with the specified symmetries applied [310. We are now in 
possession of two exact solutions, for the same theories, which have less than maximal symmetry, and which can be used as backgrounds to perturb around.

Spherically symmetric, time independent perturbations around the homogeneous, time-dependent background found in [241] are given to linear order by 304]

$$
d s^{2}=-(1+2 \psi) d t^{2}+a^{2}(t)(1-2 \phi)\left(d r^{2}+r^{2} d \Omega^{2}\right),
$$

where $a(t)=t^{\frac{\delta(1+2 \delta)}{(1-\delta)}}$. The perturbations $\phi(r)$ and $\psi(r)$ are then found to be

$$
\begin{aligned}
& \psi=-\frac{c_{1}}{r}+\frac{2 c_{2}\left(1-6 \delta+4 \delta^{2}+4 \delta^{3}\right)}{\left(5-14 \delta-12 \delta^{2}\right)} r^{2} \\
& \phi=-\frac{(1-2 \delta) c_{1}}{r}-\delta c_{2} r^{2}
\end{aligned}
$$

The corresponding perturbative analysis about the static, spherically symmetric background found in 310 gives

$$
\begin{aligned}
d s^{2}= & -r^{2 \delta \frac{(1+2 \delta)}{(1-\delta)}}(1+V(r)) d t^{2} \\
& +\frac{\left(1-2 \delta+4 \delta^{2}\right)\left(1-2 \delta-2 \delta^{2}\right)}{(1-\delta)^{2}}(1+W(r)) d r^{2}+r^{2} d \Omega^{2}
\end{aligned}
$$

where $V(r)$ and $W(r)$ are given in full generality by $V(r)=c_{3} V_{1}(r)+c_{4} V_{2}(r)+c_{5} V_{3}(r)$ and $W(r)=-c_{3} V_{1}(r)+c_{4} W_{2}(r)+c_{5} W_{3}(r)$, where

$$
V_{1}=-r^{-\frac{\left(1-2 \delta+4 \delta^{2}\right)}{(1-\delta)}}
$$

and where $V_{2}, V_{3}, W_{2}$ and $W_{3}$ are oscillatory modes [310. It can immediately be seen that the form of the linearised perturbations around these two backgrounds are quite different to each other, even though the field equations they obey are identical. One can verify that an observer in the homogeneous, time-dependent background should measure

$$
\beta=1 \quad \text { and } \quad \gamma=1-2 \delta,
$$

which gives $\delta=-1.1 \pm 1.2 \times 10^{-5}$ when the constraint derived from the Cassini space probe on $\gamma$ is applied [147]. On the other hand an observer in the static background should measure an anomalous extra gravitational force that goes like [310]

$$
F \sim-\frac{\delta}{r}
$$

When subjected to constraints from observations of the perihelion precession of Mercury, the presence of this extra force gives $\delta=2.7 \pm 4.5 \times 10^{-19} 310$. The different forms of the gravitational potentials and forces in these examples show that the choice of symmetries of the background space-time can have important consequences for its weak field limit, and the constraints on the underlying theory that are derived from it.

\section{Chameleon mechanism}


As with a variety of other modified theories of gravity, the 'Chameleon Mechanism' has been applied to $f(R)$ theories. This mechanism was outlined in Section 3.1.4 where a summary of some of the accumulated literature on it was outlined. Here we will simply reiterate the basic point that this mechanism potentially allows a means by which theories with a light effective scalar degree of freedom can evade solar system and binary pulsar tests of the PPN parameter $\gamma$ by allowing the scalar to acquire a higher mass in the locale of high mass concentrations, such as the Sun and Earth.

The chameleon mechanism has been applied to specific $f(R)$ theories, and its behaviour in this application considered further, in e.g. [267, 945, 1180, 495, 193, 1199, 244, 629. As with other applications of the Chameleon mechanism, if a 'thin shell' is present then the mass of the scalar degree of freedom in these theories is thought to be able to be supressed enough to satisfy solar system tests of gravity.

\subsubsection{Exact solutions, and general behaviour}

Having discussed the weak field solutions which are of interest for inferring constraints on $f(R)$ theories from observations of gravitational phenomena in the solar system and binary pulsar systems, let us now consider the behaviour of solutions to the full nonlinear field equations. Here we will be concerned with exact solutions, which can be obtained in some simple cases, as well as what can be inferred about the general behaviour of non-linear solutions by other methods, and what theorems can tell us about the behaviours that are possible. The relatively simple structure of $f(R)$ theories make such considerations a feasible proposition. The geodesic deviation equation in general $f(R)$ theories has been studied in [569].

\section{Isolated masses, and black holes}

Progress was made into understanding the static spherically symmetric vacuum solutions of $f(R)$ theories of gravity by Mignemi and Wiltshire in [891]. These authors consider theories with higher powers of the Ricci scalar added to the Einstein-Hilbert Lagrangian, and use a dynamical systems analysis to determine the behaviour of the general solutions with the specified symmetries. They find the asymptotes of these solutions, for a variety of different cases, and show that the only static spherically symmetric solutions of the theories they consider that have regular horizons are the Schwarzschild solutions. They further find that by dropping the requirement of regularity the Schwarzschild solution is also the only solution to these theories that is asymptotically flat.

The black hole 'no-hair' theorems have been considered in the context of $f(R)=$ $R+\alpha R^{2}$ theories by Whitt [1270]. Collapse to a black hole, however, has not been as extensively studied in $f(R)$ theories of gravity as it has in Brans-Dicke theory, where direct numerical calculations have been performed [1107]. Nevertheless, the same logic that tells us that the vacuum black hole solutions of general relativity are the only vacuum black hole solution of Brans-Dicke theory that can result from gravitational collapse, also suggest that this should be true for $f(R)$ gravity. In particular, most of the results of Hawking on this subject only rely on inequalities of the form

$$
R_{\mu \nu} l^{\mu} l^{\nu} \geq 0,
$$

where $l^{\mu}$ is a null vector, and not on the details of the field equations themselves [595]. This null energy condition is true of the conformally transformed scalar fields equations 
in Brans-Dicke theory, and is also true in $f(R)$ gravity. It therefore seems reasonable to expect that the vacuum black hole solutions of $f(R)$ gravity should also be the same as the vacuum black hole solutions of General Relativity. The subject of black hole radiation in the context of $f(R)$ gravity has been studied in [200, 322, 16, 543, 378, and [489] where it was shown that black holes in $f(R)$ gravity have a entropy given by

$$
S=\frac{f_{R} A}{4} .
$$

The subject of the de Sitter no-hair theorems and isotropisation in $f(R)$ gravity has been considered by Barrow and Ottewill [110] and Goheer, Leach and Dunsby [540, where it was shown that flat FLRW isotropic points can exist in the phase plane of Bianchi solutions, and that de Sitter space can be a stable asymptote of $f(R)$ theories of gravity. One should, however, be aware that such behaviour depends on the theory in question, and the initial conditions. For example, for theories with negative powers of $R$ in a series expansion of their Lagrangian one may generically expect such terms to become important asymptotically. In this case accelerating power-law expansion is an attractor solution [306]. This will be discussed further in the cosmology section below.

As well as the black hole solutions of General Relativity, it is known that other vacuum solutions to $f(R)$ theories of gravity that can describe isolated masses also exist. Due to the complicated nature of the field equations in these theories, however, only a few exact solutions that describe these situations have been found. For the case of $f(R)=R^{1+\delta}$ solutions are known that correspond to an isolated mass in a homogeneous and time dependent background, and an isolated mass in a static, spherically symmetric background. The former of these solutions is given by the line-element 304

$$
d s^{2}=-A_{1}(r) d t^{2}+a^{2}(t) B_{1}(r)\left(d r^{2}+r^{2} d \Omega^{2}\right),
$$

where $a(t)=t^{\delta \frac{(1+2 \delta)}{(1-\delta)}}$, and where $A_{1}(r)$ and $B_{1}(r)$ are given by

$$
A_{1}(r)=\left(\frac{1-\frac{C_{1}}{r}}{1+\frac{C_{1}}{r}}\right)^{\frac{2}{q}}, \quad \text { and } \quad B_{1}(r)=\left(1+\frac{C_{1}}{r}\right)^{4} A(r)^{q+2 \delta-1},
$$

where $q^{2}=1-2 \delta+4 \delta^{2}$. The latter solution is given by [310]

$$
d s^{2}=-A_{2}(r) d t^{2}+\frac{d r^{2}}{B_{2}(r)}+r^{2} d \Omega^{2}
$$

where

$$
\begin{aligned}
& A_{2}(r)=r^{2 \delta \frac{(1+2 \delta)}{(1-\delta)}}+\frac{C_{2}}{r^{\frac{(1-4 \delta)}{(1-\delta)}}} \\
& B_{2}(r)=\frac{(1-\delta)^{2}}{\left(1-2 \delta+4 \delta^{2}\right)(1-2 \delta(1+\delta))}\left(1+\frac{C_{2}}{r^{\frac{\left(1-2 \delta+4 \delta^{2}\right)}{(1-\delta)}}}\right) .
\end{aligned}
$$

The constants $C_{1}$ and $C_{2}$ appear in these solutions as mass parameters, and it can be seen that both Eq. 326 and Eq. 327 reduce to the Schwarzschild solution when 
$\delta \rightarrow 0$. The problem of static, spherically symmetric solutions in general $f(R)$ has been considered in [992, where a covariant formalism was developed for studying the problem, and the non-uniqueness of the Schwarzschild solution was demonstrated. The $\delta=1 / 4$ case of Eq. (327) was rediscovered in 243. Black holes coupled to Yang-Mills fields have been studied in [872], where an exact solution was found for the case $f=\sqrt{R}$.

These solutions are interesting for a number of reasons. Firstly, they show that the generalisation of the solutions of General Relativity to other theories of gravity is not always unique; i.e. there can be multiple solutions in modified theories of gravity that reduce to the same solution in the limit of general relativity. It may therefore be the case that one needs to understand the symmetries of the background space-time to a greater extent than is necessary in General Relativity, in order to fully understand which solution should be used to model a given situation. Secondly, Eq. (326) shows explicitly that Birkhoff's theorem is not valid in general, when one considers generalisations of Einstein's theory. Spherically symmetric vacuum solutions of modified theories of gravity can therefore be time-dependent, which can lead to new phenomenology. Birkhoff's theorem, in the context of $f(R)$ gravity, has been considered in [490. Thirdly, Eq. (327) displays non-trivial asymptotic behaviour as $r \rightarrow \infty$. Such behaviour is unexpected in general relativity, and again opens the window to new phenomenology. The results of Mignemi and Wiltshire 891 even suggest that such behaviour is generic. A fourth point is that the solution given in Eq. (326) has been shown in [488, to exhibit a naked singularity. This has clear implications for the applicability of the cosmic censorship hypothesis to modified theories of gravity. The Misner-Sharp energy in spherically symmetric spacetimes is considered in 225.

\section{Cosmological solutions}

A variety of cosmological solutions in $f(R)$ theories of gravity are known, and have had their stability analysed. Here we will briefly review and provide references to studies of these solutions.

The conditions for existence and stability of de Sitter solutions in $f(R)$ gravity appears to have been first studied in [110. One can show that for any theory for which there is a value of $R$ which satisfies

$$
f_{R}\left(R_{d S}\right) R_{d S}=2 f\left(R_{d S}\right)
$$

there exists a de Sitter solution with $R_{d S}=4 \Lambda$. The stability of de Sitter solutions in $f(R)$ gravity was studied in [110, 1049, 486. These solutions are of obvious importance for cosmology at both early and late times. One can note that with $f(R) \propto R^{2}$ Eq. (328) is satisfied with any value of $R$. Theories with $R^{2}$ terms in their Lagrangian's have been studied extensively, due to the naturalness of adding an $R^{2}$ term to the Einstein-Hilbert action, and due to their improved renormalisation properties [1186. They were also introduced and studied by Starobinsky for cosmological purposes, and in particular their ability to give rise to an early non-singular period of accelerating expansion in a natural way 1179 .

Less symmetric cosmological solutions than de Sitter space can also be found for some $f(R)$ theories. In particular, theories of the type $f(R)=R^{1+\delta}$ are again of interest here, as they admit simple exact solutions. As mentioned in the preceding section, a power-law 
exact solution for a spatially flat vacuum FLRW solution is known to be given by [241]

$$
a(t)=t^{\delta \frac{1+2 \delta}{1-\delta}},
$$

and a spatially flat solution in the presence of a perfect barotropic fluid with equation of state $P=w \rho$ is also known 248]

$$
a(t)=t^{\frac{2(1+\delta)}{3(1+w)}} .
$$

The stability of these solutions, and their properties as asymptotes of the general solution, have been investigated in [248] and 310]. In fact, it has been shown that these are the only power-law perfect fluid FLRW solutions that exist for any $f(R)$ gravity theory [539. Explicit non-power-law general solutions with FLRW symmetries were found in [305], both with spatial curvature, and in the presence of a perfect fluid. These solutions show explicitly that in the early universe both non-singular and inflationary behaviour are possible. The energy conditions in FLRW solutions have been considered in [1033], and braneworld cosmology in these theories have been considered in 68 .

Beyond exact solutions, FLRW cosmological solutions have also been studied in $f(R)$ theories of gravity using dynamical systems analysis. This has been done for the case of a number of particular $f(R)$ theories in $[248,310,790,5,538$, and also in the general case in 390, 306, 40, 253. The dynamical systems approach has even been applied to perturbed FLRW solutions in 247]. We will discuss perturbed FLRW further in the Cosmology section that follows. These studies find a variety of interesting cosmological behaviours at both early and late-times. In particular non-singular and accelerating behaviour in the early universe is again identified, as well as late-time accelerating expansion, and the non-sequential domination of higher powers in the Ricci curvature, for analytic $f(R)$, as the initial singularity is approached. The conditions required for a non-singular 'bounce' are given in [249], and oscillating solutions were considered in [208]. There have also been some concerns expressed as to whether a matter dominated epoch is generically expected to exist after radiation domination [242, 972, 40, 43, 479]. The inverse problem of finding particular forms of $f(R)$ that result in pre-specified cosmological evolutions has been considered in 240, 929, 242, 376, 973, 496, 251, 980. Such inversions do not always specify $f(R)$ uniquely [929], and it has been shown that to reproduce exact $\Lambda \mathrm{CDM}$ evolution with dust only one is forced towards the Einstein-Hilbert action with a cosmological constant 443].

Exact Bianchi cosmological solutions were discovered for $f(R)=R^{n}$ theories in [98, and been studied further in 778 , 540, where shear dynamics and isotropisation are discussed. The special case of $n=2$ was studied in 209. Bianchi type $I$ and $V$ solutions have been considered in [1132] and [1133], and Bianchi $V I I_{A}$ solutions in 367]. Kantowski-Sachs solutions have been studied in [786]. Other know exact solutions are the Einstein static universe [309, 790, 549, 1122, and the Gödel universe [309, 1057]. These studies explore the stability of the Einstein static universe, and the existence of closed time-like curves in the Gödel solution.

\subsubsection{Cosmology}

Much of the recent motivation for studying $f(R)$ gravity has come from the need to explain the apparent late-time accelerating expansion of the Universe. Previous motivation for studying $f(R)$ gravity has also come from cosmological considerations, including 
the presence of an initial singularity, and early universe inflationary expansion. We will therefore now present an overview of what we consider to be some of the most relevant aspects of $f(R)$ gravity for physical cosmology. In terms of the viability of FLRW geometry in $f(R)$ gravity, the Ehlers-Geren-Sachs theorem of General Relativity has been extended to cover these theories by Rippl, van Elst, Tavakol, and Taylor in [1065], and more recently by Faraoni in 487 .

\section{Field Equations}

To describe the cosmology, up to scalar perturbations, we first define line-element

$$
d s^{2}=-(1+2 \Psi) d t^{2}+a^{2}(t)(1-2 \Phi) q_{i j} d x^{i} d x^{j},
$$

and the energy-momentum tensor,

$$
\begin{aligned}
T_{0}^{0} & =-\rho-\delta \rho \\
T^{0}{ }_{i} & =-(\rho+P) \vec{\nabla}_{i} \theta \\
T^{i}{ }_{j} & =P \delta^{i}{ }_{j}+\delta P \delta^{i}{ }_{j}+(\rho+P) D^{i}{ }_{j} \Sigma,
\end{aligned}
$$

where $\theta$ is the peculiar velocity, $\delta P$ is the pressure perturbation, and $\Sigma$ is the anisotropic stress. At zeroth order the Friedmann equations are

$$
\begin{aligned}
H^{2} & =\frac{1}{3 F}\left[8 \pi \rho-\frac{1}{2}(f-R F)-3 H \dot{F}\right]-\frac{\kappa}{a^{2}} \\
\dot{H} & =-\frac{1}{2 F}(8 \pi \rho+8 \pi P+\ddot{F}-H \dot{F})+\frac{\kappa}{a^{2}}
\end{aligned}
$$

where the Ricci scalar is given by $R=6\left(2 H^{2}+\dot{H}+\kappa / a^{2}\right)$, and energy-momentum conservation gives, as usual,

$$
\dot{\rho}+3 H(\rho+P)=0,
$$

where $F=f_{R}$, over-dots denote derivatives with respect to $t$, and $\kappa$ is spatial curvature.

Now let us consider the first-order scalar perturbation equations, which are given in 637. Here it is convenient to define a new quantity

$$
\chi \equiv 3 H \Psi+3 \dot{\Phi} .
$$

The perturbation equations are then 637]

$$
\begin{aligned}
& \dot{\chi}+\left(2 H+\frac{\dot{F}}{2 F}\right) \chi+\frac{3}{2} \frac{\dot{F}}{F} \dot{\Psi}+\left[3 \dot{H}+\frac{3}{2 F}(2 \ddot{F}+H \dot{F})-\frac{k}{a^{2}}\right] \Psi \\
= & \frac{1}{2 F}\left[8 \pi \delta \rho+24 \pi \delta P+3 \delta \ddot{F}+3 H \delta \dot{F}+\left(\frac{k^{2}-6 \kappa}{a^{2}}-6 H^{2}\right) \delta F\right]
\end{aligned}
$$

and

$$
\begin{aligned}
& \delta \ddot{F}+3 H \delta \dot{F}+\left(\frac{k^{2}}{a^{2}}-\frac{R}{3}\right) \delta F \\
&= \frac{8 \pi}{3}(\delta \rho-3 \delta P)+\dot{F}(\chi+\dot{\Psi})+(2 \ddot{F}+3 H \dot{F}) \Psi-\frac{F}{3} \delta R \\
& 115
\end{aligned}
$$


with fluid evolution equations

$$
\delta \dot{\rho}+3 H(\delta \rho+\delta P)=(\rho+P)\left(\chi-3 H \Psi-\frac{k^{2} \theta}{a}\right)
$$

and

$$
\frac{\left(a^{4}(\rho+P) k \theta\right)^{\cdot}}{a^{4}(\rho+P)}=\frac{k}{a}\left[\Psi+\frac{1}{(\rho+P)}\left(\delta P-\frac{2}{3}\left(k^{2}-3 \kappa\right)(\rho+P) \Sigma\right)\right] .
$$

Here the perturbation to the Ricci scalar, $\delta R$, is given by

$$
\delta R=-2\left[\dot{\chi}+4 H \chi-\left(\frac{k^{2}}{a^{2}}-3 \dot{H}\right) \Psi+2 \frac{\left(k^{2}-3 \kappa\right)}{a^{2}} \Phi\right],
$$

and we have the constraint equations

$$
\chi+\frac{3}{2} \frac{\dot{F}}{F} \Psi=\frac{3}{2 F}[8 \pi a(\rho+P) \theta+\delta \dot{F}-H \delta F]
$$

and

$$
\begin{aligned}
& \left(H+\frac{\dot{F}}{2 F}\right) \chi+\frac{\left(k^{2}-3 \kappa\right)}{a^{2}} \Phi+\frac{3 H \dot{F}}{2 F} \Psi \\
= & -\frac{1}{2 F}\left[8 \pi \delta \rho-3 H \delta \dot{F}+\left(3 \dot{H}+3 H^{2}-\frac{k^{2}}{a^{2}}\right) \delta F\right] .
\end{aligned}
$$

Furthermore, we again have that $\Psi \neq \Phi$, in general. Instead it is the case that

$$
\Psi-\Phi=-\frac{8 \pi a^{2}(\rho+P) \Sigma}{F}-\frac{\delta F}{F} .
$$

The equivalent equations to those given above can also be derived in the covariant approach to cosmological perturbation theory [250. In the rest of this section we will consider the consequences of these equations for various cosmological phenomena.

\section{Inflation}

The existence of inflation has provided considerable motivation for the study of $f(R)$ theories of gravity. The pioneering work on this subject was that of Starobinsky in 1980, who found that theories with $R^{2}$ corrections to their gravitational Lagrangian can have an early period of de Sitter expansion [1179]. The spectrum of scalar and tensor fluctuation generated during this type of inflation have been studied in [916, 1178, 719, 636] where they were found to compatible with observations of the CMB. Quantum initial conditions ("tunnelling from nothing"), as well as the process of reheating, were also considered in [1249.

Inflation in $f(R)$ gravity is particularly transparent in the Einstein conformal frame. Here, for the Starobinsky model with [1179]

$$
f(R)=R+\frac{R^{2}}{6 M^{2}},
$$


the conformally transformed theory in vacuum is one in which the minimally coupled scalar field exists in a potential

$$
V(\phi)=\frac{3 M^{2}}{2 \chi}\left(1-e^{-\sqrt{\frac{x}{3}} \phi}\right) .
$$

This potential is displayed in Figure 4, where it can be seen that slow-roll inflation is likely to occur in the region $\phi \gtrsim m_{p l}$, and reheating is feasible during oscillations around the minimum at $\phi=0$. This is, of course, exactly the type of behaviour that one wants for a viable inflaton field.

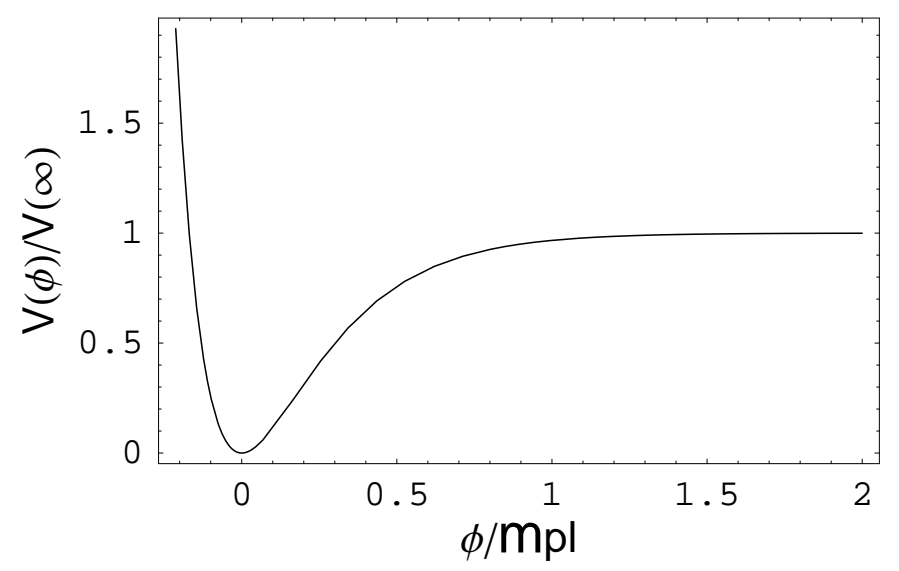

Figure 4: The potential given in Eq. 348, normalised by its asymptotic value as $\phi \rightarrow \infty$.

In fact, for the theory specified in Eq. (347) one can show that inflation is the transient attractor of the general solution 852, and that in the region $\phi \gg m_{p l}$ slow-roll inflation occurs with

$$
\epsilon=-\frac{\dot{H}}{H^{2}} \simeq \frac{M^{2}}{6 H^{2}},
$$

and proceeds for $N \simeq(2 \epsilon)^{-1}$ e-foldings. We will not proceed with showing the details of reheating in this model, but only note that around $\phi \simeq 0$ the potential given in Eq. (348) is well approximated by $V \simeq \frac{1}{2} M^{2} \phi^{2}$. For details of how reheating occurs in this potential the reader is referred to $[1249,892]$. Pre-heating in $f(R)$ inflationary models has been considered in 1222]. Quantum cosmology, instantons, and their implications for inflation have been studied in [1246, 1027.

\section{Dark Energy}

As well as accelerating expansion in the early universe, $f(R)$ theories of gravity are also capable of producing late-time accelerating expansion. There have been a large number of papers on this subject. There have also been attempts to construct quintessencelike $f(R)$ models which produce both early and late acceleration $974,976,977,975,82$.

An easy way to see the potential for late-time accelerating expansion is to consider the Friedmann equations (335) and (336) in vacuo. One can then identify effective density 
and pressure parameters by analogy to the Friedmann equations of general relativity. These are

$$
\begin{aligned}
8 \pi \rho_{\mathrm{eff}} & =\frac{R F-f-6 H \dot{F}}{2 F} \\
8 \pi P_{\mathrm{eff}} & =\frac{2 \ddot{F}+4 H \dot{F}+f-R F}{2 F} .
\end{aligned}
$$

The equation of state of this effective fluid is then given by

$$
w=\frac{2 \ddot{F}+4 H \dot{F}+f-R F}{R F-f-6 H \dot{F}},
$$

and one can then determine what is required to achieve $w<-1 / 3$, and hence accelerating expansion.

One example of this is the now much considered theory of Carroll, Duvuri, Trodden and Turner 255

$$
f(R)=R-\frac{\mu^{2(n+1)}}{R^{n}},
$$

where $\mu$ is a constant. For power-law evolution the effective equation of state, (352), is then given at late-times by [255]

$$
w=-1+\frac{2(n+2)}{3(2 n+1)(n+1)},
$$

so that if $n=1$, and the extra term in the gravitational Lagrangian is inversely proportional to $R$, then one achieves an equation of state with $w=-2 / 3$, and hence accelerating expansion. In fact, this just corresponds to the power-law solution given in Eq. 329, for a theory with $f(R) \propto R^{-n}$.

The theory specified in Eq. (353) is now known to have a number of deficiencies that make it non-viable [999, 43, 479, 428, 485, 258, 473, 1102, 122, 1157. Some of these have to do with the weak-field limit, which we have already discussed, others come from cosmology, and yet more are due to stability issues, which we will address in Section 4.1.5. Many of these problems can be traced back to the value of the effective mass in the scalar degree of freedom of this theory, which is thought to be either too small for validity of gravitational physics in the solar system, or imaginary, leading to some of the instabilities just alluded to. Models which have been constructed to try and over-come these problems, while still leading to accelerating expansion at late-times, are those of Starobinsky [1180]:

$$
f(R)=R-\mu R_{c}\left[1-\left(1+\frac{R^{2}}{R_{c}^{2}}\right)^{-n}\right],
$$

Hu and Sawicki [629]:

$$
f(R)=R-\frac{\mu R_{c}}{1+\left(R / R_{c}\right)^{-2 n}},
$$

and Battye and Appleby [56]:

$$
f(R)=R+R_{c} \log \left[\begin{array}{c}
e^{-\mu}+\left(1-e^{-\mu}\right) e^{-R / R_{c}} \\
118
\end{array}\right]
$$


where $\mu, n$ and $R_{c}$ are all positive constants. Attempts to construct viable models that include an early stage of inflationary expansion, as well as late-time accelerating expansion, have been made in [321, 468. All of these theories rely on the chameleon mechanism to satisfy solar system constraints on gravity.

\section{Observational Probes}

As with many modifications to gravity, cosmological observables can be used to constrain $f(R)$ theories of gravity. Here we will briefly survey the literature on this subject.

Primordial nucleosynthesis has been studied in $f(R)$ gravity in [310, 204, 764, 475. Due to the conformal equivalence between these theories and general relativity, the behaviour of cosmological solutions during the radiation dominated epoch are considerably simplified: They evolve in a similar way to the radiation dominated solutions of GR, but with a different value of the effective gravitational constant. This situation is familiar from studies of primordial nucleosynthesis in the scalar-tensor theories of gravity outlined in Section 3.1. In the present case the relevant effective gravitational 'constant' is inversely proportional to $f_{R}$. The value of $f_{R}$ evolves throughout the matter dominated and accelerating epochs, but is constant during radiation domination. Observations of the abundances of light elements then provide constraints on the allowed values of $f_{R}$ during the radiation dominated epoch, and hence constrain the rate of evolution of this quantity that is allowed during the rest of the Universe's history.

Other probes of the background expansion of an FLRW universe are the peak positions of the CMB spectrum of temperature fluctuations, and baryon acoustic oscillations. Observations of these quantities allow the form of $a(t)$ to be constrained, but due to the freedom in the choice of $f(R)$ are not able to falsify the most general form of these theories directly [240, 929, 242, 376, 973, 496, 251, 980. To go further using cosmological observations we must therefore consider the solutions to the perturbation equations given above.

The first thing that one may wish to consider is the growth of density perturbations, $\delta=\delta \rho / \rho$. In a spatially flat universe, manipulation of Eqs. (339)-(346) allows one to write [1299, 1219, 1226]

$$
\ddot{\delta}+2 H \dot{\delta}-\frac{4 \pi \delta \rho}{3 f_{R}} \frac{\left(4+3(a / k)^{2} M^{2}\right)}{\left(1+(a / k)^{2} M^{2}\right)}=0,
$$

where the mass parameter $M$ is given, just as in the weak field limit discussed in Section 4.1 .2 as

$$
M^{2}=\frac{f_{R}-R f_{R R}}{3 f_{R R}} .
$$

From the third term on the LHS of Eq. 358 it can be seen that the evolution of $\delta$ depends on the magnitude of $M$, and, in particular, is different in the two regimes $M^{2} \gg k^{2} / a^{2}$ and $M^{2} \ll k^{2} / a^{2}$. When the former is true, the density perturbations evolve as they do in General Relativity, with an effective Newton's constant given by $G=1 / f_{R}$. For a matter dominated universe this means

$$
\delta \propto t^{\frac{2}{3}} .
$$


In the latter regime, in which $M^{2} \ll k^{2} / a^{2}$, this is no longer true. Here, the third term in Eq. (358) is modified from its form in GR by a multiplicative factor of $4 / 3$, and the evolution of $\delta$ during the matter dominated era is consequently modified to

$$
\delta \propto t^{(\sqrt{33}-1) / 6} .
$$

The transition between these two limits is theory dependent. For studies on this subject the reader is referred to [1180, 629, 122, 377, 1219, 1226, 1043, 1220, 519, 1221, 914, 940, Interesting results are that the change in evolution between the two regimes discussed above is scale dependent. That is, modes with different wave-numbers can evolve in different ways depending on whether they are larger or smaller than $a^{2} M^{2}$. This length scale is therefore imprinted on the density perturbations. Furthermore, oscillating modes can also become present when $M^{2} \gg k^{2} / a^{2}$, which can lead to undesirable singularities [515. The inclusion of an $R^{2}$ in the gravitational Lagrangian was found to remove these singularities in [57]. One can also see that $f_{R R}>0$ is need for the stability of scalar modes.

The modified growth of structure just discussed has consequences for large-scale structure, and the cosmic microwave background, which we will now discuss. The matter power spectrum in $f(R)$ theories of gravity has been considered in 495, , 258, 1102, 122, 724, 1157, 790, 46, 45, 814, 1026, and cluster abundances have been used to constrain these theories in [1115, 818, 500]. The formation of non-linear structure has also been considered in 630, 1006, 1007, 1114, 1303. Cosmic microwave observations are considered in [790, 1158, 1157, 818, where it is shown that power on large-scales is sensitive to the modified growth of structure through the integrated Sachs-Wolfe (ISW) effect. This can lead to damped power for small deviations from GR, or amplified power if the deviations are large enough. Correlating ISW effects in the CMB with observations of galaxy number density also leads to tight constraints [1158, 818, due to the sign of the $\mathrm{CMB}$ temperature fluctuation changing if the modification to gravity is large enough.

\subsubsection{Stability issues}

There are a variety of stability issues that are of concern for $f(R)$ theories of gravity. These include ghost degrees of freedom, as evidenced in the Ostrogradski instability, as well as the instabilities found by Frolov, and Dolgov and Kawasaki. Some of these issues have been mentioned already. In this section we will discuss them further.

\section{Ghosts, and the Ostrogradski instability}

In Section 2.1.3 we discussed the problems associated with ghosts - pathological fields that admit physical states of negative energy, or negative norm when quantised. It is known that ghosts can occur in general higher derivative theories of gravity, see e.g. 1187, 236, 606, 607, 293, 944, 392, 113, 990. They are not, however, as problematic in $f(R)$ theories as they are for general fourth-order theories, as we will now outline. Let us first consider the existence of negative energy states in the context of Ostrogradski's theorem 1003 .

The Ostrogradski instability states that Lagrangians that contain second derivatives, and are non-linear in those second derivatives, are generically unstable. At first sight such a result appears to be problematic for $f(R)$ theories of gravity, which are only linear 
in second-order derivatives of the metric in the case of General Relativity with a possible cosmological constant. One can show, however, that these instabilities do not occur for $f(R)$ theories [1281]. This works in the following way: Let us consider a Lagrangian

$$
L=L(g, \dot{g}, \ddot{g}),
$$

where dots denote derivatives of $g$ with respect to some parameter $\lambda$. Now define a set of four canonical variables by $Q_{1} \equiv g, Q_{2} \equiv \dot{g}$, and

$$
P_{1} \equiv \frac{\partial L}{\partial \dot{g}}-\frac{d}{d \lambda} \frac{\partial L}{\partial \ddot{g}}, \quad \text { and } \quad P_{2} \equiv \frac{\partial L}{\partial \ddot{g}} .
$$

If it is now possible to write $\ddot{g}=f\left(Q_{1}, Q_{2}, P_{2}\right)$ then the Hamiltonian of the system can be written as

$$
H=P_{1} Q_{1}+P_{2} f-L\left(Q_{1}, Q_{2}, f\right) .
$$

This Hamiltonian, however, is only linear in the momentum $P_{1}$, and cannot therefore be stable. This is Ostrogradski's instability. Now, $f(R)$ gravity avoids this instability because one cannot write down the equivalent of $\ddot{g}=f\left(Q_{1}, Q_{2}, P_{2}\right)$ for each component of the metric. Instead, only a single scalar degree of freedom contains the higher-order derivatives, and by an appropriate field redefinition one can remove this extra field so that the redefined metric appears in the Lagrangian only linearly in its second-order derivatives. This is just the conformal transformation discussed in Section 4.1.1. The Ostrogradski instability does not, therefore, apply to $f(R)$ theories of gravity [1281].

Let us now consider ghost-like instabilities from the point of view of linear fluctuations. In generic fourth-order theories massive spin-2 degrees of freedom appear along with a scalar degree of freedom, and the familiar massless spin-2 degree of freedom from General Relativity. It is the massive spin-2 fields in this situations that present the generic problem with ghosts. Such fields are absent from $f(R)$ theories, however, which contain only the massless spin-2 fields of GR, and a single scalar field. Again, this is clear from the existence of the conformal transformations outlined in Section 4.1.1. The $f(R)$ theories of gravity therefore do not always suffer from the same problems with ghosts as more general higher-order theories, which will be discussed in more detail in Section 4.2 .5 .

\section{Frolov instability}

A potential problem with $f(R)$ theories that modify the infra-red limit of General Relativity has been identified by Frolov in [515]. This instability is caused by the fact that for the scalar degree of freedom in $f(R)$ theories curvature singularities can occur at finite field value and energy level, a phenomenon previously investigated in [4, 203.

To illustrate this problem consider the $f(R)$ proposed by Starobinsky, Eq. 355). The potential for the effective scalar field in this theory is shown in Fig. 5. During cosmological expansion, the scalar fields associated with FLRW cosmologies roll down the slope from $\phi=0$ to the local minimum at $\phi \simeq-0.1$. The short section of curve between this minimum and the singularity at $\phi=0$ is the only part of the potential the scalar field need experience in the entire history of a perfect FLRW solution. Frolov 


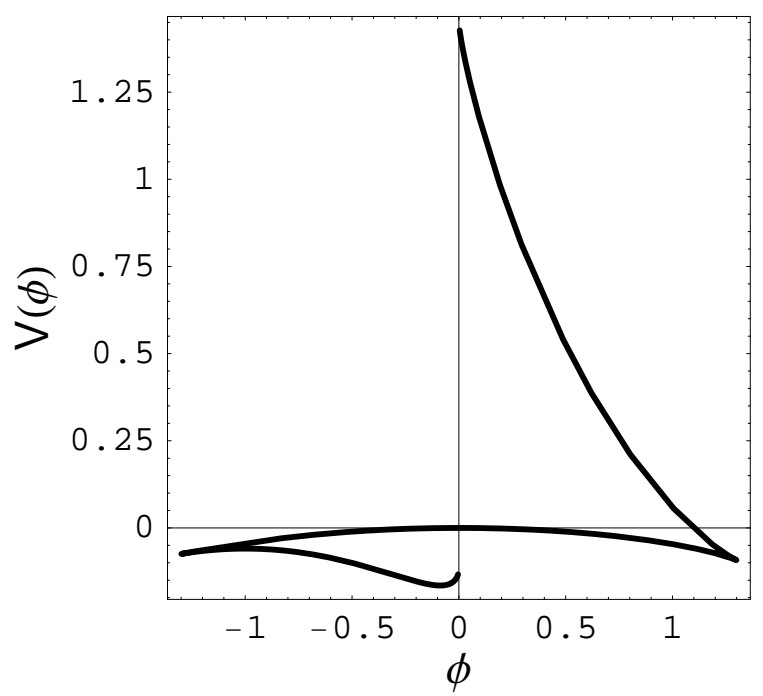

Figure 5: The potential for the scalar field in Starobinsky's theory, Eq. 355, with $R_{c}=1, n=1$ and $\mu=2$.

argues, however, that relatively small perturbation in curvature, caused by collapse of dust, are enough to push $\phi$ back up the potential to the singularity. The existence of such instability is, of course, undesirable for a physically plausible theory, although it may be mitigated by the addition of higher power of $R$ to the gravitational Lagrangian [203, 81, 57.

\section{Dolgov-Kawasaki instability}

Finally, let us consider another instability that was initially found by Dolgov and Kawasaki for the theory given by Eq. (353) with $n=1$ [428. This was later extended to more general functions of $f(R)$ that modify gravity in the infra-red limit [485] and formalised better in 1124.

The basic point here is that the trace of the field equations, 289 , acts as the propagation equation for scalar degree of freedom. For Eq. (353) with $n=1$ this equation is

$$
R-\frac{3 \mu^{4}}{R}-\square\left(\frac{3 \mu^{4}}{R^{2}}\right)=8 \pi \rho,
$$

where we have taken the matter content to be that of dust. Now, de Sitter space is a solution of this equation, with

$$
R_{d S}=\frac{1}{2}\left(8 \pi \rho+\sqrt{(8 \pi \rho)^{2}+12 \mu^{4}}\right) \simeq 8 \pi \rho,
$$

for cosmologically relevant $\mu$. If we now consider perturbations around this solution with 
$R=R_{d S}+\delta R$ then we get to lowest order that

$$
\frac{6 \mu^{4}}{R_{d S}^{3}} \square \delta R+\left(1+\frac{3 \mu^{4}}{R_{d S}^{2}}\right) \delta R=0 .
$$

Comparing this with the propagation equation of a massive scalar field gives

$$
m^{2}=-\frac{R_{d S}}{2}-\frac{R_{d S}^{3}}{6 \mu^{4}} \simeq-\frac{(8 \pi \rho)^{3}}{6 \mu^{4}} \simeq-10^{6} \mathrm{GeV},
$$

where in the last equality the density has been taken to be that of water, $\rho \simeq 10^{3} \mathrm{~kg} / \mathrm{m}^{3}$, and $\mu$ has been taken to be $\sim 10^{-33} \mathrm{eV}$, as required to account for the observed latetime accelerating expansion of the Universe. This large negative mass corresponds to a catastrophic instability that should make itself apparent on time scales of $\sim 10^{-26}$ seconds.

For more general theories it can be shown that the effective mass squared in the relevant scalar field equation takes the same sign as $f_{R R}$ 485. It is therefore the negative value of this quantity in the theory of Eq. (353) that is responsible for its exhibition of this instability. Further, one can show that the addition of higher powers of $R$ to the gravitational Lagrangian again helps defend it from instability 968, 421, 970. The Dolgov-Kawasaki instability has been shown not to occur in the Palatini approach [1165. The existence of this type of instability was rediscovered in [706, for relativistic stars. The problem in this context has been further studied in [417, 72, 1237, 1206, 707, 901, where it was shown that the instability can be avoided by changing the equation of state of the star, adding a divergence to the scalar field potential, or including chameleon effects. Neutron stars in $f(R)$ gravity have been studied in [332, 1099, and instabilities in systems with time-dependent mass have been studied in 60.

\subsection{General combinations of Ricci and Riemann curvature.}

In the previous section we considered theories that generalise the Einstein-Hilbert action by replacing the Ricci scalar, $R$, with some non-linear function, $f(R)$. Here we go further, and allow the action to be a function of not only $R$, but of any of the three linear and quadratic contractions of the Riemann curvature tensor ${ }^{26}, R, R_{\mu \nu} R^{\mu \nu}$ and $R_{\mu \nu \rho \sigma} R^{\mu \nu \rho \sigma}$. A systematic approach to studying theories of this type, based on minimal sets of curvature invariants, is proposed and studied in [646, 906].

\subsubsection{Action and field equations}

The most general weight-zero scalar density that one can construct from $g, R, R_{\mu \nu} R^{\mu \nu}$ and $R_{\mu \nu \rho \sigma} R^{\mu \nu \rho \sigma}$ alone is given by

$$
\mathcal{L}=\chi^{-1} \sqrt{-g} f\left(R, R_{\mu \nu} R^{\mu \nu}, R_{\mu \nu \rho \sigma} R^{\mu \nu \rho \sigma}\right)
$$

where $f$ is an arbitrary function of its arguments, and $\chi$ is a constant which can be determined from the Newtonian limit. The action is obtained, as usual, by integrating

\footnotetext{
${ }^{26}$ There is also a fourth possibility, namely $\epsilon^{\mu \nu \rho \sigma} R_{\epsilon \tau \mu \nu} R^{\epsilon \tau}$ 418. This contraction, however, is of limited physical interest as it does not affect the field equations, due to its parity.
} 
this density, together with that of the matter fields, over all space-time. The addition of supplementary terms to the density (369), in order to cancel total divergences, and which can be transformed to integrals on the boundary, can be problematic (see e.g. 849]) and so, for simplicity, they are usually assumed to vanish.

As with $f(R)$ theories one can proceed by using the metric variation approach (in which the connection is a priori taken to be given by the Christoffel symbols), using the Palatini formalism (in which the metric and connection are taken to be independent fields in the gravitational action), or the metric-affine formalism (in which the metric and connection are taken to be independent fields throughout the entire action). Here we will spell out the metric variational approach explicitly, as this is the most commonly considered form of the theory found in the literature. For studies involving the Palatini procedure, as applied to the these theories, the reader is referred to [792, 477, 794, 173, [795, 1002, 1070].

Varying the action, derived from integrating Eq. (369) over all space, with respect to the metric, then gives

$$
\begin{aligned}
\delta I=\chi^{-1} \int d \Omega \sqrt{-g}\left[\frac{1}{2} f g^{\mu \nu} \delta g_{\mu \nu}+f_{X} \delta X+f_{Y} \delta Y+f_{Z} \delta Z\right] \\
=\chi^{-1} \int d \Omega \sqrt{-g}\left[\frac{1}{2} f g^{\mu \nu} \delta g_{\mu \nu}-f_{X}\left(R^{\mu \nu} \delta g_{\mu \nu}-g^{\mu \nu} \delta R_{\mu \nu}\right)\right. \\
-2 f_{Y}\left(R^{\rho(\mu} R^{\nu)}{ }_{\rho} \delta g_{\mu \nu}-R^{\mu \nu} \delta R_{\mu \nu}\right) \\
\left.-2 f_{Z}\left(R_{\rho \sigma \epsilon}{ }^{(\nu} R^{\mu) \epsilon \sigma \rho} \delta g_{\mu \nu}-R_{\mu}{ }^{\nu \rho \sigma} \delta R_{\nu \rho \sigma}^{\mu}\right)\right]
\end{aligned}
$$

where we have defined $X \equiv R, Y \equiv R_{\mu \nu} R^{\mu \nu}$ and $Z \equiv R_{\mu \nu \rho \sigma} R^{\mu \nu \rho \sigma}$. Using $\delta R^{m u}{ }_{\nu \rho \sigma}=$ $\frac{1}{2} g^{\mu \epsilon}\left(\delta g_{\epsilon \sigma ; \nu \rho}+\delta g_{\epsilon \nu ; \sigma \rho}-\delta g_{\sigma \nu ; \epsilon \rho}-\delta g_{\epsilon \rho ; \nu \sigma}-\delta g_{\epsilon \nu ; \rho \sigma}+\delta g_{\rho \nu ; \epsilon \sigma}\right)$ we can then write the gravitational part of the action as

$$
\delta I=-\chi^{-1} \int d \Omega \sqrt{-g} P^{\mu \nu} \delta g_{\mu \nu}
$$

where

$$
\begin{aligned}
P^{\mu \nu} \equiv & -\frac{1}{2} f g^{\mu \nu}+f_{X} R^{\mu \nu}+2 f_{Y} R^{\rho(\mu} R_{\rho}^{\nu)_{\rho}}+2 f_{Z} R^{\epsilon \sigma \rho(\mu} R_{\rho \sigma \epsilon}^{\nu)} \\
& +f_{X ; \rho \sigma}\left(g^{\mu \nu} g^{\rho \sigma}-g^{\mu \rho} g^{\nu \sigma}\right)+\square\left(f_{Y} R^{\mu \nu}\right)+g^{\mu \nu}\left(f_{Y} R^{\rho \sigma}\right)_{; \rho \sigma} \\
& -2\left(f_{Y} R^{\rho(\mu}\right)_{; \rho}^{\nu)}-4\left(f_{Z} R^{\sigma(\mu \nu) \rho}\right)_{; \rho \sigma} .
\end{aligned}
$$

The notation $f_{N}$ here denotes the functional derivative of $f$ with respect to $N$. Looking for a stationary point of this action, by setting the first variation to zero, then gives the field equations

$$
P_{\mu \nu}=\frac{\chi}{2} T_{\mu \nu}-g_{\mu \nu} \Lambda
$$

where matter fields and a cosmological constant have been included. Here, $\Lambda$ is the cosmological constant (defined independent of $f(X, Y, Z)$ ) and $T^{\mu \nu}$ is the energy-momentum tensor of the matter fields. These field equations are generically of fourth-order, with the exception of cases in which the function $f$ is linear in second derivatives of the metric [418], as occurs in GR. 
Unlike the case of $f(R)$ theories of gravity, the theories described by the density given in Eq. 370 are not, in general, conformally related to General Relativity with a scalar field.

\subsubsection{Weak-field limit}

Let us consider the weak field limit of theories with additional terms in their action that are quadratic in curvature invariants:

$$
\mathcal{L}=\frac{\sqrt{-g}}{\chi}\left(R+\alpha R^{2}+\beta R_{\mu \nu} R^{\mu \nu}+\gamma R_{\mu \nu \rho \sigma} R^{\mu \nu \rho \sigma}\right),
$$

where $\alpha, \beta$ and $\gamma$ are constants. In this case one can use the well known result that the Gauss-Bonnet combination of curvature invariants is a total divergence, i.e.

$$
4 R_{\mu \nu} R^{\mu \nu}-R^{2}-R_{\mu \nu \rho \sigma} R^{\mu \nu \rho \sigma}=\text { total divergence, }
$$

which in the action integrates to a boundary term that is usually ignored. By redefining $\alpha$ and $\beta$ we can therefore write Eq. (374) in the equivalent form

$$
\mathcal{L}=\frac{\sqrt{-g}}{\chi}\left(R+\alpha R^{2}+\beta R_{\mu \nu} R^{\mu \nu}\right),
$$

without any loss of generality in the solutions to the resulting field equations.

If we now substitute Eq. (376) into Eq. (373), to get the field equations, we find that for the perturbed metric

$$
d s^{2}=-(1+2 \psi) d t^{2}+(1-2 \phi)\left(d x^{2}+d y^{2}+d z^{2}\right),
$$

the lowest order equations in the weak-field and slow-motion limit are

$$
\begin{aligned}
2(3 \alpha+\beta) \Delta R-R & =-\frac{\chi}{2} \rho \\
(4 \alpha+\beta) \Delta R-R-2 \Delta(\psi+\beta \Delta \psi) & =-\chi \rho,
\end{aligned}
$$

where the Ricci scalar is given as usual by $R=-2 \Delta \psi+4 \Delta \phi$. For a delta function source, $\rho=m \delta$, Eqs. (378) and 379 ) can then be seen to have the solutions

$$
\begin{aligned}
16 \pi \psi & =-\frac{\chi m}{r}\left(1+\frac{e^{-m_{1} r}}{3}-\frac{4 e^{-m_{2} r}}{3}\right) \\
16 \pi \phi & =-\frac{\chi m}{r}\left(1-\frac{e^{-m_{1} r}}{3}-\frac{2 e^{-m_{2} r}}{3}\right),
\end{aligned}
$$

where

$$
m_{1}^{2}=\frac{1}{2(3 \alpha+\beta)} \quad \text { and } \quad m_{2}^{2}=-\frac{1}{\beta} .
$$

These are the solutions found by Stelle in 1978 [1187]. These solutions can be seen to reduce to Eqs. (308) and (309) in the limit $\beta \rightarrow 0_{-}$. More generally, however, these theories can be seen to exhibit massive modes with two different mass parameters. In order to have non-oscillatory behaviour in the present case we must therefore require that both $3 \alpha+\beta \geq 0$ and $\beta \leq 0$ be simultaneously satisfied. 
If the solutions given in Eqs. 380 and (381) are the correct ones for describing the space-time geometry around approximately isolated masses, such as the Sun, then one can immediately see that if $m_{1}$ and $m_{2}$ are both large compared to $1 / r$ then one recovers the general relativistic prediction of $\gamma=1$, just as with $f(R)$ theories. For small masses, however, the situation is somewhat different from the $f(R)$ case. If both $m_{1}$ and $m_{2}$ are small compared to $1 / r$ then one has that the leading order term in Eqs. (380) and (381) is a constant (which can be absorbed into coordinate redefinitions), followed by a term proportional to $r$. This is a considerable deviation from the behaviour $\gamma \rightarrow 1 / 2$ that occurs when $m_{1} r$ is small and $m_{2} r$ is large, which is the limit of $f(R)$ gravity with a low mass parameter. It can also be seen that for $m_{1} \leq 2 m_{2}$ gravity is always attractive, while for $m_{1}>2 m_{2}$ it is attractive over large distances, while being repellent over small distances.

\subsubsection{Exact solutions, and general behaviour}

Let us now discuss what is known about the solutions to these general fourth-order theories of gravity in the context of both isolated masses, and cosmological solutions.

\section{Isolated Masses}

Motivation for a number of studies in this area has come from Einstein's particle programme, in which one looks for asymptotically flat and singularity free vacuum solutions which could be used to model particles [466. While it is known that no such solutions exist in General Relativity (Lichnerowicz's theorem [797]), it has been conjectured that they could exist in fourth-order theories 1252 .

By studying the solutions of quadratic theories of the type given in Eq. (376) with $\beta=-3 \alpha$ it has been shown that the solutions to the linearised vacuum field equations can be both asymptotically flat as $r \rightarrow \infty$, and smooth as $r \rightarrow 0$ [503. These theories are equivalent to the sum of an Einstein-Hilbert term and a Weyl term. Such results would initially appear to be encouraging for Einstein's programme, but it was later shown that there are, in fact, no solutions with the specified properties that exist within a neighbourhood of Minkowski space [1116. This means that if any non-trivial static spherically symmetric vacuum solutions to these theories exist, that are simultaneously asymptotically flat and geodesically complete, then they must correspond to very large energy densities (exceeding the energy density of neutron stars by at least 40 orders of magnitude [1116]).

The theorems of Lichnerowicz [797, and Israel 650, have more recently been considered in the context of fourth-order theories of the form given in Eq. 376 by Nelson [950. Here it is found that for static space-times with spatial curvature satisfying

$$
\begin{aligned}
m_{1}^{2}-{ }^{(3)} R & \geq 0 \\
\bar{R}_{\nu}^{\mu} \bar{R}_{\mu}^{\nu} m_{1}^{2}+\bar{R}_{\nu}^{\mu} \bar{R}_{\rho}^{\nu} \bar{R}_{\mu}^{\rho} & \geq 0,
\end{aligned}
$$

the vacuum field equations imply that all asymptotically constant solutions (or asymptotically flat, if the inequalities are saturated) obey $R_{\mu \nu}=0$. The expression for $m_{1}$ is given in Eq. (382). Over-bars here denote quantities projected into space-like hypersurfaces. The spherically symmetric solution to $R_{\mu \nu}=0$ is, of course, the Schwarzschild 
solution, which is geodesically complete only for the case of Minkowski space. Lichnerowicz's theorem can therefore be extended to all theories that obey the inequalities (383) and (384). It is then shown in [950] that if the spatial curvature satisfies

$$
\begin{aligned}
{ }^{(3)} R & \leq m_{2} \\
\frac{\bar{R}^{\mu}{ }_{\nu} \bar{R}^{\nu}{ }_{\rho} \bar{R}^{\rho}{ }_{\mu}}{\bar{R}^{\mu}{ }_{\nu} \bar{R}^{\nu}{ }_{\mu}} & \geq-m_{2},
\end{aligned}
$$

where $m_{2}$ is given by Eq. 382, and the space-time is asymptotically constant (or asymptotically flat, if the inequalities are saturated), then the only solutions with $m_{1}^{2} \geq 0$ that exist in the region exterior to a closed spherical null surface also obey $R_{\mu \nu}=0$. The only asymptotically constant vacuum solutions with a horizon, that satisfy the bounds (385) and (386), are therefore the Schwarzschild solutions. This extends the Israel's nohair theorem for black holes to quadratic fourth-order theories of gravity. It is argued in [950] that the inequalities (383)-(386) should be satisfied everywhere where the spatial curvature is smaller than the scale of the corrections to the Einstein-Hilbert action. If these corrections are motivated by quantum considerations, then we should therefore expect all of the inequalities (383)-(386) to be satisfied for astrophysically interesting systems. The stability of Schwarzschild black holes in the quadratic theories 376 has been studied in [1271, 1186].

The initial value problem for quadratic theories, of the type given in Eq. 376, , has also been studied in [967, where it was found to be well-posed.

\section{Cosmological Solutions}

There are a number of exact cosmological solutions known to exist for fourth-order theories containing $R_{\mu \nu} R^{\mu \nu}$ and $R_{\mu \nu \rho \sigma} R^{\mu \nu \rho \sigma}$. This simplest of these is, of course, de Sitter space, which exists for theories with general $f(X, Y, Z)$ in Eq. 369, and a cosmological constant, if 309.

$$
\frac{1}{2} f-\Lambda=\Lambda f_{X}+2 \Lambda^{2} f_{Y}+\frac{4}{3} \Lambda^{2} f_{z},
$$

where $f_{N}$ denotes differentiation of $f$ with respect to $N$. The stability of de Sitter space in quadratic theories, of the type given in Eq. (376), has been studied in [102], and in the more general case in 325. Other known exact homogeneous and isotropic cosmological solutions are the Einstein static universe and the Gödel universe, the existence of which has been discussed in [309] for arbitrary $f(X, Y, Z)$ (together with the conditions for the existence of closed time-like curves in the case of latter). The existence of powerlaw FLRW solutions, both in vacuum and in the presence of a perfect fluid, has been discussed by Middleton in 887. Power-law scaling FLRW solutions for theories with $L=R+\alpha \sqrt{R^{2}-4 R_{\mu \nu} R^{\mu \nu}+R_{\mu \nu \rho \sigma} R^{\mu \nu \rho \sigma}}$ have been investigated in [1233]. The extent to which the FLRW solutions of General Relativity can be reproduced in these theories is discussed in [848, 949].

As well as isotropic cosmological solutions, a number of studies have also been performed of anisotropic cosmological solutions in these theories. The simplest of these are probably the Bianchi I Kasner-like exact solutions found in [311, which were used to show that the infinite sequence of anisotropic oscillations that occurs on approach 
to the initial singularity in General Relativity does not occur in higher-order gravity theories, except in unphysical situations. This type of solution was further studied in 887. Exact Bianchi type $I I$ and $V I_{h}$ solutions were found by Barrow and Hervik in [102], for quadratic theories of the type (376), and were used to show the lack of validity of the cosmic no-hair theorems in these theories: Anisotropic inflation with positive $\Lambda$ is possible, without de Sitter space as the late-time asymptote. These authors also considered the general behaviour of Bianchi type $I$ and $I I$ solutions in quadratic theories, where the possibility of a stable isotropic singularity was discovered [103. Bianchi type $I, I V, V I_{h}$ and $V I I_{h}$ universes have been studied in [104], where it was shown that periods of anisotropic expansion can occur after a near isotropic expansion, and before re-isotropisation at late-times. Bianchi type $V I I_{A}$ solutions have been studied for quadratic theories in [367], and Bianchi type $I X$ universes have been studied by Cotsakis et al. in [340], where the Kasner solution of General Relativity was shown not to be a stable early asymptote of the quadratic theories given in Eq. (376).

Stability of past isotropic attractors has been the subject of study by Barrow and Middleton [107, 888. In the first of these papers the authors demonstrated the stability of past isotropic solutions to the quadratic theories 376 under scalar, vector and tensor inhomogeneous perturbations [107. This supports the hypothesis that small perturbations to the past isotropic attractor form part of the general cosmological solution to quadratic theories of fourth-order gravity. This study is extended to theories with powerlaw curvature terms, $\left(R_{\mu \nu} R^{\mu \nu}\right)^{n}$, in their Lagrangian in 888, where conditions are given for the stability of early isotropic states. This study also shows the instability of the exact solution found in 311, as the initial singularity is approached. The asymptotic behaviour of theories with quadratic corrections to the Einstein-Hilbert were studied, in the context of string cosmology, in [1090, 236, 1218. Exponential and power-law FLRW solutions in higher-dimensional string inspired models are found in 855, 856, 17. The evolution of FLRW solutions in generalised theories has also been studied using a dynamical systems analysis in 326 .

\subsubsection{Physical cosmology and dark energy}

Having discussed various cosmological solutions in these general fourth-order theories, let us now consider their relevance for observational cosmology and dark energy. We will proceed with this by first discussing studies of more general theories, followed by theories constructed from the Gauss-Bonnet curvature invariant. The Gauss-Bonnet invariant has special properties, which we will discuss in the Section 4.2.5

\section{General Theories}

In order to construct cosmological models that can produce late-time accelerating expansion the authors of [254] considered theories of the type

$$
L=R+\frac{\mu^{4 n+2}}{\left(a R^{2}+b R_{\mu \nu} R^{\mu \nu}+c R_{\mu \nu \rho \sigma} R^{\mu \nu \rho \sigma}\right)^{n}},
$$

where $\mu, n, a, b$ and $c$ are constants. It is found that for these theories there exist 
power-law attractors for the general spatially flat FLRW solutions, which are given by

$$
a(t)=a_{0}\left(\frac{t}{t_{0}}\right)^{\frac{8 n^{2}+10 n+2-3 \alpha \pm \sqrt{\Gamma}}{4(n+1)}}
$$

where

$$
\begin{aligned}
\alpha \equiv & \frac{12 a+4 b+4 c}{12 a+3 b+2 c} \\
\Gamma \equiv & 9 n^{2} \alpha^{2}-\left(80 n^{3}+116 n^{2}+40 n+4\right) \alpha \\
& +64 n^{4}+160 n^{3}+132 n^{2}+40 n+4
\end{aligned}
$$

The smaller of the exponents in Eq. 389 can be seen to $\rightarrow 0$ as $n \rightarrow \infty$, while the larger tends to $4 n$. For large $n$ it is therefore the case that accelerating expansion can occur at late times. This is a generalisation of the type of model considered in [255], for $f(R)$ gravity. In [880] it is shown that while the theory given in Eq. (388) is capable of explaining the supernova results, to do so and still have an acceptable age for the Universe it requires the matter content of the Universe to have an equation of state $0.07 \leq w \leq 0.21$, to $2 \sigma$. The FLRW solutions of theories with powers of $R, R_{\mu \nu} R^{\mu \nu}$ and $R_{\mu \nu \rho \sigma} R^{\mu \nu \rho \sigma}$ added to the Einstein-Hilbert action were also studied in [455], where the possibility of late-time accelerating expansion was considered.

Primordial nucleosynthesis in theories with powers of $R_{\mu \nu} R^{\mu \nu}$ added to the EinsteinHilbert action have been considered in 957, where constraints from observed element abundances are imposed. As with scalar-tensor theories, the constraints imposed from big bang nucleosynthesis are largely due to the different expansion rate during the radiation dominated period due to a different value of the effective Newton's constant.

The addition of a conformally invariant term to the Einstein-Hilbert action has been considered in [953, 954, 951, 952. In this case the gravitational Lagrangian takes the form given in Eq. $(376)$ with $3 \alpha+\beta=0$, and the resulting field equations are sometimes known as the 'Bach-Einstein equations'. The solutions to these equations have been studied in the context of inflation 953, the evolution of background cosmological models 954, the observational constraints available from pulsars [951], and weak fields and gravitational waves [952. Theories of this type are motivated, in part, from noncommutative geometry 268. For a review on short scale modifications of gravity in the context of non-commutative geometry, see 961 .

$$
\text { Theories with } L=f\left(R, R^{2}-4 R_{\mu \nu} R^{\mu \nu}+R_{\mu \nu \rho \sigma} R^{\mu \nu \rho \sigma}\right)
$$

Theories that are functions of the Ricci scalar, $R$, and the Gauss-Bonnet combination,

$$
\hat{G}=R^{2}-4 R_{\mu \nu} R^{\mu \nu}+R_{\mu \nu \rho \sigma} R^{\mu \nu \rho \sigma}
$$

have been particularly well studied, as they are motivated by string theory $886,530,529$, 981, and have improved stability properties (as will be discussed in Section 4.2.5). The linear case of $f=R+\hat{G}$ is known to be equivalent to the Einstein-Hilbert Lagrangian in 4 dimensions, up to surface terms, but more general functions, of the type $f(R, \hat{G})$, produce field equations that differ from those of General Relativity. The mathematical properties 
of the Gauss-Bonnet tensor, that occurs from varying the action of these theories, as well as the more general Lovelock tensor, have been studied in [493, 491, 492, 494.

The general behaviour of spatially flat FLRW solution in theories with $L=R+f(\hat{G})$ has been studied by Zhou, Copeland and Saffin in [1309] using a phase plane analysis. In this case the Friedmann equations become

$$
\begin{aligned}
3 H^{2} & =\hat{G} f_{\hat{G}}-f-24 H^{3} \dot{f}_{\hat{G}}+\frac{\chi}{2} \rho \\
2 \dot{H} & =8 H^{3} \dot{f}_{\hat{G}}-16 H \dot{H} \dot{f}_{\hat{G}}-8 H^{2} \ddot{f}_{\hat{G}}+\frac{\chi}{2}(\rho+P),
\end{aligned}
$$

where $R=6\left(\dot{H}+2 H^{2}\right)$ and $\hat{G}=24 H^{2}\left(\dot{H}+H^{2}\right)$. The existence of both stable de Sitter space, and phantom-like accelerating solutions to the above equations can be demonstrated, as well as trajectories in the phase space that mimic the evolution of the standard $\Lambda \mathrm{CDM}$ universe through radiation and matter dominated periods [1309]. The stability of de Sitter space, as well as radiation and matter dominated epochs has also been studied by de Felice and Tsujikawa in [371, where the conditions $f_{\hat{G} \hat{G}}>0$ and $f_{\hat{G} \hat{G}} \rightarrow 0_{+}$as $|\hat{G}| \rightarrow \infty$ were found to be required for models to be viable. These authors suggest the following functional forms for $f(\hat{G})$ as examples that satisfy these conditions, and could produce acceptable expansion histories for the Universe:

$$
\begin{aligned}
f(\hat{G}) & =\lambda \frac{\hat{G}}{\sqrt{\hat{G}_{*}}} \tan ^{-1}\left(\frac{\hat{G}}{\hat{G}_{*}}\right)-\frac{\lambda}{2} \sqrt{\hat{G}_{*}} \ln \left(1+\frac{\hat{G}^{2}}{\hat{G}_{*}^{2}}\right)-\alpha \lambda \sqrt{\hat{G}_{*}} \\
f(\hat{G}) & =\lambda \frac{\hat{G}}{\sqrt{\hat{G}_{*}}} \tan ^{-1}\left(\frac{\hat{G}}{\hat{G}_{*}}\right)-\alpha \lambda \sqrt{\hat{G}_{*}},
\end{aligned}
$$

where $\alpha, \lambda$ and $\hat{G}_{*}$ are constants. It is further claimed that these forms of $f(\hat{G})$ are compatible with solar system observations [372, producing corrections to the Schwarzschild metric that are of the form $\sim H^{2} r_{s}^{2}\left(r / r_{s}\right)^{p}$, where $r_{s}$ is the Schwarzschild radius of the Sun, $H$ is the Hubble rate and $p$ is a model dependent quantity. Much larger correction to General Relativistic predictions are claimed in 364 for theories with polynomial additions of the Gauss-Bonnet term, $\hat{G}^{n}$, to the Einstein-Hilbert action. The cosmologies of these theories, and theories with inverse powers of $\alpha \hat{G}+\beta R$ added to the Einstein-Hilbert action (where $\alpha$ and $\beta$ are constants), have been considered in [391, 979], while the FLRW solutions of other $R+f(\hat{G})$ theories have been considered in 971, 323. The 'inverse problem', of finding FLRW solutions that behave like $\Lambda$ CDM has been considered for $R+f(\hat{G})$ theories in 324. The phase space of FLRW solutions to $L=f(R, \hat{G})$ theories, and the transition from deceleration to acceleration, has also been studied in [25, 26, 24]. Supernova, $\mathrm{BAO}$ and $\mathrm{CMB}$ observations have been used to constrain $L=R+f(\hat{G})$ theories in 907 .

Linear perturbations around spatially flat FLRW universes have been studied in $L=$ $f(R, \hat{G})$ theories by de Felice, Gérard and Suyama in [368, and in $L=R+f(\hat{G})$ theories in particular by Li, Barrow and Mota [791. The former of these studies uses the velocity potentials and variational principle approach of Schutz [1119], while the latter uses the covariant formulation of Ellis and Bruni [469]. In the case of general $f(R, \hat{G})$ it is found that scalar perturbations in these theories have, in general, six degrees of freedom, two 
of which propagate on small scales with group velocity 368

$$
v_{g}^{2} \simeq-\frac{256}{3} \frac{\dot{H}^{2}\left(f_{R R} f_{\hat{G} \hat{G}}-f_{R \hat{G}}^{2}\right)}{\left(8 f_{R \hat{G}} H^{2}+16 H^{4} f_{\hat{G} \hat{G}}+f_{R R}\right)\left(f_{R}+4 H \dot{f}_{\hat{G}}\right)} \frac{k^{2}}{a^{2}} .
$$

There can be seen to be a $k$-dependence in Eq. (397), which does not occur in General Relativity. Such a relation had previously been found for the vacuum case in 393, where it was argued that the space-time is unstable if $v_{g}^{2}<0$, or has super-luminal modes in short wavelength modes if $v_{g}^{2}>0$. These features are problematic, but can be avoided in theories that satisfy $f_{R R} f_{\hat{G} \hat{G}}-f_{R \hat{G}}^{2}=0$. Such theories have scale independent propagation speeds only, and include the cases of $L=f(R), f(\hat{G}), \hat{G}+f(R)$ and $R+f(\hat{G})$. The latter case, being the subject of study in [791, has also been shown to suffer from matter instabilities. This is due to the evolution equation for perturbations to $f_{\hat{G}}$, which we write as $\epsilon$, and which obeys 791

$$
\ddot{\epsilon}+\left(\theta+\frac{4 \dot{\theta}}{\theta}\right) \dot{\epsilon}+\left[\left(1+\frac{4 \dot{\theta}}{\theta^{2}}\right) \frac{k^{2}}{a^{2}}-2\left(\dot{\theta}+\frac{\dot{\theta}^{2}}{\theta^{2}}+\frac{2 \theta^{2}}{9}\right)-\frac{27(3-4 \dot{f} \dot{G} \theta)}{48 \theta^{4} f_{\hat{G} \hat{G}}}\right] \epsilon=S,
$$

where $\theta=3 H$ is the expansion scalar, and the reader is referred to 791 for the form of the source term $S$. For stability it is required that the third term in the square brackets be positive, and remain dominant over the first, which is expected to be negative during matter domination. This requires $f_{\hat{G} \hat{G}} \geq 0$, and for $f_{\hat{G} \hat{G}} H^{6}$ to remain suitably small, in order to avoid instabilities [791, 370]. These are strong constraints on the forms of $f(\hat{G})$. It was further shown in 368 that vector modes in the general case of $f(R, \hat{G})$ decay, and that the propagation of tensor modes in these theories is model dependent.

\subsubsection{Other topics}

Let us now consider some remaining topics in fourth-order gravity, that have yet to be discussed.

Theories with $L=f\left(R, \phi, R^{2}-4 R_{\mu \nu} R^{\mu \nu}+R_{\mu \nu \rho \sigma} R^{\mu \nu \rho \sigma}\right)$

There has been some study of fourth-order theories that include a scalar field, as well as the Ricci scalar and the Gauss-Bonnet scalar, in the gravitational action. This is motivated by the dilaton that arises in string theory compactifications 886, and has been studied in terms of 'pre-big bang' cosmology in [530, 529, 264. In this scenario there is an early period of very rapid expansion due to the kinetic term of the scalar field. Black holes in these theories, and their extensions, have been studied in 890 , 889, 678, 22, 1209, 700, 229, 231, 573, 574, 994, 995, 857, 285, 228, 996, 858. Further string motivated study of FLRW cosmology in the context of these theories has also been performed in [53, 1217, 1223, 324, 575].

Late-time acceleration has also been studied in theories where a scalar field has been included, along with $R$ and $G$, in the gravitational action [982, 960, 959, 36, 728, 729, 1223, 1097, 324, 37, 983, 80]. These papers have considered the evolution of FLRW space-times, as well as inflation, structure formation, and the constraints that can be imposed upon them from supernovae, $\mathrm{CMB}, \mathrm{BAO}$, solar system observations, and primordial nucleosynthesis. These observations place strong constraints on the theories. 
Greater-than-fourth-order Theories

Another option that has been considered in the literature is that the action itself could contain derivatives of curvature invariants, so that [206]

$$
L=f\left(R, \square R, \square^{2} R, \ldots, \square^{n} R\right),
$$

where $\square$ is the D'Alembertian. Extremising the action associated with this Lagrangian, by varying the metric, gives the field equations [1117]

$$
\mathcal{Y} R_{\mu \nu}-\frac{1}{2} f g_{\mu \nu}-\mathcal{Y}_{; \mu \nu}+g_{\mu \nu} \square \mathcal{Y}+\mathcal{X}_{\mu \nu}=\frac{\chi}{2} T_{\mu \nu}
$$

where

$$
\begin{aligned}
\mathcal{X}_{\mu \nu} & \equiv \sum_{i=1}^{n}\left[\frac{1}{2} g_{\mu \nu}\left(\mathcal{Z}_{i}\left(\square^{i-1} R\right)^{; \sigma}\right)_{; \sigma}-\mathcal{Z}_{i ;(\mu}\left(\square^{i-1} R\right)_{; \nu)}\right], \\
\mathcal{Y} & \equiv \sum_{i=0}^{n} \square^{i} \frac{\partial f}{\partial\left(\square^{i} R\right)}, \\
\mathcal{Z}_{i} & \equiv \sum_{j=i}^{n} \square^{j-i} \frac{\partial f}{\partial\left(\square^{j} R\right)} .
\end{aligned}
$$

The field equations 400 can be seen to generically contain derivatives of the metric of order $2 n+4$, so that the familiar fourth-order theories discussed above are recovered when $n=0$. Theories with infinite $n$ have also been considered in the literature, and have been claimed to be ghost-free [164, 163].

Greater-than-fourth-order theories can be shown to be equivalent, under a conformal transformation, to General Relativity with two scalar fields [550], and their Newtonian limit has been considered in [657, where it was found that the familiar form of Newtonian potentials and Yukawa potentials can be present. Their consequences for inflation have been studied in [143, 551, 144, 42, 871, 977, 163, and the attractor nature of de Sitter space established. Bouncing cosmologies in these theories have been considered in [164, 162, 163], and the form of the CMB has been investigated in [162. Primordial nucleosynthesis has been considered in [957, and the consequences of this type of theory for dark energy have been considered in 977. For a more detailed overview of theories with greater than four derivatives of the metric in their field equations the reader is referred to [1118.

\section{Conformal Gravity}

One more possibility is to completely abandon the Einstein-Hilbert action, even as a limiting case of the fundamental action. Such a proposal has been advocated by Mannheim [866] in the case of conformal Gravity. Here the Einstein-Hilbert action is replaced by

$$
S_{C}=-\alpha_{G} \int d^{4} x \sqrt{-g} C_{\lambda \mu \nu \kappa} C^{\lambda \mu \nu \kappa},
$$


where $C_{\lambda \mu \nu \kappa}$ is the Weyl tensor, given by

$$
\begin{aligned}
C_{\lambda \mu \nu \kappa}= & R_{\lambda \mu \nu \kappa}+\frac{1}{6} R_{\alpha}^{\alpha}\left[g_{\lambda \nu} g_{\mu \kappa}-g_{\lambda \kappa} g_{\mu \nu}\right] \\
& -\frac{1}{2}\left[g_{\lambda \nu} R_{\mu \kappa}-g_{\lambda \kappa} R_{\mu \nu}-g_{\mu \nu} R_{\lambda \kappa}+g_{\mu \kappa} R_{\lambda \nu}\right],
\end{aligned}
$$

and $\alpha_{G}$ is a dimensionless gravitational self-coupling constant. It has been shown that such an action can be obtained from the path integral of fermionic degrees of freedom for the conformal and gauge invariant action of a fermionic field.

The case has been made that such a theory has a number of desirable properties eluding other higher derivative theories of gravity. Even though the equations are fourth order, signalling the presence of negative energy states, it has been shown that such states are completely decoupled from what the authors dub the physical sector 865. Conformal gravity can then be held up as a viable theory of quantum gravity. Furthermore, the peculiar ultraviolet properties of conformal gravity have been argued to lead to a solution to the cosmological constant problem.

At a classical level, conformal gravity has been shown to have intriguing properties. For a start, the non-relativistic limit of the field equations leads to a fourth order differential equation for the gravitational potential, $\Phi$, in which the usual Newtonian potentials that drop off as $1 / r$ are but one possibility. The general weak gravity potential is of the form

$$
\Phi(r)=-\frac{A}{r}+B r
$$

Such a form, it has been argued, can be tuned to fit a range of galaxy rotation curves. As in some other theories of modified gravity, this is achieved by fixing universal parameters. This may be contrasted with the usual dark matter prescription in which, for each galaxy, one can choose the properties of the dark matter halo.

The situation becomes more complicated once one adds couplings to matter fields 864. Conformal symmetry is spontaneously broken through a new scalar degree of freedom, $S$, such that the general (conformal) matter action is

$$
I_{M}=-\hbar \int d^{4} x \sqrt{-g}\left[\frac{1}{2} S^{\mu} S_{\mu}-\frac{1}{12} S^{2} R_{\mu}^{\mu}+\lambda S^{4}+i \bar{\psi} \gamma^{\mu} \nabla_{\mu} \psi-g S \bar{\psi} \psi\right],
$$

where $\psi$ is a fermionic field. One can extend this to more general actions containing scalar and fermionic fields. The vacuum expectation value of the scalar field is what then sets the Gravitational constant and the coupling to matter. It can also be used to renormalise the cosmological constant.

Although some of the quantum properties of conformal gravity have been worked out, a fully consistent and complete analysis of their cosmology is still lacking. In particular, and in its current incarnation, in which no dark matter is invoked, it is unclear how the correct angular diameter distance for the CMB can be obtained.

Theories with $L=f(T)$

An interesting variant on generalisations of the Einstein-Hilbert action are the $L=$ $f(T)$ theories, where $T$ is a contraction of the torsion tensor (defined below). These 
theories generalise the 'teleparallel' approach to General Relativity, which corresponds to a Lagrangian $L=T$, from which Einstein's equations can be derived [1235]. Here $T$ is defined by

$$
T=\frac{1}{4} T^{\mu \nu \rho} T_{\mu \nu \rho}+\frac{1}{2} T^{\mu \nu \rho} T_{\rho \nu \mu}-T_{\mu \nu}^{\mu} T_{\rho}^{\nu \rho},
$$

where $T_{\nu \rho}^{\mu}$ is the torsion tensor, defined in terms of the vierbein from $g_{\mu \nu}=\eta_{\alpha \beta} h_{\mu}^{\alpha} h_{\nu}^{\beta}$, as

$$
T_{\nu \rho}^{\mu}=h_{\alpha}^{\mu}\left(\partial_{\nu} h_{\rho}^{\alpha}-\partial_{\rho} h_{\nu}^{\alpha}\right) .
$$

This definition is equivalent to setting $T_{\nu \rho}^{\mu}$ equal to the antisymmetric part of the Weitzenbock connection. Now, varying the action

$$
S=\int \frac{\sqrt{-g}}{16 \pi G} f(T) d \Omega
$$

with respect to the vierbein fields gives the field equations

$$
\begin{gathered}
f_{T}\left(R_{\mu \nu}-\frac{1}{2} g_{\mu \nu} R\right)+\frac{1}{2} g_{\mu \nu}\left(f-f_{t} T\right) \\
+\quad\left(\frac{1}{2}\left(T_{\mu \nu \rho}+T_{\rho \nu \mu}+T_{\nu \mu \rho}\right)-g_{\mu \rho} T_{\nu \sigma}^{\sigma}+g_{\mu \nu} T_{\rho \sigma}^{\sigma}\right) f_{T T} \nabla^{\sigma} T=8 \pi G \Theta_{\mu \nu}
\end{gathered}
$$

where we have called the energy-momentum tensor $\Theta_{\mu \nu}$, to distinguish it from the torsion tensor. It can be seen that in the case $f(T)=T$ the field equations 405 reduce to Einstein's equations, so that the theory $L=T$ is equivalent to the Einstein-Hilbert action, as stated above. For $f \neq T$, however, the teleparallel approach outlined here gives different field equations to the fourth-order theories we have so far considered.

It was within the framework of these generalised equations that Bengochea and Ferraro suggested that the late-time accelerating expansion of the Universe could be accounted for without dark energy [135]. These authors considered the particular case

$$
f=T-\frac{\alpha}{(-T)^{n}}
$$

where $\alpha$ and $n$ are constants, and constrained the resulting FLRW cosmology they found with supernovae, BAOs and the CMB. They found the best fitting model has $n=-0.10$, $\Omega_{m}=0.27$, and has the required radiation, matter and accelerating epochs. A large number of papers have followed [135] in a short space of time, exploring the transition from deceleration to acceleration, observational constraints, conformal transformations, and structure formation [815, 1287, 932, 1288, 1227, 289, 134, 1286, 78, 933, 681, 1293, [1308, 79].

It has been shown, however, that these theories do not respect local Lorentz invariance, and have a number of extra degrees of freedom that are not present in General Relativity as a result [796]. This can seen by noticing that one can write the Ricci scalar in terms of $T_{\nu \sigma}^{\mu}$ as

$$
R=-T-2 \nabla^{\mu}\left(T_{\mu \nu}^{\nu}\right)
$$

Now, while $R$ is of course a Lorentz scalar, one can show that $\nabla^{\mu}\left(T^{\nu}{ }_{\mu \nu}\right)$ is not. It therefore follows that $T$ is not a Lorentz scalar either, and so the $f(T)$ theories do not exhibit 
local Lorentz symmetry. The exceptional case is $f=T$, in which case the non-Lorentz invariant part of the action can be seen from Eq. (407) to be a total divergence, which does not affect the field equations.

\section{Stability Issues}

There are serious concerns with the stability of general theories of the type $L=$ $f\left(R, R_{\mu \nu} R^{\mu \nu}, R_{\mu \nu \rho \sigma} R^{\mu \nu \rho \sigma}\right)$. Not least of these is the presence of 'ghosts', or perturbative modes with negative norm, as well as tachyonic instabilities in massive modes [1187, 236 , 606, 607, 293, 944, 392, 113, 990.

Let us now outline how ghost terms arise in these theories, following the discussion of 606, 607, 293. This starts by considering the quadratic theory

$$
\mathcal{L}=\sqrt{-g}\left[R+\frac{1}{6 m_{0}^{2}} R^{2}-\frac{1}{2 m_{2}^{2}} C^{2}\right],
$$

where $m_{0}$ and $m_{2}$ are constants, and $C^{2}=C_{\mu \nu \rho \sigma} C^{\mu \nu \rho \sigma}=R_{\mu \nu \rho \sigma} R^{\mu \nu \rho \sigma}-2 R_{\mu \nu} R^{\mu \nu}+R^{2}$ is the square of the Weyl tensor. Any quadratic theory can be written in this form, up to boundary terms, because of the Gauss-Bonnet identity. We now want to identify the scalar and spin-two degrees of freedom in Eq. (408), for which it is convenient to introduce auxiliary fields that play these roles, and to transform so that they have canonical form. We will do this now, following 606. By introducing an auxiliary scalar field, $\varphi$, and performing a conformal transformation $\tilde{g}_{\mu \nu}=e^{\varphi} g_{\mu \nu}$, Eq. 408) can be rewritten as 606]

$$
\mathcal{L}=\sqrt{-\tilde{g}}\left[\tilde{R}-\frac{3}{2}(\tilde{\nabla} \varphi)^{2}-\frac{3 m_{0}^{2}}{2}\left(1-e^{-\varphi}\right)^{2}-\frac{\tilde{C}^{2}}{2 m_{2}^{2}}\right] .
$$

By extremising this equation with respect to $\varphi$, and substituting the resulting value of $\phi$ back into the Lagrangian density, one recovers Eq. 408). It can be seen from Eq. 409) that $\varphi$ now has the kinetic term of a canonical scalar field. By introducing a second auxiliary field, $\pi_{\mu \nu}$, and transforming the metric so that $\bar{g}^{\mu \nu}=\tilde{g}^{\mu \rho} A_{\rho}{ }^{\nu} / \sqrt{|A|}$, where $A_{\rho}{ }^{\nu}=\left(1+\frac{1}{2} \phi\right) \delta_{\rho}{ }^{\nu}-\phi_{\rho}{ }^{\nu}$, we can then rewrite Eq. 409 as [606]

$$
\begin{aligned}
\mathcal{L}=\sqrt{-\bar{g}}\left[\bar{R}-\frac{3}{2}\left(A^{-1}\right)_{\mu}{ }_{\mu} \bar{\nabla}^{\mu} \varphi \bar{\nabla}_{\nu} \varphi-\frac{3 m_{0}^{2}}{2 \sqrt{|A|}}\left(1-e^{-\varphi}\right)^{2}\right. \\
\left.\quad-\bar{g}^{\mu \nu}\left[C^{\rho}{ }_{\mu \sigma} C^{\sigma}{ }_{\nu \rho}-C^{\rho}{ }_{\mu \nu} C^{\sigma}{ }_{\sigma \rho}\right]+\frac{m_{2}^{2}}{4 \sqrt{|A|}}\left(\phi_{\mu \nu} \phi^{\mu \nu}-\phi^{2}\right)\right] \\
\simeq \sqrt{-\bar{g}}\left[\bar{R}-\frac{3}{2} \bar{\nabla}^{\mu} \varphi \bar{\nabla}_{\mu} \varphi-\frac{3 m_{0}^{2}}{2} \varphi^{2}+\frac{m_{2}^{2}}{4}\left(\phi_{\mu \nu} \phi^{\mu \nu}-\phi^{2}\right)\right. \\
\left.-\frac{1}{4}\left(\bar{\nabla}_{\mu} \phi \bar{\nabla}^{\mu} \phi-\bar{\nabla}_{\mu} \phi_{\nu \rho} \bar{\nabla}^{\mu} \phi^{\nu \rho}+2 \bar{\nabla}^{\mu} \phi_{\mu \nu} \bar{\nabla}^{\nu} \phi-2 \bar{\nabla}_{\mu} \phi_{\nu \rho} \bar{\nabla}^{\rho} \phi^{\nu \mu}\right)\right]
\end{aligned}
$$

where in the second equality we have expanded out to quadratic order in $\varphi$ and $\phi_{\mu}{ }^{\nu}$ around zero, so that we are considering theories that are close to GR. The field $\phi_{\mu}{ }^{\nu}=\pi_{\mu}{ }^{\nu}$ 
has been introduced here to make clear with respect to which metric the indices are being raised or lowered: those of $\pi_{\mu}{ }^{\nu}$ are raised and lowered with $\tilde{g}_{\mu \nu}$, while those of $\phi_{\mu}{ }^{\nu}$ are raised and lowered with $\bar{g}_{\mu \nu}$. The quantities $C_{\nu \rho}^{\mu}$ are defined as

$$
C_{\nu \rho}^{\mu}=\frac{1}{2}\left(\tilde{g}^{-1}\right)^{\mu \sigma}\left(\bar{\nabla}_{\nu} \tilde{g}_{\rho \sigma}+\bar{\nabla}_{\rho} \tilde{g}_{\nu \sigma}-\bar{\nabla}_{\sigma} \tilde{g}_{\nu \rho}\right) \text {. }
$$

The Lagrangian given in Eq. 411) now has scalar, $\varphi$, and spin-2 modes, $\phi_{\mu}{ }^{\nu}$, both in canonical form. It can be seen from Eq. (411) that for real $\phi_{\mu}{ }^{\nu}$ the spin-2 field does indeed have the wrong sign before its kinetic term, and is therefore generically a ghost, while for real $\varphi$ the scalar mode is not [1270]. What is more, if $m_{0}^{2}<0$ or $m_{2}^{2}<0$ then the scalar or spin-2 modes exhibit tachyonic instabilities, respectively.

Having outlined the proof for the generic existence of spin-2 ghosts in quadratic fourth-order theories of gravity, (408), let us now extend this to more general theories of the form

$$
\mathcal{L}=\sqrt{-g} f(X, Y, Z)
$$

where $X \equiv R, Y \equiv R_{\mu \nu} R^{\mu \nu}$ and $Z \equiv R_{\mu \nu \rho \sigma} R^{\mu \nu \rho \sigma}$. This demonstration proceeds by showing that the particle content of theories of the type (413) are the same as the quadratic theories (408) (at least, when considering fluctuations around de Sitter space), and then using the result derived above, that these theories generically contain spin-2 ghosts. The first step here is to introduce auxiliary fields $\phi_{1}, \phi_{2}$ and $\phi_{3}$ so that the Lagrangian density 413) becomes 607, 293.

$$
\mathcal{L}=\sqrt{-g}\left[f+f_{1}\left(X-\phi_{1}\right)+f_{2}\left(Y-\phi_{2}\right)+f_{3}\left(Z-\phi_{3}\right)\right],
$$

where $f_{i} \equiv \partial f / \partial \phi_{i}$, and $i=\{1,2,3\}$. As long as $f_{i}$ is non-degenerate, extremising with respect to the these three new fields then gives $\phi_{1}=X, \phi_{2}=Y$ and $\phi_{3}=Z$, so that Eq. 413 is recovered. Using the Gauss-Bonnet combination, and discarding boundary terms, this equation can then be written as 293 .

$$
\begin{aligned}
\mathcal{L}=\sqrt{-g}[ & \left(f-\phi_{1} f_{1}-\phi_{2} f_{2}-\phi_{3} f_{3}\right)+f_{1} R+\frac{1}{3}\left(f_{1}+f_{3}\right) R^{2} \\
& +\frac{1}{2}\left(f_{2}+4 f_{3}\right) C_{\mu \nu \rho \sigma} C^{\mu \nu \rho \sigma} \\
& \left.-\frac{1}{2}\left(f_{2}+2 f_{3}\right)\left(C_{\mu \nu \rho \sigma} C^{\mu \nu \rho \sigma}-2 R_{\mu \nu} R^{\mu \nu}+\frac{2}{3} R^{2}\right)\right],
\end{aligned}
$$

where $C_{\mu \nu \rho \sigma}$ is the Weyl tensor, and the last quantity in brackets is the Gauss-Bonnet combination. At this point the theory has been shown to be equal to a scalar-scalarscalar-tensor theory, with the three scalars non-minimally coupled to quadratic curvature invariants. Finally, we can choose to expand the density above up to second order in fluctuations around a de Sitter background with constant Ricci curvature, $R_{0}$, so that 944

$$
\mathcal{L}=\sqrt{-g}\left[-\Lambda+\alpha R+\frac{1}{2 m_{0}^{2}} R^{2}-\frac{1}{2 m_{2}^{2}} C^{2}\right]
$$


where boundary terms have been ignored, and we have defined

$$
\begin{aligned}
\Lambda \equiv & \left\langle f-X f_{X}+X^{2}\left(\frac{1}{2} f_{X X}-\frac{1}{4} f_{Y}-\frac{1}{6} f_{Z}\right)\right. \\
& \left.+X^{3}\left(\frac{1}{2} f_{X Y}+\frac{1}{3} f_{X Z}\right)+X^{4}\left(\frac{1}{8} f_{Y Y}+\frac{1}{18} f_{Z Z}+\frac{1}{6} f_{Y Z}\right)\right\rangle_{0} \\
\alpha \equiv & \left\langle f_{X}-X f_{X X}-X^{2}\left(f_{X Y}+\frac{2}{3} f_{X Z}\right)\right. \\
& \left.\quad-X^{3}\left(\frac{1}{4} f_{Y Y}+\frac{1}{9} f_{Z Z}+\frac{1}{3} f_{Y Z}\right)\right\rangle_{0} \\
m_{0}^{-2} \equiv & \left\langle\left(3 f_{X X}+2 f_{Y}+2 f_{Z}\right)+X\left(3 f_{X Y}+2 f_{X Z}\right)\right. \\
& \left.+X^{2}\left(\frac{3}{4} f_{Y Y}+\frac{1}{3} f_{Z Z}+f_{Y Z}\right)\right\rangle_{0} \\
m_{2}^{-2} \equiv & -\left\langle f_{Y}+4 f_{Z}\right\rangle_{0},
\end{aligned}
$$

where $\langle\ldots\rangle_{0}$ denotes the value of the quantity inside the brackets on the de Sitter background. It should be clear that Eq. (416) is identical to Eq. (408), up to the values of $\alpha$ and $\Lambda$. The particle content of general theories of the type (413), on a de Sitter background, therefore also has a scalar mode with mass $m_{0}$, and a ghost-like spin- 2 mode with mass $m_{2}$.

As mentioned in Section 4.2.4 it has been suggested that theories that are only functions of the Ricci scalar, $R$, and the Gauss-Bonnet combination, $G=R^{2}-4 R_{\mu \nu} R^{\mu \nu}+$ $R_{\mu \nu \rho \sigma} R^{\mu \nu \rho \sigma}$, can evade the ghost problem outlined above [944. The reason given for this is that the mass term $m_{2}^{-2} \rightarrow 0$ as $f_{Y} \rightarrow 4 f_{Z}$, a condition that is satisfied for theories of the type $L=f(X, Z-4 Y)$, or, equivalently, $L=f(R, G)$. When $m_{2}^{-2}$ vanishes it can be seen from Eq. (416) that the term responsible for the ghost spin-2 fluctuations will also disappear. Further requirements for the non-existence of ghosts in $f(R, G)$ theories are discussed in [392, with particular reference to the model of 254]. Such theories may still be subjected to constraints on their parameters by the possible existence of tachyonic instabilities, if $m_{0}^{2}<0$. For theories with $L=f(R, G)$ it can be seen that $m_{0}^{-2}=\frac{1}{3} R^{2} f_{G G}$, so that the condition $m_{0}^{2}>0$ is equivalent to the stability condition $f_{G G}>0$ found in [371, 370] and 791] in the context of cosmology.

\subsection{Hor̆ava-Lifschitz Gravity}

Hořava-Lifschitz (HL) gravity was proposed as a toy model of quantum gravity 618 619, 620. The model is non-relativistic and relies on anisotropic scaling between space and time in the UV to help render the theory asymptotically safe. Furthermore, it was claimed that General Relativity could be recovered in the infra-red by including additional relevant operators. HL gravity in its various guises has been reviewed in a number of articles [1016, 1166, 1266, 926, 170].

To understand the idea behind HL gravity we must first understand why perturbative General Relativity is not UV complete. The non-renormalisability arises because the coupling constant has negative mass dimension, $[G]=-2$, and the graviton propagator scales as $1 / p^{2}$. Consider the following scalar field theory,

$$
S=\int d^{4} x\left[\begin{array}{c}
-\frac{1}{2}(\partial \varphi)^{2}+\lambda \varphi^{6} \\
137
\end{array} .\right.
$$


Again, the propagator scales like $1 / p^{2}$, and the coupling constant has mass dimension $[\lambda]=-2$, so schematically at least, one might expect this theory to be non-renormalisable too. To render the theory asymptotically safe, we need to improve the UV behaviour of the propagator. One might do this by adding relativistic higher derivative terms to the action, but this is known to introduce an additional ghost-like degree of freedom. The reason for the existence of this ghost can be traced back to higher order time derivatives as opposed to space derivatives. This observation suggests an alternative approach. Let us abandon Lorentz invariance and introduce higher order spatial derivatives without introducing any higher order time derivatives. The former should improve the UV behaviour of the propagator, whereas the latter guarantees the absence of ghosts. We therefore modify the kinetic term

$$
-\frac{1}{2}(\partial \varphi)^{2} \rightarrow \frac{1}{2} \dot{\varphi}^{2}-\frac{1}{2} \varphi(-\Delta)^{z} \varphi,
$$

where $\Delta$ is the spatial Laplacian. We now have a non-relativistic dispersion relation $w^{2} \propto k^{2 z}$, which means that time and space scale differently,

$$
x \rightarrow l x, \quad \text { and } \quad t \rightarrow l^{z} t,
$$

For large enough $z$, it follows that the coupling constant has a non-negative scaling dimension, $[\lambda]=4 z-6$ so we expect the theory to be power counting renormalisable, and ghost-free. On the flip side, we have broken Lorentz invariance, which is well tested at low energies. However, we can cope with this by adding a relevant operator of the form $\mathcal{L}_{r e l}=\frac{1}{2} c^{2} \varphi \Delta \varphi$. This leaves the good UV physics unaffected, but allows Lorentz invariance to be restored as an emergent symmetry in the IR, with an emergent speed of light $c$.

In HL gravity, one applies similar logic to the relevant perturbative degrees of freedom, schematically replacing $\phi$ with the graviton, $h_{i j}$. Since we will require time and space to scale differently in this model, we must first choose a preferred time, $t$, which in the language of General Relativity means making an ADM split 902 .

$$
d s^{2}=-N^{2} c^{2} d t^{2}+q_{i j}\left(d x^{i}+N^{i} d t\right)\left(d x^{j}+N^{j} d t\right),
$$

where $q_{i j}(x, t)$ is the spatial metric and $N^{i}(x, t)$ is the shift vector. For the lapse function we consider two separate scenarios: (i) the projectable case where the lapse $N=N(t)$ is homogeneous and (ii) the non-projectable case where the lapse $N=N(x, t)$ can depend on space. Having chosen a preferred time, we no longer have the full diffeomorphism group, $\operatorname{Diff}(\mathcal{M})$, but a subset known as foliation preserving diffeomorphisms, $\operatorname{Diff}(\mathcal{M}, \mathcal{F})$, generated by

$$
\delta t=f(t), \quad \text { and } \quad \delta x^{i}=\xi^{i}(x, t) .
$$

$\operatorname{Diff}(\mathcal{M}, \mathcal{F})$ is defined by the following set of infinitesimal transformations

$$
\begin{aligned}
\delta N & =\partial_{t}(N f)+\xi^{i} \partial_{j} N \\
\delta N^{i} & =\partial_{t}\left(N^{i} f+\xi^{i}\right)+\mathcal{L}_{\xi} N^{i} \\
\delta q_{i j} & =f \partial_{t} q_{i j}+\mathcal{L}_{\xi} q_{i j} .
\end{aligned}
$$

Note that this hard breaking of diffeomorphism invariance is at the root of many of the problems facing HL gravity as it allows additional degrees of freedom to propagate 
230, 278. To see the extra degree of freedom emerge it is convenient to perform a Stuckelberg trick [1076, and artificially restore full diffeomorphism invariance at the expense of introducing a new field - the Stuckelberg field. This field becomes strongly coupled as the parameters of the low energy theory run towards their diff-invariant values [278. (see also [167, 735, 1025]).

We can think of the lapse and shift as playing the role of gauge fields in $\operatorname{Diff}(\mathcal{M}, \mathcal{F})$. It follows that the projectable case is the more natural since then the gauge fields have the same space-time dependence as the corresponding generators. Having said that one might expect it to be easier to match the non-projectable case to General Relativity in the infra-red.

In any event, the action from these theories is built from objects that are covariant with respect to $\operatorname{Diff}(\mathcal{M}, \mathcal{F})$. These are the spatial metric, $q_{i j}$, and the extrinsic curvature, $K_{i j}=\frac{1}{2 N}\left[\dot{q}_{i j}-2 \vec{\nabla}_{(i} N_{j)}\right]$, where $\vec{\nabla}_{i}$ is the spatial covariant derivative. In the nonprojectable case one should also consider terms built from $a_{i}=\vec{\nabla}_{i} \log N$ [167]. To build the gravitational analogue of the action (417), we replace the kinetic term such that

$$
\frac{1}{2} \dot{\varphi}^{2} \rightarrow \frac{1}{\kappa_{G}} \sqrt{q} N\left(K_{i j} K^{i j}-\lambda K^{2}\right),
$$

where $\kappa_{G}$ is the gravitational coupling with scaling dimension $\left[\kappa_{G}\right]=z-3$, and $\lambda$ is a dimensionless parameter that also runs with scale. Clearly, for the $z=3$ theory the gravitational coupling constant is dimensionless, which may lead one to suspect the theory to be power counting renormalisable. For $z=3$ the leading order term in the UV part of the action becomes

$$
-\frac{1}{2} \varphi(-\Delta)^{3} \varphi \rightarrow-\kappa_{G} \sqrt{q} N V_{6}
$$

where the dimension six contribution to the potential is

$$
V_{6}=\beta \vec{\nabla}_{k} R_{i j} \vec{\nabla}^{k} R^{i j}+\ldots
$$

Here $\beta$ is a dimensionless parameter, $R_{i j}$ is the spatial Ricci tensor and "..." denotes any of the other possible dimension 6 operators that one might wish to include, e.g. $R^{3}, R \Delta R,\left(a^{i} a_{i}\right)^{3}$ etc.

Now let us consider the type of relevant operators one might add. If we demand our action to be invariant under spatial parity $x^{i} \rightarrow-x^{i}$ and time reversal $t \rightarrow-t$, then we only need consider even dimensional operators,

$$
\mathcal{L}_{r e l}=-\frac{1}{\kappa_{G}} \sqrt{q} N\left(V_{2}+\kappa_{G} V_{4}\right)
$$

At dimension four these are 692

$$
\begin{aligned}
V_{4}=A_{1} R^{2}+A_{2} R_{i j} R^{i j}++ & B_{1} R a^{i} a_{i}+B_{2} R_{i j} a^{i} a^{j}+B_{3} R a^{i}{ }_{i} \\
& +C_{1}\left(a^{i} a_{i}\right)^{2}+C_{2}\left(a^{i} a_{i}\right) a^{j}{ }_{j}+C_{3}\left(a^{i}{ }_{i}\right)^{2}+C_{4} a^{i j} a_{i j},
\end{aligned}
$$

whereas at dimension two,

$$
V_{2}=-\alpha\left(a^{i} a_{i}\right)-c^{2} R .
$$


Here we have introduced the notation $a^{i_{1} \ldots i_{n}}=\vec{\nabla}^{i_{1}} \ldots \vec{\nabla}^{i_{n}} \log N$. Of course, terms of this form are only relevant in the non-projectable case.

The full $z=3$ gravitational theory is now given by

$$
S=\int d t d^{3} x \frac{1}{\kappa_{G}} \sqrt{q} N\left[K_{i j} K^{i j}-\lambda K^{2}+c^{2} R+\alpha\left(a^{i} a_{i}\right)^{2}-\kappa_{G} V_{4}-\kappa_{G}^{2} V_{6}\right]+S_{m},
$$

where $S_{m}\left[N, N_{i}, q_{i j} ; \Psi\right]$ is the matter part of the action, and $V_{6}$ is the relevant dimension 6 operator. Note that in the absence of full diffeomorphism invariance we do not require matter to satisfy energy-momentum conservation [278. Indeed, in general we expect to see violation of energy conservation $\operatorname{since} \operatorname{Diff}(\mathcal{M})$ breaking operators in the gravity Lagrangian will induce $\operatorname{Diff}(\mathcal{M})$ breaking quantum corrections to the matter Lagrangian 692 .

Let us compare this to the Einstein-Hilbert action, written in terms of the ADM variables as

$$
S_{G R}=\frac{c^{4}}{16 \pi G} \int d t d^{3} x \sqrt{q} N\left[\frac{K_{i j} K^{i j}-K^{2}}{c^{2}}+R\right] .
$$

The claim is that $\lambda=1$ and $\alpha=0$ are the infra-red fixed points of the renormalisation group flow. Of course, the parameter $\alpha$ plays no role in the projectable theory, since any terms containing $a_{i}=\vec{\nabla}_{i} \log N$ will vanish for $N=N(t)$. For both the projectable and non-projectable theories, the free parameters run to their infra-red fixed points at low energies, so that the HL action (431) tends towards the Einstein-Hilbert action 432 with an emergent speed of light, $c$, and an emergent Newton's constant, $G=\kappa_{G} c^{2} / 16 \pi$.

Before delving further into the different manifestations of HL gravity, let us pause to make a few general comments. The first of these is with regard to the large number of terms appearing in the potential. We have not bothered to present the contributions from dimension six operators since they are two numerous ${ }^{27}$. To reduce the number of terms, Hořava originally borrowed the notion of detailed balance from condensed matter theory 619], but this has since been shown to lead to phenomenological problems [278. 1169, 1170. Of course, the large number of terms appearing in the potential is really only an aesthetic concern.

A second, more serious, concern, involves the fine tuning of light cones for each field. Since Lorentz invariance is not exact there is no symmetry guaranteeing that all fields see the same emergent light cone. We would like there to be some mechanism suppressing Lorentz violating operators at low energies but preliminary investigations suggest that fine tuning is required [1045]. It is possible that supersymmetry may be able help with this to some extent [565].

Another issue that has yet to be fully explored concerns possible equivalence principle violations in HL gravity 692]. To see how these might arise it is convenient to go to the Stuckelberg picture. The Stuckelberg trick was developed in the context of massive gauge theories 1076, but it has proven very useful in elucidating some of the key physics of HL gravity as well (see, for example, [278, 167, 692]). Recall that the anisotropic scaling of space and time requires a hard breaking of $\operatorname{Diff}(\mathcal{M})$ down to $\operatorname{Diff}(\mathcal{M}, \mathcal{F})$. We can

\footnotetext{
${ }^{27}$ Note that the full set of inequivalent terms up to dimension six has been presented for the projectable
} case in 1169,1170 . 
artificially restore full diffeomorphism invariance by redefining the ADM slicing in terms of the Stuckelberg field $\phi(x, t)$. That is, the slicing goes from

$$
t=\text { constant } \quad \rightarrow \quad \phi(x, t)=\text { constant }
$$

The unit normal to the spatial surfaces is covariant under $\operatorname{Diff}(\mathcal{M})$ and given by the spacetime gradient $n_{\mu}=\nabla_{\mu} \phi / \sqrt{-(\nabla \phi)^{2}}$. We can now express HL gravity as a relativistic theory involving the space-time metric, $g_{\mu \nu}$, and the Stuckelberg field [167, 692, 532]. We do this by defining the space-time analogue of the spatial metric and the extrinsic curvature in terms of the projection tensor $q_{\mu \nu}=g_{\mu \nu}+n_{\mu} n_{\nu}$ and its Lie derivative $\frac{1}{2} \mathcal{L}_{n} q_{\mu \nu}$ 532. Violations of the EP can occur because the Stuckelberg field can mediate a force between matter fields carrying Stuckelberg "charge". As shown in 692, Stuckelberg "charge" is a measure of violation of energy-momentum conservation, schematically given by

$$
\Gamma \sim \sqrt{\left|\frac{\nabla_{\mu} T^{\mu \nu} \nabla_{\alpha} T_{\nu}^{\alpha}}{T_{\mu \nu} T^{\mu \nu}}\right|} \neq 0 .
$$

This is allowed by foliation preserving diffeomorphisms which simply require 692 .

$$
q_{\alpha \nu} \nabla_{\mu} T^{\mu \nu}=0, \quad \text { and } \quad \frac{1}{\sqrt{-g}} \frac{\delta S_{m}}{\delta \phi}=-\frac{n_{\nu} \nabla_{\mu} T^{\mu \nu}}{\sqrt{-(\nabla \phi)^{2}}} .
$$

Even if the Stuckelberg charges, $\Gamma_{1}, \Gamma_{2}, \ldots$ are always small, violation of the EP can still be large since the relevant Eötvös parameter $\eta \sim \frac{\Gamma_{1}-\Gamma_{2}}{\Gamma_{1}+\Gamma_{2}}$ really only cares about the charge ratios.

\subsubsection{The projectable theory}

We now focus on the projectable version of HL gravity, for which the lapse function is homogeneous, $N=N(t)$. The action is then given by

$$
S=\int d t d^{3} x \frac{1}{\kappa_{G}} \sqrt{q} N\left[K_{i j} K^{i j}-\lambda K^{2}-V\right]+S_{m},
$$

where the potential $V=-c^{2} R+$ higher derivative operators. Since the condition of projectability is imposed at the level of the theory itself, it follows that the Hamiltonian constraint is non-local:

$$
\int d^{3} x \sqrt{q}\left[K_{i j} K^{i j}-\lambda K^{2}+V\right]=\frac{\delta S_{m}}{\delta N} .
$$

In comparison with GR where the Hamiltonian constraint is local, this admits a much larger class of solutions. Indeed, it has been suggested that the resulting integration constant can account for dark matter [924], although this may lead to the formation of caustics and the break down of the theory [167].

Dark matter as an integration constant 
To see how this might emerge, we rewrite the action 436 in the following form

$$
\begin{aligned}
S_{=} \frac{c^{4}}{16 \pi G} \int d t d^{3} x \sqrt{q} N & {\left[\frac{K_{i j} K^{i j}-K^{2}}{c^{2}}+R\right]+S_{m} } \\
& +(1-\lambda) \frac{c^{2}}{16 \pi G} \int d t d^{3} x \sqrt{q} N K^{2}+\text { UV corrections. }
\end{aligned}
$$

Focusing on the low energy theory, the resulting field equations are 924]

$$
\begin{aligned}
\int d^{3} x \sqrt{-g}\left(G_{\mu \nu}^{(4)}-\frac{8 \pi G}{c^{2}} T_{\mu \nu}\right) n^{\mu} n^{\nu} & =\mathcal{O}(1-\lambda) \\
\left(G_{i \mu}^{(4)}-\frac{8 \pi G}{c^{4}} T_{i \mu}\right) n^{\mu} & =\mathcal{O}(1-\lambda) \\
G_{i j}^{(4)}-\frac{8 \pi G}{c^{4}} T_{i j} & =\mathcal{O}(1-\lambda),
\end{aligned}
$$

where $g_{\mu \nu}$ is the full space-time metric, $G_{\mu \nu}^{(4)}$ is the corresponding Einstein tensor, and $n^{\mu}=\frac{1}{N}\left(1,-N^{i}\right)$ is the unit normal to hyper-surfaces of constant $t$. The stress energy tensor, $T_{\mu \nu}$, is not necessarily conserved, as previously stated. Note that the non-local Hamiltonian constraint, 439, and the local momentum constraint, 440, are preserved by the dynamical equations (441).

Now, these equations can be rewritten as follows [924]:

$$
G_{\mu \nu}^{(4)}=\frac{8 \pi G}{c^{4}}\left(T_{\mu \nu}+T_{\mu \nu}^{H L}\right)+\mathcal{O}(1-\lambda),
$$

where $T_{\mu \nu}^{H L}=\rho^{H L} n_{\mu} n_{\nu}$ and $\int d^{3} x \sqrt{q} \rho^{H L}=0$. Note that this latter condition does not require $\rho^{H L}$ to vanish at all points in space, and one might wish to identify $T_{\mu \nu}^{H L}$ with a pressureless fluid moving with 4 -velocity $n^{\mu}$. Taking $\rho^{H L}>0$ in our Hubble patch, we may associate this integration constant with dark matter [924].

This scenario has been criticised in [167, where it is argued that the cosmological fluid $T_{\mu \nu}^{H L}$ will inevitably lead to the formation of caustics and the break down of the theory. To see why this might be the case it is convenient to go to the Stuckelberg picture, where we identify the unit normal with the space-time gradient $n_{\mu}=\nabla_{\mu} \phi / \sqrt{-(\nabla \phi)^{2}}$. Now $T_{\mu \nu}^{H L}$ behaves like a pressureless fluid, and in General Relativity it is well known that this will lead to the formation of caustics, due to the attractive nature of gravity. This is not a problem for real dust, as virialisation can occur. However, in the scenario of [924], the fluid is characterised by the gradient $\nabla_{\mu} \phi$ which is problematic as the Stuckelberg field is not differentiable at the caustic. These conclusions have been disputed in 923 . where it is argued that as the putative caustic begins to form we enter a UV regime and the parameter $\lambda$ runs away from its IR fixed point at $\lambda=1$. At $\lambda \neq 1$ it is claimed that an extra repulsive force could ultimately prevent caustics from forming.

\section{Perturbation theory - ghosts, tachyons, and strong coupling}

Let us now consider linearised perturbations about a Minkowski background. In what follows we will work in units where the emergent speed of light is given by $c=1$. Given 
the residual diffeomorphism, in Eqs. 422 to 424, we can choose a gauge defined by

$$
N=1, \quad N_{i}=\partial_{i} B+n_{i}, \quad \text { and } \quad q_{i j}=(1+2 \zeta) \delta_{i j}+h_{i j},
$$

where $n_{i}$ is a divergence-free vector, and $h_{i j}$ is a transverse-tracefree tensor. To study the propagating degrees of freedom we neglect the matter contribution, and integrate out the constraints. As it is non-local, the Hamiltonian constraint does not affect the local propagating degrees of freedom. Meanwhile, the momentum constraint yields

$$
B=-\frac{1}{c_{s}^{2} \Delta} \dot{\zeta}, \quad \text { and } \quad n_{i}=0,
$$

where

$$
c_{s}^{2}=\frac{1-\lambda}{3 \lambda-1} .
$$

This will shortly be identified with the scalar speed of sound at low energies. Plugging this into the action and expanding to quadratic order one finds [1170, 926]

$$
S=\frac{1}{8 \pi G} \int d t d^{3} x\left[-\frac{1}{c_{s}^{2}}\left(\dot{\zeta}^{2}+\zeta \mathcal{O}_{s} \zeta\right)+\frac{1}{8}\left(\dot{h}_{i j}^{2}+h^{i j} \mathcal{O}_{t} h_{i j}\right)\right]+S_{\mathrm{int}},
$$

where $S_{\text {int }}$ denotes the interactions and

$$
\mathcal{O}_{s}=c_{s}^{2}\left(\Delta+\frac{\Delta^{2}}{k_{U V}^{2}}+\frac{\Delta^{3}}{k_{U V}^{4}}\right), \quad \mathcal{O}_{t}=\Delta+\mu \frac{\Delta^{2}}{k_{U V}^{2}}+\nu \frac{\Delta^{3}}{k_{U V}^{4}},
$$

where $\mu$ and $\nu$ are dimensionless parameters of order one. Here we assume that all Lorentz symmetry breaking terms in $V_{4}$ and $V_{6}$ depend on roughly the same scale, $k_{U V} \lesssim$ $1 / \sqrt{8 \pi G}$. The dispersion relation for the scalar is given by

$$
w^{2}=c_{s}^{2}\left(k^{2}+\frac{k^{4}}{k_{U V}^{2}}+\frac{k^{6}}{k_{U V}^{4}}\right) .
$$

Now we see a problem: At low energies, $k \ll k_{U V}$, we require $c_{s}^{2}>0$ to avoid a tachyonic instability, where we identify $c_{s}$ with the speed of propagation of the scalar waves. However, as we see from the action (446), $c_{s}^{2}>0$ yields a ghost, which is far more troubling. We therefore take $c_{s}^{2}<0$, but with $\left|c_{s}\right|$ being small so as to render the tachyonic instability mild. But how small does $\left|c_{s}\right|$ need to be? The timescale of this instability is $t_{s} \sim 1 /\left|c_{s}\right| k>1 /\left|c_{s}\right| k_{U V}$, and as this would need to exceed the age of the universe, we infer $\left|c_{s}\right|<H_{0} / k_{U V}$, where $H_{0}$ is the current Hubble scale 167. Furthermore, since modifications to Newton's law have been tested down to the meV scale, we may impose $k_{U V}>\mathrm{meV}$. This gives $\left|c_{s}\right|<10^{-30}$, or equivalently $|1-\lambda|<10^{-60}$, on scales $H_{0} \ll k \ll k_{U V}$.

What about the interaction terms? At low energies, $k \ll k_{U V}$, and working up to cubic order we find that [735, 1166]

$$
\begin{aligned}
S_{\mathrm{int}}=\frac{1}{8 \pi G} \int d t d^{3} x\left\{\zeta\left(\partial_{i} \zeta\right)^{2}-\frac{2}{c_{s}^{4}} \partial_{t} \zeta \partial_{i} \zeta \frac{\partial^{i}}{\Delta} \partial_{t} \zeta\right. & \\
& \left.+\frac{3}{2}\left[\frac{1}{c_{s}^{4}} \zeta\left(\frac{\partial_{i} \partial_{j}}{\Delta} \partial_{t} \zeta\right)^{2}-\left(\frac{2}{c_{s}^{2}}+\frac{1}{c_{s}^{4}}\right) \zeta\left(\partial_{t} \zeta\right)^{2}\right]\right\}+\ldots
\end{aligned}
$$


To estimate the strength of these interactions we canonically normalise the quadratic term (446) in the infra-red by redefining

$$
t=\frac{\hat{t}}{\left|c_{s}\right|}, \quad \text { and } \quad \zeta=\sqrt{8 \pi G\left|c_{S}\right|} \hat{\zeta}
$$

so that

$$
\begin{aligned}
S_{\mathrm{int}}=\sqrt{\frac{8 \pi G}{\left|c_{s}\right|^{3}} \int d \hat{t} d^{3} x\left\{c_{s}^{2} \hat{\zeta}\left(\partial_{i} \hat{\zeta}\right)^{2}-2 \partial_{\hat{t}} \hat{\zeta} \partial_{i} \hat{\zeta} \frac{\partial^{i}}{\Delta} \partial_{\hat{t}} \hat{\zeta}\right.} & \\
& \left.+\frac{3}{2}\left[\hat{\zeta}\left(\frac{\partial_{i} \partial_{j}}{\Delta} \partial_{\hat{t}} \hat{\zeta}\right)^{2}-\left(2 c_{s}^{2}+1\right) \hat{\zeta}\left(\partial_{\hat{t}} \hat{\zeta}\right)^{2}\right]\right\}+\ldots
\end{aligned}
$$

For small $\left|c_{s}\right|$, we see that the largest cubic interactions become strongly coupled at a scale $\Lambda_{s c} \sim \sqrt{\left|c_{s}\right|^{3} / 8 \pi G}$. Imposing the constraint, $\left|c_{s}\right|<10^{-30}$ and taking $1 / \sqrt{8 \pi G} \sim 10^{18}$ $\mathrm{GeV}$, we find $\Lambda_{s c} \lesssim 10^{-18} \mathrm{eV}$. This lies well below scale of the UV corrections given by $k_{U V}>\mathrm{meV}$ so we can certainly trust the effective low energy description we have used to derive this scale. The implications for the theory are profound. The scale $\Lambda_{s c}$ represents the scale at which perturbative quantum field theory breaks down in Minkowski space. For scattering processes above $\Lambda_{s c} \lesssim 10^{-18} \mathrm{eV}$ we must sum up the contribution from all multi-loop diagrams. Since the claims of renormalisability are based on the validity of the perturbative description at all energies, we see that much of the motivation for studying this theory is lost. We also note that any notion of Minkowski space is meaningless below distances $1 / \Lambda_{s c} \gtrsim 10^{8} \mathrm{~km}$ since one would require a scattering process at energies above $\Lambda_{s c}$ to probe its structure. In analogy with DGP gravity (see section 5.5) one might hope to raise the scale of strong coupling by considering fluctuations on curved backgrounds, for example, on the background gravitational field generated by the Sun 926 . However, this seems optimistic since Minkowski space is an excellent approximation ${ }^{28}$ to the background geometry at distances of order $1 / \Lambda_{s c} \gtrsim$ $10^{8} \mathrm{~km}$, so the derived scale, $\Lambda_{s c} \sim \sqrt{\left|c_{s}\right|^{3} / 8 \pi G}$ should still be reliable.

\subsubsection{The non-projectable theory}

We now consider the non-projectable theory for which the lapse function can depend on space, $N=N(x, t)$, just as in General Relativity. This means the Hamiltonian constraint is now local and that terms depending on $a_{i}=\vec{\nabla}_{i} \log N$ could play an interesting role in the dynamics [169, 168. Just as in the projectable case, the absence of full diffeomorphism invariance allows an extra scalar mode to propagate [278. The action is given by

$$
\begin{aligned}
& S_{=} \frac{c^{4}}{16 \pi G} \int d t d^{3} x \sqrt{q} N {\left[\frac{K_{i j} K^{i j}-K^{2}}{c^{2}}+R\right]+\alpha \frac{c^{2}}{16 \pi G} \int d t d^{3} x \sqrt{q} N a_{i} a^{i} } \\
&+(1-\lambda) \frac{c^{2}}{16 \pi G} \int d t d^{3} x \sqrt{q} N K^{2}+S_{m}+\text { UV corrections. }
\end{aligned}
$$

\footnotetext{
${ }^{28}$ At distances $r \gtrsim 10^{8} \mathrm{~km}$ from the Sun, the Newtonian potential $V(r) \lesssim 10^{-8}$.
} 
Again we consider small vacuum perturbations on a Minkowski background, working in units where $c=1$. Given the reduced set of diffeomorphisms, Eqs. (422) to 424), we cannot gauge away the fluctuations in the lapse function that depend on space. Instead, we choose a gauge

$$
N=1+\chi, \quad N_{i}=\partial_{i} B+n_{i}, \quad \text { and } \quad q_{i j}=(1+2 \zeta) \delta_{i j}+h_{i j},
$$

where, as before, $n_{i}$ is a divergence free vector, and $h_{i j}$ is a transverse-tracefree tensor. Now integrating out the momentum constraint, one finds

$$
B=-\frac{1}{c_{s}^{2} \Delta} \dot{\zeta}, \quad n_{i}=0
$$

where $c_{s}^{2}$ is given by equation (445), but, as we will see, we do not identify it with the speed of sound. The Hamiltonian constraint yields [169, 1025.

$$
\chi=-\frac{2}{\alpha} \zeta
$$

Expanding to quadratic order gives [169, 1025]

$$
S=\frac{1}{8 \pi G} \int d t d^{3} x\left[-\frac{1}{c_{s}^{2}}\left(\dot{\zeta}^{2}+\zeta \tilde{\mathcal{O}}_{s} \zeta\right)+\frac{1}{8}\left(\dot{h}_{i j}^{2}+h^{i j} \tilde{\mathcal{O}}_{t} h_{i j}\right)\right]+S_{\text {int }},
$$

where $S_{\text {int }}$ denotes the interactions and

$$
\tilde{\mathcal{O}}_{s}=c_{s}^{2}\left(\frac{\alpha-2}{\alpha} \Delta+\frac{\Delta^{2}}{k_{U V}^{2}}+\frac{\Delta^{3}}{k_{U V}^{4}}\right), \quad \text { and } \quad \tilde{\mathcal{O}}_{t}=\Delta+\tilde{\mu} \frac{\Delta^{2}}{k_{U V}^{2}}+\tilde{\nu} \frac{\Delta^{3}}{k_{U V}^{4}},
$$

where $\tilde{\mu}$ and $\tilde{\nu}$ are dimensionless parameters of order one. Again we assume that all Lorentz symmetry breaking terms in $V_{4}$ and $V_{6}$ depend on roughly the same scale, $k_{U V} \lesssim$ $1 / \sqrt{8 \pi G}$. We now write down the dispersion relation for the scalar

$$
w^{2}=c_{s}^{2}\left(\frac{\alpha-2}{\alpha} k^{2}+\frac{k^{4}}{k_{U V}^{2}}+\frac{k^{6}}{k_{U V}^{4}}\right) .
$$

It follows that the speed of propagation of the scalar waves in the non-projectable theory are given by

$$
\tilde{c}_{s}^{2}=c_{s}^{2}\left(\frac{\alpha-2}{\alpha}\right) .
$$

The ghost and the tachyon problems can now be avoided by simultaneously taking [169, 1025

$$
c_{s}^{2}<0, \quad \text { and } \quad 0<\alpha<2 .
$$

Recall that this was not possible in the projectable case, where we had to accept the tachyonic instability and use the fact that this should be slow relative to a Hubble time to place strong bounds on $|1-\lambda|$. Since in the non-projectable case we no longer have such concerns regarding tachyonic instabilities, the strongest bounds on $|1-\lambda|$ and $|\alpha|$ come from preferred frame effects in the Solar System, requiring [1272]

$$
|1-\lambda|,|\alpha| \lesssim 10^{-7} .
$$


For $\lambda$ and $\alpha$ satisfying this bound, the speed of propagation of the scalar is

$$
\tilde{c}_{s}^{2} \approx \frac{\lambda-1}{\alpha}
$$

which should not be too slow. If the scalar graviton fluctuations propagated significantly slower than other fields to which they couple, then those fields would decay into scalar gravitons via the Cerenkov process. This would be particularly worrying for light since it would result in photon decay and the absence of cosmic rays. To avoid this problem we take $\tilde{c}_{s}^{2} \sim 1$, and so $|1-\lambda| \sim|\alpha|$. Solar system constraints on these theories are considered further in [643, 644, 645], based on the solutions presented in [684], and black holes are studied in [226, 227, 232.

What about the interactions? Again, focusing on the low energy theory we find that the quantum fluctuations on Minkowski space become strongly coupled at the scale $\tilde{\Lambda}_{s c} \sim \sqrt{|\lambda-1| / 8 \pi G} \sim \sqrt{|\alpha| / 8 \pi G}[1025,692$. Note that this result can be derived using a direct method similar to the one presented for the projectable case in the previous section [1025], or using the Stuckelberg method in the decoupling limit [692]. Now given the bounds in Eq. 459, it follows that the strong coupling scale is $\tilde{\Lambda}_{s c} \lesssim 10^{15} \mathrm{GeV}$. If $k_{U V}>\Lambda_{s c}$ we can trust our low energy description, and the derivation of this scale. As explained in the projectable case, strong coupling casts serious doubts on the claims of renormalisability as these rely on the validity of perturbative quantum field theory. However, if $k_{U V}<\Lambda_{s c}$, we cannot trust our derivation of the strong coupling scale, since the low energy description would not be valid there [168. In this scenario, new physics that softens the interactions kicks in at $k \sim k_{U V}$. This situation is reminiscent of the case in string theory where we introduce the string scale just below the Planck scale, where strong coupling would otherwise occur.

Whilst this seems promising there are some issues facing the non-projectable theory. One of these relates to the formal structure of the theory, and in particular the constraint algebra, which is dynamically inconsistent. This manifests itself through the lapse function vanishing asymptotically for generic solutions to the constraint equations 603. The asymptotically flat solutions we have just discussed represent a non-generic subset of measure zero in the space of all solutions.

In the quantum version of the theory, its has been claimed that one must take $\lambda<1 / 3$ in order to have a stable vacuum [1144. This is incompatible with phenomenological requirements for the following reason: We expect there to be 3 fixed points in the renormalisation group flow for $\lambda$. These are $\lambda=1$ (diff invariance), $\lambda=1 / 3$ (conformal invariance) and $\lambda=\infty$ 619]. Now, at low energies we require $|\lambda-1|<10^{-7}$ and $c_{s}^{2}=\frac{1-\lambda}{3 \lambda-1}<0$. This suggests that $\lambda$ flows from infinity in the UV to $\lambda=1$ in the IR.

\subsubsection{Aspects of Hořava-Lifschitz cosmology}

HL gravity was first applied to cosmology in [698, 235. As we have seen, in the UV, the relevant degrees of freedom have an anisotropic dispersion relation $w^{2} \approx k^{6} / k_{U V}^{4}$. This is often at the root of much of the interesting cosmology that has subsequently arisen, including (i) a scale invariant spectrum of cosmological perturbations, without early time acceleration [925, 284, (ii) cosmological bounces [179, 234, 522, 854, 351, (iii) dark matter as an integration constant [924, (iv) chirality of primordial gravity waves [1196] and (v) enhancement of baryon asymmetry, abundance of gravity waves, dark matter, and so on [927. These latter effects occur because the modified dispersion 
relation results in radiation scaling like $1 / a^{6}$ as opposed to $1 / a^{4}$ in the UV regime. We refer the reader to the following review articles on this subject [926, 1098.

For an FLRW universe, the background cosmology in both the projectable and nonprojectable theories is qualitatively very similar. Choosing units where the emergent speed of light is $c=1$, the Friedmann equation takes the following form:

$$
\frac{3 \lambda-1}{2} H^{2}=\frac{8 \pi G}{3}\left(\rho+\frac{C(t)}{a^{3}}\right)+V(a),
$$

where

$$
V(a)=\frac{\kappa}{a^{2}}\left[1+m\left(\frac{\kappa}{k_{U V}^{2} a^{2}}\right)+n\left(\frac{\kappa}{k_{U V}^{2} a^{2}}\right)^{2}\right],
$$

and where $m$ and $n$ are dimensionless parameters of order one. Here $\kappa=0, \pm 1$ is the spatial curvature. We assume that the matter component with energy density, $\rho$, and pressure, $P$, satisfies the usual energy-conservation law, $\dot{\rho}+3 H(\rho+P)=0$, although this is not necessarily required in HL gravity, as we have already discussed.

The contribution from $C(t) / a^{3}$ depends on the theory in question. For the projectable theory it corresponds to the "dark matter integration constant" 924, with $C(t) \rightarrow$ constant at low energies. For the non-projectable theory there is no such contribution and $C(t) \equiv 0$.

We immediately notice that the effective Newton's constant seen by cosmology differs from the one derived by comparing the low energy effective action to the Einstein-Hilbert action:

$$
G_{\text {cosmo }}=\frac{2}{3 \lambda-1} G \text {. }
$$

Although as $\lambda \rightarrow 1$, at low energies, we see that $G_{\text {cosmo }} \rightarrow G$.

To see how HL cosmology can admit a bounce, consider the limiting behaviour of the right hand side of Eq. 460). Neglecting $C(t) / a^{3}$ and assuming $\rho$ scales like $1 / a^{3}$ or $1 / a^{4}$ we see that this goes like $\sim n k_{U V}^{-4}\left(\kappa / a^{2}\right)^{3}$, which is negative if $n \kappa<0$. By continuity, this suggests that there exists $a_{*}$ for which the right-hand side of Eq. 460 is zero at $a=a_{*}$. This corresponds to the position of the bounce, since at this point $H=0$.

Observational constraints on $|\lambda-1|$ coming from the background cosmology have been studied in [45, 446] using $\mathrm{BAO}+\mathrm{CMB}+\mathrm{SN1}$, but they are not particularly strong. At $1 \sigma$ confidence level they find that $|\lambda-1| \lesssim 0.02$, which is far weaker than the bounds presented in previous sections. Recall that in the projectable theory, stability considerations require $|\lambda-1| \lesssim 10^{-60}$, whereas in the non-projectable theory preferred frame effects require $|\lambda-1| \lesssim 10^{-7}$.

Cosmological perturbations in HL gravity have also been considered (see, for example, [925, 284, 521, 1257, 712, 1258, 713, 1256, 632, 652). Indeed, for the projectable theory, it has been claimed that scalar fluctuations on cosmological backgrounds are stable. This is in contrast to the corresponding fluctuations on Minkowski space 632 which are known to suffer from either a ghost or tachyonic instability, as we saw in section 4.3.1. Whilst this may be relevant to long wavelength modes, it is of no consequence on sub-horizon scales where we can trust the perturbative analysis about Minkowski space, to a good approximation. Also, gravity waves produced during inflation have been found to be chiral in HL gravity, thereby representing a robust prediction of the theory [1196]. 


\subsubsection{The $\Theta C D M$ model}

HL gravity represents the UV completion of an interesting cosmological model, dubbed $\Theta C D M$ [171. In this model, it is assumed that the old cosmological constant problem is solved in some way, such that the net contribution to the cosmological constant is vanishing. The model then seeks to explain the tiny, but non-zero, amount of cosmic acceleration that is currently observed, without any fine tuning. Indeed, it is shown that the model allows for a technically natural small contribution to cosmic acceleration, without any corrections from other scales in the theory.

A key assumption corresponds to the fact that Lorentz invariance is broken in the gravitational sector. Thus the theory contains a unit time-like vector field which may be generic (as in Einstein-Aether theory) or expressed in terms of the gradient of a scalar field defining a global time (sometimes called the khronon ${ }^{29}$ ). The proposed acceleration mechanism appears generically when we assume the existence of another field, $\Theta$, which is taken to be invariant under shift transformations. The model is a valid effective field theory up to a high cut-off just a few orders of magnitude below the Planck scale, with a UV completion offered by HL gravity in the khronon case.

In the absence of any matter sources (including the cosmological constant) the model possesses two solutions corresponding to Minkowski and de Sitter space-times. The former solution is unstable and the presence of an arbitrarily small amount of matter destroys it. The cosmological evolution of a matter-filled universe is driven to the de Sitter attractor, with effective equation of state $w=-1$. The value of the effective cosmological constant on the de Sitter branch is determined by the lowest dimension coupling between the Goldstone field and the khronon. Remarkably, it is technically natural to assume this coupling to be small as it is protected from radiative corrections by a discrete symmetry. Thus, in the absence of a contribution from the cosmological constant, the current value of cosmic acceleration would not present any fine-tuning problem.

Interestingly, the evolution of cosmological perturbations is different in the $\Theta \mathrm{CDM}$ and $\Lambda$ CDM models. In particular, the growth of linear perturbations is enhanced in $\Theta C D M$ as compared to the standard $\Lambda \mathrm{CDM}$ case. The enhancement is most prominent at very large scales of order a few gigaparsecs, but extends also to shorter scales. Another difference is the appearance of an effective anisotropic stress, resulting in a nontrivial gravitational slip at very large scales. In principle, these effects may allow one to discriminate between $\Theta \mathrm{CDM}$ and $\Lambda \mathrm{CDM}$ in the near future.

\subsubsection{HMT-da Silva theory}

We have discussed in Sections 4.3 .1 and 4.3 .2 how the original versions of HL gravity are plagued with problems at the level of both theory and phenomenology. The root of this is the breaking of diffeomorphism invariance and the additional scalar degree of freedom that propagates as a result. With this in mind, Hořava and Melby-Thompson (HMT) proposed a modified version of the projectable theory possessing an additional $U(1)$ symmetry [621]. It is claimed that this extra symmetry removes the troublesome scalar degree of freedom, so that one is left with a spin-2 graviton as the only propagating

\footnotetext{
${ }^{29}$ The khronon field is naturally identified with the Stuckelberg mode in HL gravity at low energies.
} 
mode. The HMT action is given by

$$
\begin{aligned}
S_{H M T}=\frac{1}{\kappa_{G}} \int d t d^{3} x \sqrt{q}\left\{N \left[K_{i j} K^{i j}\right.\right. & -K^{2}-V\left(q_{i j}, R_{i j}, \vec{\nabla}_{k} R_{i j}\right) \\
& \left.\left.+\nu \Theta^{i j}\left(2 K_{i j}+\vec{\nabla}_{i} \vec{\nabla}_{j} \nu\right)\right]-A(R-2 \Omega)\right\},
\end{aligned}
$$

where $\Theta^{i j}=R^{i j}-\frac{1}{2} R q^{i j}+\Omega q^{i j}$, and $\Omega$ is a dimensionful coupling constant that can run with scale. This constant controls the scalar curvature of spatial slices, and can be thought of as a second cosmological constant. As with the original projectable theory, we assume $N=N(t)$, with a potential $V=-c^{2} R+\ldots$ containing the usual terms up to dimension six in order to guarantee the $z=3$ scaling in the UV. In addition, however, HMT theory contains two new fields given by $A=A(x, t)$ and $\nu=\nu(x, t)$. These are important in extending the symmetry group to $U(1) \times \operatorname{Diff}(\mathcal{M}, \mathcal{F})$.

The action is invariant under $\operatorname{Diff}(\mathcal{M}, \mathcal{F})$, with

$$
\begin{aligned}
\delta N & =\partial_{t}(N f) \\
\delta N^{i} & =\partial_{t}\left(N^{i} f+\xi^{i}\right)+\mathcal{L}_{\xi} N^{i} \\
\delta q_{i j} & =f \partial_{t} q_{i j}+\mathcal{L}_{\xi} q_{i j} \\
\delta A & =\partial_{t}(A f)+\xi^{i} \partial_{j} A \\
\delta \nu & =f \partial_{t} \nu+\xi^{i} \partial_{j} \nu .
\end{aligned}
$$

Note that $\nu$ transforms as a scalar, whereas $A$ transforms like a spatial scalar and a temporal vector. Indeed, $A$ transforms exactly as the lapse function would in a nonprojectable theory. This is not a coincidence. One can think of $A$ as being the next to leading order term in the non-relativistic expansion of the lapse. Of course, one ought to ask why we have not included the parameter $\lambda$ in front of the $K^{2}$ term in the action, as in previous versions of HL gravity. According to [621], the parameter $\lambda$ is fixed to be equal to one by requiring the action to be invariant under a local $U(1)$ symmetry:

$$
\begin{aligned}
\delta A & =\dot{\psi}-N^{i} \vec{\nabla}_{i} \psi \\
\delta \nu & =-\psi \\
\delta N^{i} & =N \vec{\nabla}^{i} \psi .
\end{aligned}
$$

It is this symmetry that removes the scalar graviton. Furthermore, fixing $\lambda=1$ ensures no conflict with observational tests of Lorentz violation at low energies. The HMT model has been applied to cosmology in [1259.

Recently, da Silva has argued that in contrast to the claims of [621, one can accommodate $\lambda \neq 1$ and still retain the $U(1) \times \operatorname{Diff}(\mathcal{M}, \mathcal{F})$ invariance 353. Indeed, he proposed the following action:

$$
\begin{aligned}
S_{\text {daSilva }}= & \frac{1}{\kappa_{G}} \int d t d^{3} x \sqrt{q}\left\{N \left[K_{i j} K^{i j}-\lambda K^{2}-V\left(q_{i j}, R_{i j}, \vec{\nabla}_{k} R_{i j}\right)\right.\right. \\
& \left.\left.+\nu \Theta^{i j}\left(2 K_{i j}+\vec{\nabla}_{i} \vec{\nabla}_{j} \nu\right)+(1-\lambda)\left[(\Delta \nu)^{2}+2 K \Delta \nu\right]\right]-A(R-2 \Omega)\right\},
\end{aligned}
$$

which is invariant under the same symmetries as 463). Now we must subject $\lambda$ to the same constraints as before, in particular those coming from preferred frame effects. There 
are claims that the extra symmetry will eliminate the scalar graviton even when $\lambda \neq 1$ [353, although more detailed study is required to be sure. Preliminary investigations on this subject have been carried out in 631. In any event, strong coupling problems have recently been shown to infect the matter sector of this theory [811, unless one introduces a low scale of Lorentz violation, in a way that is reminiscent of [169, 168.

\subsection{Galileons}

Galileon theory [963] was originally developed by Nicolis et al. to facilitate a model independent analysis of a large class of modified gravity models. In each case, General Relativity on perturbed Minkowski space is modified by an additional single scalar field, the galileon, with derivative self-interactions. Although the galileon and the graviton both couple to matter, any direct coupling between them is neglected to leading order. The resulting vacuum Lagrangian is invariant under the following shift in the galileon field

$$
\pi \rightarrow \pi+b_{\mu} x^{\mu}+c .
$$

This symmetry corresponds to a generalisation of Galilean invariance, hence the name. The inspiration for the model comes from DGP gravity [454. In Section 5.5, we will see how the boundary effective theory on the DGP brane is well described by the following action,

$$
S_{\mathrm{eff}}^{\mathrm{DGP}}=\int d^{4} x\left[\mathcal{L}_{G R}+\mathcal{L}_{\pi}^{\mathrm{DGP}}\right],
$$

where

$$
\begin{aligned}
\mathcal{L}_{G R} & =\frac{1}{16 \pi G}\left\{\frac{1}{4} \tilde{h}^{\mu \nu}\left[\partial^{2}\left(\tilde{h}_{\mu \nu}-\frac{1}{2} \tilde{h} \eta_{\mu \nu}\right)+\ldots\right]\right\}+\frac{1}{2} \tilde{h}_{\mu \nu} T^{\mu \nu}, \\
\mathcal{L}_{\pi}^{\text {DGP }} & =\frac{1}{16 \pi G}\left\{\frac{1}{2}\left[3 \pi \partial^{2} \pi-r_{c}^{2}(\partial \pi)^{2} \partial^{2} \pi\right]\right\}+\frac{1}{2} \pi T .
\end{aligned}
$$

The Lagrangian (474) has two components: a linearised GR piece, $\mathcal{L}_{G R}$, and a modification due to the brane bending mode, $\mathcal{L}_{\pi}^{\text {DGP }}$. It is valid in the so-called decoupling limit in which all interactions go to zero except the scalar self-interactions. Focusing on the $\pi$-Lagrangian, $\mathcal{L}_{\pi}^{\text {DGP }}$, Nicolis et al. observed that the vacuum field equations are built exclusively out of second derivatives, $\partial_{\mu} \partial_{\nu} \pi$. In particular, this means that there are no terms higher than second order, ensuring a well defined Cauchy problem and avoiding any of the potential problems arising from ghosts in higher derivative theories. In addition there are no first or zero derivative terms which means that the $\pi$ Lagrangian possesses the Galilean symmetry. This is inherited from Poincaré invariance in the bulk [1017.

One might expect that almost any co-dimension one braneworld model with large distance deviations from GR will be described, in part, and in some appropriate limit, by a generalised $\pi$ Lagrangian possessing the Galilean symmetry. This essentially follows from the fact that the extrinsic curvature of the brane is $K_{\mu \nu} \approx \partial_{\mu} \partial_{\nu} \pi$, on scales where we can neglect background curvature.

We should also note that even if there is no direct coupling to matter and therefore no modification of gravity, galileons are of interest in their own right as a source of energy-momentum. In particular, one can potentially obtain violations of the null energy 
condition without introducing any instability [964, 343. Generically, however, a single galileon will result in superluminality, although the situation may be improved by going to multi-galileon theory (see section 4.4.5).

\subsubsection{Galileon modification of gravity}

To see how to generalise the decoupling limit of DGP to a larger class of modified gravity theories, let us consider the amplitude, $\mathcal{A}$, for the exchange of one graviton between two conserved sources, $T_{\mu \nu}$ and $T_{\mu \nu}^{\prime}$. In General Relativity, this amplitude is given by

$$
\mathcal{A}_{G R}=\frac{16 \pi G}{p^{2}}\left[T_{\mu \nu} T^{\prime \mu \nu}-\frac{1}{2} T T^{\prime}\right],
$$

where $T=T_{\mu}^{\mu}$. We are interested in the case where gravity is modified by an additional scalar, so that locally we have

$$
\delta \mathcal{A}=\mathcal{A}-\mathcal{A}_{G R}=-\frac{1}{\alpha p^{2}} T T^{\prime} .
$$

Such a theory can be described by the following action

$$
S=\int d^{4} x \frac{1}{16 \pi G} \sqrt{-\tilde{g}} R(\tilde{g})-\frac{\alpha}{2} \pi \partial^{2} \pi+\frac{1}{2} \tilde{h}_{\mu \nu} T^{\mu \nu}+\pi T+\text { interactions }
$$

where $\tilde{g}_{\mu \nu}=\eta_{\mu \nu}+\tilde{h}_{\mu \nu}$. The fluctuation $\tilde{h}_{\mu \nu}$ is identified with the GR graviton, and as such, for a given source and boundary conditions, it coincides with the linearised solutions of GR. This statement is true to all orders in the "decoupling" limit

$$
M_{p l}, \alpha, T^{\mu \nu} \rightarrow \infty, \quad \frac{\alpha}{M_{p l}^{2}}=\text { const }, \quad \text { and } \quad \frac{T^{\mu \nu}}{M_{p l}}=\text { const }
$$

where $M_{p l}=\sqrt{1 / 8 \pi G}$. Note that matter is minimally coupled to the metric $g_{\mu \nu}=$ $\eta_{\mu \nu}+h_{\mu \nu}$, where the physical graviton is $h_{\mu \nu}=\tilde{h}_{\mu \nu}+2 \pi \eta_{\mu \nu}$.

\section{Galileon Action and Equations of Motion}

Now suppose we consider the decoupling limit 480 with the additional assumption that the strength of some of the scalar self-interactions can be held fixed. This amounts to neglecting the back-reaction of the scalar onto the geometry so that we can consider it as a field on Minkowski space. We retain some of the scalar self-interactions for the following reason: we are interested in an $\mathcal{O}(1)$ modification of GR on cosmological scales, but we would like this to be screened down to $\lesssim \mathcal{O}\left(10^{-5}\right)$ on solar system scales. As we will see, the derivative self-interactions can help shut down the scalar at short distances through Vainshtein screening 30 In the decoupling limit, the action is given by

$$
S\left[\tilde{h}_{\mu \nu}, \pi\right]=\int d^{4} x \mathcal{L}_{G R}+\mathcal{L}_{\pi},
$$

\footnotetext{
${ }^{30}$ See section 5.5 .4 for a detailed discussion of the Vainshtein mechanism in DGP gravity.
} 
where $\mathcal{L}_{\pi}=\mathcal{L}_{\text {gal }}(\pi, \partial \pi, \partial \partial \pi)+\pi T$ represents the generalisation of the $\pi$-Lagrangian in DGP gravity.

The vacuum part of the generalised $\pi$-Lagrangian, $\mathcal{L}_{g a l}(\pi, \partial \pi, \partial \partial \pi)$ gives second order field equations, and is assumed to be Galilean invariant in the sense that $\mathcal{L}_{\text {gal }} \rightarrow \mathcal{L}_{\text {gal }}+$ total derivative, when $\pi \rightarrow \pi+b_{\mu} x^{\mu}+c$. What is the most general Lagrangian with this property? The answer is remarkably simple, and in four dimensions is given by 963,400 .

$$
\mathcal{L}_{\text {gal }}(\pi, \partial \pi, \partial \partial \pi)=\sum_{i=1}^{5} c_{i} \mathcal{L}_{i}(\pi, \partial \pi, \partial \partial \pi)
$$

where the $c_{i}$ are constants, and 31

$$
\begin{aligned}
\mathcal{L}_{1} & =\pi \\
\mathcal{L}_{2} & =-\frac{1}{2}(\partial \pi)^{2} \\
\mathcal{L}_{3} & =-\frac{1}{2} \partial^{2} \pi(\partial \pi)^{2} \\
\mathcal{L}_{4} & =-\frac{1}{2}\left[\left(\partial^{2} \pi\right)^{2}-(\partial \partial \pi)^{2}\right](\partial \pi)^{2} \\
\mathcal{L}_{5} & =-\frac{1}{2}\left[\left(\partial^{2} \pi\right)^{3}-3\left(\partial^{2} \pi\right)(\partial \partial \pi)^{2}+2(\partial \partial \pi)^{3}\right](\partial \pi)^{2} .
\end{aligned}
$$

By construction, the variation of each component is built exclusively out of second derivatives,

$$
\frac{\delta}{\delta \pi} \int d^{4} x \mathcal{L}_{i}(\pi, \partial \pi, \partial \partial \pi)=\mathcal{E}_{i}(\partial \partial \pi),
$$

where

$$
\mathcal{E}_{i}(\partial \partial \pi)=(i-1) ! \delta_{\left[\nu_{1}\right.}^{\mu_{1}} \ldots \delta_{\left.\nu_{i-1}\right]}^{\mu_{i-1}}\left(\partial_{\mu_{1}} \partial^{\nu_{1}} \pi\right) \ldots\left(\partial_{\mu_{i-1}} \partial^{\nu_{i-1}} \pi\right)
$$

Specifically,

$$
\begin{aligned}
& \mathcal{E}_{1}=1 \\
& \mathcal{E}_{2}=\partial^{2} \pi \\
& \mathcal{E}_{3}=\left(\partial^{2} \pi\right)^{2}-(\partial \partial \pi)^{2} \\
& \mathcal{E}_{4}=\left(\partial^{2} \pi\right)^{3}-3 \partial^{2} \pi(\partial \partial \pi)^{2}+2(\partial \partial \pi)^{3} \\
& \mathcal{E}_{5}=\left(\partial^{2} \pi\right)^{4}-6\left(\partial^{2} \pi\right)^{2}(\partial \partial \pi)^{2}+8 \partial^{2} \pi(\partial \partial \pi)^{3}+3\left[(\partial \partial \pi)^{2}\right]^{2}-6(\partial \partial \pi)^{4} .
\end{aligned}
$$

It follows that the field equations for the galileon model are therefore given by the following:

$$
\begin{aligned}
-\frac{1}{2} \partial^{2}\left(\tilde{h}_{\mu \nu}-\frac{1}{2} \tilde{h} \eta_{\mu \nu}\right)+\ldots & =8 \pi G T_{\mu \nu}, \\
\sum_{i=1}^{5} c_{i} \mathcal{E}_{i}(\pi, \partial \pi, \partial \partial \pi) & =-T .
\end{aligned}
$$

\footnotetext{
${ }^{31}$ We define $(\partial \partial \pi)^{n}=\left(\partial_{\alpha_{1}} \partial^{\alpha_{2}} \pi\right)\left(\partial_{\alpha_{2}} \partial^{\alpha_{3}} \pi\right) \ldots\left(\partial_{\alpha_{n}} \partial^{\alpha_{1}} \pi\right)$. Note that we have presented the simpler expressions as suggested by [400].
} 
Equation 493 corresponds to the linearised Einstein equations, and so their solution, $\tilde{h}_{\mu \nu}$, corresponds to the standard GR solution for a given source and boundary conditions. The modification of GR is encoded entirely in the solution of the scalar equation of motion, 494).

\section{Galileon cosmology as a weak field}

The galileon theory has been constructed in terms of a tensor and a scalar propagating on a Minkowski background. Whilst it is straightforward to understand the weak gravitational field in the solar system using this description, it is not clear how one should describe cosmology. Fortunately, at distances below the curvature scale any metric is well approximated by a local perturbation about Minkowski space. In what follows, local will mean local in both space and time, which for cosmological solutions will correspond to sub-Hubble distances and sub-Hubble times.

Let us consider a spatially flat FLRW space-time. If we take our position to be given by $\vec{x}=\vec{y}=0$ and $t=\tau=0$, then for $|\vec{x}| \ll H^{-1}$ and $|t| \ll H$ we have 963 .

$$
d s^{2}=-d \tau^{2}+a(\tau)^{2} d \vec{y}^{2} \approx\left[1-\frac{1}{2} H^{2}|\vec{x}|^{2}+\frac{1}{2}\left(2 \dot{H}+H^{2}\right) t^{2}\right]\left(-d t^{2}+d \vec{x}^{2}\right)
$$

where the Hubble scale $H$ and its time derivative $\dot{H}$ are evaluated now. We recognise this as a perturbation on Minkowski space in Newtonian gauge,

$$
d s^{2} \approx-(1+2 \Psi) d t^{2}+(1-2 \Phi) d \vec{x}^{2},
$$

where the Newtonian potentials are 32

$$
\Psi=-\frac{1}{4} H^{2}|\vec{x}|^{2}+\frac{1}{4}\left(2 \dot{H}+H^{2}\right) t^{2}, \quad \text { and } \quad \Phi=-\Psi .
$$

For a given cosmological fluid, the corresponding GR solutions have Hubble parameter $H_{G R}$. Since $\tilde{h}_{\mu \nu}$ agrees with the linearised GR solution, we have $\tilde{h}_{t t}=-2 \Psi_{G R}$, and $\tilde{h}_{i j}=2 \Psi_{G R} \delta_{i j}$, where

$$
\Psi_{G R}=-\frac{1}{4} H_{G R}^{2}|\vec{x}|^{2}+\frac{1}{4}\left(2 \dot{H}_{G R}+H_{G R}^{2}\right) t^{2} .
$$

Now in our modified theory the physical Hubble parameter (associated with $h_{\mu \nu}$ ) differs from the corresponding GR value, $H \neq H_{G R}$. Since $h_{\mu \nu}=\tilde{h}_{\mu \nu}+2 \pi \eta_{\mu \nu}$, we have a non-trivial scalar

$$
\pi=\Psi-\Psi_{G R} .
$$

Note that a Galilean transformation $\pi \rightarrow \pi+b_{\mu} x^{\mu}+c$ merely corresponds to a coordinate transformation $x^{\mu} \rightarrow x^{\mu}-c x^{\mu}+\frac{1}{2}\left(x_{\nu} x^{\nu} b^{\mu}-2 b_{\nu} x^{\nu} x^{\mu}\right)$ in the physical metric.

\footnotetext{
${ }^{32}$ It may look like that since $\Phi-\Psi=2 \Phi \neq 0$ that this construction introduces anisotropic stress This is not the case, however, as strictly speaking the condition for the absence of anisotropic stress is $D^{i}{ }_{j}(\Phi-\Psi)=0$. It is easy to show that $D^{i}{ }_{j}|\vec{x}|^{2}=0$, hence, no anisotropic stress is present.
} 


\section{Self-accelerating solutions}

Of particular interest are self-accelerating solutions. A self-accelerating vacuum is one that accelerates even in the absence of any sources for the fields $\tilde{h}_{\mu \nu}$ and $\pi$. These are familiar to us from DGP gravity (see Section 5.5), where the self-accelerating solution is haunted by ghosts. Is the same true in a general galileon scenario? Or can we identify a scenario that admits a consistent self-accelerating solution?

There is some ambiguity as to what is actually meant by 'self-acceleration' if the tadpole term $\int d^{4} x c_{1} \pi$ is present in the galileon Lagrangian. The point is that at the level of the graviton equations of motion, the source corresponds to the vacuum energy, $\lambda$. However, at the level of the scalar equations of motion the tadpole term, $\int d^{4} x c_{1} \pi$, has the effect of renormalising the vacuum energy seen by the $\pi$ field, $\lambda \rightarrow \lambda+c_{1}$. Indeed, in a braneworld context, one might associate the tadpole with the vacuum energy in the bulk. To avoid considering a simple cosmological constant, we set the bare vacuum energy $\lambda=0$, and require the $\pi$-tadpole term to vanish, $c_{1}=0$. This guarantees that Minkowski space is a solution for the physical metric since the field equations are solved by $\tilde{h}_{\mu \nu}=0$, and $\pi=0$. Note that this Minkowski solution need not be stable, as in the ghost condensate scenario. On the contrary, our interest is in stable de Sitter solutions. Given the constraints $\lambda=0$, and $c_{1}=0$, any de Sitter solution is necessarily self-accelerating.

We now consider maximally symmetric vacua in the absence of vacuum energy, $\lambda=0$, and the tadpole, $c_{1}=0$. The corresponding GR solution is always Minkowski space, with $\tilde{h}_{\mu \nu}=0$. Non-trivial solutions for the scalar $\pi=\bar{\pi}(x)$, however, could give rise to self-acceleration. A self-accelerating vacuum with de Sitter curvature $H^{2}$ would have $\bar{\pi}=-\frac{1}{4} H^{2} x_{\mu} x^{\mu}$. Plugging this into the field equations (494), with $c_{1}=0$, and $T=0$, gives 963

$$
-2 c_{2} H^{2}+3 c_{3} H^{4}-3 c_{4} H^{6}+\frac{3}{2} c_{5} H^{8}=0 .
$$

Clearly non-trivial solutions exist for suitable choices of the parameters $c_{i}$, so selfaccelerating solutions also exist.

Are these vacua consistent? To investigate this we need to consider fluctuations $\tilde{h}_{\mu \nu}$, and $\delta \pi=\pi-\bar{\pi}$ about the self-accelerating vacuum. Because of the Galilean symmetry, the galileon structure is preserved in the effective theory describing fluctuations,

$$
S\left[\tilde{h}_{\mu \nu}, \delta \pi\right]=\int d^{4} x \mathcal{L}_{G R}+\mathcal{L}_{\delta \pi}
$$

where $\mathcal{L}_{\delta \pi}=\sum_{i=1}^{5} d_{i} \mathcal{L}_{i}(\delta \pi, \partial \delta \pi, \partial \partial \delta \pi)+\delta \pi T$. The coefficients can be obtained from the coefficients in the underlying theory via a linear map $d_{i}=\sum_{i} M_{i j} c_{j}$, where the matrix $M_{i j}$ depends on the background curvature, $H^{2}$ [963.

There are two immediate things to consider: (i) does the spectrum of fluctuations contain a ghost, and (ii) does the scalar get screened on solar system scales? For a general galileon theory, to avoid the ghost we must choose parameters such that $d_{2}>0$. In DGP gravity, where the ghost is known to be present on the self-accelerating branch, one would find $d_{2}^{\text {DGP }}<0$ (see Section 5.5.3 for further details).

In order to screen the scalar at solar system scales one must appeal to the Vainshtein mechanism. Again, we discuss the Vainshtein mechanism in detail in the context of DGP 
gravity in Section 5.5.4. The mechanism works in exactly the same way in a general galileon theory. For simplicity we assume spherical symmetry for fluctuations on the self-accelerating background and consider the profile outside of a heavy non-relativistic source, $T_{\mu \nu}=\operatorname{diag}(\rho(r), 0,0,0)$. Now, it is well known that the GR solution is given by the standard Newtonian potential

$$
\left|\tilde{h}_{\mu \nu}(r)\right| \sim \frac{G M}{r},
$$

where the mass of the source $M=\int \rho(r) d V$. The galileon solution $\delta \pi(r)$ is given by [963, 223 .

$$
d_{2}\left(\frac{\delta \pi^{\prime}}{r}\right)+2 d_{3}\left(\frac{\delta \pi^{\prime}}{r}\right)^{2}+2 d_{4}\left(\frac{\delta \pi^{\prime}}{r}\right)^{3}=\frac{M}{4 \pi r^{3}} .
$$

Note that $\mathcal{E}_{5}$ is identically zero when evaluated on a spherically symmetric field. At large distances one can neglect the higher order terms in Eq. 502 and derive the linearised solution

$$
\delta \pi^{l i n}(r)=-\frac{M}{4 \pi d_{2} r} .
$$

Now $\left|\tilde{h}_{\mu \nu}(r)\right| \sim\left|\delta \pi^{l i n}(r)\right|$ so we have an $\mathcal{O}(1)$ modification of GR. At shorter distances, the non-linear terms in Equation 502 become important and start to dominate. This happens at the so-called 'Vainshtein radius', given by 223 .

$$
r_{V} \sim \max \left\{\left(\frac{d_{3} M}{d_{2}^{2}}\right)^{1 / 3},\left(\frac{d_{4} M^{2}}{d_{2}^{3}}\right)^{1 / 6}\right\} .
$$

Depending on which of the non-linear terms dominates, the profile of the galileon field changes to

$$
\delta \pi^{\text {nonlin }}(r) \sim \begin{cases}\left(\frac{M}{d_{3}}\right)^{1 / 2} \sqrt{r} & \text { if the term with } d_{3} \text { dominates } \\ \left(\frac{M}{d_{4}}\right)^{1 / 3} r & \text { if the term with } d_{4} \text { dominates }\end{cases}
$$

For a suitable choice of parameters one can have $\left|\tilde{h}_{\mu \nu}(r)\right| \gg\left|\delta \pi^{\text {nonlin }}(r)\right|$ on solar system scales, and might claim that the modification of gravity does indeed get screened. However, it is important to note that the Vainshtein mechanism itself has yet to be properly understood in a well defined and fully covariant theory. We discuss some aspects of this at the end of Section 5.5.4.

Nonetheless, our galileon analysis indicates that self-accelerating solutions that are ghost-free and exhibit some form of Vainshtein screening on solar system scales could exist. However, there are also other concerns. Firstly, we should consider the question of back-reaction. Our galileon description holds provided we can neglect the effect of the scalar field back onto the geometry. This turns out not to be problematic provided we take $\left|d_{i}\right| \lesssim M_{p l}^{2} / H^{2 i-4}[963$.

More serious concerns appear when we study fluctuations about the spherically symmetric solutions we have just described. These can cause problems at both the classical and the quantum levels. At the quantum level, one must identify the scale at which 
the quantum fluctuations become strongly coupled, and the radius at which one can no longer trust the classical background. As the background solution changes with scale, so does the strong coupling scale. This means that there exists a critical radius at which the quantum effects start to dominate and one can no longer trust the classical solution. Aspects of strong coupling in DGP gravity are discussed in Section 5.5.4. Here we note that for a general galileon model the critical radius at which the theory enters a quantum fog can sometimes be unacceptably larg $£^{33}$ At the classical level, we find that fluctuations at short distances can sometimes propagate extremely slowly, so much so that a huge amount of Cerenkov radiation would be emitted as the earth moves through the solar galileon field. Indeed, to simultaneously avoid problems with Cerenkov emission and a low scale of strong coupling in a ghost-free theory with self-accelerating solutions, one must introduce a tadpole. As we have already explained, this could be considered undesirable as a tadpole will renormalise the vacuum energy seen by the galileon.

Yet another problem concerns radial fluctuations at large distances. These can propagate at superluminal speeds, indicating problems for causality ${ }^{34}$. This is known to be a problem in DGP gravity [9, 609] and can only be avoided in the general case by eliminating all of the interaction terms. This is unacceptable since the interaction terms are crucial to the successful implementation of the Vainshtein mechanism.

In summary then, while it is possible to obtain self-acceleration in a general galileon model that avoids some of the problems facing DGP gravity, one cannot find a completely consistent scenario. However, the situation can be improved by the introduction of a second galileon [1018, as we will discuss in Section 4.4.5

\section{Conformal galileon}

The conformal galileon is constructed in much the same way as the pure galileon we have just described, except now we demand that the relevant vacuum Lagrangian, $\mathcal{L}_{\text {gal }}^{\text {conformal }}(\pi, \partial \pi, \partial \partial \pi)$, is invariant under the conformal group:

$$
\begin{array}{rll}
\text { dilations } & : & \pi\left(x^{\mu}\right) \rightarrow \pi\left(b x^{\mu}\right)+\log b \\
\text { translations } & : & \pi\left(x^{\mu}\right) \rightarrow \pi\left(x^{\mu}+a^{\mu}\right) \\
\text { boosts } & : & \pi\left(x^{\mu}\right) \rightarrow \pi\left(\Lambda^{\mu}{ }_{\nu} x^{\nu}\right) \\
\text { special conformal } & : & \pi\left(x^{\mu}\right) \rightarrow \pi\left(x^{\mu}+c^{\mu}|x|^{2}-2(c \cdot x) x^{\mu}\right)-2 c \cdot x . \\
\text { transformations } & &
\end{array}
$$$$
\text { transformations }
$$

\footnotetext{
${ }^{33}$ Larger than the Schwarzschild radius of the Sun

${ }^{34}$ Note, however, that it has been suggested that causal paradoxes associated with superluminality do not always manifest themselves in theories with non-linear scalar interactions [73].
} 
It turns out that $\mathcal{L}_{\text {gal }}^{\text {conformal }}=\sum_{i} c_{i} \mathcal{L}_{i}^{\text {conformal }}$, where 963 , 389

$$
\begin{aligned}
\mathcal{L}_{1}^{\text {conformal }}= & e^{4 \pi} \\
\mathcal{L}_{2}^{\text {conformal }}= & -\frac{1}{2} e^{2 \pi}(\partial \pi)^{2} \\
\mathcal{L}_{3}^{\text {conformal }}= & -\frac{1}{2}\left[\partial^{2} \pi+\frac{1}{2}(\partial \pi)^{2}\right](\partial \pi)^{2} \\
\mathcal{L}_{4}^{\text {conformal }}= & e^{-2 \pi} \mathcal{L}_{4}-\frac{1}{20} e^{-2 \pi}(\partial \pi)^{2}\{4(\partial \pi) \cdot(\partial \partial \pi) \cdot(\partial \pi) \\
& \left.\quad-4(\partial \pi)^{2} \partial^{2} \pi+3\left[(\partial \pi)^{2}\right]^{2}\right\} \\
\mathcal{L}_{5}^{\text {conformal }}= & e^{-4 \pi} \mathcal{L}_{5}+3 e^{-4 \pi}(\partial \pi)^{2}\left\{\mathcal{L}_{4}+\frac{1}{56}\left[(\partial \pi)^{2}\right]^{3}\right. \\
& \left.+\frac{5}{7}(\partial \pi)^{2}\left[(\partial \pi)^{2} \partial^{2} \pi-(\partial \pi) \cdot(\partial \partial \pi) \cdot(\partial \pi)\right]\right\} .
\end{aligned}
$$

Aspects of the conformal galileon model are studied in [343, 964]. Violations of the null energy condition here can drive inflationary expansion without introducing instabilities. There are, however, some issues with superluminality.

A supersymmetric version of the conformal galileon has been obtained in [687] as a consistent completion of the supersymmetric ghost condensate.

\subsubsection{Covariant galileon}

The galileon action 481 describes fields propagating on a Minkowski background, and does not represent a fully covariant theory. Although galileon theory was originally motivated by co-dimension one braneworld models, it is interesting to consider the four dimensional covariant completion of the theory in its own right. This has been worked out in [400, 396], and is given by

$$
S\left[g_{\mu \nu}, \pi\right]=\int d^{4} x \sqrt{-g}\left[\frac{1}{16 \pi G} R+\mathcal{L}_{\text {gal }}^{\text {cov }}\right]+S_{\text {matter }}\left[\hat{g}_{\mu \nu}, \psi_{i}\right] .
$$

Naturally we recognise the first term in brackets as the standard Einstein-Hilbert action, $\frac{1}{16 \pi G} \int d^{4} x \sqrt{-g} R$. The last term corresponds to the matter action. Note that the matter fields are minimally coupled to the metric $\hat{g}_{\mu \nu}=f(\pi) g_{\mu \nu}$, where the conformal factor depends on $\pi$. An obvious example would be $\hat{g}_{\mu \nu}=e^{2 \pi} g_{\mu \nu}$ although this is by no means a unique choice. Neglecting the tadpole, the covariant completion of $\mathcal{L}_{\text {gal }}=\sum_{i} c_{i} \mathcal{L}_{i}$ is

\footnotetext{
${ }^{35}$ Our sign conventions agree with [963], rather than 389 .
} 
given by $\mathcal{L}_{\text {gal }}^{\text {cov }}=\sum_{i} c_{i} \mathcal{L}_{i}^{\text {cov }}$, where

$$
\begin{aligned}
& \mathcal{L}_{2}^{c o v}=-\frac{1}{2}(\nabla \pi)^{2} \\
& \mathcal{L}_{3}^{c o v}=-\frac{1}{2} \square \pi(\nabla \pi)^{2} \\
& \mathcal{L}_{4}^{c o v}=-\frac{1}{2}\left[(\square \pi)^{2}-(\nabla \nabla \pi)^{2}-\frac{1}{4} R(\nabla \pi)^{2}\right](\nabla \pi)^{2} \\
& \mathcal{L}_{5}^{c o v}=-\frac{1}{2}\left[(\square \pi)^{3}-3(\square \pi)(\nabla \nabla \pi)^{2}+2(\nabla \nabla \pi)^{3}\right. \\
&\left.\left.\quad-6 G_{\mu \nu}\left(\nabla^{\mu} \pi\right)\left(\nabla^{\nu} \nabla_{\alpha} \pi\right) \nabla^{\alpha} \pi\right)\right](\nabla \pi)^{2} .
\end{aligned}
$$

Note that for the 4 th and 5 th order terms one must introduce some non-minimal gravitational coupling to $\pi$. This is necessary since the naive covariant completion of $\mathcal{L}_{4}$ and $\mathcal{L}_{5}$, with minimal couplings, results in equations of motion containing higher derivatives. The non-minimal coupling helps to eliminate those higher derivatives. Now although the field equations in our covariant theory remain at most second order in derivatives, Galilean invariance is broken. We will not present the field equations here since they are long and complicated, especially for the higher-order terms. The interested reader can find them in [400, but should be mindful of the fact that the formulae for $\mathcal{L}_{4}^{\text {cov }}$ and $\mathcal{L}_{5}^{\text {cov }}$ presented here differ from those in 400 by an overall factor of 4 and 5 , respectively.

In a very recent paper, covariant galileon terms are seen to arise in Kaluza-Klein compactifications of Lovelock actions [1242]. This might have been expected since the underlying theory has at most second-order fields, and this is inherited by the dimensionally reduced theory. We discuss aspects of Lovelock gravity, and in particular, Gauss-Bonnet gravity, in Section 5.7 .

\subsubsection{DBI galileon}

The galileon Lagrangian $\mathcal{L}_{\text {gal }}$ can also be obtained from the non-relativistic limit of a probe brane in five dimensional Minkowski space [389. The probe brane action corresponds to a generalisation of the DBI action, as we will now explain. We take our bulk coordinates to be $\left(x^{\mu}, y\right)$, and place the probe brane at $y=\pi(x)$. The induced metric on the brane is then given by $g_{\mu \nu}=\eta_{\mu \nu}+\partial_{\mu} \pi \partial_{\nu} \pi$, from which we deduce that the DBI action is

$$
S_{\mathrm{DBI}}=-\lambda \int d^{4} x \sqrt{-g}=\int d^{4} x-\lambda \sqrt{1+(\partial \pi)^{2}} .
$$

For a slowly moving brane $(\partial \pi)^{2} \ll 1$, the leading order dynamical piece goes like $-\frac{\lambda}{2}(\partial \pi)^{2}$. To generalise this, we first consider objects that transform covariant on the brane, and then build a Lagrangian from them that gives rises to field equations that are at most second-order. The relevant covariant objects are the extrinsic curvature, $K_{\mu \nu}$

\footnotetext{
${ }^{36}$ We define $(\nabla \nabla \pi)^{n}=\left(\nabla_{\alpha_{1}} \nabla^{\alpha_{2}} \pi\right)\left(\nabla_{\alpha_{2}} \nabla^{\alpha_{3}} \pi\right) \ldots\left(\nabla_{a \alpha_{n}} \nabla^{\alpha_{1}} \pi\right), \square \pi=g^{\mu \nu} \nabla_{\mu} \nabla_{\nu} \pi$, and $(\nabla \pi)^{2}=$ $g^{\mu \nu} \nabla_{\mu} \pi \nabla_{\nu} \pi$.
} 
the induced curvature, $R_{\mu \nu \alpha \beta}$, and the covariant derivatives of these quantities. The generalised DBI action required to guarantee second-order field equations is

$$
S_{\text {gen-DBI }}=\sum_{i} c_{i} S_{i}
$$

where 37

$$
\begin{aligned}
S_{2} & =-\int d^{4} x \sqrt{-g} \\
& \rightarrow-\int d^{4} x \sqrt{1+(\partial \pi)^{2}} \\
S_{3} & =\int d^{4} x \sqrt{-g} K \\
& \rightarrow-\int d^{4} x \gamma\left[\partial^{2} \pi-\gamma^{2}(\partial \pi) \cdot(\partial \partial \pi) \cdot(\partial \pi)\right]
\end{aligned}
$$

and

$$
\begin{aligned}
S_{4}= & -\int d^{4} x \sqrt{-g} R \\
\rightarrow & -\int d^{4} x \gamma\left[\left(\partial^{2} \pi\right)^{2}-(\partial \partial \pi)^{2}\right. \\
& \left.+2 \gamma^{2}\left((\partial \pi) \cdot(\partial \partial \pi)^{2} \cdot(\partial \pi)-\partial^{2} \pi(\partial \pi) \cdot(\partial \partial \pi) \cdot(\partial \pi)\right)\right] \\
S_{5}= & \frac{3}{2} \int d^{4} x \sqrt{-g}\left(J-2 G^{\mu \nu} K_{\mu \nu}\right) \\
\rightarrow & -\int d^{4} x \gamma^{2}\left[\left(\partial^{2} \pi\right)^{3}+2(\partial \partial \pi)^{3}-3\left(\partial^{2} \pi\right)(\partial \partial \pi)^{2}\right. \\
& +6 \gamma^{2}\left(\left(\partial^{2} \pi\right)(\partial \pi) \cdot(\partial \partial \pi)^{2} \cdot(\partial \pi)-(\partial \pi) \cdot(\partial \partial \pi)^{3} \cdot(\partial \pi)\right) \\
& \left.-3 \gamma^{2}\left(\left(\partial^{2} \pi\right)^{2}-(\partial \partial \pi)^{2}\right)(\partial \pi) \cdot(\partial \partial \pi) \cdot(\partial \pi)\right],
\end{aligned}
$$

with the Lorentz factor $\gamma=1 / \sqrt{1+(\partial \pi)^{2}}$. The expressions for $S_{3}$ and $S_{5}$ can be identified with the boundary terms in General Relativity and in Gauss-Bonnet gravity, respectively. Of course, the former is the Gibbons-Hawking term, and the latter is the Myers boundary term 931, discussed in more detail in Section 5.7.3. Now, for a slowly moving probe in Minkowski space, it can be shown that $S_{i} \approx \int d^{4} x \mathcal{L}_{i}$, which means that, neglecting the tadpole, $S_{\text {gen-DBI }} \approx \int d^{4} x \mathcal{L}_{\text {gal }} 389$. One can then recover the conformal galileon by considering a probe brane in AdS, and the covariant galileon by considering a general bulk geometry [389.

This procedure has recently been extended to probe branes that are curved, giving rise to a more general class of effective theories on curved space [545, 544] (see, also [221]). These represent the analogues of galileons and DBI theories living on $d S_{4}$ and $A d S_{4}$, retaining the same number of symmetries as their flat space counterparts. There is a rich structure and in some cases the symmetries can even admit non-trivial potentials beyond the usual tadpoles.

${ }^{37}$ We write $\left(\partial^{\mu_{1}} \pi\right)\left(\partial_{\mu_{1}} \partial^{\mu_{2}} \pi\right) \ldots\left(\partial_{\mu_{n}} \partial^{\mu_{n+1}} \pi\right)\left(\partial_{\mu_{n+1}} \pi\right)=(\partial \pi) \cdot(\partial \partial \pi)^{n} \cdot(\partial \pi)$. 


\subsubsection{Galileon cosmology}

Galileon cosmology encompasses much more than the original model and its covariant completion. The cosmological behaviour of a number of models that are inspired by the galileon have also been investigated (see, for example, 1145, 711, 704, 375, 714, 404). These include the braiding model [714, 404, 1046, which is described by the following action

$$
S=\int d^{4} x \sqrt{-g}\left[\frac{1}{16 \pi G} R+K(\phi, X)+G(\phi, X) \square \phi\right],
$$

where $X=-(\nabla \phi)^{2}$. Note that for $K=\frac{c_{2}}{2} X$ and $G=\frac{c_{2}}{2} X$ we recover the covariant galileon action up to cubic order. This model still gives rise to second-order field equations and admits some rich phenomenology. It is claimed that the scalar equation of state can cross the phantom divide without introducing any instabilities, and results in a blue tilt for the spectrum tensor perturbations. Constraints on the model coming from large scale structure and non-Gaussianity have been obtained in 693, 903, respectively. NonGaussianity in DBI galileon inflation has been studied in [1059]. An even more general class of scalar tensor theories yielding second order field equations has recently been presented in [402, and is now known to be equivalent to Horndeski's general theory 623 in four dimensions 710 .

It has been argued that some of these generalised models are perhaps too general 222. The point is that there is no symmetry protecting the theory from large radiative corrections. This can spoil the functional form of the Lagrangian so much so that we require more input parameters than we can measure, and we lose all predictivity. In contrast, the pure galileon, conformal galileon and DBI galileon theories are safe against radiative corrections since they possess additional symmetries that control the form of the derivative interactions. For this reason, in the remainder of this section we will restrict attention to those models for which the Galilean invariance is only weakly broken, so that any radiative corrections that break the galileon symmetry are suppressed.

Let us begin with early universe cosmology and the covariant galileon. This can give rise to inflation even in the absence of a potential [222. The theory is radiatively safe because the terms that break Galilean symmetry are suppressed by powers of $\Lambda / M_{p l}$, where $\Lambda$ is the naive cut-of ${ }^{38}$ As with DBI inflation, fluctuations about the quasi de Sitter background will result in large non-Gaussianities at low sound speeds [903, 222. It has been argued that what sets this model apart is the fact that the non-Gaussianity is not constrained to obey $f_{N L} \sim 1 / c_{s}^{2}$, making it distinguishable from DBI inflation 222, although this claim has been disputed in 341. Indeed, it is interesting to note that the authors of 341 adopt the effective field theory approach to inflation, imposing Galilean symmetry on the small perturbations around the inflationary background. This permits additional interactions compared with 222 , but maintains stability against radiative corrections. They find that one can have large (observable) four point functions even when the three point function is small.

We now turn to the cosmology of the late universe. The late time cosmology of the covariant galileon has been studied for up to cubic [299, 911, quartic [520, 23], and quintic scalar interactions [373, 956, 369. In the latter model, we focus on the role of the galileon as a dark energy field - it deviates slightly from the original galileon scenario

\footnotetext{
${ }^{38}$ This corresponds to the scale of the galileon self-interactions.
} 
963. because the galileon coupling to matter disappears as $M_{p l} \rightarrow \infty$. In any event, the model is described by the following action

$$
S=\int d^{4} x \sqrt{-g}\left[\frac{1}{16 \pi G} R+\mathcal{L}_{\phi}+\mathcal{L}_{m}\left(g_{\mu \nu}, \psi\right)\right],
$$

where

$$
\begin{gathered}
\mathcal{L}_{\phi}=\frac{c_{2}}{2}(\nabla \phi)^{2}+\frac{c_{3}}{2 \Lambda^{3}}(\nabla \phi)^{2} \square \phi+\frac{c_{4}}{2 \Lambda^{6}}(\nabla \phi)^{2}\left[2(\square \phi)^{2}-2(\nabla \nabla \phi)^{2}-\frac{1}{2} R(\nabla \phi)^{2}\right] \\
+\frac{c_{5}}{2 \Lambda^{9}}(\nabla \phi)^{2}\left[(\square \phi)^{3}-3(\square \phi)(\nabla \nabla \phi)^{2}+2(\nabla \nabla \phi)^{3}-6 G_{\mu \nu}\left(\nabla^{\mu} \phi\right)\left(\nabla^{\nu} \nabla_{\alpha} \phi\right) \nabla^{\alpha} \phi\right] .
\end{gathered}
$$

As there is no potential, late time acceleration must be driven by the kinetic terms. There is a late time de Sitter solution characterised by $H=H_{d S}=$ constant and $\dot{\phi}=$ $\dot{\phi}_{d S}=$ constant. The existence of this fixes a relationship between $c_{2}, c_{3}, c_{4}$ and $c_{5}$ such that there are only two free parameters, given by

$$
\alpha=c_{4} x_{d S}^{4}, \quad \text { and } \quad \beta=c_{5} x_{d S}^{5},
$$

where $x_{d S}=\sqrt{8 \pi G}\left(\dot{\phi}_{d S} / H_{d S}\right)$. In 373] conditions are derived that guarantee the absence of ghosts and imaginary sounds speeds in both the tensor and the scalar sector. The viable region of parameter space, $(\alpha, \beta)$, where these conditions are met is presented. As regards the cosmological evolution, we see that there exists a tracker solution that approaches the late time de Sitter attractor. In Figure 6, we plot the evolution of the galileon equation of state for the tracker solution, and for generic initial conditions. Note that the tracker has a phantom equation of state. Indeed, even for generic initial conditions, the galileon field is drawn into a phantom phase by the tracker. It turns out that the tracker solution is disfavoured by a combined data analysis ( $\mathrm{SNe}$, BAO, $\mathrm{CMB}$. The generic case fares rather better, especially if we have non-zero curvature, $\Omega_{k}$, although it is still disfavoured with respect to $\Lambda$ CDM [956.

Matter density perturbations have been studied in detail within the context of this model in 369, where it is shown that the growth rate of matter perturbations is larger than in $\Lambda$ CDM. In the generic case, for suitable choices of $\alpha$ and $\beta$, we typically find that the growth index today is $\gamma_{0}<0.4$, with large variations at earlier times. This makes the model easily distinguishable from $\Lambda$ CDM. Another distinguishing feature is the effective gravitational potential changing with time, even during matter domination.

\subsubsection{Multi-galileons}

The extension of the galileon scenario to include multiple scalar fields [1017, 1018. 1019, 396] and even arbitrary p-forms 397. has recently been developed (see [481, 480 for earlier work). A general multi-galileon theory, in four dimensions, with $N$ real scalar degrees of freedom is given by the Lagrangian [1019, 1310.

$$
\mathcal{L}_{\text {N-gal }}=\sum_{m=1}^{5} \alpha^{i_{1} \ldots i_{m}} \delta_{\left[\nu_{2} \ldots \nu_{m}\right]}^{\mu_{2} \ldots \mu_{m}} \pi_{i_{1}} \partial_{\mu_{2}} \partial^{\nu_{2}} \pi_{i_{2}} \ldots \partial_{\mu_{m}} \partial^{\nu_{m}} \pi_{i_{m}}
$$

where $\left\{\alpha^{i_{1} \ldots i_{m}}\right\}$ are free parameters of the theory and $\delta_{\left[\nu_{1} \ldots \nu_{m}\right]}^{\mu_{1} \ldots \mu_{m}}=m ! \delta_{\left[\nu_{1}\right.}^{\mu_{1}} \ldots \delta_{\left.\nu_{m}\right]}^{\mu_{m}}$. As usual, summation over repeated Lorentz (Greek) and galileon indices (Latin) should be 


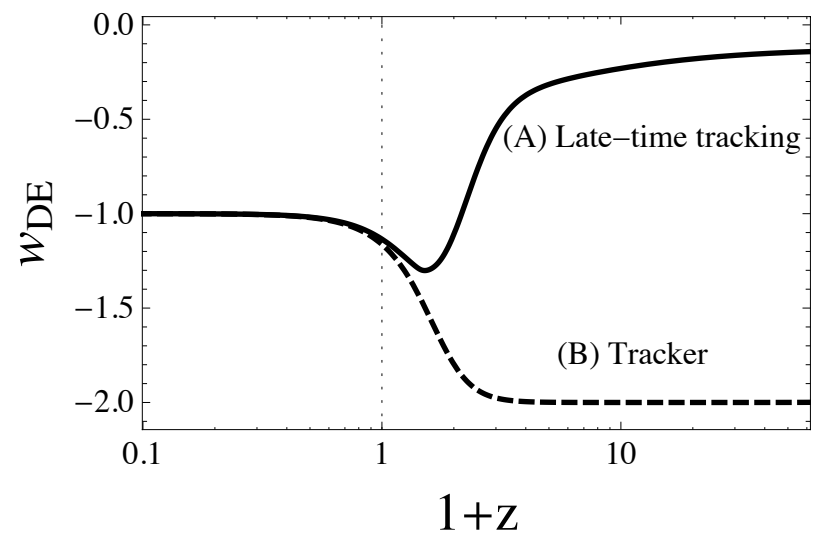

Figure 6: Taken from Figure 1 in 369. The equation of state of the galileon field, $w_{D E}$, versus redshift, $z$, for $\alpha=1.37$ and $\beta=0.44$. The evolution is given for generic initial conditions (A) and for the tracker (B)

understood to be implicit. Note further that we define the $m=1$ term of expression (531) to be $\alpha^{i_{1}} \pi_{i_{1}}$. The Lagrangian 531) is constructed so that it is invariant under

$$
\pi_{i} \rightarrow \pi_{i}+\left(b_{i}\right)_{\mu} x^{\mu}+c_{i}, \quad i=1, \ldots, N
$$

One might expect this to appear in the decoupling limit of some co-dimension $N$ braneworld scenarios, with $\pi_{1}, \ldots, \pi_{N}$ corresponding to the position of the brane in the $N$ transverse directions [1017]. Indeed, one can generalise the formalism discussed in Section 4.4.3 to probe a brane of co-dimension $N$ and recover the multi-galileon theory in the nonrelativistic limit 610 .

How many free parameters are there in this theory? We can always choose $\alpha^{i_{1} \ldots i_{m}}$ to be symmetric so the total number free parameters is given by

$$
\sum_{m=1}^{5}\left(\begin{array}{c}
N+m-1 \\
m
\end{array}\right)=\sum_{m=1}^{5} \frac{(N+m-1) !}{m !(N-1) !} .
$$

Even for $N=3$, this corresponds to 55 free parameters. To reduce the number of parameters one can consider imposing internal symmetries on the galileon fields [1019], although this will break the galileon symmetry (532). The phenomenology of spherically symmetric solutions with an internal $\mathrm{SO}(\mathrm{N})$ has been studied and found to suffer from problems with instabilities and superluminality, at least for standard non-derivative matter coupling [50]. One can also prove a generalised form a Goldstone's theorem when internal symmetries are present [1310]. 
Of course, the large number of free parameters is less of an issue in the simple case of $N=2$, dubbed bigalileon theory. The phenomenology of this theory was developed in detail in 1018. Let us summarise the main results. In direct analogy with the single galileon case, the bigalileon theory is formulated on sub-horizon scales as fields propagating on Minkowski space. This time we have our GR graviton, $\tilde{h}_{\mu \nu}$, and two scalar galileons, $\pi$ and $\xi$. Only one of the scalars, $\pi$, say, couples directly to the trace of the energy-momentum tensor. The other scalar, $\xi$, couples indirectly through its mixing with $\pi$, so it still has an important role to play. The governing action is given by 1017, 1018

$$
S\left[\tilde{h}_{\mu \nu}, \pi, \xi\right]=\int d^{4} x \mathcal{L}_{G R}+\mathcal{L}_{\pi, \xi}
$$

where $\mathcal{L}_{G R}$ is given by Eq. 475, and

$$
\mathcal{L}_{\pi, \xi}=\sum_{0 \leqslant m+n \leqslant 4}\left(\alpha_{m, n} \pi+\beta_{m, n} \xi\right) \mathcal{E}_{m, n}(\partial \partial \pi, \partial \partial \xi)+\pi T,
$$

with

$$
\begin{array}{r}
\mathcal{E}_{m, n}=(m+n) ! \delta_{\left[\nu_{1}\right.}^{\mu_{1}} \ldots \delta_{\nu_{m}}^{\mu_{m}} \delta_{\sigma_{1}}^{\rho_{1}} \ldots \delta_{\left.\sigma_{n}\right]}^{\rho_{n}}\left(\partial_{\mu_{1}} \partial^{\nu_{1}} \pi\right) \ldots \\
\ldots\left(\partial_{\mu_{m}} \partial^{\nu_{m}} \pi\right)\left(\partial_{\rho_{1}} \partial^{\sigma_{1}} \xi\right) \ldots\left(\partial_{\rho_{n}} \partial^{\sigma_{n}} \xi\right) .
\end{array}
$$

The physical metric is given by $g_{\mu \nu}=\eta_{\mu \nu}+h_{\mu \nu}$, where $h_{\mu \nu}=\tilde{h}_{\mu \nu}+2 \pi \eta_{\mu \nu}$. Given a source $T_{\mu \nu}, \tilde{h}_{\mu \nu}$ gives the usual perturbative GR solution, and so $2 \pi \eta_{\mu \nu}$ gives the modified gravity correction. The field equations for the scalars are

$$
T+\sum_{0 \leqslant m+n \leqslant 4} a_{m, n} \mathcal{E}_{m, n}=0, \quad \text { and } \sum_{0 \leqslant m+n \leqslant 4} b_{m, n} \mathcal{E}_{m, n}=0,
$$

where $39 a_{m, n}=(m+1)\left(\alpha_{m, n}+\beta_{m+1, n-1}\right)$ and $b_{m, n}=(n+1)\left(\beta_{m, n}+\alpha_{m-1, n+1}\right)$.

In contrast to the single galileon case, self-accelerating solutions can be consistent in some bigalileon theories. Indeed, one can choose parameters such that we simultaneously satisfy each of the following: (i) there is no tadpole, (ii) there is a self-accelerating vacuum, (iii) fluctuations about the self-accelerating vacuum do not contain a ghost, (iv) spherically symmetric excitations about the self-accelerating vacuum undergo Vainshtein screening in the solar system, (v) fluctuations about the spherically symmetric solutions are never superluminal, (vi) fluctuations about the spherically symmetric solutions never lead to trouble with excessive emission of Cerenkov radiation, (vii) there is not an unacceptably low momentum scale for strong coupling on the spherically symmetric solution, and (viii) there are no problems with back-reaction on the spherically symmetric solution (or the vacuum). This supports the case for considering bigalileon theories as a viable alternative to dark energy.

One can also develop models of self-tuning in bigalileon theory, where the vacuum energy does not affect the four dimensional curvature. These models get around Weinberg's no-go theorem by breaking Poincaré invariance. Unfortunately, in order for them to remain compatible with solar system tests one must limit the amount of vacuum energy to be $\lesssim \mathrm{meV}$.

\footnotetext{
${ }^{39}$ We define $\alpha_{-1, n}=\beta_{m,-1}=0$.
} 


\subsection{Other Theories}

Let us now consider some further theories that have yet to be discussed. These are ghost condensate theories, non-metric theories, and the dark energy from curvature corrections approach of Piazza.

\subsubsection{Ghost condensates}

Ghost condensate theories involve introducing into the gravitational sector an extra scalar field, $\phi$, with shift symmetry

$$
\phi \rightarrow \phi+\text { constant. }
$$

The only terms in the action that can obey this symmetry are derivative ones, and so the building block for this theory is taken to be

$$
X=\partial_{\mu} \phi \partial^{\mu} \phi
$$

In [590] it was shown that if the leading order term in the action has the wrong sign, so that $\phi$ is a ghost field, it is still possible to construct a theory that is stable to small fluctuations by including terms that push $X$ to a fixed value, so that

$$
\langle X\rangle=C .
$$

Theories of this type have a number of interesting properties. For a start, the non-zero vacuum expectation value of the ghost field signals a spontaneous breaking of Lorentz invariance. What is more, fluctuations in the ghost field about the vacuum expectation value appear linearly in the energy-momentum tensor, meaning that anti-gravity is possible. A further interesting point is that in the weak field limit large ghost condensate clumps move more slowly than small clumps, with potentially interesting phenomenological consequences.

It has been argued in [590] that ghost condensate fields act like the gravitational counterpart to the Higgs field of the standard model of particle physics. This is because gravitational fields propagating through the condensate acquire a massive mode, much like particles acquire mass while propagating through the Higgs field. Ghost condensates also introduce oscillatory correction to the gravitational potential, with a Jeans instability that grows with time. For mass parameters of the order $10^{-3} \mathrm{eV}$, these corrections occur on spatial and temporal scales greater than $H_{0}^{-1}$. If the massive modes are of order $10 \mathrm{MeV}$, however, then corrections can occur on length scales as small as $1000 \mathrm{~km}$, but, again, only on time scales greater than $H_{0}^{-1}$.

The action for ghost condensate theories can be written

$$
S=\int d^{4} x \sqrt{-g}\left(\frac{R}{16 \pi G}+M^{4} P(X)\right),
$$

where $M$ is a mass scale (confined to be $1 \mathrm{meV} \leq M \leq 10 \mathrm{MeV}$ ), and $P(X)$ is a function that must have a non-zero minimum at $X=C$ in order to be a ghost. For stability we then require

$$
\begin{aligned}
P^{\prime}(C) & \geq 0 \\
P^{\prime}(C)+2 C P^{\prime \prime}(C) & \geq 0 . \\
164 &
\end{aligned}
$$


Extremisation of the action (541), with respect to the metric, yields field equations of the form

$$
G_{\mu \nu}=8 \pi G T_{\phi, \mu \nu}
$$

where

$$
T_{\phi, \mu \nu}=M^{4}\left(P(X) g_{\mu \nu}+2 P^{\prime}(X) \partial_{\mu} \phi \partial_{\nu} \phi\right),
$$

and for simplicity we have not included a term in the action for normal matter fields.

To see why this theory is considered a modified theory of gravity we look at the perturbed field equations. Writing the metric as $g_{\mu \nu}=\eta_{\mu \nu}+h_{\mu \nu}$, and the ghost field as $\phi=\sqrt{C} t+\pi$, the Lagrangian density of the theory becomes

$$
\mathcal{L}=\sqrt{-\gamma}\left[F_{0}(X)+F_{1}(X) K^{2}+F_{2}(X) K^{i j} K_{i j}+\cdots\right],
$$

where we have chosen a unitary gauge, and where $\pi$ has been set to zero. The $F_{n}$ here are functions of $X$ that are derived from $P(X)$, and $K$ is the extrinsic curvature of the 3 dimensional hyper-surfaces of constant $\phi$. Diffeomorphism invariance in Eq. (546) can be seen to have been explicitly broken.

Let us now consider cosmology. For a homogeneous and isotropic Universe, the equation of motion for $\phi$ is

$$
\frac{d}{d t}\left(a^{3} \dot{\phi} P^{\prime}(X)\right)=0
$$

If we assume that $X \rightarrow 0$ and $P^{\prime}(C) \rightarrow 0$, as $t \rightarrow \infty$, then Eq. (547) tells us that $\phi \rightarrow \pm \sqrt{C} t$. The Friedmann equation for this theory is

$$
H^{2}=\frac{m^{2}}{3}\left(2 X P^{\prime}(X)-P(X)\right),
$$

where the new mass parameter is $m \equiv \sqrt{8 \pi G} M^{2}=M^{2} / M_{P l}$. The Raychaudhuri equation is

$$
\frac{\ddot{a}}{a}=-\frac{m^{2}}{3}\left(X P^{\prime}(X)+P(X)\right) .
$$

Now, it can be shown that the effect of the ghost field on the expansion of the Universe is such it can mimic radiation domination, matter domination and vacuum domination. Indeed, the simple choice of $P(X)=\frac{1}{2}(X-C)^{2}$ leads to $a(t) \propto(C m t)^{1 / 2}$ at early times, and $a(t) \propto(C m t)^{2 / 3}$ at late times. Adding a constant, such that $P(X)=\frac{1}{2}(X-C)^{2}+$ $\Lambda / m^{2}$, leads to a period of vacuum domination.

A general class of solutions, with matter sources included, has been studied in 755. Some of these solutions combine dark matter and dark energy-like behaviour, at the background level. The behaviour of scalar perturbations in the ghost condensate theory was worked out in detail in 922. Modified Newtonian potentials were discovered with

$$
\begin{aligned}
& \Phi=\Phi_{G R}+\Phi_{\text {mod }} \\
& \Psi=\Psi_{G R}+\Psi_{m o d}, \\
& 165
\end{aligned}
$$


where $\Phi_{G R}$ and $\Psi_{G R}$ take their standard form from General Relativity, while $\Phi_{m o d}$ and $\Psi_{\text {mod }}$ are corrections due to the ghost condensate that occur in the limit where the wavelength of the fluctuation is larger than the symmetry breaking scale. If we consider the case of de Sitter space, where $\Phi_{\text {mod }}=\Psi_{m o d}$, then the evolution equations are

$$
\partial_{t}^{2} \Phi_{m o d}+3 H_{0} \partial_{t} \Phi_{m o d}+\left(\frac{\alpha}{M^{2}} \frac{k^{4}}{a^{4}}-\frac{\alpha M^{2}}{2 M_{P l}^{2}} \frac{k^{2}}{a^{2}}+2 H_{0}^{2}\right) \Phi_{m o d}=\frac{\alpha M^{2}}{2 M_{P l}^{2}} \frac{k^{2}}{a^{2}} \Phi_{G R}
$$

where $\alpha$ is a combination of dimensionless coefficients of $\mathcal{O}(1)$ from the action. These equations shows that the Newtonian part of the potential seeds the modified part.

\subsubsection{Non-metric gravity}

We will now describe the non-metric gravity theory that deforms GR while keeping only two dynamical degrees of freedom [136, 137, 138, 747, 748. In this theory the fundamental gravitational object is no longer the metric but a triple of 2 -forms $\underline{\underline{B}}^{i}=$ $B_{\mu \nu}^{i} d x^{\mu} \wedge d x^{\nu}$, where lower-case Latin indices denote internal $S U(2)$ indices and take values from 1 to 3 . The space-time metric is an emergent variable and is given in terms of $B_{\mu \nu}^{i}$ as

$$
\sqrt{-g} g_{\mu \nu} \propto \tilde{\epsilon}^{\alpha \beta \gamma \delta} B_{\mu \alpha}^{i} B_{\nu \beta}^{j} B_{\gamma \delta}^{k} \epsilon_{i j k}
$$

where $\tilde{\epsilon}^{\alpha \beta \gamma \delta}$ is the completely antisymmetric tensor density having components \pm 1 in any coordinate system. The proportionality symbol is used above, rather than equality, because the metric is defined only up to conformal rescalings. The reason for this is that $B_{\mu \nu}^{i}$ is self-dual, i.e. $\frac{1}{2} \epsilon_{\mu \nu}^{\rho \sigma} B_{\rho \sigma}^{i}=i B_{\mu \nu}^{i}$ is a conformally invariant relation.

The class of theories we will now describe contains only two propagating degrees of freedom [139, 513, just like GR. Spherically symmetric solutions, as well as black holes, have been studied [751, 752, 648], and extensions of these ideas to bimetric theories have also been considered [1175]. Let us now describe the kinematical setup of the theory before proceeding to discuss its dynamics.

\section{Kinematics}

Consider the set of 1 -forms $\left\{\underline{e}^{0}, \underline{e}^{I}\right\}$, the tetrad, where the capital Latin indices denote internal $S O(3)$ indices (note that this is a different space than $S U(2)$ considered above) such that

$$
d s^{2}=g_{\mu \nu} d x^{\mu} d x^{\nu}=-\underline{e}^{0} \underline{e}^{0}+\delta_{I J} \underline{e}^{I} \underline{e}^{J} .
$$

From the tetrad we can also define the self-dual 2-forms $\underline{\underline{\Sigma}}_{(+)}^{I}$, and similarly the anti self-dual 2-forms $\left.\underline{\underline{\Sigma}}_{(-)}^{I}\right)$, by

$$
\underline{\underline{\Sigma}}_{ \pm}^{I}=i \underline{e}^{0} \wedge \underline{e}^{I} \mp \frac{1}{2} \epsilon^{I}{ }_{J K} \underline{e}^{J} \wedge \underline{e}^{K}
$$

Any other self-dual 2-form can then be decomposed in terms of $\underline{\underline{\Sigma}}_{(+)}^{I}$. In particular, we may write

$$
\underline{\underline{B}}^{i}=B_{I}^{i} \underline{\underline{\Sigma}}_{(+)}^{I} .
$$


From $\underline{\underline{B}}^{i}$ we can then define the connection one-forms $\underline{A}^{i}$ as ${ }^{40}$

$$
d \underline{\underline{B}}^{i}+\epsilon^{i j k} \underline{A}^{j} \wedge \underline{\underline{B}}^{k}=0
$$

The above equation can be solved to get

$$
A_{\mu}^{i}=\frac{1}{2 \operatorname{det} B} B^{i \alpha \beta} \delta_{j k} B_{\mu \alpha}^{j} \nabla^{\nu} B_{\nu \beta}^{k},
$$

where $\operatorname{det} B=-\frac{1}{24} \epsilon^{i j k} B_{\mu}^{i \nu} B_{\nu}^{j \rho} B_{\rho}^{k \mu}$. Now, since $\underline{\underline{B}}^{i}$ is conformally invariant, we know that $\underline{A}^{i}$ is too. We can now proceed and define the curvature two-forms $\underline{\underline{F}}^{i}$ of $\underline{A}^{i}$ as

$$
\underline{\underline{F}}^{i}=d \underline{A}^{i}+\frac{1}{2} \epsilon_{j k}^{i} \underline{A}^{j} \wedge \underline{A}^{k}
$$

\section{Dynamics}

The action for this theory takes on the form of the well known BF-theory:

$$
S[B, A]=\frac{i}{8 \pi G} \int\left[\delta_{i j} \underline{\underline{B}}^{i} \wedge \underline{\underline{F}}^{j}[A]-\frac{1}{2} V\left(\underline{\underline{B}}^{i} \wedge \underline{\underline{B}}^{j}\right)\right]+S_{m},
$$

where $V(\mathbf{M})$ is a holomorphic function of a complex symmetric $3 \times 3$ matrix, $\mathbf{M}$, that is required to be homogeneous of degree one (i.e. $V(\lambda \mathbf{M})=\lambda V(\mathbf{M}))$ so that when it is applied to a four-form such as $\underline{B}^{i} \wedge \underline{\underline{B}}^{j}$ the result is also a four-form. We introduce the internal metric $h^{i j}=B_{I}^{i} B^{j}{ }_{J} \delta^{I J}$ and further decompose it into trace and traceless parts as $h^{i j}=\frac{1}{3} h\left(\delta^{i j}+H^{i j}\right)$, where $h=\delta_{i j} h^{i j}$ and $\delta_{i j} H^{i j}=0$. We can then write $V$ as $V\left(h^{i j}\right)=\frac{1}{3} h U\left(H^{i j}\right)$, and expand $U$ as

$$
U(\mathbf{H})=\Lambda_{0}-\frac{1}{8 \ell^{2}} \operatorname{tr} \mathbf{H}^{2}+\mathcal{O}\left(\mathbf{H}^{3}\right),
$$

where the constant $\Lambda_{0}$ plays the role of the cosmological constant, while the constant $\ell$ is a new scale that describes deviations from GR. The minus sign in the 2 nd term above is required to avoid instabilities. In particular, as $\ell \rightarrow 0$ the theory reduces to the Plebanski formulation of GR with a cosmological constant (see Section 2.3.3).

As discussed above, the metric here is defined only up to conformal transformations. In order to couple the theory to matter fields we have to fix this ambiguity, which can be achieved by the introduction of a new function $R\left(h^{i j}\right)$ that is also homogeneous of degree one. The conformal freedom is then fixed by requiring ${ }^{41} R(\mathbf{h})=1$. In a similar fashion to $V$, we can then decompose $R$ as $R=\frac{1}{3} h U_{m}(\mathbf{H})$, and expand $U_{m}$ as

$$
U_{m}=1-\frac{g}{2} \operatorname{tr} \mathbf{H}^{2}+\mathcal{O}\left(\mathbf{H}^{3}\right),
$$

\footnotetext{
${ }^{40}$ Strictly speaking this defines a three-form which is then dualised to a one-form.

${ }^{41}$ This method of fixing the conformal ambiguity can be shown to arise naturally by considering the motion of a test body 749 .
} 
where $g$ is a dimensionless constant that can be of any sign. This new parameter measures the departure from the Urbantke metric, given by an equality in Eq. (553). Rather than the two new parameters, $g$ and $\ell$, it is sometimes convenient to use the two dimensionless parameters $\beta$ and $\gamma$, defined by

$$
\beta=g-\frac{1}{3}, \quad \text { and } \quad \gamma=\frac{1}{\ell^{2} \Lambda_{0}}-\frac{4}{3} .
$$

Cosmological consideration then tell us that $0<g<1$, and hence $-\frac{1}{3}<\beta<\frac{2}{3}$ and $\gamma>0$ [753. General Relativity is recovered in the limit $\gamma \rightarrow \infty$.

Variation of Eq. (560) with respect to $\underline{A}$ gives Eq. (557), while variation with respect to $\underline{\underline{B}}$ gives

$$
\delta_{i j} B_{I}^{i} \underline{\underline{F}}^{j}=B_{I}^{i}\left[\frac{\partial U}{\partial H^{i j}}+\frac{1}{3} \Lambda \delta^{i j}-2 \pi G T\left(\frac{\partial U_{m}}{\partial H^{i j}}+\frac{1}{3} \Lambda_{m} \delta^{i j}\right)\right] B_{J \underline{\underline{\Sigma}}_{(+)}^{J}-8 \pi G T_{I J} \underline{\underline{\Sigma}}_{(-)}^{J}},
$$

where $\Lambda$ and $\Lambda_{m}$ are the Legendre transforms of $U$ and $U_{m}$ respectively, i.e.

$$
\Lambda=U-\frac{\partial U}{\partial H^{i j}} H^{i j} \quad \text { and } \quad \Lambda_{m}=U_{m}-\frac{\partial U_{m}}{\partial H^{i j}} H^{i j},
$$

and where $T^{I J}=\tilde{T}_{\nu}^{\mu} \Sigma_{\mu \lambda}^{I} \Sigma^{J \nu \lambda}$, where $\tilde{T}_{\nu}^{\mu}$ is the traceless part of the energy-momentum tensor, $T_{\mu \nu}$ [750].

\section{Cosmology}

The cosmology of this theory has been analysed by Krasnov and Shtanov at the level of perturbed FLRW solutions 753. Let us first consider the FLRW solutions of this theory. For homogeneous and isotropic spaces we have $B^{i I}=\delta^{i I}$. Hence, we can drop the distinction between $i$ and $I$ and let $\underline{B}^{i}=\underline{\Sigma}^{i}$. We also have $H^{i j}=0$, so $U=\Lambda_{0}$ and $U_{m}=1$, resulting in $R=\frac{1}{3} h$ and $V=\frac{1}{3} h \Lambda_{0}$. The fixing condition $R=1$ then gives $h=3$ and $V=\Lambda_{0}$. Under these conditions the field equations can be written

$$
\underline{\underline{F}}^{i}=\frac{1}{3} \Lambda_{0} \underline{\underline{\Sigma}}^{i}-2 \pi G\left(P-\frac{\rho}{3}\right) \underline{\underline{\Sigma}}^{i}-2 \pi G(\rho+P) \underline{\underline{\Sigma}}^{i} .
$$

For the homogeneous and isotropic space-time we also have $\underline{e}^{0}=a d \tau$ and $\underline{e}^{i}=a d x^{i}$. After some algebra this gives $\underline{A}^{j}=i \mathcal{H} d x^{j}$ and $\underline{F}^{i}=i \mathcal{H}^{\prime} d \tau \wedge \overline{d x} x^{j}-\frac{1}{2} \epsilon^{i j k} \mathcal{H}^{2} d x^{j} \wedge d x^{k}$. The field equations can then be written

$$
3 \mathcal{H}^{2}=8 \pi G a^{2}\left(\rho+\rho_{\Lambda}\right),
$$

where $P_{\Lambda}=-\rho_{\Lambda}$ with $\Lambda_{0}=8 \pi G \rho_{\Lambda}$, and

$$
-2 \mathcal{H}^{\prime}-\mathcal{H}^{2}=8 \pi G a^{2}\left(P+P_{\Lambda}\right),
$$

where $\rho$ and $P$ do not include the cosmological constant. Thus, for metric backgrounds the FLRW solutions of this theory are the same as those of General Relativity. The situation changes, however, when we consider linear fluctuations. In this case one gets departures from $\Lambda$ CDM that depend on $g$ and $\ell$. 
We now consider the perturbed space-time metric in the conformal Newtonian gauge. The perturbation for $B_{j}^{i}$ is then given (after some convenient gauge-fixing) in terms of a new scalar mode $\chi$ as

$$
B^{i}{ }_{j}=\delta^{i}{ }_{j}+\frac{1}{2 a^{2}} D_{j}^{i} \chi .
$$

The perturbed field equations are then given by 753 .

$$
\begin{aligned}
& -k^{2} \Phi=4 \pi G a^{2} \rho[\delta+3 \mathcal{H}(1+w) \theta]+\frac{1}{3 a^{2}} k^{2}\left[k^{2} \chi-3 \mathcal{H} \chi^{\prime}\right], \\
& \Phi^{\prime}+\mathcal{H} \Psi=4 \pi G a^{2}(\rho+P) \theta-\frac{1}{3 a^{2}} k^{2} \chi^{\prime} \\
& \Phi^{\prime \prime}+2 \mathcal{H} \Phi^{\prime}+\mathcal{H} \Psi^{\prime}+\left(2 \mathcal{H}^{\prime}+\mathcal{H}^{2}\right) \Psi+\frac{k^{2}}{3}(\Phi-\Psi)=4 \pi G a^{2} \delta P+\frac{k^{2}}{9 a^{2}} \chi, \\
& \Phi-\Psi=8 \pi G a^{2}(\rho+P) \Sigma+\frac{1}{a^{2}}\left(\chi^{\prime \prime}+\frac{k^{2}}{3} \chi\right),
\end{aligned}
$$

and one can show that the Bianchi identities are satisfied independently of the $\chi$ terms. In this sense the $\chi$ field is non-dynamical. The remaining equations determine $\chi$ in terms of $\Phi$ and $\Psi$ as

$$
\chi^{\prime \prime}-2 \mathcal{H} \chi^{\prime}-\left[\Delta+4 \mathcal{H}^{2}+\Lambda_{0} \gamma+8 \pi G \beta(\rho-3 P)\right] \chi+a^{2}(\Phi+\Psi)=0,
$$

where $\rho$ and $P$ does not include the cosmological constant. This equation can be solved to get $\chi$ in terms of $\Phi+\Psi$, hence the $\chi$ terms in the field equations can be thought of as non-local modifications of the Einstein equations.

Krasnov and Shtanov also find the vector and tensor mode equations 753 . Furthermore, they study the evolution of perturbations during inflation, a well as radiation, matter and $\Lambda$ dominated epochs, and estimate the effects of the modifications on the matter power spectrum.

\subsubsection{Dark energy from curvature corrections}

A proposal for IR modifications of gravity has been put forward by Piazza 1036, 1037. The starting point for this is the usual semi-classical gravity, where matter fields are quantised on a curved background manifold. The operators of the matter field theory are then modified in the IR in a way we will now describe. Schematically, in a cosmological setup, operators corresponding to Fourier modes of physical momentum $k$ are corrected by terms of order $H^{2} / k^{2}$, where $H$ is the Hubble parameter. These modifications lead to the apparent existence of Dark Energy, but without introducing a new scale in the problem.

To illustrate this idea consider the vacuum expectation value of the local energy density of a massless field 42

$$
\begin{aligned}
\left\langle T_{0}^{0}(t, \vec{x})\right\rangle_{\text {bare }} & =\int d^{3} k\left[k+\frac{f_{\text {quad }}(t)}{k}+\frac{f_{\log }(t)}{k^{3}}+\ldots\right] \\
& =\text { local terms }+ \text { non-local terms }
\end{aligned}
$$

\footnotetext{
${ }^{42}$ see e.g. 1298,516 for the explicit expression of a massive scalar on flat FLRW background.
} 
where spatial homogeneity has been assumed for simplicity. The local terms can be removed by local gravitational counter-terms, while the non-local pieces represent the genuine particle/energy content of the chosen "vacuum" state. The first term contributes to the cosmological constant, and in flat space-time can be removed by the usual procedure of normal-ordering (the $f$ 's vanish in flat space-time). In curved space-time, however, the presence of the time-dependent $f$ 's makes the normal-ordering procedure meaningless. The conjecture of [1036, 1037] is that there exists a theory that resembles semi-classical GR on small scales, but that has an IR-completion that prohibits the time dependent pieces in (575). If that is the case then one can still deal with the cosmological constant term by the usual procedure of normal-ordering, as in flat space-time.

To try to construct such a theory [1036, 1037, propose what they call the Ultra Strong Equivalence Principle: For each matter field or sector sufficiently decoupled from all other matter fields, there exists a state (the "vacuum") for which the expectation value of the (bare) energy-momentum tensor is the same as in flat space, regardless of the configuration of the gravitational field.

What this principle aims to achieve is to remove the time-dependent terms in Eq. (575) by appropriate modifications of semi-classical gravity that manifest themselves when the Fourier modes have wavelengths comparable to the inverse extrinsic curvature (i.e. the inverse Hubble radius $H^{-1}$ ). At the present, a complete theory that implements this idea is lacking, but a toy-model with massive scalar fields has been considered in 1036, 1037. Letting $\vec{n}$ be the comoving momentum that labels operators in Fourier space (related to physical momentum as $\vec{n} / a)$, the modification to $O\left(H^{2} a^{2} / n^{2}\right)$ is given by the modified commutation relation

$$
\left[A_{\vec{n}}^{(1)}, A_{\vec{n}^{\prime}}^{(1) \dagger}\right]=\delta^{(3)}\left(\vec{n}-\vec{n}^{\prime}\right)\left(1-\frac{H^{2} a^{2}}{2 n^{2}}+\ldots\right),
$$

where $A_{\vec{n}}^{(1)}$ is the annihilation operator. This prescription is equivalent to using the standard commutation relation $\left[A_{\vec{n}}^{(0)}, A_{\vec{n}^{\prime}}^{(0) \dagger}\right]=\delta^{(3)}\left(\vec{n}-\vec{n}^{\prime}\right)$ for the standard operator $A_{\vec{n}}^{(0)}$, but with a modified comoving momentum given by $\vec{k}=\vec{n}\left(1-\frac{H^{2} a^{2}}{2 n^{2}}\right)$ that locally defines the infinitesimal translations. In a local neighbourhood (smaller than a Hubble patch) the above prescription can be shown to cancel the quadratically divergent piece $f_{\text {quad }}(t)$ in Eq. 575 . Note that the momentum $\vec{k}$ is not conserved, but $\vec{n}$ is.

To extend the above to the global picture one can use the translation operator $e^{-i \lambda P^{(1)}}$, where $\vec{P}^{(1)}=\int d^{3} n \vec{n} A_{\vec{n}}^{(1) \dagger} A_{\vec{n}}^{(1)}=\vec{P}^{(1)}=\int d^{3} n \vec{k} A_{\vec{n}}^{(0) \dagger} A_{\vec{n}}^{(0)}$ is the momentum operator constructed with the modified Fourier modes, and $\lambda$ is the comoving proper distance to a point far away from the origin. In GR the comoving distance $\lambda=d(t) / a(t)$ is a constant given by the ratio of the physical distance, $d(t)$, to the scale factor, $a(t)$. However, in the present theory one finds instead that

$$
\dot{\lambda}=\frac{1}{4} \lambda^{3} \frac{d}{d t}\left(a^{2} H^{2}\right) \quad+\text { higher orders. }
$$

Comoving distances obeying Eq. (577) are, in fact, already strongly disfavoured by observations 958 . One may, however, try to explore further whether the dynamical Hubble scale $H(t)$ itself could provide the scale required by cosmic acceleration by considering 
the more general expansion

$$
\begin{aligned}
\dot{\lambda}=A_{1} & \lambda H+A_{2}(\lambda H)^{2}+\ldots \\
& +B_{1} \lambda^{2} \frac{d}{d t}(a H)+B_{2} \lambda^{3} \frac{d}{d t}\left(a^{2} H^{2}\right)+\ldots,
\end{aligned}
$$

where $A_{i}$ and $B_{i}$ are a set of constants. The authors find that certain regions of the resulting parameter space can fit the data as well as $\Lambda$ CDM. 


\section{Higher Dimensional Theories of Gravity}

The first systematic studies of higher dimensional geometry date back to the likes of Riemann, Cayley and Grassmann in the mid nineteenth century. It lies at the heart of General Relativity, where space and time form part of a curved $3+1$ dimensional manifold, as described in Section 2. Of course, Riemannian geometry is not restricted to $3+1$ dimensions, so we have the tools to study gravitational theories in higher dimensions. Indeed, this is more than just a theoretical curiosity. Superstring theory, arguably our best candidate for a quantum theory of gravity, can only be formulated consistently in 10 dimensions.

The problem now is a phenomenological one: Gravity does not behave like a 10 dimensional force in our experiments and observations. Perhaps the simplest observation along these lines is the stability of earth's orbit. In $D$ dimensions of space-time, the Newtonian potential due to a point source will typically go like $1 / r^{D-3}$. For $D \neq 4$, it follows that we cannot have stable planetary orbits, and so it is clear that gravity should not appear 10 dimensional on solar system scales. We use the word appear, because there exist gravitational models where the extra dimensions are hidden from experiment, but which open up at shorter and/or larger distances.

In this section we will review various models of higher dimensional gravity that have been proposed. We will only discuss the case of extra spatial dimensions, although extra temporal dimensions have been studied (see eg [1141]). One might worry that extra temporal dimensions lead to problems with causality, as they permit closed time-like curves in the form of circles in the plane of the two temporal directions.

\subsection{Kaluza-Klein Theories of Gravity}

Kaluza-Klein (KK) theory grew out of an attempt to unify gravity and electrodynamics $[985,673,701,702$. The basic idea was to consider General Relativity on a $4+1$ dimensional manifold where one of the spatial dimensions was taken to be small and compact. One can perform a harmonic expansion of all fields along the extra dimension, and compute an effective $3+1$ dimensional theory by integrating out the heavy modes. This idea has been embraced by string theorists who compactify 10 dimensional string theories and 11 dimensional supergravity/M-theory on compact manifolds of 6 or 7 dimensions respectively, often switching on fluxes and wrapping branes on the compact space (see [552] for a review). Each different compactification gives a different effective 4-dimensional theory, so much so that we now talk about an entire landscape of effective theories 1194.

Assuming that the extra dimensions have been stabilised, the late-time dynamics of KK theories is most easily understood at the level of the $4 D$ effective theory. As we will show, this will generically correspond to a $4 D$ gravity theory with extra fields, examples of which are studied in detail in Section 3 . At early times, when the 3 dimensional space is comparable in size to the extra dimensions, the effective description clearly breaks down. This forms the basis of KK cosmology where one can ask the deeply profound question of why and how the 3 extended dimensions of space were able to grow large, while the extra dimensions remained microscopically small. It seems fair to say that a fully satisfactory answer to this question has yet to emerge. 
We will now discuss some aspects of KK theory, starting with an overview of dimensional reduction and effective theory before moving on to a discussion of KK cosmology at early times. For a more detailed review of KK theory see [76, 1005].

\subsubsection{Kaluza-Klein compactifications}

To understand the generic features of KK compactifications, it is sufficient to describe the dimensional reduction of General Relativity on a circle, $S^{1}$. We first define General Relativity in $D=d+1$ dimensions, via the generalised Einstein-Hilbert action

$$
S[\gamma]=\frac{1}{16 \pi G_{D}} \int d^{D} X \sqrt{-\gamma} \mathcal{R},
$$

where $G_{D}$ is Newton's constant in $D$ dimensions, $\gamma_{A B}$ is the $D$ dimensional metric with corresponding Ricci tensor, $\mathcal{R}_{A B}$, and Ricci scalar, $\mathcal{R}=\gamma^{A B} \mathcal{R}_{A B}$. Note that we are neglecting the matter Lagrangian for brevity. We are assuming that one of the spatial dimensions is compactified on a circle of radius $L / 2 \pi$. To this end we can define coordinates $X^{A}=\left(x^{\mu}, z\right)$, where the coordinate $z$ lies along the compact direction, such that $0 \leq z<L$.

We can expand the metric as a Fourier series of the form

$$
\gamma_{A B}(x, z)=\sum_{n} \gamma_{A B}^{(n)}(x) e^{i n z / L} .
$$

We find that this gives an infinite number of extra fields in $d$ dimensions. Modes with $n \neq 0$ correspond to massive fields with mass $|n| / L$, whereas the zero mode corresponds to a massless field. As we take $L$ to be smaller and smaller we see that the mass of the first massive field becomes very large. This means that if we compactify on a small enough circle we can truncate to massless modes in the 4-dimensional theory. Massive modes will only get excited by scattering processes whose energy lies at or above the compactification scale $1 / L$. This also applies to matter fields arising in particle physics. Indeed, particle physics imposes by far the strongest constraints on the size of the extra dimension. Standard Model processes have been well tested with great precision down to distances of the order $\sim(\mathrm{TeV})^{-1}$, with no evidence of extra dimensions yet emerging 941. Assuming that the extra dimensions are universal, that is the Standard Model fields can extend all the way into them, we infer that $L \lesssim 10^{-19} \mathrm{~m}$. The natural scale of the compact dimensions is usually taken be Planckian, $L \sim l_{p l}$.

Let us now focus on the zero modes, $\gamma_{A B}^{(0)}(x)$. We could define $\gamma_{\mu \nu}^{(0)}, \gamma_{\mu z}^{(0)}$ and $\gamma_{z z}^{(0)}$ to be the $d$-dimensional fields $g_{\mu \nu}(x), A_{\mu}(x)$ and $\phi(x)$. In effective field theory language, these will correspond to the metric, gauge field, and dilaton, respectively. In order that our results are more transparent we will actually define the components of the metric in the following way:

$$
\gamma_{\mu \nu}^{(0)}=e^{2 \alpha \phi} g_{\mu \nu}+e^{2 \beta \phi} A_{\mu} A_{\nu}, \quad \gamma_{\mu z}^{(0)}=e^{2 \beta \phi} A_{\mu}, \quad \gamma_{z z}^{(0)}=e^{2 \beta \phi},
$$

where $\alpha=1 / \sqrt{2(d-1)(d-2)}$, and $\beta=-(d-2) \alpha$. Since we have truncated to the massless fields, we can integrate out the $z$ part of the action given in Eq. 580. We find that the $d$-dimensional effective action is then given by

$$
S_{\mathrm{eff}}[g, A, \phi]=\frac{L}{16 \pi G_{D}} \int d^{d} x \sqrt{-g}\left({ }_{173}^{\left.R-\frac{1}{2}(\nabla \phi)^{2}-\frac{1}{4} e^{-2(d-1) \alpha \phi} F^{2}\right),}\right.
$$


where $F^{2}=F_{\mu \nu} F^{\mu \nu}$ and $F_{\mu \nu}=\nabla_{\mu} A_{\nu}-\nabla_{\nu} A_{\mu}$ is the electromagnetic field strength. The curvature associated with the $d$ dimensional metric, $g_{\mu \nu}$, is described by the Ricci tensor, $R_{\mu \nu}$, and Ricci scalar, $R=g^{\mu \nu} R_{\mu \nu}$. What we now have is an Einstein-Maxwell-Dilaton system in $d$ dimensions. Of course, Kaluza and Klein were particularly interested in the case of $d=4$. They were frustrated by the presence of the dilaton, $\phi$, in the resulting 4-dimensional effective theory. The point is that one cannot simply set the dilaton to zero and retain a non-trivial Maxwell field, since this would be in conflict with the field equations arising from Eq. (580),

$$
\begin{aligned}
& G_{\mu \nu}=\frac{1}{2}\left[\nabla_{\mu} \phi \nabla_{\nu} \phi-\frac{1}{2}(\nabla \phi)^{2} g_{\mu \nu}+e^{-2(d-1) \alpha \phi}\left(F_{\mu \alpha} F_{\nu}^{\alpha}-\frac{1}{4} F^{2} g_{\mu \nu}\right)\right], \\
& \nabla^{\mu}\left(e^{-2(d-1) \alpha \phi} F_{\mu \nu}\right)=0, \\
& \square \phi=-\frac{1}{2}(d-1) \alpha e^{-2(d-1) \alpha \phi} F^{2},
\end{aligned}
$$

where $G_{\mu \nu}=R_{\mu \nu}-\frac{1}{2} R g_{\mu \nu}$ is the Einstein tensor in $d$ dimensions. In the usual jargon, switching off the dilaton does not represent a consistent truncation of the higher dimensional theory [440]. We should also note that the physical size of the compact dimension is not necessarily given by $L$, but by $L e^{\beta \phi(x)}$. If $L$ is to represent an accurate measure of the compactification scale, we are therefore implicitly assuming that $\phi$ is stabilised close to zero. For this to happen we need to generate a potential for $\phi$ that admits a stable solution- this is known as the problem of moduli stabilisation. In more general compactifications, moduli potentials can be generated by Casimir effects of fields in the compact space [54, 55, 238, 335, 336, but the moduli remain unstable [336]. In fact, the problem of moduli stabilisation has only recently been solved by switching on fluxes to stabilise the volume of the compact space 662, 661.

There are, of course, many different compactifications that have been studied in the literature, a detailed analysis of which is clearly beyond the scope of this review (see [552]). However, aside from details such as the inclusion of fluxes and branes on the compact space, the general scheme of each compactification is the same as the one we have just described. Typically, a compactification of, say, 11 dimensional super-gravity down to four dimensions will give rise to a gravity theory with a plethora of extra fields. These extra fields include scalars, pseudo-scalars, vectors, pseudo-vectors, and arbitrary p-forms. Modifications of gravity due to extra fields are studied in detail in Section 3.

\subsubsection{Kaluza-Klein cosmology}

As we have just discussed, for a phenomenologically viable theory with compact (and stabilised) extra dimensions, the characteristic size, $L$, of the compact manifold should not exceed the scale probed by modern collider experiments, which is currently around $10^{-19} \mathrm{~m}$ [941]. It is amusing to compare this to the characteristic size of the 3 large spatial dimensions, which is at least a Hubble length $c / H_{0} \sim 10^{26} \mathrm{~m}$, or in other words at least 45 orders of magnitude greater. Of course, it was not always like that. In the very early universe, at times $t \lesssim L / c$, one might expect that all spatial dimensions were of the same scale, each playing an equally important role in the dynamical evolution. This begs the question: Why did the Universe evolve into a state where just 3 spatial dimensions grew to macroscopic scales? Put another way, how does one achieve a dynamical compactification 
mechanism in the early Universe such that 3 spatial dimensions expand exponentially to an extremely large size, in contrast to the remaining spatial dimensions? Were those extra dimensions somehow prevented from growing beyond a certain size, or did they grow initially and later contract towards their current state?

These questions have led many authors (see, e.g. [514, 33, 730, 1084, 1083, 1085, 1, 3, 2, 997, 555]) to consider the dynamics of anisotropic cosmologies in $D=d+1$ dimensions, where $d=n+\tilde{n}$. Indeed, consider the Bianchi-type metric

$$
d s^{2}=\gamma_{a b} d X^{a} d X^{b}=-d t^{2}+a^{2}(t) q_{i j}(x) d x^{i} d x^{j}+\tilde{a}^{2}(t) \tilde{q}_{m n}(\tilde{x}) d \tilde{x}^{m} d \tilde{x}^{n},
$$

where the coordinates $x^{i}$ run over the $n$ spatial dimensions and the coordinates $\tilde{x}^{m}$ run over the $\tilde{n}$ spatial dimensions. The $n$-dimensional metric, $q_{i j}(x)$, is taken to have constant curvature $\kappa$, whereas the $\tilde{n}$-dimensional metric, $\tilde{q}_{m n}(\tilde{x})$, is taken to have constant curvature $\tilde{\kappa}$. The growth of these two spaces is controlled by the relevant scale factors $a(t)$ and $\tilde{a}(t)$. Naturally, we will be interested in the case of $n=3$, but for the moment let us keep things general.

We now apply Einstein's equations in $D=d+1$ dimensions,

$$
\mathcal{G}_{a b} \equiv \mathcal{R}_{a b}-\frac{1}{2} \mathcal{R} \gamma_{a b}=8 \pi G_{D} T_{a b},
$$

where the energy-momentum tensor is given by an anisotropic fluid,

$$
T_{b}^{a}=\operatorname{diag}(-\rho, \overbrace{P, \ldots, P}^{n}, \overbrace{\tilde{P}, \ldots, \tilde{P}}^{n}) .
$$

As usual, $\rho(t)$ is the energy density, whereas $p(t)$ is the pressure along the $n$ dimensions and $\tilde{P}(t)$ is the pressure along the $\tilde{n}$ dimensions. Einstein's Equations 587 then yield the following $[514$

$$
\begin{gathered}
\frac{n}{2}(n-1)\left(H^{2}+\frac{\kappa}{a^{2}}\right)+\frac{\tilde{n}}{2}(\tilde{n}-1)\left(\tilde{H}^{2}+\frac{\tilde{\tilde{\kappa}}}{\tilde{a}^{2}}\right)+n \tilde{n} H \tilde{H}=8 \pi G_{D} \rho, \\
\frac{\ddot{a}}{a}+(n-1)\left(H^{2}+\frac{\kappa}{a^{2}}\right)+\tilde{n} H \tilde{H}=\frac{8 \pi G_{D}}{d-1}[\rho+(\tilde{n}-1) P-\tilde{n} \tilde{P}], \\
\ddot{\tilde{\tilde{a}}}+(\tilde{n}-1)\left(\tilde{H}^{2}+\frac{\tilde{\kappa}}{\tilde{a}^{2}}\right)+n H \tilde{H}=\frac{8 \pi G_{D}}{d-1}[\rho-n P+(n-1) \tilde{P}],
\end{gathered}
$$

where $H=\dot{a} / a$ and $\tilde{H}=\dot{\tilde{a}} / \tilde{a}$ are the Hubble parameters of the two expanding/contracting spaces. Of course, we also have energy conservation, which gives

$$
\dot{\rho}+n H(\rho+P)+\tilde{n} \tilde{H}(\rho+\tilde{P})=0 .
$$

Note that we do not necessarily have to assume that the cosmological dynamics is governed by $D$-dimensional General Relativity. We can also consider modifications of GR where additional fields are present. For example, in string gas cosmology [183, 1216], we consider the action

$$
S=\frac{1}{16 \pi G_{D}} \int d^{D} x \sqrt{-\gamma} e^{-2 \phi}\left(\mathcal{R}-4(\nabla \phi)^{2}-c\right)+S_{m}\left[\gamma, \Psi_{n}\right],
$$


where $S_{m}$ is the matter part of the action, containing the string gas, and the constant $c$ vanishes in the critical case 43 , but not otherwise. The resulting field equations can be written in the form $G_{a b}=8 \pi G_{D} T_{a b}$, where

$$
T_{a b}=\frac{1}{8 \pi G_{D}}\left[-\frac{c}{2} \gamma_{a b}+8 \nabla_{a} \phi \nabla_{b} \phi-6 \gamma_{a b}(\nabla \phi)^{2}-2\left(\nabla_{a} \nabla_{b}-\gamma_{a b} \square\right) \phi\right]+e^{2 \phi} T_{a b}^{(m)},
$$

and $T_{a b}^{(m)}=-\frac{2}{\sqrt{-\gamma}} \frac{\delta S_{m}}{\delta \gamma^{a b}}$. The scalar equation of motion just follows from energy conservation, $\nabla^{a} T_{a b}=0$.

Let us return to Equations (588)- 590 with a view toward dynamical compactification. Many of the earlier works [33, 730, 1084, 1083, 1085, 1, 3, focus on isotropic perfect fluids, for which $P=\tilde{P}=w \rho$. For simplicity and definiteness, let us follow the analysis of Abbott, Barr and Ellis [1. We consider an epoch in which we have radiation domination, $w=1 /(n+\tilde{n})$, so that the evolution equations read

$$
\begin{gathered}
\frac{n}{2}(n-1)\left(H^{2}+\frac{\kappa}{a^{2}}\right)+\frac{\tilde{n}}{2}(\tilde{n}-1)\left(\tilde{H}^{2}+\frac{\tilde{\kappa}}{\tilde{a}^{2}}\right)+n \tilde{n} H \tilde{H}=8 \pi G_{D} \rho, \\
\frac{\ddot{a}}{a}+(n-1)\left(H^{2}+\frac{\kappa}{a^{2}}\right)+\tilde{n} H \tilde{H}=\frac{8 \pi G_{D}}{d} \rho, \\
\frac{\ddot{\tilde{a}}}{\tilde{a}}+(\tilde{n}-1)\left(\tilde{H}^{2}+\frac{\tilde{\kappa}}{\tilde{a}^{2}}\right)+n H \tilde{H}=\frac{8 \pi G_{D}}{d} \rho .
\end{gathered}
$$

Now, if the $\tilde{n}$ dimensions are taken to be an $\tilde{n}$-sphere $(\tilde{\kappa}>0)$, it is clear from Equation 596 that they will reach a maximum size when $\tilde{H}=0$, and will subsequently start to recollapse. In contrast, we can take the $n$ dimensions to be flat or hyperbolic $(\kappa \leq 0)$, so that these dimensions will never turn around. In fact, one can show that as we start to approach the singularity of the collapsing sub-space $(\tilde{a}(t) \rightarrow 0)$, the $n$ dimensions enter a phase of accelerated expansion. To see this note that $\tilde{H}$ starts to become large and negative, and so it is clear from Equation (595) that we will enter a phase with $\ddot{a}>0$. The typical evolution of the two scale factors is shown in Figure 7 . Note that we can even allow for a sufficiently small $\kappa>0$ and still retain this qualitative behaviour. The upper bound on $\kappa$ follows from demanding that the turnaround in $a(t)$ occurs after the turnaround in $\tilde{a}(t)$. The bound is not strong enough to be interesting: It merely implies that today's Universe is larger than the horizon [1].

Of course, it is clear that the classical equations will start to break down in the neighbourhood of the singular point. The physical radius of the $\tilde{n}$-sphere is $\tilde{a} / \sqrt{\tilde{\kappa}}$, so we certainly would not expect to trust our field equations when $\tilde{a}(t) \lesssim \sqrt{\tilde{\kappa}} L_{D}$, where $L_{D} \propto G_{D}^{1 /(D-2)}$ is the fundamental Planck length in $D$ dimensions. In [1], it is assumed that quantum gravity effects will ultimately stabilise the size of the internal space, ending the inflationary phase at some time $t_{c}$, where $\tilde{a}\left(t_{c}\right)=\sqrt{\tilde{\kappa}} L$, for some compactification scale $L \gtrsim L_{D}$. Albeit without much justification, let us accept this assumption for the moment, and consider the physically interesting case of $n=3$. One might hope that the inflationary phase is sufficiently long to offer a solution to the flatness, entropy and horizon problems of the standard cosmology. Consider the entropy problem in particular. Entropy is indeed released from the extra dimensions into the usual 3 dimensions of

43 For the bosonic string the critical dimension is $D=26$, whereas for the superstring the critical dimension is $D=10$. 


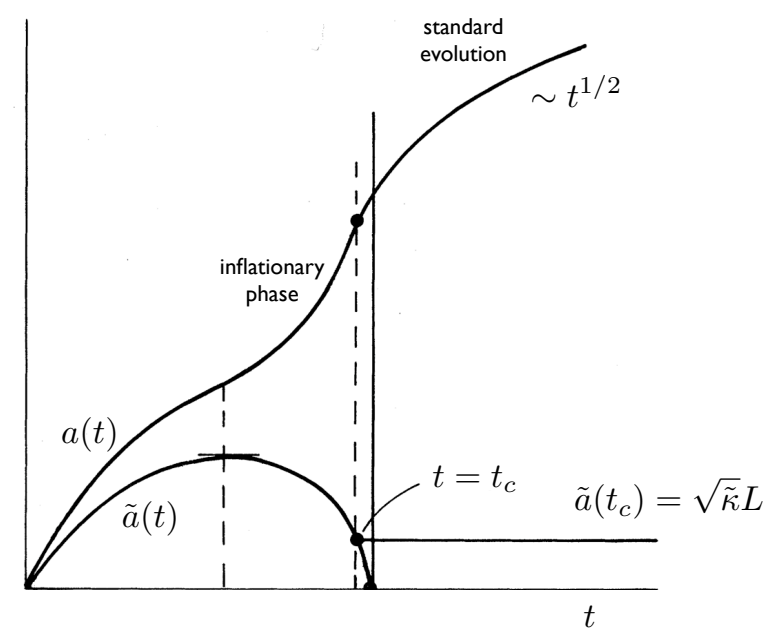

Figure 7: Adapted from Figure 1 in 1. The typical evolution of the scale factors in the two different sub-spaces. The scale factor $a(t)$ along the $n$ dimensions grows large, entering an inflationary phase as the remaining $\tilde{n}$ dimensions begin to recollapse. The scale factor $\tilde{a}(t)$ along the $\tilde{n}$ dimension is assumed to be stabilised by quantum gravity effects at some time $t_{c}$.

space [33, 1, but only as much as $\log S \sim|\mathcal{O}(1)| \log \left(L_{D} / L\right)$ [30. Since we demand that $L \gtrsim L_{D}$, this is clearly way short of the total required to solve the entropy problem, $\log S \sim 88$. In short, KK inflation does not last long enough to provide an alternative to scalar field driven inflation.

To get the required expansion of 3-dimensional space, we must therefore include some additional scalar fields. Adapting 615] slightly, we can mimic a period of slow-roll inflation by plugging a cosmological constant, $\Lambda$, into the Field Equations (588)-(590). Setting $P=\tilde{P}=-\rho$, where $\rho=\Lambda / 8 \pi G_{D}$, we find

$$
\begin{gathered}
\frac{n}{2}(n-1)\left(H^{2}+\frac{\kappa}{a^{2}}\right)+\frac{\tilde{n}}{2}(\tilde{n}-1)\left(\tilde{H}^{2}+\frac{\tilde{\kappa}}{\tilde{a}^{2}}\right)+n \tilde{n} H \tilde{H}=\Lambda, \\
\frac{\ddot{a}}{a}+(n-1)\left(H^{2}+\frac{\kappa}{a^{2}}\right)+\tilde{n} H \tilde{H}=\frac{8 \Lambda}{d-1}, \\
\frac{\ddot{\tilde{a}}}{\tilde{a}}+(\tilde{n}-1)\left(\tilde{H}^{2}+\frac{\tilde{\kappa}}{\tilde{a}^{2}}\right)+n H \tilde{H}=\frac{2 \Lambda}{d-1} .
\end{gathered}
$$

Again, by taking the $n$ dimensions to be flat $(\kappa=0)$, and the $\tilde{n}$ dimensions to be positively curved $(\tilde{\kappa}>0)$, we find a solution for which the flat directions grow exponentially, and the spherical dimensions remain fixed with $H=H_{*}$ at $\tilde{a}=\tilde{a}_{*}$, where $H_{*}^{2}=\frac{\tilde{n}-1}{n} \frac{\tilde{\kappa}}{\tilde{a}_{*}^{2}}=\frac{2 \Lambda}{n(d-1)}$. The radius of the extra dimensions lies at the maximum of its potential, so this solution is unstable. Indeed, fluctuations reveal that the spherical dimensions collapse to zero size over a time scale $\Delta t \sim(1+\sqrt{1+8 / n}) / 4 H_{*}$, after which we cannot count on exponential growth in the flat directions.

We now consider the phenomenologically interesting case of $n=3$. To get the required 
number of $\sim 65$ e-folds of inflation along the 3 flat directions we need $H_{*} \Delta t \sim 65$. For $n=3, H_{*} \Delta t \sim 0.729$, so once again inflation is cut short far too early. We could imagine getting around this problem if we could alter the potential for the radius $\tilde{a}$, such that it develops a minimum as well as a maximum, by switching on fluxes 662, 661. Generically, it is still very difficult to get enough exponential growth along the familiar 3 dimensions without causing the extra dimensions to grow alongside them [615]. For further details on the latest attempts to embed inflation in higher dimensional theories, we refer the reader to $807,1048,812,666,218,873,166$. Bounds on the variation of fundamental constants for dynamical compactifications have been studied in [571, 572, while PPN parameters for KK models in the solar system were computed in [461. Note that Kaluza-Klein cosmologies have also recently been applied to the dark energy problem [119, 120.

We end our discussion of Kaluza-Klein cosmology by asking the question: Why are there 3 large spatial dimensions? We have already alluded to an anthropic explanation demanding the existence of stable planetary orbits 44 . To this we could add the existence of stable atoms and chemistry, both key to the development of intelligent life, and both requiring no more than 3 (large) spatial dimensions.

Modern attempts at a dynamical understanding of the dimensionality of space include String Gas Cosmology [183, 1216] (for reviews see, e.g., [180, 116, 181]). Here the spatial dimensions are taken to be compact and precisely 3 dimensions are allowed to grow large due to the annihilation of strings wrapping around those dimensions. The point is that strings winding around compact dimensions oppose their expansion since the energy of the string winding modes increases with radius. To allow the compact dimensions to grow large the winding modes must therefore collide and annihilate with the anti-winding modes. Generically, we would only expect collisions of $1+1$ dimensional strings in at most $3+1$ dimensions. Thus, the dimensionality of the string controls the dimensionality of space by allowing at most 3 spatial dimensions to grow to macroscopic scales. Note that this result is not spoilt by the inclusion of branes wrapping compact directions, as these happen to fall out of equilibrium before the strings [21.

Whilst this idea has some appeal at first glance, it has not stood up to intense scrutiny. More detailed quantitative analyses suggest that the desired outcome is not at all generic, and requires highly fine tuned initial conditions 456, 457, 458. Whilst one can engineer an anisotropic set-up allowing 3 dimensions to grow large as desired, typically the internal dimensions also grow to large sizes, just at a slower rate [456, 457]. In fact, it turns out that either all dimensions grow large since the string gas eventually annihilates completely, or all dimensions stay small since the string gas gets frozen out 458. There are also problems at the level of cosmology. For example, when properly calculated, the scalar perturbations have a blue power spectrum with $n=5$, which is strongly ruled out by observations 670]. It has been argued that a near scale invariant spectrum can be obtained if the dilaton gets frozen during the strong coupled Hagedorn phase in the very early Universe [182. However, such claims still rely on a semi-classical treatment of cosmological perturbations that cannot be trusted during the Hagedorn phase, as the strings are strongly interacting.

In the context of 10 dimensional string theory, other attempts to explain the dimen-

\footnotetext{
${ }^{44} \mathrm{On}$ the subject of planetary orbits, it is amusing to note that Kepler himself reasoned that the 3-fold nature of the Holy Trinity was responsible for the perceived dimensionality of space. Ptolemy is reputed to have offered some alternative ideas in his work On Dimensionality, but they have since been lost.
} 
sionality of our Universe consider that for integer values of $n$, the inequality $2 n<10 \Longrightarrow$ $n \leq 4$ [44, 683. This is interesting because it means that the world volume of $3+1$ dimensional branes (known as 3-branes) are less likely to intersect than those of larger branes. In particular, Karch and Randall 683, have shown that an FLRW universe initially filled with equal numbers of branes and anti-branes will ultimately come to be dominated by 3-branes and 7-branes. This analysis accounts for the fact that larger branes dilute more slowly, as well as the likelihood of intersections and annihilations (hence the importance of 7-branes). In a braneworld scenario, this could explain why we might be more likely to find ourselves living on a 3-brane, as opposed to a larger brane. The consequences of living on a 3-brane are discussed in detail in the Section 5.2

Finally, we note that for toroidal compactifications, 3 large spatial dimensions can be linked to the stability of the small extra dimensions, at least in the presence of solitonic strings/branes that correspond to point masses in the large dimensions [462].

\subsection{The Braneworld Paradigm}

The braneworld paradigm [15, 1074, 62, 52, 63, represents a radical alternative to the standard Kaluza-Klein scenario, discussed in the previous section. In the KK scenario, the extra dimensions must be small and compact, the size of the internal space constrained by collider experiments to be below the inverse $\mathrm{TeV}$ scale. In the braneworld scenario the extra dimensions can be much larger, perhaps even infinite in extent. This is made possible by relaxing the assumption of universal extra dimensions.

In the braneworld picture the Standard Model fields are not universal, rather they are confined to lie on a $3+1$ dimensional hyper-surface, known as the brane, embedded in some higher dimensional space-time, known as the bulk. Tests of Standard Model processes can only constrain how far the brane may extend into the bulk, or, in other words, the brane thickness. They do not constrain the size of the bulk itself. Such constraints can only come from gravitational experiments, as gravity is the only force that extends into the bulk space-time. As is well known, on small scales gravity is much weaker than the other three fundamental forces, making it difficult to test at short distances. In fact, the gravitational interaction has only been probed down to $\sim 0.1 \mathrm{~mm}$, with torsion-balance tests of the inverse square law [10]. It is too simplistic, however, to suggest that this translates into an upper bound on the radius of the bulk. Gravity is intimately related to geometry, and, as we shall see, one can warp the bulk geometry such that an infinitely large extra dimension is still allowed by experiment. For an excellent introduction to large extra dimensions see [1072].

Before delving into a detailed discussion of the various models, we note in passing that the braneworld paradigm is well motivated by string theory [622, 838, 52. As well as fundamental strings, string theory contains fundamental objects known as D-branes 1044. These are extended objects upon which open strings can end. The braneworld set-up therefore has a natural interpretation in terms of a stack of D-branes embedded in a higher dimensional target space (see Figure 8). Open strings, with their ends attached to the D-branes, can be identified with the Standard Model fields bound to the brane. Only closed strings can propagate through the bulk, and these are identified with the gravitational interactions. 


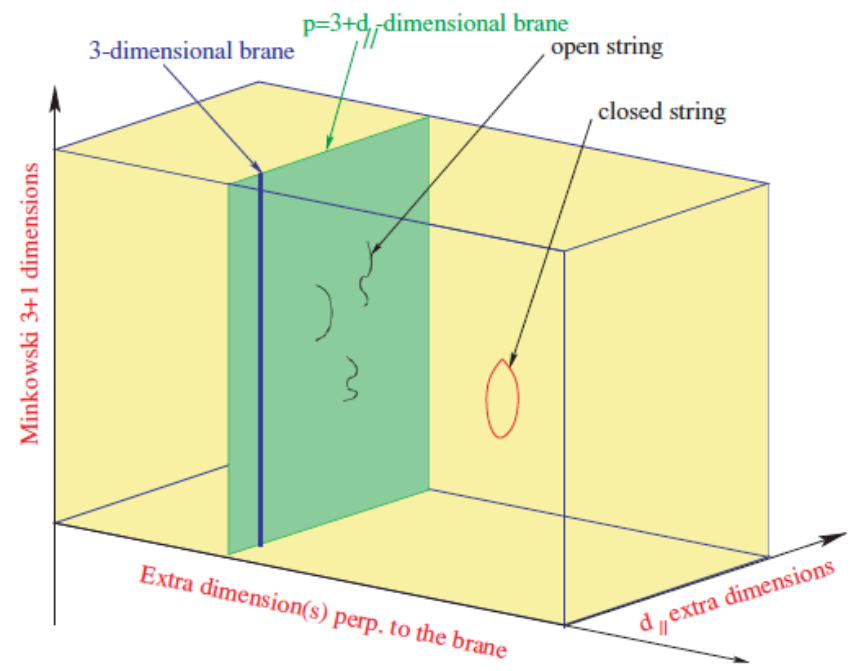

Figure 8: Taken from [51. Here the brane has 3 macroscopic dimensions, and $d_{\|}$compact dimensions. The open strings end on the D branes, whereas the closed strings propagate through the bulk.

\subsubsection{The ADD model}

The braneworld paradigm really began to gather momentum with the seminal work of Arkani-Hamed, Dimopoulos and Dvali 62, in which the large extra dimension is exploited in order to explain the vast hierarchy between the electro-weak scale, $M_{E W} \sim$ $\mathrm{TeV}$, and the Planck scale, $M_{p l} \sim 10^{16} \mathrm{TeV}$. In this scenario the hierarchy does not go away completely, rather it is reformulated as a hierarchy between the scale of the extra dimensions, $\mu \sim 1 / L$, and the electro-weak scale. The set-up is as follows: Standard Model fermions and gauge bosons are localised on a $3+1$ dimensional domain wall, in an (effective) $D=4+n$ dimensional space-time. We should clarify that $D$ counts the number of macroscopic dimensions in this scenario. Any microscopic dimensions, with characteristic size given by the fundamental Planck length, behave as in the standard KK scenario described previously.

Now, the width of the wall cannot exceed the inverse TeV scale, as explained above. The bulk space transverse to the wall is compact but much larger than the width of the wall $\left(L \gg \mathrm{TeV}^{-1}\right)$, so much so that we can treat the wall as an infinitely thin 3brane. In the simplest construction, the bulk action is then described by the generalised Einstein-Hilbert action

$$
S_{\text {bulk }}=\frac{M_{D}^{D-2}}{2} \int d^{D} X \sqrt{-\gamma} \mathcal{R},
$$

where $M_{D}$ is the fundamental Planck scale in $D$ dimensions, $\gamma_{A B}$ is the $D$ dimensional metric with corresponding Ricci tensor, $\mathcal{R}_{A B}$, and Ricci scalar, $\mathcal{R}=\gamma^{A B} \mathcal{R}_{A B}$. The Planck mass is related to the fundamental Newton's constant in $D$ dimensions by 
$8 \pi G_{D}=M_{D}^{2-D}$. At large distances, gravitational interactions along the brane are mediated by the graviton zero mode, which has a homogeneous profile over the extra dimensions. Truncating to the zero mode, we can compute the four-dimensional effective action describing long distance gravity along the brane by integrating out the macroscopic extra dimensions. This result in

$$
S_{\text {eff }}=\frac{M_{D}^{D-2} V_{n}}{2} \int d^{4} x \sqrt{-g} R,
$$

where $g_{\mu \nu}$ is the four-dimensional metric on the brane, with Ricci tensor $R_{\mu \nu}$, and Ricci scalar $R=g^{\mu \nu} R_{\mu \nu}$, and where the volume of the extra dimensions is given by $V_{n} \propto L^{n}$. The effective four-dimensional Planck scale, as seen by an observer on the brane, is then given by

$$
M_{p l}^{2} \sim M_{D}^{2+n} L^{n} .
$$

By taking the macroscopic extra dimensions to be sufficiently large, we can eliminate the standard hierarchy in $D$ dimensions, $M_{D} \sim M_{E W} \sim 1 \mathrm{TeV}$, and replace it with a new hierarchy involving the scale of the extra dimensions, $\mu \sim 1 / L \ll M_{E W}$. This is not in violation of short distance gravity tests, at least in $D \geq 6$ dimensions. Indeed, in six dimensions one can eliminate the hierarchy even for millimetre size extra dimensions.

In actual fact, the strongest constraints on the ADD model do not come from short distance gravity tests, but from astrophysics and cosmology 63. The problem arises because the Kaluza-Klein modes can be extremely light, $m_{K K} \gtrsim 1 / L \gtrsim 10^{-4} \mathrm{eV}$, and extremely numerous, $N_{K K} \sim M_{p l}^{2} / M_{D}^{2} \lesssim 10^{32}$. This means that even though each mode is only very weakly coupled, with strength $1 / M_{p l}$, scattering processes along the brane, at energies $E \gtrsim m_{K K}$, can produce a copious number of KK gravitons.

The strongest astrophysical constraint comes from the possible emission of KK modes during the collapse of SN1987a. Requiring this to not be the dominant cooling processes imposes a lower bound on the fundamental Planck scale. For example, with $n=2$ we have $M_{D} \geq 50 \mathrm{TeV}$, whereas for $n=3$ we have $M_{D} \geq 3 \mathrm{TeV}$ [350].

In cosmology, one has to worry about over-production of KK modes at high temperatures, since this may destroy the standard Big Bang picture. In order to be consistent with Big Bang Nucleosynthesis, and the current composition of the Universe, one must identify a maximum temperature for the early Universe for a given fundamental scale. Taking the fundamental scale to be $M_{D} \sim 1 \mathrm{TeV}$ imposes a temperature bound $T \leq 10$ $\mathrm{MeV}$ for $n=2$, rising to $T \leq 10 \mathrm{GeV}$ for $n=6$ [588]. A higher fundamental scale will raise the maximum temperature, but then one loses much of the appeal of the original model. While a low maximum temperature is not in contradiction with cosmological data, it does present a challenge to models of baryogenesis and inflation. The temperature bounds can be weakened considerably if one does not require the bulk to be flat. For example, when the bulk manifold is a compact hyperbolic space, the maximum temperature can be pushed beyond the GeV scale even for $n=2671$.

\subsection{Randall-Sundrum Gravity}

As we have already suggested, in a generic braneworld set-up there is no obvious reason why one should demand that the bulk space should be flat, as in the ADD model. In perhaps the most celebrated braneworld model, developed soon after ADD by Randall and Sundrum [1051, 1052, the bulk is an anti-de Sitter space. There are two versions 
of the Randall-Sundrum model, generally referred to as Randall-Sundrum I (RS1) 1051] and Randall-Sundrum II (RS2) [1052]. Somewhat confusingly, the RS1 model contains two branes, whereas the RS2 model only contains a single brane.

The RS1 model [1051] was also proposed as a resolution to the hierarchy problem. It improves on the ADD model as the compact extra dimension need not be so large as to introduce a new hierarchy. This is achieved by exploiting the exponential warp factor to generate a large bulk volume from a small compactification radius. In contrast, the RS2 model 1052 contains a single brane and a non-compact extra dimension - it is infinite in extent. This time the bulk warp factor ensures that gravity is localised close to the brane, so that a brane observer only sees the gravitational effects of the extra dimension above scales set by the bulk curvature.

Although the phrase Randall-Sundrum gravity really refers to these two original models, here we extend the definition to include any model with similar features. In this section we are particularly interested in five-dimensional models containing 3-branes, with some non-trivial geometry, or "warping", present in the bulk. We begin with an overview of some of the models. For further details see, for example, [1008, 845].

\subsubsection{The RS1 model}

In RS1, we have two 3-branes separated by a region of five-dimensional anti-de Sitter space 1051. The branes are located at $z=0$, and $z=z_{c}$, and we impose $\mathbb{Z}_{2}$ symmetry across each brane. Neglecting Gibbons-Hawking boundary terms [536], the action describing this model is given by

$$
\begin{aligned}
S=\frac{M_{5}^{3}}{2} \int d^{4} x \int_{-z_{c}}^{z_{c}} d z \sqrt{-\gamma}(\mathcal{R} & -2 \Lambda) \\
& -\sigma_{+} \int_{z=0} d^{4} x \sqrt{-g^{(+)}}-\sigma_{-} \int_{z=z_{c}} d^{4} x \sqrt{-g^{(-)}},
\end{aligned}
$$

where $\gamma_{a b}$ is the bulk metric, and $g_{\mu \nu}^{(+)}$and $g_{\mu \nu}^{(-)}$are the metrics on the branes at $z=0$ and $z=z_{c}$, respectively. $M_{5}$ is the five-dimensional Planck scale and is related to the five-dimensional Newton's constant via the standard relation $G_{5}=1 / 8 \pi M_{5}^{3}$. We also include a negative bulk cosmological constant, $\Lambda=-6 / l^{2}$. If we fine-tune the brane tensions against $\Lambda$, such that

$$
\sigma_{+}=-\sigma_{-}=\frac{6 M_{5}^{3}}{l}=\frac{3}{4 \pi G_{5} l},
$$

then we admit a background solution in which the branes exhibit four-dimensional Poincaré invariance:

$$
d s^{2}=e^{-2|z| / l} \eta_{\mu \nu} d x^{\mu} d x^{\nu}+d z^{2},
$$

for $-z_{c} \leq z \leq z_{c}$. The $\mathbb{Z}_{2}$ symmetry about $z=0$ is explicit, whereas the other boundary condition should be understood to be implicit. The line-element given in Eq. 604 contains an exponential warp factor that is displayed graphically in Figure 9. In between the branes we recognise the geometry to be anti-de Sitter space, written in Poincaré coordinates. Notice the peak in the warp factor at the positive tension brane, and the trough at the negative tension brane. Although only a toy model, the RS1 set-up is well motivated by a number of string theory/super-gravity constructions [622, 838, 439]. 


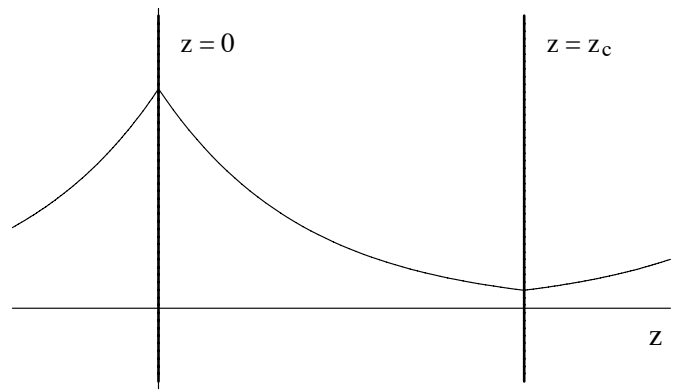

Figure 9: The behaviour of the warp factor in the RS1 model.

By integrating out the $4 D$ zero-mode we are able to derive the $4 D$ effective Planck scale on a given brane [1051, 1008:

$$
M_{ \pm}^{2}= \pm M_{5}^{3} l\left(1-e^{\mp 2 z_{c} / l}\right)
$$

where \pm labels the sign of the corresponding brane tension. In terms of the effective Newton's constants we have

$$
G_{ \pm}=\frac{G_{5}}{l}\left(\frac{ \pm 1}{1-e^{\mp 2 z_{c} / l}}\right) .
$$

Now suppose we live on the negative tension brane. If we take the fundamental Planck scale $M_{5} \sim \mathrm{TeV}$, the bulk curvature scale to be just below $1 / l \sim 0.01 M_{5}$, and the distance between the branes to be such that $z_{c} / l \sim 35$, we recover the desired effective Planck scale, $M_{-} \sim m_{p l} \sim 10^{16} \mathrm{TeV}$. Thus, the hierarchy problem has been eliminated altogether, and not just shifted around, as in the ADD model. In contrast, the hierarchy is not eliminated if we live on the positive tension brane since then the effective Planck mass is given by $M_{+} \sim e^{-z_{c} / l} M_{-}$[1051, 1008.

As it stands, the RS1 model is incomplete. The problem is that on either brane the low energy $4 D$ effective theory is not GR, but Brans-Dicke gravity. The extra scalar comes from fluctuations in the brane separation, and is sometimes referred to as the radion [541, 528, 275]. The value of the Brans-Dicke parameter depends on the brane, and is given by 528 .

$$
w_{B D}^{( \pm)}=\frac{3}{2}\left(e^{ \pm 2 z_{c} / l}-1\right) .
$$

Observations require this parameter to be large $\left(w_{B D}>40000\right.$, see Section 3.1.2). Note that for the positive tension brane $w_{B D}^{(+)}$can be made arbitrarily large with increasing brane separation. The same cannot be said for $w_{B D}^{(-)}$on the negative tension brane. If we want to live on the negative tension brane we must generate a mass for the radion to 


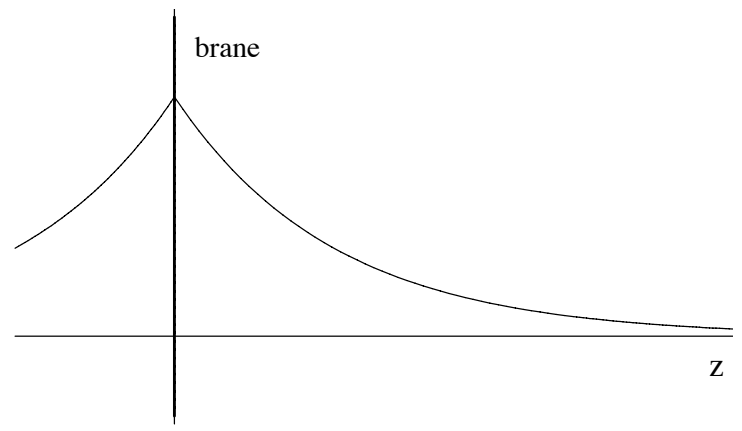

Figure 10: The behaviour of the warp factor in the RS2 model.

suppress its fluctuations. The Goldberger-Wise mechanism does exactly that, and thus stabilises the distance between the branes [542].

\subsubsection{The RS2 model}

The RS2 model is obtained from RS1 by taking the negative tension brane off to infinity 1052. The geometry is then described by the metric in Eq. (604) with $z_{c} \rightarrow \infty$. The corresponding warp factor is shown in Figure 10, the single peak at $z=0$ indicating that we have a single brane with positive tension. In this limit $M_{+} \rightarrow M_{5}^{3} l$, so we cannot eliminate the hierarchy problem as in the RS1 model. Rather, the situation is more akin to the five-dimensional ADD model, with the curvature scale, $1 / l$, playing the role of the compactification scale, $\mu \sim 1 / L$.

What makes the RS2 model interesting is the way in which $4 D \mathrm{GR}$ is recovered on the brane. As we have just seen, in RS1 the observer on the positive tension brane sees a low energy gravity theory corresponding to Brans-Dicke gravity, with a BD parameter $w_{B D}^{(+)}=$ $\frac{3}{2}\left(e^{2 z_{c} / l}-1\right)$. In the RS2 limit of infinite brane separation the BD scalar decouples and one is left with $4 D$ GR. As we will see in detail in Section 5.3.5, even though the bulk is infinite in extent, gravity is localised on the brane at energies below the bulk curvature scale, $1 / l$. As a method to screen the extra dimensions from the low energy observer, this represents a radical alternative to the standard method of Kaluza-Klein compactifications.

The key to gravity localisation is that the bulk volume is finite even though it has infinite extent. This ensures that there is a normalisable graviton zero mode, which, in the absence of any other massless modes, guarantees $4 D \mathrm{GR}$ at low enough energies. The finite bulk volume arises because the warp factor falls off exponentially as we move away from the brane. Intuitively, gravity localisation occurs because the warping makes it difficult for the graviton to propagate too far away from the brane, so much so that the region of the bulk with $z \gtrsim l$ has no influence on low energy brane interactions.

Since $1 / l$ sets the scale at which the brane observer starts to become sensitive to the 
bulk, table top experiments of the inverse square law impose the limit $1 / l \gtrsim 10^{-4} \mathrm{eV}$. This translates into a lower bound on the fundamental Planck scale, $M_{5} \gtrsim 10^{5} \mathrm{TeV}$, which is well above the electro-weak scale. Since TeV scale gravity is not phenomenologically viable in this case, we abandon any discussion of the hierarchy problem for the single brane scenario.

Up until now our discussion has centred around weak gravity on the brane. What about strong gravity in the presence of localised sources? Whilst there have been some interesting numerical studies (see, e.g., [1278, 758]) exact strong gravity solutions are rare in RS2. One exception are the solutions for a domain wall localised on the brane [561, 560]. In contrast, an exact solution describing a braneworld black hole remains elusive 45 The difficulty arises because the brane is an accelerated surface, so any black hole residing on the brane must follow an accelerated trajectory. Such an accelerated black hole requires knowledge of the AdS C-metric, but this solution is unknown in five dimensions. For a nice review of the search for braneworld black hole solutions see [558, and more recently [1200].

\section{RS2 and $A d S / C F T$}

Emparan, Fabbri and Kaloper (EFK) have suggested that a static braneworld black hole does not exist [470. To understand their argument we must first recall the $A d S / C F T$ correspondence in which type IIB string theory on $A d S_{5} \times S^{5}$ is conjectured to be dual to $\mathcal{N}=4 S U(N)$ super-Yang-Mills, in the large $N$ limit 862 . This suggests an alternative description of RS2 gravity [570: 'Gravity on an RS2 brane is dual to a strongly coupled conformal field theory (CFT) cut off in the UV, minimally coupled to $4 D$ gravity'. There is plenty of evidence for this holographic description of RS2 (see, for example, 570, [100, 438, 1009, 556, 1014, 596, 1138, 695], and for a review [1008]). Another way of describing the correspondence is to say [470]: 'A classical source on the brane is dual to a quantum corrected source in four dimensions, with the quantum corrections coming from the strongly coupled CFT'. The quantum corrections are large because of the large number of degrees of freedom in the large- $N$ limit. When applied to the problem of finding a black hole on the brane, this suggests that the solution should not be static since it should include the back-reaction of the Hawking radiation [470] (see also [1201]). Fitzpatrick, Randall and Wiseman have disputed this interpretation, pointing out that the CFT is strongly coupled and may therefore carry fewer degrees of freedom [508. At this point it is fair to say that as yet there has been no consensus, and the subject remains an active area of debate (see also [564, 562, 966, 505, 506]).

Note that if we accept the EFK conjecture, we can improve the bound on the bulk curvature by an order of magnitude, $1 / l \gtrsim 10^{-3} \mathrm{eV}$. This is based on the existence of long-lived black hole X-ray binaries [471]. For smaller values of $1 / l$ these binaries would have already decayed.

\subsubsection{Other RS-like models}

One of the characteristic features of the Randall-Sundrum models is the structure of the bulk geometry, described by a non-factorisable, or warped, metric. One can embrace

${ }^{45}$ There have been many attempts to find such a solution, most of which remain unpublished. 
this structure and consider a whole slew of interesting generalisations. Here we consider a class of models described by warped geometries of the form

$$
d s^{2}=a^{2}(z) \bar{g}_{\mu \nu}(x) d x^{\mu} d x^{\nu}+d z^{2} .
$$

Many of the most interesting RS-like models exhibit quasi-localisation and give rise to large-distance modifications of gravity. Perhaps the most celebrated of these is the DGP model [454, which will be discussed in detail in Section 5.5. Other interesting examples include the GRS model [563, the asymmetric brane model [1012, 1013] and the CGP model [274]. We begin with the simplest generalisation, however, proposed by Karch and Randall 682] (see also 667]).

\section{The Karch-Randall model}

Here we take the RS2 model and de-tune the brane tension, $\sigma_{+} \neq 6 M_{5}^{3} / l$, so that one no longer has Poincaré invariance along the brane. For an excess tension, $\sigma_{+}>6 M_{5}^{3} / l$, the metric $\bar{g}_{\mu \nu}$ is de Sitter, whereas for a tension deficit, $\sigma_{+}<6 M_{5}^{3} / l, \bar{g}_{\mu \nu}$ is anti-de Sitter. The effective cosmological constant on the brane is given by

$$
\Lambda_{4}=3\left[\left(\frac{\sigma_{+}}{6 M_{5}^{3}}\right)^{2}-\frac{1}{l^{2}}\right] .
$$

The behaviour of the warp factor also changes. We find that

$$
\begin{array}{ll}
a(z)=A \cosh (c-|z| / l), & c=\cosh ^{-1}(1 / A), \\
a(z)=A \sinh (c-|z| / l), & c=\sinh ^{-1}(1 / A),
\end{array}
$$

where $A=l \sqrt{\left|\Lambda_{4} / 3\right|}$. The decay of the warp factor away from the brane is greatest for the dS brane, cutting off the space-time at $z=l c$. This means that gravity is more strongly localised than in the standard RS2 scenario. In contrast, for the AdS brane the warp factor turns around at some finite value of $z$. This means the bulk volume is infinite, and gravity is not localised at all on the AdS brane as there is no normalisable zero mode. Actually, when $\left|\Lambda_{4}\right|$ is small (compared with $M_{5}$ ), the AdS brane exhibits quasi-localisation. This is because there is a normalisable mode that is ultra-light, with mass $m_{\text {ultra-light }}^{2} \sim \Lambda_{4}^{2} / M_{5} \ll\left|\Lambda_{4}\right|[1120$. At intermediate energies, $k \gg E \gg m_{\text {ultra-light }}$, the light mode behaves as if it were effectively massless and one recovers $4 D$ gravity. Note that there is no issue with the vDVZ discontinuity in AdS when $m_{\text {ultra-light }}^{2} /|| \Lambda_{4} \mid \lesssim 0.1[721$. We say that gravity is only quasi-localised because the extra-dimension opens up in the far infra-red, at energies $E \lesssim m_{\text {ultra-light }}$.

Now consider what happens when we introduce a second AdS brane, along the lines of an AdS generalisation of the RS1 model [720]. The first thing to note is that both branes can have positive tension since the warp factor turns around. The second thing to note is that the bulk volume is rendered finite, and so we have a zero mode as well as the ultra-light mode. At energies above the mass of both modes gravity is mediated by the exchange of two spin-2 fields, one massless and one massive. This corresponds to a braneworld realisation of the bigravity scenarios discussed in Section 3.3 (see also [723, 722, 1011). 


\section{The GRS model}

The GRS model [563] was developed by Gregory, Rubakov and Sibiryakov. The setup contains three Minkowski branes, one with positive tension and two with negative tension. The positive tension brane is $\mathbb{Z}_{2}$ symmetric and is flanked on either side by a section of anti-de Sitter space as far as a negative tension brane. Beyond the negative tension brane lies an infinite region of Minkowski space. The warp factor goes like 563

$$
a(z)= \begin{cases}e^{-|z| / l} & |z|<z_{c} \\ e^{-z_{c} / l} & |z|>z_{c} .\end{cases}
$$

As the bulk volume is infinite, gravity is not localised, although the decaying warp factor around the positive tension brane gives some degree of quasi-localisation. Again, although there is no zero mode, there is an ultra-light mode, and the extra dimension opens up at very large distances. Unfortunately, the GRS model is known to be unstable due to the presence of a ghost in the spectrum of linearised fluctuations [1038].

\section{The asymmetric brane model}

The asymmetric brane model [1012, 1013 (see also [1189]) is a single brane mode,l like RS2, only without $\mathbb{Z}_{2}$ symmetry imposed across the brane. Indeed, the fundamental parameters in the bulk are allowed to differ on either side of the brane, including the bulk cosmological constant and the bulk Planck scales. Allowing the bulk Planck scales to differ might seem strange, but not if we imagine a string compactification down to five dimensions in which the dilaton is stabilised at different values on either side of a domain wall (the brane). If the bulk cosmological constant and Planck scales are given by

$$
\Lambda=\left\{\begin{array}{ll}
-6 / l_{1}^{2} & z>0 \\
-6 / l_{2}^{2} & z<0
\end{array}, \quad M= \begin{cases}M_{1} & z>0 \\
M_{2} & z<0 .\end{cases}\right.
$$

The asymmetric model then admits Minkowski branes for a suitably tuned brane tension, $\sigma=3\left(\epsilon_{1} M_{1}^{3} / l_{1}+\epsilon_{2} M_{2}^{3} / l_{2}\right)$, where $\epsilon_{1}= \pm 1$ and $\epsilon_{2}= \pm 1$. The corresponding solutions have a warp factor of the form 1013 .

$$
a(z)= \begin{cases}e^{-\epsilon_{1} z / l_{1}} & z>0 \\ e^{\epsilon_{2} z / l_{2}} & z<0 .\end{cases}
$$

The parameters $\epsilon_{1}$ and $\epsilon_{2}$ control whether the warp factor grows $(\epsilon=-1)$ or decays $(\epsilon=-1)$ away from the brane in a given direction. The model includes RS2 as a special case.

It is, however, more interesting to consider the case where one of the warp factors grows away from the brane while the other decays (e.g. $\epsilon_{2}=-\epsilon_{1}=1$ ). The bulk volume is then infinite so that there is no zero mode, but by choosing the scales appropriately one can engineer a degree of quasi-localisation. The point is that on the growing side the graviton localises at the AdS boundary, where its sees an effective $4 D$ Planck scale $M_{1}^{3} l_{1}$. By taking this scale to be very large, the effect of localisation close to the AdS boundary 
is almost decoupled from the gravitational dynamics near the brane. We should note that the asymmetric model also admits self-accelerating solutions, just as in the DGP model. In fact, the model shares a number of features with the DGP model. This is no coincidence, since the DGP model can be obtained as a limiting case of the asymmetric model [736. It is known that the self-accelerating solutions of DGP contain ghost-like instabilities [273, 1015, 559, 546], so the same is expected to be true for the asymmetric model.

\section{The CGP model}

The CGP model 274, developed by Charmousis, Gregory and Padilla, also exhibits quasi-localisation. It combines the main features of both the asymmetric model and the DGP model in that there is an induced gravity term, and asymmetry across the brane. Interestingly, the model contains a new type of cosmological solution that tends to Minkowski space at very late times, but undergoes an intermediate period of cosmic acceleration in the presence of ordinary matter. In fact, it corresponds to a braneworld realisation of the Cardassian cosmology, with $H^{2} \approx \frac{8 \pi G}{3}(\rho+c \sqrt{\rho})[512$. Although a ghost is present when we introduce a small positive vacuum energy, this decouples in the Minkowski limit [740].

\subsubsection{Action and equations of motion}

In each of the models described in our overview, we have a five dimensional bulk space split into a series of domains separated from each another by 3-branes. Here we consider the action and field equations for generic models of this type. The 3-branes may be thought of as the boundaries of the various domains so that the action is given by

$$
S=\int_{\text {bulk }} d^{5} x \sqrt{-\gamma}\left(\frac{M_{5}^{3}}{2} \mathcal{R}+\mathcal{L}_{\text {bulk }}\right)+\sum_{\text {branes }} \int_{\text {brane }} d^{4} x \sqrt{-g}\left[-\Delta\left(M_{5}^{3} K\right)+\mathcal{L}_{\text {brane }}\right]
$$

where $\gamma_{a b}$ is the bulk metric with corresponding Ricci scalar, $\mathcal{R}, M_{5}$ is the bulk Planck scale, and $\mathcal{L}_{\text {bulk }}$ is the Lagrangian density describing the bulk field content. In principle both $M_{5}$ and $\mathcal{L}_{\text {bulk }}$ can vary from domain to domain. For each brane $g_{\mu \nu}$ is the induced metric and $\mathcal{L}_{\text {brane }}$ is the Lagrangian density describing the field content on that particular brane. $K=g^{\mu \nu} K_{\mu \nu}$ is the trace of extrinsic curvature, $K_{\mu \nu}$. This should be evaluated on either side of the brane as it can differ from side to side. Labelling the two sides of a given brane, using $L$ and $R$, we define $\left.K_{\mu \nu}\right|_{L, R}=\frac{1}{2} \mathcal{L}_{\left.n\right|_{L, R}} g_{\mu \nu}$, i.e. extrinsic curvature is given by the Lie derivative of the induced metric, with respect to the unit normal $\left.n^{a}\right|_{L, R}$. The unit normal on both sides points from $L$ to $R$. Note that what appears in the action is the jump ${ }^{46}$

$$
\Delta\left(M_{5}^{3} K\right)=\left.M_{5}^{3} K\right|_{R}-\left.M_{5}^{3} K\right|_{L} .
$$

This corresponds to the Gibbons-Hawking boundary term [536 for the bulk domains on each side of the brane. The $\Delta$ here is not to be confused with the 3-dimensional Laplacian.

Now there are two (completely equivalent) ways to treat the brane contributions at the level of the field equations. One approach is to treat them as delta-function sources in

\footnotetext{
${ }^{46}$ Henceforth we define the jump of any quantity $Q$ across a brane as $\Delta Q=\left.Q\right|_{R}-\left.Q\right|_{L}$.
} 
the Einstein equations. However, our preferred approach is to explicitly separate the field equations in the bulk from the boundary conditions at the brane. The bulk equations of motion are then given by the bulk Einstein equations

$$
\mathcal{G}_{a b}=\mathcal{R}_{a b}-\frac{1}{2} \mathcal{R} \gamma_{a b}=\frac{1}{M_{5}^{3}} T_{a b}^{\text {bulk }},
$$

where $T_{a b}^{\text {bulk }}=-\frac{2}{\sqrt{-\gamma}} \frac{\delta}{\delta \gamma^{a b}} \int_{\text {bulk }} d^{5} x \sqrt{-\gamma} \mathcal{L}_{\text {bulk }}$ is the bulk energy-momentum tensor. The boundary conditions at $\Sigma_{i}$ are given by the Israel junction conditions 649.

$$
\Delta\left[M_{5}^{2}\left(K_{\mu \nu}-K g_{\mu \nu}\right)\right]=-T_{\mu \nu}^{\mathrm{brane}},
$$

where $T_{\mu \nu}^{(i)}=-\frac{2}{\sqrt{-g}} \frac{\delta}{\delta g^{\mu \nu}} \int_{\text {brane }} d^{4} x \sqrt{-g} \mathcal{L}_{\text {brane }}$ is the brane energy-momentum tensor. Note that in each of these examples, the bulk geometry is only sourced by a cosmological constant, $T_{a b}^{\mathrm{bulk}}=-M_{5}^{3} \Lambda \gamma_{a b}$.

\subsubsection{Linear perturbations in RS1 and RS2}

We now consider the theory of linear perturbations. For brevity, we will restrict attention to RS1 and RS2, although the methods we use are fairly standard, and should apply to all RS-like models (for further details, see [528, 275, 1038, 1011]).

\section{Weak gravity on a RS1 brane}

It is enough to consider RS1, as RS2 can be readily obtained by taking the negative tension brane to infinity. Recall that the background metric, $\bar{\gamma}_{a b}$, is given by Equation 604, with the positive tension brane (the "+" brane) fixed at $z=0$ and the negative tension brane (the "-" brane) fixed at $z=z_{c}$. We see that the induced metric on the "+" brane is given by $\bar{g}_{\mu \nu}^{(+)}=\eta_{\mu \nu}$, and on the "-" brane by $\bar{g}_{\mu \nu}^{(-)}=e^{-2 z_{c} / l} \eta_{\mu \nu}$. Note that we have $\mathbb{Z}_{2}$ symmetry across the branes, so we can restrict attention to $0 \leq z \leq z_{c}$.

We now consider small perturbations about the background, so that the metric is given by $\gamma_{a b}=\bar{\gamma}_{a b}+\delta \gamma_{a b}$. It is convenient to choose Gaussian Normal (GN) gauge, defined by

$$
\delta \gamma_{\mu z}=\delta \gamma_{z z}=0
$$

Actually, this is only a partial gauge fixing. Since we have no additional bulk matter, we can also take the metric to be transverse and trace-free in the bulk. In other words, $\delta \gamma_{\mu \nu}=\chi_{\mu \nu}(x, z)$, where

$$
\partial_{\nu} \chi_{\mu}^{\nu}=\chi_{\mu}^{\mu}=0 .
$$

This is known as Randall-Sundrum (RS) gauge [1052]. In RS gauge, the linearised bulk equations of motion, $\delta \mathcal{G}_{a b}=\frac{6}{l^{2}} \delta \gamma_{a b}$, yield

$$
\left[e^{2 z / l} \partial^{2}+\frac{\partial^{2}}{\partial z^{2}}-\frac{4}{l^{2}}\right] \chi_{\mu \nu}=0,
$$

where $\partial^{2}=\partial_{\mu} \partial^{\mu}$.

Unfortunately, we can no longer assume that the branes are fixed at $z=0$ and $z=z_{c}$. The presence of matter on the branes will cause them to bend [528] so that they will now 
be positioned at $z=f_{+}(x)$ and $z=z_{c}+f_{-}(x)$, for some functions $f_{ \pm}$that depend only on the coordinates $x^{\mu}$. This makes it difficult to apply the Israel junction conditions at the branes. To get round this we can apply a gauge transformation that fixes the position of the "+" brane, and another that fixes the position of the "-" brane [275], without spoiling the Gaussian Normal condition (618). This gives rise to two coordinate patches that are related by a gauge transformation in the region of overlap. We will call them the "+" patch and the "-" patch accordingly.

We first consider the "+" brane. To fix its position we make the following coordinate transformation

$$
z \rightarrow z-f_{+}(x), \quad x_{\mu} \rightarrow x_{\mu}+\frac{l}{2}\left(1-e^{-2 z / l}\right) \partial_{\mu} f_{+} .
$$

The "+" brane is now fixed at $z=0$, although the other brane is now at $z=z_{c}+f_{-}-f_{+}$. It follows that the metric perturbation in the "+" patch is given by

$$
\delta \gamma_{\mu \nu}=\chi_{\mu \nu}^{(+)}(x, z)=\chi_{\mu \nu}(x, z)-l\left(1-e^{-2 z / l}\right) \partial_{\mu} \partial_{\nu} f_{+}-\frac{2}{l} f_{+} \bar{\gamma}_{\mu \nu} .
$$

Similarly, to fix the position of the "_" brane we let

$$
z \rightarrow z-f_{-}(x), \quad x_{\mu} \rightarrow x_{\mu}+\frac{l}{2}\left(1-e^{-2\left(z-z_{c}\right) / l}\right) \partial_{\mu} f_{-} .
$$

Now we have the "-" brane at $z=z_{c}$, but with the "+" brane at $z=f_{+}-f_{-}$. The metric perturbation in the "-" patch is given by

$$
\delta \gamma_{\mu \nu}=\chi_{\mu \nu}^{(-)}(x, z)=\chi_{\mu \nu}(x, z)-l\left(1-e^{-2\left(z-z_{c}\right) / l}\right) \partial_{\mu} \partial_{\nu} f_{-}-\frac{2}{l} f_{-} \bar{\gamma}_{\mu \nu} .
$$

Now the induced metric on the "+" brane is given by $g_{\mu \nu}^{(+)}=\bar{g}_{\mu \nu}^{(+)}+\delta g_{\mu \nu}^{(+)}$, where

$$
\delta g_{\mu \nu}^{(+)}=\chi_{\mu \nu}^{(+)}(x, 0)=\chi_{\mu \nu}(x, 0)-\frac{2}{l} f_{+} \bar{g}_{\mu \nu}^{(+)},
$$

whereas on the "-_" brane it is given by $g_{\mu \nu}^{(-)}=\bar{g}_{\mu \nu}^{(-)}+\delta g_{\mu \nu}^{(-)}$, where

$$
\delta g_{\mu \nu}^{(-)}=\chi_{\mu \nu}^{(-)}\left(x, z_{c}\right)=\chi_{\mu \nu}\left(x, z_{c}\right)-\frac{2}{l} f_{-} \bar{g}_{\mu \nu}^{(-)} .
$$

We are now ready to make use of the linearised Israel junction conditions given by Eq. (617) at each brane to find

$$
\Delta\left[M_{5}^{2} \delta\left(K_{\mu \nu}-K g_{\mu \nu}\right)\right]^{( \pm)}=\sigma_{ \pm} \delta g_{\mu \nu}^{( \pm)}-\mathcal{T}_{\mu \nu}^{( \pm)},
$$

where $\sigma_{ \pm}= \pm 6 M_{5}^{3} / l$ is the tension on the ' \pm ' brane, and $\mathcal{T}_{\mu \nu}^{( \pm)}$is the energy-momentum tensor for matter excitations. Now, owing to the $\mathbb{Z}_{2}$ symmetry, the extrinsic curvature simply changes by a sign when evaluated on either side of a given brane. It follows that the linearised boundary conditions at each brane are given by

$$
\begin{aligned}
\left.\left(\frac{\partial}{\partial z}+\frac{2}{l}\right) \chi_{\mu \nu}\right|_{z=0} & =-S_{\mu \nu}^{(+)} \\
\left.\left(\frac{\partial}{\partial z}+\frac{2}{l}\right) \chi_{\mu \nu}\right|_{z=z_{c}} & =-S_{\mu \nu}^{(-)}, \\
190 &
\end{aligned}
$$


where

$$
S_{\mu \nu}^{ \pm}(x)= \pm \frac{1}{M_{5}^{3}}\left[\mathcal{T}_{\mu \nu}^{( \pm)}-\frac{1}{3} \mathcal{T}^{( \pm)} \bar{g}_{\mu \nu}^{( \pm)}\right]-2 \partial_{\mu} \partial_{\nu} f_{ \pm}
$$

and where $\mathcal{T}^{( \pm)}=g_{( \pm)}^{\mu \nu} \mathcal{T}_{\mu \nu}^{( \pm)}$is the trace of the appropriate energy-momentum tensor. Indeed, taking the trace of these equations, and using the fact that $\chi_{\mu \nu}$ is traceless, we clearly see that matter on a brane causes it to be bend, such that

$$
\frac{\partial^{2} f_{ \pm}}{a_{ \pm}^{2}}=\mp \frac{\mathcal{T}^{( \pm)}}{6 M_{5}^{3}}
$$

where $a_{ \pm}$gives the warp factor at the " \pm " brane (i.e. $a_{+}=1$ and $a_{-}=e^{-z_{c} / l}$ ). Equations 620, 628 and 629 give the governing differential equations, and a complete set of boundary conditions for the graviton mode $\chi_{\mu \nu}$. We now take Fourier transforms along the brane directions, $Q(x, \ldots) \rightarrow \tilde{Q}(p, \ldots)=\frac{1}{(2 \pi)^{2}} \int d^{4} x e^{-i p_{\mu} x^{\mu}} Q(x, \ldots)$, to find that

$$
\chi_{\mu \nu}(x, z)=\frac{1}{(2 \pi)^{2}} \int d^{4} p e^{i p_{\mu} x^{\mu}} \tilde{\chi}_{\mu \nu}(p, z)
$$

where

$$
\left[-p^{2} e^{2 z / l}+\frac{\partial^{2}}{\partial z^{2}}-\frac{4}{l^{2}}\right] \tilde{\chi}_{\mu \nu}=0
$$

and

$$
\left.\left(\frac{\partial}{\partial z}+\frac{2}{l}\right) \tilde{\chi}_{\mu \nu}\right|_{z=0}=-\tilde{S}_{\mu \nu}^{(+)}(p),\left.\quad\left(\frac{\partial}{\partial z}+\frac{2}{l}\right) \tilde{\chi}_{\mu \nu}\right|_{z=z_{c}}=-\tilde{S}_{\mu \nu}^{(-)}(p) .
$$

This system is easily solved to give

$$
\tilde{\chi}_{\mu \nu}(p, z)=C^{(+)}(p, z) \tilde{S}_{\mu \nu}^{(+)}-C^{(-)}(p, z) \tilde{S}_{\mu \nu}^{(-)},
$$

where

$$
C^{(+)}(p, z)=-\frac{1}{p \operatorname{det} A(p)}\left[I_{1}\left(p l e^{z_{c} / l}\right) K_{2}\left(p l e^{z / l}\right)+K_{1}\left(p l e^{z_{c} / l}\right) I_{2}\left(p l e^{z / l}\right)\right],
$$

and

$$
C^{(-)}(p, z)=-\frac{1}{p \operatorname{det} A(p)} e^{-z_{c} / l}\left[I_{1}(p l) K_{2}\left(p l e^{z / l}\right)+K_{1}(p l) I_{2}\left(p l e^{z / l}\right)\right] .
$$

Note that $I_{n}$, and $K_{n}$ are modified Bessel's functions of integer order $n[6]$, and

$$
\operatorname{det} A(p)=I_{1}(p l) K_{1}\left(p l e^{z_{c} / l}\right)-K_{1}(p l) I_{1}\left(p l e^{z_{c} / l}\right) .
$$

From Eq. 631 we also have

$$
f_{ \pm}(x)= \pm \frac{1}{(2 \pi)^{2}} \frac{a_{ \pm}^{2}}{M_{5}^{3}} \int d^{4} p e^{i p_{\mu} x^{\mu}} \frac{\tilde{\mathcal{T}}^{( \pm)}}{6 p^{2}} .
$$


To compute the metric perturbation on each of the branes we simply use Eqs. 622 and (624), given our knowledge of $\chi_{\mu \nu}(x, z)$ and $f_{ \pm}(x)$. At the positive tension brane we then have

$$
\begin{aligned}
& \chi_{\mu \nu}^{(+)}=\frac{1}{(2 \pi)^{2}} \frac{1}{M_{5}^{3}} \int d^{4} p e^{i p_{\mu} x^{\mu}}\left\{C^{(+)}(p, 0)\left[\tilde{\mathcal{T}}_{\mu \nu}^{(+)}-\frac{\alpha_{+}}{2} \tilde{\mathcal{T}}^{(+)} \bar{g}_{\mu \nu}^{(+)}\right]\right. \\
&\left.-\frac{e^{-z_{c} / l}}{l p^{2} \operatorname{det} A(p)}\left[\tilde{\mathcal{T}}_{\mu \nu}^{(-)}-\frac{1}{3} \tilde{\mathcal{T}}^{(-)} \bar{g}_{\mu \nu}^{(-)}\right]\right\}+ \text {pure gauge terms }
\end{aligned}
$$

whereas at the negative tension brane we have

$$
\begin{aligned}
& \chi_{\mu \nu}^{(-)}=\frac{1}{(2 \pi)^{2}} \frac{1}{M_{5}^{3}} \int d^{4} p e^{i p_{\mu} x^{\mu}}\left\{C^{(-)}\left(p, z_{c}\right)\left[\tilde{\mathcal{T}}_{\mu \nu}^{(-)}-\frac{\alpha_{-}}{2} \tilde{\mathcal{T}}^{(-)} \bar{g}_{\mu \nu}^{(-)}\right]\right. \\
&\left.-\frac{e^{-z_{c} / l}}{l p^{2} \operatorname{det} A(p)}\left[\tilde{\mathcal{T}}_{\mu \nu}^{(+)}-\frac{1}{3} \tilde{\mathcal{T}}^{(+)} \bar{g}_{\mu \nu}^{(+)}\right]\right\}+ \text {pure gauge terms. }
\end{aligned}
$$

The parameters $\alpha_{ \pm}$are crucial as they control the tensor structure of the propagator on the " \pm " branes. They are given by

$$
\alpha_{+}=\frac{2}{3}\left(1+\frac{1}{l p^{2} C^{(+)}(p, 0)}\right), \quad \alpha_{-}=\frac{2}{3}\left(1-\frac{e^{-2 z_{c} / l}}{l p^{2} C^{(-)}\left(p, z_{c}\right)}\right) .
$$

Using the properties of modified Bessel functions [6], we can show that at low energies, $p \ll k e^{-z_{c} / l}$, we have 528 .

$$
\begin{aligned}
\chi_{\mu \nu}^{(+)} \approx \frac{1}{(2 \pi)^{2}} \int d^{4} p e^{i p_{\mu} x^{\mu}} & \left\{\frac{2}{M_{+}^{2} p^{2}}\left[\tilde{\mathcal{T}}_{\mu \nu}^{(+)}-\left(\frac{w_{B D}^{+}+1}{2 w_{B D}^{+}+3}\right) \tilde{\mathcal{T}}^{(+)} \bar{g}_{\mu \nu}^{(+)}\right]\right. \\
& \left.+\frac{2}{M_{-}^{2} p^{2}}\left[\tilde{\mathcal{T}}_{\mu \nu}^{(-)}-\frac{1}{3} \tilde{\mathcal{T}}^{(-)} \bar{g}_{\mu \nu}^{(-)}\right]\right\}+ \text {pure gauge terms }
\end{aligned}
$$

and

$$
\begin{aligned}
\chi_{\mu \nu}^{(-)} \approx \frac{e^{-2 z_{c} / l}}{(2 \pi)^{2}} \int d^{4} p e^{i p_{\mu} x^{\mu}}\left\{\frac{2}{M_{-}^{2} p^{2}}\left[\tilde{\mathcal{T}}_{\mu \nu}^{(-)}-\left(\frac{w_{B D}^{-}+1}{2 w_{B D}^{-}+3}\right) \tilde{\mathcal{T}}^{(-)} \bar{g}_{\mu \nu}^{(-)}\right]\right. \\
\left.+\frac{2}{M_{+}^{2} p^{2}}\left[\tilde{\mathcal{T}}_{\mu \nu}^{(+)}-\frac{1}{3} \tilde{\mathcal{T}}^{(+)} \bar{g}_{\mu \nu}^{(+)}\right]\right\}+ \text {pure gauge terms }
\end{aligned}
$$

where $M_{ \pm}$and $w_{B D}^{ \pm}$are the $4 D$ effective Planck scale and Brans-Dicke parameter on the " \pm " brane, respectively. They are given by Equations (605) and (607). We can now see explicitly how BD gravity emerges as the low energy effective theory in RS1, on both branes, as claimed in Section 5.3 .1

\section{Graviton spectrum}

Let us now pause to comment on the mass spectrum for the graviton. The spectrum can be obtained by identifying the poles in the propagator. These are given by the 
solutions to $p^{2}=-m^{2}$, where $\operatorname{det} A(p)=0$. This gives

$$
\begin{aligned}
I_{1}(i m l) K_{1}\left(i m l e^{z_{c} / l}\right)- & K_{1}(i m l) I_{1}\left(i m l e^{z_{c} / l}\right)=0 \\
& \Longrightarrow J_{1}(m l) Y_{1}\left(m l e^{z_{c} / l}\right)-Y_{1}(m l) J_{1}\left(m l e^{z_{c} / l}\right)=0,
\end{aligned}
$$

where $J_{1}$ and $Y_{1}$ are Bessel's functions of order one. By solving this equation we see that there is a zero mode, and a tower of heavy Kaluza-Klein modes with mass splitting $\Delta m \sim 1 / l\left(e^{z_{c} / l}-1\right)$.

\section{Radion effective action}

The finite Brans-Dicke parameter indicates the presence of a massless scalar in addition to the massless graviton. This is due to the radion, or brane bending mode, $\delta f=f_{+}-f_{-}$. In some RS-like models (e.g. GRS), the radion can exhibit pathological behaviour that can only be revealed by computing the effective action 1038. We will now briefly outline the procedure for doing this.

Let us consider vacuum fluctuations $\left(\mathcal{T}_{\mu \nu}^{( \pm)}=0\right)$ in the scalar sector. The first thing to note is that the Field Equations 620, (628) and 629) admit a solution of the form 275

$$
\chi_{\mu \nu}^{(\mathrm{rad})}=-\frac{l^{2}}{4} e^{2 z / l} \partial_{\mu} \partial_{\nu} \psi,
$$

where $\partial^{2} \psi=0$ and the vacuum boundary conditions require

$$
f_{ \pm}^{\mathrm{vac}}=-\frac{l}{2 a_{ \pm}^{2}} \psi
$$

where $f_{ \pm}^{\text {vac }}$ is the vacuum fluctuation in $f_{ \pm}$. Note that Equation (631) implies $\partial^{2} f_{ \pm}^{\text {vac }}=0$, and so Equation (647) is consistent with $\partial^{2} \psi=0$. Equation (647) also imposes a relation between $f_{+}^{\text {vac }}$ and $f_{-}^{\text {vac }}$, resulting in a single free scalar degree of freedom, which we take to be the physical radion mode, $\psi$. Note that the radion profile in the bulk is localised close to the "-" brane, in contrast to the graviton zero mode which is localised close to the "+" brane. We now work in the "+" patch, which has the branes positioned at $z=0$ and $z=z_{c}-\delta f^{\mathrm{vac}}$. Focusing solely on the scalar sector, the metric perturbation is given by

$$
\delta \gamma_{\mu \nu}=-\frac{l^{2}}{4} e^{2 z / l} \partial_{\mu} \partial_{\nu} \psi-l\left(1-e^{-2 z / l}\right) \partial_{\mu} \partial_{\nu} f_{+}^{\mathrm{vac}}-\frac{2}{l} f_{+}^{\mathrm{vac}} \bar{\gamma}_{\mu \nu}, \quad \delta \gamma_{\mu z}=\delta \gamma_{z z}=0 .
$$

In order to integrate out the extra dimension, it is convenient to have both branes fixed. We can do this with the following coordinate transformation

$$
z \rightarrow z+B(z) \delta f^{\mathrm{vac}}, \quad x_{\mu} \rightarrow x_{\mu}-e^{-2 z / l} \partial_{\mu}\left(\delta f^{\mathrm{vac}}\right) \int_{0}^{z} e^{2 y / l} B(y) d y,
$$

where $B(z)$ is some differentiable function for $0 \leq z \leq z_{c}$, satisfying $B(0)=0$ and $B\left(z_{c}\right)=1$. While this transformation ensures that $\delta \gamma_{\mu z}$ is still zero, the price we pay for 
fixed branes is that we now have non-vanishing $\delta \gamma_{z z}$. More precisely,

$$
\begin{aligned}
\delta \gamma_{\mu \nu}=h_{\mu \nu}=-\frac{l^{2}}{4} e^{2 z / l} \partial_{\mu} \partial_{\nu} \psi- & l\left(1-e^{-2 z / l}\right) \partial_{\mu} \partial_{\nu} f_{+}^{\mathrm{vac}}-\frac{2}{l}\left[f_{+}^{\mathrm{vac}}-\left(\delta f^{\mathrm{vac}}\right) B(z)\right] \bar{\gamma}_{\mu \nu} \\
& +2 e^{-2 z / l} \partial_{\mu} \partial_{\nu}\left(\delta f^{\mathrm{vac}}\right) \int_{0}^{z} e^{2 y / l} B(y) d y \\
\delta \gamma_{z z}=h_{\mu z}=-2 \delta f^{\mathrm{vac}} B^{\prime}(z) . &
\end{aligned}
$$

To quadratic order, the effective action is given by

$$
\begin{aligned}
S_{\mathrm{eff}}=-\frac{M_{5}^{3}}{2} \int_{0}^{z_{c}} d z \int d^{4} x \sqrt{-\bar{\gamma}} h^{a b} \delta\left[\mathcal{G}_{a b}-\frac{6}{l^{2}} \gamma_{a b}\right] \\
+\frac{1}{2} \int_{z=0} d^{4} x \sqrt{-\bar{g}^{(+)}} h^{\mu \nu}\left[\Delta\left(M_{5}^{2} \delta\left(K_{\mu \nu}-K g_{\mu \nu}\right)\right)^{(+)}-\sigma_{+} h_{\mu \nu}\right] \\
+\frac{1}{2} \int_{z=z_{c}} d^{4} x \sqrt{-\bar{g}^{(-)}} h^{\mu \nu}\left[\Delta\left(M_{5}^{2} \delta\left(K_{\mu \nu}-K g_{\mu \nu}\right)\right)^{(-)}-\sigma_{-} h_{\mu \nu}\right] .
\end{aligned}
$$

It turns out that $\delta\left[\mathcal{G}_{\mu \nu}-\frac{6}{l^{2}} \gamma_{\mu \nu}\right]$ and $\Delta\left(M_{5}^{2} \delta\left(K_{\mu \nu}-K g_{\mu \nu}\right)\right)^{( \pm)}-\sigma_{ \pm} h_{\mu \nu}$ are identically zero [1038. After integrating out a total derivative in $z$, we arrive at the following effective action for the radion:

$$
S_{\text {radion }}=-\frac{3}{2} M_{-}^{2} \int d^{4} x\left(\partial_{\mu} \psi\right)^{2},
$$

where $M_{-}$is given by Equation (605). Thus, as expected, the radion, $\psi$, behaves as a massless scalar, and, being localised close to the "-" brane, its coupling strength is controlled by the scale $M_{-}$. As we move the branes further and further away from one another, $M_{-}$increases and the radion starts to decouple (decoupling completely in the RS2 limit). Note that in some models, such as GRS [563, the radion effective action comes in with the wrong overall sign, signalling the presence of a physical ghost [1038. There is no such pathology in RS1 or RS2.

Weak gravity on a RS2 brane

Let us now focus on RS2 by taking the negative tension brane to infinity. As $z_{c} \rightarrow \infty$, the metric on the remaining positive tension brane is given by

$$
\begin{aligned}
& \chi_{\mu \nu}^{(+)}=\frac{1}{(2 \pi)^{2}} \int d^{4} p e^{i p_{\mu} x^{\mu}} \frac{K_{2}(p l)}{M_{5}^{3} p K_{1}(p l)}\left[\tilde{\mathcal{T}}_{\mu \nu}^{(+)}-\frac{1+\frac{K_{1}(p l)}{p l K_{2}(p l)}}{3} \tilde{\mathcal{T}}^{(+)} \bar{g}_{\mu \nu}^{(+)}\right] \\
& + \text {pure gauge terms. }
\end{aligned}
$$

At low energies, $p \ll k$, we again use the properties of modified Bessel functions [6] to show that

$$
\chi_{\mu \nu}^{(+)} \approx \frac{1}{(2 \pi)^{2}} \int d^{4} p e^{i p_{\mu} x^{\mu}} \frac{2}{M_{4}^{2} p^{2}}\left[\tilde{\mathcal{T}}_{\mu \nu}^{(+)}-\frac{1}{2} \tilde{\mathcal{T}}^{(+)} \bar{g}_{\mu \nu}^{(+)}\right]+\text {pure gauge terms. }
$$


It follows that gravity is indeed localised on the brane at low energies, with a $4 D$ effective Planck scale $M_{4}^{2}=M_{5}^{3} l$, or, equivalently, with an effective Newton's constant $G_{4}=G_{5} / l$. Note that the tensor structure of the propagator matches that of General Relativity, with a factor of $-\frac{1}{2}$ in front of the trace term. This is where the brane bending mode $f_{+}(x)$ plays a crucial role. It cancels part of the graviton zero mode in just the right way to guarantee good agreement with solar system gravity tests. It is worth noting that this neat cancellation of terms does not always happen, even in single brane scenarios. We will see this in DGP gravity, for example, where one has to argue for some sort of Vainshtein effect to pass observational tests.

Let us finally consider massive modes. In RS2 there is actually a continuum of massive modes, consistent with the fact that the extra dimension is no longer compact. However, as we have seen, we still recover $4 D$ GR to leading order. The next to leading order corrections are obtained by integrating over the continuum, or, equivalently, by considering the next to leading order expansion in $p l$ above. The result is that the Newtonian potential reads 47

$$
V(r) \propto \frac{1}{r}\left(1+\frac{2 l^{2}}{3 r^{2}}+\mathcal{O}\left(l^{3} / r^{3}\right)\right) .
$$

We refer the reader to $\left[528,1008,438\right.$, for details of the derivation. Note that the $1 / r^{3}$ correction can be obtained in a dual picture as the one loop CFT correction to the graviton propagator 438 .

\subsection{Brane Cosmology}

There are two obvious reasons why cosmology offers an interesting arena in which to develop the braneworld paradigm. The first is that cosmological branes possess a high degree of symmetry, and this makes it possible to solve the field equations. The second is that cosmological physics can be tested by a number of observations, ranging from supernova data to the abundance of light elements. In this section we will study the cosmology of co-dimension one branes, focusing on the RS2 scenario with a single $\mathbb{Z}_{2}$ symmetric brane. We will review the background dynamics [159, 178] before moving on to cosmological perturbation theory [716, 1244]. Further details, including generalisations to multi-brane scenarios with bulk scalar fields, can be found in the following review articles [1048, 845, 639, 770, 189, 195] (see also [1008, 346, 347, 1139]). Other generalisations include: the cosmology of branes without $\mathbb{Z}_{2}$ symmetry [1012, 1189, 1143]; anisotropic braneworlds 843, 966; and branes for which energy is explicitly transferred between bulk and brane 694, 695, 1234. Note that any anisotropy is seen to dissipate on the brane in RS gravity, a feature that can be identified with CFT particle production in the holographic picture [966]. Bulk-brane energy transfer has been used to account for dark energy 694, 695, 1234.

Braneworld cosmology can be studied using two different formalisms: The brane based formalism, and the bulk based formalism. These two approaches are completely equivalent and yield a background cosmology governed by the following Friedmann equations [159,

\footnotetext{
${ }^{47}$ Note that this result differs slightly from the original one quoted in 1052 .
} 


$$
\begin{aligned}
H^{2}+\frac{\kappa}{a^{2}} & =\frac{\Lambda_{4}}{3}+\frac{8 \pi G_{4}}{3} \rho\left(1+\frac{\rho}{2 \sigma}\right)+\frac{\mu}{a^{4}}, \\
\dot{H}-\frac{\kappa}{a^{2}} & =-4 \pi G_{4}(\rho+P)\left(1+\frac{\rho}{\sigma}\right)-\frac{2 \mu}{a^{4}},
\end{aligned}
$$

where $H=\dot{a} / a$ is the Hubble parameter along the brane, $a(t)$ is the scale factor, and $\kappa=0, \pm 1$ describes the spatial curvature. The brane is sourced by a tension, $\sigma$, and a cosmological fluid with energy density, $\rho(t)$, and pressure, $P(t)$. The parameters $\Lambda_{4}$ and $G_{4}$ denote the effective cosmological constant and Newton's constant on the brane, respectively. As in the standard scenario, the Raychaudhuri Equation 657) follows from the Friedmann Equation (656) and energy conservation,

$$
\dot{\rho}+3 H(\rho+P)=0 .
$$

We will now review the derivation of this cosmology using the two equivalent formalisms. For the moment, however, let us comment on a few of its important features. From Equations (656) and (657) we see that the corrections to the standard cosmology manifest themselves in a term $\propto \rho^{2}$, and a dark radiation term $\frac{\mu}{a^{4}}=\frac{8 \pi G_{4}}{3} \rho_{\text {weyl }}$. The latter corresponds to a non-local "Weyl" contribution and can only be fixed by specifying the bulk geometry. In the holographic description of RS2, the $\rho^{2}$ corrections contribute to the conformal anomaly [1138, while the dark radiation is identified with thermal excitations of the CFT [570, 1100, 1009, 556, 1014, 596.

Both corrections will strongly affect Big Bang nucleosynthesis (BBN), so their magnitude can be constrained by the abundance of light elements. These require that the dark radiation can be at most $10 \%$ of the photon energy density, $\rho_{\text {weyl }} / \rho_{\gamma} \lesssim 0.01$, in the period following BBN 638. In addition, the $\rho^{2}$ corrections to the cosmological evolution should be negligible after BBN, which imposes a constraint ${ }^{48}$ on the tension $\sigma \gtrsim(\mathrm{MeV})^{4}$ [159.

The $\rho^{2}$ corrections play the dominant role in the very early universe and will have a big impact on the inflationary dynamics. Assuming the inflaton is confined to the brane along with all the Standard Model fields, one finds that the slow roll parameters are given by 847 .

$$
\begin{aligned}
& \epsilon=-\frac{\dot{H}}{H^{2}}=\frac{1}{16 \pi G_{4}}\left(\frac{V^{\prime}}{V}\right)^{2}\left[\frac{1+\frac{V}{\sigma}}{\left(1+\frac{V}{2 \sigma}\right)^{2}}\right], \\
& \eta=-\frac{\ddot{\phi}}{H \dot{\phi}}=\frac{1}{8 \pi G_{4}}\left(\frac{V^{\prime \prime}}{V}\right)\left[\frac{1}{1+\frac{V}{2 \sigma}}\right],
\end{aligned}
$$

where $V(\phi)$ is the inflaton potential. At low energies, $V \ll \sigma$, these match the standard formulae of $4 D$ General Relativity,

$$
\epsilon \sim \epsilon_{G R}=\frac{1}{16 \pi G_{4}}\left(\frac{V^{\prime}}{V}\right)^{2}, \quad \eta \sim \eta_{G R}=\frac{1}{8 \pi G_{4}}\left(\frac{V^{\prime \prime}}{V}\right) .
$$

\footnotetext{
${ }^{48}$ Assuming a RS2 scenario with fine tuned tension, $\sigma=12 M_{5}^{3} / l$, the BBN constraint is significantly weaker than the constraint arising from table top experiments quoted in Section 5.3 .2 i.e. $1 / l \gtrsim 10^{-4}$ $\mathrm{eV}$, giving $\sigma \gtrsim(100 \mathrm{GeV})^{4}\left[189\right.$. Here we have used the fact that $M_{4}^{2}=M_{5}^{3} l \sim 10^{10} \mathrm{TeV}$.
} 
However, at high energies we have

$$
\epsilon \sim \epsilon_{G R} \frac{4 \sigma}{V} \ll \epsilon_{G R}, \quad \eta \sim \eta_{G R} \frac{2 \sigma}{V} \ll \eta_{G R}
$$

This means we can get away with steeper potentials in the braneworld case 847. This is essentially because Hubble friction gets enhanced by the $\rho^{2}$ terms. However, such potentials are incompatible with observational constraints as they lead to a large tensorto-scalar ratio 800.

\subsubsection{Brane based formalism - covariant formulation}

We shall now derive the background cosmology, Eqs. (656) and (657), using the brane based formalism. This makes use of the Gauss-Codazzi equations [531, 320], and the Israel junction conditions to derive the Einstein tensor on the brane [1140. We will assume $\mathbb{Z}_{2}$ symmetry, although we refer the reader to [117. for a non- $\mathbb{Z}_{2}$ symmetric generalisation.

The Gauss-Codazzi equations are the fundamental equations of embedded hypersurfaces. The brane can be thought of as an embedding,

$$
x^{a}=X^{a}\left(\xi^{\mu}\right)
$$

in the bulk geometry, $\gamma_{a b}(x)$. By $\mathbb{Z}_{2}$ symmetry this is the same on both sides of the brane. We can now define tangent vectors $V_{\mu}^{a}=\partial X^{a} / \partial \xi^{\mu}$, and the outward pointing unit normal, $n^{a}$, satisfying

$$
\gamma_{a b} n^{a} n^{b}=1, \quad \gamma_{a b} n^{a} V_{\mu}^{b}=0
$$

It follows that the induced metric on the brane is given by

$$
g_{\mu \nu}=\gamma_{a b}(X) V_{\mu}^{a} V_{\nu}^{b}
$$

For such an embedding, the Gauss-Codazzi equations [531, 320] give

$$
\begin{aligned}
R_{\mu \nu \alpha \beta} & =\mathcal{R}_{a b c d} V_{\mu}^{a} V_{\nu}^{b} V_{\alpha}^{c} V_{\beta}^{d}+K_{\mu \alpha} K_{\nu \beta}-K_{\mu \beta} K_{\nu \alpha}, \\
\nabla^{\mu}\left(K_{\mu \nu}-K g_{\mu \nu}\right) & =\mathcal{R}_{a b} n^{a} V_{\nu}^{b} \\
R-K^{2}+K_{\mu \nu} K^{\mu \nu} & =-2 \mathcal{G}_{a b} n^{a} n^{b}
\end{aligned}
$$

where $\mathcal{R}_{a b c d}$ is the Riemann tensor in the bulk, $R_{\mu \nu \alpha \beta}$ is the Riemann tensor on the brane, and we recall that the extrinsic curvature of the brane is given by $K_{\mu \nu}=\frac{1}{2} \mathcal{L}_{n} g_{\mu \nu}$, the Lie derivative of the induced metric, with respect to the normal.

Now, in the RS2 scenario the dynamics of the brane are governed by the bulk equations of motion and the $\mathbb{Z}_{2}$ symmetric junction conditions

$$
\mathcal{G}_{a b}=\frac{6}{l^{2}} \gamma_{a b}, \quad K_{\mu \nu}-K \gamma_{\mu \nu}=3 \sigma_{*} g_{\mu \nu}-4 \pi G_{5} \mathcal{T}_{\mu \nu},
$$

with $\sigma_{*}=\frac{4 \pi G_{5} \sigma}{3}$, where $\sigma$ is the brane tension and $\mathcal{T}_{\mu \nu}$ is the energy-momentum tensor of additional matter excitations. Of course, in the RS2 scenario we have a fine-tuned tension such that $\sigma_{*}=1 / l$, but we will keep things general for the moment. 
Consider the Codazzi Equation (665). Because the bulk is only sourced by a cosmological constant, the right hand side of this equation is identically zero. This is important, because the junction conditions in Eq. (667) now imply the usual conservation law along the brane, $\nabla^{\mu} \mathcal{T}_{\mu \nu}=0$. To extract information about the Einstein tensor on the brane, we contract the Gauss Equation (664), and plug in Eq. (667), to give [1140]

$$
G_{\mu \nu}=-\Lambda_{4} g_{\mu \nu}+8 \pi G_{4} \mathcal{T}_{\mu \nu}+\left(4 \pi G_{5}\right)^{2} \Pi_{\mu \nu}-E_{\mu \nu}
$$

where $\Lambda_{4}=3\left(\sigma_{*}^{2}-\frac{1}{l^{2}}\right)$ is the effective cosmological cosmological constant on the brane, and the effective Newton's constant is given by

$$
G_{4}=G_{5} \sigma_{*}
$$

The corrections to standard $4 D$ gravity are encoded in a local contribution, $\Pi_{\mu \nu}$, and a non-local "Weyl" contribution, $E_{\mu \nu}$. The local piece is a quadratic combination of the energy-momentum tensor

$$
\Pi_{\mu \nu}=-\mathcal{T}_{\mu}^{\alpha} T_{\nu \alpha}+\frac{1}{3} \mathcal{T} \mathcal{T}_{\mu \nu}+\frac{1}{2} \mathcal{T}^{\alpha \beta} \mathcal{T}_{\alpha \beta} g_{\mu \nu}-\frac{1}{6} \mathcal{T}^{2} g_{\mu \nu}
$$

whereas the non-local "Weyl" piece is

$$
E_{\mu \nu}=C_{a b c d} n^{a} V_{\mu}^{b} n^{c} V_{\nu}^{d}
$$

where $C_{a b c d}$ is the Weyl tensor of the bulk. It is often referred to as the electric part of the Weyl tensor, and in general one must solve the bulk equations of motion first in order to evaluate it in full. We should also note that $E_{\mu \nu}$ has vanishing trace, and its divergence is sourced by the local quadratic contribution

$$
g^{\mu \nu} E_{\mu \nu}=0, \quad \nabla_{\mu} E_{\nu}^{\mu}=\left(4 \pi G_{5}\right)^{2} \nabla_{\mu} \Pi_{\nu}^{\mu} .
$$

The latter equation follows from the divergence of Equation (668), making use of the Bianchi identity and the local conservation of energy-momentum, $\nabla^{\mu} \mathcal{T}_{\mu \nu}=0$.

Our interest here lies in the cosmology, so let us now assume spatial homogeneity and isotropy on the brane. The induced metric is given by the usual Friedmann-LemaitreRobertson-Walker (FLRW) metric

$$
d s^{2}=g_{\mu \nu} d \xi^{\mu} d \xi^{\nu}=-d t^{2}+a(t)^{2} q_{i j} d x^{i} d x^{j},
$$

where $q_{i j}(x)$ is the metric of a hyper-surface of constant curvature, $\kappa=0, \pm 1$. The matter excitations contribute a cosmological fluid with energy density, $\rho(t)$, and pressure, $P(t)$, such that its energy-momentum tensor is given by

$$
\mathcal{T}_{\nu}^{\mu}=\operatorname{diag}(-\rho(t), P(t), P(t), P(t))
$$

In the absence of non-trivial sources in the bulk $T_{\nu}^{\mu}$ is conserved, which means we have the standard relation given by Equation 658.

It remains to compute the non-local piece, $E_{\mu \nu}$. Although in general we must solve the bulk equations of motion to evaluate this, we can exploit the large amount of symmetry 
to avoid doing so in the current instance. Making use of the Constraints Equations 672, we can show that

$$
E_{\nu}^{\mu}=\frac{\mu}{a^{4}} \operatorname{diag}\left(-1, \frac{1}{3}, \frac{1}{3}, \frac{1}{3}\right)
$$

where $\mu$ is an integration constant that should be fixed by the bulk geometry (we will see in the next section that it can be identified with the mass of a bulk black hole). Note that $E_{\nu}^{\mu}$ is conserved on a cosmological background, by virtue of the fact that $\nabla_{\mu} \Pi_{\nu}^{\mu}=0$.

The modified Friedmann Equations (656) and 657) now follow automatically from Equation 668.

\subsubsection{Bulk based formalism - moving branes in a static bulk}

The principle limitation of the brane based formalism we have just described is that it suppresses physics deep inside the bulk. This can be dangerous since it is not at all obvious if a particular choice of the non-local "Weyl" term, $E_{\mu \nu}$, at the brane will evolve into a pathological bulk geometry. Such problems can be avoided by solving for the bulk geometry first. For example, in the brane based formalism, the parameter $\mu$ giving rise to dark radiation, is just an integration constant and can take any sign. Using the bulk based formalism, we are able to identify $\mu$ with the mass of a bulk black hole, and use this to constrain it to be positive.

We will now develop the bulk based formalism for cosmological branes. Again, we will assume $\mathbb{Z}_{2}$ symmetry across the branes, for brevity. The reader can refer to [1008. for the non- $\mathbb{Z}_{2}$ symmetric generalisation. The bulk based formalism requires us to solve for the bulk geometry. Since we are interested in cosmological branes (with constant curvature Euclidean 3 -spaces), we study the Einstein equations, $\mathcal{G}_{a b}=\frac{6}{l^{2}} \gamma_{a b}$, with the following metric ansatz [178]

$$
d s^{2}=\gamma_{a b} d x^{a} d x^{b}=e^{2 \nu} A^{-2 / 3}\left(-d t^{2}+d z^{2}\right)+A^{2 / 3} q_{i j} d x^{i} d x^{j},
$$

where $A$ and $\nu$ are undetermined functions of $t$ and $z$, and as before $q_{i j}(x)$ is the metric of a hyper-surface of constant curvature, $\kappa=0, \pm 1$. Now, in an extremely elegant calculation, Bowcock et al. [178] were able to prove a generalised form of Birkhoff's theorem, showing that the bulk geometry is necessarily given by

$$
d s^{2}=-V(r) d \tau^{2}+\frac{d r^{2}}{V(r)}+r^{2} q_{i j} d x^{i} d x^{j},
$$

where

$$
V(r)=\frac{r^{2}}{l^{2}}+\kappa-\frac{\mu}{r^{2}} .
$$

For $\mu>0$, the metric in Eq. 676 takes the form of a (topological) Schwarzschild black hole in anti-de Sitter space. Here we have written the solution in an explicitly time independent coordinate system, meaning that we can no longer say that we have a static brane sitting at a fixed coordinate position. On the contrary, we now have a dynamic brane, whose trajectory in the these coordinates is more complicated. Braneworld cosmology from this perspective was first studied by Ida 639], although moving branes in a static anti-de Sitter bulk were considered earlier by Kraus [754]. 
To construct the brane solution, we treat it as an embedding,

$$
\tau=\tau(t), \quad r=a(t),
$$

of the bulk geometry given in Eq. (676). The induced metric on the brane is then

$$
d s^{2}=g_{\mu \nu} d \xi^{\mu} d \xi^{\nu}=\left(-V(a) \dot{\tau}^{2}+\frac{\dot{a}^{2}}{V(a)}\right) d t^{2}+a^{2}(t) q_{i j} d x^{i} d x^{j}
$$

where over-dots denote $\partial / \partial t$. We are free to choose $t$ to correspond to the proper time with respect to an observer comoving with the brane. This imposes the condition

$$
-V(a) \dot{\tau}^{2}+\frac{\dot{a}^{2}}{V(a)}=-1
$$

ensuring that the brane takes the standard FLRW form, as in Eq. 673). The function $a(t)$ is then immediately identified with the scale factor along the brane.

The boundary condition at the brane are given in Eq. 667). We must compute the extrinsic curvature, $K_{\mu \nu}=\frac{1}{2} \mathcal{L}_{n} g_{\mu \nu}$, defined as the Lie derivative of the normal pointing into the bulk. Assuming we cut away the AdS boundary and retain the region $r<a(t)$, we find that the inward pointing unit normal is given by

$$
n_{a}=(-\dot{a}, \dot{\tau}, 0,0,0),
$$

where we are free to specify that $\dot{\tau}>0$. The components of extrinsic curvature are then given by

$$
K_{j}^{i}=\frac{V \dot{\tau}}{a} \delta_{j}^{i}, \quad K_{t}^{t}=-\left(\frac{\ddot{a}+V^{\prime} / 2}{V \dot{\tau}}\right) .
$$

In the presence of a cosmological fluid, as given by Equation (674), the junction conditions in Eq. 667) yield the following:

$$
\begin{gathered}
\frac{V \dot{\tau}}{a}=\sigma_{*}\left(1+\frac{\rho}{\sigma}\right), \\
\frac{\ddot{a}+\frac{1}{2} V^{\prime}}{V \dot{\tau}}=\sigma_{*}\left[1-2 \frac{\rho}{\sigma}-3 \frac{P}{\sigma}\right] .
\end{gathered}
$$

Making use of Equation (680) we then arrive at the modified Friedmann Equations 656 and (657).

\subsubsection{Cosmological perturbations}

While the theory describing cosmological perturbations in braneworld gravity has been well developed in recent years (see, for example, 842, 771, 847, 334, 1087, 991, 801, 202, 201, 548, 772, 547, 918, 919, 921, 920, 596, 716, 768, 1244, 742, 1245, 703, 705, 715, 769, 597, 408, 194, 430, 955, 286, 300, 395, 1062, 787, 245), even approximate solutions to the resulting field equations have been notoriously hard to come by. The problem stems from the fact that one has to solve the fully coupled system of brane and bulk, which is, in general, a far from trivial task. Indeed, this will generically render the brane based formalism somewhat incomplete without making ad hoc assumptions about the 
perturbations of the non-local "Weyl" contribution, $E_{\mu \nu}$. The bulk based formalism is better in this respect, but the resulting system is virtually intractable, and only in some special cases, where the bulk and brane equations become separable, has progress been made [528, 596, 202, 201, 772]. Note that, unlike in other sections, we will on occasion include vector and tensor perturbations, as well as scalars. This is because the bulk can source vector and tensor modes on the brane, giving qualitatively different behaviour to that seen in standard $4 D$ cosmology.

\section{Cosmological perturbations in the brane based formalism}

We shall now review some aspects of cosmological perturbation theory using the brane based formalism introduced in Section 5.4.1. Further details can be found in [845, 842]. The dynamics on the brane are governed by Equation (668), and so perturbations about the background cosmology on the brane satisfy

$$
\delta G_{\nu}^{\mu}=8 \pi G_{4} \delta \mathcal{T}_{\nu}^{\mu}+\left(4 \pi G_{5}\right)^{2} \delta \Pi_{\nu}^{\mu}-\delta E_{\nu}^{\mu} .
$$

To study this we use the standard four-dimensional formalism [90, 717, 469, 917, decomposing the system into scalar, vector and tensor perturbations with respect to the spatial diffeomorphism group in the background cosmology. Working with conformal time as opposed to proper time, the perturbed metric is given by

$$
\begin{aligned}
d s^{2}=a^{2}\left[-(1+2 \Psi) d \tau^{2}-2\right. & \left(\beta_{i}+\vec{\nabla}_{i} \beta\right) d x^{i} d \tau \\
& \left.+\left[(1-2 \Phi) q_{i j}+D_{i j} \nu+2 \vec{\nabla}_{(i} A_{j)}+h_{i j}\right] d x^{i} d x^{j}\right],
\end{aligned}
$$

where $A_{i}$ and $\beta_{i}$ are transverse vectors on $q_{i j}$, and $h_{i j}$ is a transverse and trace-free tensor. Recall that the operator $D_{i j}=\vec{\nabla}_{i} \vec{\nabla}_{j}-\frac{1}{3} q_{i j} \Delta$. The fluctuations energy-momentum on the brane are written in terms of the fluctuations in density, $\delta$, pressure, $\delta P$, fluid 3-velocity, $v_{i}$, and anisotropic stress, $\Sigma_{j}^{i}$, in the usual way,

$$
\delta \mathcal{T}_{0}^{0}=-\rho \delta, \quad \delta \mathcal{T}_{i}^{0}=-(\rho+P) v_{i}, \quad \delta \mathcal{T}_{j}^{i}=\delta P \delta_{j}^{i}+(\rho+P) \Sigma_{j}^{i} .
$$

The fluid 3-velocity and anisotropic stress can then be decomposed with respect to their scalar, vector and tensor components,

$$
\begin{aligned}
v_{i} & =\theta_{i}^{\text {(vector) }}+\vec{\nabla}_{i} \theta, \\
\Sigma_{i j} & =\Sigma_{i j}^{\text {(tensor })}+\vec{\nabla}_{(i} \Sigma_{j)}^{\text {(vector })}+D_{i j} \Sigma .
\end{aligned}
$$

The fluctuation in the quadratic piece is also given in terms of $\rho \delta, \delta P \ldots$ etc., according to

$$
\begin{aligned}
& \delta \Pi_{0}^{0}=\frac{2}{3} \rho \delta \mathcal{T}_{0}^{0}, \quad \delta \Pi_{i}^{0}=\frac{2}{3} \rho \delta \mathcal{T}_{i}^{0}, \\
& \delta \Pi_{j}^{i}=-\frac{1}{3}(\rho+3 P) \delta \mathcal{T}_{j}^{i}-\frac{2}{3}(\rho+P) \delta_{j}^{i}\left(\delta \mathcal{T}_{0}^{0}-\frac{1}{2} \delta \mathcal{T}_{k}^{k}\right),
\end{aligned}
$$

where $\delta T_{\nu}^{\mu}$ are given by Equations 687. 
Now we consider the contribution from the non-local "Weyl" perturbation, and identify it with the fluctuation in some dark energy energy-momentum tensor

$$
\delta E_{\mu \nu}=-8 \pi G_{4} \delta \mathcal{T}_{\mu \nu}^{\text {weyl }}
$$

In direct analogy with Equations 687), we can read off corresponding fluctuations in Weyl energy density, $\delta^{\text {weyl }}$, Weyl pressure, $\delta P^{\text {weyl }}$, Weyl fluid 3 -velocity, $v_{i}^{\text {weyl }}$, and Weyl anisotropic stress, $\Sigma_{i j}^{\text {weyl }}$. We might hope to determine each of these in terms of the local matter fluctuations, $\delta, \delta P \ldots$ etc., by making use of the Constraints Eqs. 672 to linear order. We find that for scalar perturbations $\delta E_{\mu}^{\mu}=0$ gives ${ }^{49}$

$$
\delta P^{\mathrm{weyl}}=\frac{1}{3} \rho^{\mathrm{weyl}} \delta^{\mathrm{weyl}}
$$

and $\nabla_{\mu}\left[\delta E_{\nu}^{\mu}-\left(4 \pi G_{5}\right)^{2} \Pi_{\nu}^{\mu}\right]=0$ gives

$$
\begin{gathered}
\left(\delta^{\text {weyl }}\right)^{\prime}+4 \Psi^{\prime}+\frac{4}{3} \Delta\left[\beta+\frac{1}{2} \nu^{\prime}-\theta^{\text {weyl }}\right]=0 \\
-\frac{4}{3} \rho^{\text {weyl }}\left[\left(\theta^{\text {weyl }}\right)^{\prime}+\Phi-\frac{2}{3}(\Delta+3 \kappa) \Sigma^{\text {weyl }}-\frac{\delta^{\text {weyl }}}{4}\right]= \\
\frac{\rho+P}{\sigma}\{\rho \delta+(\rho+P)[3 \mathcal{H} \theta-(\Delta+3 \kappa) \Sigma]\},
\end{gathered}
$$

where' denotes differentiation with respect to conformal time, and $\mathcal{H}=a^{\prime} / a=a H$. Clearly we have too many Weyl unknowns and not enough equations. The bottom line is that we need to know the Weyl anisotropic stress $\delta \pi_{i j}^{\text {weyl }}$ explicitly, and for that we need to abandon the brane based formalism and solve the bulk equations of motion [842.

Some progress can be made at super-horizon scales, since then we can neglect the spatial gradients in Eqs. (692) and 693), and solve for the Weyl energy density and Weyl momentum in terms of $\rho \delta, \delta P \ldots$ etc., thereby closing the system [842, 548, 771]. This simplification has been applied to the study of both density perturbations and vector perturbations at large scales, revealing qualitatively different behaviour to that in General Relativity [548, 844]. Regarding density perturbations, it can be shown that the quantity $\rho a^{4} \Delta \delta$ will grow during slow-roll inflation on super-horizon scales (it stays constant in GR) [548. It can be shown that vector perturbations can be non-vanishing, even in the absence of vorticity 844 .

We can also solve for the (total) curvature perturbation on large scales 771 . Unfortunately, this does not mean we can compute large-scale CMB anisotropies. The problem is that to evaluate the (non-integrated) Sachs-Wolfe equation we need knowledge of the metric perturbations. These are sourced by the dark anisotropic stress, according to

$$
\hat{\Phi}-\hat{\Psi}=8 \pi G_{4} a^{2}\left(\frac{4}{3} \rho^{\text {weyl }}\right) \Sigma^{\text {weyl }},
$$

\footnotetext{
${ }^{49}$ The remaining formulae in this section are taken from [771, where one should identify $\delta \rho=\rho \delta, \delta q=$ $-(\rho+P) \theta, \delta \pi=(\rho+P) \Sigma, \mathcal{R}=\Psi, A=-\Phi, E=\frac{1}{2} \nu, B=-\beta$.
} 
where $\hat{\Psi}$ and $\hat{\Phi}$ are the Bardeen gauge invariants for the metric perturbations, and we have neglected the local anisotropic stress. The braneworld corrections to the SachsWolfe effect are given by 771 .

$$
\begin{aligned}
\frac{\delta T}{T}=\left.\frac{\delta T}{T}\right|_{G R} & -\frac{8}{3}\left(\frac{\rho_{\gamma}}{\rho_{c d m}}\right) \mathcal{S}_{\text {weyl }} \\
& -8 \pi G_{4} a^{2}\left(\frac{4}{3} \rho^{\text {weyl }}\right) \Sigma^{\text {weyl }}+\frac{16 \pi G_{4}}{a^{5 / 2}} \int d a a^{7 / 2}\left(\frac{4}{3} \rho^{\text {weyl }}\right) \Sigma^{\text {weyl }},
\end{aligned}
$$

where $\mathcal{S}_{\text {weyl }}$ is the Weyl entropy perturbation, determined by $\rho \delta^{\text {weyl }}$.

\section{Cosmological perturbations in the bulk based formalism}

As we saw in the previous section, a proper treatment of cosmological perturbation theory in brane cosmology requires us to solve the coupled system of brane and bulk. We will now present the details of this in the bulk based formalism, essentially following [716] (see also [1244, 918, 919, 921, 920]). Note that in this section we deviate from our usual convention of treating cosmological perturbations with respect to conformal time on the brane, preferring instead to use proper time of comoving observers, in keeping with the majority of the relevant literature. For consistency, however, we do define the brane quantities as in the previous section.

We begin with some notation on the background. The bulk metric is given by

$$
d s^{2}=\bar{\gamma}_{a b} d x^{a} d x^{b}=\lambda_{\alpha \beta} d x^{\alpha} d x^{\beta}+r^{2} q_{i j} d x^{i} d x^{j},
$$

where $\lambda_{\alpha \beta}$ is some two-dimensional metric, and $\lambda_{\alpha \beta}$ and $r$ depend only on the first two coordinates, $x^{\alpha}$. This corresponds to a section of (topological) AdS-Schwarzschild, and one can choose a gauge such that

$$
\lambda_{\alpha \beta} d x^{\alpha} d x^{\beta}=-V(r) d \tau^{2}+\frac{d r^{2}}{V(r)}, \quad V(r)=\frac{r^{2}}{l^{2}}+\kappa-\frac{\mu}{r^{2}} .
$$

However, here we will leave the choice of gauge unspecified so that our analysis can be applied in other gauges (e.g. Gaussian-Normal gauge). The background embedding equation is now given by $x^{a}=\bar{X}^{a}\left(\xi^{\mu}\right)$, where $\xi^{\mu}=\left(t, \xi^{i}\right)$ are the coordinates along the brane. We take

$$
\bar{X}^{\alpha}=\bar{X}^{\alpha}(t), \quad \bar{X}^{i}=\xi^{i},
$$

and $\lambda_{\alpha \beta}(\bar{X}) \dot{\bar{X}}^{\alpha} \dot{\bar{X}}^{\beta}=-1$ to ensure that the induced metric on the brane has the standard FLRW form, as in Eq. 673), with scale factor $a(t)=r(\bar{X})$. Over-dots here denote differentiation with respect to proper time on the brane, $t$.

We shall now specify the perturbations in the bulk. Decomposing the bulk metric in terms of scalar, vectors and tensor components (with respect to $q_{i j}$ ), we write $\delta \gamma_{a b}=$ $h_{a b}^{\text {scalar }}+h_{a b}^{\text {vector }}+h_{a b}^{\text {tensor }}$, where,

$$
\begin{aligned}
& h_{\alpha \beta}^{\text {scalar }}=\chi_{\alpha \beta}, \quad h_{\alpha i}^{\text {scalar }}=r \vec{\nabla}_{i} \chi_{\alpha}, \quad h_{i j}^{\text {scalar }}=2 r^{2}\left[A q_{i j}+D_{i j} E\right], \\
& h_{\alpha \beta}^{\text {vector }}=0, \quad h_{\alpha i}^{\text {vector }}=r B_{\alpha i}, \quad h_{i j}^{\text {vector }}=2 r^{2} \vec{\nabla}_{(i} H_{j)}, \\
& h_{\alpha \beta}^{\text {tensor }}=0, \quad h_{\alpha i}^{\text {tensor }}=0, \quad h_{i j}^{\text {tensor }}=r^{2} H_{i j},
\end{aligned}
$$


where $D_{i j}=\vec{\nabla}_{i} \vec{\nabla}_{j}-\frac{1}{3} q_{i j} \Delta$. Here $B_{\alpha i}$ and $H_{i}$ are transverse, and $H_{i j}$ is transverse and trace-free. One can identify the following gauge invariants in the bulk [716]:

$$
\begin{array}{ll}
\text { scalars: } & Y_{\alpha \beta}=\chi_{\alpha \beta}-2 D_{(\alpha} Q_{\beta}, \quad Z=A-\frac{1}{3} \Delta E-Q^{\alpha} \partial_{\alpha} \ln r, \\
\text { vectors: } & F_{\alpha i}=B_{\alpha i}-r D_{\alpha} H_{i}, \\
\text { tensors: } & H_{i j},
\end{array}
$$

where $D_{\alpha}$ is the covariant derivative on $\lambda_{\alpha \beta}$, and $Q_{\alpha}=r\left(\chi_{\alpha}-r \partial_{\alpha} E\right)$. For simplicity we will assume that there are no exceptional modes [716] in any sector. Now, the bulk equations of motion, $\delta G_{b}^{a}=0$, are extremely complex in general (see [716]). From a practical perspective, the only way to proceed is to assume that the background bulk is maximally symmetric anti de Sitter space, so there is no bulk black hole (i.e. $\mu=0$ ) 918. The vector and scalar perturbations in the bulk can then be expressed in terms of a corresponding 'master variable'. For example, the scalar gauge invariants $Y_{\alpha \beta}$ and $Z$ can be written as

$$
Y_{\alpha \beta}=\frac{1}{r}\left[D_{\alpha} D_{\beta}-\frac{2}{3} \lambda_{\alpha \beta}\left(D^{2}-\frac{1}{2 l^{2}}\right)\right] \Omega, \quad Z=-\frac{1}{2} Y=\frac{1}{6 r}\left(D^{2}-\frac{2}{l^{2}}\right) \Omega,
$$

where the scalar master variable $\Omega$ satisfies

$$
\left[D^{2}-3 \partial_{\alpha} \ln r D^{\alpha}+\frac{\Delta+3 \kappa}{r^{2}}+\frac{1}{l^{2}}\right] \Omega=0 .
$$

For the vectors, we introduce the vector master variable, writing the gauge invariant as

$$
F_{\alpha i}=\frac{1}{r^{2}} \epsilon_{\alpha \beta} D^{\beta} \Omega_{i},
$$

where $\epsilon_{\alpha \beta}$ is the total antisymmetric Levi-Civita tensor on $\lambda_{\alpha \beta}$, and $\Omega_{i}$ satisfies

$$
D^{\alpha}\left[r^{5} D_{\beta}\left(\frac{D^{\beta} \Omega_{i}}{r^{3}}\right)+(\Delta+2 \kappa) \Omega_{i}\right]=0 .
$$

The tensor gauge invariant, $H_{i j}$, is essentially its own master variable, and satisfies

$$
\left[D^{2}+3 D^{\alpha} \ln r D_{\alpha}+\frac{1}{r^{2}}(\Delta-2 \kappa)\right] H_{i j}=0 .
$$

To solve for these master variables we need to specify boundary conditions at the brane, as well as the asymptotic boundary conditions. The latter correspond to the condition of normalisability. Let us now consider the boundary conditions at the brane. The first thing to note here is that the brane position can fluctuate, so that it now corresponds to an embedding $x^{a}=\bar{X}^{a}+f^{a}\left(\xi^{\mu}\right)$. We decompose this fluctuation in terms of scalars $f^{\alpha}, \epsilon$, and a transverse vector $\epsilon^{i}$, such that $f^{a}=\left(f^{\alpha}, \epsilon^{i}+\vec{\nabla}^{i} \epsilon\right)$. Secondly, in applying the boundary conditions, it is important to express all fields in terms of covariant objects along the (background) brane. This suggests that all fields of the form $W^{\alpha}$ and $W^{\alpha \beta}$ should be decomposed in terms of their components parallel to the tangent vector $\dot{\bar{X}}^{\alpha}$ and along the normal $\bar{n}^{\alpha}$, as follows:

$$
\begin{aligned}
W^{\alpha} & =-W_{\|} \dot{\bar{X}}^{\alpha}+W_{\perp} \bar{n}^{\alpha}, \\
W^{\alpha \beta} & =W_{\|\| \|} \dot{\bar{X}}^{\alpha} \dot{\bar{X}}^{\beta}-W_{\| \perp} \dot{\bar{X}}^{\alpha} \bar{n}^{\beta}-W_{\perp \|} \dot{\bar{X}}^{\beta} \bar{n}^{\alpha}+W_{\perp \perp} \bar{n}^{\alpha} \bar{n}^{\beta} . \\
& 204
\end{aligned}
$$


The induced metric on the brane then takes the form

$$
\begin{aligned}
d s^{2}=-(1+2 \Psi) d t^{2}-2 a\left(\beta_{i}\right. & \left.+\vec{\nabla}_{i} \beta\right) d x^{i} d t \\
& +a^{2}\left[(1-2 \Phi) q_{i j}+D_{i j} \nu+2 \vec{\nabla}_{(i} A_{j)}+h_{i j}\right] d x^{i} d x^{j}
\end{aligned}
$$

with

$$
\begin{array}{ll}
\Psi=-\frac{1}{2} Y_{\|\|}-\dot{U}_{\|}+\bar{K}_{t}^{t} U_{\perp}, & \beta=-\frac{U_{\|}}{a}-\frac{a \dot{\nu}}{2}, \\
\Phi=-Z-\frac{1}{3} \Delta \nu+H U_{\|}-\left(D_{\perp} \ln r\right) U_{\perp}, & \nu=2(E+\epsilon), \\
\beta_{i}=-F_{\| i}-a \dot{A}_{i}, & A_{i}=H_{i}+\epsilon_{i}, \\
h_{i j}=H_{i j}, &
\end{array}
$$

where $U^{\alpha}=Q^{\alpha}+f^{\alpha}$. Note that $U^{\alpha}, \nu$ and $A_{i}$ are invariant under gauge transformations in the bulk, so these expressions have been written entirely in terms of bulk gauge invariants.

The gauge invariants on the brane are the ones familiar to us from standard cosmological perturbation theory in four dimensions. Given the fluctuation in the brane energy-momentum tensor

$$
\delta \mathcal{T}_{t}^{t}=-\rho \delta, \quad \delta \mathcal{T}_{i}^{t}=-a(\rho+P) v_{i}, \quad \delta \mathcal{T}_{j}^{i}=\delta P \delta_{j}^{i}+(\rho+P) \Sigma_{j}^{i},
$$

and Equations (688) and (689), we note that the components of the anisotropic stress $\Sigma, \Sigma^{\text {(vector) }}$ and $\Sigma_{i j}^{\text {(tensor) }}$ are all gauge invariant on the brane, along with the following scalars,

$$
\begin{aligned}
\hat{\Phi} & =\Phi+\frac{1}{6} \Delta \nu+\dot{a} \sigma_{g}=-Z-\left(D_{\perp} \ln r\right) U_{\perp}, \\
\hat{\Psi} & =\Psi-\left(a \sigma_{g}\right)=-\frac{1}{2} Y_{\|\|}+\bar{K}_{t}^{t} U_{\perp}, \\
\hat{\theta} & =\theta-\sigma_{g}, \\
\hat{\delta} & =\delta+3 H a\left(1+\frac{P}{\rho}\right) \theta, \\
\Gamma & =\rho \delta-c_{s}^{2} \delta P,
\end{aligned}
$$

the vectors $\theta_{i}$ and $\hat{\beta}_{i}=\beta_{i}+a \dot{A}_{i}=-F_{\| i}$, and the tensor, $h_{i j}$. Note that

$$
\sigma_{g}=\frac{a \dot{\nu}}{2}+\beta=-\frac{U_{\|}}{a} .
$$

The goal is now to solve for the master variables, subject to boundary conditions set by normalisability and the values of the non-dynamical gauge invariants on the brane, namely, $\Gamma, \Sigma, \Sigma_{i}^{\text {(vector) }}$ and $\Sigma_{i j}^{\text {(tensor) }}$. We can then use this knowledge to derive the dynamical gauge invariants, $\hat{\Phi}, \hat{\Psi}, \hat{\beta}_{i}, h_{i j}, \hat{\theta}, \hat{\delta}$, and $\theta_{i}$. We are already able to express the 
metric invariants in terms of the master variables (and $U_{\perp}$ ) as follows:

$$
\begin{aligned}
\hat{\Phi}= & \frac{1}{2 a}\left[H \dot{\Omega}-\left(D_{\perp} \ln r\right) D_{\perp} \Omega+\left(\frac{1}{l^{2}}+\frac{(\Delta+3 \kappa)}{3 a^{2}}\right) \Omega\right] \\
& \quad-\left(D_{\perp} \ln r\right) U_{\perp}, \\
\hat{\Psi}= & -\frac{1}{2 a}\left[\ddot{\Omega}-2 H \dot{\Omega}+\left(2 D_{\perp} \ln r-\bar{K}_{t}^{t}\right) D_{\perp} \Omega-\left(\frac{1}{l^{2}}+\frac{2(\Delta+3 \kappa)}{3 a^{2}}\right) \Omega\right] \\
\quad & \quad \bar{K}_{t}^{t} U_{\perp}, \\
\hat{\beta}_{i}= & -\frac{1}{a^{2}} D_{\perp} \Omega_{i} .
\end{aligned}
$$

To fix $U_{\perp}$, and the remaining dynamical gauge invariants, we need to impose boundary conditions at the brane, given by the linearised Israel junction conditions $\delta\left(K_{\nu}^{\mu}-K \delta_{\nu}^{\mu}\right)=$ $-4 \pi G_{5} \delta \mathcal{T}_{\nu}^{\mu}$. For the scalars this gives

$$
\begin{aligned}
(\Delta+3 \kappa) D_{\perp}\left(\frac{\Omega}{r}\right) & =8 \pi G_{5} a^{2}[\rho \hat{\delta}-(\Delta+3 \kappa)[(\rho+P) \Sigma]], \\
\left(D_{\perp} \Omega\right)^{\cdot}-\bar{K}_{t}^{t} \dot{\Omega} & =8 \pi G a^{2}\left[(\rho+P) \hat{\theta}-\partial_{t}[a(\rho+P) \Sigma]\right], \\
U_{\perp} & =4 \pi G_{5} a^{2}(\rho+P) \Sigma \\
-\Gamma & =c_{s}^{2} \rho \hat{\delta}+(\rho+P)\left(\hat{\Psi}-\partial_{t}(a \hat{\theta})+\frac{2}{3}(\Delta+3 \kappa) \Sigma\right) .
\end{aligned}
$$

We should now view these equations as follows [716: Equations 725 and 726 fix $\hat{\delta}$ and $\hat{\theta}$ given knowledge of the anisotropic stress, $\Sigma$ and the master variable, $\Omega$, while Equation 727 fixes $U_{\perp}$. One can then substitute the expression for $\hat{\Psi}, \hat{\delta}$ and $\hat{\theta}$ (in terms of $\Omega$ and $\Sigma$ ) into Equation $(728$ to derive a Neumann type boundary condition on $\Omega$. In principle, one should now be able to solve the Master Equation (705), with a suitable boundary condition derived from Eq. (728), and feed the solution back into the expressions for $\hat{\Phi}, \hat{\Psi}, \hat{\theta}$ and $\hat{\delta}$.

For vectors, the Israel junction conditions yield

$$
\begin{aligned}
(\Delta+2 \kappa) \Omega_{i} & =8 \pi G_{5} a^{4}(\rho+P) \theta_{i} \\
\dot{\Omega}_{i} & =4 \pi G_{5} a^{3}(\rho+P) \Sigma_{i}^{(\text {vector })} .
\end{aligned}
$$

This time we view Equation 730 as a Dirichlet type boundary condition on the master variable. We then solve for $\Omega_{i}$ using Equation (707), and subsequently fix $\hat{\beta}_{i}$ and $\theta_{i}$ using Equations 724 and 729.

Finally, we consider the tensor. Here we solve Equation 708 , subject to the following junction condition at the brane:

$$
D_{\perp} H_{i j}=-4 \pi G_{5}(\rho+P) \Sigma_{i j}^{(\text {tensor })} .
$$

We have now presented the formalism in full, but the task of solving the system explicitly is another matter altogether. To do so one must choose coordinates for the background bulk. Whilst the static coordinate system might seem the simplest from 
the bulk perspective, it is rarely used owing to the fact that a Gaussian Normal (GN) coordinate system makes it much easier to specify the boundary conditions. For example, when $\mu=0$ and $\kappa=0$, the metric in GN coordinates is given by [158]

$$
\begin{aligned}
& \lambda_{\alpha \beta} d x^{\alpha} d x^{\beta}=d z^{2}-N^{2}(t, z) d t^{2}, \\
& r(t, z)=a(t)\left[\cosh (|z| / l)-\left(1+\frac{\rho(t)}{\sigma}\right) \sinh (|z| / l)\right],
\end{aligned}
$$

where $N=\frac{\dot{r}(t, z)}{\dot{a}(t)}$. Even in this case analytic solutions can only be obtained in the case of a background de Sitter brane, when the Master Equation 705 becomes separable 202, 772. Numerical solutions, however, have been obtained for scalar perturbations on a radiation dominated brane 245. It was found that short wavelength density perturbations are amplified relative to their value GR during horizon reentry, but not so much that they cause an observable effect in the CMB or in large-scale structure.

\subsection{Dvali-Gabadadze-Porrati Gravity}

The most celebrated braneworld model exhibiting quasi-localisation and an infra-red modification of gravity is without doubt the DGP model, developed by Dvali, Gabadadze and Porrati 454. The model admits two distinct sectors: The normal branch and the self-accelerating branch. The latter has generated plenty of interest since it gives rise to cosmic acceleration without the need for dark energy [394, 398. However, as we will see, fluctuations about the self-accelerating vacuum suffer from ghost-like instabilities [273, 1015, 559, 546]. Although the normal branch is less interesting phenomenologically, it is fundamentally more healthy and is the closest thing we have to a consistent non-linear completion of massive gravity. Here the graviton is a resonance of finite width, $1 / r_{c}$, as opposed to a massive field. At short distances, $r \ll r_{c}$, the brane dynamics do not feel the width of the resonance, and the theory resembles $4 \mathrm{D}$ GR. At large distances, $r \gg r_{c}$, however, the theory becomes five dimensional as the resonance effectively decays into continuum Kaluza-Klein modes. It is claimed that the Vainshtein mechanism works well on the normal branch of DGP, screening the longitudinal graviton without introducing any new pathological modes, in contrast to massive gravity [405, 399. The breakdown of classical perturbation theory at the Vainshtein scale can be linked to quantum fluctuations on the vacuum becoming strongly coupled at around $1000 \mathrm{~km}$ [839, 1073, 447.

\subsubsection{Action, equations of motion, and vacua}

The DGP model contains a single 3-brane embedded in an otherwise empty five dimensional bulk space-time. Generalisations that include a bulk cosmological constant and/or bulk branes have been studied [697, 1011. However, the original DGP action is given by

$$
S=M_{5}^{3} \int_{\mathcal{M}} d^{5} x \sqrt{-\gamma} \mathcal{R}+\int_{\partial \mathcal{M}} d^{4} x \sqrt{-g}\left[-2 M_{5}^{3} K+\frac{M_{4}^{2}}{2} R-\sigma+\mathcal{L}_{\text {matter }}\right],
$$

where, by $\mathbb{Z}_{2}$ symmetry across the brane, we identify the entire bulk space-time with two identical copies of $\mathcal{M}$, and the brane with the common boundary, $\partial \mathcal{M}$. The bulk metric is given by $\gamma_{a b}$, with corresponding Ricci scalar, $\mathcal{R}$, and $M_{5}$ is the Planck scale in the 
bulk. The induced metric on the brane is given by $g_{\mu \nu}$, and $K=g^{\mu \nu} K_{\mu \nu}$ is the trace of extrinsic curvature, $K_{\mu \nu}=\frac{1}{2} \mathcal{L}_{n} g_{\mu \nu}$. Here we define the unit normal to point into $\mathcal{M}$.

The brane has a bare vacuum energy, or tension, $\sigma$, and additional matter contributions contained in $\mathcal{L}_{\text {matter. }}$. However, the key feature in the brane Lagrangian is the induced curvature term, given by $M_{4}^{2} R$. Such a term can be generated by matter loop corrections [161, or finite width effects [263. The mass scale, $M_{4}$, is taken to be Planckian, $\sim 10^{18} \mathrm{GeV}$. There is a hierarchy between this scale and the bulk Planck scale, $M_{4} \gg M_{5}$, which has proven difficult to derive from fundamental theory. Nevertheless, the hierarchy enables us to identify a crossover scale, $r_{c} \sim M_{4}^{2} / 2 M_{5}^{3}$, below which the theory looks four dimensional, and above which it looks five dimensional.

The bulk equations of motion are given by the vacuum Einstein equations,

$$
\mathcal{G}_{a b}=\mathcal{R}_{a b}-\frac{1}{2} \mathcal{R} \gamma_{a b}=0,
$$

and the boundary conditions at the brane are given by the Israel junction conditions,

$$
2 M_{5}^{2}\left(K_{\mu \nu}-K g_{\mu \nu}\right)=M_{4}^{2} G_{\mu \nu}+\sigma g_{\mu \nu}-\mathcal{T}_{\mu \nu},
$$

where $\mathcal{T}_{\mu \nu}=-\frac{2}{\sqrt{-g}} \frac{\delta}{\delta g^{\mu \nu}} \int_{\partial \mathcal{M}} d^{4} x \sqrt{-g} \mathcal{L}_{\text {matter }}$ is the energy-momentum tensor for the additional matter.

Let us now consider the vacua of this theory, corresponding to maximally symmetric brane solutions with $\mathcal{T}_{\mu \nu}=0$. For a given tension, there exist two distinct vacua [394, 398. Assuming $\sigma>0$ for definiteness, one finds that these correspond to de Sitter branes with intrinsic curvature,

$$
H=\frac{M_{5}^{3}}{M_{4}^{2}}\left[\epsilon+\sqrt{1+\frac{M_{4}^{2} \sigma}{6 M_{5}^{3}}}\right],
$$

where $\epsilon= \pm 1$. In conformal coordinates, the full solution can be written as

$$
d s^{2}=\bar{\gamma}_{a b} d x^{a} d x^{b}=e^{2 \epsilon H y}\left(d y^{2}+\bar{g}_{\mu \nu} d x^{\mu} d x^{\nu}\right),
$$

where $\bar{g}_{\mu \nu} d x^{\mu} d x^{\nu}=-d t^{2}+e^{2 H t} d \mathbf{x}^{2}$ is the $4 D$ de Sitter line-element written in Poincaré coordinates. The domain $\mathcal{M}$ corresponds to $0<y<\infty$, while the brane is located on the boundary at $y=0$.

The two branches of this solution are often referred to as the normal branch $(\epsilon=-1)$, and the self-accelerating branch $(\epsilon=1)$. The latter is so-called because as we take the limit of vanishing vacuum energy, $\sigma \rightarrow 0$, the metric on the brane is still asymptotically de Sitter. The limiting de Sitter curvature is given by the cross-over scale, $H=2 M_{5}^{3} / M_{4}^{2} \sim$ $1 / r_{c}$. If this were to account for dark energy today, the crossover from four to five dimensions would have to occur at the horizon scale, placing the fundamental Planck scale at $M_{5} \sim 10 \mathrm{MeV}$ or so. However, as we will discuss in Section 5.5.3, this branch of solution is unstable as it contains physical ghost excitations. The normal branch is asymptotically Minkowski in the limit $\sigma \rightarrow 0$, and does not suffer from a ghostly pathology. On this branch it is phenomenologically more interesting to have the crossover scale at distances below the horizon scale.

The distinction between branches is best understood by considering their embedding in the $5 D$ Minkowski bulk. In each case, the brane can be viewed as a $4 D$ hyperboloid 


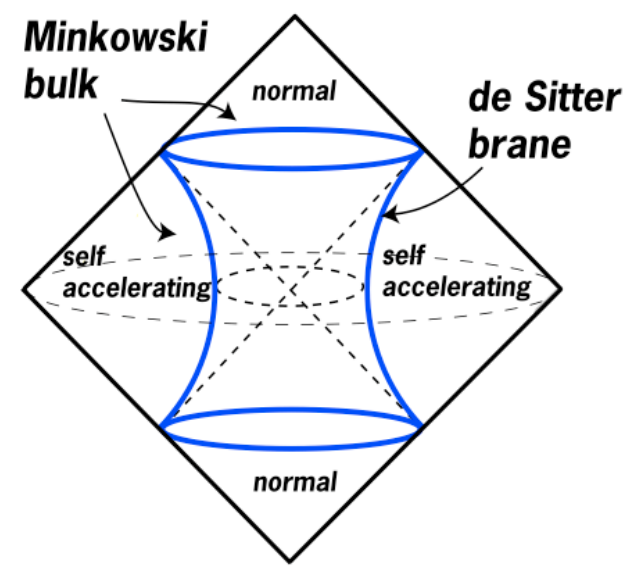

Figure 11: Embedding of a de Sitter brane in a flat 5D bulk, taken from 273 559. The braneworld volume is the hyperboloid in the Minkowski bulk. The normal branch corresponds to keeping the interior of the hyperboloid, while the self-accelerating branch corresponds to the exterior.

of radius $1 / H$ (see Figure 11). The choice of sign, $\epsilon= \pm 1$, corresponds to the choice in whether one retains the interior $(\epsilon=-1)$ or the exterior $(\epsilon=1)$ of the hyperboloid. Note that retaining the exterior ensures that the brane behaves like a domain wall with negative effective tension, even when $\sigma \geq 0$ [559]. This is an early warning sign that the self-accelerating branch could be pathological.

\subsubsection{Linear perturbations on the normal branch}

We now consider linear perturbations on the normal branch. For simplicity and brevity, let us consider the limiting case of vanishing vacuum energy, $\sigma=0$, corresponding to fluctuations about a Minkowski brane in a $5 D$ Minkowski bulk, $\gamma_{a b}=\eta_{a b}+h_{a b}$. It is convenient to choose harmonic gauge,

$$
\partial_{a} h_{b}^{a}=\frac{1}{2} \partial_{b} h_{a}^{a}
$$

so that the linearised bulk equations of motion take the simple form

$$
\left[\partial_{y}^{2}+\partial^{2}\right] h_{a b}=0
$$

where $\partial^{2}=\partial_{\mu} \partial^{\mu}$. Taking Fourier transforms with respect to the brane coordinates, so that

$$
Q(x, y) \rightarrow \tilde{Q}(p, y)=\frac{1}{(2 \pi)^{2}} \int d^{4} x e^{i p_{\mu} x^{\mu}} Q(x, y)
$$

it can be seen that the normalisable solution is given by

$$
\tilde{h}_{a b}(p, y)=e^{-p y} \tilde{h}_{a b}(p, 0) \text {. }
$$


We have not yet fully fixed the gauge as there is still a residual symmetry corresponding to gauge transformations of the form $x^{a} \rightarrow x^{a}+\xi^{a}$, where $\left(\partial_{y}^{2}+\partial^{2}\right) \xi^{a}=0$, or, equivalently, $\tilde{\xi}^{a}(p, y)=e^{-p y} \tilde{\xi}^{a}(p, 0)$. We now use $\xi^{y}$ to fix the brane position to lie at $y=0$, and $\xi^{\mu}$ to set $h_{\mu y}=0$. It follows from the $y$ component of Equation 739 that $h_{y y}=h$, where $h=\eta^{\mu \nu} h_{\mu \nu}$. The $\nu$ component of Eq. (739) then gives $\partial^{\mu} h_{\mu \nu}=\partial_{\nu} h$.

The junction conditions at the brane,

$$
2 M_{5}^{3} \delta\left(K_{\mu \nu}-K g_{\mu \nu}\right)=M_{4}^{2} \delta G_{\mu \nu}-\mathcal{T}_{\mu \nu},
$$

now yield the following solution for the metric on the brane:

$$
\tilde{h}_{\mu \nu}(p, 0)=\frac{2}{M_{4}^{2} p^{2}+2 M_{5}^{3} p}\left[\tilde{\mathcal{T}}_{\mu \nu}-\frac{1}{3} \tilde{\mathcal{T}} \eta_{\mu \nu}\right]+\text { pure gauge pieces. }
$$

At low energies, $p \ll 1 / r_{c}=2 M_{5}^{3} / M_{4}^{2}$, this becomes

$$
\tilde{h}_{\mu \nu}(p, 0) \sim \frac{1}{M_{5}^{3} p}\left[\tilde{\mathcal{T}}_{\mu \nu}-\frac{1}{3} \tilde{\mathcal{T}} \eta_{\mu \nu}\right],
$$

which suggests that the potential for a point mass will scale like $V(r) \propto 1 / r^{2}$ at large distances, $r \gg r_{c}$. This is consistent with $5 D$ gravity, and is to be expected for an infinitely large 5 th dimension. In contrast, at high energies, $p \gg 1 / r_{c}$, we have

$$
\tilde{h}_{\mu \nu}(p, 0) \sim \frac{2}{M_{4}^{2} p^{2}}\left[\tilde{\mathcal{T}}_{\mu \nu}-\frac{1}{3} \tilde{\mathcal{T}} \eta_{\mu \nu}\right]
$$

This can be compared with the propagator in four-dimensional General Relativity, $\tilde{h}_{\mu \nu}^{G R}=$ $\frac{2}{M_{p l}^{2} p^{2}}\left[\tilde{\mathcal{T}}_{\mu \nu}-\frac{1}{2} \tilde{\mathcal{T}} \eta_{\mu \nu}\right]$. Thus we almost have agreement. Indeed, since the scaling with momenta is the same, so the potential for a point mass will go like $V(r) \propto 1 / r$ for $r \ll r_{c}$. We can understand this as follows: The graviton mediating the interaction between two point particles along the brane is bound close to the brane by the induced curvature there. It is only at very large distances, when the induced curvature is insignificant, that the graviton is able to probe the extra dimension.

However, at this stage it would be premature to say that we have localisation of gravity at short distances. The problem lies with the tensor structure of the propagator. As we can see, the GR result differs from DGP in that it has a factor of $\frac{1}{2}$ as opposed to $\frac{1}{3}$. This is due to the longitudinal mode of the graviton propagating in DGP but not in GR, and will inevitably lead to disagreement with observation. To see this, consider the amplitude for the interaction between two sources, $\mathcal{T}_{\mu \nu}$ and $T_{\mu \nu}^{\prime}$, which is given by $\mathcal{A}=h_{\mu \nu} \mathcal{T}^{\prime \mu \nu}$. In GR, we have

$$
\tilde{\mathcal{A}}_{G R}=\frac{2}{M_{p l}^{2} p^{2}}\left[\tilde{T}_{\mu \nu}(p) \tilde{T}^{\prime \mu \nu}(-p)-\frac{1}{2} \tilde{\mathcal{T}}(p) \tilde{\mathcal{T}}^{\prime}(-p)\right],
$$

whereas in DGP, at high energies, we have

$$
\tilde{\mathcal{A}}_{D G P} \sim \frac{2}{M_{4}^{2} p^{2}}\left[\begin{array}{c}
\left.\tilde{T}_{\mu \nu}(p) \tilde{T}^{\prime \mu \nu}(-p)-\frac{1}{3} \tilde{\mathcal{T}}(p) \tilde{\mathcal{T}}^{\prime}(-p)\right] . \\
210
\end{array}\right.
$$


To get the same potential for the interaction of two non-relativistic sources, we require that ${ }^{50} M_{4}^{2}=\frac{4}{3} M_{p l}^{2}$. Now consider what happens when one of the sources is relativistic, say $\mathcal{T}_{\mu \nu}^{\prime}$. We then have $T^{\prime}=0$, and so the amplitudes differ by a factor of $\frac{3}{4}$. So, although we can recover the standard Newtonian potential, the prediction for light bending around the Sun differs from GR by a factor of $\frac{3}{4}$.

This is reminiscent of the vDVZ discontinuity in massive gravity [1243, 1296. In fact, it is sometimes said that DGP gravity (on the normal branch) is the closest thing we have to a non-linear completion of massive gravity. Strictly speaking, the behaviour is more like a massive resonance of width $1 / r_{c}$, with decay into a continuum of massive modes occurring at $r>r_{c}$. However, the similarities with massive gravity do suggest a possible resolution to this issue of tensor structure, and the resulting phenomenological problems. In massive gravity, it has been argued that the linearised analysis around a heavy source breaks down at the so-called Vainshtein radius, which can be much larger than the Schwarzschild radius [1241. Below the Vainshtein radius, it is claimed that non-linear effects help to screen the longitudinal scalar and match the theory to GR without a vDVZ discontinuity. Similar claims have been made in DGP gravity [399], as we will discuss in Section 5.5.4.

\subsubsection{Linear perturbations (and ghosts) on the self-accelerating branch}

Let us consider linearised perturbations and ghosts about the self-accelerating vacuum described in Section 5.5.1 (see also [273, 559, 1015, 546, 734]). To reveal the ghost in the cleanest way possible, it is convenient to retain non-zero tension on the background. It is also enough to consider vacuum fluctuations, so we will set $\mathcal{T}_{\mu \nu}=0$ for brevity (a more complete discussion including matter fluctuations can be found in [273]).

Recall that the self-accelerating background solution is given by the geometry in Eq. (738), with $\epsilon=+1$ and the brane positioned at $y=0$. A generic perturbation can be described by $\gamma_{a b}=\bar{\gamma}_{a b}+\delta \gamma_{a b}$, with the brane position shifted to $y=F(x)$. We will work in Gaussian Normal (GN) coordinates, so that

$$
\delta \gamma_{y y}=\delta \gamma_{\mu y}=0, \quad \text { and } \quad \delta \gamma_{\mu \nu}=e^{H y / 2} h_{\mu \nu}(x, y)
$$

The tensor $h_{\mu \nu}$ can be decomposed in terms of the irreducible representations of the $4 D$ de Sitter diffeomorphism group as

$$
h_{\mu \nu}=h_{\mu \nu}^{T T}+\nabla_{\mu} A_{\nu}+\nabla_{\nu} A_{\mu}+\nabla_{\mu} \nabla_{\nu} \phi-\frac{1}{4} \bar{g}_{\mu \nu} \square \phi+\frac{h}{4} \bar{g}_{\mu \nu}
$$

where $\nabla$ is the covariant derivative associated with the $4 D$ de Sitter metric, $\bar{g}_{\mu \nu}$. The tensor $h_{\mu \nu}^{T T}$ is transverse and trace-free, $\nabla^{\mu} h^{T T}{ }_{\mu \nu}=\bar{g}^{\mu \nu} h_{\mu \nu}^{T T}=0$, and $A_{\mu}$ is transverse, $\nabla^{\mu} A_{\mu}=0$. In addition, we have two scalars, $\phi$ and $h=\bar{g}^{\mu \nu} h_{\mu \nu}$.

Following a similar approach to the one outlined for Randall-Sundrum gravity in Section 5.3.5. we can now fix the position of the brane to be at $y=0$ while remaining in GN gauge by making the following gauge transformation:

$$
y \rightarrow y-F e^{-H y}, \quad \text { and } \quad x^{\mu} \rightarrow x^{\mu}-\frac{e^{-H y}}{H} \nabla^{\mu} F .
$$

\footnotetext{
${ }^{50}$ To verify this result, simply insert the appropriate energy-momentum tensors for non-relativistic sources, $\mathcal{T}_{\mu \nu}=\operatorname{diag}(\rho, 0,0,0)$, and $\mathcal{T}_{\mu \nu}^{\prime}=\operatorname{diag}\left(\rho^{\prime}, 0,0,0\right)$, in each expression for the amplitude, and match the two results.
} 
Although the brane position is now fixed at $y=0$, the original brane position $F(x)$ still enters the dynamics through a book-keeping term, $h_{\mu \nu}^{(F)}$, that modifies the metric perturbation as

$$
h_{\mu \nu} \rightarrow h_{\mu \nu}^{T T}+\nabla_{\mu} A_{\nu}+\nabla_{\nu} A_{\mu}+\nabla_{\mu} \nabla_{\nu} \phi-\frac{1}{4} \bar{g}_{\mu \nu} \square \phi+\frac{h}{4} \bar{g}_{\mu \nu}+h_{\mu \nu}^{(F)} .
$$

The book-keeping term is pure gauge in the bulk, and is given by

$$
h_{\mu \nu}^{(F)}=\frac{2}{H} e^{H y / 2}\left(\nabla_{\mu} \nabla_{\nu}+H^{2} \bar{g}_{\mu \nu}\right) F .
$$

We can now substitute our modified expression for $h_{\mu \nu}$ into the linearised fields equations in the bulk, $\delta \mathcal{G}_{a b}=0$, and on the brane, $2 M_{5}^{3} \delta\left(K_{\mu \nu}-K g_{\mu \nu}\right)=M_{4}^{2} \delta G_{\mu \nu}$. It turns out that the transverse vector, $A_{\mu}$, is a free field in the linearised theory and can be set to zero. In addition, the $y y$ and $y \mu$ equations in the bulk imply that one can consistently choose a gauge for which

$$
h=0, \quad \text { and } \quad\left(\square+4 H^{2}\right) \phi=0 .
$$

Note that we now have $h_{\mu \nu}=h_{\mu \nu}^{T T}+h_{\mu \nu}^{(\phi)}+h_{\mu \nu}^{(F)}$, where the contribution from $\phi(x, y)$ has been rewritten as

$$
h_{\mu \nu}^{(\phi)}=\left(\nabla_{\mu} \nabla_{\nu}+H^{2} \bar{g}_{\mu \nu}\right) \phi(x, y) .
$$

This mode is now entirely transverse and trace-free in its own right. In the absence of any additional matter on the brane, $\mathcal{T}_{\mu \nu}=0$, the same is true of the book-keeping mode, $h_{\mu \nu}^{(F)}$. This is because the trace of the Israel equation now implies that

$$
\left(\square+4 H^{2}\right) F=0 .
$$

The entire perturbation $h_{\mu \nu}(x, y)$ is therefore now completely transverse and trace-free. This greatly simplifies the bulk and brane equations of motion, giving

$$
\begin{aligned}
{\left[\square-2 H^{2}+\partial_{y}^{2}-\frac{9 H^{2}}{4}\right] h_{\mu \nu}(x, y)=0 } & \text { for } y>0, \\
{\left[M_{4}^{2}\left(\square-2 H^{2}\right)+2 M_{5}^{3}\left(\partial_{y}-\frac{3 H}{2}\right)\right] h_{\mu \nu}=0 } & \text { at } y=0^{+} .
\end{aligned}
$$

Variables in the tensor and scalar fields can now be separated as follows:

$$
h_{\mu \nu}^{T T}(x, y)=\sum_{m} u_{m}(y) \chi_{\mu \nu}^{(m)}(x), \quad \phi(x, y)=W(y) \hat{\phi}(x)
$$

where $\chi_{\mu \nu}^{(m)}$ is $4 D$ tensor field of mass $m$ satisfying $\left(\square-2 H^{2}\right) \chi_{\mu \nu}^{(m)}=m^{2} \chi_{\mu \nu}^{(m)}$. Note that $\hat{\phi}$ is a $4 D$ tachyonic field satisfying $\left(\square+4 H^{2}\right) \hat{\phi}=0$. This is a mild instability, related to the repulsive nature of inflating domain walls.

Assuming that the tensor and scalar equations of motion can be treated independently, we find that there is a continuum of normalisable tensor modes with mass $m^{2} \geq 9 H^{2} / 4$. In addition, there is also a discrete tensor mode with mass

$$
m_{d}^{2}=\frac{1}{r_{c}} \underset{212}{\left(3 H-\frac{1}{r_{c}}\right)},
$$


and normalisable wave-function $u_{m_{d}}(y)=\alpha_{m_{d}} e^{-y \sqrt{\frac{9 H^{2}}{4}-m_{d}^{2}}}$. Now, for positive brane tension, $\sigma>0$, one can easily check that $0<m_{d}^{2}<2 H^{2}$. For massive gravitons propagating in $4 D$ de Sitter, it is well known that masses lying in this range result in the graviton containing a helicity-0 ghost [605]. The lightest tensor mode in this case is therefore perturbatively unstable. For negative brane tension we find $m_{d}^{2}>2 H^{2}$, and there is no helicity- 0 ghost in the lightest tensor.

Now consider the scalar equations of motion. The first thing to note is that $h_{\mu \nu}^{(\phi)}$ behaves like a transverse trace-free mode with mass $m_{\phi}^{2}=2 H^{2}$, because $\left(\square-2 H^{2}\right) h_{\mu \nu}^{(\phi)}=$ $2 H^{2} h_{\mu \nu}^{(\phi)}$. Since none of the tensor modes have this mass, they are all orthogonal to $h_{\mu \nu}^{(\phi)}$. This means it was consistent to assume that the scalar and tensor equations of motion could indeed be treated independently. It turns out that the scalar has a normalisable wave-function $W(y)=e^{-H y / 2}$, and the $4 D$ scalar $\hat{\phi}$ is sourced by $F$ by the relation

$$
\hat{\phi}(x)=\alpha F(x), \quad \alpha=-\left[\frac{2 H-\frac{1}{r_{c}}}{H\left(H-\frac{1}{r_{c}}\right)}\right] .
$$

This is well defined for $\sigma \neq 0$, as in this case $H \neq 1 / r_{c} . h_{\mu \nu}^{(\phi)}(x, y)$ may now be thought of as a genuine radion mode, measuring the physical motion of the brane with respect to infinity. It does not decouple even though we only have a single brane. This property is related to the fact that the background warp factor, $e^{2 H y}$, grows as we move deeper into the bulk.

We have now identified the helicity-0 mode of the lightest tensor as a ghost when $\sigma>0$. When $\sigma<0$, a calculation of the $4 D$ effective action reveals the ghost to be the radion (see [273, 1015]). Given that there is always a ghost for non-zero tension, by continuity one may expect this to remain the case when $\sigma=0$.

\section{The limit of vanishing tension}

To study this limit more closely, let us first ask whether we can trust the above solutions in the limit where $\sigma \rightarrow 0$. In this limit $H \rightarrow 1 / r_{c}$, and the quantity $\alpha$ becomes ill-defined. To understand what has gone wrong, note that the mass of the lightest tensor has the limit $m_{d}^{2} \rightarrow 2 H^{2}$. This means that it is no longer orthogonal to the radion, $h_{\mu \nu}^{(\phi)}$, and so we cannot treat the tensor and scalar equations of motion independently. This behaviour can be traced back to an additional symmetry that appears in the linearised theory in the limit of vanishing brane tension. It is analogous to the "partially massless limit" in the theory of a massive graviton propagating in de Sitter space 605. In that theory, the equations of motion are invariant under the following redefinition of the graviton field:

$$
\chi_{\mu \nu}^{(\sqrt{2} H)}(x) \rightarrow \chi_{\mu \nu}^{(\sqrt{2} H)}(x)+\left(\nabla_{\mu} \nabla_{\nu}+H^{2} \bar{g}_{\mu \nu}\right) \psi(x) .
$$

This field redefinition has the effect of extracting out part of the helicity- 0 mode from $\chi_{\mu \nu}^{(\sqrt{2} H)}$, and as a result of the symmetry this mode disappears from the spectrum. In DGP gravity this shift must be accompanied by a shift in the scalar field, $\phi$,

$$
\begin{gathered}
\phi(x, y) \rightarrow \phi(x, y)-\alpha_{\sqrt{2} H} e^{-H y / 2} \psi(x)=\phi(x, y)-\lim _{\sigma \rightarrow 0} \alpha_{m_{d}} e^{-y \sqrt{\frac{9 H^{2}}{4}-m_{d}^{2}}} \psi(x), \\
213
\end{gathered}
$$


in order to render the overall perturbation, $h_{\mu \nu}(x, y)$, invariant. These $\psi$ shifts can be understood as extracting part of the helicity-0 mode from $\chi_{\mu \nu}^{(\sqrt{2} H)}$, and absorbing it into a renormalisation of $\phi$. The symmetry will have the effect of combining the helicity-0 mode and the radion into a single degree of freedom. It is only after fixing this $\psi$ symmetry that we can treat the scalar and tensor equations of motion independently of each another. We could also consider extracting the entire helicity- 0 mode and absorbing it into $\phi$, or vice versa.

Actually, there exists a clever choice of gauge that enables us to take a smooth limit as $\sigma \rightarrow 0$ [546. One can then readily calculate the $4 D$ effective action [273], and derive the corresponding Hamiltonian [546]. It turns out that the Hamiltonian is unbounded from below, signalling a ghost-like instability 546. This ghost is a combination of the radion and helicity- 0 mode, and represents the residual scalar degree of freedom left over after fixing the aforementioned $\psi$ symmetry.

\section{Ghosts}

We have shown that for any value of the brane tension, perturbations about the selfaccelerating branch of DGP contain a ghost. As explained in Section 2.1.3, the ghost will generate catastrophic instabilities as it couples universally with gravitational strength to the Standard Model fields and the remaining gravity modes. The existence of the ghost can be trusted as long as we can trust our effective description. It had been argued that this breaks down at the Hubble energy scale, casting doubt on the ghost's existence at sub-horizon distances [401. However the analysis of [741] suggests that the cut-off for the effective theory is actually at a much higher energy, being the same as that on the normal branch. As we will see in the next section, for fluctuations on the vacuum this corresponds to $\Lambda_{\text {cut-off }} \sim 10^{-13} \mathrm{eV}$. As this is well above the characteristic scale of the self-accelerating vacuum, $H_{0} \sim 10^{-33} \mathrm{eV}$, the ghost will cause this vacuum to be infinitesimally short-lived due to a divergent rate of particle creation ${ }^{51}$

It is also worth pointing out that the ghost can manifest itself beyond perturbation theory. In the presence of a self-accelerating brane one can accommodate a Schwarzschild bulk with negative mass, without introducing any naked singularities. This demonstrates that the five-dimensional energy is unbounded from below, as suggested by the perturbative analysis. Furthermore, standard Euclidean techniques indicate that the spontaneous nucleation of self-accelerating branes is unsuppressed. This is problematic even on the normal branch, and suggests that self-accelerating branes should be projected out the from the theory altogether [559].

\subsubsection{From strong coupling to the Vainshtein mechanism}

We now return to the normal branch of DGP gravity. In Section 5.5.2, we saw some similarities between this theory and massive gravity. Indeed, it happens that the brane to brane graviton propagator is given by

$$
D_{\mu \nu \alpha \beta}^{D G P}(p)=D_{\mu \nu \alpha \beta}^{\operatorname{massive}}\left(p, p / r_{c}\right),
$$

\footnotetext{
${ }^{51}$ Note that it has been argued that this choice of vacuum state explicitly breaks de Sitter invariance 653], and so one is free to impose a Lorentz non-invariant cut-off in the 3-momentum such that the creation rate is no longer divergent.
} 
where $D_{\mu \nu \alpha \beta}^{\operatorname{massive}}\left(p, m^{2}\right)$ is the propagator for $4 D$ massive gravity. Thus, just as massive gravity suffers from the vDVZ discontinuity [1243, 1296] as $m \rightarrow 0$, so does DGP gravity (normal branch) in the limit $p \ll 1 / r_{c}$. This means the linearised theory is not reduced to GR at short distances, but to a four-dimensional scalar-tensor theory. If this description can be trusted at solar system scales, then it leads to wildly inaccurate predictions for the bending of light around the Sun.

In massive gravity, it turns out that the linearised theory cannot necessarily be trusted at solar system scales. This is because it breaks down at the Vainshtein radius, $r_{V}^{\text {massive }} \sim\left(r_{s} / \mathrm{m}^{4}\right)^{1 / 5}$, where $r_{s} \sim 3 \mathrm{~km}$ is the Schwarzschild radius of the Sun. For quantum fluctuations in vacuo, the breakdown of classical perturbation theory at $r_{V}^{\text {massive }}$ translates into a strong coupling scale $\Lambda_{\text {cut-off }} \sim\left(M_{p l} m^{4}\right)^{1 / 5}$ [64]. Note that the equivalent scales in four-dimensional General Relativity are simply the Schwarzschild radius and the Planck scale.

\section{Strong coupling in DGP gravity}

The situation in DGP gravity (normal branch) is similar to the case of massive gravity, as we will now show. While aspects of the strong coupling problem were first identified in 696, 1073, the derivation of the strong coupling scale for quantum fluctuations on the Minkowski brane is most elegantly presented in 839. Here we review the results of this study ${ }^{52}$ (see also [1017]). Their analysis involves a computation of the boundary effective action 53 which is found to be

$$
\begin{array}{r}
\Gamma\left[h_{\mu \nu}, N_{\mu}, h_{y y}\right]=\int d^{4} x \frac{M_{4}^{2}}{4}\left[\frac{1}{2} h^{\mu \nu}\left(\partial^{2}-\frac{\sqrt{-\partial^{2}}}{r_{c}}\right) h_{\mu \nu}-\frac{1}{4} h\left(\partial^{2}-\frac{\sqrt{-\partial^{2}}}{r_{c}}\right) h\right. \\
\left.-\frac{1}{r_{c}} N^{\mu}\left(\sqrt{-\partial^{2}}+\frac{1}{r_{c}}\right) N_{\mu}+\frac{1}{2 r_{c}} h \sqrt{-\partial^{2}} h_{y y}-\frac{1}{r_{c}} N^{\mu} \partial_{\mu} h_{y y}-\frac{1}{4 r_{c}} h_{y y} \sqrt{-\partial^{2}} h_{y y}\right] \\
+\frac{1}{2} \int d^{4} x h_{\mu \nu} \mathcal{T}^{\mu \nu}+\Gamma_{i n t}\left[h_{\mu \nu}, N_{\mu}, h_{y y}\right],
\end{array}
$$

where $N_{\mu}=h_{\mu y}$ and $\partial^{2}=\partial_{\mu} \partial^{\mu}$. The function $\Gamma_{i n t}$ contains all the higher-order interaction terms to be discussed shortly. For now let us focus on the quadratic term. This can be diagonalised by means of the following field redefinition

$$
h_{\mu \nu}=\tilde{h}_{\mu \nu}+\pi \eta_{\mu \nu}, \quad N_{\mu}=\tilde{N}_{\mu}+r_{c} \partial_{\mu} \pi, \quad h_{y y}=-2 r_{c}\left(\sqrt{-\partial^{2}}+\frac{1}{r_{c}}\right) \pi .
$$

\footnotetext{
${ }^{52}$ Correcting a few typos along the way.

${ }^{53}$ The boundary effective action, $\Gamma[\phi]$, is obtained by integrating the full action, $S_{\mathcal{M}}[\Phi]+S_{\partial \mathcal{M}}[\phi]$, over bulk fields, $\Phi$, satisfying the boundary condition $\left.\Phi\right|_{\partial \mathcal{M}}=\phi$, i.e.

$$
e^{i \Gamma[\phi]}=\int_{\left.\Phi\right|_{\partial \mathcal{M}}=\phi} d[\Phi] e^{i\left(S_{\mathcal{M}}[\Phi]+S_{\partial \mathcal{M}}[\phi]\right)}
$$


The effective action can then be written as

$$
\begin{aligned}
\Gamma\left[\tilde{h}_{\mu \nu}, \tilde{N}_{\mu}, \pi\right]= & \int d^{4} x \frac{M_{4}^{2}}{4}\left[\frac{1}{2} \tilde{h}^{\mu \nu}\left(\partial^{2}-\frac{\sqrt{-\partial^{2}}}{r_{c}}\right) \tilde{h}_{\mu \nu}-\frac{1}{4} \tilde{h}\left(\partial^{2}-\frac{\sqrt{-\partial^{2}}}{r_{c}}\right) \tilde{h}\right. \\
& \left.-\frac{1}{r_{c}} \tilde{N}^{\mu}\left(\sqrt{-\partial^{2}}+\frac{1}{r_{c}}\right) \tilde{N}_{\mu}+3 \pi\left(\partial^{2}-\frac{\sqrt{-\partial^{2}}}{r_{c}}\right) \pi\right] \\
& +\frac{1}{2} \int d^{4} x\left(\tilde{h}_{\mu \nu} \mathcal{T}^{\mu \nu}+\pi \mathcal{T}\right)+\Gamma_{i n t}\left[\tilde{h}_{\mu \nu}, \tilde{N}_{\mu}, \pi\right]
\end{aligned}
$$

The next step is to write everything in terms of the canonically normalised fields

$$
\hat{h}_{\mu \nu}=\frac{M_{4}}{2} \tilde{h}_{\mu \nu}, \quad \hat{N}_{\mu}=\frac{M_{4}}{\sqrt{2 r_{c}}} \tilde{N}_{\mu}, \quad \hat{\pi}=\sqrt{\frac{3}{2}} M_{4} \pi .
$$

This gives

$$
\begin{aligned}
\Gamma\left[\hat{h}_{\mu \nu}, \hat{N}_{\mu}, \hat{\pi}\right]= & \int d^{4} x \frac{1}{2} \hat{h}^{\mu \nu}\left(\partial^{2}-\frac{\sqrt{-\partial^{2}}}{r_{c}}\right) \hat{h}_{\mu \nu}-\frac{1}{4} \hat{h}\left(\partial^{2}-\frac{\sqrt{-\partial^{2}}}{r_{c}}\right) \hat{h} \\
& -\frac{1}{2} \hat{N}^{\mu}\left(\sqrt{-\partial^{2}}+\frac{1}{r_{c}}\right) \hat{N}_{\mu}+\frac{1}{2} \hat{\pi}\left(\partial^{2}-\frac{\sqrt{-\partial^{2}}}{r_{c}}\right) \hat{\pi} \\
& +\int d^{4} x\left(\frac{1}{M_{4}} \hat{h}_{\mu \nu} \mathcal{T}^{\mu \nu}+\frac{1}{\sqrt{6} M_{4}} \hat{\pi} \mathcal{T}\right)+\Gamma_{\text {int }}\left[\hat{h}_{\mu \nu}, \hat{N}_{\mu}, \hat{\pi}\right] .
\end{aligned}
$$

Turning our attention to the interaction piece, we note that it contains terms that schematically take the form

$$
\int d^{4} x M_{5}^{3} \sqrt{-\partial^{2}}\left(h_{\mu \nu}\right)^{a}\left(N_{\mu}\right)^{b}\left(h_{y y}\right)^{c}, \quad \int d^{4} x M_{4}^{2}{\sqrt{-\partial^{2}}}^{2}\left(h_{\mu \nu}\right)^{d},
$$

where $a+b+c \geq 3$, and $d \geq 3$. By writing these in terms of the canonically normalised fields one can easily check that the largest interaction comes from the term with $a=0$ and $b+c=3$. Indeed, for $\sqrt{-\partial^{2}} \gg 1 / r_{c}$, the interaction term goes like [839, 1017]

$$
\Gamma_{\text {int }}=-\frac{1}{3 \sqrt{6} \Lambda^{3}} \int d^{4} x(\partial \hat{\pi})^{2} \partial^{2} \hat{\pi}+\text { sub-leading interactions, }
$$

where

$$
\Lambda=\left(M_{4} / r_{c}^{2}\right)^{1 / 3} .
$$

This corresponds to the scale at which quantum fluctuations in vacuum become strongly coupled. For $M_{4} \sim M_{p l}$, and $r_{c} \sim 1 / H_{0}$, we have $\Lambda \sim 10^{-13} \mathrm{eV} \sim 1 /(1000 \mathrm{~km})$. In other words, for scattering processes above $10^{-13} \mathrm{eV}$ perturbative quantum field theory in a vacuum is no longer well defined. Of course, it is important to realise that we do not actually live in a vacuum, and it has been demonstrated that the strong coupling scale can be raised on curved backgrounds 962. At the surface of the earth, the new scale corresponds to about $10^{-5} \mathrm{eV}$, indicating the presence of large quantum corrections to the $\pi$ field at distances below a centimetre. This will not impact much on table 
top experiments, however, as the $\pi$ field is expected to be overwhelmed by the classical graviton at this scale, due to Vainshtein screening.

\section{The $\pi$-Lagrangian}

To see the emergence of the new strong coupling scale most succinctly, it is convenient to take the limit in which the troublesome $\pi$ field decouples from the graviton. This will also help us in our discussion of the breakdown of classical perturbation theory around heavy sources and the Vainshtein effect. In any event, we take the so-called decoupling limit 962

$$
M_{4}, r_{c}, \mathcal{T}_{\mu \nu} \rightarrow \infty, \quad \text { and } \quad \Lambda, \frac{\mathcal{T}_{\mu \nu}}{M_{4}}=\text { fixed }
$$

so that all the sub-leading interactions in Eq. 770 go to zero. The limiting theory should be valid at intermediate scales,

$$
\max \left(L_{4}, r_{s}\right) \ll r \ll r_{c},
$$

where $L_{4} \sim 1 / M_{4}$ is the Planck length, and $r_{s}$ is the Schwarzschild radius of the source (if present). The action goes like

$$
\Gamma \sim \int d^{4} x\left[\mathcal{L}_{G R}+\mathcal{L}_{\pi}\right]
$$

where

$$
\begin{aligned}
\mathcal{L}_{G R} & =\frac{M_{4}^{2}}{4}\left[\frac{1}{2} \tilde{h}^{\mu \nu} \partial^{2} \tilde{h}_{\mu \nu}-\frac{1}{4} \tilde{h} \partial^{2} \tilde{h}\right]+\frac{1}{2} \tilde{h}_{\mu \nu} \mathcal{T}^{\mu \nu}, \\
\mathcal{L}_{\pi} & =\frac{M_{4}^{2}}{4}\left[3 \pi \partial^{2} \pi-r_{c}^{2}(\partial \pi)^{2} \partial^{2} \pi\right]+\frac{1}{2} \pi \mathcal{T},
\end{aligned}
$$

and we have set the free field $N_{\mu}=0$. Note that $\mathcal{L}_{G R}$ is just the standard Einstein-Hilbert Lagrangian, expanded to quadratic order about Minkowski space. The modification to GR is encoded in $\mathcal{L}_{\pi}$. This is often referred to as the $\pi$-Lagrangian and much of the interesting phenomenology of DGP gravity, from strong coupling and Vainshtein effects on the normal branch to ghosts on the self-accelerating branch, can be studied using this Lagrangian.

In vacuum $\left(\mathcal{T}_{\mu \nu}=0\right)$ the $\pi$-Lagrangian possesses a symmetry $\pi \rightarrow \pi+a_{\mu} x^{\mu}+b$, where $a_{\mu}$ and $b$ are constants. This is sometimes referred to as Galilean invariance and is the inspiration for galileon models 963. The most general Galilean invariant Lagrangians and will be studied in detail in Section 4.4.

We now return to the question of the strong coupling scale in the theory described by Eq. 774 in the presence of a classical source. The first thing to note is that our classical background is no longer the vacuum, but the solution $\pi=\pi_{c l}(x)$ of the classical field equations

$$
3 \partial^{2} \pi-r_{c}^{2}\left[\left(\partial_{\mu} \partial_{\nu} \pi\right)\left(\partial^{\mu} \partial^{\nu} \pi\right)-\left(\partial^{2} \pi\right)^{2}\right]=-\frac{\mathcal{T}}{M_{4}^{2}} .
$$

Our interest now lies in the quantum fluctuations, $\pi=\pi_{c l}(x)+\varphi(x)$, about this solution. Plugging this into the Lagrangian given in Eq. 774 we find

$$
\mathcal{L}_{\pi}=\frac{M_{4}^{2}}{4}\left[-Z^{\mu \nu}(x) \partial_{\mu} \varphi \partial_{\nu} \varphi-r_{c}^{2}(\partial \varphi)^{2} \partial^{2} \varphi\right]+\frac{1}{2} \varphi \delta \mathcal{T},
$$


where

$$
Z^{\mu \nu}(x)=3 \eta^{\mu \nu}-2 r_{c}^{2}\left(\partial^{\mu} \partial^{\nu}-\eta^{\mu \nu} \partial^{2}\right) \pi_{c l} .
$$

For non-trivial solutions $\pi_{c l}(x)$ is not constant, and so neither is $Z^{\mu \nu}(x)$. However, assuming the background varies slowly (relative to the fluctuations) we can treat $Z^{\mu \nu}$ as approximately constant in a neighbourhood of a point in space-time. Further assuming that the eigen-values of $Z_{\nu}^{\mu}(x)$ are all of similar magnitude, $\sim Z(x)$, we can identify a localised strong coupling scale,

$$
\Lambda_{*}(x) \sim \Lambda \sqrt{Z(x)} \gg \Lambda .
$$

As we will see shortly, for spherically symmetric solutions inside the Vainshtein radius, $r_{V} \sim\left(r_{s} r_{c}^{2}\right)^{1 / 3}$, we have $\pi_{c l} \sim \sqrt{r_{s} r} / r_{c}$, and so $Z(r) \sim\left(r_{V} / r\right)^{3 / 2}$. On the sphere of radius $r$ the local strong-coupling scale is

$$
\Lambda_{*}(r) \sim \Lambda\left(\frac{r_{V}}{r}\right)^{3 / 4} .
$$

It follows that the classical background, $\pi_{c l}$, ceases to make sense below a critical length, $r_{\min }$, where $r_{\min } \Lambda_{*}\left(r_{\min }\right) \sim 1$. This is because scattering processes would need to exceed the local strong coupling scale to probe the structure of the background at $r<r_{\min }$. This short distance cut-off will typically lie well within the Schwarzschild radius of the source, and therefore outside of the regime of validity of the $\pi$-Lagrangian.

We should also consider the implication of the local strong coupling scale for energetic processes taking place on the surface of the Earth. Here the Earth's gravitational field has the dominant effect, so we have $\Lambda_{*} \sim\left(\sqrt{r_{c} / r_{H}}\right) 10^{-5} \mathrm{eV}$, where $r_{H} \sim 1 / H_{0} \sim 10^{26} \mathrm{~m}$ is the current Hubble radius. For $r_{c} \sim r_{H}$ this means the fluctuations in the $\pi$ field becomes strongly coupled at around $1 \mathrm{~cm}$. However it is important to realise that it is only the $\varphi$ self-interactions that grow large at this scale. The direct coupling to the graviton and the coupling to matter are negligible, with the latter going like $G_{4} / Z(r) \sim 10^{-15} G_{4}$. Although the scalar fluctuations enter a quantum fog at the centimetre scale they do not spoil our classical description as this is dominated by the graviton, which is fifteen orders of magnitude larger 962 .

Finally, let us note another important feature of the scalar dynamics: As Poincaré invariance is broken by the classical background, $\pi_{c l}$, the fluctuations no longer have to propagate on the light cone. Indeed, one can explicitly show that angular fluctuations about spherically solutions will be super-luminal, causing problems for causality [9, 609].

\section{The Vainshtein effect in DGP gravity}

Let us now consider the Vainshtein effect directly. As we will see, this is a mechanism in which an additional scalar mode is screened at short distances by non-linear interactions, thereby eliminating the troublesome vDVZ discontinuity. To see how the scalar is screened in DGP gravity, we consider the classical solution to the field equations around a heavy non-relativistic source, $T_{\mu \nu}=\operatorname{diag}(\rho(r), 0,0,0)$, assuming spherical symmetry for simplicity. If we are to screen the scalar and recover GR at a given scale, the classical graviton solution, $\tilde{h}_{\mu \nu}^{(c l)}$, should dominate over the corresponding scalar solution, $\pi_{c l}$. In other words, we should schematically have

$$
\begin{gathered}
\left|\tilde{h}_{\mu \nu}^{(c l)}\right| \gg\left|\pi_{c l}\right| . \\
218
\end{gathered}
$$


Since $\tilde{h}_{\mu \nu}^{(c l)}$ is derived from Eq. 773 , it is just the standard linearised GR solution given by the Newtonian potential,

$$
\left|h_{\mu \nu}^{(c l)}\right| \sim \frac{r_{s}}{r},
$$

where $r_{s}=2 G_{4} M$ is the Schwarzschild radius of the source, $M=\int \rho(r) d V$ is its mass, and $G_{4}=1 / 8 \pi M_{4}^{2}$ is the four dimensional Newton's constant.

In general, the dynamics of the scalar are governed by the Field Equations (775). It follows that the spherically symmetric solution, $\pi_{c l}(r)$, around our heavy source satisfies

$$
\pi_{c l}^{\prime}+\frac{2 r_{c}^{2}}{3 r} \pi_{c l}^{\prime 2}=\frac{r_{s}}{3 r^{2}}
$$

where we have integrated once over a sphere centred at the origin and enclosing the entire source. Now at large distances we expect the linear term to dominate, giving a solution

$$
\pi_{c l}^{(\operatorname{lin})}(r)=-\frac{r_{s}}{3 r} .
$$

This scales in the same way as the corresponding graviton solution in Eq. (779), and so we have an $\mathcal{O}(1)$ modification of General Relativity. However, the linearised description breaks down once the non-linear piece becomes comparable, or, equivalently, when

$$
\left|\left(\pi_{c l}^{(\mathrm{lin})}\right)^{\prime}\right| \sim\left|\frac{r_{c}^{2}}{r}\left(\pi_{c l}^{(\mathrm{lin})}\right)^{\prime 2}\right| .
$$

Substituting our solution from Eq. 781, we see that the linearised theory breaks down at the Vainshtein radius,

$$
r_{V} \sim\left(r_{s} r_{c}^{2}\right)^{1 / 3}
$$

For the Sun we have $r_{s}^{\odot} \sim 3 \mathrm{~km}$, and so

$$
r_{V}^{\odot} \sim\left(\frac{r_{c}}{r_{H}}\right)^{2 / 3} 10^{18} \mathrm{~m}
$$

As long as the cross-over scale is not too far inside the cosmological horizon, the Vainshtein effect extends beyond the edges of the solar system, given by the Oort cloud at an average of $10^{16} \mathrm{~m}$ or so. For the earth we have $r_{s}^{\oplus} \sim 9 \mathrm{~mm}$, and so

$$
r_{V}^{\oplus} \sim\left(\frac{r_{c}}{r_{H}}\right)^{2 / 3} 10^{17} \mathrm{~m}
$$

In each case, the breakdown of classical perturbation theory has the same origin as the strong coupling of quantum fluctuations in vacuum, namely the self-interaction of the scalar, $\pi$, with scale-dependent coupling. Note that the Vainshtein scale can always be obtained from the strong coupling scale by trading the Planck length for the Schwarzschild radius of the source.

Now let us ask whether or not the scalar gets screened below the Vainshtein radius. For $r \ll r_{V}$, the linearised solution in Eq. (781) is no longer a good approximation. 
On the contrary, the non-linear part of Equation 780 will dominate, so that at short distances we have the solution

$$
\pi_{c l}^{(\text {nonlin) }}(r)=\frac{\sqrt{2 r_{s} r}}{r_{c}} .
$$

This implies that at short distances the scalar correction to the Schwarzschild solution goes like 567 .

$$
\frac{\delta V}{V} \sim \frac{\left|\pi_{c l}^{(\text {nonlin })}\right|}{\left|h_{\mu \nu}^{(c l)}\right|} \sim\left(\frac{r}{r_{V}}\right)^{3 / 2} .
$$

Given that $r_{V} \rightarrow \infty$ as $r_{c} \rightarrow \infty$, we see that the scalar does appear to get screened in this limit, and that one is able to recover GR without any vDVZ discontinuity.

We can now use the correction to the Newtonian force to place bounds on the crossover scale. At $r \sim 5 \mathrm{AU}$ fractional corrections to the Sun's gravitational field should be $\lesssim 10^{-8}$ [1197], which implies $r_{c} \gtrsim 10^{-4} r_{H}$. Similarly, the corrections to the Earth's gravitational field may have an observable effect on the precession of the moon, where corrections to the potential go like $\delta V /\left.V\right|_{\text {moon }} \sim 10^{-13} r_{H} / r_{c}$ [451.

While we have demonstrated aspects of the Vainshtein mechanism at the level of the $\pi$-Lagrangian, for simplicity, it is worth noting that it was originally discussed using the full theory [399, 567]. In [399] it was emphasised how one must choose the correct expansion parameter at a given scale. Standard perturbation theory corresponds to performing an expansion in $r_{s} / r$, but this is not a good expansion parameter in the limit $r_{c} \rightarrow \infty$, as then the next-to-leading order terms become singular. At short distances, the claim is that one should expand in powers of $r / r_{V}$ around the Schwarzschild solution [567, so the result can be smoothly patched onto the standard perturbative solution in some neighbourhood of the Vainshtein radius 399.

It is fair to say that the Vainshtein mechanism has not yet been fully explored as a viable concept. While it is clear that there is a breakdown of linear perturbation theory at the Vainshtein radius in both massive gravity and DGP, the arguments for a smooth transition to GR are at best promising, but hardly conclusive. N-body simulations of large-scale structure in DGP gravity, however, support the case for its successful implementation [1121, 269]. We will discuss this further in the next section.

One should also worry about the elephant problem ${ }^{54}$ An elephant is an extended object made up of many point particles each with their own Vainshtein radius. Is the Vainshtein radius of the elephant the same as the Vainshtein radius of a point particle with the same mass located at the centre of mass of the elephant? While this may not be a problem when the size of the source particle distribution is much smaller than the Vainshtein radius, it clearly becomes an issue when the particles are well distributed over the whole Vainshtein sphere. How does one account for such many particle systems? The answer is not known and given the role of non-linearities in the Vainshtein mechanism the problem may well be very complicated, especially in the full covariant theory. See 672 for a recent discussion.

\footnotetext{
${ }^{54}$ We thank Nemanja Kaloper for this colourful observation.
} 


\subsubsection{DGP cosmology}

In this section we describe DGP cosmology, from the FLRW background, to linear perturbations and non-linear studies. A thorough review of DGP cosmology up to 2006 can be found in 834 .

As discussed in Section 5.4.1, Shiromizu, Maeda and Sasaki 1140 found the $4 D$ Einstein equations of a 3 -brane world embedded in a $5 D$ bulk with $Z_{2}$ symmetry. Applying this formalism to DGP we get

$$
G_{\mu \nu}=\left(16 \pi G r_{c}\right)^{2} \Pi_{\mu \nu}-E_{\mu \nu}
$$

where

$$
\Pi_{\mu \nu}=-\frac{1}{4} \tilde{T}_{\mu \lambda} \tilde{T}_{\nu}^{\lambda}+\frac{1}{12} \tilde{T} \tilde{T}_{\mu \nu}+\frac{1}{24}\left[3 \tilde{T}^{\alpha \beta} \tilde{T}_{\alpha \beta}-\tilde{T}^{2}\right] g_{\mu \nu}
$$

and

$$
\tilde{T}_{\mu \nu}=\mathcal{T}_{\mu \nu}-\frac{1}{8 \pi G} G_{\mu \nu}
$$

The Bianchi identities give

$$
\nabla_{\nu} E_{\mu}^{\nu}=\left(16 \pi G r_{c}\right)^{2} \nabla_{\nu} \Pi_{\mu}^{\nu}
$$

while the matter stress-energy tensor satisfies local energy-momentum conservation: $\nabla_{\nu} T_{\mu}^{\nu}=0$.

The cosmological solutions for an FLRW space-time were found by Deffayet 394] by considering the embedding of the DGP brane into a Minkowski bulk. This corresponds to setting $E_{\mu \nu}=0$ and plugging an FLRW metric into our field equations $(788)$. Note that the global structure of the cosmological solutions has been investigated by Lue 833, while some exact solutions in special cases have been found by Dick [420. If one were to generalise the bulk to include $5 D$ Schwarzschild, one can run into an unusual class of singularity on account of the branch cut in Friedmann equation [1142, 559. Cosmological branes in generalised DGP gravity can also mimic a phantom equation of state [1086, 835.

Staying with the simplest case of a Minkowski bulk and a tensionless brane, the 00 component of the $4 D$ field equations (with $E_{\mu \nu}=0$ ) gives the Friedmann equation as

$$
H^{2}+\frac{\kappa}{a^{2}}-\frac{\epsilon}{r_{c}} \sqrt{H^{2}+\frac{\kappa}{a^{2}}}=\frac{8 \pi G}{3} \rho,
$$

where $\epsilon=-1$ for the normal branch, and $\epsilon=1$ for the self-accelerating branch. Clearly, if $\rho=0$ then the normal branch implies Minkowski space, while the self-accelerating branch gives de Sitter space with Hubble constant $H_{\infty}=\frac{1}{r}$. This self-accelerating model has been proposed as an alternative to dark energy [398], but as we have already seen, the theory contains a ghost in its perturbative spectrum. A phenomenological extension of Eq. 792 has been considered by Dvali and Turner [453, where for $\kappa=0$ and $\epsilon=1$ the term $\frac{H^{2 \alpha}}{r_{c}^{2-\alpha}}$ is added to Eq. 792 .

After using Eq. 792 the $i j$ component can be re-worked into a modified Raychaudhuri equation, as

$$
2 \frac{d H}{d t}+3 H^{2}+\frac{\kappa}{a^{2}}=-\frac{3 H^{2}+\frac{3 \kappa}{a^{2}}-2 \epsilon r_{c} \sqrt{H^{2}+\frac{\kappa}{a^{2}}} 8 \pi G P}{1-2 \epsilon r_{c} \sqrt{H^{2}+\frac{\kappa}{a^{2}}}} .
$$


It is instructive to cast the background equations into a form resembling an effective dark-energy fluid with density $\rho_{E}$ and pressure $P_{E}$. This gives

$$
X=8 \pi G \rho_{E}=\frac{3 \epsilon}{r_{c}} \sqrt{H^{2}+\frac{\kappa}{a^{2}}},
$$

and

$$
Y=8 \pi G P_{E}=-\epsilon \frac{\frac{d H}{d t}+3 H^{2}+\frac{2 \kappa}{a^{2}}}{r_{c} \sqrt{H^{2}+\frac{\kappa}{a^{2}}}}
$$

so that we can define the equation of state, $w_{E}$, as

$$
w_{E}=\frac{P_{E}}{\rho_{E}}=-\frac{\frac{d H}{d t}+3 H^{2}+\frac{2 \kappa}{a^{2}}}{3 H^{2}+\frac{3 \kappa}{a^{2}}} .
$$

The Bianchi identities, and energy-momentum conservation, ensure that

$$
\frac{d \rho_{E}}{d t}+3 H\left(\rho_{E}+P_{E}\right) \equiv 0
$$

As a consequence, during radiation domination we get $w_{E} \approx-\frac{1}{3}$, during matter domination we get $w_{E} \approx-\frac{1}{2}$, during a possible spatial curvature dominated era we get $w_{E} \approx-\frac{2}{3}$, and eventually during the self-accelerating era we get $w_{E} \rightarrow-1$.

Let us now look at the perturbed FLRW universe in DGP. Perturbation theory for DGP was originally worked out in detail by Deffayet [395, and has since been developed by a number of authors. Although braneworld cosmological perturbation theory is discussed in detail in Section 5.4.3, we will now present the formalism for DGP gravity explicitly, in the interests of self-containment.

Let us consider scalar perturbations in the conformal Newtonian gauge. The components of $E_{\mu \nu}$ are ther 5

$$
\begin{aligned}
E^{0}{ }_{0} & =-\mu_{E}, \\
E^{0}{ }_{i} & =-\vec{\nabla}_{i} \Theta_{E}, \\
E^{i}{ }_{j} & =\frac{1}{3} \mu_{E} \delta^{i}{ }_{j}+D^{i}{ }_{j} \Sigma_{E} .
\end{aligned}
$$

We find the perturbed Einstein equations are then given by

$$
\begin{gathered}
2(\Delta+3 \kappa)-6 \mathcal{H}\left(\Phi^{\prime}+\mathcal{H} \Psi\right)=A_{D} 8 \pi G a^{2} \rho \delta+B_{D} a^{2} \mu_{E} \\
\left(\Phi^{\prime}+\mathcal{H} \Psi\right)=A_{D} 8 \pi G a^{2}(\rho+P) \theta+B_{D} a^{2} \Theta_{E} \\
\Phi^{\prime \prime}+\mathcal{H} \Psi^{\prime}+2 \mathcal{H} \Phi^{\prime}+\left(2 \mathcal{H}^{\prime}+\mathcal{H}^{2}+\frac{1}{3} \Delta\right) \Psi-\left(\frac{1}{3} \Delta+\kappa\right) \Phi \\
=A_{D} 4 \pi G a^{2} \delta P-\frac{B_{D}}{2}\left[\left(1+w_{E}\right) A_{D}\left(8 \pi G a^{2} \rho \delta+a^{2} \mu_{E}\right)-\frac{a^{2}}{2} \mu_{E}\right],
\end{gathered}
$$

\footnotetext{
${ }^{55}$ Note that this is consistent with our general analysis for brane world cosmological perturbations, provided one identifies $\mu_{E}=-8 \pi G_{4} \rho^{\text {weyl }} \delta^{\text {weyl }}, \Theta_{E}=-8 \pi G_{4}\left(\frac{4}{3} \rho^{\text {weyl }}\right) \theta^{\text {weyl }}, \Sigma_{E}=$ $-8 \pi G_{4}\left(\frac{4}{3} \rho^{w e y l}\right) \Sigma^{w e y l}$.
} 


$$
\Phi-\Psi=a^{2} \frac{r_{c}^{2}(X+3 Y) 8 \pi G(\rho+P) \Sigma-3 \Sigma_{E}}{3+r_{c}^{2}(X+3 Y)},
$$

where we recall that primes denote differentiation with respect to conformal time, and $\mathcal{H}=\frac{a^{\prime}}{a}=a H$. Note that we have set $A_{D}=\frac{2 X r_{c}^{2}}{2 X r_{-}^{2}-3}$ and $B_{D}=\frac{3}{2 X r_{-}^{2}-3}=A_{D}-1$, for simplicity. In the limit $r_{c} \rightarrow \infty$ one recovers the familiar perturbation equations of General Relativity from the above.

We can now use the Bianchi identities to find the field equations for $\mu_{E}$ and $\Theta_{E}$. They are

$$
\mu_{E}^{\prime}+4 \mathcal{H} \mu_{E}-\Delta \Theta_{E}=0
$$

and

$$
\begin{aligned}
& \Theta_{E}^{\prime}+4 \mathcal{H} \Theta_{E}-\frac{1}{3} \mu_{E}+\left(1+w_{E}\right)\left(\mu_{E}+3 \frac{\mathcal{H}}{a} \Theta_{E}\right) \\
& +\frac{\Delta+3 \kappa}{1+3 w_{E}}\left\{\frac{4}{3} \Sigma_{E}+2 \frac{1+w_{E}}{a^{2}}\left[\left(2+3 w_{E}\right) \Phi-\Psi\right]\right\}=0 .
\end{aligned}
$$

It is clear that the above equations are not closed due to the presence of the bulk anisotropic stress, $\Sigma_{E}$. Several authors have applied a variety of approximations to solve the linear perturbations in the DGP model. Sawicki and Carroll 1101 assumed that the Weyl perturbations are zero. It is clear, however, from Eq. 805 that this is not a consistent approximation.

To fully determine the DGP perturbations one must use the five-dimensional equations. For maximally symmetric $5 D$ space-times, the bulk scalar mode perturbations can be deduced using a single master variable, $\Omega$, as we discussed in Section 5.4 Assuming that the bulk cosmological constant is zero, the bulk metric is given by Equation 696, with

$$
\begin{gathered}
\lambda_{\alpha \beta}=d z^{2}-n^{2}(t, z) d t^{2}, \quad n(t, z)=1+\frac{\epsilon\left(H^{2}+\frac{d H}{d t}\right)}{\sqrt{H^{2}+\frac{\kappa}{a^{2}}}}|z|, \\
r(t, z)=a\left(1+\epsilon \sqrt{H^{2}+\frac{\kappa}{a^{2}}}|z|\right) .
\end{gathered}
$$

Using the Master Equation 705, we find

$$
\frac{\partial}{\partial t}\left[\frac{1}{n b^{3}} \frac{\partial \Omega}{\partial t}\right]-\frac{\partial}{\partial z}\left[\frac{n}{b^{3}} \frac{\partial \Omega}{\partial z}\right]-\frac{\Delta+3 \kappa}{b^{2}} \frac{n}{b^{3}} \Omega=0 .
$$

The energy-momentum tensor of the Weyl fluid on the brane can then be related to $\Omega$. For the case of a spatially flat universe $(\kappa=0)$ one finds

$$
\begin{aligned}
\mu_{E} & =-\left.\frac{1}{3} \frac{k^{4}}{a^{5}} \Omega\right|_{z=0}, \\
\Theta_{E} & =\left.\frac{1}{3} \frac{k^{2}}{a^{4}}\left(\frac{\partial \Omega}{\partial t}-H \Omega\right)\right|_{z=0}, \\
\Sigma_{E} & =-\left.\frac{1}{6 a^{3}}\left(3 \frac{\partial^{2} \Omega}{\partial t^{2}}-3 H \frac{\partial \Omega}{\partial t}+\frac{k^{2}}{a^{2}} \Omega-\frac{3}{H} \frac{d H}{d t} \frac{\partial \Omega}{\partial z}\right)\right|_{z=0},
\end{aligned}
$$


where $k$ is the 3 -momentum on the homogeneous background. Thus, in general one has to solve Eq. 808 with appropriate initial and boundary conditions, and then use Eq. (811) in the perturbed Einstein equations. In practise, one can however apply various approximations.

Koyama and Maartens 737 assume the small-scale approximation $k / a \gg r_{c}, \mathcal{H}$. Under this assumption Eq. (804) implies that $\Theta_{E}=0$. In quasi-static situations we also have $\partial_{t} \Omega \approx 0$ and $\frac{1}{H} \frac{d H}{d t} \ll \frac{1}{\mathcal{H}}$, so that the master equation becomes $\partial_{z}^{2} \Omega-\frac{2 \epsilon H}{n} \partial_{z} \Omega-$ $\frac{k^{2}}{a^{2} n^{2}} \Omega=0$. Assuming that the solution of this last equation is regular as $z \rightarrow \infty$, it can then be shown that $\Omega=\Omega_{b r}(1+\epsilon H z)^{-\frac{k}{a H}}$ when $a H / k \ll 1$. Therefore, using Eq. 811 one finds that $\mu_{E}=2 k^{2} \Sigma_{E}$ on the brane, in the quasi-static limit. Inserting this into Eq. 805 gives $\Sigma_{E}$, and therefore $\mu_{E}$, in terms of the potentials. This in turn allows us to eliminate all the Weyl perturbations, to get

$$
-k^{2} \Phi=4 \pi G\left(1-\frac{1}{3 \beta}\right) \rho a^{2} \delta_{M},
$$

and

$$
-k^{2} \Psi=4 \pi G\left(1+\frac{1}{3 \beta}\right) \rho a^{2} \delta_{M},
$$

where $\beta=1+2 \epsilon H r_{c} w_{E}$.

Sawicki, Song and $\mathrm{Hu} 1103$ propose a scaling ansatz to close the Bianchi identities, and solve the master equation on the self-accelerating branch near the cosmological horizon. This allows them to move away from the quasi-static regime. The ansatz they use is $\Omega=A(p) a^{s} G\left(z / z_{h o r}\right)$, where $z_{\text {hor }}=a H \int_{0}^{a} \frac{d \tilde{a}}{\tilde{a}^{2} H(\tilde{a})^{2}}$, and $s$ is an exponent that is approximately constant during times when a particular fluid dominates the expansion. The master equation then becomes an ordinary differential equation with independent variable $z / z_{\text {hor }}$, and can be solved iteratively by assuming the boundary conditions $G(0)=1$ and $G(1)=0$. They find that for super-horizon modes $s=6$ during radiation domination, decreasing to $s=4$ during matter domination, and finally approaching $s=1$ during $\Lambda$ domination. For all sub-horizon modes $s=3$, which reproduces the quasistatic approximation of Koyama and Maartens [737. By iteration, one can correct for the time-dependence of $s$, as is necessary during transitions between cosmological eras. Song has performed a similar analysis on the normal branch [1160.

Cardoso et al. 246 solve the master equation numerically by employing null coordinates in the $\{t, z\}$ plane, thus obtaining the linearised DGP solutions without resorting to any approximations. They find that the quasi-static approximation is valid to within $5 \%$ for $k \geq 0.01 h M p c^{-1}$. Seahra and $\mathrm{Hu}$ [1123] develop analytic solutions for both branches of DGP based on the scaling ansatz, and compare these with numerical solutions in 246. They find that the analytic/scaling solutions are accurate to within a few percent all the way to the horizon, and therefore that the use of the scaling ansatz in the observational constraints imposed in [482, 817] is justifiable.

The FLRW background evolution on the self-accelerating branch has been extensively tested, using a variety of different cosmological observations. Alcaniz [20] used measurements of the angular size of high redshift compact radio sources [577] to place constraints on $r_{c}$. He finds that $\frac{1}{4 r_{c}^{2} H_{0}^{2}} \geq 0.29$ at $1 \sigma$, with a best-fit value of $r_{c} \sim 0.94 H_{0}^{-1}$. Jain, Dev and Alcaniz [658] find that gravitationally lensed QSOs require $r_{c} \geq 1.14 H_{0}^{-1}$ 
at $1 \sigma$. Deffayet et al. 403 use SN-Ia from [1034, and the angular diameter distance to recombination of pre-WMAP CMB data, while assuming a flat universe. Their SN analysis gives $\frac{1}{4 r_{c}^{2} H_{0}^{2}}=0.17_{-0.02}^{+0.03}$ at $1 \sigma$, which translates to $r_{c}=1.21_{-0.09}^{+0.09} H_{0}^{-1}$. Including CMB data increases the preferred value of $\Omega_{M}$, and leads to a best fit model with $r \sim 1.4 H_{0}^{-1}$. Fairbairn and Goobar [478] use the SNLS SN-Ia data 67] and BAO data 467] to show that the self-accelerating model is not compatible with a flat universe at the $99 \%$ level. For the generalised model of [453] they find that $-0.8<\alpha<0.3$ at $1 \sigma$ (the self-accelerating model corresponds to $\alpha=1$ ). Maartens and Majerotto [846] used SN-Ia [1064, 67], a CMB shift parameter [1261]), and BAO data 467] to place constraints on the self-accelerating FLRW background. They find that the self-accelerating model is consistent with these data sets to within $2 \sigma$, but is worse fit than $\Lambda$ CDM. This puts tension on the self-accelerating model, but as they point out, it is not necessarily reliable to use the BAO data for models other than $\Lambda \mathrm{CDM}$, as $\Lambda \mathrm{CDM}$ is used throughout the analysis in [467. Rydbeck, Fairnbairm and Goobar [1080. repeated the analysis of 467 by including SN-Ia data from ESSENCE [893, 1280], and constraints on the CMB shift parameter from WMAP-3 1173] to disfavour the self-accelerating model at the $1-2 \sigma$ level, depending on whether a peculiar velocity error on the SN-Ia data is included or not. The inclusion of BAO data from [467] further supports this result.

Moving away from using distance measurements alone, a number of studies have been performed on the growth of linear structure using the small-scale quasi-static approximation of Koyama and Maartens [737. Song, Sawicki and $\mathrm{Hu}$ 1161] used the angular diameter distance to recombination from WMAP-3 [1173, SN-Ia data from 1064, 67, and constraints on the Hubble constant to exclude the spatially flat self-accelerating model at $3 \sigma$. By allowing non-zero spatial curvature, however, they show that consistency with the data can be improved, but is still marginally worse than spatially flat $\Lambda$ CDM. They then use data coming from BAOs, the linear growth of structure, and ISW and galaxy-ISW correlations to show that any self-accelerating models that shares the same expansion history as a quintessence-CDM models are strongly disfavoured. As these authors argue, one must properly take into account the perturbations on the selfaccelerating branch in order to make firm conclusion. Xia considered the generalised model of Dvali and Turner [453] in conjunction with Union SN-Ia data set [733], CMB distance measurements, GRB data [1106, and a collection of data on the growth of linear structure [598, 1248, 1203, 1071, 585, 352, 877]. Using the quasi-static approximation of Koyama- Maartens [737] to calculate the growth function, Xia finds $\alpha<0.686$ at $2 \sigma$. This excludes the self-accelerating value of $\alpha=1$.

Fang et al.. 482 perform a thorough test of the self-accelerating model using the PPF approach of 627, 630. Using CMB and large-scale structure data these authors find that the spatially flat model is a $5.3 \sigma$ poorer fit than $\Lambda \mathrm{CDM}$, while that open model is $4.8 \sigma$ worse. In the latter case non-zero spatial curvature improves the fits for distance measurements, but worsens those involving the growth of linear structure. One may speculate that changes to the initial power spectrum may be able to improve the this situation, but the required reduction in large-scale power also produces unacceptable reductions in power in the large-scale CMB polarisation spectrum. Changes of this type cannot therefore save the model. In a follow-up study Lombriser et al. 817] constrained both branches of the DGP model by employing the PPF description of the CMB and large-scale structure. These authors find that either brane tension or $\Lambda$ is required for 
these models to fit the data well, but that both cases the best fitting models are practically indistinguishable from $\Lambda \mathrm{CDM}$. They further find that the cross-over scale is $H_{0} r_{c}>3$ if spatial curvature is included, and $H_{0} r_{c}>3.5$ in the spatially flat case.

The growth index on small scales, $\gamma$, has been calculated by Wei using a Taylor series expansion in the fractional density parameters $\Omega_{D G P}$ and $\Omega_{\kappa}[1263$. Ferreira and Skordis have also calculated this quantity, within a more general frame-work of modified gravity models, to find $\gamma=\frac{11}{16}-\frac{7}{5632} \Omega_{D G P}+\frac{93}{4096} \Omega_{D G P}^{2}+O\left(\Omega_{D G P}^{3}\right)$ [501]. This analytic result is in excellent agreement with numerical studie $\$ 56$.

Although the DGP model is strongly constrained by the observations we have already discussed, it is still instructive to consider the substantial work that has been performed on constructing and studying non-linear structure formation scenarios in these models 836, 743, 1121, 269, 1112, 1111, 690, 1290, 1113, 1123. Lue, Scoccimarro and Starkman [836] have considered spherical perturbations on sub-horizon scales, and derived the gravitational force law in a collapsing top-hat model embedded in an expanding background. They find that the non-linear CDM density contrast evolves as

$$
\frac{d^{2} \delta_{M}}{d t^{2}}+\left[2 H-\frac{4}{3} \frac{1}{1+\delta_{M}} \frac{d \delta_{M}}{d t}\right] \frac{d \delta_{M}}{d t}=4 \pi G_{\mathrm{eff}} \bar{\rho}_{M} \delta_{M}\left(1+\delta_{M}\right)
$$

where

$$
G_{\mathrm{eff}}=G\left[1+\frac{2}{3 \beta} \frac{1}{\epsilon_{D}}\left(\sqrt{1+\epsilon_{D}}-1\right)\right],
$$

and $\epsilon_{D}=\frac{8}{9 \beta^{2}} \frac{\Omega_{M}}{\Omega_{D G P}^{2}} \delta_{M}=\frac{8}{9} \frac{\left(1+\Omega_{M}\right)^{2}}{\left(1+\Omega_{M}^{2}\right)^{2}} \Omega_{M} \delta_{M}$. Their model uncovers a transition point at $\epsilon_{D} \sim 1$, below which gravity behaves as in GR (in accordance with the Vainshtein mechanism). This gives a Vainshtein radius of $r_{\star}=\left(\frac{16 G M r_{c}^{2}}{9 \beta^{2}}\right)^{1 / 3}$, where $M$ is the mass of the spherically symmetric object. This result was subsequently re-derived by Koyama and Silva [741] without the restrictive assumptions of [836. It can be shown that the solutions to Eq. (814) are a factor of two larger than the corresponding solutions in theories that obey Birkhoff's law, and that have similar expansion histories.

The form of $G_{\text {eff }}$ in Eq. 815 is due to an additional degree of freedom in DGP: The brane-bending mode, as uncovered in 839,963 . To expand upon this we may rewrite the RHS of Eq. 814 using $4 \pi G_{\text {eff }} \delta \rho_{M}=\Delta \Psi$. The potential $\Psi$ can then be written in terms of a Poisson potential, $\Psi_{P}$, and a brane-bending mode, $\varphi$, as $\Psi=\Psi_{P}+\frac{1}{2} \varphi$. These two new potentials, $\Psi_{P}$ and $\varphi$, satisfy $\Delta \Psi_{P}=8 \pi G \delta \rho_{M}$, and $\Delta \varphi=8 \pi\left(G_{\text {eff }}-G\right) \delta \rho_{M}$. Ignoring non-local contributions, the brane-bending mode can then be shown to obey the following equation in the decoupling limit, and in the sub-horizon and quasi-static regime:

$$
\Delta \varphi+\frac{r_{c}^{2}}{3 \beta}\left[(\Delta \varphi)^{2}-\left(\vec{\nabla}_{i} \vec{\nabla}_{j} \varphi\right)\left(\vec{\nabla}^{i} \vec{\nabla}^{j} \varphi\right)\right]=\frac{8 \pi G}{3 \beta} \delta \rho_{M} .
$$

This equation is closely related to the Field Equation (775), following from the $\pi$ Lagrangian discussed in Section 5.5.4

\footnotetext{
${ }^{56}$ The terms $\frac{3}{16} \Omega_{D G P}$ and $\frac{1}{16} \Omega_{D G P}^{2}$ in the Wei 1263 result are incorrect, and appear to have come from erroneously dropping terms proportional to $\Omega_{D G P}$ when going from Eq. (21) to Eq. (22) of that paper.
} 
Schmidt, Hu and Lima [1113] used the spherical collapse model in DGP to study the halo mass function, bias and the non-linear matter power spectrum. They find that top-hat spherical collapse in DGP requires a new, more general method for defining the virial radius that does not rely on energy conservation. To obtain the comoving number density of halos per logarithmic interval in the virial mass, and the linear bias, they use the Sheth-Tormen method [1137, while they use the Navarro-Frenk-White 946] form for halo profiles. In this way they find that the spherical collapse model agrees well with the halo mass function and bias obtained from N-body simulations, for both the normal and the self-accelerating branch. For the non-linear power spectrum, the spherical collapse model in the self-accelerating branch also matches the simulation results very well. This is not true, however, for the normal branch, although even in this case the spherical collapse model predictions are better than those obtained from HALOFIT [1156].

N-body simulations of DGP have been conducted by three independent groups: Schmidt [1112, 1111, Chan and Scoccimarro [269, and Khoury and Wyman 690. The general result, common to all of these studies, is that the brane-bending mode on the self-accelerating branch provides a repulsive force that greatly suppresses the growth of structure, while the opposite effect occurs on the normal branch. All three simulations also display the Vainshtein effect.

The N-body simulations conducted by Schmidt were on both the self-accelerating 1112, and the normal branch [1111. Rather than assuming Eq. 815, Schmidt use a relaxation solver on Eq. 816 to show that the Vainshtein effect is recovered without making any assumptions about symmetry. Specifically, he finds that the Vainshtein effect is weakened for non-spherically symmetric situations, and in general sets in at smaller scales than is found in 690]. Like [690, however, he also finds that the HALOFIT model does not correctly describe the non-linear DGP matter power spectrum. Schmidt then proceeds to calculate the halo mass function, and shows that the abundance of massive halos in self-accelerating DGP is much smaller than in CDM models. This last result puts strong constraints on the self-accelerating model from cluster abundance measurements, independent from of the CMB and large-scale structure constraints discussed above [482, 817]). For the normal branch, structure is enhanced, and the abundance of massive halos is larger than a CDM model [1111. In this case, the halo profiles were also obtained, and departures from the predictions of GR were seen outside of the halo virial radius. Finally, Schmidt calculates the bispectrum in both the normal and selfaccelerating branches. The self-accelerating (normal) branch bispectrum is found to be enhanced (suppressed) for equilateral configurations, but not for squeezed configurations. This is in agreement with [269, and illustrates the diminishing strength of the Vainshtein effect for squashed matter configurations.

Scoccimarro [1121] derives the linear and non-linear equations for the growth of structure in DGP without using the Mukohyama formalism. This results in a set of equations that includes non-local terms. For example, in the quasi-static limit it is found that

$$
\left[\Delta-\frac{a}{r_{c}} \sqrt{-\Delta}\right](\Phi+\Psi)=8 \pi G a^{2} \rho \delta,
$$

rather than $\Delta(\Phi+\Psi)=8 \pi G a^{2} \rho \delta$, as implied by adding the quasi-static expressions given in Eqs. 812 and 813 . Scoccimarro finds that in the linearised quasi-static limit the bulk behaviour decouples from the brane behaviour, and thus that the nonlocal operators can be safely ignored. This ensures the validity of the Koyama-Maartens 
result 737 on small scales. On larger scales, however, the non-local terms become more important. On very scales the linear approximation for the brane-bending mode breaks down, and Scoccimarro finds a non-local and non-linear equation for the potential, $\Psi$, and the density perturbation, $\delta$. Chan and Scoccimarro 269] perform N-body simulations by accounting for the non-local operators $\sqrt{-\Delta}$ and $1 / \sqrt{-\Delta}$. These operators contribute to the equation for the brane bending mode, $\varphi$, and the potential, $\Psi$ [1121. They uncover the Vainshtein mechanism through a broad transition around $k \sim 2 h \mathrm{Mpc}^{-1}$ for $z=1$, and $k \sim 1 h \mathrm{Mpc}^{-1}$ for $z=0$. They also compute the non-linear matter power spectrum and bi-spectrum, the CDM mass function, and the halo bias. The results of all this are in broad agreement with those of Schmidt [1112, 1111.

The simulations of Khoury and Wyman [690] were improved upon in [1290, where Eq. 816 was solved. It was found that in DGP, and higher dimensional cascading gravity models, peculiar velocities are enhanced by $24-34 \%$ compared to CDM [780]. This corresponds to an enhancement by four orders of magnitude in the probability of the occurrence of high velocity merging system such as the "bullet cluster".

Scoccimarro [1121] and Koyama, Taruya and Hiramatsu [743] have also developed two independent techniques based on higher-order perturbation theory in order to find the non-linear power spectrum. Scoccimarro [1121] shows that the non-linearities coming from the brane-bending mode can be described by a time and space dependent gravitational constant. He then goes on to develop a re-summation scheme to calculate the non-linear power spectrum and the bi-spectrum. Koyama, Taruya and Hiramatsu 743 , develop a general perturbation theory method that can be applied in the quasi non-linear regime of any theory that has an additional scalar degree of freedom (such as Brans-Dicke, $f(R)$ and DGP). For the case of DGP, they find that their perturbative method recovers the extreme non-linearity of the Vainshtein mechanism. Their technique has compared with the HALOFIT mapping [1156], and the non-linear PPF fit of Hu and Sawicki [630]. It is found that HALOFIT over-predicts power on small-scales, in agreement with the findings of [690, 1112, 1111, while the non-linear PPF fit works well within the regime of validity of the perturbation theory.

\subsection{Higher Co-Dimension Braneworlds}

An important characteristic of any braneworld model is the brane's co-dimension. This is given by the difference between the dimension of the bulk and the dimension of the brane. Up to this point we have been concentrating on co-dimension one models. These are by far the most well developed, mainly because they are much more tractable. In this section we will discuss braneworld models with co-dimension $n \geq 2$. In this case the gravitational description typically becomes much more complicated.

We have already seen higher co-dimension branes in Section 5.2.1, when discussing the ADD model 62$]$ in six or more dimensions. This is a particularly simple example of a higher co-dimension braneworld set-up, corresponding to a tension-less brane in a Minkowski bulk. In general, of course, one must consider more complicated scenarios, including the full gravitational back-reaction of brane sources, both in the bulk and on the brane. Indeed, attempts to solve for a higher co-dimension delta function source in Einstein gravity will generically result in a singular bulk geometry unless the energymomentum of the source is given by pure tension [316. This is problematic if we insist on using that infinitely thin defect as a model of our 4-dimensional Universe. However, in a realistic set-up the defect will not really be a delta function source (it will have some finite 
thickness, and the would-be singularity will be automatically resolved). Alternatively, one could consider a genuine delta function source and avoid issues with the singularity by including higher-order operators in the bulk gravity theory [449, 174, 283, 282 .

Despite being extremely difficult to study, higher co-dimension braneworld models have been discussed in the literature (see, for example, [256, 316, 1250, 965, 13, 448, 449, 450, 518, 436, 669, 668, 381, 386, 388, 379, 387, 900, 12, 338, 339, 174, 280, 281, 277, 1023, 1022, 348, 349]). Much of the interest in this field lies in the fact that these models offer new ways to think about the cosmological constant problem [256, 316, 1250, 13, 450, 518. For example, in Section 5.6.2 we will briefly review the importance of higher co-dimension branes in the degravitation scenario 61 .

Co-dimension two branes are particularly interesting as there is reason to believe that within them one may be able to realise self-tuning of the vacuum energy. This is because a maximally symmetric co-dimension two brane generically behaves like a cosmic string in the bulk by forming a conical singularity 57 . Changes in tension then alter the deficit angle, as opposed to the geometry, of the defect [316]. Braneworld models with two extra dimensions shaped like a rugby ball have been developed with this in mind [256]. The curvature of the brane is now completely determined by the bulk cosmological constant and the magnetic flux, independent of the brane tension. One might then infer that phase transitions on the brane that alter the brane tension do not alter the curvature. This is not the case, however, as such a transition can affect the brane curvature via the backdoor, by altering magnetic flux, so there is no self-tuning [1250, 965]. The situation can sometimes (but not always [214, 215]) be improved by including super-symmetry and dilaton dynamics in the bulk to protect the relationship between the bulk cosmological constant and the flux [13, 216, 217, 214, 215]. A low scale of bulk super-symmetry breaking, set by the size of the rugby ball, can then account for a small amount of dark energy [216]. Other notable contributions to the literature on rugby ball compactifications with fluxes and/or scalars include the following: A detailed discussion of the brane-bulk matching conditions [213, 121, an analysis of the low energy effective theory [708, and a study of cosmological evolution on the brane 1021 .

The rugby ball described above represents a compact extra dimension, with potentially troublesome/interesting light moduli fields [18, 19. In any event, it is worth pursuing alternative braneworld models with non-compact extra dimensions if they are able to reproduce $4 D$ gravity at some scale. Co-dimension two branes in Gauss-Bonnet gravity will be discussed in this context in Section 5.7.4. On the other hand, one might expect that an infinite bulk should be compatible with some form of quasi-localisation of gravity by considering generalisations of DGP gravity 454 in any number of dimensions. Such generalisations are sometimes referred to as Brane Induced Gravity (BIG) models $[448,449,450,518,436,381,386,379,338,339,669,668$. In the simplest scenario we can consider BIG models on a single 3 -brane of co-dimension $n$, described by the following action 448]:

$$
S=\frac{M_{*}^{2+n}}{2} \int_{\text {bulk }} d^{4+n} x \sqrt{-\gamma} \mathcal{R}+\frac{M_{p l}^{2}}{2} \int_{\text {bulk }} d^{4} x \sqrt{-g} R .
$$

Using higher derivative operators to resolve the singularity in the bulk propagator 58 , we

\footnotetext{
${ }^{57}$ There are exceptions that give rise to curvature singularities as opposed to conical, even for maximal symmetry on the brane 1208 .

${ }^{58}$ Note that this theory becomes higher-dimensional at large distances, so the finer details of how the
} 
find that for $n=2,3$ the Green's function in momentum space along the brane takes the form 449 .

$$
\tilde{G}(p, 0)=\frac{2}{M_{p l}^{2} p^{2}+\frac{M_{*}^{2+n}}{D(p, 0)}},
$$

where

$$
D(p, 0)=\int \frac{d^{n} q}{(2 \pi)^{n}} \frac{1}{p^{2}+q^{2}+\left(p^{2}+q^{2}\right)^{2} / M_{u v}^{2}},
$$

and where $M_{u v} \lesssim M_{*}$ represents the regularisation scale. Taking $M_{u v} \sim 10^{-3} \mathrm{eV}$, this theory reproduces normal $4 D$ gravity at intermediate scales $M_{u v}^{-1} \ll r \ll r_{c}$, but is modified at sub-millimetre scales and at very large distances. It has therefore been dubbed seesaw gravity in [449]. The large distance cross-over scale can be computed from Equation (819),

$$
r_{c} \sim \begin{cases}\frac{M_{p l}}{M_{*}^{2}} \sqrt{\ln \left(M_{u v} r_{c}\right)} & \text { for } n=2 \\ \frac{M_{p l}}{M_{*}^{2}} \sqrt{\frac{M_{u v}}{M_{*}}} & \text { for } n=3 .\end{cases}
$$

Unfortunately, these constructions suffer from the presence of ghosts in the spectrum of fluctuations [436].

\subsubsection{Cascading gravity}

More sophisticated BIG proposals make the transition from $4+n$ dimensional gravity to 4 dimensional gravity via a series of intermediate steps, $(4+n) D \rightarrow(4+n-1) D \rightarrow$ $\ldots \rightarrow 5 D \rightarrow 4 D[381,386,379,338,339,669,668$. This could potentially help with the ghost problem. These are sometimes referred to as cascading gravity models as the effective description tumbles down a cascade of extra dimensions as we come in from large distances, and increase the resolution of our description. Let us now turn our attention to this class of gravity models.

\section{The Kaloper-Kiley model}

The first braneworld model to explore a cascade from $6 D \rightarrow 5 D \rightarrow 4 D$ gravity was developed by Kaloper and Kiley 669, 668. They considered a landscape of models describing BIG on a 3 -brane in six dimensions. The singularities are resolved by modelling the 3 -brane as a cylindrical 4-brane with compact radius $r_{0}$. The brane has tension $\sigma_{5}$, and some induced curvature weighted by a $5 D$ Planck scale, $M_{5}$. The effective $4 D$ tension and Planck scale at distances $r \gg r_{0}$ are then given by $\sigma_{4}^{\text {eff }}=2 \pi r_{0} \sigma_{5}$ and $M_{4}^{\text {eff }}=2 \pi r_{0} M_{5}^{3}$, respectively. Note that an axion flux is used to cancel the vacuum pressure in the compact direction $q^{2}=2 \sigma_{5} r_{0}^{2}$.

The resulting vacua resemble a cone in the bulk, with the conical singularity cut away (see Figure 12. The metric is given by

$$
d s_{6}^{2}=\eta_{\mu \nu} d x^{\mu} d x^{\nu}+d \rho^{2}+f(\rho)^{2} d \phi^{2},
$$

theory behaves there will be sensitive to how we resolve the singularity in the bulk [448. 


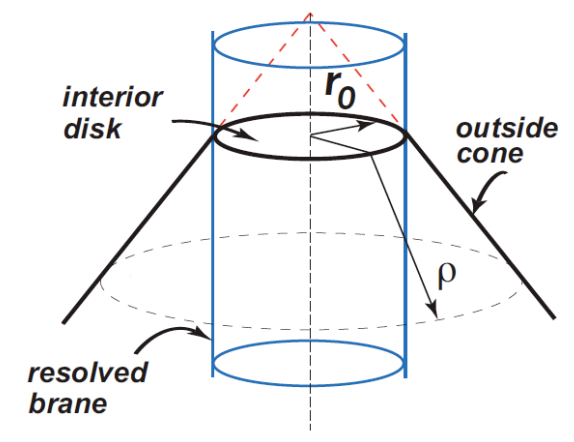

Figure 12: Taken from 669. An illustration of the $2 D$ bulk geometry formed around the resolved brane in vacuum.

where

$$
f(\rho)= \begin{cases}\rho & 0 \leq \rho<r_{0} \\ (1-b)\left(\rho+\frac{b}{1-b} r_{0}\right) & \rho>r_{0} .\end{cases}
$$

The brane tension does not affect the geometry along the brane's non-compact directions. Instead, it controls the deficit angle measured at infinity, according to $b=$ $2 \sigma_{5} r_{0} / M_{6}^{2}$, where $M_{6}$ is the fundamental scale of gravity in the bulk. When the tension lies below a critical value, $\sigma_{\text {crit }}=M_{6}^{2} / 2 r_{0}$, the deficit angle is less than $2 \pi$, and the bulk space is infinite. For sub-critical branes, $\sigma_{5}<\sigma_{\text {crit }}$, the theory generically resembles the seesaw gravity theory. We have $4 D$ (Brans-Dicke) gravity at intermediate scales $r_{0}<r<r_{c}$, and a crossover to $6 D$ gravity at a scale $r_{c} \sim \frac{M_{4}^{\text {eff }}}{(1-b) M_{6}^{2}} \sqrt{\ln \left(2(1-b) r_{c} / r_{0}\right)}$. Note that the scalar fluctuations naively indicate the possible presence of a ghost, but this conclusion cannot be trusted since the perturbative theory breaks down due to strong coupling 669. This is consistent with the conclusions drawn in [435] for thick brane regularisations of seesaw gravity.

Things are much more interesting in the near critical limit, for which $b=1-\epsilon$, where $0<\epsilon \ll 1$. In this case the brane lies inside a very deep throat, such that the angular dimension is effectively compactified up to distances of the order $r_{0} / \epsilon$ (see Figure 13). It turns out that perturbation theory is under much better control in this limit, and that there are no ghosts. The improved behaviour is due to the fact that we make a series of transitions from $4 D \rightarrow 5 D \rightarrow 6 D$ gravity. Indeed, the theory looks like four dimensional Brans-Dicke gravity at scales $r_{0}<r<r_{c}$, becoming five dimensional beyond $r_{c} \sim M_{4}^{\text {eff }} / M_{6}^{4} r_{0}$. The transition to six dimensions only occurs at very large distances, $r>r_{0} / \epsilon$, at which point the cylinder decompactifies.

Note that this model does not represent a solution to the cosmological constant problem. Instead it recasts it in terms of a fine-tuning of the hierarchy between cross-over scales at each transition. 


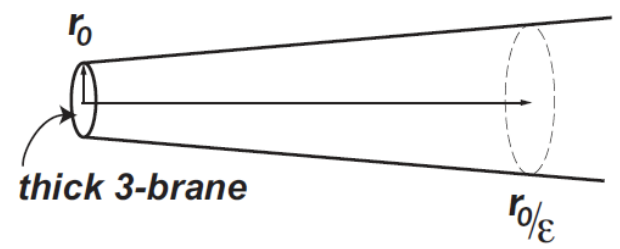

Figure 13: Taken from 669. An illustration of the $2 D$ bulk geometry formed around the resolved brane in vacuum, in the near critical limit.

\section{The cascading DGP model}

A more recent BIG model exploring these ideas was developed by de Rham et al., who coined the phrase cascading gravity [381, 386, 379]. In the simplest model [381, one has a DGP 3-brane within a DGP 4-brane within a six dimensional bulk. This situation is described by the following action:

$$
S=\frac{M_{6}^{4}}{2} \int_{\text {bulk }} d^{6} x \sqrt{-g_{6}} R_{6}+\frac{M_{5}^{3}}{2} \int_{4-\text { brane }} d^{5} x \sqrt{-g_{5}} R_{5}+\frac{M_{4}^{2}}{2} \int_{3 \text {-brane }} d^{4} x \sqrt{-g_{4}} R_{4},
$$

where $\left(g_{6}\right)_{A B},\left(g_{5}\right)_{a b}$ and $\left(g_{4}\right)_{\mu \nu}$ are the metrics in the bulk, on the 4-brane and on the 3 -brane, respectively. The corresponding Ricci scalars are given by $R_{6}, R_{5}$ and $R_{4}$. The model contains two important mass scales corresponding to the following ratios:

$$
m_{5}=\frac{M_{5}^{3}}{M_{4}^{2}}, \quad \text { and } \quad m_{6}=\frac{M_{6}^{4}}{M_{5}^{3}} .
$$

The claim is that the intermediate DGP 4-brane can help to resolve the singularity in the bulk propagator at the location of the 3-brane 381. Let us now consider fluctuations due to a conserved source, $T_{\mu \nu}$, about a bulk and branes that are all Minkowski. The field can then be decomposed in terms of a scalar, $\pi$, and a transverse and trace-free tensor, $h_{\mu \nu}^{\mathrm{TT}}$, such that

$$
h_{\mu \nu}=h_{\mu \nu}^{\mathrm{TT}}+\pi \eta_{\mu \nu}+\text { gauge terms. }
$$

Working in momentum space one finds that the Fourier transformed fields are given by 381, 386

$$
\begin{aligned}
\tilde{h}_{\mu \nu}^{\mathrm{TT}}(p) & =\frac{2}{M_{4}^{2}}\left(\frac{1}{p^{2}+g\left(p^{2}\right)}\right)\left[\tilde{T}_{\mu \nu}-\frac{1}{3} \tilde{T} \eta_{\mu \nu}+\frac{p_{\mu} p_{\nu}}{p^{2}} \tilde{T}\right] \\
\tilde{\pi}(p) & =-\frac{1}{3 M_{4}^{2}}\left(\frac{1}{p^{2}-\underset{232}{2 g\left(p^{2}\right)}}\right) \tilde{T}
\end{aligned}
$$


where 59

$$
g\left(p^{2}\right)= \begin{cases}\frac{\pi m_{5}}{2} \frac{\sqrt{\left|p^{2}-4 m_{6}^{2}\right|}}{\tanh ^{-1}\left(\sqrt{\frac{\left|p-2 m_{6}\right|}{p+2 m_{6}}}\right)} & p<2 m_{6} \\ \frac{\pi m_{5}}{2} \frac{\sqrt{\left|p^{2}-4 m_{6}^{2}\right|}}{\tan ^{-1}\left(\sqrt{\frac{\left|p-2 m_{6}\right|}{p+2 m_{6}}}\right)} & p>2 m_{6} .\end{cases}
$$

The amplitude between two conserved sources on the brane, $T_{\mu \nu}$ and $T_{\mu \nu}^{\prime}$, now takes the surprisingly simple form

$$
\mathcal{A}=\frac{2}{M_{4}^{2}}\left(\frac{1}{p^{2}+g\left(p^{2}\right)}\right)\left[\tilde{T}_{\mu \nu} \tilde{T}^{\prime \mu \nu}-\frac{1}{3} \tilde{T} \tilde{T}^{\prime}-\frac{1}{6} \frac{p^{2}+g\left(p^{2}\right)}{p^{2}-2 g\left(p^{2}\right)} \tilde{T} \tilde{T}^{\prime}\right] .
$$

The last term represents the contribution from the scalar amplitude, whereas the remainder is the contribution from the massive spin-2 field. The coefficient of scalar amplitude changes sign as we flow from the IR to the UV,

$$
\frac{p^{2}+g\left(p^{2}\right)}{p^{2}-2 g\left(p^{2}\right)} \rightarrow \begin{cases}-\frac{1}{2} & \text { as } p \rightarrow 0 \\ 1 & \text { as } p \rightarrow \infty\end{cases}
$$

indicating the presence of a ghost in the UV theory, consistent with the results of 436]. To eliminate this ghost we must add additional operators to the 3-brane action that modify the scalar propagator in the UV ${ }^{60}$. It turns out that this can be achieved simply by adding tension, $\lambda$, to the 3 -brane 381 , 388. As the 3-brane is co-dimension 2, its vacuum solution is unaffected by the change in tension (it is still Minkowski). In contrast, the 3-brane tension does alter the profile of the fields in the bulk by creating a deficit angle. This means that kinetic terms describing fluctuations on the new vacuum now receive corrections from the non-linear bulk interactions. The result is that the scalar propagator on the 3 -brane is modified. One might expect that such effects will necessarily be suppressed by the five and six dimensional Planck scales, but this is not necessarily so because of strong coupling of the scalar in the five dimensional boundary effective field theory [381, 388].

In any event, at higher energies, $p \gg m_{6}$, the modified amplitude goes like

$$
\mathcal{A}_{\lambda} \sim \frac{2}{M_{4}^{2}}\left(\frac{1}{p^{2}+2 m_{5} p}\right)\left[\tilde{T}_{\mu \nu} \tilde{T}^{\prime \mu \nu}-\frac{1}{3} \tilde{T} \tilde{T}^{\prime}-\frac{1}{6} \frac{p^{2}+2 m_{5} p}{p^{2}\left(1-\frac{3 \lambda}{2 m_{6}^{2} M_{4}^{2}}\right)-4 m_{5} p} \tilde{T} \tilde{T}^{\prime}\right] .
$$

For large enough tension, $\lambda>\frac{2 m_{6}^{2} M_{4}^{2}}{3}$, the scalar amplitude always has the correct sign, so there is no ghost. However, the tension cannot be arbitrarily large since we require

\footnotetext{
${ }^{59}$ Note that the various formulae for propagators and amplitudes in 381,386 differ. One must take $m_{5,6} \rightarrow \frac{1}{2} m_{5,6}$ in going from 381 to [386. The difference is because only one half of the $6 \mathrm{D}$ bulk, and one half of the 4-brane are considered in [386. This is a perfectly legitimate truncation by $\mathbb{Z}_{2}$ symmetry across each of the branes. Here, however, we will adopt the conventions of 381 when expressing our formulae.

${ }^{60}$ It has also been argued that thickening of the brane can help to eliminate the ghost in this model 386 .
} 
that the bulk deficit angle is less than $2 \pi$. This places an upper bound $\lambda<2 \pi M_{6}^{4}=$ $2 \pi m_{5} m_{6} M_{4}^{2}$. Provided $m_{6}<m_{5}$ we therefore have a window,

$$
\frac{2 m_{6}^{2} M_{4}^{2}}{3}<\lambda<2 \pi m_{5} m_{6} M_{4}^{2},
$$

for which the theory is ghost-free in the UV [381, 388. The condition $m_{6}<m_{5}$ has added significance in that it permits a transition from $4 D$ to $5 D$ at energies $p \sim m_{5}$. In particular, we have $4 D$ scalar-tensor gravity in the far UV, for $p>m_{5}>m_{6}$,

$$
\mathcal{A}_{\lambda}^{(4 D)} \sim \frac{2}{M_{4}^{2} p^{2}}\left[\tilde{T}_{\mu \nu} \tilde{T}^{\prime \mu \nu}-\frac{1}{2}\left(\frac{1-\frac{\lambda}{m_{6}^{2} M_{4}^{2}}}{1-\frac{3 \lambda}{2 m_{6}^{2} M_{4}^{2}}}\right) \tilde{T} \tilde{T}^{\prime}\right],
$$

and $5 D$ scalar-tensor gravity for $m_{5}>p>m_{6}$,

$$
\mathcal{A}_{\lambda}^{(5 D)} \sim \frac{1}{M_{5}^{3} p}\left[\tilde{T}_{\mu \nu} \tilde{T}^{\prime \mu \nu}-\frac{1}{4} \tilde{T} \tilde{T}^{\prime}\right] .
$$

In the far infra-red, $p<m_{6}$, we recover $6 D$ gravity,

$$
\mathcal{A}^{(6 D)} \sim \frac{\ln \left(m_{6} / p\right)}{\pi M_{6}^{4}}\left[\tilde{T}_{\mu \nu} \tilde{T}^{\prime \mu \nu}-\frac{1}{4} \tilde{T} \tilde{T}^{\prime}\right] .
$$

Thus we have a cascade from $6 D \rightarrow 5 D \rightarrow 4 D$ gravity as we move from large to short distances. It is interesting to note that the existence of this cascade seems to be closely related to the absence of ghosts. By taking $M_{4} \sim M_{p l}, M_{5} \sim 10 \mathrm{MeV}$ and $M_{6} \sim \mathrm{meV}$, the cascade occurs at the current horizon scale, as $m_{6} \sim m_{5} \sim H_{0}$. Note that a cascade from seven dimensions has also been studied in some detail in [387.

For tensions in the range allowed by Eq. 832 the $4 D$ theory at short distances is a Brans-Dicke theory that is incompatible with solar system constraints. The authors of 381 suggest that some sort of Vainshtein mechanism could account for the recovery of GR at short scales. Indeed, the role of non-linearities in cascading DGP has not yet been properly investigated, and one may be concerned that there are issues surrounding the validity of the linearised results (as was found in the sub-critical regime of the KaloperKiley model 669).

Another aspect of cascading DGP that requires further study is cosmology (see 1900, 12 for some preliminary work). In 12 a five dimensional proxy theory is used that is expected to capture many of the salient features of the original model. This proxy model corresponds to a $5 D$ scalar-tensor theory that, in the limit where the scalar and tensor degrees of freedom decouple, agrees with the decoupling limit of the $5 D$ boundary effective field theory of cascading DGP presented in 381. Of course, it is by no means unique in this respect.

\section{Induced gravity on intersecting braneworlds}

A third BIG model that is very closely related to cascading DGP was developed by Corradini, Koyama and Tasinato [338, 339. Here a six dimensional bulk contains two intersecting DGP 4-branes, each with induced curvature. At the intersection we place a 
DGP 3-brane, also with some induced curvature. The cosmological evolution is derived using a formalism based on mirage cosmology [685]. The resulting Friedmann equation on the 3-brane is then given by 339

$$
\rho=2 M_{4}^{2} H^{2}+6 M_{5}^{3} k_{2} \sqrt{1+\frac{H^{2}}{k_{2}^{2}}}+4 M_{6}^{4} \tan ^{-1}\left[\tan \alpha \sqrt{1+\frac{H^{2}}{k_{2}^{2}}}\right],
$$

where $\alpha$ is the angle between the two 4-branes, and $k_{2}$ is a constant that encodes information about the warped geometry in the bulk (and can be derived in a non-trivial way from the parameters of the theory). The model claims to admit self-accelerating and self-tuning vacua. For fluctuations on a Minkowski 3-brane one may also expect a cascade from $6 D \rightarrow 5 D \rightarrow 4 D$ gravity as we move from large to short distances, for suitably chosen scales. A thorough perturbative analysis has yet to be done that confirms this expectation, or the possible existence of ghosts and strong coupling.

\subsubsection{Degravitation}

Higher co-dimension braneworld models and cascading gravity are expected to play an important role in realising the degravitation scenario [61. The idea of degravitation is best understood by rephrasing the cosmological constant problem. Instead of asking "why is the vacuum energy so small?", we ask "why does the vacuum energy hardly gravitate at all?". In other words, we accept what our particle physics models are saying and take the vacuum energy to be up at the $(\mathrm{TeV})^{4}$ scale or beyond. We then try to develop a gravity theory that prevents this large vacuum energy from generating a large amount of curvature.

A phenomenological description of degravitation is given by 61 .

$$
\mathcal{G}^{-1}\left(L^{2} \square\right) G_{\mu \nu}=8 \pi T_{\mu \nu},
$$

where Newton's constant, $G$, has been promoted to a differential operator, $\mathcal{G}\left(L^{2} \square\right)$, depending on a length scale, $L$, and the covariant d'Alembertian operator, $\square$. The idea is that this operator behaves like a high pass filter characterised by the scale $L$. Sources with short characteristic wavelengths, $l \ll L$, pass through the filter and gravitate normally. Sources with long characteristic wavelengths, $l \gg L$, such as the cosmological constant, are filtered out and hardly gravitate at all. These considerations amount to imposing the following limits: $\mathcal{G} \rightarrow G$ for $L^{2}(-\square) \rightarrow \infty$ and $\mathcal{G} \rightarrow 0$ for $L^{2}(-\square) \rightarrow 0$.

Since the covariant derivative does not commute with the d'Alembertian, Equation (837) suggests that the energy-momentum tensor is not conserved. However, it is important to realise that this equation is only expected to describe the linearised dynamics of the helicity- 2 mode of the graviton, given by $g_{\mu \nu}=\eta_{\mu \nu}+h_{\mu \nu}$. The source is conserved with respect to the full metric, $\hat{g}_{\mu \nu}=\eta_{\mu \nu}+h_{\mu \nu}+\ldots$, where the ellipsis denotes the additional (Stuckelberg) modes that necessarily appear in a fully covariant theory 452 . It is clear that the extra modes must play an important role in the filtering process at long wavelengths. The transition to normal gravity for short wavelength modes occurs precisely because these modes get screened by the Vainshtein mechanism [1241] (see our discussion of the Vainshtein mechanism in the context of DGP gravity in Section 5.5.4).

The filter operator is often parametrised as follows:

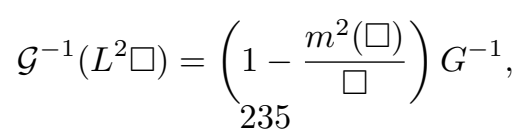


where $m^{2}(\square) \propto L^{2(\alpha-1)} \square^{\alpha}$, and $0 \leq \alpha<1$. The upper limit is a necessary, but not sufficient, condition for degravitation, whereas the lower limit is required by unitarity [452. Massive gravity (see Section 3.3.3 corresponds to $\alpha=0$, whereas DGP gravity ${ }^{61}$ (see Section 5.5 corresponds to $\alpha=1 / 2$. It turns out that models with co-dimension $n>2$ are expected to correspond to $\alpha=0$ [386].

Using Equation (837) as its starting point, degravitation can be demonstrated in a cosmological context by the ratio of scalar curvature to $\Lambda$ scaling as $t^{-1 / 2}$ for a broad class of filters, where $t$ is the proper time of comoving observers 452 . However, the corresponding cosmological solution arising from a higher co-dimension brane scenario has yet to be found explicitly.

Working at the level of the phenomenological equations, N-body simulations for degravitation scenarios have been studied by Khoury and Wyman 690. Their simulation is based on a modified Poisson equation, given by

$$
-\left[\frac{k^{2}}{a^{2}}+\frac{1}{L^{2}}\left(\frac{k L}{a}\right)^{2 \alpha}\right] \Psi=4 \pi G \rho .
$$

Thus, the emergence of the Vainshtein mechanism is encoded in the simulations via Eq. 815), rather than being recovered from the simulation itself. They find that the matter power spectrum determined from the simulation agrees well with the HALOFIT [1156] formula for $k<\sim 0.2 \mathrm{hppc}^{-1}$. However, for smaller scales the HALOFIT formula overpredicts power because HALOFIT does not capture the Vainshtein effect. Nevertheless, they find a way to recalibrate the HALOFIT parameters such that it agrees with their simulation on all scales.

\subsection{Einstein Gauss-Bonnet Gravity}

In Section 2.4.1 we outlined how Lovelock's theorem 831, 832 constrains the class of theories that can be constructed from the metric tensor alone: In four dimensions, the most general rank-2 tensor that can be derived from a variational principle and is (i) symmetric, (ii) divergence free, and (iii) built out of the metric and its first two derivatives only is given by a linear combination of the metric and the Einstein tensor, i.e.

$$
\mathcal{E}_{\mu \nu}^{(4 D)}=-\frac{1}{2} \alpha_{0} g_{\mu \nu}+\alpha_{1} G_{\mu \nu}
$$

Such a term arises from the variation of the Einstein-Hilbert action in the presence of a cosmological constant,

$$
\mathcal{E}_{\mu \nu}^{(4 D)}=\frac{1}{\sqrt{-g}} \frac{\delta}{\delta g^{\mu \nu}} \int d^{4} x \sqrt{-g}\left(\alpha_{0}+\alpha_{1} R\right) .
$$

In four dimensions the Einstein-Hilbert action therefore gives the most general field equations with the desired properties. In more than four dimensions, however, this result

\footnotetext{
${ }^{61}$ The full DGP theory does not exhibit degravitation, but this is not necessarily the unique theory with $\alpha=1 / 2$.
} 
no longer holds. For $D=5$ or 6 dimensions the most general rank-2 tensor satisfying the same three conditions is given by [831] (see also [767, 1313])

$$
\mathcal{E}_{a b}^{(5 D / 6 D)}=-\frac{1}{2} \alpha_{0} \gamma_{a b}+\alpha_{1} \mathcal{G}_{a b}+\alpha_{2} \mathcal{H}_{a b},
$$

where (in $D$ dimensions) $\gamma_{a b}$ is the metric, $\mathcal{G}_{a b}=\mathcal{R}_{a b}-\frac{1}{2} \mathcal{R} \gamma_{a b}$ is the Einstein tensor, and we have introduced the Lovelock tensor 831]

$$
\mathcal{H}_{a b}=2 \mathcal{R}_{a b}-4 \mathcal{R}_{a \alpha} \mathcal{R}_{b}^{c}-4 \mathcal{R}_{a c b d} \mathcal{R}^{c d}+2 \mathcal{R}_{a c d e} \mathcal{R}_{b}{ }^{c d e}-\frac{1}{2} \hat{G} \gamma_{a b},
$$

where $\hat{G}=\mathcal{R}_{a b c d} \mathcal{R}^{a b c d}-4 \mathcal{R}_{a b} \mathcal{R}^{a b}+\mathcal{R}^{2}$, and $\mathcal{R}_{a b c d}$ is the Riemann tensor constructed from $\gamma_{a b}$. The Lovelock tensor is obtained by variation of the Gauss-Bonnet action,

$$
\mathcal{H}_{a b}=\frac{1}{\sqrt{-\gamma}} \frac{\delta}{\delta \gamma^{a b}} \int d^{D} x \sqrt{-g} \hat{G} .
$$

These results can generalised still further as the dimensionality of space-time is increased. To see how, note that $d^{D} x \sqrt{-\gamma} \mathcal{R}$ and $d^{D} x \sqrt{-\gamma} \hat{G}$ correspond to the Euler classes of order one and order two [936, and are topological in $D \leq 2$ and $D \leq 4$ respectively ${ }^{62}$. To generalise Lovelock's theorem to even higher dimensions we must then add the higher order Euler classes to the corresponding action, and compute the metric variation. The Euler class at order $k$ depends on $k$ th powers of curvature. In $D$ dimensions we must therefore include Euler classes up to order $[(D-1) / 2]$, where the square brackets denote the integer part. The reader is referred to [270], and references therein, for further details.

\subsubsection{Action, equations of motion, and vacua}

In five or six dimensions General Relativity is just a special case of a broader class of theories commonly referred to as Einstein-Gauss-Bonnet (EGB) gravity. These theories are described by the action

$$
S=\frac{1}{16 \pi G_{D}} \int d^{D} x \sqrt{-\gamma}(\mathcal{R}-2 \Lambda+\alpha \hat{G})+\int d^{D} x \mathcal{L}_{m}\left(\gamma_{a b}, \psi\right),
$$

where $\mathcal{L}_{m}$ is the Lagrangian density of the higher dimensional matter fields that are minimally coupled to the metric. The corresponding field equations are given by

$$
\mathcal{G}_{a b}+\Lambda \gamma_{a b}+\alpha \mathcal{H}_{a b}=8 \pi G_{D} T_{a b},
$$

where $T_{a b}=-\frac{2}{\sqrt{-\gamma}} \frac{\delta}{\delta \gamma^{a b}} \int d^{D} x \mathcal{L}_{m}\left(\gamma_{a b}, \psi\right)$ is the energy-momentum tensor of the matter fields. As we will see shortly, although $\Lambda$ acts like a bare cosmological constant, it differs from the effective cosmological constant, $\Lambda_{\text {eff }}$, seen by the geometry.

The Gauss-Bonnet corrections are weighted by the parameter $\alpha$. This has dimensions of $[\text { length }]^{2}$ and is often associated with the slope parameter, $\alpha^{\prime}$, in heterotic string theory. To see why, consider the effective theory describing the dynamics of the heterotic

\footnotetext{
${ }^{62}$ One can check explicitly that the Einstein tensor is identically zero in two dimensions, and that the Lovelock tensor is identically zero in four dimensions.
} 
string on the 10 dimensional target space. Working at tree level in the string coupling and performing a perturbative expansion in the inverse string tension, $\mu_{F}^{-1} \sim 2 \pi \alpha^{\prime}$, we see that the leading order term gives the standard Einstein-Hilbert action, and the next to leading order term, after some field redefinitions, gives the Gauss-Bonnet action [566, 886]. In fact, one may have expected such a result based on the fact that string theory is known to be ghost free. As noted by Zwiebach [1316, second-order corrections to the Einstein-Hilbert action will necessarily give rise to ghosts unless they appear in the Gauss-Bonnet combination. Note that if we do identify EGB with a stringy generalisation of General Relativity, we should restrict attention to $\alpha \geq 0$ for consistency.

\section{Maximally symmetric vacua}

We now consider the maximally symmetric vacuum solutions, $\bar{\gamma}_{a b}$, satisfying $\overline{\mathcal{R}}_{a b c d}=$ $\frac{2 \Lambda_{\text {eff }}}{(D-1)(D-2)}\left(\bar{\gamma}_{a c} \bar{\gamma}_{b d}-\bar{\gamma}_{a d} \bar{\gamma}_{b c}\right)$, where $\Lambda_{\text {eff }}$ is the effective cosmological constant seen by the curvature. There are two possible values for $\Lambda_{\text {eff }}$, given by [279]

$$
\Lambda_{\mathrm{eff}}^{ \pm}=\Lambda_{C S}\left(1 \pm \sqrt{1-\frac{2 \Lambda}{\Lambda_{C S}}}\right),
$$

where

$$
\Lambda_{C S}=-\frac{1}{4 \alpha} \frac{(D-1)(D-2)}{(D-3)(D-4)} .
$$

For these vacua to be well defined the bare cosmological constant must satisfy the bound $\Lambda / \Lambda_{C S} \leq 1 / 2$. It is easy to check that $\Lambda_{\text {eff }}^{+} / \Lambda_{C S} \geq 1 \geq \Lambda_{\text {eff }}^{-} / \Lambda_{C S}$, with equality when $\Lambda=\Lambda_{C S} / 2$. This is known as the Chern-Simons limit (at least in odd dimensions) [1297, 344, and corresponds to the case where the two roots coincide. Note that only the lower root has a smooth limit, $\Lambda_{\text {eff }}^{-} \rightarrow \Lambda$, as $\alpha \rightarrow 0$, and as such is often referred to as the "Einstein" branch. In contrast, the upper root, $\Lambda_{\mathrm{eff}}^{+}$, is not smooth as $\alpha \rightarrow 0$, and represents a distinct new feature of EGB gravity that is completely absent in higher dimensional General Relativity. For this reason, this branch is often referred to as the "stringy", or "Gauss-Bonnet", branch.

We now consider metric perturbations about these vacua, $\gamma_{a b}=\bar{\gamma}_{a b}+\delta \gamma_{a b}$. The linearised field equations then take the remarkably simply form

$$
\delta \mathcal{G}_{a b}+\Lambda_{\mathrm{eff}} \delta \gamma_{a b}=8 \pi G_{\mathrm{eff}} T_{a b},
$$

where $\delta \mathcal{G}_{a b}$ is the linearised Einstein tensor. Thus, we have perturbative Einstein gravity with an effective Newton's constant given by

$$
G_{\text {eff }}=\frac{G_{D}}{1-\frac{\Lambda_{\text {eff }}}{\Lambda_{C S}}}
$$

Assuming that the bare Newton's constant is positive, $G_{D}>0$, it follows that perturbative gravity on the Einstein branch $\left(\Lambda_{\text {eff }} / \Lambda_{C S}<1\right)$ is essentially well behaved, as $G_{\text {eff }}>0$. In contrast, on the Gauss-Bonnet branch $\left(\Lambda_{\text {eff }} / \Lambda_{C S}>1\right)$, we have $G_{\text {eff }}<0$ indicating the presence of a perturbative ghost [279]. We refer the reader to the closing paragraphs of Section 5.5.3 for a discussion of the pathologies associated with ghosts. 
The above conclusions regarding stability are robust provided we can trust our effective perturbative description. Here we expect this description to be valid at energies up to a cut-off, $E_{\text {cut-off }} \sim 1 /\left(G_{\text {eff }}\right)^{1 /(D-2}$. As we approach the Chern-Simons limit $\left(\Lambda \rightarrow \Lambda_{C S} / 2\right)$, where the two branches coincide, it follows that the cut-off for the effective description should have the limit $E_{\text {cut-off }} \rightarrow 0$. This indicates strong coupling, and a breakdown of perturbation theory. To analyse the stability of either branch close to this limit one must study non-perturbative phenomena such as instanton transitions. This reveals that transitions between branches are unsuppressed in the near Chern-Simons regime, and that there is very strong mixing between the two (almost degenerate) vacua. We conclude that neither of them can accurately describe the true quantum vacuum state in this regime, as both will quickly become littered with bubbles of the other vacuum [279].

\section{Spherically symmetric solutions}

Static spherically symmetric solutions to the vacuum field equations were first discovered by Boulware and Deser [176,

$$
d s^{2}=-V(r) d t^{2}+\frac{d r^{2}}{V(r)}+r^{2} d \Omega_{D-2},
$$

where $d \Omega_{D-2}$ is the metric on a unit $(D-2)$-sphere. We have two branches for the potential, $V(r)$, given by [176, 279 .

$$
V_{ \pm}(r)=1+\frac{2 \Lambda_{C S} r^{2}}{(D-1)(D-2)}\left(1 \pm \sqrt{1-\frac{2 \Lambda}{\Lambda_{C S}}-\frac{M(D-1)}{\Lambda_{C S} \Omega_{D-2} r^{D-1}}}\right)
$$

where $\Omega_{D-2}$ is the volume of the unit $(D-2)$-sphere. $M$ is an integration constant that one can identify with the mass of a spherically symmetric source (possibly pointlike). As above, the lower root corresponds to the Einstein branch, and has a smooth limit as $\alpha \rightarrow 0$. The upper root corresponds to the Gauss-Bonnet branch, and does not have a smooth limit. Of course, the two roots are degenerate in the Chern-Simons case $\left(\Lambda=\Lambda_{C S} / 2\right)$.

We shall stay away from the Chern-Simons limit in the remainder of this section. Note that a generalised form of Birkhoff's theorem now holds that guarantees that Equation (851) represents the most general spherically symmetric solution, even without the assumption of staticity [272]. Let us consider the properties of this solution for a source of positive mass, $M>0$. On the Einstein branch the singularity at $r=0$ is shielded by an event horizon, and there are no obvious pathologies. This is not the case on the Gauss-Bonnet branch, where we have a naked time-like singularity.

At asymptotically large radii the metric functions take the form

$$
V_{ \pm} \approx-\frac{2 \Lambda_{\mathrm{eff}}^{ \pm} r^{2}}{(D-1)(D-2)}+1 \pm \frac{M}{\Lambda_{C S} \Omega_{D-2} r^{D-3}}
$$

The asymptotic solution here can be seen to resemble the generalised SchwarzschildTangherlini solution in $D$ dimensional GR [1202, with cosmological constant $\Lambda_{\text {eff }}^{ \pm}$, and mass $\pm M$. In particular, on the Gauss-Bonnet branch, it appears as if our solution has 
negative gravitational energy even for a positive energy source. This conclusion, however, is incorrect. A proper computation of the gravitational energy taking into account all of the Gauss-Bonnet corrections reveals the mass of the solution in Eq. (851) is $+M$ on both branches 415, 416, 1010. For an in-depth discussion of this, and related stability issues, the reader is referred to [279]. For an excellent review of Lovelock gravities, and their black hole solutions, see [270].

\subsubsection{Kaluza-Klein reduction of EGB gravity}

In Section 5.1.1 we discussed compactification of higher dimensional GR on a circle. The same operation can now be performed with the EGB action, given in Eq. (845). We start in five dimensions with coordinates $\left(x^{\mu}, z\right)$, where the $z$ direction is compact. We can then dimensionally reduce down to four dimensions using the following ansatz

$$
\gamma_{\mu \nu}=g_{\mu \nu}+e^{2 \phi} A_{\mu} A_{\nu}, \quad \gamma_{\mu z}=e^{2 \phi} A_{\mu}, \quad \text { and } \quad \gamma_{z z}=e^{2 \phi},
$$

where the metric, $g_{\mu \nu}$, gauge field, $A_{\mu}$ and the dilaton, $\phi$, are all independent of $z$. Integrating out the compact dimension we arrive at the following effective theory [915]:

$$
S_{\text {eff }}=\frac{1}{16 \pi G_{5}} \int d^{4} x \sqrt{-g} \mathcal{L}_{\mathrm{eff}}
$$

where

$$
\begin{gathered}
\mathcal{L}_{\mathrm{eff}}=e^{\phi}\left[R-\frac{1}{4} e^{2 \phi} F^{2}-2 \Lambda+\alpha\left(R_{\mu \nu \alpha \beta} R^{\mu \nu \alpha \beta}-4 R_{\mu \nu} R^{\mu \nu}+R^{2}\right)\right] \\
+\alpha e^{3 \phi}\left[-\frac{3}{8} e^{2 \phi}\left(F_{\nu}^{\mu} F_{\alpha}^{\nu} F_{\beta}^{\alpha} F_{\mu}^{\beta}-\frac{1}{2}\left(F^{2}\right)^{2}\right)\right. \\
-\left(F^{\mu}{ }_{\alpha} F^{\nu}{ }_{\beta}+F^{\mu \nu} F_{\alpha \beta}\right)\left(R^{\alpha \beta}{ }_{\mu \nu}-4 R_{[\mu}^{\alpha} \delta_{\nu]}^{\beta}+R \delta_{[\mu}^{\alpha} \delta_{\nu]}^{\beta}\right) \\
-2\left(\nabla_{\mu} F_{\alpha \beta}\right)\left(\nabla^{\alpha} F^{\beta \mu}\right)-2\left(\nabla^{\mu} F_{\alpha \mu}\right)\left(\nabla_{\nu} F^{\alpha \nu}\right)-12\left(F_{\mu \alpha} \nabla^{\alpha} \phi\right)\left(F^{\mu \beta} \nabla_{\beta} \phi\right)+6 F^{2}(\nabla \phi)^{2} \\
\left.+4\left(F_{\alpha \beta} \nabla_{\mu} \phi\right) \nabla^{\mu} F^{\alpha \beta}-4\left(F_{\alpha \beta} \nabla_{\mu} \phi\right) \nabla^{\alpha} F^{\beta \mu}-12\left(F_{\alpha \beta} \nabla^{\beta} \phi\right) \nabla_{\mu} F^{\alpha \mu}\right] \\
+\alpha e^{\phi}\left[8 e^{-\phi} \nabla_{\mu} \nabla_{\nu} e^{\phi}-2 e^{2 \phi} F_{\mu \alpha} F_{\nu}{ }^{\alpha}\right] G^{\mu \nu}+3 \alpha e^{2 \phi}\left[2 F^{\alpha \beta} F_{\beta}{ }^{\mu} \nabla_{\mu} \nabla_{\alpha} e^{\phi}+F^{2} \square e^{\phi}\right]
\end{gathered}
$$

and $F^{2}=F_{\mu \nu} F^{\mu \nu}$, with $F_{\mu \nu}=\nabla_{\mu} A_{\nu}-\nabla_{\nu} A_{\mu}$. Note that for $\Lambda=0$ and $\alpha=0$ we recover the results of Section 5.1.1, provided we perform a conformal transformation to the Einstein frame and canonically normalise the dilaton.

One property of the Lagrangian in Eq. 856 is that it gives rise to field equations that are at most second order in derivatives. This is, of course, inherited from the underlying theory, and enables us to consider an interesting class of scalar-tensor theories by freezing the gauge field such that $A_{\mu}=0$. We refer the reader to Section 3.1, and 38, for further discussions on four-dimensional theories of this type.

\subsubsection{Co-dimension one branes in EGB gravity}

Interest in EGB gravity has really taken off in recent years, largely due to its application to braneworlds. We discussed the braneworld paradigm in some detail in Section 
5.2 Schematically, the physics of 3-branes in five dimensional EGB gravity is more or less the same as in General Relativity, as we will now discuss.

\section{Generic action and equations of motion}

As in the GR case discussed in Section 5.3.4. we split our five dimensional bulk into a series of domains separated by a series of 3-branes, so that the action is given by

$$
\begin{aligned}
S=\int_{\text {bulk }} & d^{5} x \sqrt{-\gamma}\left[\frac{M_{5}^{3}}{2}(\mathcal{R}+\alpha \hat{G})+\mathcal{L}_{\text {bulk }}\right] \\
& +\sum_{\text {branes }} \int_{\text {brane }} d^{4} x \sqrt{-g}\left\{-\Delta\left[M_{5}^{3}\left(K+2 \alpha\left(J-2 G^{\mu \nu} K_{\mu \nu}\right)\right)\right]+\mathcal{L}_{\text {brane }}\right\}
\end{aligned}
$$

where $M_{5}=\left(1 / 8 \pi G_{5}\right)^{1 / 3}$ is the bulk Planck scale. In comparison with Equation 615 we have a Gauss-Bonnet correction in the bulk and the corresponding Myers boundary term 931 on each brane. The latter depends on the extrinsic curvature, $K_{\mu \nu}$, the induced Einstein tensor $G_{\mu \nu}=R_{\mu \nu}-\frac{1}{2} R g_{\mu \nu}$, and the trace $J=g^{\mu \nu} J_{\mu \nu}$, where 363 .

$$
J_{\mu \nu}=\frac{1}{3}\left(2 K K_{\mu \alpha} K_{\nu}^{\alpha}+K_{\alpha \beta} K^{\alpha \beta} K_{\mu \nu}-2 K_{\mu \alpha} K^{\alpha \beta} K_{\beta \nu}-K^{2} K_{\mu \nu}\right) .
$$

The bulk field equations are then given by

$$
\mathcal{R}_{a b}-\frac{1}{2} R \gamma_{a b}+\alpha \mathcal{H}_{a b}=\frac{1}{M_{5}^{3}} T_{a b}^{\mathrm{bulk}},
$$

with the boundary conditions at the brane given by the "DGW" junction conditions [363, 553. (see also [409]),

$$
\Delta\left\{M_{5}^{2}\left[K_{\mu \nu}-K g_{\mu \nu}+2 \alpha\left(3 J_{\mu \nu}-J g_{\mu \nu}-2 P_{\mu \alpha \nu \beta} K^{\alpha \beta}\right)\right]\right\}=-T_{\mu \nu}^{\text {brane }} .
$$

Here we introduce the double dual of the Riemann tensor, defined as 902 ]

$$
P_{\alpha \beta}^{\mu \nu}=-R_{\alpha \beta}^{\mu \nu}+2 R_{[\alpha}^{\mu} \delta_{\beta]}^{\nu}-2 R_{[\alpha}^{\nu} \delta_{\beta]}^{\mu}-R \delta_{[\alpha}^{\mu} \delta_{\beta]}^{\nu} .
$$

In what follows we will assume that the bulk geometry is only ${ }^{63}$ sourced by a cosmological constant $T_{a b}^{\mathrm{bulk}}=M_{5}^{3} \Lambda \gamma_{a b}$, which we take to be negative, as in the Randall-Sundrum model, $\Lambda=-6 k^{2}$. We will consider a single brane with tension $\sigma$, and some induced curvature, $M_{\text {ind }}^{2} R$. Its energy momentum tensor is then given by

$$
T_{\mu \nu}^{\text {brane }}=-\sigma g_{\mu \nu}-M_{\text {ind }}^{2} G_{\mu \nu}+\mathcal{T}_{\mu \nu}
$$

where $\mathcal{T}_{\mu \nu}$ denotes the contribution from any additional matter excitations on the brane. For simplicity we will impose $\mathbb{Z}_{2}$ symmetry across the brane (see [1012, 1013] for a discussion of asymmetric configurations).

\footnotetext{
${ }^{63}$ For generalisations with bulk scalar fields and bulk Maxwell fields see [271] and [805], respectively.
} 
Weak gravity on a Minkowski brane

Let us seek Randall-Sundrum-like vacua, corresponding to a Minkowski brane in an AdS bulk,

$$
d s^{2}=\bar{\gamma}_{a b} d x^{a} d x^{b}=d z^{2}+e^{-2|z| / l_{\text {eff }}} \eta_{\mu \nu} d x^{\mu} d x^{\nu}
$$

The effective AdS curvature in the bulk is then determined by the bulk equations of motion (859). We find

$$
\frac{1}{l_{\mathrm{eff}}^{2}}=\frac{1}{4 \alpha}\left(1 \pm \sqrt{1-\frac{8 \alpha}{l^{2}}}\right)
$$

where the lower root corresponds to the Einstein branch and the upper root to the GB branch. Meanwhile, the vacuum DGW junction conditions at $z=0$ impose the following constraint on the brane tension

$$
\sigma=6 \frac{M_{5}^{3}}{l_{\mathrm{eff}}}\left(1-\frac{4 \alpha}{3 l_{\mathrm{eff}}^{2}}\right) .
$$

Since we are interested in weak gravity on the brane, we consider small fluctuations in the metric and the brane position. As we saw previously, the linearised equations of motion are identical to those found in perturbative General Relativity,

$$
\delta \mathcal{G}_{a b}-\frac{6}{l_{\text {eff }}^{2}} \delta \gamma_{a b}=0
$$

The linearised junction conditions also take a remarkably simple form,

$$
2\left(M_{5}^{\mathrm{eff}}\right)^{3} \delta\left(K_{\nu}^{\mu}-K \delta_{\nu}^{\mu}\right)=M_{\mathrm{ren}}^{2} \delta G_{\nu}^{\mu}-\mathcal{T}_{\nu}^{\mu}
$$

where we identify the effective five dimensional Planck scale,

$$
\left(M_{5}^{\mathrm{eff}}\right)^{3}=M_{5}^{3}\left(1-\frac{4 \alpha}{l_{\mathrm{eff}}^{2}}\right)
$$

and the renormalised induced curvature scale,

$$
M_{\mathrm{ren}}^{2}=M_{\mathrm{ind}}^{2}+\frac{8 \alpha M_{5}^{3}}{l_{\mathrm{eff}}} .
$$

The effective Planck scale is consistent with the effective Newton's constant introduced in Equation $850,\left(M_{5}^{\text {eff }}\right)=\left(1 / 8 \pi G_{\text {eff }}\right)^{1 / 3}$. It follows that our weak gravity description is identical to the corresponding description in General Relativity, provided we make use of the effective cosmological constant and Planck scale in the bulk, and renormalise the induced curvature on the brane. For further details the reader is referred to [270], and references therein (see also 413 ).

The characteristic behaviour of weak gravity on the brane is as follows [270]: At large distances we recover four dimensional General Relativity, with an effective four dimensional Planck scale

$$
M_{p l}^{\mathrm{IR}}=\sqrt{M_{\mathrm{ind}}^{2}+M_{5}^{3} l_{\mathrm{eff}}\left(1+4 \alpha / l_{\mathrm{eff}}^{2}\right)}=\sqrt{M_{\mathrm{ren}}^{2}+\left(M_{5}^{\mathrm{eff}}\right)^{3} l_{\mathrm{eff}}} .
$$


This holds provided $1 / l_{\text {eff }} \neq 0$. For $1 / l_{\text {eff }}=0$ the large distance behaviour is five dimensional owing to the absence of a normalisable zero mode in the Minkowski bulk.

At short distances we recover four dimensional Brans-Dicke gravity, with a different effective Planck scale ${ }^{64}, M_{p l}^{\mathrm{UV}}=\sqrt{M_{\text {ind }}^{2}+8 \alpha M_{5}^{3} / l_{\mathrm{eff}}}=M_{\mathrm{ren}}$, and a Brans-Dicke parameter given by

$$
2 w+3=\frac{3}{4}\left(\frac{M_{\mathrm{ind}}^{2} / l_{\mathrm{eff}}+M_{5}^{3}\left(1+4 \alpha / l_{\mathrm{eff}}^{2}\right)}{M_{5}^{3}\left(1-4 \alpha / l_{\mathrm{eff}}^{2}\right)}\right)=\frac{3}{4}\left(\frac{\left(M_{p l}^{\mathrm{IR}}\right)^{2}}{\left(M_{5}^{\mathrm{eff}}\right)^{3} l_{\mathrm{eff}}}\right) .
$$

This holds provided $M_{\text {ren }} \neq 0$. If $M_{\text {ren }}=0$ then the short distance behaviour is five dimensional since there is no effective induced curvature giving rise to quasi-localisation near the brane.

These results are consistent with the behaviour that would be expected in linearised theory on a brane in five dimensional General Relativity, with bulk cosmological constant, $\Lambda_{\text {eff }}=6 / l_{\text {eff }}^{2}$, bulk Planck scale, $M_{5}^{\text {eff }}$, and brane induced curvature scale, $M_{\text {ren }}$. It is interesting to note that the Brans-Dicke parameter gets large close to the ChernSimons limit as $M_{5}^{\text {eff }} \rightarrow 0$. However, as we saw earlier, quantum fluctuations in the bulk become strongly coupled at $\sim M_{5}^{\text {eff }}$, so this prediction may be unreliable.

Brane cosmology in EGB gravity

The methods used in Section 5.4 to derive the cosmological evolution of co-dimension one branes apply equally well to branes in EGB gravity, provided we use the DGW junction conditions give in Eq. (860). Recall that brane cosmology can be studied using either the brane-based formalism or the bulk-based formalism. The brane-based formalism relies on the covariant formulation of the effective Einstein equation on the brane. This has been worked out for EGB gravity and applied to cosmological branes [859] (see also [709]). In the bulk based formalism, the generalised form of Birkhoff's theorem [272] ensures that the bulk geometry around a FLRW brane is given by

$$
d s^{2}=-V(r) d \tau^{2}+\frac{d r^{2}}{V(r)}+r^{2} q_{i j} d x^{i} d x^{j},
$$

where $V(r)$ takes the form

$$
V_{ \pm}(r)=\kappa+\frac{r^{2}}{4 \alpha}\left(1 \pm \sqrt{1-\frac{8 \alpha}{l^{2}}-\frac{8 \alpha \mu}{r^{4}}}\right)
$$

and $q_{i j}(x)$ is the metric of a 3 -space of constant curvature, $\kappa=0, \pm 1$. Note that this generalises the black hole solution given in Eq. 852 to different "horizon" topologies 233. Let us once again assume $\mathbb{Z}_{2}$ symmetry across the brane for simplicity. As in Section 5.4.2 we treat the brane as an embedding $\tau=\tau(t)$, and $r=a(t)$ in the bulk geometry, identifying $t$ with the proper time of comoving observers on the brane, and $a(t)$ with the scale factor. After imposing the DGW boundary conditions given in Eq.

${ }^{64}$ There is a typo in the corresponding expression for $M_{p l}^{\mathrm{UV}}$ in [270]. 
(860), we find that the Hubble parameter, $H=\dot{a} / a$, obeys [270] (see also [363, 272, 557, 851, 718, )

$$
\begin{aligned}
{\left[1+\frac{4 \alpha}{3}\left(2\left(H^{2}+\frac{\kappa}{a^{2}}\right)+\frac{\kappa-V(a)}{a^{2}}\right)\right] \sqrt{H^{2}+\frac{V(a)}{a^{2}}} } & =\frac{\rho_{\text {brane }}}{6 M_{5}^{3}} \\
& =\frac{\sigma+\rho-3 M_{\text {ind }}^{2}\left(H^{2}+\frac{\kappa}{a^{2}}\right)}{6 M_{5}^{3}}(874
\end{aligned}
$$

This can be recast as a cubic equation in $H^{2}+\frac{\kappa}{a^{2}}$, and solved analytically to give a modified Friedmann equation. The most commonly studied scenario has $M_{\text {ind }}=0$, with the bulk taken to lie on the Einstein branch. We then find 363

$$
H^{2}+\frac{\kappa}{a^{2}}=\frac{c_{+}+c_{-}-2}{8 \alpha}
$$

where

$$
c_{ \pm}=\left[\sqrt{\left(1-\frac{8 \alpha}{l^{2}}+\frac{8 \alpha \mu}{a^{4}}\right)^{3 / 2}+\frac{\alpha(\sigma+\rho)^{2}}{2 M_{5}^{6}}} \pm \frac{(\sigma+\rho)}{M_{5}^{3}} \sqrt{\frac{\alpha}{2}}\right]^{2 / 3} .
$$

This cosmology can give rise to rapid inflation, just as in Randall-Sundrum cosmology [806. However, the Gauss-Bonnet corrections do introduce some new features at the level of cosmological perturbations. For example, in Randall-Sundrum cosmology the consistency relation between the tensor-to-scalar ratio, $r$, and the tensor spectral index, $n_{T}$, agrees with the standard GR result, $r=-8 n_{T}$. This relationship is broken by the GB corrections as the amplitude for the tensor perturbations are no longer monotonically increasing with scale [437. We also find that observational constraints of GB brane inflation are typically softened relative to Randall-Sundrum cosmology, such that certain "steep" potentials are no longer ruled out [1224.

\subsubsection{Co-dimension two branes in EGB gravity}

In Section 5.6 we mentioned that higher-order operators could be added to the bulk gravity to regularise the singularities that appear when we have an infinitely thin braneworld of co-dimension $\geq 2$. One of the ways to do this is to add Gauss-Bonnet corrections in the bulk [174, 283, 282. The simplest and most well studied scenario is to consider co-dimension-2 branes in six dimensional EGB gravity without any additional sources in the bulk (see, for example, [174, 277, 676, 675, 281, 280, 348, 349]). This gives

$$
S=\int_{\text {bulk }} d^{6} x \sqrt{-\gamma}\left[\frac{1}{16 \pi G_{6}}(\mathcal{R}+\alpha \hat{G})\right]+\int_{\text {brane }} d^{4} x \mathcal{L}_{\text {brane }}
$$

If we assume axial symmetry in the bulk we get

$$
d s^{2}=\gamma_{A B} d x^{A} d x^{B}=d r^{2}+L^{2}(x, r) d \theta^{2}+g_{\mu \nu}(x, r) d x^{\mu} d x^{\nu},
$$

and it turns out that the Einstein tensor on the brane is given by [174, 277]

$$
G_{\mu \nu}=-\frac{1}{4 \alpha} g_{\mu \nu}+\frac{G_{6}}{\alpha\left(\frac{1-\beta)}{244}\right.} T_{\mu \nu}^{\text {brane }}+f(\beta) W_{\mu \nu}
$$


where

$$
W_{\mu \nu}=K_{\mu}^{\lambda} K_{\nu \lambda}-K K_{\mu \nu}+\frac{1}{2} g_{\mu \nu}\left(K^{2}-K_{\lambda \sigma} K^{\lambda \sigma}\right),
$$

and $K_{\mu \nu}=\frac{1}{2} \partial_{r} g_{\mu \nu}$. The parameter $\beta$ is the deficit angle on the brane, and is often assumed to be constant, although this need not be case [277. A field dependent deficit angle, $\beta=\beta(x)$, will lead to two important effects: Transfer of energy between bulk and brane, and an effective four dimensional Newton's "constant" that can vary as $G_{\text {eff }}=$ $\frac{G_{6}}{8 \pi \alpha(1-\beta(x))}$ 277. These can clearly be constrained by observation, but it would be very interesting to study the role varying $\beta$ could play in attempts to self-tune the vacuum curvature in co-dimension 2 models.

The function $f(\beta)$ depends on the mathematical technique used to derive the boundary conditions at the distributional source [277, 676, 675]. The boundary conditions derived in [174] lead to the condition $\left.W_{\mu \nu}\right|_{\text {brane }}=0$, suggesting that Einstein gravity should be recovered on the brane at all scales, even for an infinitely large bulk. However, it is now understood that these conclusions rely on the assumption $\partial_{r} K_{\mu \nu}=0$ at the brane, which is too constraining. For constant $\beta$ the Friedmann equation on the brane is given by 277 .

$$
H^{2}+\frac{\kappa}{a^{2}}=\frac{8 \pi G_{\text {eff }}}{3} \rho_{\text {brane }}-\frac{1}{12 \alpha}+\frac{c^{2}}{\rho_{\text {brane }}^{2} a^{8}},
$$

where $c^{2}$ is an integration constant. However, as we already emphasised, in general $\beta$ can vary, and in this case one cannot find a closed system of equations on the brane 277. 


\section{Parameterised Post-Friedmannian Approaches and Observational Constraints}

As expounded in this review, there now exists a vast range of candidate theories of gravity that modify Einstein's theory of General Relativity in one way or another. It is also seems clear that many more such theories are likely to be proposed in the future. If these theories are to be of any value in understanding and resolving the problems associated with the Dark Universe then they must be confronted with cosmological data. This is, in principle, straightforward but time-consuming. It involves working out the perturbation equations for each and every theory, incorporating them into the EinsteinBoltzmann solvers and N-body codes, and calculating a list of observables.

This situation is analogous to the experimental study of General Relativity in the early 1970s, during what has become known as the 'Golden Age' of General Relativity. There, one had a plethora of alternative theories of gravity that needed to be confronted with constraints from Solar System measurements. The Parameterised Post-Newtonian (PPN) method was invented in this case as an intermediate step between theory and experiment. It involves a set of generic parameters that can be easily constrained by experiments [1207]. Using the PPN method one can then take any given theory, calculate the PPN parameters it predicts, and compare them with observational constraints. This process is outlined in Section 2.5

Over the last few years, the idea of creating such an intermediate step when considering cosmological constraints has starting taking hold. It has been dubbed by some the 'Parameterised Post-Friedmannian approach', and it attempts to encompass, at the linear level, the behaviour of a wide array of alternative theories of gravity. We will now outline the basic idea behind this approach.

The bulk of the cosmological data that can be used to constrain modifications of gravity can be interpreted in terms of perturbations about a Friedmann-Lemaître-RobertsonWalker universe. Throughout this review we have presented how the evolution of cosmological perturbations is modified in these theories, relative to their behaviour in General Relativity. One can now ask oneself if there is a general way of modifying the equations of cosmological perturbations such that it will encompass all the theories we have previously discussed.

The simplest approach, that has been in vogue for the past few years, is to modify two of the four Einstein field equations as follows:

$$
\begin{aligned}
-2 k^{2} \Phi & =8 \pi \mu G a^{2} \sum_{X} \rho_{X}\left[\delta_{X}+3\left(1+w_{X}\right) \mathcal{H} \theta_{X}\right] \\
\Phi-\Psi & =\zeta \Phi,
\end{aligned}
$$

where two new functions have been introduced: The effective Newton constant, $G_{\text {eff }}=$ $\mu G$, and the gravitational slip, $\zeta$. One can interpret $G_{\text {eff }}$ as the inclusion of a form of gravitational screening, reducing (or enhancing) the local gravitational force on cosmological scales. The gravitational slip phenomenologically parametrises the shear that seems to arise frequently in scenarios of modified gravity. This parametrisation is incredibly useful for quantifying deviations from General Relativity, and a number of authors have used it in their analyses of cosmological data [148, 237, 630, 627, 41, 360, 361, 123, 1302.

It is instructive to see in what circumstances such a parametrisation might arise, and to do this we will develop a consistent formalism in what follows. 


\subsection{The Formalism}

In order to generalise the perturbed Einstein equations we follow the approach and notation used in 1149, 1151, 501, 77. Here we split the field equations of the theory in question into a set of evolution equations for the metric, evolution equations for the additional gravitational fields (if any are present), and a set of constraint equations. The evolution equations for the metric, and the constraint equations, can then be written schematically as

$$
\delta G_{\mu \nu}^{m o d} \equiv \delta G_{\mu \nu}-\delta U_{\mu \nu}=8 \pi G\left[\delta T_{\mu \nu}+\delta T_{\mu \nu}^{E}\right],
$$

where $\delta G_{\mu \nu}$ is the perturbed Einstein tensor, $\delta U_{\mu \nu}$ is the contribution of any other terms that involve perturbations of the metric, $\delta T_{\mu \nu}$ is the perturbed energy-momentum tensor of matter fields in the space-time, and $\delta T_{\mu \nu}^{E}$ is the contribution of any terms that involve perturbations to the additional gravitational fields.

Let us now be more specific. We can define the following new variables: $U_{\Delta} \equiv$ $-a^{2} \delta U_{0}^{0}, \vec{\nabla}_{i} U_{\Theta} \equiv-a^{2} \delta U^{0}{ }_{i}, U_{P} \equiv \delta U^{i}{ }_{i}$ and $D^{i}{ }_{j} U_{\Sigma} \equiv a^{2}\left(\delta U^{i}{ }_{j}-\frac{1}{3} \delta U_{k}^{k}{ }_{k}{ }_{j}{ }_{j}\right)$, as well as the new gauge invariant

$$
\hat{\Gamma} \equiv \frac{1}{k}\left(\hat{\Phi}^{\prime}+\mathcal{H} \hat{\Psi}\right)
$$

where the Bardeen potentials $\hat{\Phi}$ and $\hat{\Psi}$ are defined in Eqs. 85 and 86 of Section 2.6 Dropping the hats (i.e. working in the conformal Newtonian gauge), we can write the two constraint equations coming from $G_{00}$ and $G_{0 i}$ as

$$
\begin{aligned}
-2 k^{2}\left(\Phi+3 \mathcal{H}_{k} \Gamma\right) & =8 \pi G a^{2} \sum_{X} \rho_{X} \delta_{X}+U_{\Delta} \\
2 k \Gamma & =8 \pi G a^{2} \sum_{X}\left(\rho_{X}+P_{X}\right) \theta_{X}+U_{\Theta},
\end{aligned}
$$

and the two evolution equations coming from the trace and traceless parts of $G_{i j}$ as

$$
\begin{aligned}
k \Gamma^{\prime}+2 k \mathcal{H} \Gamma+\left(\mathcal{H}^{\prime}-\mathcal{H}^{2}-\frac{k^{2}}{3}\right) \Psi+\frac{k^{2}}{3} \Phi & =4 \pi G a^{2} \sum_{X} \delta P_{X}+\frac{1}{6} U_{P} \\
\Phi-\Psi & =8 \pi G a^{2} \sum_{X}\left(\rho_{X}+P_{X}\right) \Sigma_{X}+U_{\Sigma}(8898
\end{aligned}
$$

We can then combine Eqs. 886 and 887 to find a modified Poisson equation:

$$
-2 k^{2} \Phi=8 \pi G a^{2} \sum_{X} \rho_{X}\left[\delta_{X}+3 \mathcal{H}\left(1+w_{X}\right) \theta_{X}\right]+U_{\Delta}+3 \mathcal{H} U_{\Theta}
$$

Assuming that the theory in question has at most $N$-time derivatives in its field equations, and bearing in mind from Eqs. 85,86 and 885 that $\hat{\Phi}$ and $\hat{\Gamma}$ have one time derivative when expressed in an arbitrary gauge, the components of the tensor $U_{\alpha \beta}$ 
can be written as

$$
\begin{aligned}
U_{\Delta} & =\sum_{n=0}^{N-2} k^{2-n}\left[A_{n} \hat{\Phi}^{(n)}+E_{n} \hat{\Gamma}^{(n)}\right], \\
U_{\Theta} & =\sum_{n=0}^{N-2} k^{1-n}\left[B_{n} \hat{\Phi}^{(n)}+F_{n} \hat{\Gamma}^{(n)}\right], \\
U_{P} & =\sum_{n=0}^{N-1} k^{2-n}\left[C_{n} \hat{\Phi}^{(n)}+I_{n} \hat{\Gamma}^{(n)}\right], \\
U_{\Sigma} & =\sum_{n=0}^{N-1} k^{-n}\left[D_{n} \hat{\Phi}^{(n)}+J_{n} \hat{\Gamma}^{(n)}\right],
\end{aligned}
$$

where $\hat{\Phi}^{(n)} \equiv \frac{d^{n}}{d \tau^{n}} \hat{\Phi}$, and similarly for $\hat{\Gamma}$. The coefficients $A_{n^{-}} J_{n}$ depend on time and scale through the scale factor, $a$, and wavenumber, $k$. For the sake of brevity we will refrain from explicitly stating these dependences.

Now, although we have defined $U$ above in the Newtonian gauge, the individual terms appearing in the expressions above are all gauge invariant. This fact, however, imposes further constraints because the gauge-invariant variable $\hat{\Gamma}$ contains second derivatives of the scale factor, when expressed in an arbitrary gauge. Hence, to avoid higher derivatives of the background appearing in the field equations, we have to set

$$
E_{N-2}=F_{N-2}=I_{N-1}=J_{N-1}=0 \text {. }
$$

We assume that the evolution equations for the matter fields remain unchanged, and that these equations are supplemented by additional evolution equations for the extra gravitational fields. Finally, the field equations are closed by imposing the Bianchi identities. This imposes one of two possible options: (i) $\nabla^{\alpha} T_{\alpha \beta}^{E}=\nabla^{\alpha} U_{\alpha \beta}=0$, which imposes a series of constraints such that the theory remains consistent, or (ii) $\nabla^{\alpha}\left(T_{\alpha \beta}^{E}+U_{\alpha \beta}\right)=$ 0 , which is the more general situation. For a detailed discussion of these issues see 1149, 1151, 77.

\subsubsection{Evolution of perturbations on super-horizon scales}

In principle it may seem that one should explore the long wavelength behaviour of cosmological perturbations on a case by case basis. It turns out, however, that the infinite wavelength mode can be studied simply by considering the evolution of the background equations. This observation is due to Bertschinger [148], and proceeds as follows. Consider a background with scale factor $a(\tau, \kappa)$ where $\kappa$ is the spatial curvature of a hyper-surface of constant $\tau$ :

$$
d s^{2}=a^{2}\left[-d \tau^{2}+d \chi^{2}+\frac{1}{\kappa} \sinh _{\kappa}^{2}(\sqrt{\kappa} \chi) d \Omega\right]
$$

where $\sinh _{\kappa}(x)$ equals $\sin (x)$ for $\kappa>0$, equals $x$ for $\kappa=0$, or equals $\sinh (x)$ for $\kappa<0$. We can now perturb this space-time as $\kappa \rightarrow \kappa\left(1+\delta_{\kappa}\right)$, and compensate by a change the coordinates $\tau \rightarrow \tau+\alpha$ and $\chi \rightarrow \chi\left(1-\frac{1}{2} \delta_{\kappa}\right)$. Here $\delta_{\kappa}$ is a constant, while $\alpha=\alpha(\tau)$. Note that in this case the scale factor $a(\tau, \kappa)$ is perturbed as $a\left(\tau+\alpha, \kappa\left(1+\delta_{\kappa}\right)\right)$. In words, the 
scale factor will come out as the solution of some generalised set of Friedmann equations, and will depend on the spatial curvature, $\kappa$.

We can now write this new geometry in the form of a perturbed FLRW metric, with background curvature $\kappa$ :

$$
d s^{2}=a^{2}\left\{-(1+2 \Psi) d \tau^{2}+(1-2 \Phi)\left[d \chi^{2}+\frac{1}{\kappa} \sinh _{\kappa}^{2}(\sqrt{\kappa} \chi) d \Omega\right]\right\},
$$

where

$$
\begin{aligned}
\Psi(\tau) & =\frac{\partial \ln a}{\partial \ln \kappa} \delta_{\kappa}+\alpha^{\prime}+\mathcal{H} \alpha, \\
\Phi(\tau) & =\left(\frac{1}{2}-\frac{\partial \ln a}{\partial \ln \kappa}\right) \delta_{\kappa}-\mathcal{H} \alpha .
\end{aligned}
$$

One can now eliminate $\alpha$ to find a generic evolution equation that relates $\delta_{\kappa}$ with $\Phi$ and $\Psi$ without specifying any particular theory of gravity:

$$
\frac{1}{a^{2}} \frac{d}{d \tau}\left(\frac{a^{2} \Phi}{\mathcal{H}}\right)=\Phi-\Psi+\left[\frac{1}{2 a} \frac{d}{d \tau}\left(\frac{a}{\mathcal{H}}\right)-\frac{\partial \ln \mathcal{H}}{\partial \ln \kappa}\right] \delta_{\kappa},
$$

where entropy perturbations have been neglected 65 . The constant $\delta_{\kappa}$ that remains in the equation above has a direct physical interpretation: It is twice the comoving curvature perturbation. Note that we have also assumed that shear perturbations are negligible on large scales, that local energy-momentum us conserved, and that spatial gradients can be discarded. By choosing $\mathcal{H}(a, \kappa)$, and a relation between $\Phi$ and $\Psi$, one now has the evolution equation completely defined. This is a powerful statement, as it means it is possible to determine the evolution of large-scale perturbations without delving into the details of the theory.

However, in order to complete the system here one still needs to specify a relation between $\Phi$ and $\Psi$. As we will describe below, it has become standard practise to assume the simplest form of the PPF parametrisation, that we described above, on super-horizon scales. Within this approach comparisons have been made between choices of $\mu$ and $\zeta$, and the outcomes of numerical solutions for specific theories. These comparisons show reasonable agreement, but, as yet, there is no compelling argument for applying the simplest PPF parametrisation on large scales. In other words, there is no guarantee that a simple relation of the form $\Psi=\zeta \Phi$ can encompass all possible theories of gravity. In fact, it would appear that Eq. 900 does not allow such an interpretation.

\subsubsection{The simplified PPF approach, and its extensions}

Let us now revisit the simplest, and by far the most popular, version of the Parameterised Post Friedmannian approach [148, 237, 630, 627, 41]. Here one considers only Eqs. 889 and 890 . The further assumption that perturbations have no anisotropic stress (i.e. $\Sigma_{X}=0$ ) then allows one to reorganise them in the form of Eqs. 882 and 883). These equations can then be rewritten in terms of the $U_{\alpha \beta}$ tensor by choosing

$$
\begin{aligned}
U_{\Delta}+3 \mathcal{H} U_{\theta} & =2 k^{2}\left[\frac{1-\mu}{\mu}\right] \Phi, \\
U_{\Sigma} & =\zeta \Phi .
\end{aligned}
$$

\footnotetext{
${ }^{65}$ Including entropy perturbations is straightforward, and for this the reader is referred to 148 .
} 
The coefficients $A_{i}, B_{i}, C_{i}$ and $D_{i}$ must now be chosen to satisfy this condition.

It is understandable why this approach is popular, as it has a number of benefits. Firstly, it is applicable in the quasi-static regime the arises when $k \rightarrow \infty$ (i.e. on small scales relative to the cosmological horizon). Furthermore, the perturbation equations now form a closed system. This means that if, for example, we restrict ourselves to a dust-filled universe then Eqs. 882 and 883 , together with

$$
\begin{aligned}
& \delta_{M}^{\prime}=-k^{2} \theta_{M}+3 \Phi^{\prime} \\
& \theta_{M}^{\prime}=-\mathcal{H} \theta_{M}+\Psi,
\end{aligned}
$$

form a complete system of differential equations that can be straightforwardly solved.

It is instructive at this point to specify the various versions of the simplified PPF formalism that are currently in use:

- Caldwell, Cooray and Melchiorri [237, 360, introduced the system 66

$$
\begin{aligned}
\mu & =1 \\
\zeta & \equiv \varpi=\varpi_{0} a^{3},
\end{aligned}
$$

which was later extended to 361

$$
\mu=1+\mu_{0} a^{3} .
$$

- Bertschinger and Zukin [148, 151 proposed a reduced parametrisation that takes into account the conservation of long wavelength curvature perturbations. Only one parameter is considered 67

$$
\gamma_{B Z}=1-\zeta
$$

that is further refined as $\gamma_{B Z}=1+\beta a^{s}$, where $\beta$ and $s$ are constants. These authors make the additional assumptions that $\gamma_{B Z}$ depends only on time, even on sub-horizon scales, and that the Bertschinger long-wavelength construction (see Section 6.1.1 can also be extended to sub-horizon scales. The latter of these assumptions allows them to solve for $\Phi$, from which $\Psi$ and $\delta$ are then determined. The assumption of scale-independence is later relaxed further, such that the following parameterisation can be made

$$
\begin{aligned}
\gamma_{B Z} & =\frac{1+\beta_{1} k^{2} a^{s}}{1+\beta_{2} k^{2} a^{s}} \\
G_{\Phi} & =\mu(1-\zeta)=G \frac{1+\alpha_{1} k^{2} a^{s}}{1+\alpha_{2} k^{2} a^{s}},
\end{aligned}
$$

where $\alpha_{i}$ and $\beta_{i}$ are constants 68

\footnotetext{
${ }^{66}$ The initial convention was that $\varpi=\varpi_{0} \frac{\Omega_{0 D E}}{\Omega_{0 M}} a^{3}$, but the authors changed their convention in subsequent publications.

${ }^{67}$ The symbol used was actually $\gamma$, to which we have added a subscript "BZ" to distinguish it from other parameters with the same name. A similar approach will be used with the other frame-works presented here.

${ }^{68}$ We kept the original symbol $G_{\Phi}$ here, although under our conventions it would be more accurately written as $G_{\Psi}$, as it plays the role of an effective gravitational constant for a modified Poisson equation for $\Psi$.
} 
- Amendola, Kunz and Sapone [41] modify the Poisson equation and the slip by considering the two functions $Q_{A}$ and $\eta_{A}$ :

$$
\begin{aligned}
Q_{A} & =\mu, \\
\eta_{A} & =-\zeta,
\end{aligned}
$$

while a third variable, $\Sigma_{A}=Q_{A}\left(1+\eta_{A} / 2\right)$, is also introduced in order to simplify the relevant expression for gravitational lensing: $\Phi+\Psi=2 \Sigma_{A} \Phi$. A comparison of this approach is made with DGP and scalar-tensor theories, both on sub-horizon scales.

- Zhang et al. 1301 make the parameterisation

$$
\begin{aligned}
\eta_{Z L} & =\frac{1}{1-\zeta}, \\
\tilde{G}_{e f f} & =\mu(1-\zeta / 2) .
\end{aligned}
$$

These authors also introduce the $E_{G}$ statistic, which we describe further below.

- Zhao et al. 1302 modify the Poisson equation using $\Psi$. They introduce the two parameters

$$
\begin{aligned}
\eta_{Z G} & =\frac{1}{1-\zeta}, \\
\mu_{Z G} & =\mu(1-\zeta),
\end{aligned}
$$

as well as a third derived parameter $\Sigma_{Z G}=\mu_{Z G}\left(1+\eta_{Z G}\right) / 2=\Sigma_{A}$. Further refinements are made by considering two specific models: A case where $\mu_{Z G}=$ $\mu_{Z G}(\tau)$ and $\eta_{Z G}=\eta_{Z G}(\tau)$ transit from their GR values in the early universe to modified constant values today, and a case where the two functions are pixelised in the $\{\tau, k\}$ plane by $2 \times 2$ pixels per function.

- A parametrisation specifically designed for small scales was proposed by Amin, Blandford and Wagoner [44, where

$$
\begin{aligned}
B_{A}\left(\mathcal{H}_{k}, a\right) & =(1-\zeta) \mu=\beta_{0}(a)+\beta_{1}(a) \mathcal{H}_{k}+\beta_{2}(a) \mathcal{H}_{k}^{2}+\ldots \\
\Gamma_{A}\left(\mathcal{H}_{k}, a\right) & =\mu=\gamma_{0}(a)+\gamma_{1}(a) \mathcal{H}_{k}+\gamma_{2}(a) \mathcal{H}_{k}^{2}+\ldots
\end{aligned}
$$

while the matter density fluctuation is likewise parametrised by

$$
\delta_{m}(a, k)=\delta(k)_{i}\left[\delta_{0}(a)+\delta_{1}(a) \mathcal{H}_{k}+\delta_{2}(a) \mathcal{H}_{k}^{2}\right],
$$

where $\delta(k)_{i}$ is specified by initial conditions. In this approach different theories correspond to different sets of the functions $\left\{\beta_{i}, \gamma_{i}, \delta_{i}\right\}$. The authors find the appropriate functions for $\Lambda \mathrm{CDM}$, scalar-tensor theories, quintessence models, $f(R)$ theories, and DGP. They also note, however, that not every theory can be adequately matched to this expansion (e.g. k-essence). 
The simplified PPF approach, though useful as a phenomenological tool that can be used to constrain $\mu$ and $\zeta$, is not without its problems. For a start, it is not clear what theories a specific choice of parameters actually encompasses. In [1149, 501, 77, this problem has been addressed in detail, and it is shown that if one wishes to consider theories with second order field equations only then the PPF equations become:

$$
\begin{aligned}
-2 k^{2} \Phi & =8 \pi \mu G a^{2} \sum_{X} \rho_{X}\left[\delta_{X}+3\left(1+w_{X}\right) \mathcal{H} \theta_{X}\right] \\
\Phi-\Psi & =\zeta \Phi+\frac{g}{k} \Phi^{\prime}
\end{aligned}
$$

with the constraint $\mu=1-\frac{g}{k} \mathcal{H}$. In other words, if the field equations of the theory are second order then the simplified PPF approach is not applicable.

Let us now consider what the simplified PPF approach does correspond to in the field equations. Taking the expansion for $U_{\alpha \beta}$, and considering terms up to the lowest acceptable order, we have:

$$
\begin{aligned}
& U_{\Delta}=A_{0} k^{2} \Phi+A_{1} k \Phi^{\prime}+E_{0} k^{2} \Gamma, \\
& U_{\Theta}=B_{0} k \Phi+B_{1} \Phi^{\prime}+F_{0} k \Gamma, \\
& U_{P}=C_{0} k^{2} \Phi+C_{1} k \Phi^{\prime}+C_{2} \Phi^{\prime \prime}+I_{0} k^{2} \Gamma+I_{1} k \Gamma^{\prime}, \\
& U_{\Sigma}=D_{0} \Phi .
\end{aligned}
$$

Applying the Bianchi identities one can then determine the above functions in terms of $\zeta=D_{0}$ and $\tilde{g}=\frac{1}{\mu}-1$. This gives $F_{0}=E_{0}=I_{0}=I_{1}=0$ (i.e. no $\Gamma$ terms appearing), and

$$
\begin{aligned}
& k B_{0}=2 k^{2} \frac{\tilde{g}^{\prime}+\mathcal{H}(\tilde{g}+\zeta)}{3 \mathcal{H}^{\prime}-3 \mathcal{H}^{2}-k^{2}}, \quad A_{0}=2 \tilde{g}-3 \mathcal{H}_{k} B_{0}, \quad C_{0}=2 \zeta+3\left(\frac{1}{k} B_{0}^{\prime}+2 \mathcal{H}_{k} B_{0}\right), \\
& B_{1}=2 k^{2} \frac{\tilde{g}}{3 \mathcal{H}^{\prime}-3 \mathcal{H}^{2}-k^{2}}, \quad A_{1}=-3 \mathcal{H}_{k} B_{1}, \quad k C_{1}=3\left(B_{1}^{\prime}+2 \mathcal{H} B_{1}+k B_{0}\right) .
\end{aligned}
$$

Since $A_{1}+3 \mathcal{H}_{k} B_{1}=E_{0}=F_{0}=0$, the form of the Poisson Equation 890 is retained. However, since $A_{1}, B_{1}$ and $C_{2}$ do not vanish, the field equations contain third time derivatives in arbitrary gauge. This is due to the presence of $\Phi^{\prime \prime}$, and corresponds to a higher-order gravitational theory 69 . In other words, one to simplify the equations for the gravitational slip, but only at the cost of introducing higher-order terms in the other evolution equations (to enforce consistency).

The above way of reconstructing the $U$ 's from the simplified approach is not unique. One can also relax the condition $\nabla^{\alpha} T_{\alpha \beta}^{E}=\nabla^{\alpha} U_{\alpha \beta}=0$, but this moves us further away from the simplified approach.

A further problem of the simplified approach is that it is impossible to determine $\mu$ and $\zeta$ for a specific theory without solving the field equations for a specific choice of initial conditions. What we would like to have is a one-to-one correspondence between the functions that appear in the parametrised frame-work and those that appear in the theories themselves, without having to solve the field equations every time (just as in the PPN formalism, discussed in Section 21. This can be achieved by constructing the field equations as in Section 6.1.

\footnotetext{
${ }^{69}$ Since the form of the Poisson equation is retained, however, both $\Phi$ and $\Psi$ remain non-dynamical. Hence, the higher derivatives do not introduce additional propagating degrees of freedom.
} 


\subsubsection{The Hu-Sawicki frame-work}

$\mathrm{Hu}$ and Sawicki introduced a frame-work [630, later generalised by $\mathrm{Hu}$ 627, that depends on a function of time and space, $g_{H S}(\tau, k)$, two functions of time, $f_{\zeta}(\tau)$ and $f_{G}(\tau)$, and a constant, $c_{\Gamma}$. Their frame-work goes beyond the simplified PPF frameworks described above, and tends to them in either the super-horizon or sub-horizon limits of the late Universe ${ }^{70}$. The field equations in this case are written as

$$
\begin{aligned}
\Phi+\Psi & =-\frac{8 \pi G a^{2}}{c_{\kappa} k^{2}} \sum_{X} \rho_{X}\left[\delta_{X}+3 \mathcal{H}\left(1+w_{X}\right) \theta_{X}\right]-8 \pi G a^{2} \sum_{X}\left(\rho_{X}+P_{X}\right) \Sigma_{X}+2 \Gamma_{H S}, \\
\Phi-\Psi & =8 \pi G a^{2} \sum_{X}\left(\rho_{X}+P_{X}\right) \Sigma_{X}+g_{H S}(\Phi+\Psi),
\end{aligned}
$$

where $c_{\kappa}=1-\frac{3 \kappa}{k^{2}}$. The variable $\Gamma_{H S}$ is obtained by solving the differential equation

$$
\begin{aligned}
& 2\left(1+g_{H S}\right)\left(1+\frac{c_{\Gamma}}{\mathcal{H}_{k}^{2}}\right)\left[\Gamma_{H S}^{\prime}+\mathcal{H} \Gamma_{H S}+\frac{c_{\Gamma} k}{\mathcal{H}_{k}}\left(\Gamma_{H S}+\frac{1}{2} f_{G}(\Phi+\Psi)\right)\right] \\
= & \left(2 \mathcal{H} g_{H S}-g_{H S}^{\prime}\right)(\Phi+\Psi)+8 \pi G a^{2} g_{H S} \sum_{X}\left[\left(\left(\rho_{X}+P_{X}\right) \Sigma_{X}\right)^{\prime}+\mathcal{H}\left(\rho_{X}+P_{X}\right) \Sigma_{X}\right] \\
& -\frac{8 \pi G a^{2}}{k^{2}}\left[g_{H S}\left(1+f_{\zeta}\right)+f_{G}\right] \sum_{X}\left(\rho_{X}+P_{X}\right) \theta_{X}+\frac{8 \pi G a^{2}}{k^{2}}\left(\rho_{E}+P_{E}\right) \theta_{T},
\end{aligned}
$$

where $\theta_{T}=\frac{\sum_{X}\left(\rho_{X}+P_{X}\right) \theta_{X}}{\sum_{X} \rho_{X}+P_{X}}$ is calculated in conformal Newtonian gaug $\oint^{71}$. In both Eq. (913) and Eq. (914) $\rho_{X}$ and $P_{X}$ do not include contributions from the dark energy. Taking the sub-horizon limit, for which $\mathcal{H}_{k} \rightarrow 0$, we get that $\Gamma_{H S} \rightarrow-\frac{1}{2} f_{G}(\Phi+\Psi)$, and the system then reduces to the simplified frame-work with

$$
\begin{aligned}
\mu & =\frac{1+g_{H S}}{1+f_{G}}, \\
\zeta & =\frac{2 g_{H S}}{1+g_{H S}} .
\end{aligned}
$$

In the super-horizon limit the system obeys the Bertschinger construction (see Section 6.1.1, with $g_{H S}=g_{H S}(\tau)$ while $f_{\zeta}(\tau)$ provides the next order correction (beyond the Bertschinger construction). We see that the sub-horizon limit depends only on $g_{H S}$ and $f_{G}$, while the super-horizon limit depends only on $g_{H S}$ and $f_{\zeta}$. The constant $c_{\Gamma}$ controls the transition scale between these two limits.

As in the simplified approaches, it is not clear from the outset what kind of theories this frame-work encompasses. One can find fitting functions $g_{H S}, f_{G}$ and $f_{\zeta}$ that reproduce the solutions for specific theories, but only after some experimentation.

\footnotetext{
${ }^{70}$ At late times the matter content of the Universe is effectively described by dust and dark energy, for which the dust has no anisotropic stress.

${ }^{71}$ In the original formulation the comoving gauge was used, but we translated the equations here to make it more consistent with the other approaches we described.
} 


\subsection{Models for $\mu$ and $\zeta$ on Sub-Horizon Scales}

On sub-horizon scales the situation is greatly simplified. In this case one can make a quasi-static approximation, and discard all time variations in the perturbed fields. Let us now consider this regime in a few different models.

To start with we can consider $f(R)$ theories. Following [1043] we then have

$$
\begin{aligned}
-k^{2}(\Phi+\Psi) & =4 \pi \frac{G a^{2} \rho_{M}}{f_{R}} \delta_{M}, \\
\Psi-\Phi & =-\frac{2 f_{R R} k^{2}}{3 f_{R R} k^{2}+a^{2} f_{R}} f_{R}(\Phi+\Psi),
\end{aligned}
$$

which can be rewritten in the $\mu-\zeta$ form given above. Note that there is a scale in these equations, the "Compton wavelength" given by

$$
Q \equiv 3 \frac{k^{2}}{a^{2}} \frac{f_{R R}}{f_{R}} .
$$

For large wavelengths $Q \rightarrow 0$, and $\Psi-\Phi \rightarrow 0$. For small wavelengths $Q \gg 1$, and $\Psi-\Phi \simeq-(2 / 3) f_{R}(\Phi+\Psi)$.

Let us now consider DGP models. In this case the expression for gravitational slip can be found to be 737 .

$$
\begin{aligned}
& \mu=1-\frac{1}{3 \beta}, \\
& \zeta=\frac{3 \beta-2}{3 \beta-1},
\end{aligned}
$$

where $\beta=1+2 \epsilon H r_{c} w_{E}$ (for more details see Section 5.5, and in particular Eqs. 812, and $(813)$ ).

Finally, let us consider scalar-tensor theories. For a generalised coupling parameter, $F(\phi)$, it can be shown that [41]

$$
\begin{aligned}
\mu & =\frac{G^{*}}{F G_{\mathrm{cav}}} \frac{2\left(F+F^{\prime 2}\right)}{2 F+3 F^{\prime 2}} \\
\zeta & =\frac{F^{\prime 2}}{F+F^{\prime 2}}
\end{aligned}
$$

The expression above can be very useful in studying the evolution of structure on observable scales. They can, for example, be used to correctly reproduce the growth rate of structure, and the effects of lensing, on scales of up to hundreds of Mpc.

\subsubsection{The importance of shear}

An important issue arises if one wishes to distinguish between the effects of dark energy and the effects of modified gravity. In [759] the authors constructed an explicit example of how anisotropic stress could mimic the effect of gravitational slip. To see this let us consider Eq. 889 with $U_{\Sigma}=D_{0} \Phi$. There is clearly a degeneracy between the two terms on the left hand side. In particular, if we focus on DGP then we have 
that $\Sigma=0$, but $U_{\Sigma}=\frac{3 \beta-2}{3 \beta-1} \Phi^{72}$. However, we can of course consider an alternative model where gravity is unmodified, but the dark energy has anisotropic stress given by $\left(1+w_{E}\right) \Sigma_{E}=-\frac{1}{2 k^{2}} \frac{3 \beta-2}{3 \beta-1} \delta_{M}$. This would be an odd form of dark energy, but it could also explain observations that may otherwise have been attributed to gravitational slip. Distinguishing between such models is, of course, made easier when considering a wide variety of different scales.

\subsubsection{The growth function}

The primary effect of modified gravity will be on the growth of structure. The time evolution of the density field can be a sensitive probe of not only the expansion rate of the Universe but also its matter content. In a flat, matter dominated universe we have that $\delta_{M}$, the density contrast of matter, evolves as $\delta_{M} \propto a$. We can parametrise deviations from this behaviour in terms of the growth function, $f$, given by

$$
f \equiv \frac{\delta_{M}^{\prime}}{\mathcal{H} \delta_{M}},
$$

or by the introduction of a parameter $\gamma$ through [1031, 813, 783, 784]

$$
f=\Omega_{M}^{\gamma}
$$

Note that $\gamma$ and $f$ are not parameters in the usual sense, but are derived quantities (indirect observables, to some extent). It is, however, sometimes convenient to parameterise some results or processes in terms of these quantities.

For standard growth in the presence of a cosmological constant one has $\gamma \approx 6 / 11$ to a very good approximation, although this can change with $\Omega_{M}$. For the case of General Relativity with a dark energy component with equation of state $P=w \rho$ (where $w$ is constant) we have

$$
\gamma=\gamma_{0}+\gamma_{1} \Omega_{E}+O\left(\Omega_{E}^{2}\right)
$$

where $\Omega_{E}=1-\Omega_{M}$, and where $\gamma_{0}$ and $\gamma_{1}$ are given by

$$
\begin{aligned}
\gamma_{0} & =\frac{3(1-w)}{5-6 w} \\
\gamma_{1} & =\frac{\gamma_{0}}{2 w}\left[\frac{6 w^{2}-7 w+2}{5-12 w}-\frac{2-3 w}{3} \gamma_{0}\right] .
\end{aligned}
$$

In the case of a cosmological constant (i.e. with $w=-1$ ) these expressions reduce to

$$
\gamma=\frac{6}{11}+\frac{15}{2057} \Omega_{E}+\ldots
$$

which gives the first order correction to the expression $\gamma \approx 6 / 11$, given above.

\footnotetext{
${ }^{72}$ Actually, as discussed in Section 5.5 DGP has shear but the quasi-static limit imposes the condition $\mu_{E}=2 k^{2} \Sigma_{E}$ between energy density associated with the perturbation of the Weyl tensor and the shear. This in turn generates the term $U_{\Sigma}$ above.
} 
A natural question to ask is how the growth parameter, $\gamma$, depends on modifications of gravity. There have been a number of attempts at finding analytic expressions that relate $\gamma$ to parameters in the underlying theory. This has focused on specific theories, as well as the extended PPF approach. We now discuss some of the results.

An expression for the growth parameter in the quasi-static limit of $f(R)$ theories was found in [58]. Here one can define a time dependent mass scale:

$$
M^{2}(a)=\frac{1}{3 \bar{f}_{R R}},
$$

where $\bar{f}_{R R}$ is the second functional derivative of $f$ with respect to the Ricci scalar, $R$, evaluated at the General Relativistic value of $R$. A non-local expression for $\gamma$ can then be found of the form

$$
\gamma=\frac{6}{11}-\frac{\Omega_{\Lambda} k^{2}}{2 \Omega_{M}} a^{-11 / 2} \int_{0}^{a} \frac{\tilde{a}^{3 / 2} d \tilde{a}}{k^{2}+\tilde{a}^{2} M^{2}(\tilde{a})} .
$$

One can see that for $k \ll a M(a)$ this expression for $\gamma$ remains the same as in General Relativity, but that once $k$ crosses the mass threshold modifications start to kick-in.

In DGP models the correct expression for $\gamma$ is given by ${ }^{73}$ [501]

$$
\gamma=\frac{11}{16}+\frac{7}{5632} \Omega_{E}-\frac{93}{4096} \Omega_{E}^{2}+O\left(\Omega_{E}^{3}\right) .
$$

This result is in excellent agreement with numerical studies (to within $2 \%$ or better for $\Omega_{E}<0.8$, and to $5 \%$ for $\left.\Omega_{E}<0.9\right)$.

An attempt at finding approximate analytic expressions for $\gamma$ was presented in 501. In this report we will restrict ourselves to limiting cases where $\zeta \simeq \zeta_{1} \Omega_{E}$. If we then assume that we can Taylor expand $\gamma$ as in Eq. (919) then we find the coefficients

$$
\begin{aligned}
\gamma_{0} & =\frac{3\left(1-w+\zeta_{1}\right)}{5-6 w}, \\
\gamma_{1} & =\frac{3 w}{2}\left[-3 Y_{1}-(2-3 w) Y_{1}^{2}+4 Y_{2}\right],
\end{aligned}
$$

where we have defined

$$
\begin{aligned}
Y_{1} & \equiv \frac{1-w+\zeta_{1}}{w(5-6 w)} \\
Y_{2} & \equiv \frac{(1-w)\left(15 w^{2}-4 w-1\right)+\zeta_{1}\left(9 w^{2}+2 w-2\right)}{2 w^{2}(12 w-5)(5-6 w)}-\frac{\zeta_{1}^{2}}{2 w^{2}(12 w-5)(5-6 w)} .
\end{aligned}
$$

A comparison between the approximation described above and the exact numerical value of $\gamma$ is shown in Figure 14. More general expression for $\gamma$ that include the impact of $\mu$, varying $w$, higher-order terms in $\Omega_{E}$, as well as scale dependent correction in $\mathcal{H}_{k}$ are presented in [501].

\footnotetext{
${ }^{73}$ Incorrect values for $\gamma$ in DGP models have been presented in 816 41, 1263.
} 


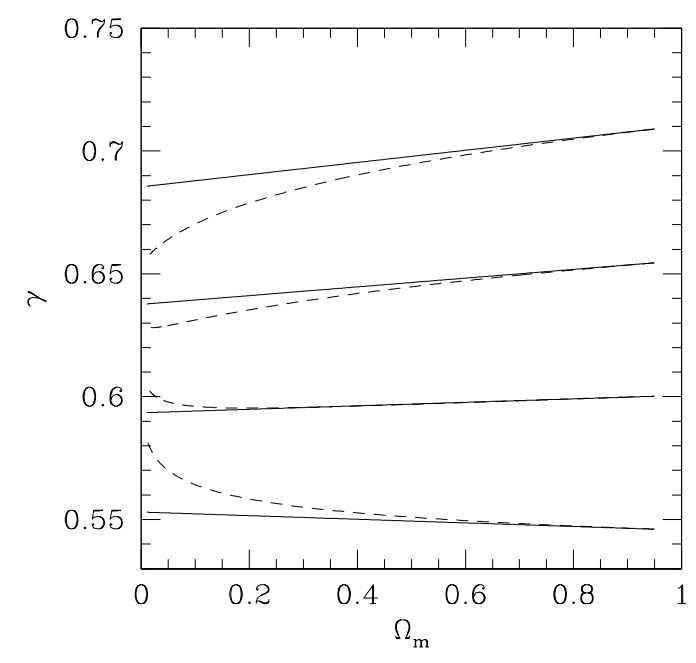

Figure 14: The growth parameter, $\gamma$, for a selection of gravitational slip parameters of the form $\zeta=\zeta_{1} \Omega_{E}$, as a function of $\Omega_{M}$. The dashed curves are the numerical results for $\zeta_{1}=0,0.2,0.4$ and 0.6 in ascending order, and the corresponding analytic approximations are plotted as solid lines.

\subsubsection{Current constraints on the PPF parameters}

At the time of writing this report, there have only been a few attempts at constraining the PPF parameters using existing cosmological data sets [123, 361, 1302. Let us now consider each of these in turn.

In [123] the authors find constraints on $Q=\mu$ and $R=1-\zeta$ using the Union set of Supernovae from the Supernovae Cosmology Project (with an additional sample), the joint 2dF and DR7 SDSS survey estimate of the Baryon Acoustic Oscillations, the WMAP 7-year CMB Temperature and Polarisation data, the matter power spectrum from the SDSS DR7 release, auto and cross-correlation functions from 2MASS and the SDSS LRG catalogue with ISW and the COSMOS weak lensing survey.

A scale and time dependent form of the PPF parameters is proposed:

$$
\begin{gathered}
Q(k, a)=1+\left[Q_{0} e^{k / k_{c}}+Q_{\infty}\left(1-e^{k / k_{c}}\right)-1\right] a^{s}, \\
R(k, a)=1+\left[R_{0} e^{k / k_{c}}+R_{\infty}\left(1-e^{k / k_{c}}\right)-1\right] a^{s} .
\end{gathered}
$$

Setting $k_{c} \rightarrow \infty$ and $s=0$ the authors then find time independent constraints on the parameters: $0.97<Q<1.01$ and $0.99<R<1.02$ (both at the $95 \%$ confidence level). Their analysis shows that primary constraints come from the WMAP7 data, and that current weak lensing and cross correlations between galaxies and ISW have a minimal effect. Allowing time dependence greatly relaxes the constraints on the parameters: $1.04<Q<2.66$ and $-0.22<R<1.44$ (both at the $95 \%$ confidence level). If a transition scale of $k_{c}=0.01 \mathrm{Mpc}^{-1}$ is considered, parameters are then constrained to be $0.47<Q_{0}<3.49,-0.80<R_{0}<2.52,0.97<Q_{\infty}<2.65$, and $-0.43<R_{\infty}<1.76$.

The analysis of Daniel et al 361 extends the above analysis to include an incarnation of the CFHTLS lensing survey, which has substantially larger coverage than that of the COSMOS survey. An earlier release of WMAP data is used, and cross correlations 
between large scale structure and ISW are not included. The authors focus on scaleindependent parameters, $\zeta=\tilde{\omega}=\tilde{\omega}_{0} a^{3}$ and $\mu=\mu_{0} a^{3}$, and find $-1.4<\tilde{\omega}_{0}<2.8$ and $-0.67<\mu_{0}<2.0$. The authors also consider a more elaborate time evolution of both $\tilde{\omega}$ and $\mu$ by dividing it up into bins in the following redshift ranges: (a) $[0,1]$, (b) $[1,2]$ and (c) $[2,9]$. Setting $\tilde{\omega}=0$ they then find that $\mu$ is constrained to be: $-0.074<\mu_{0 a}<0.08$, $-0.058<\mu_{0 b}<0.14,-0.023<\mu_{0 c}<0.22$. Setting $\mu=1$ they find that $\tilde{\omega}$ is constrained to be: $-0.074<\tilde{\omega}_{0 a}<0.08,-0.058<\tilde{\omega}_{0 b}<0.14,-0.023<\tilde{\omega}_{0 c}<0.22$.

In 1302 the authors consider two different parameterisations. In the first they consider a scale independent but time evolving parametrisation for $\eta_{Z G}$ and $\mu_{Z G}$, given by Eq. 908 as

$$
\begin{gathered}
\mu_{Z G}(z)=\frac{1-\mu_{0}}{2}\left(1+\tanh \frac{z-z_{s}}{\Delta z}\right)+\mu_{0}, \\
\eta_{Z G}(z)=\frac{1-\eta_{0}}{2}\left(1+\tanh \frac{z-z_{s}}{\Delta z}\right)+\eta_{0} .
\end{gathered}
$$

These authors use essentially the same data sets as in [123, albeit with an earlier release of the WMAP data (5 year) and without BAO constraints. They find that if $z_{s}=1$ then the constraints are $0.65<\mu_{0}<1.9$ and $-0.41<\eta_{0}<2.18$, while if $z_{s}=1$ the constraints are $0.68<\mu_{0}<1.11$ and $0.7<\eta_{0}<1.9$.

In a second parametrisation the authors bin the parameters in both time and scale. They choose not to bin in $\eta$, but rather in $\Sigma_{Z G}=\frac{\mu_{Z G}\left(1+\eta_{Z G}\right)}{2}$. This is the combination that enters into the calculation of both the ISW effect and weak lensing through their dependence on $\Phi+\Psi$. They consider the following bins: (a) $k \in[0,0.1], z \in[1,2]$, (b) $k \in[0,0.1], z \in[0,1]$, (c) $k \in[0.1,0.2], z \in[1,1]$, and (d) $k \in[0.1,0.2], z \in[1,2]$. The resulting constraints are then as follows: In bin (a) $0.96<\Sigma_{1}<1.04,0.64<\mu_{1}<1.42$, in bin (b) $0.93<\Sigma_{2}<1.07,0.65<\mu_{2}<1.34$, in bin (c) $0.58<\Sigma_{3}<1.02,0.24<\mu_{3}<$ 2.24 , in bin (d) $0<\Sigma_{4}<2.23,0.05<\mu_{4}<2.46$.

\subsubsection{Constraining the growth rate}

Over the past few years there have been attempts to target the growth rate directly using redshift space distortions. This method involves measuring either the redshift space correlation function, $\xi^{s}\left(r_{\|}, r_{\perp}\right)$ (where the $r_{\|}$and $r_{\perp}$ correspond to parallel or perpendicular directions to the line of sight), or the redshift space power spectrum, $P_{g}^{s}(k)$. The power spectrum can be related to the real space power spectrum through an extension of what is known as the 'Kaiser formula':

$$
P^{s}(\mathbf{k})=P(\mathbf{k})\left[1+2 \mu^{2} \beta^{2}+\mu^{4} \beta^{2}\right] G\left[\frac{k^{2} \mu^{2} \sigma_{v}^{2}}{H^{2}(z)}\right],
$$

where $\beta=\frac{f}{b}, b$ is the bias factor, $G(x)$ encodes the non-linear effect due to velocity dispersion, and $\mu$ is the cosine of the $\mathbf{k}$ vector with the line of sight. Note that $P^{s}(k)$ becomes anisotropic (as does the correlation function) and it is through this anisotropy that one can measure $\beta$ and hence $f$.

Until recently, measurements of $\beta$ were seen as constraints on $\Omega_{M}$. The reason for this is that in $\Lambda C D M$ we have $\beta \simeq \Omega_{M}^{6 / 11}(z) / b$, and hence measurements of $\beta$ at different redshifts can be used to reconstruct the history of $\Omega_{M}$. In [585] it is found that the wide 
part of the VIMOS-VLT Deep Survey (VVDS) can be used to obtain $\beta=0.70 \pm 0.26$ at $z \simeq 0.8$. This result is then combined with the constraint from the 2dFGRS, $\beta=$ $0.49 \pm 0.09$ at $z \simeq 0.15$, and the 2dF-SDSS LRG and QSO (2SLAQ) constraint (shown in Figure 15). The emphasis in [585] was on finding deviations from growth rate in $\Lambda C D M$, and although not done systematically, their analysis showed that a number of specific models could be ruled out.

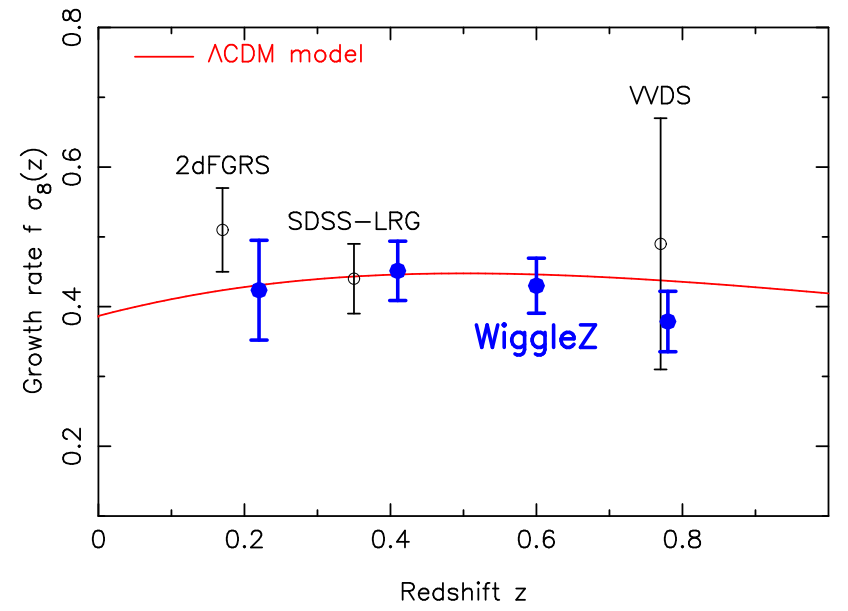

Figure 15: The growth rate, $f$, as a function of redshift, $z$, for a number of different models. The symbols and error bars correspond to constraints from VVDS, 2dFGRS and WiggleZ. Taken from [165.

The analysis of [585] have led to a number of upcoming redshift surveys focusing, in part, on redshift space distortions. In particular, WiggleZ, FMOS, VIPERS, GAMA and BOSS, many of whom were primarily targeting large scale structure and the Baryon Acoustic Oscillations, will now all deliver constraints on $\beta$ in the near to medium future.

In [1159] it was argued that measurements of the growth of structure through constraints on the large scale peculiar velocity dispersion, $\sigma_{v}^{2}$ (the large-scale redshift space distortion), can be combined with weak lensing measurements to break the degeneracies, and target $\mu$ more accurately. With measurements at different redshifts it should be possible to reconstruct the time evolution of $\mu$.

\subsubsection{The $E_{G}$ diagnostic}

The effect of modified gravity on the gravitational potentials, $\Phi$ and $\Psi$, can in principle be teased out with a careful combination of measurements. In 1301] it was argued that this should be possible using a cross-correlation between an estimate of the local velocity field from redshift space distortions, and the gravitational potentials inferred from a lensing map from background galaxies. More specifically, from a more general form of 
Eq. 924,

$$
P^{s}(\mathbf{k})=P^{s}(\mathbf{k})\left[P_{g}(\mathbf{k})+2 \mu^{2} P_{g \theta}(\mathbf{k})+\mu^{4} P_{\theta}(\mathbf{k})\right] G\left[\frac{k^{2} \mu^{2} \sigma_{v}^{2}}{H^{2}(z)}\right],
$$

it is possible to estimate the galaxy-velocity power spectrum, $P_{g \theta}$, in bandpowers $P_{\alpha}$, and the galaxy-galaxy power spectrum, $P_{g}$, in bandpowers $P_{i}$. From a weak lensing measurement of the convergence

$$
\kappa=\frac{1}{2} \int_{0}^{\chi_{s}} \nabla^{2}(\Psi-\Phi) W\left(\chi, \chi_{s}\right) d \chi
$$

where $W$ is the lensing kernel, it is possible to construct a cross power spectrum with galaxies, $C_{\kappa g}(\ell) \simeq \sum_{\alpha} f_{\alpha}(\ell) P_{\alpha}^{(2)}$, where $P_{\alpha}^{(2)}$ is the band power estimate of $P_{\nabla^{2}(\Psi-\Phi) g}$. These band powers can all be collected into one estimator,

$$
\hat{E}_{G} \equiv \frac{C_{\kappa g}(\ell, \Delta \ell)}{3 H_{0}^{2} a^{-1} \sum_{\alpha} f_{\alpha}\left(\ell, \Delta_{\ell}\right) P_{\alpha}},
$$

where a band averaging over bins of width $\Delta \ell$ has been assumed. It can be shown that

$$
\left\langle\hat{E}_{G}\right\rangle=\left[\frac{\nabla^{2}(\Psi-\Phi)}{3 H_{0}^{2} a^{-1} \beta \delta}\right]_{k=\ell / \bar{\chi}} .
$$

The diagnostic $E_{G}$ will take different values depending on the theory of gravity: $E_{G}=\Omega_{0} / \beta$ in $\Lambda$ CDM and DGP (with different $\Omega_{0}$ in either case), and $E_{G}=\Omega_{0} /\left(1+f_{R}\right) \beta$ in $F(R)$. In TeVeS $E_{G}$ is significantly different from the $\Lambda C D M$ value, and is scale dependent. Hence, $E_{G}$ is in principle a good diagnostic of the underlying gravitational theory.

In [1060, the authors attempted to extract an estimate of $E_{G}$ from a sample of 70,205 luminous red galaxies (LRGs) from the SDSS. Their estimate was concentrated at a mean redshift of $z \simeq 0.32$ and they found 8 estimates of $E_{G}$ across a range of between $2 h^{-1}$ to $50 h^{-1} \mathrm{Mpc}$, with a mean of $E_{G}=0.40 \pm 0.07$. This should be compared with the $\Lambda$ CDM prediction of $E_{G}=0.408 \pm 0.029$, the $F(R)$ prediction of $E_{G}=0.328-0.365$, and an approximate TeVeS prediction of $E_{G} \simeq 0.22$. The authors of 1060 have argued that this is evidence for the validity of General Relativity on cosmological scales. The results from 1060 are somewhat tentative and preliminary, but nonetheless promising. A judicious choice of cosmological parameters may indeed be able to tease out the particularities of how $\Phi$ and $\Psi$ evolve in different theories of gravity. With planned mega surveys such LAMOST, LSST and SKA it is likely that very tight constraints on $E_{G}$ will be achievable in the future.

\subsection{Forecasting Constraints from Future Surveys}

There is still quite a way to go in terms of constraining deviations from General Relativity. As mentioned in the previous section, with the planned mega surveys it should be possible to reconstruct the time evolution of $\mu$ and $\zeta$ with ever increasing precision [431, 432]. With the projected quality of the data, it also makes sense to consider model independent parameterisations of $\mu$ and $\zeta$ using some form of Principal 
Component Analysis (PCA). The authors in 1304 have done just this, and attempted to quantify how many eigen-modes in an expansion of $\mu$ and $\zeta$ can be accurately constrained with surveys such as Planck, DES, LSST, SNAP and JDEM. They find that while with DES it should be possible to constrain up to 20 independent eigen-modes, LSST will improve this by a factor of 5 , allowing detailed reconstruction of the time evolution in modified gravity. They find that in the best case scenario LSST will accurately constrain the time and scale dependence of $\mu$ and $\zeta$ in the range $0.5<z<2$ and $0.04<k 0.16 \mathrm{~h}^{-1}$ Mpc. 


\section{Discussion}

In this review we have discussed recent advances in gravitational physics, many of which have been driven by the discovery of the dark side of the Universe in the late 1990s. In this final section let us now briefly consider the outlook for future gravity research, and, in particular, what we consider to be some of the upcoming highlights.

From a theoretical perspective, model building is an important part of understanding and explaining existing data, as well as making predictions for the future. Tight constraints already available on solar system and astrophysical scales, however, mean that model builders are presented with a choice: They can either study minimal deviations away from General Relativity, or must otherwise look for mechanisms that hide modifications to gravity on the scales probed by experiment. The former of these has value for understanding the special nature of General Relativity, and the consequences of moving away from it, while the latter provides an exciting opportunity to try and solve some of the cosmological puzzles that have arisen with the discovery of dark matter and energy.

Modified gravity necessarily involves additional fields, extra dimensions, or broken symmetries, since we know that GR is the unique diffeomorphism invariant theory of a single rank-2 tensor that can be constructed from the metric variation of an action in four dimensions. An important consideration is then whether or not these deviations manifest themselves at the level of the background cosmology, or merely at the level of perturbations. Of course, if we wish to account for dark energy, or solve the cosmological constant problem using modified gravity, these deviations must be manifest in the solutions of the Friedmann equations. We must also require, however, that they do not spoil the successful predictions of the standard cosmology, such as the abundance of light elements, the peak positions of the $\mathrm{CMB}$ acoustic spectrum, or the predictions for baryon acoustic oscillations. This requires the background FLRW cosmology of the modified theories to closely mimic the standard evolution of $\Lambda \mathrm{CDM}$ from nucleosynthesis through to matter domination.

Let us now consider the assumptions that go into the standard cosmology. These include [149]

- Einstein's field equations.

- The universality of free fall.

- Local Lorentz invariance.

- Three spatial dimensions (below the electro-weak scale, at least).

- Conservation of energy-momentum.

- Homogeneity and isotropy of space on large scales.

- Matter fields being well modelled by fluids of dust and radiation.

These assumptions, when confronted with observations, require the presence of dark matter and dark energy. From a particle physics perspective this is very discomforting, as we have discussed in Section 2.6.6, as it requires the vacuum energy to be at least 60 orders of magnitude smaller than expected from particle theory. This problem is exacerbated by the fact that contributions from the zero-point energy of massive particles changes as 
the universe cools and goes through phase transitions. To circumvent the difficulties that this entails, many model builders therefore assume some unknown symmetry that sets the vacuum energy, plus any contribution from a bare cosmological constant, to zero. To account for cosmic acceleration they then require modifications to gravity on large scale:74.

In some respects addressing the puzzles in cosmology is the easy part of the model builders job. More difficult is to do this in a way that is consistent with the well established observations of relativistic gravitational phenomena. This often involves identifying a screening mechanism that can work on solar system scales. The point here is that in order to modify the cosmological evolution we have to deviate considerably from GR in the IR, but IR modifications of gravity are known to cause strong deviations from the predictions of General Relativity for experiments that involve, for example, the bending or time delay of light. Deviations on the scales that these observations are made must therefore be suppressed. Currently the most popular examples of this type of mechanism are the Vainshtein mechanism and the chameleon mechanism, both of which we have discussed in this review, and neither of these is without its problems. It is therefore important for theorists to identify new ways to screen the deviations from GR at short distances, and we expect this to be an important avenue of future research.

It is also crucial to ensure that our models obey some basic requirements of stability. There is little motivation for carrying out complicated simulations, and devising expensive experiments, to probe theories that are fundamentally sick. For example, we may choose to ask if the theory contains ghosts, which can lead to catastrophic instabilities unless the mass of the ghost lies above the effective theory cut-off, as described in Section 2.1.3 Ghosts are rife in modified theories of gravity, and dealing with them is one of the principle challenges faced by model builders. Another important thing to establish is the cut-off for our effective classical theory. Modified theories of gravity can become strongly coupled at lower than expected energies, and enter a quantum fog. If we want an effective theory description of gravitational fields at the surface of the earth to be classical, we need to impose that the cut-off is at least of the order of a few $m e V$, since classical gravity has been tested in the lab at this scale. Conservatively, one may also wish to impose that the classical description of gravitational fields around the Sun should be trustworthy, at least down to its Schwarzschild radius.

Let us now consider the prospect of future input into this field from experiments and observations. Tests of Lorentz invariance and the weak equivalence principle, that are already well established on Earth, are now being proposed and planned as space missions. Space offers a number of benefits over Earth-based experiments, including a lack of seismic noise, and the fact that cooled atoms stay in interferometers longer. There are also theoretical reasons for wanting to test these foundational issues of gravity in space, such as the proposal by Khoury and Weltman that extra gravitational degrees of freedom could have an environmental dependence. Space based tests of Lorentz invariance offer the possibility of improving constraints on violations of this symmetry by orders of magnitudes (see, e.g. [172, 869]). Space based tests of the equivalence are being planned by the French space agency CNES, under the name MICROSCOPE (MICRO-Satellite à trainée Compensée pour l'Observation du Principe d'Équivalence), and by ESA and

\footnotetext{
${ }^{74}$ Some approaches, such as degravitation, (see Section 5.6.2 even use modifications to gravity in order to screen the effects of the large vacuum energy, thereby removing the need for the unknown symmetry. 
NASA, under the name STEP (Satellite Test of the Equivalence Principle). These two missions promise to increase bounds on violations of the weak equivalence principle to the level of 1 part in $10^{15}$, and 1 part in $10^{18}$, respectively.

With regards to space based tests of metric theories of gravity, there is also hope for further improvement on current bounds. The Bepi-Columbo Mercury orbiter being planned by ESA will, after a two year mission, be capable of placing constraints on PPN parameters of the order $\gamma-1 \sim 3 \times 10^{-5}, \beta-1 \sim 3 \times 10^{-4}$, and $\alpha_{1} \sim 10^{-5}$ [894. The bounds on $\gamma$ could be improved further by Gaia, a high precision space telescope that could constrain $\gamma-1$ to around 1 part in $10^{6}$. Still greater constraints may be possible with LATOR (Laser Astronomic Test of Relativity) [1230. This mission consists of two satellites that orbit the Sun at $1 \mathrm{AU}$, and will have the potential of being able to constrain $\gamma-1$ to around 1 part in $10^{8}$, and solar frame-dragging to the level of $\sim 1 \%$. Such constraints are orders of magnitude greater than those that are currently available.

Lunar laser ranging has played an important part in testing gravity over the past 4 decades, since retroreflectors were placed on the moon by Apollo astronauts, and the Soviet Lunokhod rovers [885. Improvements in ground based technology during this time have improved the bounds on PPN parameters that reflected lasers have been able to impose, but we are now reaching the stage where further gains will be limited by the retroreflectors themselves. Tightening the current bounds using this technology will therefore require new space missions, and, in particular, the possibility of planting retroreflectors on other planets has great potential. Laser ranging of Mars over a 10 year period would allow $\gamma-1$ to be constrained to around 1 part in $10^{6}$ [1231], and the Nordtvedt parameter $\eta=4 \beta-\gamma-3$ to the level of $\sim 6-2 \times 10^{-6}$ [47. Again, these are order of magnitude improvements on current bounds.

Moving beyond the solar system, binary pulsars are an excellent test of relativistic gravity, and offer the possibility of becoming more constraining than solar system test in the near future [1177]. Pulsars also offer the opportunity to test gravity through the emission of gravitational waves. In particular, pulsar-white dwarf systems have great potential to constrain the emission of dipolar gravitational, which is a generic prediction of a large number of modified theories of gravity. Binary systems PSR J1141-6545, J0751+1807 and J1757-5322 are all recently discovered pulsar-white dwarf systems. What is more, continued observation of existing pulsars also offer the possibility of new tests of gravity, as, for example, the perihelion of PSR B1913+16 precesses it may soon allow for tests of the Shapiro time-delay effect. Future prospects for testing gravitational physics using pulsars are also bright due to the large numbers of these objects that are expected to be found by the Parkes, Arecibo, and Green Bank telescopes, as well, of course, as the SKA (Square Kilometre Array). The chance of detecting a pulsar-black hole systems increases dramatically with large-scale observations of this kind. Such a system would be potentially of great importance for testing strong field gravity. Finally, the double pulsar PSR J0737-3039A,B also offers a unique test of gravitational physics, with excellent prospects for improving constraints on gravity in the future as observations of it continue.

In all of these areas it is likely that the bounds on deviations from General Relativity will continue to be tightened, with lab tests too promising continued improvement. The Eöt-Wash group at the University of Washington, and others, continue to increase bounds at ever smaller scales, and even particle experiments using the LHC at CERN are looking for the signs of the extra-dimensions that are crucial for so many modern theories of gravity. The future prospects for constraining all of these aspects of gravity means that 
the extra degrees of freedom in modified theories of gravity will have ever smaller regions of parameter space in which to hide. This also, of course, means that the possibility of making a detection of a deviation from General Relativity is improved, if any such deviations really do exist in nature.

It is, of course, the case that there have been tremendous developments in observational cosmology over the past couple of decades, and these observations have put gravity in the spotlight once again. With measurements of the CMB, weak lensing, and galaxy surveys, as well as probes of the expansion rate with distant supernovae $I_{A} \mathrm{~s}$, a strange Universe has been uncovered in which more than $95 \%$ of the energy budget is in some exotic dark form. The quality of these observations are such that it is difficult to avoid such a conclusion if the gravitational force arises from Einstein's theory of gravity. An alternative point of view is that these observations are pointing at a flaw in our understanding of the behaviour of the Universe on the largest scales, and of behaviour of gravity at these distances in particular. Indeed, these observations may be a sign that we must think beyond Einstein's General Theory of Relativity, and his field equations.

In the same way that cosmological observations may be hinting at new physics in the gravitational sector, they can also be used to constrain and even rule out alternatives. With a vast range of experiments being planned and constructed throughout the world, it seems that we are a critical juncture in the path to understanding gravity. At ESA, the satellite mission Euclid is under assessment. This mission could map out vast regions of space, probing the growth rate and morphology of large-scale structure, both key observables for constraining theories of gravity through their effects on gravitational collapse. The SKA is in a planning phase with path finders being constructed on two continents. SKA will be a vast radio telescope that will generate a survey of up to a billion radio galaxies, mapping out the evolution of structure back to extremely high redshifts. These observatories will also be competing against the Large Synoptic Survey Telescope (LSST) that will image the sky over a period of ten years, building up a survey of galaxies that will primarily be used for weak lensing.

These larger experiments will be complemented by smaller, more rapid surveys such FastSound, Weave, Boss, Viper, KIDS, DES and a host of other collaborations that will produce galaxy and weak lensing surveys on smaller scales, targeting different redshifts. Interestingly enough, while the original scientific driver for many of these surveys was to constrain dark energy, they have all taken on board the need to test gravity. Indeed measuring the growth rate of gravitational collapse, as a test of modified gravity, has become the core business in all of these surveys.

Einstein developed his General Theory of Relativity almost a century ago, and, although it remains a cornerstone of modern physics, one could argue that of all the fundamental forces of nature it is gravity that remains the least well understood. This is almost certainly due to the weakness of the gravitational interaction, which makes it incredibly difficult to test in the lab experimentally. Inevitably, experiments on the scale of planets, stars, galaxies, and beyond cannot be performed with the same level of precision and control as those conducted for the other forces on Earth. Never the less, technology is now starting to catch up with gravity. The latter half of the twentieth century may have belonged to the Standard Model of particle physics, but there is every reason to suspect that the twenty first century will belong to gravity. 


\section{Acknowledgements}

TC and PGF acknowledge the support of the STFC, CERN, the BIPAC, the Oxford Martin School and Jesus College, Oxford. AP and CS are supported by Royal Society University Research Fellowships. For comments, discussion and support we wish to thank Tessa Baker, John Barrow, Cliff Burgess, Christos Charmousis, Ed Copeland, Kenny Dalglish, Gregory Gabadadze, Nemanja Kaloper, Ian Kimpton, Kazuya Koyama, Ed Macaulay, João Magueijo, Gustavo Niz, Claudia de Rham, Paul Saffin, Thomas Sotiriou, Glenn Starkman, Reza Tavakol, Anzhong Wang, Shuang Yong Zhou, Tom Zlosnik, Joe Zuntz, and Jessica Padilla and her Mum. 


\section{References}

\section{References}

[1] R. B. Abbott, S. M. Barr, and S. D. Ellis. Kaluza-Klein Cosmologies and Inflation. Phys. Rev., D30:720, 1984.

[2] R. B. Abbott, B. Bednarz, and S. D. Ellis. Cosmological perturbations in Kaluza-Klein models. Phys. Rev., D33:2147, 1986.

[3] R. B. Abbott, S. D. Ellis, and S. M. Barr. Kaluza-Klein Cosmologies and Inflation. 2. Phys. Rev., D31:673, 1985.

[4] M. C. B. Abdalla, S. Nojiri, and S. D. Odintsov. Consistent modified gravity: dark energy, acceleration and the absence of cosmic doomsday. Classical and Quantum Gravity, 22:L35-42, Mar. 2005, arXiv:hep-th/0409177

[5] M. Abdelwahab, S. Carloni, and P. K. S. Dunsby. Cosmological dynamics of 'exponential gravity'. Classical and Quantum Gravity, 25(13):135002, July 2008, 0706.1375.

[6] M. Abramowitz and I. A. Stegun. Handbook of Mathematical Functions with Formulas, Graphs, and Mathematical Tables. Dover, New York, 1964.

[7] V. Acquaviva, C. Baccigalupi, S. M. Leach, A. R. Liddle, and F. Perrotta. Structure formation constraints on the Jordan-Brans-Dicke theory. Phys. Rev. D, 71(10):104025, May 2005, arXiv:astro-ph/0412052

[8] W. Adamowicz and A. Trautman. The equivalence principle for spin. Bull. Acad. Pol. Sci., Ser. Sci. Math. Astron. Phys., 23: 339, 1975.

[9] A. Adams, N. Arkani-Hamed, S. Dubovsky, A. Nicolis, and R. Rattazzi. Causality, analyticity and an IR obstruction to UV completion. JHEP, 0610:014, 2006, hep-th/0602178.

[10] E. G. Adelberger, J. H. Gundlach, B. R. Heckel, S. Hoedl, and S. Schlamminger. Torsion balance experiments: A low-energy frontier of particle physics. Prog. Part. Nucl. Phys., 62:102-134, 2009.

[11] E. G. Adelberger, B. R. Heckel, and A. E. Nelson. Tests of the Gravitational Inverse-Square Law. Annual Review of Nuclear and Particle Science, 53:77-121, Dec. 2003, arXiv:hep-ph/0307284

[12] N. Agarwal, R. Bean, J. Khoury, and M. Trodden. Cascading Cosmology. Phys. Rev., D81:084020, 2010, 0912.3798.

[13] Y. Aghababaie, C. P. Burgess, S. L. Parameswaran, and F. Quevedo. Towards a naturally small cosmological constant from branes in 6D supergravity. Nucl. Phys., B680:389-414, 2004, hepth/0304256

[14] M. Ahlers, A. Lindner, A. Ringwald, L. Schrempp, and C. Weniger. Alpenglow: A signature for chameleons in axionlike particle search experiments. Phys. Rev. D, 77(1):015018, Jan. 2008, 0710.1555 .

[15] K. Akama. An early proposal of 'brane world'. Lect. Notes Phys., 176:267-271, 1982, hepth/0001113

[16] M. Akbar and R. Cai. Friedmann equations of FRW universe in scalar tensor gravity, $\mathrm{f}(\mathrm{R})$ gravity and first law of thermodynamics. Physics Letters B, 635:7-10, Mar. 2006, arXiv:hep-th/0602156

[17] K. Akune, K.-i. Maeda, and N. Ohta. Inflation from superstring and M-theory compactification with higher order corrections. - II. - Case of quartic Weyl terms. Phys. Rev. D, 73(10):103506, May 2006, hep-th/0602242

[18] A. Albrecht, C. Burgess, F. Ravndal, and C. Skordis. Exponentially large extra dimensions. Phys.Rev., D65:123506, 2002, hep-th/0105261

[19] A. Albrecht, C. Burgess, F. Ravndal, and C. Skordis. Natural quintessence and large extra dimensions. Phys.Rev., D65:123507, 2002, astro-ph/0107573

[20] J. Alcaniz. Some observational consequences of brane world cosmologies. Phys.Rev., D65:123514, 2002, astro-ph/0202492

[21] S. Alexander, R. H. Brandenberger, and D. A. Easson. Brane gases in the early universe. Phys. Rev., D62:103509, 2000, hep-th/0005212

[22] S. O. Alexeyev and M. V. Pomazanov. Black hole solutions with dilatonic hair in higher curvature gravity. Phys. Rev. D, 55:2110-2118, Feb. 1997, arXiv:hep-th/9605106

[23] A. Ali, R. Gannouji, and M. Sami. Modified gravity a la Galileon: Late time cosmic acceleration and observational constraints. Phys.Rev., D82:103015, 2010, 1008.1588.

[24] M. Alimohammadi and N. Agharafiei. Quantum attractors of generalized Gauss-Bonnet dark energy. ArXiv e-prints, Dec. 2009, 0912.0589. 
[25] M. Alimohammadi and A. Ghalee. Phase space of generalized Gauss-Bonnet dark energy. Phys. Rev. D, 80(4):043006, Aug. 2009, 0908.1150.

[26] M. Alimohammadi and A. Ghalee. Remarks on generalized Gauss-Bonnet dark energy. Phys. Rev. D, 79(6):063006, Mar. 2009, 0811.1286.

[27] R. Allahverdi, B. Dutta, and K. Sinha. Cladogenesis: Baryon-dark matter coincidence from branchings in moduli decay. Phys. Rev. D, 83(8):083502, Apr 2011.

[28] G. Allemandi, A. Borowiec, and M. Francaviglia. Accelerated cosmological models in first-order nonlinear gravity. Phys. Rev. D, 70(4):043524, Aug. 2004, arXiv:hep-th/0403264.

[29] G. Allemandi, A. Borowiec, M. Francaviglia, and S. D. Odintsov. Dark energy dominance and cosmic acceleration in first-order formalism. Phys. Rev. D, 72(6):063505, Sept. 2005, arXiv:gr$\mathrm{qc} / 0504057$

[30] G. Allemandi, M. Francaviglia, M. L. Ruggiero, and A. Tartaglia. Post-newtonian parameters from alternative theories of gravity. General Relativity and Gravitation, 37:1891-1904, Nov. 2005, arXiv:gr-qc/0506123

[31] G. Allemandi and M. L. Ruggiero. Constraining extended theories of gravity using Solar System tests. General Relativity and Gravitation, 39:1381-1388, Sept. 2007, arXiv:astro-ph/0610661.

[32] S. W. Allen, A. E. Evrard, and A. B. Mantz. Cosmological Parameters from Observations of Galaxy Clusters. ArXiv e-prints, Mar. 2011, 1103.4829.

[33] E. Alvarez and M. Belen Gavela. Entropy from Extra Dimensions. Phys. Rev. Lett., 51:931, 1983.

[34] M. Amarzguioui, Ø. Elgarøy, D. F. Mota, and T. Multamäki. Cosmological constraints on $\mathrm{f}(\mathrm{R})$ gravity theories within the Palatini approach. $A \mathscr{E} A, 454: 707-714$, Aug. 2006, arXiv:astro$\mathrm{ph} / 0510519$

[35] L. Amendola. Dark energy and the boomerang data. Phys. Rev. Lett., 86(2):196-199, Jan 2001.

[36] L. Amendola, C. Charmousis, and S. C. Davis. Constraints on Gauss Bonnet gravity in dark energy cosmologies. J. Cosmology Astropart. Phys., 12:20, Dec. 2006, arXiv:hep-th/0506137

[37] L. Amendola, C. Charmousis, and S. C. Davis. Solar system constraints on Gauss Bonnet mediated dark energy. J. Cosmology Astropart. Phys., 10:4, Oct. 2007, 0704.0175.

[38] L. Amendola, C. Charmousis, and S. C. Davis. Solar System Constraints on Gauss-Bonnet Mediated Dark Energy. JCAP, 0710:004, 2007, 0704.0175.

[39] L. Amendola, K. Enqvist, and T. Koivisto. Unifying Einstein and Palatini gravities. Phys. Rev. D, 83(4):044016, 2011, 1010.4776.

[40] L. Amendola, R. Gannouji, D. Polarski, and S. Tsujikawa. Conditions for the cosmological viability of $\mathrm{f}(\mathrm{R})$ dark energy models. Phys. Rev. D, 75(8):083504, Apr 2007.

[41] L. Amendola, M. Kunz, and D. Sapone. Measuring the dark side (with weak lensing). J. Cosmology Astropart. Phys., 4:13, Apr. 2008, 0704.2421.

[42] L. Amendola, A. B. Mayer, S. Capozziello, S. Gottlober, V. Muller, F. Occhionero, and H. Schmidt. Generalized sixth-order gravity and inflation. Classical and Quantum Gravity, 10:L43-L47, May 1993.

[43] L. Amendola, D. Polarski, and S. Tsujikawa. Are f(R) Dark Energy Models Cosmologically Viable? Physical Review Letters, 98(13):131302, Mar. 2007, arXiv:astro-ph/0603703

[44] M. A. Amin, R. V. Wagoner, and R. D. Blandford. A subhorizon framework for probing the relationship between the cosmological matter distribution and metric perturbations. MNRAS, 390:131-142, Oct. 2008, 0708.1793.

[45] K. N. Ananda, S. Carloni, and P. K. S. Dunsby. A characteristic signature of fourth order gravity. ArXiv e-prints, Dec. 2008, 0812.2028.

[46] K. N. Ananda, S. Carloni, and P. K. S. Dunsby. Structure growth in $\mathrm{f}(\mathrm{R})$ theories of gravity with a dust equation of state. Classical and Quantum Gravity, 26(23):235018, Dec. 2009, 0809.3673.

[47] J. D. Anderson, M. Gross, K. L. Nordtvedt, and S. G. Turyshev. The Solar Test of the Equivalence Principle. ApJ, 459:365, Mar. 1996, arXiv:gr-qc/9510029

[48] J. D. Anderson, M. A. Slade, R. F. Jurgens, E. L. Lau, X. X. Newhall, and E. Myles. Radar and spacecraft ranging to Mercury between 1966 and 1988. Proceedings of the Astronomical Society of Australia, 9:324, 1991.

[49] J. D. Anderson et al. in The Sixth Marcel Grossmann Meeting on general relativity, Kyoto, Japan. 1992

[50] M. Andrews, K. Hinterbichler, J. Khoury, and M. Trodden. Instabilities of Spherical Solutions with Multiple Galileons and SO(N) Symmetry. 2010, 1008.4128.

[51] I. Antoniadis. Gravity modifications from extra dimensions. J. Phys., A40:6657-6667, 2007.

[52] I. Antoniadis, N. Arkani-Hamed, S. Dimopoulos, and G. R. Dvali. New dimensions at a millimeter to a Fermi and superstrings at a TeV. Phys. Lett., B436:257-263, 1998, hep-ph/9804398 
[53] I. Antoniadis, J. Rizos, and K. Tamvakis. Singularity-free cosmological solutions of the superstring effective action. Nuclear Physics B, 415:497-514, Mar. 1994, arXiv:hep-th/9305025

[54] T. Appelquist and A. Chodos. Quantum Effects in Kaluza-Klein Theories. Phys. Rev. Lett. 50:141, 1983.

[55] T. Appelquist and A. Chodos. The Quantum Dynamics of Kaluza-Klein Theories. Phys. Rev., D28:772, 1983.

[56] S. A. Appleby and R. A. Battye. Do consistent $f(R)$ models mimic General Relativity plus Lambda? Phys.Lett., B654:7-12, 2007, 0705.3199.

[57] S. A. Appleby, R. A. Battye, and A. A. Starobinsky. Curing singularities in cosmological evolution of f(R) gravity. J. Cosmology Astropart. Phys., 6:5, June 2010, 0909.1737.

[58] S. A. Appleby and J. Weller. Parameterizing scalar-tensor theories for cosmological probes. ArXiv e-prints, Aug. 2010, 1008.2693.

[59] K. Arai, M. Hashimoto, and T. Fukui. Primordial nucleosynthesis in the Brans-Dicke theory with a variable cosmological term. $A \& A, 179: 17-22$, June 1987.

[60] E. V. Arbuzova, and A. D. Dolgov. Explosive phenomena in modified gravity. Phys. Lett., B700:289-293, 2011, 1012.1963.

[61] N. Arkani-Hamed, S. Dimopoulos, G. Dvali, and G. Gabadadze. Nonlocal modification of gravity and the cosmological constant problem. 2002, hep-th/0209227

[62] N. Arkani-Hamed, S. Dimopoulos, and G. R. Dvali. The hierarchy problem and new dimensions at a millimeter. Phys. Lett., B429:263-272, 1998, hep-ph/9803315.

[63] N. Arkani-Hamed, S. Dimopoulos, and G. R. Dvali. Phenomenology, astrophysics and cosmology of theories with sub-millimeter dimensions and $\mathrm{TeV}$ scale quantum gravity. Phys. Rev., D59:086004, 1999, hep-ph/9807344

[64] N. Arkani-Hamed, H. Georgi, and M. D. Schwartz. Effective field theory for massive gravitons and gravity in theory space. Annals Phys., 305:96-118, 2003, hep-th/0210184

[65] C. Armendariz-Picon, T. Damour, and V. F. Mukhanov. k-Inflation. Phys. Lett., B458:209-218, 1999, hep-th/9904075

[66] C. Armendariz-Picon, V. F. Mukhanov, and P. J. Steinhardt. A dynamical solution to the problem of a small cosmological constant and late-time cosmic acceleration. Phys. Rev. Lett., 85:4438-4441, 2000, astro-ph/0004134

[67] P. Astier et al. The Supernova legacy survey: Measurement of $\Omega_{m}, \Omega_{\Lambda}$ and $w$ from the first year data set. Astron.Astrophys., 447:31-48, 2006, astro-ph/0510447

[68] K. Atazadeh, M. Farhoudi, and H. R. Sepangi. Accelerating universe in f(R) brane gravity. Phys. Lett., B660:275-281, 2008, 0801.1398.

[69] M. Bañados and P. G. Ferreira. Eddington's Theory of Gravity and Its Progeny. Physical Review Letters, 105(1):011101, July 2010, 1006.1769.

[70] M. Bañados, P. G. Ferreira, and C. Skordis. Eddington-Born-Infeld gravity and the large scale structure of the Universe. Phys. Rev. D, 79(6):063511, Mar. 2009, 0811.1272.

[71] M. Bañados, A. Gomberoff, and M. Pino. The bigravity black hole and its thermodynamics. ArXiv e-prints, May 2011, 1105.1172.

[72] E. Babichev and D. Langlois. Relativistic stars in $\mathrm{f}(\mathrm{R})$ gravity. Phys. Rev. D, 80(12):121501, Dec. 2009, 0904.1382

[73] E. Babichev, V. Mukhanov, and A. Vikman. k-Essence, superluminal propagation, causality and emergent geometry. JHEP, 0802:101, 2008, 0708.0561.

[74] C. Baccigalupi, S. Matarrese, and F. Perrotta. Tracking extended quintessence. Phys. Rev. D, 62(12):123510, Nov 2000

[75] A. F. Bahrehbakhsh, M. Farhoudi, and H. Shojaie. FRW cosmology from five dimensional vacuum Brans-Dicke theory. Gen. Rel. Grav. 43:847-869, 2011, 1005.2501.

[76] D. Bailin and A. Love. Kaluza-Klein theories. Rept. Prog. Phys., 50:1087-1170, 1987.

[77] T. Baker, P. G. Ferreira, C. Skordis, and J. Zuntz. A fully consistent parameterization of modified gravity. Phys. Rev. D, 84(12):124018, 2011, 1107.0491.

[78] K. Bamba, C. Geng, and C. Lee. Comment on "Einstein's Other Gravity and the Acceleration of the Universe". ArXiv e-prints, Aug. 2010, 1008.4036.

[79] K. Bamba, C. Geng, C. Lee, and L. Luo. Equation of state for dark energy in f(T) gravity. ArXiv e-prints, Nov. 2010, 1011.0508.

[80] K. Bamba, Z.-K. Guo, and N. Ohta. Accelerating Cosmologies in the Einstein-Gauss-Bonnet Theory with Dilaton. Prog. Theo. Phys., 118:879-892, 2007, 0707.4334.

[81] K. Bamba, S. Nojiri, and S. D. Odintsov. The future of the universe in modified gravitational theories: approaching a finite-time future singularity. J. Cosmology Astropart. Phys., 10:45, Oct. 
2008, 0807.2575

[82] K. Bamba and S. D. Odintsov. Inflation and late-time cosmic acceleration in non-minimal Maxwell-f(R) gravity and the generation of large-scale magnetic fields. J. Cosmology Astropart. Phys., 4:24, Apr. 2008, 0801.0954.

[83] C. Bambi, M. Giannotti, and F. L. Villante. Response of primordial abundances to a general modification of $g_{N}$ and/or of the early universe expansion rate. Phys. Rev. D, 71(12):123524, Jun 2005.

[84] M. Bañados. Eddington-Born-Infeld action for dark matter and dark energy. Phys.Rev. D77:123534, 2008, 0801.4103.

[85] M. Banados, A. Gomberoff, D. C. Rodrigues, and C. Skordis. A note on bigravity and dark matter. Phys. Rev., D79:063515, 2009, 0811.1270.

[86] A. Banerjee, S. B. Dutta Choudhury, N. Banerjee, and A. Sil. Inflationary solutions in general scalar tensor theory of gravitation. Pramana, 40:31-34, 1993.

[87] E. Barausse, T. P. Sotiriou, and J. C. Miller. A no-go theorem for polytropic spheres in Palatini f(R) gravity. Class. Quant. Grav., 25:062001, 2008, gr-qc/0703132

[88] E. Barausse, T. P. Sotiriou, and J. C. Miller. Curvature singularities, tidal forces and the viability of Palatini f(R) gravity. Class. Quant. Grav., 25:105008, 2008, 0712.1141.

[89] E. Barausse, T. P. Sotiriou, and J. C. Miller. Polytropic spheres in Palatini f(R) gravity. EAS Publ. Ser., 30:189-192, 2008, 0801.4852.

[90] J. M. Bardeen. Gauge Invariant Cosmological Perturbations. Phys.Rev., D22:1882-1905, 1980.

[91] S. M. Barr, R. S. Chivukula, and E. Farhi. Electroweak fermion number violation and the production of stable particles in the early universe. Physics Letters B, 241(3):387 - 391, 1990.

[92] D. E. Barraco and V. H. Hamity. Spherically symmetric solutions in $f(R)$ theories of gravity obtained using the first order formalism. Phys. Rev. D, 62(4):044027, Aug. 2000.

[93] J. D. Barrow. Gravitational memory? Phys. Rev. D, 46(8):R3227-R3230, Oct 1992.

[94] J. D. Barrow. Nonsingular scalar-tensor cosmologies. Phys. Rev. D, 48:3592-3595, Oct. 1993.

[95] J. D. Barrow. Scalar-tensor cosmologies. Phys. Rev. D, 47(12):5329-5335, Jun 1993.

[96] J. D. Barrow. Slow-roll inflation in scalar-tensor theories. Phys. Rev. D, 51:2729-2732, Mar. 1995.

[97] J. D. Barrow and B. J. Carr. Formation and evaporation of primordial black holes in scalar-tensor gravity theories. Phys. Rev. D, 54(6):3920-3931, Sep 1996.

[98] J. D. Barrow and T. Clifton. Exact cosmological solutions of scale-invariant gravity theories. Classical and Quantum Gravity, 23:L1-L6, Jan. 2006, arXiv:gr-qc/0509085

[99] J. D. Barrow, E. J. Copeland, E. W. Kolb, and A. R. Liddle. Baryogenesis in extended inflation. I. Baryogenesis via production and decay of supermassive bosons. Phys. Rev. D, 43:977-983, Feb. 1991.

[100] J. D. Barrow, E. J. Copeland, E. W. Kolb, and A. R. Liddle. Baryogenesis in extended inflation. II. Baryogenesis via primordial black holes. Phys. Rev. D, 43:984-994, Feb. 1991.

[101] J. D. Barrow and S. Cotsakis. Inflation and the conformal structure of higher-order gravity theories. Physics Letters B, 214:515-518, Dec. 1988.

[102] J. D. Barrow and S. Hervik. Anisotropically inflating universes. Phys. Rev., D73:023007, 2006, gr-qc/0511127.

[103] J. D. Barrow and S. Hervik. Evolution of universes in quadratic theories of gravity. Phys. Rev. D, 74(12):124017, Dec. 2006, arXiv:gr-qc/0610013

[104] J. D. Barrow and S. Hervik. Simple types of anisotropic inflation. Phys. Rev. D, 81(2):023513, Jan. 2010, 0911.3805.

[105] J. D. Barrow, D. Kimberly, and J. Magueijo. Bouncing universes with varying constants. Classical and Quantum Gravity, 21:4289-4296, Sept. 2004, arXiv:astro-ph/0406369

[106] J. D. Barrow and K. Maeda. Extended inflationary universes. Nuclear Physics B, 341:294-308, Sept. 1990.

[107] J. D. Barrow and J. Middleton. Stable isotropic cosmological singularities in quadratic gravity. Phys. Rev. D, 75(12):123515, June 2007, arXiv:gr-qc/0702098

[108] J. D. Barrow and J. P. Mimoso. Perfect fluid scalar-tensor cosmologies. Phys. Rev. D, 50(6):37463754, Sep 1994.

[109] J. D. Barrow, J. P. Mimoso, and M. R. de Garcia Maia. Amplification of gravitational waves in scalar-tensor theories of gravity. Phys. Rev. D, 48:3630-3640, Oct. 1993.

[110] J. D. Barrow and A. C. Ottewill. The stability of general relativistic cosmological theory. Journal of Physics A: Mathematical and General, 16(12):2757, 1983

[111] J. D. Barrow and P. Parsons. Behavior of cosmological models with varying g. Phys. Rev. D, 
55(4):1906-1936, Feb 1997.

[112] J. D. Barrow and D. J. Shaw. Some late-time asymptotics of general scalar-tensor cosmologies. Classical and Quantum Gravity, 25(8):085012, 2008.

[113] N. H. Barth and S. M. Christensen. Quantizing fourth-order gravity theories: The functional integral. Phys. Rev. D, 28:1876-1893, Oct. 1983.

[114] Y. V. Baryshev, I. N. Taganov and P. Teerikorpi. Energy-momentum of the gravitational field: crucial point for gravitation physics and cosmology. Problems of Practical Cosmology, Volume 1, p.276-286, 2008, gr-qc/0809.2323.

[115] Y. V. Baryshev, I. N. Taganov and P. Teerikorpi. New possibilities for observational distinction between geometrical and field gravity theories. Problems of Practical Cosmology, Volume 1 , p.347-359, 2008, gr-qc/0809.2328.

[116] T. Battefeld and S. Watson. String gas cosmology. Rev. Mod. Phys., 78:435-454, 2006, hepth/0510022

[117] R. A. Battye, B. Carter, A. Mennim, and J.-P. Uzan. Einstein equations for an asymmetric brane-world. Phys. Rev., D64:124007, 2001, hep-th/0105091

[118] A. Bauch and S. Weyers. New experimental limit on the validity of local position invariance. Phys. Rev. D, 65(8):081101, Apr. 2002.

[119] V. Baukh, A. Zhuk, Sp-brane accelerating cosmologies Phys. Rev. D73 (2006) 104016. hepth/0601205

[120] V. Baukh, A. Zhuk, Dynamical dark energy from extra dimensions Kinematics Phys. Celest. Bodies 25 (2009) 57-63. arXiv:0809.1385 [astro-ph]]

[121] A. Bayntun, C. Burgess, and L. van Nierop. Codimension-2 Brane-Bulk Matching: Examples from Six and Ten Dimensions. New J.Phys., 12:075015, 2010, 0912.3039.

[122] R. Bean, D. Bernat, L. Pogosian, A. Silvestri, and M. Trodden. Dynamics of linear perturbations in $\mathrm{f}(\mathrm{R})$ gravity. Phys. Rev. D, 75(6):064020, Mar. 2007, arXiv:astro-ph/0611321

[123] R. Bean and M. Tangmatitham. Current constraints on the cosmic growth history. Phys. Rev. D, 81(8):083534, Apr. 2010, 1002.4197.

[124] J. Bekenstein and J. Magueijo. MOND habitats within the solar system. Phys. Rev., D73:103513, 2006, astro-ph/0602266

[125] J. Bekenstein and M. Milgrom. Does the missing mass problem signal the breakdown of Newtonian gravity? Astrophys. J., 286:7-14, 1984.

[126] J. D. Bekenstein. Phase coupling gravitation: symmetries and gauge fields. Phys. Lett., B202:497500, 1988.

[127] J. D. Bekenstein. The Relation between physical and gravitational geometry. Phys. Rev., D48:3641-3647, 1993, gr-qc/9211017

[128] J. D. Bekenstein. Relativistic gravitation theory for the MOND paradigm. Phys. Rev., D70:083509, 2004

[129] J. D. Bekenstein and E. Sagi. Do Newton's G and Milgrom's $a_{0}$ vary with cosmological epoch? Phys. Rev., D77:103512, 2008, 0802.1526.

[130] J. D. Bekenstein and R. H. Sanders. Gravitational lenses and unconventional gravity theories. Astrophys. J., 429:480, 1994, astro-ph/9311062

[131] J. A. Belinchon. Bianchi $V I_{0}$ in Scalar and Scalar-Tensor cosmologies. ArXiv e-prints, Sep. 2011, 1109.2880 .

[132] J. A. Belinchon. Generalized Self-similar Scalar-Tensor Theories. ArXiv e-prints, Sep. 2011, 1109.2877.

[133] V. A. Belinskij and I. M. Khalatnikov. On the effect of scalar and vector fields on the nature of the cosmological singularity. Zhurnal Eksperimental noi i Teoreticheskoi Fiziki, 63:1121-1134, 1972 .

[134] G. R. Bengochea. Observational information for $\mathrm{f}(\mathrm{T})$ theories and Dark Torsion. ArXiv e-prints, Aug. 2010, 1008.3188.

[135] G. R. Bengochea and R. Ferraro. Dark torsion as the cosmic speed-up. Phys. Rev. D, 79(12):124019, June 2009, 0812.1205.

[136] I. Bengtsson. The Cosmological constants. Phys.Lett., B254:55-60, 1991.

[137] I. Bengtsson. Form connections. 1993, gr-qc/9305004.

[138] I. Bengtsson. Form geometry and the 't Hooft-Plebanski action. 1995, gr-qc/9502010

[139] I. Bengtsson. Note on non-metric gravity. Mod.Phys.Lett., A22:1643-1649, 2007, gr-qc/0703114

[140] Z. Berezhiani, D. Comelli, F. Nesti, and L. Pilo. Spontaneous Lorentz Breaking and Massive Gravity. Phys. Rev. Lett, 99:131101, 2007, hep-th/0703264

[141] Z. Berezhiani, D. Comelli, F. Nesti, and L. Pilo. Exact Spherically Symmetric Solutions in 
Massive Gravity. JHEP, 0807:130, 2008, 0803.1687.

[142] P. G. Bergmann. Comments on the scalar tensor theory. Int. J. Theor. Phys., 1:25-36, 1968.

[143] A. L. Berkin and K. Maeda. Effects of $R^{3}$ and $R \square R$ terms on $R^{2}$ inflation. Physics Letters B, 245:348-354, Aug. 1990.

[144] A. L. Berkin and K. Maeda. Inflation in generalized Einstein theories. Phys. Rev. D, 44:16911704, Sept. 1991.

[145] M. S. Berman and L. A. Trevisan. Amplification of Gravitational Waves During Inflation in Brans-Dicke Theory. ArXiv General Relativity and Quantum Cosmology e-prints, Nov. 2001, arXiv:gr-qc/0111100

[146] C. P. L. Berry and J. R. Gair. Linearized f(R) Gravity: Gravitational Radiation and Solar System Tests. ArXiv e-prints, Apr. 2011, 1104.0819.

[147] B. Bertotti, L. Iess, and P. Tortora. A test of general relativity using radio links with the Cassini spacecraft. Nature, 425:374-376, Sept. 2003.

[148] E. Bertschinger. On the Growth of Perturbations as a Test of Dark Energy and Gravity. ApJ, 648:797-806, Sept. 2006, arXiv:astro-ph/0604485

[149] E. Bertschinger. Testing General Relativity with Cosmology. The Royal Society, February 2011.

[150] E. Bertschinger. One Gravitational Potential or Two? Forecasts and Tests. Phil. Trans. R. Soc. A, 369:4947, 2011, 1111.4659.

[151] E. Bertschinger and P. Zukin. Distinguishing modified gravity from dark energy. Phys. Rev. D, 78(2):024015, July 2008, 0801.2431.

[152] A. Bhadra and K. K. Nandi. On the equivalence of the Buchdahl and the Janis-Newman- Winnicour solutions. Int. J. Mod. Phys., A16:4543-4545, 2001.

[153] A. Bhadra and K. Sarkar. On static spherically symmetric solutions of the vacuum Brans-Dicke theory. Gen. Rel. Grav., 37:2189-2199, 2005, gr-qc/0505141

[154] N. D. R. Bhat, M. Bailes, and J. P. W. Verbiest. Gravitational-radiation losses from the pulsar white-dwarf binary PSR J1141 6545. Phys. Rev. D, 77(12):124017, June 2008, 0804.0956.

[155] G. V. Bicknell. A geometrical Lagrangian for the neutral scalar meson field. Journal of Physics A Mathematical General, 7:341-345, Feb. 1974.

[156] A. Billyard, A. Coley, and J. Ibáñez. Asymptotic behavior of cosmological models in scalar-tensor theories of gravity. Phys. Rev. D, 59(2):023507, Jan. 1999, arXiv:gr-qc/9807055

[157] A. Billyard, A. Coley, J. Ibáñez, and I. Olasagasti. Inhomogeneous cosmological models in scalartensor theories. Europhysics Letters, 46:832-837, June 1999.

[158] P. Binetruy, C. Deffayet, U. Ellwanger, and D. Langlois. Brane cosmological evolution in a bulk with cosmological constant. Phys. Lett., B477:285-291, 2000, hep-th/9910219

[159] P. Binetruy, C. Deffayet, and D. Langlois. Non-conventional cosmology from a brane-universe. Nucl. Phys., B565:269-287, 2000, hep-th/9905012

[160] G. D. Birkhoff. Relativity and Modern Physics: Harvard University Press. 1923.

[161] N. Birrell and P. Davies. Quantum fields in curved space. Cambridge Univ Pr, 1984.

[162] T. Biswas, R. Brandenberger, A. Mazumdar, and W. Siegel. Non-perturbative gravity, the Hagedorn bounce and the cosmic microwave background. J. Cosmology Astropart. Phys., 12:11, Dec. 2007, arXiv:hep-th/0610274

[163] T. Biswas, T. Koivisto, and A. Mazumdar. Towards a resolution of the cosmological singularity in non-local higher derivative theories of gravity. J. Cosmology Astropart. Phys., 11:8, Nov. 2010, 1005.0590.

[164] T. Biswas, A. Mazumdar, and W. Siegel. Bouncing universes in string-inspired gravity. J. Cosmology Astropart. Phys., 3:9, Mar. 2006, arXiv:hep-th/0508194

[165] C. Blake et al. The WiggleZ Dark Energy Survey: the growth rate of cosmic structure since redshift $\mathrm{z}=0.9$. 2011, 1104.2948.

[166] J. J. Blanco-Pillado, D. Buck, E. J. Copeland, M. Gomez-Reino, and N. J. Nunes. Kahler Moduli Inflation Revisited. JHEP, 01:081, 2010, 0906.3711.

[167] D. Blas, O. Pujolas, and S. Sibiryakov. On the Extra Mode and Inconsistency of Horava Gravity. JHEP, 0910:029, 2009, 0906.3046.

[168] D. Blas, O. Pujolas, and S. Sibiryakov. Comment on 'Strong coupling in extended Horava-Lifshitz gravity'. Phys.Lett., B688:350-355, 2010, 0912.0550.

[169] D. Blas, O. Pujolas, and S. Sibiryakov. Consistent Extension of Horava Gravity. Phys.Rev.Lett., 104:181302, 2010, 0909.3525.

[170] D. Blas, O. Pujolas, and S. Sibiryakov. Models of non-relativistic quantum gravity: The Good, the bad and the healthy. 2010, 1007.3503.

[171] D. Blas, S. Sibiryakov, Technically natural dark energy from Lorentz breaking JCAP 1107 
(2011) 026. arXiv:1104.3579 [hep-th]].

[172] R. Bluhm, V. A. Kostelecký, C. D. Lane, and N. Russell. Probing Lorentz and CPT violation with space-based experiments. Phys. Rev. D, 68(12):125008, Dec. 2003, arXiv:hep-ph/0306190

[173] M. Borunda, B. Janssen, and M. Bastero-Gil. Palatini versus metric formulation in highercurvature gravity. J. Cosmology Astropart. Phys., 11:8, Nov. 2008, 0804.4440.

[174] P. Bostock, R. Gregory, I. Navarro, and J. Santiago. Einstein gravity on the codimension 2-brane? Phys.Rev.Lett., 92:221601, 2004, hep-th/0311074

[175] D. Boulware and S. Deser. Can gravitation have a finite range? Phys.Rev., D6:3368-3382, 1972.

[176] D. G. Boulware and S. Deser. String Generated Gravity Models. Phys. Rev. Lett., 55:2656, 1985.

[177] F. Bourliot, P. G. Ferreira, D. F. Mota, and C. Skordis. The cosmological behavior of Bekenstein's modified theory of gravity. Phys. Rev., D75:063508, 2007, astro-ph/0611255

[178] P. Bowcock, C. Charmousis, and R. Gregory. General brane cosmologies and their global spacetime structure. Class. Quant. Grav., 17:4745-4764, 2000, hep-th/0007177

[179] R. Brandenberger. Matter Bounce in Horava-Lifshitz Cosmology. Phys. Rev., D80:043516, 2009, 0904.2835.

[180] R. H. Brandenberger. Challenges for string gas cosmology. 2005, hep-th/0509099

[181] R. H. Brandenberger. String Gas Cosmology. 2008, 0808.0746.

[182] R. H. Brandenberger et al. More on the Spectrum of Perturbations in String Gas Cosmology. JCAP, 0611:009, 2006, hep-th/0608186

[183] R. H. Brandenberger and C. Vafa. Superstrings in the Early Universe. Nucl. Phys., B316:391, 1989.

[184] C. Brans and R. H. Dicke. Mach's principle and a relativistic theory of gravitation. Phys. Rev., 124:925-935, 1961.

[185] C. H. Brans. Mach's principle and a relativistic theory of gravitation. ii. Phys. Rev., 125(6):21942201, Mar 1962.

[186] P. Brax and C. Burrage. Chameleon Induced Atomic Afterglow. ArXiv e-prints, Sept. 2010, 1009.1065

[187] P. Brax, C. Burrage, A. Davis, D. Seery, and A. Weltman. Higgs production as a probe of chameleon dark energy. Phys. Rev. D, 81(10):103524, May 2010, 0911.1267.

[188] P. Brax, R. Rosenfeld, and D. A. Steer. Spherical collapse in chameleon models. J. Cosmology Astropart. Phys., 8:33, Aug. 2010, 1005.2051.

[189] P. Brax and C. van de Bruck. Cosmology and brane worlds: A review. Class. Quant. Grav., 20:R201-R232, 2003, hep-th/0303095

[190] P. Brax, C. van de Bruck, and A. Davis. Compatibility of the Chameleon-Field Model with Fifth-Force Experiments, Cosmology, and PVLAS and CAST Results. Physical Review Letters, 99(12):121103, Sept. 2007, arXiv:hep-ph/0703243

[191] P. Brax, C. van de Bruck, A. Davis, J. Khoury, and A. Weltman. Detecting dark energy in orbit: The cosmological chameleon. Phys. Rev. D, 70(12):123518, Dec. 2004, arXiv:astro-ph/0408415.

[192] P. Brax, C. van de Bruck, A. Davis, D. F. Mota, and D. Shaw. Testing chameleon theories with light propagating through a magnetic field. Phys. Rev. D, 76(8):085010, Oct. 2007, 0707.2801.

[193] P. Brax, C. van de Bruck, A. Davis, and D. J. Shaw. f(R) gravity and chameleon theories. Phys. Rev. D, 78(10):104021, Nov. 2008, 0806.3415.

[194] P. Brax, C. van de Bruck, and A. C. Davis. Brane-world cosmology, bulk scalars and perturbations. JHEP, 10:026, 2001, hep-th/0108215

[195] P. Brax, C. van de Bruck, and A.-C. Davis. Brane world cosmology. Rept. Prog. Phys., 67:21832232, 2004, hep-th/0404011

[196] P. Brax, C. van de Bruck, A. C. Davis, and A. M. Green. Small scale structure formation in chameleon cosmology. Physics Letters B, 633:441-446, Feb. 2006, arXiv:astro-ph/0509878

[197] P. Brax, C. van de Bruck, A. C. Davis, J. Khoury, and A. Weltman. Chameleon Dark Energy. In C. J. A. P. Martins, P. P. Avelino, M. S. Costa, K. Mack, M. F. Mota, \& M. Parry, editor Phi in the Sky: The Quest for Cosmological Scalar Fields, volume 736 of American Institute of Physics Conference Series, pages 105-110, Nov. 2004, arXiv:astro-ph/0410103

[198] P. Brax, C. van de Bruck, D. F. Mota, N. J. Nunes, and H. A. Winther. Chameleons with Field Dependent Couplings. ArXiv e-prints, June 2010, 1006.2796.

[199] P. Brax and K. Zioutas. Solar chameleons. Phys. Rev. D, 82(4):043007, Aug. 2010, 1004.1846.

[200] I. Brevik, S. Nojiri, S. D. Odintsov, and L. Vanzo. Entropy and universality of the Cardy-Verlinde formula in a dark energy universe. Phys. Rev. D, 70(4):043520, Aug. 2004, arXiv:hep-th/0401073

[201] H. A. Bridgman, K. A. Malik, and D. Wands. Cosmic vorticity on the brane. Phys. Rev. D63:084012, 2001, hep-th/0010133 
[202] H. A. Bridgman, K. A. Malik, and D. Wands. Cosmological perturbations in the bulk and on the brane. Phys. Rev., D65:043502, 2002, astro-ph/0107245

[203] F. Briscese, E. Elizalde, S. Nojiri and S. D. Odintsov. Phantom scalar dark energy as modified gravity: Understanding the origin of the Big Rip singularity. Phys. Lett. B, 646:105-111, Mar. 2007, arXiv:hep-th/0612220

[204] A. W. Brookfield, C. van de Bruck, and L. M. H. Hall. Viability of $\mathrm{f}(\mathrm{R})$ theories with additional powers of curvature. Phys. Rev. D, 74(6):064028, Sept. 2006, arXiv:hep-th/0608015

[205] J.-P. Bruneton. On causality and superluminal behavior in classical field theories. Applications to k-essence theories and MOND-like theories of gravity. Phys. Rev., D75:085013, 2007, grqc/0607055

[206] H. Buchdahl. ber die variationsableitung von fundamental-invarianten beliebig hoher ordnung. Acta Mathematica, 85:63-72, 1964. 10.1007/BF02395741.

[207] H. A. Buchdahl. Reciprocal Static Metrics and Scalar Fields in the General Theory of Relativity. Phys. Rev., 115:1325-1328, 1959.

[208] H. A. Buchdahl. Non-linear Lagrangians and cosmological theory. MNRAS, 150:1, 1970.

[209] H. A. Buchdahl. The field equations generated by the square of the scalar curvature: solutions of Kasner type. Journal of Physics A Mathematical General, 11:871-876, May 1978.

[210] M. R. Buckley and L. Randall. Xogenesis. 2010, 1009.0270.

[211] J. S. Bullock. Notes on the Missing Satellites Problem. 2010, 1009.4505.

[212] A. Burd and A. Coley. Extended inflation and generalised scalar-tensor theories. Physics Letters B, 267:330-336, Sept. 1991 .

[213] C. Burgess, D. Hoover, C. de Rham, and G. Tasinato. Effective Field Theories and Matching for Codimension-2 Branes. JHEP, 0903:124, 2009, 0812.3820.

[214] C. Burgess and L. van Nierop. Bulk Axions, Brane Back-reaction and Fluxes. 2010, 1012.2638.

[215] C. Burgess and L. van Nierop. Large Dimensions and Small Curvatures from Supersymmetric Brane Back-reaction. 2011, 1101.0152.

[216] C. P. Burgess. Supersymmetric large extra dimensions and the cosmological constant: An update. Ann. Phys., 313:283-401, 2004, hep-th/0402200

[217] C. P. Burgess. Towards a natural theory of dark energy: Supersymmetric large extra dimensions. AIP Conf. Proc., 743:417-449, 2005, hep-th/0411140

[218] C. P. Burgess. Lectures on Cosmic Inflation and its Potential Stringy Realizations. PoS, P2GC:008, 2006, 0708.2865.

[219] C. Burrage. Supernova brightening from chameleon-photon mixing. Phys. Rev. D, 77(4):043009, Feb. 2008, 0711.2966.

[220] C. Burrage, A. Davis, and D. J. Shaw. Detecting chameleons: The astronomical polarization produced by chameleonlike scalar fields. Phys. Rev. D, 79(4):044028, Feb. 2009, 0809.1763.

[221] C. Burrage, C. de Rham, and L. Heisenberg. de Sitter Galileon. 2011, 1104.0155.

[222] C. Burrage, C. de Rham, D. Seery, and A. J. Tolley. Galileon inflation. JCAP, 1101:014, 2011, 1009.2497.

[223] C. Burrage and D. Seery. Revisiting fifth forces in the Galileon model. JCAP, 1008:011, 2010, 1005.1927.

[224] A. J. Bustelo and D. E. Barraco. Hydrostatic equilibrium equation and Newtonian limit of the singular f(R) gravity. Classical and Quantum Gravity, 24:2333-2342, May 2007, arXiv:grqc/0611149

[225] R.-G. Cai, L.-M. Cao, Y.-P. Hu, and N. Ohta. Generalized Misner-Sharp energy in f(R) gravity. Phys. Rev. D, 80(10):104016, Nov. 2009, 0910.2387.

[226] R.-G. Cai, L.-M. Cao, and N. Ohta. Topological black holes in Horava-Lifshitz gravity. Phys. Rev. D, 80(2):024003, Jul. 2009, 0904.3670.

[227] R.-G. Cai, L.-M. Cao, and N. Ohta. Thermodynamics of black holes in Horava-Lifshitz gravity. Phys. Lett. B, 679:504-509, Sep. 2009, 0905.0751.

[228] R.-G. Cai, L.-M. Cao, and N. Ohta. Black holes without mass and entropy in Lovelock gravity. Phys. Rev. D, 81(2):024018, Jan. 2010, 0911.0245

[229] R.-G. Cai, C.-M. Chen, K.-I. Maeda, N. Ohta, and D.-W. Pang. Entropy function and universality of entropy-area relation for small black holes. Phys. Rev. D, 77(6):064030, Mar. 2008, 0712.4212.

[230] R.-G. Cai, B. Hu, and H.-B. Zhang. Dynamical Scalar Degree of Freedom in Horava-Lifshitz Gravity. Phys.Rev., D80:041501, 2009, 0905.0255.

[231] R.-G. Cai, and N. Ohta. Quantum aspects of black holes, evaporation, thermodynamics, Gravity in more than four dimensions, Kaluza-Klein theory, unified field theories; alternative theories of gravity, Classical black holes. Phys. Rev. D, 74(6):064001, Sep. 2006, hep-th/0604088. 
[232] R.-G. Cai, and N. Ohta. Horizon thermodynamics and gravitational field equations in HoravaLifshitz gravity. Phys. Rev. D, 81(8):084061, Apr. 2010, 0910.2307.

[233] R.-G. Cai and K.-S. Soh. Topological black holes in the dimensionally continued gravity. Phys.Rev., D59:044013, 1999, gr-qc/9808067

[234] Y.-F. Cai and E. N. Saridakis. Non-singular cosmology in a model of non-relativistic gravity. JCAP, 0910:020, 2009, 0906.1789.

[235] G. Calcagni. Cosmology of the Lifshitz universe. JHEP, 0909:112, 2009, 0904.0829.

[236] G. Calcagni, S. Tsujikawa, and M. Sami. Dark energy and cosmological solutions in second-order string gravity. Classical and Quantum Gravity, 22:3977-4006, Oct. 2005, arXiv:hep-th/0505193

[237] R. Caldwell, A. Cooray, and A. Melchiorri. Constraints on a new post-general relativity cosmological parameter. Phys. Rev. D, 76(2):023507, July 2007, arXiv:astro-ph/0703375

[238] P. Candelas and S. Weinberg. Calculation of Gauge Couplings and Compact Circumferences from Selfconsistent Dimensional Reduction. Nucl. Phys., B237:397, 1984.

[239] F. Cannata and A. Y. Kamenshchik. Chameleon Cosmology Model Describing the Phantom Divide Line Crossing. ArXiv e-prints, May 2010, 1005.1878.

[240] S. Capozziello, V. F. Cardone, and A. Troisi. Reconciling dark energy models with $\mathrm{f}(\mathrm{R})$ theories. Phys. Rev. D, 71(4):043503, Feb. 2005, arXiv:astro-ph/0501426

[241] S. Capozziello, S. Carloni, and A. Troisi. Quintessence without scalar fields. ArXiv Astrophysics e-prints, Mar. 2003, arXiv:astro-ph/0303041

[242] S. Capozziello, S. Nojiri, S. D. Odintsov, and A. Troisi. Cosmological viability of f(R)-gravity as an ideal fluid and its compatibility with a matter dominated phase. Physics Letters B, 639:135143, Aug. 2006, arXiv:astro-ph/0604431

[243] S. Capozziello, A. Stabile, and A. Troisi. Spherically symmetric solutions in $\mathrm{f}(\mathrm{R})$ gravity via the Noether symmetry approach. Classical and Quantum Gravity, 24(8):2153, 2007.

[244] S. Capozziello and S. Tsujikawa. Solar system and equivalence principle constraints on $\mathrm{f}(\mathrm{R})$ gravity by the chameleon approach. Phys. Rev. D, 77(10):107501, May 2008, 0712.2268.

[245] A. Cardoso, T. Hiramatsu, K. Koyama, and S. S. Seahra. Scalar perturbations in braneworld cosmology. JCAP, 0707:008, 2007, 0705.1685.

[246] A. Cardoso, K. Koyama, S. S. Seahra, and F. P. Silva. Cosmological perturbations in the DGP braneworld: Numeric solution. Phys.Rev., D77:083512, 2008, 0711.2563.

[247] S. Carloni, K. N. Ananda, P. K. S. Dunsby, and M. E. S. Abdelwahab. Unifying the study of background dynamics and perturbations in $\mathrm{f}(\mathrm{R})$ gravity. ArXiv e-prints, Dec. 2008, 0812.2211.

[248] S. Carloni, P. K. S. Dunsby, S. Capozziello, and A. Troisi. Cosmological dynamics of $R^{n}$ gravity. Classical and Quantum Gravity, 22:4839-4868, Nov. 2005, arXiv:gr-qc/0410046

[249] S. Carloni, P. K. S. Dunsby, and D. Solomons. Bounce conditions in $\mathrm{f}(\mathrm{R})$ cosmologies. Classical and Quantum Gravity, 23:1913-1922, Mar. 2006, arXiv:gr-qc/0510130

[250] S. Carloni, P. K. S. Dunsby, and A. Troisi. Evolution of density perturbations in $\mathrm{f}(\mathrm{R})$ gravity. Phys. Rev. D, 77(2):024024, Jan. 2008, 0707.0106.

[251] S. Carloni, R. Goswami, and P. K. S. Dunsby. A new approach to reconstruction methods in $\mathrm{f}(\mathrm{R})$ gravity. ArXiv e-prints, May 2010, 1005.1840.

[252] S. Carloni, J. A. Leach, S. Capozziello, and P. K. S. Dunsby. Cosmological dynamics of scalar tensor gravity. Classical and Quantum Gravity, 25(3):035008, Feb. 2008, arXiv:gr-qc/0701009

[253] S. Carloni, A. Troisi, and P. K. S. Dunsby. Some remarks on the dynamical systems approach to fourth order gravity. General Relativity and Gravitation, 41:1757-1776, Aug. 2009, 0706.0452.

[254] S. M. Carroll, A. de Felice, V. Duvvuri, D. A. Easson, M. Trodden, and M. S. Turner. Cosmology of generalized modified gravity models. Phys. Rev. D, 71(6):063513, Mar. 2005, arXiv:astro$\mathrm{ph} / 0410031$

[255] S. M. Carroll, V. Duvvuri, M. Trodden, and M. S. Turner. Is cosmic speed-up due to new gravitational physics? Phys. Rev. D, 70(4):043528, Aug. 2004, arXiv:astro-ph/0306438

[256] S. M. Carroll and M. M. Guica. Sidestepping the cosmological constant with football shaped extra dimensions. 2003, hep-th/0302067

[257] S. M. Carroll and E. A. Lim. Lorentz-violating vector fields slow the universe down. Phys. Rev. D, 70(12):123525, Dec. 2004, arXiv:hep-th/0407149.

[258] S. M. Carroll, I. Sawicki, A. Silvestri, and M. Trodden. Modified-source gravity and cosmological structure formation. New Journal of Physics, 8:323, Dec. 2006, arXiv:astro-ph/0607458

[259] É. Cartan. Sur une généralisation de la notion de courbure de Riemann et les espaces à torsion. C. R. Acad. Sci. , 174, 593, 1922

[260] É. Cartan. Sur les variétés à connexion affine et la théorie de la relativitée généralisée I. Ann. 
Ec. Norm. Sup., 40, 325, 1923.

[261] É. Cartan. Sur les variétés à connexion affine et la théorie de la relativitée généralisée II. Ann. Ec. Norm. Sup. , 42, 17, 1925.

[262] B. Carter. Axisymmetric black hole has only two degrees of freedom. Phys. Rev. Lett., 26(6):331333, Feb 1971

[263] B. Carter and R. Gregory. Curvature corrections to dynamics of domain walls. Phys. Rev., D51:5839-5846, 1995, hep-th/9410095

[264] C. Cartier, E. J. Copeland, and R. Madden. The graceful exit in string cosmology. Journal of High Energy Physics, 1:35, Jan. 2000, arXiv:hep-th/9910169

[265] J. A. Casas, J. García-Bellido, and M. Quirós. Nucleosynthesis Bounds on Jordan-Brans Theories of Gravity. Modern Physics Letters A, 7:447-456, 1992.

[266] J. A. Casas, J. García-Bellido, and M. Quirós. Updating nucleosynthesis bounds on JordanBrans-Dicke theories of gravity. Physics Letters B, 278:94-96, Mar. 1992.

[267] J. A. R. Cembranos. The newtonian limit at intermediate energies. Phys. Rev. D, 73(6):064029, Mar 2006.

[268] A. H. Chamseddine, A. Connes, and M. Marcolli. Gravity and the standard model with neutrino mixing. Adv. Theor. Math. Phys., 11:991-1089, 2007, hep-th/0610241

[269] K. Chan and R. Scoccimarro. Large-Scale Structure in Brane-Induced Gravity II. Numerical Simulations. Phys.Rev., D80:104005, 2009, 0906.4548.

[270] C. Charmousis. Higher order gravity theories and their black hole solutions. Lect.Notes Phys., 769:299-346, 2009, 0805.0568.

[271] C. Charmousis, S. C. Davis, and J.-F. Dufaux. Scalar brane backgrounds in higher order curvature gravity. JHEP, 0312:029, 2003, hep-th/0309083

[272] C. Charmousis and J.-F. Dufaux. General Gauss-Bonnet brane cosmology. Class.Quant.Grav., 19:4671-4682, 2002, hep-th/0202107

[273] C. Charmousis, R. Gregory, N. Kaloper, and A. Padilla. DGP specteroscopy. JHEP, 10:066 2006, hep-th/0604086

[274] C. Charmousis, R. Gregory, and A. Padilla. Stealth Acceleration and Modified Gravity. JCAP, 0710:006, 2007, 0706.0857.

[275] C. Charmousis, R. Gregory, and V. A. Rubakov. Wave function of the radion in a brane world. Phys. Rev., D62:067505, 2000, hep-th/9912160

[276] C. Charmousis, C. E. J., P. Antonia, and P. M. Saffin. General second order scalar-tensor theory, self tuning, and the fab four. 2011. submitted.

[277] C. Charmousis, G. Kofinas, and A. Papazoglou. The consistency of codimension-2 braneworlds and their cosmology. JCAP, 1001:022, 2010, 0907.1640.

[278] C. Charmousis, G. Niz, A. Padilla, and P. M. Saffin. Strong coupling in Horava gravity. JHEP, 0908:070, 2009, 0905.2579.

[279] C. Charmousis and A. Padilla. The Instability of Vacua in Gauss-Bonnet Gravity. JHEP, 0812:038, 2008, 0807.2864.

[280] C. Charmousis and A. Papazoglou. Self-properties of codimension-2 braneworlds. JHEP, 07:062, $2008,0804.2121$.

[281] C. Charmousis and A. Papazoglou. Properties of codimension-2 braneworlds in six-dimensional Lovelock theory. J. Phys. Conf. Ser., 189:012007, 2009, 0902.2174.

[282] C. Charmousis and R. Zegers. Einstein gravity on an even codimension brane. Phys.Rev. D72:064005, 2005, hep-th/0502171

[283] C. Charmousis and R. Zegers. Matching conditions for a brane of arbitrary codimension. JHEP, 0508:075, 2005, hep-th/0502170

[284] B. Chen, S. Pi, and J.-Z. Tang. Scale Invariant Power Spectrum in Horava-Lifshitz Cosmology without Matter. JCAP, 0908:007, 2009, 0905.2300.

[285] C.-M. Chen, D. V. Gal'Tsov, N. Ohta, and D. G. Orlov. Global solutions for higher-dimensional stretched small black holes. Phys. Rev. D, 81(2):024002, Jan. 2010, 0910.3488

[286] C.-M. Chen, T. Harko, W. F. Kao, and M. K. Mak. Rotational perturbations of FriedmannRobertson-Walker type brane-world cosmological models. Nucl. Phys., B636:159-178, 2002, hepth/0201012

[287] D.-M. Chen. Strong lensing probability in TeVeS theory. JCAP, 0801:006, 2008, 0712.1633.

[288] D.-M. Chen and H. Zhao. Strong gravitational lens probability in TeVeS. Astrophys. J., 650:L9L12, 2006, astro-ph/0606506.

[289] S. Chen, J. B. Dent, S. Dutta, and E. N. Saridakis. Cosmological perturbations in $\mathrm{f}(\mathrm{T})$ gravity. ArXiv e-prints, Aug. 2010, 1008.1250. 
[290] X. Chen and M. Kamionkowski. Cosmic microwave background temperature and polarization anisotropy in Brans-Dicke cosmology. Phys. Rev. D, 60(10):104036, Nov. 1999, arXiv:astroph/9905368

[291] J. Chiaverini, S. J. Smullin, A. A. Geraci, D. M. Weld, and A. Kapitulnik. New Experimental Constraints on Non-Newtonian Forces below $100 \mu \mathrm{m}$. Physical Review Letters, 90(15):151101, Apr. 2003, arXiv:hep-ph/0209325

[292] T. Chiba. 1/R gravity and scalar-tensor gravity. Physics Letters B, 575:1-3, Nov. 2003, arXiv:astro-ph/0307338

[293] T. Chiba. Generalized gravity and a ghost. J. Cosmology Astropart. Phys., 3:8, Mar. 2005, arXiv:gr-qc/0502070

[294] T. Chiba, T. L. Smith, and A. L. Erickcek. Solar system constraints to general $f(r)$ gravity. Phys. Rev. D, 75(12):124014, Jun 2007.

[295] M.-C. Chiu, C.-M. Ko, and Y. Tian. Theoretical aspects of gravitational lensing in TeVeS. Astrophys. J., 636:565-574, 2006, astro-ph/0507332.

[296] M. C. Chiu, Y. Tian, and C. M. Ko. Necessity of Dark Matter in Modified Newtonian Dynamics within Galactic Scales? - Testing the Covariant MOND in Elliptical Lenses. 2008, 0812.5011.

[297] G. Chkareuli and D. Pirtskhalava. Vainshtein Mechanism In $\Lambda_{3}$ - Theories. 2011, 1105.1783.

[298] A. S. Chou, W. Wester, A. Baumbaugh, H. R. Gustafson, Y. Irizarry-Valle, P. O. Mazur, J. H. Steffen, R. Tomlin, A. Upadhye, A. Weltman, X. Yang, and J. Yoo. Search for Chameleon Particles Using a Photon-Regeneration Technique. Physical Review Letters, 102(3):030402, Jan. 2009, 0806.2438.

[299] N. Chow and J. Khoury. Galileon Cosmology. Phys.Rev., D80:024037, 2009, 0905.1325.

[300] D. J. H. Chung and K. Freese. Lensed density perturbations in braneworlds: An alternative to perturbations from inflation. Phys. Rev., D67:103505, 2003, astro-ph/0202066

[301] T. E. Chupp, R. J. Hoare, R. A. Loveman, E. R. Oteiza, J. M. Richardson, M. E. Wagshul, and A. K. Thompson. Results of a new test of local Lorentz invariance: A search for mass anisotropy in ${ }^{21}$ Ne. Physical Review Letters, 63:1541-1545, Oct. 1989.

[302] I. Ciufolini and E. C. Pavlis. A confirmation of the general relativistic prediction of the LenseThirring effect. Nature, 431:958-960, Oct. 2004.

[303] C. A. Clarkson, A. A. Coley, and E. S. D. O'Neill. Cosmic microwave background and scalartensor theories of gravity. Phys. Rev. D, 64(6):063510, Sept. 2001, arXiv:gr-qc/0105026

[304] T. Clifton. Spherically symmetric solutions to fourth-order theories of gravity. Classical and Quantum Gravity, 23:7445-7453, Dec. 2006, arXiv:gr-qc/0607096

[305] T. Clifton. Exact Friedmann solutions in higher-order gravity theories. Classical and Quantum Gravity, 24:5073-5091, Oct. 2007, arXiv:gr-qc/0703126

[306] T. Clifton. Higher powers in gravitation. Phys. Rev. D, 78(8):083501, Oct. 2008, 0807.4682.

[307] T. Clifton. Parametrized post-Newtonian limit of fourth-order theories of gravity. Phys. Rev. D, 77(2):024041, Jan. 2008, 0801.0983.

[308] T. Clifton, M. Bañados, and C. Skordis. The Parameterized Post-Newtonian Limit of Bimetric Theories of Gravity. ArXiv e-prints, June 2010, 1006.5619.

[309] T. Clifton and J. D. Barrow. The existence of Gödel, Einstein, and de Sitter universes. Phys. Rev. D, 72(12):123003, Dec. 2005, arXiv:gr-qc/0511076

[310] T. Clifton and J. D. Barrow. The power of general relativity. Phys. Rev. D, 72(10):103005, Nov. 2005, arXiv:gr-qc/0509059

[311] T. Clifton and J. D. Barrow. Further exact cosmological solutions to higher-order gravity theories. Classical and Quantum Gravity, 23:2951-2962, May 2006, arXiv:gr-qc/0601118

[312] T. Clifton, J. D. Barrow, and R. J. Scherrer. Constraints on the Variation of G from Primordial Nucleosynthesis. Phys. Rev., D71:123526, 2005, astro-ph/0504418.

[313] T. Clifton, D. F. Mota, and J. D. Barrow. Inhomogeneous gravity. Mon. Not. Roy. Astron. Soc., 358:601, 2005, gr-qc/0406001

[314] T. Clifton and J. M. Weisberg. A Simple Model for Pulse Profiles from Precessing Pulsars, with Special Application to Relativistic Binary PSR B1913+16. Astrophys. J., 679:687, 2008, 0708.0993.

[315] T. Clifton and T. G. Zlosnik. FRW cosmology in Milgrom's bimetric theory of gravity. Phys. Rev. D, 81(10):103525, May 2010, 1002.1448

[316] J. M. Cline, J. Descheneau, M. Giovannini, and J. Vinet. Cosmology of codimension-two braneworlds. JHEP, 06:048, 2003, hep-th/0304147

[317] J. M. Cline, S. Jeon, and G. D. Moore. The phantom menaced: Constraints on low-energy effective ghosts. Phys. Rev., D70:043543, 2004, hep-ph/0311312 
[318] D. Clowe, M. Bradac, A. H. Gonzalez, M. Markevitch, S. W. Randall, et al. A direct empirical proof of the existence of dark matter. Astrophys.J., 648:L109-L113, 2006, astro-ph/0608407

[319] A. Coc, K. A. Olive, J. Uzan, and E. Vangioni. Big bang nucleosynthesis constraints on scalartensor theories of gravity. Phys. Rev. D, 73(8):083525, Apr. 2006, arXiv:astro-ph/0601299

[320] D. Codazzi. Sulle coordinate curvilinee d'una superficie e dello spazio. Annali di Matematica Pura ed Applicata (1867-1897), 1(1):293-316, 1867.

[321] G. Cognola, E. Elizalde, S. Nojiri, S. D. Odintsov, L. Sebastiani, and S. Zerbini. Class of viable modified $\mathrm{f}(\mathrm{R})$ gravities describing inflation and the onset of accelerated expansion. Phys. Rev. D, 77(4):046009, Feb. 2008, 0712.4017.

[322] G. Cognola, E. Elizalde, S. Nojiri, S. D. Odintsov, and S. Zerbini. One-loop f(R) gravity in de Sitter universe. J. Cosmology Astropart. Phys., 2:10, Feb. 2005, arXiv:hep-th/0501096

[323] G. Cognola, E. Elizalde, S. Nojiri, S. D. Odintsov, and S. Zerbini. Dark energy in modified GaussBonnet gravity: Late-time acceleration and the hierarchy problem. Phys. Rev. D, 73(8):084007, Apr. 2006, arXiv:hep-th/0601008

[324] G. Cognola, E. Elizalde, S. Nojiri, S. D. Odintsov, and S. Zerbini. String-inspired Gauss-Bonnet gravity reconstructed from the universe expansion history and yielding the transition from matter dominance to dark energy. Phys. Rev. D, 75(8):086002, Apr. 2007, arXiv:hep-th/0611198

[325] G. Cognola, M. Gastaldi, and S. Zerbini. On the Stability of a Class of Modified Gravitational Models. International Journal of Theoretical Physics, 47:898-910, Apr. 2008, arXiv:grqc/0701138

[326] G. Cognola and S. Zerbini. Homogeneous Cosmologies in Generalized Modified Gravity. International Journal of Theoretical Physics, 47:3186-3200, Dec. 2008, 0802.3967.

[327] A. A. Coley. Qualitative Properties of Scalar-Tensor Theories of Gravity. General Relativity and Gravitation, 31:1295, Sept. 1999, arXiv:astro-ph/9910395

[328] P. D. B. Collins and R. F. Langbein. Thermodynamic aspects of extended inflation. Phys. Rev. D, 47:2302-2312, Mar. 1993.

[329] D. Comelli, M. Crisostomi, F. Nesti, and L. Pilo. Finite energy for a gravitational potential falling slower than 1/r. Phys. Rev. D, 84(10):104026, Nov. 2011, 1105.3010.

[330] A. Conley, J. Guy, M. Sullivan, N. Regnault, P. Astier, C. Balland, S. Basa, R. G. Carlberg, D. Fouchez, D. Hardin, I. M. Hook, D. A. Howell, R. Pain, N. Palanque-Delabrouille, K. M. Perrett, C. J. Pritchet, J. Rich, V. Ruhlmann-Kleider, D. Balam, S. Baumont, R. S. Ellis, S. Fabbro, H. K. Fakhouri, N. Fourmanoit, S. González-Gaitán, M. L. Graham, M. J. Hudson, E. Hsiao, T. Kronborg, C. Lidman, A. M. Mourao, J. D. Neill, S. Perlmutter, P. Ripoche, N. Suzuki, and E. S. Walker. Supernova Constraints and Systematic Uncertainties from the First Three Years of the Supernova Legacy Survey. ApJS, 192:1, Jan. 2011

[331] C. R. Contaldi, T. Wiseman, and B. Withers. TeVeS gets caught on caustics. Phys. Rev., D78:044034, 2008, 0802.1215.

[332] A. Cooney, S. Dedeo, and D. Psaltis. Neutron stars in $\mathrm{f}(\mathrm{R})$ gravity with perturbative constraints. Phys. Rev. D, 82(6):064033, Sept. 2010, 0910.5480.

[333] E. J. Copeland, E. W. Kolb, and A. R. Liddle. Topological defects in extended inflation. Phys. Rev. D, 42:2911-2914, Oct. 1990.

[334] E. J. Copeland, A. R. Liddle, and J. E. Lidsey. Steep inflation: Ending braneworld inflation by gravitational particle production. Phys. Rev., D64:023509, 2001, astro-ph/0006421

[335] E. J. Copeland and D. J. Toms. Quantized Antisymmetric Tensor Fields And Selfconsistent Dimensional Reduction In Higher Dimensional Space-Times. Nucl. Phys., B255:201, 1985.

[336] E. J. Copeland and D. J. Toms. Stability Of Selfconsistent Higher Dimensional Cosmological Solutions. Phys. Rev., D32:1921, 1985.

[337] C. J. Copi, D. Huterer, D. J. Schwarz, and G. D. Starkman. Large angle anomalies in the CMB. Adv.Astron., 2010:847541, 2010, 1004.5602.

[338] O. Corradini, K. Koyama, and G. Tasinato. Induced gravity on intersecting brane-worlds Part I: Maximally symmetric solutions. Phys. Rev., D77:084006, 2008, 0712.0385.

[339] O. Corradini, K. Koyama, and G. Tasinato. Induced gravity on intersecting brane-worlds Part II: Cosmology. Phys. Rev., D78:124002, 2008, 0803.1850.

[340] S. Cotsakis, J. Demaret, Y. De Rop, and L. Querella. Mixmaster universe in fourth-order gravity theories. Phys. Rev. D, 48(10):4595-4603, Nov 1993.

[341] P. Creminelli, G. D'Amico, M. Musso, J. Norena, and E. Trincherini. Galilean symmetry in the effective theory of inflation: new shapes of non-Gaussianity. 2010, 1011.3004.

[342] P. Creminelli, A. Nicolis, M. Papucci, and E. Trincherini. Ghosts in massive gravity. JHEP, 0509:003, 2005, hep-th/0505147 
[343] P. Creminelli, A. Nicolis, and E. Trincherini. Galilean Genesis: An Alternative to inflation. JCAP, 1011:021, 2010, 1007.0027.

[344] J. Crisostomo, R. Troncoso, and J. Zanelli. Black hole scan. Phys.Rev., D62:084013, 2000, hep-th/0003271

[345] R. Crittenden and P. J. Steinhardt. Graceful exit in extended inflation and implications for density perturbations. Physics Letters B, 293:32-36, Oct. 1992, arXiv:astro-ph/9207002

[346] C. Csaki, M. Graesser, L. Randall, and J. Terning. Cosmology of brane models with radion stabilization. Phys. Rev., D62:045015, 2000, hep-ph/9911406

[347] C. Csaki, M. L. Graesser, and G. D. Kribs. Radion dynamics and electroweak physics. Phys. Rev., D63:065002, 2001, hep-th/0008151

[348] B. Cuadros-Melgar, E. Papantonopoulos, M. Tsoukalas, and V. Zamarias. Black Holes on Thin 3-branes of Codimension-2 and their Extension into the Bulk. Nucl. Phys., B810:246-265, 2009, 0804.4459 .

[349] B. Cuadros-Melgar, E. Papantonopoulos, M. Tsoukalas, and V. Zamarias. Perturbations of Gauss-Bonnet Black Strings in Codimension-2 Braneworlds. 2010, 1012.4747.

[350] S. Cullen and M. Perelstein. SN1987A constraints on large compact dimensions. Phys. Rev. Lett., 83:268-271, 1999, hep-ph/9903422

[351] E. Czuchry. Bounce scenarios in modified Horava-Lifshitz cosmology. 2010, 1008.3410.

[352] J. da Angela, T. Shanks, S. Croom, P. Weilbacher, R. Brunner, et al. The 2dF-SDSS LRG and QSO Survey: QSO clustering and the L-z degeneracy. Mon.Not.Roy.Astron.Soc., 383:565-580, 2008, astro-ph/0612401

[353] A. M. da Silva. An Alternative Approach for General Covariant Horava-Lifshitz Gravity and Matter Coupling. 2010, 1009.4885.

[354] T. Damour and C. Gundlach. Nucleosynthesis constraints on an extended Jordan-Brans-Dicke theory. Phys. Rev. D, 43(12):3873-3877, Jun 1991.

[355] T. Damour, I. I. Kogan, and A. Papazoglou. Nonlinear bigravity and cosmic acceleration. Phys. Rev. D, 66(10):104025, Nov. 2002, arXiv:hep-th/0206044.

[356] T. Damour and K. Nordtvedt. General relativity as a cosmological attractor of tensor-scalar theories. Phys. Rev. Lett., 70(15):2217-2219, Apr 1993.

[357] T. Damour and K. Nordtvedt. Tensor-scalar cosmological models and their relaxation toward general relativity. Phys. Rev. D, 48(8):3436-3450, Oct 1993.

[358] T. Damour and B. Pichon. Big bang nucleosynthesis and tensor-scalar gravity. Phys. Rev. D, 59(12):123502, May 1999.

[359] T. Damour and J. Taylor. Strong-field tests of relativistic gravity and binary pulsars. Phys. Rev. D, 45:1840-1868, 1992

[360] S. F. Daniel, R. R. Caldwell, A. Cooray, and A. Melchiorri. Large scale structure as a probe of gravitational slip. Phys. Rev. D, 77(10):103513, May 2008, 0802.1068.

[361] S. F. Daniel et al. Testing general relativity with current cosmological data. Phys. Rev., D81:123508, 2010. arXiv:1002.1962

[362] A. Davis, C. A. O. Schelpe, and D. J. Shaw. The Chameleonic Contribution to the SZ Radial Profile of the Coma Cluster. ArXiv e-prints, Aug. 2010, 1008.1880.

[363] S. C. Davis. Generalized Israel junction conditions for a Gauss-Bonnet brane world. Phys.Rev. D67:024030, 2003, hep-th/0208205

[364] S. C. Davis. Solar System Constraints on f(G) Dark Energy. ArXiv e-prints, Sept. 2007, 0709.4453.

[365] H. Davoudiasl, D. E. Morrissey, K. Sigurdson, and S. Tulin. Unified origin for baryonic visible matter and antibaryonic dark matter. Phys. Rev. Lett., 105(21):211304, Nov 2010.

[366] W. de Blok. The Core-Cusp Problem. Advances in Astronomy, page 789293, 2010, 0910.3538.

[367] J. A. de Deus and D. Müller. Bianchi VIIA solutions of quadratic gravity. ArXiv e-prints, Mar. $2011,1103.5450$

[368] A. De Felice, J.-M. Gérard, and T. Suyama. Cosmological perturbation in $f(R, G)$ theories with a perfect fluid. Phys. Rev. D, 82(6):063526, Sep 2010.

[369] A. De Felice, R. Kase, and S. Tsujikawa. Matter perturbations in Galileon cosmology. 2010, 1011.6132 .

[370] A. de Felice, D. F. Mota, and S. Tsujikawa. Matter instabilities in general Gauss-Bonnet gravity. Phys. Rev. D, 81(2):023532, Jan. 2010, 0911.1811.

[371] A. de Felice and S. Tsujikawa. Construction of cosmologically viable $\mathrm{f}(\mathrm{G})$ gravity models. Physics Letters B, 675:1-8, May 2009, 0810.5712.

[372] A. de Felice and S. Tsujikawa. Solar system constraints on $\mathrm{f}(\mathrm{G})$ gravity models. Phys. Rev. D, 80(6):063516, Sept. 2009, 0907.1830. 
[373] A. De Felice and S. Tsujikawa. Cosmology of a covariant Galileon field. Phys.Rev.Lett., 105:111301, 2010, 1007.2700.

[374] A. de Felice and S. Tsujikawa. f(R) Theories. Living Reviews in Relativity, 13:3, June 2010, 1002.4928 .

[375] A. De Felice and S. Tsujikawa. Generalized Galileon cosmology. 2010, 1008.4236.

[376] Á. de La Cruz-Dombriz and A. Dobado. $\mathrm{f}(\mathrm{R})$ gravity without a cosmological constant. Phys. Rev. D, 74(8):087501, Oct. 2006, arXiv:gr-qc/0607118

[377] A. de La Cruz-Dombriz, A. Dobado, and A. L. Maroto. Evolution of density perturbations in $\mathrm{f}(\mathrm{R})$ theories of gravity. Phys. Rev. D, 77(12):123515, June 2008, 0802.2999.

[378] A. de La Cruz-Dombriz, A. Dobado, and A. L. Maroto. Black holes in f(R) theories. Phys. Rev. D, 80(12):124011, Dec. 2009, 0907.3872.

[379] C. de Rham. An Introduction to Cascading Gravity and Degravitation. Can. J. Phys., 87:201203, 2009, 0810.0269.

[380] C. de Rham. Massive gravity from Dirichlet boundary conditions. Phys.Lett., B688:137-141, 2010, 0910.5474.

[381] C. de Rham et al. Cascading gravity: Extending the Dvali-Gabadadze-Porrati model to higher dimension. Phys. Rev. Lett., 100:251603, 2008, 0711.2072.

[382] C. de Rham and G. Gabadadze. Generalization of the Fierz-Pauli Action. Phys.Rev., D82:044020, 2010, 1007.0443.

[383] C. de Rham and G. Gabadadze. Selftuned Massive Spin-2. Phys.Lett., B693:334-338, 2010, 1006.4367 .

[384] C. de Rham, G. Gabadadze, L. Heisenberg, and D. Pirtskhalava. Cosmic Acceleration and the Helicity-0 Graviton. 2010, 1010.1780.

[385] C. de Rham, G. Gabadadze, and A. J. Tolley. Resummation of Massive Gravity. 2010, 1011.1232.

[386] C. de Rham, S. Hofmann, J. Khoury, and A. J. Tolley. Cascading Gravity and Degravitation. JCAP, 0802:011, 2008, 0712.2821.

[387] C. de Rham, J. Khoury, and A. J. Tolley. Flat 3-Brane with Tension in Cascading Gravity. Phys. Rev. Lett., 103:161601, 2009, 0907.0473.

[388] C. de Rham, J. Khoury, and A. J. Tolley. Cascading Gravity is Ghost Free. Phys.Rev., D81:124027, 2010, 1002.1075.

[389] C. de Rham and A. J. Tolley. DBI and the Galileon reunited. JCAP, 1005:015, $2010,1003.5917$.

[390] J. C. C. de Souza and V. Faraoni. The phase-space view of $\mathrm{f}(\mathrm{R})$ gravity. Classical and Quantum Gravity, 24:3637-3648, July 2007, 0706.1223.

[391] A. DeFelice and M. Hindmarsh. Unsuccessful cosmology with modified gravity models. J. Cosmology Astropart. Phys., 6:28, June 2007, 0705.3375.

[392] A. DeFelice, M. Hindmarsh, and M. Trodden. Ghosts, instabilities, and superluminal propagation in modified gravity models. J. Cosmology Astropart. Phys., 8:5, Aug. 2006, arXiv:astro$\mathrm{ph} / 0604154$

[393] A. DeFelice and T. Suyama. Vacuum structure for scalar cosmological perturbations in modified gravity models. J. Cosmology Astropart. Phys., 6:34, June 2009, 0904.2092.

[394] C. Deffayet. Cosmology on a brane in Minkowski bulk. Phys.Lett., B502:199-208, 2001, hepth/0010186

[395] C. Deffayet. On brane world cosmological perturbations. Phys.Rev., D66:103504, 2002, hepth/0205084

[396] C. Deffayet, S. Deser, and G. Esposito-Farese. Generalized Galileons: All scalar models whose curved background extensions maintain second-order field equations and stress-tensors. Phys.Rev., D80:064015, 2009, 0906.1967.

[397] C. Deffayet, S. Deser, and G. Esposito-Farese. Arbitrary p-form Galileons. Phys.Rev., D82:061501, 2010, 1007.5278.

[398] C. Deffayet, G. R. Dvali, and G. Gabadadze. Accelerated universe from gravity leaking to extra dimensions. Phys. Rev., D65:044023, 2002, astro-ph/0105068

[399] C. Deffayet, G. R. Dvali, G. Gabadadze, and A. I. Vainshtein. Nonperturbative continuity in graviton mass versus perturbative discontinuity. Phys. Rev., D65:044026, 2002, hep-th/0106001

[400] C. Deffayet, G. Esposito-Farese, and A. Vikman. Covariant Galileon. Phys.Rev., D79:084003, 2009, 0901.1314.

[401] C. Deffayet, G. Gabadadze, and A. Iglesias. Perturbations of Self-Accelerated Universe. JCAP, 0608:012, 2006, hep-th/0607099

[402] C. Deffayet, X. Gao, D. A. Steer, and G. Zahariade. From k-essence to generalised Galileons. 
2011, 1103.3260 .

[403] C. Deffayet, S. J. Landau, J. Raux, M. Zaldarriaga, and P. Astier. Supernovae, CMB, and gravitational leakage into extra dimensions. Phys.Rev., D66:024019, 2002, astro-ph/0201164.

[404] C. Deffayet, O. Pujolas, I. Sawicki, and A. Vikman. Imperfect Dark Energy from Kinetic Gravity Braiding. JCAP, 1010:026, 2010, 1008.0048.

[405] C. Deffayet and J.-W. Rombouts. Ghosts, strong coupling and accidental symmetries in massive gravity. Phys. Rev., D72:044003, 2005, gr-qc/0505134

[406] S. del Campo. Initial conditions for anisotropic extended-type inflationary universes. Phys. Rev. D, 45:3386-3393, May 1992.

[407] S. del Campo and A. Vilenkin. Initial conditions for extended inflation. Phys. Rev. D, 40:688-690, July 1989.

[408] N. Deruelle, T. Dolezel, and J. Katz. Perturbations of brane worlds. Phys. Rev., D63:083513, 2001, hep-th/0010215

[409] N. Deruelle and C. Germani. Smooth branes and junction conditions in Einstein Gauss-Bonnet gravity. Nuovo Cim., B118:977-988, 2003, gr-qc/0306116

[410] N. Deruelle, C. Gundlach, and D. Langlois. Perturbations of extended inflation. Phys. Rev. D, 45:3301, May 1992.

[411] N. Deruelle, C. Gundlach, and D. Langlois. Vacuum density fluctuations in extended chaotic inflation. Phys. Rev. D, 46:5337-5345, Dec. 1992.

[412] N. Deruelle, C. Gundlach, and D. Polarski. The growth of density fluctuations in a simplified model of extended inflation. Classical and Quantum Gravity, 9:1511-1524, June 1992.

[413] N. Deruelle and M. Sasaki. Newton's law on an Einstein 'Gauss-Bonnet' brane. Prog. Theor.Phys., 110:441-456, 2003, gr-qc/0306032

[414] S. Desai, E. O. Kahya, and R. P. Woodard. Reduced time delay for gravitational waves with dark matter emulators. Phys. Rev., D77:124041, 2008, 0804.3804.

[415] S. Deser and B. Tekin. Gravitational energy in quadratic curvature gravities. Phys.Rev.Lett., 89:101101, 2002, hep-th/0205318.

[416] S. Deser and B. Tekin. Energy in generic higher curvature gravity theories. Phys.Rev. D67:084009, 2003, hep-th/0212292

[417] A. Dev, D. Jain, S. Jhingan, S. Nojiri, M. Sami, and I. Thongkool. Delicate $f(R)$ gravity models with a disappearing cosmological constant and observational constraints on the model parameters. Phys. Rev. D, 78(8):083515, Oct. 2008, 0807.3445.

[418] B. DeWitt. Dynamical theory of groups and fields. Gordon and Breach, 1965.

[419] L. M. Diaz-Rivera, L. Samushia, and B. Ratra. Inflation and accelerated expansion TeVeS cosmological solutions. Phys. Rev., D73:083503, 2006, astro-ph/0601153

[420] R. Dick. Standard cosmology in the DGP brane model. Acta Phys.Polon., B32:3669-3682, 2001, hep-th/0110162

[421] R. Dick. Letter: On the Newtonian Limit in Gravity Models with Inverse Powers of R. General Relativity and Gravitation, 36:217-224, Jan. 2004, arXiv:gr-qc/0307052

[422] R. H. Dicke. Mach's principle and invariance under transformation of units. Phys. Rev., 125:2163$2167,1962$.

[423] R. H. Dicke. The Theoretical Significance of Experimental Relativity. American Journal of Physics, 34:369-370, Apr. 1966.

[424] P. A. M. Dirac. A new classical theory of electrons I. Proc. Roy. Soc., A209:291, 1951.

[425] P. A. M. Dirac. A new classical theory of electrons II. Proc. Roy. Soc., A212:330, 1952.

[426] P. A. M. Dirac. A new classical theory of electrons III. Proc. Roy. Soc., A223:438, 1954.

[427] S. Dodelson and M. Liguori. Can Cosmic Structure form without Dark Matter? Phys. Rev. Lett., 97:231301, 2006, astro-ph/0608602.

[428] A. D. Dolgov and M. Kawasaki. Can modified gravity explain accelerated cosmic expansion? Physics Letters B, 573:1-4, Oct. 2003, arXiv:astro-ph/0307285

[429] A. E. Domínguez and D. E. Barraco. Newtonian limit of the singular $\mathrm{f}(\mathrm{R})$ gravity in the Palatini formalism. Phys. Rev. D, 70(4):043505, Aug. 2004, arXiv:gr-qc/0408069.

[430] M. Dorca and C. van de Bruck. Cosmological perturbations in brane worlds: Brane bending and anisotropic stresses. Nucl. Phys., B605:215-233, 2001, hep-th/0012116

[431] J. Dossett, M. Ishak, J. Moldenauer, Y. Gong and A. Wang. Constraints on growth index parameters from current and future observations. JCAP, 1004:022, 2010, arXiv:1004.3086

[432] J. Dossett, J. Moldenauer and M. Ishak. Figures of merit and constraints from testing general relativity using the latest cosmological data set including refined COSMOS 3D weak lensing. Phys. Rev. D., 84(2):023012, 2011, arXiv:1103.1195 
[433] R. W. P. Drever. A search for anisotropy of inertial mass using a free precession technique. Philosophical Magazine, 6:683-687, May 1961.

[434] I. T. Drummond. Bimetric gravity and "dark matter". Phys. Rev. D, 63(4):043503, Feb. 2001, arXiv:astro-ph/0008234

[435] S. Dubovsky and V. Rubakov. On models of gauge field localization on a brane. Int.J.Mod.Phys., A16:4331-4350, 2001, hep-th/0105243

[436] S. Dubovsky and V. Rubakov. Brane induced gravity in more than one extra dimensions: Violation of equivalence principle and ghost. Phys.Rev., D67:104014, 2003, hep-th/0212222

[437] J.-F. Dufaux, J. E. Lidsey, R. Maartens, and M. Sami. Cosmological perturbations from brane inflation with a Gauss-Bonnet term. Phys.Rev., D70:083525, 2004, hep-th/0404161

[438] M. J. Duff and J. T. Liu. Complementarity of the Maldacena and Randall-Sundrum pictures. Class. Quant. Grav., 18:3207-3214, 2001, hep-th/0003237

[439] M. J. Duff, J. T. Liu, and K. S. Stelle. A supersymmetric type IIB Randall-Sundrum realization. J. Math. Phys., 42:3027-3047, 2001, hep-th/0007120

[440] M. J. Duff and C. N. Pope. Consistent truncations in Kaluza-Klein theories. Nucl. Phys., B255:355-364, 1985.

[441] M. J. Duncan and L. Gerhard Jensen. Extended inflation in closed cosmologies. Physics Letters B, 247:246-250, Sept. 1990 .

[442] J. Dunkley, R. Hlozek, J. Sievers, V. Acquaviva, P. A. R. Ade, P. Aguirre, M. Amiri, J. W. Appel, L. F. Barrientos, E. S. Battistelli, J. R. Bond, B. Brown, B. Burger, J. Chervenak, S. Das, M. J. Devlin, S. R. Dicker, W. Bertrand Doriese, R. Dunner, T. Essinger-Hileman, R. P. Fisher, J. W. Fowler, A. Hajian, M. Halpern, M. Hasselfield, C. Hernandez-Monteagudo, G. C. Hilton, M. Hilton, A. D. Hincks, K. M. Huffenberger, D. H. Hughes, J. P. Hughes, L. Infante, K. D. Irwin, J. B. Juin, M. Kaul, J. Klein, A. Kosowsky, J. M. Lau, M. Limon, Y. Lin, R. H. Lupton, T. A. Marriage, D. Marsden, P. Mauskopf, F. Menanteau, K. Moodley, H. Moseley, C. B. Netterfield, M. D. Niemack, M. R. Nolta, L. A. Page, L. Parker, B. Partridge, B. Reid, N. Sehgal, B. Sherwin, D. N. Spergel, S. T. Staggs, D. S. Swetz, E. R. Switzer, R. Thornton, H. Trac, C. Tucker, R. Warne, E. Wollack, and Y. Zhao. The Atacama Cosmology Telescope: Cosmological Parameters from the 2008 Power Spectra. ArXiv e-prints, Sept. 2010, 1009.0866.

[443] P. K. S. Dunsby, E. Elizalde, R. Goswami, S. Odintsov, and D. Saez-Gomez. $\Lambda$ CDM universe in f(R) gravity. Phys. Rev. D, 82(2):023519, July 2010, 1005.2205.

[444] R. Durrer, M. Kunz, and M. Sakellariadou. Why do we live in $3+1$ dimensions? Phys. Lett., B614:125-130, 2005, hep-th/0501163

[445] S. Dutta and E. N. Saridakis. Observational constraints on Horava-Lifshitz cosmology. JCAP, 1001:013, 2010, 0911.1435.

[446] S. Dutta and E. N. Saridakis. Overall observational constraints on the running parameter $\lambda$ of Horava-Lifshitz gravity. JCAP, 1005:013, 2010, 1002.3373.

[447] G. Dvali. Predictive Power of Strong Coupling in Theories with Large Distance Modified Gravity. New J. Phys., 8:326, 2006, hep-th/0610013

[448] G. Dvali and G. Gabadadze. Gravity on a brane in infinite volume extra space. Phys.Rev., D63:065007, 2001, hep-th/0008054

[449] G. Dvali, G. Gabadadze, X.-r. Hou, and E. Sefusatti. See-saw modification of gravity. Phys. Rev., D67:044019, 2003, hep-th/0111266

[450] G. Dvali, G. Gabadadze, and M. Shifman. Diluting cosmological constant in infinite volume extra dimensions. Phys.Rev., D67:044020, 2003, hep-th/0202174

[451] G. Dvali, A. Gruzinov, and M. Zaldarriaga. The Accelerated universe and the moon. Phys.Rev., D68:024012, 2003, hep-ph/0212069

[452] G. Dvali, S. Hofmann, and J. Khoury. Degravitation of the cosmological constant and graviton width. Phys.Rev., D76:084006, 2007, hep-th/0703027

[453] G. Dvali and M. S. Turner. Dark energy as a modification of the Friedmann equation. 2003, astro-ph/0301510

[454] G. R. Dvali, G. Gabadadze, and M. Porrati. 4D gravity on a brane in 5D Minkowski space. Phys. Lett., B485:208-214, 2000, hep-th/0005016

[455] D. A. Easson. Modified Gravitational Theories and Cosmic Acceleration. International Journal of Modern Physics A, 19:5343-5350, 2004, arXiv:astro-ph/0411209

[456] R. Easther, B. R. Greene, M. G. Jackson, and D. N. Kabat. Brane gas cosmology in M-theory: Late time behavior. Phys. Rev., D67:123501, 2003, hep-th/0211124

[457] R. Easther, B. R. Greene, M. G. Jackson, and D. N. Kabat. Brane gases in the early universe: Thermodynamics and cosmology. JCAP, 0401:006, 2004, hep-th/0307233 
[458] R. Easther, B. R. Greene, M. G. Jackson, and D. N. Kabat. String windings in the early universe. JCAP, 0502:009, 2005, hep-th/0409121

[459] D. H. Eckhardt, C. Jekeli, A. R. Lazarewicz, A. J. Romaides, and R. W. Sands. Tower gravity experiment - Evidence for non-Newtonian gravity. Physical Review Letters, 60:2567-2570, June 1988.

[460] A. S. Eddington. The Mathematical Theory of Relativity. 1923.

[461] M. Eingorn, A. Zhuk, Kaluza-Klein models: can we construct a viable example? Phys. Rev. D83 (2011) 044005. arXiv:1010.5740 [gr-qc]].

[462] M. Eingorn, O. R. de Medeiros, L. C. B. Crispino, A. Zhuk, Latent solitons, black strings, black branes, and equations of state in Kaluza-Klein models Phys. Rev. D84 (2011) 024031. arXiv:1101.3910 [gr-qc]].

[463] G. Efstathiou, W. J. Sutherland, and S. J. Maddox. The cosmological constant and cold dark matter. Nature, 348:705-707, Dec. 1990.

[464] A. Einstein. On The influence of gravitation on the propagation of light. Annalen Phys., 35:898908, 1911.

[465] A. Einstein. The foundation of the general theory of relativity. Annalen Phys., 49:769-822, 1916.

[466] A. Einstein and W. Pauli. On the non-existence of regular stationary solutions of relativistic field equations. The Annals of Mathematics, 44(2):pp. 131-137, 1943.

[467] D. J. Eisenstein et al. Detection of the baryon acoustic peak in the large-scale correlation function of SDSS luminous red galaxies. Astrophys.J., 633:560-574, 2005, astro-ph/0501171

[468] E. Elizalde, S. Nojiri, S. D. Odintsov, L. Sebastiani, and S. Zerbini. Nonsingular exponential gravity: A simple theory for early- and late-time accelerated expansion. Phys. Rev. D, 83(8):086006, $2011,1012.2280$.

[469] G. F. R. Ellis and M. Bruni. Covariant and gauge-invariant approach to cosmological density fluctuations. Phys. Rev. D, 40:1804-1818, Sept. 1989.

[470] R. Emparan, A. Fabbri, and N. Kaloper. Quantum black holes as holograms in AdS braneworlds. JHEP, 08:043, 2002, hep-th/0206155

[471] R. Emparan, J. Garcia-Bellido, and N. Kaloper. Black hole astrophysics in AdS braneworlds. JHEP, 01:079, 2003, hep-th/0212132

[472] R. V. Eotvos, D. Pekar, and E. Fekete. Contributions to the law of propertionality of inertia and gravity. Annalen Phys., 68:11-66, 1922.

[473] A. L. Erickcek, T. L. Smith, and M. Kamionkowski. Solar system tests do rule out 1/R gravity. Phys. Rev. D, 74(12):121501, Dec. 2006, arXiv:astro-ph/0610483

[474] J. Etherington. Phil. Mag., 15:761, 1933.

[475] J. D. Evans, L. M. H. Hall, and P. Caillol. Standard cosmological evolution in a wide range of f(R) models. Phys. Rev. D, 77(8):083514, Apr. 2008, 0711.3695.

[476] C. W. F. Everitt et al. Gravity Probe B: Final Results of a Space Experiment to Test General Relativity. Phys. Rev. Lett., 106:221101, 2011, 1105.3456.

[477] Q. Exirifard and M. M. Sheikh-Jabbari. Lovelock gravity at the crossroads of Palatini and metric formulations. Physics Letters B, 661:158-161, Mar. 2008, 0705.1879.

[478] M. Fairbairn and A. Goobar. Supernova limits on brane world cosmology. Phys.Lett., B642:432435, 2006, astro-ph/0511029

[479] M. Fairbairn and S. Rydbeck. Expansion history and $\mathrm{f}(\mathrm{R})$ modified gravity. J. Cosmology Astropart. Phys., 12:5, Dec. 2007, arXiv:astro-ph/0701900

[480] D. Fairlie and J. Govaerts. Euler hierarchies and universal equations. J.Math.Phys., 33:35433566, 1992, hep-th/9204074. Revised version.

[481] D. Fairlie, J. Govaerts, and A. Morozov. Universal field equations with covariant solutions. Nucl.Phys., B373:214-232, 1992, hep-th/9110022

[482] W. Fang et al. Challenges to the DGP Model from Horizon-Scale Growth and Geometry. Phys. Rev., D78:103509, 2008, 0808.2208.

[483] H. Farajollahi, M. Farhoudi, A. Salehi, and H. Shojaie. Chameleonic Generalized Brans-Dicke model and late-time acceleration. ArXiv e-prints, Sep. 2010, 1009.5059.

[484] H. Farajollahi and A. Salehi. Cosmic Dynamics in Chameleon Cosmology. International Journal of Modern Physics D, 19:621-633, 2010, 1004.3508.

[485] V. Faraoni. Matter instability in modified gravity. Phys. Rev. D, 74(10):104017, Nov. 2006, arXiv:astro-ph/0610734

[486] V. Faraoni. de Sitter space and the equivalence between $f(R)$ and scalar-tensor gravity. Phys. Rev. D, 75(6):067302, Mar. 2007, arXiv:gr-qc/0703044.

[487] V. Faraoni. Extension of the EGS theorem to metric and Palatini f(R) gravity. ArXiv e-prints, 
Nov. 2008, 0811.1870.

[488] V. Faraoni. Clifton's spherical solution in $\mathrm{f}(\mathrm{R})$ vacuum harbours a naked singularity. Classical and Quantum Gravity, 26(19):195013, Oct. 2009, 0909.0514.

[489] V. Faraoni. Black hole entropy in scalar-tensor and $\mathrm{f}(\mathrm{R})$ gravity: an overview. ArXiv e-prints, May 2010, 1005.2327.

[490] V. Faraoni. Jebsen-Birkhoff theorem in alternative gravity. Phys. Rev. D, 81(4):044002, Feb. 2010, 1001.2287.

[491] M. Farhoudi. Classical Trace Anomaly. Int. J. Mod. Phys. D, 14:1233-1250, 2005.

[492] M. Farhoudi. On higher order gravities, their analogy to GR, and dimensional dependent version of Duff's trace anomaly relation. Gen. Rel. Grav., 38:1261-1284, Aug 2006.

[493] M. Farhoudi. Lovelock tensor as generalized Einstein tensor. Gen. Rel. Grav., 41:117-129, Jan 2009.

[494] M. Farhoudi. On Third Order Lagrangians, Weyl Invariants \& Classical Trace Anomaly in Six Dimensions. ArXiv e-prints, Jul. 2011, 1107.1034.

[495] T. Faulkner, M. Tegmark, E. F. Bunn, and Y. Mao. Constraining f(R) gravity as a scalar-tensor theory. Phys. Rev. D, 76(6):063505, Sep 2007.

[496] S. Fay, S. Nesseris, and L. Perivolaropoulos. Can $\mathrm{f}(\mathrm{R})$ modified gravity theories mimic a $\Lambda \mathrm{CDM}$ cosmology? Phys. Rev. D, 76(6):063504, Sept. 2007, arXiv:gr-qc/0703006

[497] S. Fay, R. Tavakol, and S. Tsujikawa. f(R) gravity theories in Palatini formalism: Cosmological dynamics and observational constraints. Phys. Rev. D, 75(6):063509, Mar. 2007, arXiv:astro$\mathrm{ph} / 0701479$

[498] M. Feix, C. Fedeli, and M. Bartelmann. Asymmetric Gravitational Lenses in TeVeS and Application to the Bullet Cluster. Astron. Astrophys., 480:313, 2008, 0707.0790.

[499] B. Feldman and A. E. Nelson. New regions for a chameleon to hide. Journal of High Energy Physics, 8:2, Aug. 2006, arXiv:hep-ph/0603057

[500] S. Ferraro, F. Schmidt, and W. Hu. Cluster Abundance in f(R) Gravity Models. ArXiv e-prints, Nov. 2010, 1011.0992.

[501] P. G. Ferreira and C. Skordis. The linear growth rate of structure in Parametrized Post Friedmannian Universes. Phys.Rev., D81:104020, 2010, arXiv:1003.4231

[502] P. G. Ferreira, C. Skordis, and C. Zunckel. Dark Matter, Modified Gravity and the Mass of the Neutrino. Phys. Rev., D78:044043, 2008, 0806.0116.

[503] B. Fiedler and R. Schimming. Singularity-free static centrally symmetric solutions of some fourth order gravitational field equations. Astronomische Nachrichten, 304:221-229, 1983.

[504] M. Fierz and W. Pauli. On relativistic wave equations for particles of arbitrary spin in an electromagnetic field. Proc.Roy.Soc.Lond., A173:211-232, 1939.

[505] P. Figueras, J. Lucietti, and T. Wiseman. Ricci solitons, Ricci flow, and strongly coupled CFT in the Schwarzschild Unruh or Boulware vacua. 2011, 1104.4489.

[506] P. Figueras and T. Wiseman. Gravity and large black holes in Randall-Sundrum II braneworlds. $2011,1105.2558$

[507] E. Fischbach and C. Talmadge. Six years of the fifth force. Nature, 356:207-215, Mar. 1992.

[508] A. L. Fitzpatrick, L. Randall, and T. Wiseman. On the existence and dynamics of braneworld black holes. JHEP, 11:033, 2006, hep-th/0608208

[509] E. E. Flanagan. Palatini Form of 1/R Gravity. Phys. Rev. Lett., 92(7):071101, Feb 2004

[510] J. Forero-Romero, S. Gottloeber, and G. Yepes. Bullet Clusters in the MareNostrum Universe. Astrophys.J., 725:598-604, 2010, 1007.3902.

[511] E. Fradkin and M. A. Vasiliev. On the Gravitational Interaction of Massless Higher Spin Fields. Phys.Lett., B189:89-95, 1987.

[512] K. Freese and M. Lewis. Cardassian Expansion: a Model in which the Universe is Flat, Matter Dominated, and Accelerating. Phys. Lett., B540:1-8, 2002, astro-ph/0201229

[513] L. Freidel. Modified gravity without new degrees of freedom. 2008, 0812.3200

[514] P. G. O. Freund. Kaluza-Klein Cosmologies. Nucl. Phys., B209:146, 1982.

[515] A. V. Frolov. Singularity Problem with $\mathrm{f}(\mathrm{R})$ Models for Dark Energy. Physical Review Letters, 101(6):061103, Aug. 2008, 0803.2500.

[516] S. Fulling and L. Parker. Renormalization in the theory of a quantized scalar field interacting with a robertson-walker spacetime. Annals Phys., 87:176-204, 1974.

[517] G. Gabadadze. General Relativity With An Auxiliary Dimension. Phys.Lett., B681:89-95, 2009, 0908.1112 .

[518] G. Gabadadze and M. Shifman. Softly massive gravity. Phys.Rev., D69:124032, 2004, hepth/0312289 
[519] R. Gannouji, B. Moraes, and D. Polarski. The growth of matter perturbations in $\mathrm{f}(\mathrm{R})$ models. J. Cosmology Astropart. Phys., 2:34, Feb. 2009, 0809.3374.

[520] R. Gannouji and M. Sami. Galileon gravity and its relevance to late time cosmic acceleration. Phys.Rev., D82:024011, 2010, 1004.2808.

[521] X. Gao, Y. Wang, R. Brandenberger, and A. Riotto. Cosmological Perturbations in HořavaLifshitz Gravity. Phys. Rev., D81:083508, 2010, 0905.3821.

[522] X. Gao, Y. Wang, W. Xue, and R. Brandenberger. Fluctuations in a Hořava-Lifshitz Bouncing Cosmology. JCAP, 1002:020, 2010, 0911.3196.

[523] J. García-Bellido. Jordan-Brans-Dicke stochastic inflation. Nuclear Physics B, 423:221-242, July 1994, arXiv:astro-ph/9401042

[524] J. García-Bellido and A. Linde. Stationary solutions in Brans-Dicke stochastic inflationary cosmology. Phys. Rev. D, 52:6730-6738, Dec. 1995, arXiv:gr-qc/9504022

[525] J. García-Bellido, A. Linde, and D. Linde. Fluctuations of the gravitational constant in the inflationary Brans-Dicke cosmology. Phys. Rev. D, 50:730-750, July 1994, arXiv:astro-ph/9312039

[526] J. García-Bellido and M. Quirós. Extended inflation in scalar-tensor theories of gravity. Physics Letters B, 243:45-51, June 1990.

[527] J. García-Bellido and D. Wands. Constraints from inflation on scalar-tensor gravity theories. Phys. Rev. D, 52:6739-6749, Dec. 1995, arXiv:gr-qc/9506050

[528] J. Garriga and T. Tanaka. Gravity in the brane-world. Phys. Rev. Lett., 84:2778-2781, 2000, hep-th/9911055

[529] M. Gasperini, M. Maggiore, and G. Veneziano. Towards a non-singular pre-big-bang cosmology. Nuclear Physics B, 494:315-328, Feb. 1997, arXiv:hep-th/9611039

[530] M. Gasperini and G. Veneziano. Pre-big-bang in string cosmology. Astroparticle Physics, 1:317339, July 1993, arXiv:hep-th/9211021

[531] C. Gauss. Disquisitiones generales circa superficies curvas. Typis Dieterichianis, 1828.

[532] C. Germani, A. Kehagias, and K. Sfetsos. Relativistic Quantum Gravity at a Lifshitz Point. JHEP, 0909:060, 2009, 0906.1201.

[533] B. Geyer, S. D. Odintsov, and S. Zerbini. Inflationary Brans-Dicke quantum universe. Physics Letters B, 460:58-62, Aug. 1999, arXiv:gr-qc/9905073

[534] T. Giannantonio, R. Scranton, R. G. Crittenden, R. C. Nichol, S. P. Boughn, A. D. Myers, and G. T. Richards. Combined analysis of the integrated Sachs-Wolfe effect and cosmological implications. Phys. Rev. D, 77(12):123520, June 2008, 0801.4380.

[535] D. Giannios. Spherically symmetric, static spacetimes in TeVeS. Phys. Rev., D71:103511, 2005, gr-qc/0502122

[536] G. W. Gibbons and S. W. Hawking. Action Integrals and Partition Functions in Quantum Gravity. Phys. Rev., D15:2752-2756, 1977.

[537] H. Gies, D. F. Mota, and D. J. Shaw. Hidden in the light: Magnetically induced afterglow from trapped chameleon fields. Phys. Rev. D, 77(2):025016, Jan. 2008, 0710.1556.

[538] N. Goheer, R. Goswami, and P. K. S. Dunsby. Dynamics of f(R)-cosmologies containing Einstein static models. Classical and Quantum Gravity, 26(10):105003, May 2009, 0809.5247.

[539] N. Goheer, J. Larena, and P. K. S. Dunsby. Power-law cosmic expansion in f(R) gravity models. Phys. Rev. D, 80(6):061301, Sept. 2009, 0906.3860.

[540] N. Goheer, J. A. Leach, and P. K. S. Dunsby. Dynamical systems analysis of anisotropic cosmologies in $R^{n}$-gravity. Classical and Quantum Gravity, 24:5689-5708, Nov. 2007, 0710.0814.

[541] W. D. Goldberger and M. B. Wise. Bulk fields in the Randall-Sundrum compactification scenario. Phys. Rev., D60:107505, 1999, hep-ph/9907218

[542] W. D. Goldberger and M. B. Wise. Modulus stabilization with bulk fields. Phys. Rev. Lett., 83:4922-4925, 1999, hep-ph/9907447

[543] Y. Gong and A. Wang. Friedmann Equations and Thermodynamics of Apparent Horizons. Physical Review Letters, 99(21):211301, Nov. 2007, 0704.0793.

[544] G. Goon, K. Hinterbichler, and M. Trodden. General Embedded Brane Effective Field Theories. 2011, 1103.6029

[545] G. Goon, K. Hinterbichler, and M. Trodden. Symmetries for Galileons and DBI scalars on curved space. 2011, 1103.5745

[546] D. Gorbunov, K. Koyama, and S. Sibiryakov. More on ghosts in DGP model. Phys. Rev. D73:044016, 2006, hep-th/0512097

[547] D. S. Gorbunov, V. A. Rubakov, and S. M. Sibiryakov. Gravity waves from inflating brane or mirrors moving in adS(5). JHEP, 10:015, 2001, hep-th/0108017

[548] C. Gordon and R. Maartens. Density perturbations in the brane world. Phys. Rev., D63:044022, 


\section{1, hep-th/0009010}

[549] R. Goswami, N. Goheer, and P. K. S. Dunsby. Existence of Einstein static universes and their stability in fourth-order theories of gravity. Phys. Rev. D, 78(4):044011, Aug. 2008, 0804.3528.

[550] S. Gottlober, H. Schmidt, and A. A. Starobinsky. Sixth-order gravity and conformal transformations. Classical and Quantum Gravity, 7:893-900, May 1990.

[551] S. Gottloeber, V. Mueller, and H. Schmidt. Generalized inflation from R-cubed and R square R terms. Astronomische Nachrichten, 312:291-297, July 1991.

[552] M. Grana. Flux compactifications in string theory: A comprehensive review. Phys. Rept., 423:91158, 2006, hep-th/0509003

[553] E. Gravanis and S. Willison. Israel conditions for the Gauss-Bonnet theory and the Friedmann equation on the brane universe. Phys.Lett., B562:118-126, 2003, hep-th/0209076.

[554] A. M. Green and A. R. Liddle. Conditions for successful extended inflation. Phys. Rev. D, 54:2557-2563, Aug. 1996, arXiv:astro-ph/9604001

[555] B. R. Greene and J. Levin. Dark Energy and Stabilization of Extra Dimensions. JHEP, 11:096, 2007, 0707.1062.

[556] J. P. Gregory and A. Padilla. Exact braneworld cosmology induced from bulk black holes. Class. Quant. Grav., 19:4071-4083, 2002, hep-th/0204218

[557] J. P. Gregory and A. Padilla. Brane world holography in Gauss-Bonnet gravity. Class.Quant.Grav., 20:4221-4238, 2003, hep-th/0304250

[558] R. Gregory. Braneworld black holes. Lect. Notes Phys., 769:259-298, 2009, 0804.2595.

[559] R. Gregory, N. Kaloper, R. C. Myers, and A. Padilla. A New Perspective on DGP Gravity. JHEP, 10:069, 2007, 0707.2666.

[560] R. Gregory and A. Padilla. Braneworld instantons. Class. Quant. Grav., 19:279-302, 2002, hep-th/0107108

[561] R. Gregory and A. Padilla. Nested braneworlds and strong brane gravity. Phys. Rev., D65:084013, 2002, hep-th/0104262

[562] R. Gregory, S. F. Ross, and R. Zegers. Classical and quantum gravity of brane black holes. JHEP, 09:029, 2008, 0802.2037.

[563] R. Gregory, V. A. Rubakov, and S. M. Sibiryakov. Opening up extra dimensions at ultra-large scales. Phys. Rev. Lett., 84:5928-5931, 2000, hep-th/0002072

[564] L. Grisa and O. Pujolas. Dressed Domain Walls and Holography. JHEP, 06:059, 2008, 0712.2786.

[565] S. Groot Nibbelink and M. Pospelov. Lorentz violation in supersymmetric field theories. Phys.Rev.Lett., 94:081601, 2005, hep-ph/0404271

[566] D. J. Gross and J. H. Sloan. The Quartic Effective Action for the Heterotic String. Nucl. Phys., B291:41, 1987.

[567] A. Gruzinov. On the graviton mass. New Astron., 10:311-314, 2005, astro-ph/0112246

[568] A. Guarnizo, L. Castaeda, and J. M. Tejeiro. Boundary term in metric $f(R)$ gravity: field equations in the metric formalism. Gen. Rel. Grav. 42:2713, 2010, 1002.0617.

[569] A. Guarnizo, L. Castaeda, and J. M. Tejeiro. Geodesic deviation equation in $\mathrm{f}(\mathrm{R})$ gravity. Gen. Rel. Grav. 43:2713, 2011, 1010.5279.

[570] S. S. Gubser. AdS/CFT and gravity. Phys. Rev., D63:084017, 2001, hep-th/9912001

[571] U. Gunther, and A. Zhuk, A Note on dynamical stabilization of internal spaces in multidimensional cosmology. Class. Quant. Grav., 18:1441-1460, 2001, hep-ph/0006283

[572] U. Gunther, A. Starobinsky, and A. Zhuk. Multidimensional cosmological models: Cosmological and astrophysical implications. Phys. Rev. D, 69:044003, 2004, hep-ph/0306191.

[573] Z.-K. Guo, N. Ohta, and T. Torii. Black Holes in the Dilatonic Einstein-Gauss-Bonnet Theory in Various Dimensions. I - Asymptotically Flat Black Holes. Prog. Theo. Phys., 120:581-607, Sep. 2008, 0806.2481.

[574] Z.-K. Guo, N. Ohta, and T. Torii. Black Holes in the Dilatonic Einstein-Gauss-Bonnet Theory in Various Dimensions. II - Asymptotically AdS Topological Black Holes. Prog. Theo. Phys., 121:253-273, Feb. 2009, 0811.3068.

[575] Z.-K. Guo, N. Ohta, and S. Tsujikawa. Realizing scale-invariant density perturbations in lowenergy effective string theory. Phys. Rev. D, 75(2):023520, Jan. 2007, hep-th/0610336

[576] L. E. Gurevich, A. M. Finkelstein, and V. A. Ruban. On the Problem of the Initial State in the Isotropic Scalar-Tensor Cosmology of Brans-Dicke. Ap\&SSS, 22:231-242, June 1973.

[577] L. Gurvits, K. Kellermann, and S. Frey. The "angular size - redshift" relation for compact radio structures in quasars and radio galaxies. 1998, astro-ph/9812018.

[578] A. H. Guth and B. Jain. Density fluctuations in extended inflation. Phys. Rev. D, 45:426-432, Jan. 1992. 
[579] E. Guzman. Exact Brans-Dicke-Bianchi type-VII(h) perfect fluid solutions. ApESSS, 152:171-173, Feb. 1989.

[580] E. Guzmán. General Vacuum Solution for Brans-Dicke-Bianchi Type-II. Ap\&SS, 179:331-334, May 1991.

[581] E. Guzmán. General vacuum solution for Brans-Dicke-Bianchi type V. General Relativity and Gravitation, 23:1007-1010, Sept. 1991.

[582] E. Guzman. Cosmic no-hair theorem in Brans-Dicke cosmology. Physics Letters B, 391:267-270, Feb. 1997.

[583] E. Guzmán. Homogeneous Universes in Extended Inflation I. Ap\&SSS, 249:179-188, Apr. 1997.

[584] E. Guzmán. Homogeneous Universes in Extended Inflation II. ApESSS, 249:211-221, Apr. 1997.

[585] L. Guzzo et al. A test of the nature of cosmic acceleration using galaxy redshift distortions. Nature, 451:541, 2008.

[586] N. Haba and S. Matsumoto. Baryogenesis from Dark Sector. 2010, 1008.2487.

[587] L. J. Hall, J. March-Russell, and S. M. West. A Unified Theory of Matter Genesis: Asymmetric Freeze- In. 2010, 1010.0245.

[588] L. J. Hall and D. Tucker-Smith. Cosmological constraints on theories with large extra dimensions. Phys. Rev., D60:085008, 1999, hep-ph/9904267

[589] A. Halle, H. Zhao, and B. Li. Perturbations In A Non-Uniform Dark Energy Fluid: Equations Reveal Effects of Modified Gravity and Dark Matter. ArXiv e-prints, Nov. 2007, 0711.0958.

[590] N. A. Hamed, H. S. Cheng, M. A. Luty, and S. Mukohyama. Ghost Condensation and a Consistent IR Modification of Gravity. Journal of High Energy Physics, 5:74, May 2004, arXiv:hepth/0312099

[591] F. Hansen, A. Banday, K. Gorski, H. Eriksen, and P. Lilje. Power Asymmetry in Cosmic Microwave Background Fluctuations from Full Sky to Sub-degree Scales: Is the Universe Isotropic? Astrophys.J., 704:1448-1458, 2009, 0812.3795.

[592] J. G. Hao and R. Akhoury. Can relativistic MOND theory resolve both the dark matter and dark energy paradigms? 2005, astro-ph/0504130

[593] T. Harada, C. Goymer, and B. J. Carr. Tolman-bondi collapse in scalar-tensor theories as a probe of gravitational memory. Phys. Rev. D, 66(10):104023, Nov 2002.

[594] M. Haugan. Energy conservation and the principle of equivalence. Annals Phys., 118:156-186, 1979.

[595] S. W. Hawking. Black holes in the Brans-Dicke theory of gravitation. Comm. Math. Phys., 25:167-171, 1972 .

[596] S. W. Hawking, T. Hertog, and H. S. Reall. Brane new world. Phys. Rev., D62:043501, 2000, hep-th/0003052

[597] S. W. Hawking, T. Hertog, and H. S. Reall. Trace anomaly driven inflation. Phys. Rev., D63:083504, 2001, hep-th/0010232

[598] E. Hawkins, S. Maddox, S. Cole, D. Madgwick, P. Norberg, et al. The 2dF Galaxy Redshift Survey: Correlation functions, peculiar velocities and the matter density of the universe. Mon.Not.Roy.Astron.Soc., 346:78, 2003, astro-ph/0212375

[599] F. W. Hehl. How does one measure torsion of spacetime? Phys. Lett., A36: 225, 1971.

[600] F. W. Hehl and G. D. Kerlick. Metric-affine variational principles in general relativity. I - Riemannian space-time. General Relativity and Gravitation, 9:691-710, Aug. 1978.

[601] F. W. Hehl, P. Von Der Heyde, G. D. Kerlick and J. M. Nester. General Relativity with Spin and Torsion: Foundations and Prospects. Rev. Mod. Phys., 48, 393, 1976.

[602] F. W. Hehl and P. Von Der Heyde. Spin and the structure of space-time Annales Poincare Phys. Theor., 19: 179, 1973.

[603] M. Henneaux, A. Kleinschmidt, and G. L. Gomez. A dynamical inconsistency of Horava gravity. Phys.Rev., D81:064002, 2010, 0912.0399.

[604] A. Hewish, S. J. Bell, J. D. H. Pilkington, P. F. Scott, and R. A. Collins. Observation of a rapidly pulsating radio source. Nature, 217:709-713, 1968.

[605] A. Higuchi. Forbidden mass range for spin-2 field theory in de Sitter space-time. Nucl. Phys., B282:397, 1987.

[606] A. Hindawi, B. A. Ovrut, and D. Waldram. Consistent spin-two coupling and quadratic gravitation. Phys. Rev. D, 53:5583-5596, May 1996, arXiv:hep-th/9509142

[607] A. Hindawi, B. A. Ovrut, and D. Waldram. Nontrivial vacua in higher-derivative gravitation. Phys. Rev. D, 53:5597-5608, May 1996, arXiv:hep-th/9509147

[608] K. Hinterbichler and J. Khoury. Symmetron Fields: Screening Long-Range Forces Through Local Symmetry Restoration. Phys. Rev. Lett., 104:231301, 2010, 1001.4525. 
[609] K. Hinterbichler, A. Nicolis, and M. Porrati. Superluminality in DGP. JHEP, 0909:089, 2009, 0905.2359.

[610] K. Hinterbichler, M. Trodden, and D. Wesley. Multi-field galileons and higher co-dimension branes. Phys.Rev., D82:124018, 2010, 1008.1305.

[611] S. Ho, C. Hirata, N. Padmanabhan, U. Seljak, and N. Bahcall. Correlation of CMB with large-scale structure. I. Integrated Sachs-Wolfe tomography and cosmological implications. Phys. Rev. D, 78(4):043519, Aug. 2008, 0801.0642.

[612] D. W. Hogg. Distance measures in cosmology. ArXiv Astrophysics e-prints, May 1999 arXiv:astro-ph/9905116

613] D. J. Holden and D. Wands. Phase-plane analysis of friedmann-robertson-walker cosmologies in brans-dicke gravity. Classical and Quantum Gravity, 15(10):3271, 1998.

[614] R. Holman, E. W. Kolb, S. L. Vadas, and Y. Wang. False-vacuum decay in generalized extended inflation. Physics Letters B, 250:24-28, Nov. 1990.

[615] R. Holman, E. W. Kolb, S. L. Vadas, and Y. Wang. Extended inflation from higher dimensional theories. Phys. Rev., D43:995-1004, 1991.

[616] R. Holman, E. W. Kolb, S. L. Vadas, and Y. Wang. Scale-invariant extended inflation. Phys. Rev. D, 43:3833-3845, June 1991.

[617] R. Holman, Y. Wang, and E. W. Kolb. Gravitational couplings of the inflaton in extended inflation. Physical Review Letters, 65:17-20, July 1990

[618] P. Horava. Membranes at Quantum Criticality. JHEP, 0903:020, 2009, 0812.4287.

[619] P. Horava. Quantum Gravity at a Lifshitz Point. Phys.Rev., D79:084008, 2009, 0901.3775.

[620] P. Horava. Spectral Dimension of the Universe in Quantum Gravity at a Lifshitz Point. Phys.Rev.Lett., 102:161301, 2009, 0902.3657.

[621] P. Horava and C. M. Melby-Thompson. General Covariance in Quantum Gravity at a Lifshitz Point. Phys.Rev., D82:064027, 2010, 1007.2410.

[622] P. Horava and E. Witten. Eleven-Dimensional Supergravity on a Manifold with Boundary. Nucl. Phys., B475:94-114, 1996, hep-th/9603142

[623] G. Horndeski. Second-order scalar-tensor field equations in a four-dimensional space. International Journal of Theoretical Physics, 10(6):363-384, 1974.

[624] C. D. Hoyle, U. Schmidt, B. R. Heckel, E. G. Adelberger, J. H. Gundlach, D. J. Kapner, and H. E. Swanson. Submillimeter Test of the Gravitational Inverse-Square Law: A Search for "Large" Extra Dimensions. Physical Review Letters, 86:1418-1421, Feb. 2001, arXiv:hep-ph/0011014

[625] S. D. H. Hsu. Black holes from extended inflation. Physics Letters B, 251:343-348, Nov. 1990.

[626] W. Hu. Structure Formation with Generalized Dark Matter. ApJ, 506:485-494, Oct. 1998, arXiv:astro-ph/9801234

[627] W. Hu. Parametrized Post-Friedmann Signatures of Acceleration in the CMB. Phys. Rev., D77:103524, 2008, 0801.2433.

[628] W. Hu, R. Barkana, and A. Gruzinov. Cold and fuzzy dark matter. Phys. Rev. Lett., 85:11581161, 2000, astro-ph/0003365

[629] W. Hu and I. Sawicki. Models of $\mathrm{f}(\mathrm{R})$ cosmic acceleration that evade solar system tests. Phys. Rev. D, 76(6):064004, Sep 2007.

[630] W. Hu and I. Sawicki. Parametrized post-Friedmann framework for modified gravity. Phys. Rev. D, 76(10):104043, Nov. 2007, 0708.1190.

[631] Y. Huang and A. Wang. Nonrelativistic general covariant theory of gravity with a running constant $\lambda .2010,1011.0739$.

[632] Y. Huang, A. Wang, and Q. Wu. Stability of the de Sitter spacetime in Horava-Lifshitz theory Mod.Phys.Lett., A25:2267-2279, 2010, 1003.2003.

[633] V. W. Hughes, H. G. Robinson, and V. Beltran-Lopez. Upper Limit for the Anisotropy of Inertial Mass from Nuclear Resonance Experiments. Physical Review Letters, 4:342-344, Apr. 1960.

[634] R. A. Hulse and J. H. Taylor. Discovery of a pulsar in a binary system. ApJ, 195:L51-L53, Jan. 1975.

[635] V. Husain, E. A. Martinez, and D. Nunez. Exact solution for scalar field collapse. Phys. Rev., D50:3783-3786, 1994, gr-qc/9402021

[636] J. Hwang and H. Noh. f(R) gravity theory and CMBR constraints. Physics Letters B, 506:13-19, May 2001, arXiv:astro-ph/0102423

[637] J.-c. Hwang and H. Noh. Gauge-ready formulation of the cosmological kinetic theory in generalized gravity theories. Phys. Rev. D, 65(2):023512, Dec 2001

[638] K. Ichiki, M. Yahiro, T. Kajino, M. Orito, and G. J. Mathews. Observational constraints on dark radiation in brane cosmology. Phys. Rev., D66:043521, 2002, astro-ph/0203272 
[639] D. Ida. Brane-world cosmology. JHEP, 09:014, 2000, gr-qc/9912002

[640] A. Iglesias, N. Kaloper, A. Padilla, and M. Park. How (not) to use the palatini formulation of scalar-tensor gravity. Phys. Rev. D, 76(10):104001, Nov 2007.

[641] L. Iorio. On the reliability of the so-far performed tests for measuring the Lense Thirring effect with the LAGEOS satellites. New Astronomy, 10:603-615, Aug. 2005, arXiv:gr-qc/0411024

[642] L. Iorio, H. I. M. Lichtenegger, M. L. Ruggiero, and C. Corda. Phenomenology of the LenseThirring effect in the solar system. Astrophys. Sp. Sci., 331:351-395, Feb. 2011.

[643] L. Iorio, and M. L. Ruggiero. Phenomenological Constraints on the Kehagias-Sfetsos Solution in the HORAVA-LIFSHITZ Gravity from Solar System Orbital Motions. Int. J. Mod. Phys. A, 25:5399-5408, 2010, 0909.2562.

[644] L. Iorio, and M. L. Ruggiero. Constraining the Kehagias-Sfetsos Solution of the Horava-Lifshitz Modified Gravity with Extrasolar Planets. Open. Ast. J., 3:167-171, Dec. 2010, 0909.5355.

[645] L. Iorio, and M. L. Ruggiero. HORAVA-LIFSHITZ Gravity:. Tighter Constraints for the Kehagias-Sfetsos Solution from New Solar System Data. Int J. Mod. Phys. D, 20:1079-1093, $2011,1012.2822$.

[646] M. Ishak and J. Moldenhauer. A minimal set of invariants as a systematic approach to higher order gravity models. JCAP, 1:24, Jan. 2009, 0808.0951.

[647] C. J. Isham, A. Salam, and J. Strathdee. Relativity-f gravity and gravitational collapse. Nature, 244:82, Aug. 1973.

[648] A. Ishibashi and S. Speziale. Spherically symmetric black holes in minimally modified self-dual gravity. Class.Quant.Grav., 26:175005, 2009, 0904.3914.

[649] W. Israel. Singular hypersurfaces and thin shells in general relativity. Nuovo Cim., B44S10:1, 1966 .

[650] W. Israel. Event horizons in static vacuum space-times. Phys. Rev., 164(5):1776-1779, Dec 1967.

[651] W. Israel. Event horizons in static electrovac space-times. Communications in Mathematical Physics, 8:245-260, Sept. 1968.

[652] K. Izumi and S. Mukohyama. Nonlinear superhorizon perturbations in Horava-Lifshitz gravity. $2011,1105.0246$

[653] K. Izumi and T. Tanaka. No de Sitter invariant vacuum in massive gravity theory with ghost. Prog.Theor.Phys., 121:419-426, 2009, 0709.0199.

[654] T. Jacobson. Primordial black hole evolution in tensor-scalar cosmology. Phys. Rev. Lett., 83(14):2699-2702, Oct 1999

[655] T. Jacobson. Einstein-aether gravity: a status report. PoS, QG-PH:020, 2007, 0801.1547.

[656] T. Jacobson and D. Mattingly. Gravity with a dynamical preferred frame. Phys. Rev., D64:024028, 2001, gr-qc/0007031

[657] H. Jaichan. Cosmological perturbations in a sixth-order gravity. Classical and Quantum Gravity, 8:L133-L136, June 1991

[658] D. Jain, A. Dev, and J. Alcaniz. Brane world cosmologies and statistical properties of gravitational lenses. Phys.Rev., D66:083511, 2002, astro-ph/0206224.

[659] J. T. Jebsen. Über die allgemeinen kugelsymmetrischen Lösungen der Einsteinschen Gravitationsgleichungen im Vakuum. Ark. Mat. Astr. Fys., 15:1, 1921.

[660] X.-h. Jin and X.-z. Li. Oppenheimer-Volkoff Equation in Relativistic MOND Theory. 2006, gr-qc/0605046

[661] S. Kachru, R. Kallosh, A. D. Linde, and S. P. Trivedi. De Sitter vacua in string theory. Phys. Rev., D68:046005, 2003, hep-th/0301240

[662] S. Kachru, M. B. Schulz, and S. Trivedi. Moduli stabilization from fluxes in a simple IIB orientifold. JHEP, 10:007, 2003, hep-th/0201028

[663] E. O. Kahya. A Decisive test to confirm or rule out existence of dark matter using gravitational wave observations. Class. Quant. Grav., 25:184008, 2008, 0801.1984.

[664] E. O. Kahya and R. P. Woodard. A generic test of modified gravity models which emulate dark matter. Phys. Lett., B652:213-216, 2007, 0705.0153.

[665] K. Kainulainen, V. Reijonen, and D. Sunhede. Interior spacetimes of stars in Palatini $\mathrm{f}(\mathrm{R})$ gravity. Phys. Rev. D, 76(4):043503, Aug. 2007, arXiv:gr-qc/0611132

[666] R. Kallosh. On Inflation in String Theory. Lect. Notes Phys., 738:119-156, 2008, hep-th/0702059

[667] N. Kaloper. Bent domain walls as braneworlds. Phys. Rev., D60:123506, 1999, hep-th/9905210

[668] N. Kaloper. Brane Induced Gravity: Codimension-2. Mod. Phys. Lett., A23:781-796, 2008, 0711.3210 .

[669] N. Kaloper and D. Kiley. Charting the Landscape of Modified Gravity. JHEP, 05:045, 2007, hep-th/0703190 
[670] N. Kaloper, L. Kofman, A. D. Linde, and V. Mukhanov. On the new string theory inspired mechanism of generation of cosmological perturbations. JCAP, 0610:006, 2006, hep-th/0608200

[671] N. Kaloper, J. March-Russell, G. D. Starkman, and M. Trodden. Compact hyperbolic extra dimensions: Branes, Kaluza-Klein modes and cosmology. Phys. Rev. Lett., 85:928-931, 2000, hep-ph/0002001

[672] N. Kaloper, A. Padilla, N. Tanahashi, Galileon Hairs of Dyson Spheres, Vainshtein's Coiffure and Hirsute Bubbles, arXiv:1106.4827 [hep-th]].

[673] T. Kaluza. On the Problem of Unity in Physics. Sitzungsber. Preuss. Akad. Wiss. Berlin (Math. Phys. ), 1921:966-972, 1921.

[674] G. Kang. Black hole area in brans-dicke theory. Phys. Rev. D, 54(12):7483-7489, Dec 1996.

[675] S. Kanno and J. Soda. On the higher codimension braneworld. 2004, gr-qc/0410067

[676] S. Kanno and J. Soda. Quasi-thick codimension 2 braneworld. JCAP, 0407:002, 2004, hepth/0404207

[677] S. Kanno and J. Soda. Lorentz violating inflation. Phys. Rev. D, 74(6):063505, Sept. 2006, arXiv:hep-th/0604192

[678] P. Kanti, N. E. Mavromatos, J. Rizos, K. Tamvakis, and E. Winstanley. Dilatonic black holes in higher curvature string gravity. Phys. Rev. D, 54:5049-5058, Oct. 1996, arXiv:hep-th/9511071

[679] D. B. Kaplan. Single explanation for both baryon and dark matter densities. Phys. Rev. Lett., 68(6):741-743, Feb 1992

[680] D. E. Kaplan, M. A. Luty, and K. M. Zurek. Asymmetric dark matter. Phys. Rev. D, 79(11):115016, Jun 2009.

[681] K. Karami and A. Abdolmaleki. Original and entropy-corrected versions of the holographic and new agegraphic $\mathrm{f}(\mathrm{T})$-gravity models. ArXiv e-prints, Sept. 2010, 1009.2459.

[682] A. Karch and L. Randall. Locally localized gravity. JHEP, 05:008, 2001, hep-th/0011156

[683] A. Karch and L. Randall. Relaxing to three dimensions. Phys. Rev. Lett., 95:161601, 2005, hep-th $/ 0506053$

[684] A. Karch and K. Sfetsos. The black hole and FRW geometries of non-relativistic gravity. Phys Lett. B, 678:123-126, Jul. 2009, 0905.0477.

[685] A. Kehagias and E. Kiritsis. Mirage cosmology. JHEP, 9911:022, 1999, hep-th/9910174

[686] G. D. Kerlick. How does one measure torsion of spacetime? Phys. Lett., A36: 225, 1971

[687] J. Khoury, J.-L. Lehners, and B. A. Ovrut. Supersymmetric Galileons. 2011, 1103.0003.

[688] J. Khoury and A. Weltman. Chameleon cosmology. Phys. Rev. D, 69(4):044026, Feb. 2004, arXiv:astro-ph/0309411

[689] J. Khoury and A. Weltman. Chameleon Fields: Awaiting Surprises for Tests of Gravity in Space. Physical Review Letters, 93(17):171104, Oct. 2004, arXiv:astro-ph/0309300

[690] J. Khoury and M. Wyman. N-Body Simulations of DGP and Degravitation Theories. Phys.Rev., D80:064023, 2009, 0903.1292.

[691] T. W. B. Kibble. Lorentz invariance and the gravitational field. J. Math. Phys., 2, 212, 1961.

[692] I. Kimpton and A. Padilla. Lessons from the decoupling limit of Horava gravity. JHEP, 1007:014, $2010,1003.5666$.

[693] R. Kimura and K. Yamamoto. Large Scale Structures in Kinetic Gravity Braiding Model. 2010, 1011.2006.

[694] E. Kiritsis, G. Kofinas, N. Tetradis, T. N. Tomaras, V. Zarikas, Cosmological evolution with brane bulk energy exchange. JHEP 0302 (2003) 035. hep-th/0207060.

[695] E. Kiritsis, Holography and brane-bulk energy exchange. JCAP 0510 (2005) 014. hepth/0504219.

[696] E. Kiritsis, N. Tetradis, T. N. Tomaras, Induced brane gravity: Realizations and limitations. JHEP 0108 (2001) 012. hep-th/0106050.

[697] E. Kiritsis, N. Tetradis, T. N. Tomaras, Induced gravity on RS branes. JHEP 0203 (2002) 019. hep-th/0202037.

[698] E. Kiritsis and G. Kofinas. Horava-Lifshitz Cosmology. Nucl.Phys., B821:467-480, 2009, 0904.1334.

[699] R. Kitano, H. Murayama, and M. Ratz. Unified origin of baryons and dark matter. Physics Letters B, 669(2):145 - 149, 2008.

[700] B. Kleihaus, J. Kunz, and E. Radu. Rotating Black Holes in Dilatonic Einstein-Gauss-Bonnet Theory. ArXiv e-prints, Jan. 2011, 1101.2868.

[701] O. Klein. Quantentheorie und fünfdimensionale Relativitätstheorie. Zeitschrift fur Physik, 37:895-906, Dec. 1926

[702] O. Klein. The Atomicity of Electricity as a Quantum Theory Law. Nature, 118:516, Oct. 1926. 
[703] S. Kobayashi, K. Koyama, and J. Soda. Quantum fluctuations of bulk inflaton in inflationary brane world. Phys. Lett., B501:157-164, 2001, hep-th/0009160

[704] T. Kobayashi. Cosmic expansion and growth histories in Galileon scalar-tensor models of dark energy. Phys.Rev., D81:103533, 2010, 1003.3281.

[705] T. Kobayashi, H. Kudoh, and T. Tanaka. Primordial gravitational waves in inflationary braneworld. Phys. Rev., D68:044025, 2003, gr-qc/0305006

[706] T. Kobayashi and K. Maeda. Relativistic stars in $\mathrm{f}(\mathrm{R})$ gravity, and absence thereof. Phys. Rev. D, 78(6):064019, Sept. 2008, 0807.2503.

[707] T. Kobayashi and K. Maeda. Can higher curvature corrections cure the singularity problem in f(R) gravity? Phys. Rev. D, 79(2):024009, Jan. 2009, 0810.5664.

[708] T. Kobayashi, T. Shiromizu, and C. de Rham. Curvature corrections to the low energy effective theory in 6D regularized braneworlds. Phys.Rev., D77:124012, 2008, 0802.0103.

[709] T. Kobayashi, T. Shiromizu, and N. Deruelle. Low energy effective gravitational equations on a Gauss-Bonnet brane. Phys.Rev., D74:104031, 2006, hep-th/0608166

[710] T. Kobayashi, M. Yamaguchi, J. 'i. Yokoyama, Generalized G-inflation: Inflation with the most general second-order field equations, arXiv:1105.5723 [hep-th]].

[711] T. Kobayashi, H. Tashiro, and D. Suzuki. Evolution of linear cosmological perturbations and its observational implications in Galileon-type modified gravity. Phys.Rev., D81:063513, 2010, 0912.4641.

[712] T. Kobayashi, Y. Urakawa, and M. Yamaguchi. Large scale evolution of the curvature perturbation in Horava-Lifshitz cosmology. JCAP, 0911:015, 2009, 0908.1005.

[713] T. Kobayashi, Y. Urakawa, and M. Yamaguchi. Cosmological perturbations in a healthy extension of Horava gravity. JCAP, 1004:025, 2010, 1002.3101.

[714] T. Kobayashi, M. Yamaguchi, and J. Yokoyama. G-inflation: Inflation driven by the Galileon field. Phys.Rev.Lett., 105:231302, 2010, 1008.0603.

[715] H. Kodama. Behavior of cosmological perturbations in the brane-world model. 2000, hepth/0012132

[716] H. Kodama, A. Ishibashi, and O. Seto. Brane world cosmology: Gauge-invariant formalism for perturbation. Phys. Rev., D62:064022, 2000, hep-th/0004160

[717] H. Kodama and M. Sasaki. Cosmological Perturbation Theory. Prog.Theor.Phys.Suppl., 78:1$166,1984$.

[718] G. Kofinas, R. Maartens, and E. Papantonopoulos. Brane cosmology with curvature corrections. JHEP, 0310:066, 2003, hep-th/0307138

[719] L. A. Kofman, V. F. Mukhanov, and D. I. Pogosian. The evolution of inhomogeneities in inflationary models in the theory of gravitation with higher derivatives. Zhurnal Eksperimental noi $i$ Teoreticheskoi Fiziki, 93:769-783, Sept. 1987.

[720] I. I. Kogan, S. Mouslopoulos, and A. Papazoglou. A new bigravity model with exclusively positive branes. Phys. Lett., B501:140-149, 2001, hep-th/0011141

[721] I. I. Kogan, S. Mouslopoulos, and A. Papazoglou. The $\mathrm{m} \rightarrow 0$ limit for massive graviton in dS(4) and $\operatorname{AdS}(4)$ : How to circumvent the van Dam-Veltman-Zakharov discontinuity. Phys. Lett., B503:173-180, 2001, hep-th/0011138

[722] I. I. Kogan, S. Mouslopoulos, A. Papazoglou, and G. G. Ross. Multi-brane worlds and modification of gravity at large scales. Nucl. Phys., B595:225-249, 2001, hep-th/0006030

[723] I. I. Kogan, S. Mouslopoulos, A. Papazoglou, G. G. Ross, and J. Santiago. A three three-brane universe: New phenomenology for the new millennium? Nucl. Phys., B584:313-328, 2000, hep$\mathrm{ph} / 9912552$

[724] T. Koivisto. Matter power spectrum in $\mathrm{f}(\mathrm{R})$ gravity. Phys. Rev. D, 73(8):083517, Apr. 2006, arXiv:astro-ph/0602031

[725] T. Koivisto. Viable Palatini-f(R) cosmologies with generalized dark matter. Phys. Rev. D, 76(4):043527, Aug. 2007, 0706.0974.

[726] T. Koivisto. New variational principles as alternatives to the Palatini method. Phys. Rev. D, 83(10):101501, 2011, 1103.2743.

[727] T. Koivisto and H. Kurki-Suonio. Cosmological perturbations in the Palatini formulation of modified gravity. Class. Quant. Grav., 23:2355-2369, 2006, astro-ph/0509422

[728] T. Koivisto and D. F. Mota. Cosmology and astrophysical constraints of Gauss Bonnet dark energy. Physics Letters B, 644:104-108, Jan. 2007, arXiv:astro-ph/0606078

[729] T. Koivisto and D. F. Mota. Gauss-Bonnet quintessence: Background evolution, large scale structure, and cosmological constraints. Phys. Rev. D, 75(2):023518, Jan. 2007, arXiv:hep-th/0609155

[730] E. W. Kolb, D. Lindley, and D. Seckel. More Dimensions - Less Entropy. Phys. Rev., D30:1205, 
1984.

[731] S. J. Kolitch and D. M. Eardley. Behavior of Friedmann-Robertson-Walker cosmological models in scalar-tensor gravity. Annals of Physics, 241:128-151, July 1995, arXiv:gr-qc/9405016

[732] E. Komatsu, K. M. Smith, J. Dunkley, C. L. Bennett, B. Gold, G. Hinshaw, N. Jarosik, D. Larson, M. R. Nolta, L. Page, D. N. Spergel, M. Halpern, R. S. Hill, A. Kogut, M. Limon, S. S. Meyer, N. Odegard, G. S. Tucker, J. L. Weiland, E. Wollack, and E. L. Wright. Seven-year Wilkinson Microwave Anisotropy Probe (WMAP) Observations: Cosmological Interpretation. ApJS, 192:18, Feb. 2011, 1001.4538.

[733] M. Kowalski et al. Improved Cosmological Constraints from New, Old and Combined Supernova Datasets. Astrophys.J., 686:749-778, 2008, 0804.4142.

[734] K. Koyama. Ghosts in the self-accelerating universe. Class. Quant. Grav., 24:R231-R253, 2007, 0709.2399

[735] K. Koyama and F. Arroja. Pathological behaviour of the scalar graviton in Horava-Lifshitz gravity. JHEP, 1003:061, 2010, 0910.1998.

[736] K. Koyama and K. Koyama. Brane induced gravity from asymmetric compactification. Phys. Rev., D72:043511, 2005, hep-th/0501232

[737] K. Koyama and R. Maartens. Structure formation in the Dvali-Gabadadze-Porrati cosmological model. JCAP, 0601:016, 2006, astro-ph/0511634

[738] K. Koyama, G. Niz, and G. Tasinato. Analytic solutions in non-linear massive gravity. 2011, 1103.4708 .

[739] K. Koyama, G. Niz, and G. Tasinato. Strong interactions and exact solutions in non-linear massive gravity. 2011, 1104.2143.

[740] K. Koyama, A. Padilla, and F. P. Silva. Ghosts in asymmetric brane gravity and the decoupled stealth limit. JHEP, 03:134, 2009, 0901.0713.

[741] K. Koyama and F. P. Silva. Non-linear interactions in a cosmological background in the DGP braneworld. Phys.Rev., D75:084040, 2007, hep-th/0702169

[742] K. Koyama and J. Soda. Evolution of cosmological perturbations in the brane world. Phys. Rev., D62:123502, 2000, hep-th/0005239

[743] K. Koyama, A. Taruya, and T. Hiramatsu. Non-linear Evolution of Matter Power Spectrum in Modified Theory of Gravity. Phys.Rev., D79:123512, 2009, 0902.0618.

[744] M. Kramer. Determination of the geometry of the psr b1913+16 system by geodetic precession. Astrophys. J., 509:856-860, 1998.

[745] M. Kramer, I. H. Stairs, R. N. Manchester, M. A. McLaughlin, A. G. Lyne, R. D. Ferdman, M. Burgay, D. R. Lorimer, A. Possenti, N. D'Amico, J. M. Sarkissian, G. B. Hobbs, J. E. Reynolds, P. C. C. Freire, and F. Camilo. Tests of General Relativity from Timing the Double Pulsar. Science, 314:97-102, Oct. 2006, arXiv:astro-ph/0609417

[746] G. A. Krasinskii, E. V. Pit'eva, M. L. Sveshnikov, and L. I. Chuniaeva. The motion of major planets from observations 1769-1988 and some astronomical constants. Celestial Mechanics and Dynamical Astronomy, 55:1-23, Jan. 1993.

[747] K. Krasnov. Non-Metric Gravity. I. Field Equations. Class.Quant.Grav., 25:025001, 2008, gr$\mathrm{qc} / 0703002$

[748] K. Krasnov. On deformations of Ashtekar's constraint algebra. Phys.Rev.Lett., 100:081102, 2008, 0711.0090 .

[749] K. Krasnov. Motion of a 'small body' in non-metric gravity. Phys.Rev., D79:044017, 2009, 0812.3603.

[750] K. Krasnov. Plebanski Formulation of General Relativity: A Practical Introduction. Gen.Rel.Grav., 43:1-15, 2011, 0904.0423.

[751] K. Krasnov and Y. Shtanov. Non-Metric Gravity. II. Spherically Symmetric Solution, Missing Mass and Redshifts of Quasars. Class.Quant.Grav., 25:025002, 2008, 0705.2047.

[752] K. Krasnov and Y. Shtanov. Halos of Modified Gravity. Int.J.Mod.Phys., D17:2555-2562, 2009, 0805.2668

[753] K. Krasnov and Y. Shtanov. Cosmological perturbations in a family of deformations of general relativity. JCAP, 1006:006, 2010, 1002.1210

[754] P. Kraus. Dynamics of anti-de Sitter domain walls. JHEP, 12:011, 1999, hep-th/9910149

[755] A. Krause and S. Ng. Ghost Cosmology:. Exact Solutions, Transitions Between Standard Cosmologies and Ghost Evolution. International Journal of Modern Physics A, 21:1091-1122, 2006 arXiv:hep-th/0409241

[756] P. Kroupa, B. Famaey, K. de Boer, J. Dabringhausen, M. Pawlowski, et al. Local-Group tests of dark-matter Concordance Cosmology: Towards a new paradigm for structure formation? $A s$ - 
tron.Astrophys., 523:A32, 2010, 1006.1647.

[757] J. Kuchowicz. The Einstein-Cartan equations in astrophysically interesting situations. I The case of spherical symmetry.. Act. Phys. Pol., B5: 555, 1975.

[758] H. Kudoh, T. Tanaka, and T. Nakamura. Small localized black holes in braneworld: Formulation and numerical method. Phys. Rev., D68:024035, 2003, gr-qc/0301089

[759] M. Kunz and D. Sapone. Dark energy versus modified gravity. Phys. Rev. Lett., 98:121301, 2007, astro-ph/0612452

[760] M. Kuwahara, H. Suzuki, and E. Takasugi. Analytic solutions for cosmological fluctuation equations in extended inflation models. Phys. Rev. D, 50:661-670, July 1994.

[761] D. La and P. J. Steinhardt. Bubble percolation in extended inflationary models. Physics Letters B, 220:375-378, Apr. 1989.

[762] D. La and P. J. Steinhardt. Extended inflationary cosmology. Physical Review Letters, 62:376378, Jan. 1989.

[763] D. La, P. J. Steinhardt, and E. W. Bertschinger. Prescription for successful extended inflation. Physics Letters B, 231:231-236, Nov. 1989.

[764] G. Lambiase and G. Scarpetta. Baryogenesis in $\mathrm{f}(\mathrm{R})$ theories of gravity. Phys. Rev. D, 74(8):087504, Oct. 2006, arXiv:astro-ph/0610367

[765] S. K. Lamoreaux, J. P. Jacobs, B. R. Heckel, F. J. Raab, and E. N. Fortson. New limits on spatial anisotropy from optically-pumped ${ }^{201} \mathrm{Hg}$ and ${ }^{199} \mathrm{Hg}$. Physical Review Letters, 57:31253128, Dec. 1986.

[766] N. Lanahan-Tremblay and V. Faraoni. The Cauchy problem of $\mathrm{f}(\mathrm{R})$ gravity. Classical and Quantum Gravity, 24:5667-5679, Nov. 2007, 0709.4414.

[767] C. Lanczos. A remarkable property of the Riemann-Christoffel tensor in four dimensions. Annals of Mathematics, 39(4):842-850, 1938.

[768] D. Langlois. Brane cosmological perturbations. Phys. Rev., D62:126012, 2000, hep-th/0005025

[769] D. Langlois. Evolution of cosmological perturbations in a brane- universe. Phys. Rev. Lett., 86:2212-2215, 2001, hep-th/0010063.

[770] D. Langlois. Brane cosmology: An introduction. Prog. Theor. Phys. Suppl., 148:181-212, 2003, hep-th/0209261

[771] D. Langlois, R. Maartens, M. Sasaki, and D. Wands. Large-scale cosmological perturbations on the brane. Phys. Rev., D63:084009, 2001, hep-th/0012044

[772] D. Langlois, R. Maartens, and D. Wands. Gravitational waves from inflation on the brane. Phys. Lett., B489:259-267, 2000, hep-th/0006007.

[773] J. Larena, J. Alimi, and A. Serna. Big Bang Nucleosynthesis in Scalar Tensor Gravity: The Key Problem of the Primordial ${ }^{7} \mathrm{Li}$ Abundance. ApJ, 658:1-10, Mar. 2007, arXiv:astro-ph/0511693

[774] P. D. Lasky, H. Sotani, and D. Giannios. Structure of neutron stars in tensor-vector-scalar theory. Phys. Rev., D78:104019, 2008, 0811.2006.

[775] A. M. Laycock and A. R. Liddle. Extended inflation with a curvature-coupled inflaton. Phys. Rev. D, 49:1827-1839, Feb. 1994, arXiv:astro-ph/9306030

[776] K. Lazaridis, N. Wex, A. Jessner, M. Kramer, B. W. Stappers, G. H. Janssen, G. Desvignes, M. B. Purver, I. Cognard, G. Theureau, A. G. Lyne, C. A. Jordan, and J. A. Zensus. Generic tests of the existence of the gravitational dipole radiation and the variation of the gravitational constant. MNRAS, 400:805-814, Dec. 2009, 0908.0285.

[777] U. J. Le Verrier. Theorie DU mouvement de Mercure. Annales de l'Observatoire de Paris, 5:1, 1859.

[778] J. A. Leach, S. Carloni, and P. K. S. Dunsby. Shear dynamics in Bianchi I cosmologies with $R^{n}$-gravity. Classical and Quantum Gravity, 23:4915-4937, Aug. 2006, arXiv:gr-qc/0603012

[779] D. L. Lee, W. Ni, C. M. Caves, and C. M. Will. Theoretical frameworks for testing relativistic gravity. V - Post-Newtonian limit of Rosen's theory. ApJ, 206:555-558, June 1976.

[780] J. Lee and E. Komatsu. Bullet Cluster: A Challenge to LCDM Cosmology. Astrophys. J., 718:6065, 2010, 1003.0939.

[781] S. Lee. Stability of Palatini-f(R) cosmology. ArXiv e-prints, Oct. 2007, 0710.2395.

[782] S. Lee. Palatini f(R) Cosmology. Modern Physics Letters A, 23:1388-1396, 2008, 0801.4606.

[783] S. Lee and K. Ng. Growth index with the exact analytic solution of sub-horizon scale linear perturbation for dark energy models with constant equation of state. Physics Letters B, 688:1-3, Apr. 2010, 0906.1643.

[784] S. Lee and K. Ng. Properties of the exact analytic solution of the growth factor and its applications. Phys. Rev. D, 82(4):043004, Aug. 2010, 0907.2108.

[785] J. Lense and H. Thirring. Über den Einfluß der Eigenrotation der Zentralkörper auf die Bewegung 
der Planeten und Monde nach der Einsteinschen Gravitationstheorie. Physikalische Zeitschrift, 19:156, 1918.

[786] G. Leon and E. N. Saridakis. Dynamics of anisotropic f(R) cosmology. ArXiv e-prints, July 2010, 1007.3956.

[787] B. Leong, P. Dunsby, A. Challinor, and A. Lasenby. 1+3 covariant dynamics of scalar perturbations in braneworlds. Phys. Rev., D65:104012, 2002, gr-qc/0111033

[788] S. A. Levshakov, A. V. Lapinov, C. Henkel, P. Molaro, D. Reimers, M. G. Kozlov, and I. I. Agafonova. Searching for chameleon-like scalar fields with the ammonia method. II. Mapping of cold molecular cores in NH3 and HC3N lines. ArXiv e-prints, Aug. 2010, 1008.1160.

[789] S. A. Levshakov, P. Molaro, A. V. Lapinov, D. Reimers, C. Henkel, and T. Sakai. Searching for chameleon-like scalar fields with the ammonia method. A\&BA, 512:A44+, Mar. 2010, 0911.3732.

[790] B. Li and J. D. Barrow. Cosmology of $f(R)$ gravity in the metric variational approach Phys. Rev. D, 75(8):084010, Apr. 2007, arXiv:gr-qc/0701111.

[791] B. Li, J. D. Barrow, and D. F. Mota. Cosmology of modified Gauss-Bonnet gravity. Phys. Rev. D, 76(4):044027, Aug. 2007, 0705.3795.

[792] B. Li, J. D. Barrow, and D. F. Mota. Cosmology of Ricci-tensor-squared gravity in the Palatini variational approach. Phys. Rev. D, 76(10):104047, Nov. 2007, 0707.2664.

[793] B. Li, K. C. Chan, and M. Chu. Constraints on $f(R)$ cosmology in the Palatini formalism. Phys. Rev. D, 76(2):024002, July 2007, arXiv:astro-ph/0610794

[794] B. Li, D. F. Mota, and D. J. Shaw. Microscopic and macroscopic behaviors of Palatini modified gravity theories. Phys. Rev. D, 78(6):064018, Sept. 2008, 0805.3428.

[795] B. Li, D. F. Mota, and D. J. Shaw. Indistinguishable Macroscopic Behaviour of Palatini Gravities and General Relativity. Class. Quant. Grav., 26:055018, 2009, 0801.0603.

[796] B. Li, T. P. Sotiriou, and J. D. Barrow. f(T) gravity and local Lorentz invariance. ArXiv e-prints, Oct. 2010, 1010.1041

[797] A. Lichnerowicz. Theories Relativistes de la Gravitation et de l'Electromagnétisme. Masson, Paris, 1955.

[798] A. R. Liddle, D. H. Lyth, and W. Sutherland. Structure formation from power law (and extended) inflation. Physics Letters B, 279:244-249, Apr. 1992.

[799] A. R. Liddle, A. Mazumdar, and J. D. Barrow. Radiation-matter transition in jordan-brans-dicke theory. Phys. Rev. D, 58(2):027302, Jun 1998.

[800] A. R. Liddle and A. J. Smith. Observational constraints on braneworld chaotic inflation. Phys. Rev., D68:061301, 2003, astro-ph/0307017

[801] A. R. Liddle and L. A. Urena-Lopez. Curvaton reheating: An application to braneworld inflation. Phys. Rev., D68:043517, 2003, astro-ph/0302054

[802] A. R. Liddle and D. Wands. Microwave background constraints on extended inflation voids. MNRAS, 253:637-648, Dec. 1991

[803] A. R. Liddle and D. Wands. Hyperextended inflation: Dynamics and constraints. Phys. Rev. D, 45:2665-2673, Apr. 1992.

[804] A. R. Liddle and D. Wands. There is a big-bubble problem in extended inflation. Phys. Rev. D, 46:3655-3658, Oct. 1992.

[805] J. E. Lidsey, S. Nojiri, and S. D. Odintsov. Brane world cosmology in (anti)-de Sitter EinsteinGauss-Bonnet-Maxwell gravity. JHEP, 0206:026, 2002, hep-th/0202198

[806] J. E. Lidsey and N. J. Nunes. Inflation in Gauss-Bonnet brane cosmology. Phys.Rev., D67:103510, 2003, astro-ph/0303168

[807] J. E. Lidsey, D. Wands, and E. J. Copeland. Superstring cosmology. Phys. Rept., 337:343-492, 2000, hep-th/9909061

[808] A. P. Lightman and D. L. Lee. New Two-Metric Theory of Gravity with Prior Geometry. Phys. Rev. D, 8:3293-3302, Nov. 1973.

[809] E. A. Lim. Can we see Lorentz-violating vector fields in the CMB? Phys. Rev. D, 71(6):063504, Mar. 2005, arXiv:astro-ph/0407437

[810] C. Limbach, D. Psaltis, and F. Ozel. The Redshift Evolution of the Tully-Fisher Relation as a Test of Modified Gravity. 2008, 0809.2790.

[811] K. Lin, A. Wang, Q. Wu, and T. Zhu. On strong coupling in nonrelativistic general covariant theory of gravity. 2011, 1106.1486.

[812] A. D. Linde. Inflation and string cosmology. ECONF, C040802:L024, 2004, hep-th/0503195

[813] E. V. Linder. Cosmic growth history and expansion history. Phys. Rev. D, 72(4):043529, Aug. 2005, arXiv:astro-ph/0507263

[814] E. V. Linder. Exponential gravity. Phys. Rev. D, 80(12):123528, Dec. 2009, 0905.2962. 
[815] E. V. Linder. Einstein's other gravity and the acceleration of the Universe. Phys. Rev. D, 81(12):127301, June 2010, 1005.3039.

[816] E. V. Linder and R. N. Cahn. Parameterized Beyond-Einstein Growth. Astropart.Phys., 28:481488, 2007, astro-ph/0701317

[817] L. Lombriser, W. Hu, W. Fang, and U. Seljak. Cosmological Constraints on DGP Braneworld Gravity with Brane Tension. Phys.Rev., D80:063536, 2009, 0905.1112.

[818] L. Lombriser, A. Slosar, U. Seljak, and W. Hu. Constraints on $f(R)$ gravity from probing the large-scale structure. ArXiv e-prints, Mar. 2010, 1003.3009.

[819] J. C. Long, H. W. Chan, A. B. Churnside, E. A. Gulbis, M. C. M. Varney, and J. C. Price. Upper limits to submillimetre-range forces from extra space-time dimensions. Nature, 421:922-925, Feb. 2003, arXiv:hep-ph/0210004

[820] D. Lorenz. Exact Bianchi type-VIII and type- $I X$ cosmological models with matter and electromagnetic fields. Phys. Rev. D, 22:1848-1852, Oct. 1980.

[821] D. Lorenz-Petzold. The general vacuum Bianchi type- $V$ solution in the Brans-Dicke theory. Mathematical Proceedings of the Cambridge Philosophical Society, 95:175, 1983.

[822] D. Lorenz-Petzold. Exact Brans-Dicke-Bianchi Type-II Solutions. Progress of Theoretical Physics, 71:406-408, Feb. 1984

[823] D. Lorenz-Petzold. Exact Brans-Dicke-Bianchi type-V solutions. Mathematical Proceedings of the Cambridge Philosophical Society, 96:183, 1984.

[824] D. Lorenz-Petzold. Exact Brans-Dicke-Bianchi type-VII(h) solutions. Ap\&SS, 106:409-413, Nov. 1984 .

[825] D. Lorenz-Petzold. Exact Brans-Dicke-Bianchi types- $I I, V I I I$, and $I X$ stiff matter solutions. ApESS, 98:281-286, Jan. 1984.

[826] D. Lorenz-Petzold. Exact Brans-Dicke Friedmann-Robertson-Walker-Bianchi-Kantowski-Sachs vacuum solutions. ApESSS, 98:101-113, Jan. 1984

[827] D. Lorenz-Petzold. The general non-LRS-BDT-Bianchi type- $V$ radiation solution. Ap\&SSS, 103:397-399, Aug. 1984.

[828] D. Lorenz-Petzold. Electromagnetic Brans-Dicke-Bianchi type- $V$ solutions. Ap $\& S S, 114: 277-284$, Sept. 1985.

[829] D. Lorenz-Petzold. Exact Brans-Dicke-Bianchi type-VI $I_{h}$ solutions. Acta Physica Polonica B, 16:171-178, 1985

[830] D. Lorenz-Petzold. Bianchi type- $V I_{0}$ and type- $V I_{h}$ perfect fluid solutions. Ap\&SS $134: 415-416$, June 1987.

[831] D. Lovelock. The Einstein Tensor and Its Generalizations. Journal of Mathematical Physics, 12:498-501, Mar. 1971.

[832] D. Lovelock. The Four-Dimensionality of Space and the Einstein Tensor. Journal of Mathematical Physics, 13:874-876, June 1972 .

[833] A. Lue. Global structure of Deffayet (Dvali-Gabadadze-Porrati) cosmologies. Phys.Rev., D67:064004, 2003, hep-th/0208169

[834] A. Lue. The phenomenology of dvali-gabadadze-porrati cosmologies. Phys.Rept., 423:1-48, 2006, astro-ph/0510068

[835] A. Lue, G. D. Starkman, How a brane cosmological constant can trick us into thinking that W -1 Phys. Rev. D70 (2004) 101501. arXiv:astro-ph/0408246 [astro-ph]].

[836] A. Lue, R. Scoccimarro, and G. D. Starkman. Probing Newton's constant on vast scales: DGP gravity, cosmic acceleration and large scale structure. Phys. Rev., D69:124015, 2004, astro$\mathrm{ph} / 0401515$

[837] A. Lue and G. D. Starkman. Squeezing MOND into a Cosmological Scenario. Physical Review Letters, 92(13):131102, Apr. 2004, arXiv:astro-ph/0310005

[838] A. Lukas, B. A. Ovrut, K. S. Stelle, and D. Waldram. The universe as a domain wall. Phys. Rev., D59:086001, 1999, hep-th/9803235

[839] M. A. Luty, M. Porrati, and R. Rattazzi. Strong interactions and stability in the DGP model. JHEP, 0309:029, 2003, hep-th/0303116

840] A. G. Lyne, M. Burgay, M. Kramer, A. Possenti, R. N. Manchester, F. Camilo, M. A. McLaughlin, D. R. Lorimer, N. D'Amico, B. C. Joshi, J. Reynolds, and P. C. C. Freire. A Double-Pulsar System: A Rare Laboratory for Relativistic Gravity and Plasma Physics. Science, 303:11531157, Feb. 2004, arXiv:astro-ph/0401086

[841] C.-P. Ma and E. Bertschinger. Cosmological perturbation theory in the synchronous and conformal newtonian gauges. Astrophys. J., 455:7, 1995.

[842] R. Maartens. Cosmological dynamics on the brane. Phys. Rev., D62:084023, 2000, hep- 
th/0004166

[843] R. Maartens, V. Sahni, T. D. Saini, Anisotropy dissipation in brane world inflation Phys. Rev. D63 (2001) 063509. gr-qc/0011105.

[844] R. Maartens. Geometry and dynamics of the brane-world. 2001, gr-qc/0101059

[845] R. Maartens and K. Koyama. Brane-World Gravity. Living Rev. Rel., 13:5, 2010, 1004.3962.

[846] R. Maartens and E. Majerotto. Observational constraints on self-accelerating cosmology. Phys.Rev., D74:023004, 2006, astro-ph/0603353.

[847] R. Maartens, D. Wands, B. A. Bassett, and I. Heard. Chaotic inflation on the brane. Phys. Rev., D62:041301, 2000, hep-ph/9912464

[848] K. I. Macrae and R. J. Riegert. Effect of curvature-squared terms on cosmology. Phys. Rev. D, 24:2555-2560, Nov. 1981.

[849] M. S. Madsen and J. D. Barrow. De Sitter ground states and boundary terms in generalised gravity. Nuclear Physics B, 323:242-252, Aug. 1989.

[850] H. Maeda and G. Giribet. Lifshitz black holes in Brans-Dicke theory. ArXiv e-prints, May 2011, 1105.1331.

[851] H. Maeda, V. Sahni, and Y. Shtanov. Braneworld dynamics in Einstein-Gauss-Bonnet gravity. Phys.Rev., D76:104028, 2007, 0708.3237.

[852] K. Maeda. Inflation as a transient attractor in $R^{2}$ cosmology. Phys. Rev. D, 37:858-862, Feb. 1988.

[853] K. Maeda. Towards the Einstein-Hilbert action via conformal transformation. Phys. Rev. D, 39:3159-3162, May 1989.

[854] K.-i. Maeda, Y. Misonoh, and T. Kobayashi. Oscillating Universe in Horava-Lifshitz Gravity. Phys. Rev., D82:064024, 2010, 1006.2739.

[855] K.-i. Maeda, and N. Ohta. Inflation from M-theory with fourth-order corrections and large extra dimensions. Phys. Lett. B, 597:400-407, Sep. 2004, hep-th/0405205

[856] K.-i. Maeda, and N. Ohta. Inflation from superstring and M-theory compactification with higher order corrections. Phys. Rev. D, 71(6):063520, Mar. 2005, hep-th/0411093

[857] K.-i. Maeda, N. Ohta, and Y. Sasagawa. Black hole solutions in string theory with Gauss-Bonnet curvature correction. Phys. Rev. D, 80(10):104032, Nov. 2009, 0908.4151.

[858] K.-i. Maeda, N. Ohta, and Y. Sasagawa. AdS black hole solutions in dilatonic Einstein-GaussBonnet gravity. Phys. Rev. D, 83(4):044051, Feb. 2011, 1012.0568.

[859] K.-i. Maeda and T. Torii. Covariant gravitational equations on brane world with Gauss-Bonnet term. Phys.Rev., D69:024002, 2004, hep-th/0309152

[860] A. S. Majumdar, D. Gangopadhyay, and L. P. Singh. Evolution of primordial black holes in Jordan-Brans-Dicke cosmology. MNRAS, 385:1467-1470, Apr. 2008, 0709.3193.

[861] N. Makino and M. Sasaki. The Density Perturbation in the Chaotic Inflation with Non-Minimal Coupling. Progress of Theoretical Physics, 86:103-118, July 1991.

[862] J. M. Maldacena. The large N limit of superconformal field theories and supergravity. Adv. Theor. Math. Phys., 2:231-252, 1998, hep-th/9711200

[863] S. Mallik and D. Rai Chaudhuri. Density perturbation in extended inflation. Phys. Rev. D, 56:625-630, July 1997, arXiv:hep-ph/9612427

[864] P. D. Mannheim. Cosmic Acceleration as the Solution to the Cosmological Constant Problem. ApJ, 561:1-12, Nov. 2001, arXiv:astro-ph/9910093

[865] P. D. Mannheim. Comprehensive solution to the cosmological constant, zero-point energy, and quantum gravity problems. General Relativity and Gravitation, 43:703-750, Mar. 2011, 0909.0212.

[866] P. D. Mannheim. Making the Case for Conformal Gravity. ArXiv e-prints, Jan. 2011, 1101.2186.

[867] M. Mateo. Dwarf Galaxies of the Local Group. Ann. Rev. Astron. Astrophys., 36:435-506, 1998, astro-ph/9810070

[868] M. Mathisson. Neue mechanik materieller systemes. Acta Phys.Polon., 6: 163, 1937.

[869] D. Mattingly. Modern Tests of Lorentz Invariance. Living Reviews in Relativity, 8:5, Sept. 2005, arXiv:gr-qc/0502097

[870] N. E. Mavromatos, M. Sakellariadou, and M. F. Yusaf. Can TeVeS avoid Dark Matter on galactic scales? 2009, 0901.3932.

[871] A. B. Mayer and H. Schmidt. The de Sitter spacetime as attractor solution in eighth-order gravity. Classical and Quantum Gravity, 10:2441-2446, Nov. 1993.

[872] S. H. Mazharimousavi and M. Halilsoy. Black hole solutions in $f(R)$ gravity coupled with nonlinear Yang-Mills field. ArXiv e-prints, May 2011, 1105.3659.

[873] L. McAllister and E. Silverstein. String Cosmology: A Review. Gen. Rel. Grav., 40:565-605, 
$2008,0710.2951$.

[874] J. McDonald. Dark matter from an oscillating Brans-Dicke scalar in the extended inflation model. Phys. Rev. D, 44:2325-2334, Oct. 1991.

[875] J. McDonald. Can a Brans-Dicke scalar account for dark matter in extended inflation models? Phys. Rev. D, 48:2462-2476, Sept. 1993.

[876] J. McDonald. Baryomorphosis: Relating the Baryon Asymmetry to the 'WIMP Miracle'. 2010, 1009.3227.

[877] P. McDonald et al. The Linear theory power spectrum from the Lyman-alpha forest in the Sloan Digital Sky Survey. Astrophys.J., 635:761-783, 2005, astro-ph/0407377

[878] S. S. McGaugh. A Novel Test of the Modified Newtonian Dynamics with Gas Rich Galaxies. Phys.Rev.Lett., 106:121303, 2011, 1102.3913.

[879] S. S. McGaugh, J. M. Schombert, G. D. Bothun, and W. de Blok. The Baryonic Tully-Fisher relation. Astrophys.J., 533:L99-L102, 2000, astro-ph/0003001

[880] O. Mena, J. Santiago, and J. Weller. Constraining Inverse-Curvature Gravity with Supernovae. Physical Review Letters, 96(4):041103, Feb. 2006, arXiv:astro-ph/0510453

[881] X. Meng and P. Wang. Letter: Gravitational Potential in the Palatini Formulation of Modified Gravity. General Relativity and Gravitation, 36:1947-1954, Aug. 2004, arXiv:gr-qc/0311019

[882] X. Meng and P. Wang. Palatini Formulation of Modified Gravity with Squared Scalar Curvature. General Relativity and Gravitation, 36:2673-2680, Dec. 2004, arXiv:astro-ph/0308284.

[883] X. Meng and P. Wang. $R^{2}$ corrections to the cosmological dynamics of inflation in the Palatini formulation. Classical and Quantum Gravity, 21:2029-2036, Apr. 2004, arXiv:gr-qc/0402011

[884] X. Meng and P. Wang. Palatini formulation of the $R^{-1}$-modified gravity with an additional squared scalar curvature term. Classical and Quantum Gravity, 22:23-32, Jan. 2005, arXiv:grqc/0411007

[885] S. M. Merkowitz. Tests of Gravity Using Lunar Laser Ranging. Living Reviews in Relativity, 13:7, Nov. 2010

[886] R. R. Metsaev and A. A. Tseytlin. Order $\alpha^{\prime}$ (two-loop) equivalence of the string equations of motion and the $\sigma$-model Weyl invariance conditions Dependence on the dilaton and the antisymmetric tensor. Nuclear Physics B, 293:385-419, 1987.

[887] J. Middleton. On the existence of anisotropic cosmological models in higher order theories of gravity. Classical and Quantum Gravity, 27(22):225013, Nov. 2010, 1007.4669.

[888] J. Middleton and J. D. Barrow. Stability of an isotropic cosmological singularity in higher-order gravity. Phys. Rev. D, 77(10):103523, May 2008, 0801.4090.

[889] S. Mignemi. Dyonic black holes in effective string theory. Phys. Rev. D, 51:934-937, Jan. 1995, arXiv:hep-th/9303102

[890] S. Mignemi and N. R. Stewart. Charged black holes in effective string theory. Phys. Rev. D, 47:5259-5269, June 1993, arXiv:hep-th/9212146

[891] S. Mignemi and D. L. Wiltshire. Black holes in higher-derivative gravity theories. Phys. Rev. D, 46(4):1475-1506, Aug 1992.

[892] M. B. Mijić, M. S. Morris, and W. Suen. The $R^{2}$ cosmology: Inflation without a phase transition. Phys. Rev. D, 34:2934-2946, Nov. 1986.

[893] G. Miknaitis, G. Pignata, A. Rest, W. Wood-Vasey, S. Blondin, et al. The ESSENCE Supernova Survey: Survey Optimization, Observations, and Supernova Photometry. Astrophys.J., 666:674693, 2007, astro-ph/0701043

[894] A. Milani, D. Vokrouhlický, D. Villani, C. Bonanno, and A. Rossi. Testing general relativity with the BepiColombo radio science experiment. Phys. Rev. D, 66(8):082001, Oct. 2002.

[895] M. Milgrom. A Modification of the Newtonian dynamics as a possible alternative to the hidden mass hypothesis. Astrophys.J., 270:365-370, 1983.

[896] M. Milgrom. Bimetric MOND gravity. Phys. Rev., D80:123536, 2009, 0912.0790.

[897] M. Milgrom. Cosmological fluctuation growth in bimetric MOND. Phys. Rev. D, 82(4):043523, Aug. 2010, 1006.3809.

[898] J. P. Mimoso and D. Wands. Anisotropic scalar-tensor cosmologies. Phys. Rev. D, 52:5612-5627, Nov. 1995, arXiv:gr-qc/9501039

[899] J. P. Mimoso and D. Wands. Massless fields in scalar-tensor cosmologies. Phys. Rev. D, 51(2):477489, Jan 1995.

[900] M. Minamitsuji. Self-accelerating solutions in cascading DGP braneworld. Phys. Lett., B684:9295, 2010, 0806.2390.

[901] V. Miranda, S. E. Jorás, I. Waga, and M. Quartin. Viable Singularity-Free f(R) Gravity without a Cosmological Constant. Physical Review Letters, 102(22):221101, June 2009, 0905.1941. 
[902] C. Misner, K. Thorne, and J. Wheeler. Gravitation. WH Freeman \& co, 1973.

[903] S. Mizuno and K. Koyama. Primordial non-Gaussianity from the DBI Galileons. Phys.Rev., D82:103518, 2010, 1009.0677.

[904] N. Moeller and B. Zwiebach. Dynamics with infinitely many time derivatives and rolling tachyons. JHEP, 10:034, 2002, hep-th/0207107

[905] J. W. Moffat. Scalar tensor vector gravity theory. J. Cosmology Astropart. Phys., 3:4, Mar. 2006, arXiv:gr-qc/0506021

[906] J. Moldenhauer and M. Ishak. A minimal set of invariants as a systematic approach to higher order gravity models: physical and cosmological constraints. J. Cosmology Astropart. Phys. 12:20, Dec. 2009, 0912.5332.

[907] J. Moldenhauer, M. Ishak, J. Thompson and D. A. Easson. Supernova, baryon acoustic oscillations, and CMB surface distance constraints on $\mathrm{f}(\mathrm{G})$ higher order gravity models. Phys. Rev. D, 81:063514, Mar. 2010, 1004.2459.

[908] S. Mollerach and S. Matarrese. Non-scale-invariant density perturbations from chaotic extended inflation. Phys. Rev. D, 45:1961-1970, Mar. 1992.

[909] B. Moore, S. Ghigna, F. Governato, G. Lake, T. R. Quinn, et al. Dark matter substructure in galactic halos. Astrophys.J., 524:L19-L22, 1999, astro-ph/9907411

[910] D. F. Mota, P. G. Ferreira, and C. Skordis. Cosmic Microwave Background in TeVeS theory. Work presented at the Alternative gravities workshop in Edinburgh, 2006.

[911] D. F. Mota, M. Sandstad, and T. Zlosnik. Cosmology of the selfaccelerating third order Galileon. JHEP, 1012:051, 2010, 1009.6151.

[912] D. F. Mota and D. J. Shaw. Strongly Coupled Chameleon Fields: New Horizons in Scalar Field Theory. Physical Review Letters, 97(15):151102, Oct. 2006, arXiv:hep-ph/0606204.

[913] D. F. Mota and D. J. Shaw. Evading equivalence principle violations, cosmological, and other experimental constraints in scalar field theories with a strong coupling to matter. Phys. Rev. D, 75(6):063501, Mar 2007.

[914] H. Motohashi, A. A. Starobinsky, and J. Yokoyama. Analytic Solution for Matter Density Perturbations in a Class of Viable Cosmological $\mathrm{f}(\mathrm{R})$ Models. International Journal of Modern Physics D, 18:1731-1740, 2009, 0905.0730.

[915] F. Mueller-Hoissen. Dimensionally continued Euler forms, Kaluza-Klein cosmology and dimensional reduction. Class. Quant. Grav., 3:665, 1986.

[916] V. F. Mukhanov and G. V. Chibisov. Quantum fluctuations and a nonsingular universe. Soviet Journal of Experimental and Theoretical Physics Letters, 33:532, May 1981.

[917] V. F. Mukhanov, H. Feldman, and R. H. Brandenberger. Theory of cosmological perturbations. Part 1. Classical perturbations. Part 2. Quantum theory of perturbations. Part 3. Extensions. Phys.Rept., 215:203-333, 1992.

[918] S. Mukohyama. Gauge-invariant gravitational perturbations of maximally symmetric spacetimes. Phys. Rev., D62:084015, 2000, hep-th/0004067

[919] S. Mukohyama. Perturbation of junction condition and doubly gauge- invariant variables. Class. Quant. Grav., 17:4777-4798, 2000, hep-th/0006146

[920] S. Mukohyama. Integro-differential equation for brane-world cosmological perturbations. Phys. Rev., D64:064006, 2001, hep-th/0104185

[921] S. Mukohyama. Doubly-gauge-invariant formalism of brane-world cosmological perturbations. 2002, hep-th/0202100

[922] S. Mukohyama. An accelerating universe and cosmological perturbation in the ghost condensate. J. Cosmology Astropart. Phys., 10:11, Oct. 2006, arXiv:hep-th/0607181

[923] S. Mukohyama. Caustic avoidance in Horava-Lifshitz gravity. JCAP, 0909:005, 2009, 0906.5069.

[924] S. Mukohyama. Dark matter as integration constant in Horava-Lifshitz gravity. Phys.Rev. D80:064005, 2009, 0905.3563.

[925] S. Mukohyama. Scale-invariant cosmological perturbations from Horava-Lifshitz gravity without inflation. JCAP, 0906:001, 2009, 0904.2190.

[926] S. Mukohyama. Horava-Lifshitz Cosmology: A Review. Class.Quant.Grav., 27:223101, 2010, 1007.5199.

[927] S. Mukohyama, K. Nakayama, F. Takahashi, and S. Yokoyama. Phenomenological Aspects of Horava-Lifshitz Cosmology. Phys.Lett., B679:6-9, 2009, 0905.0055

[928] J. Müller, K. Nordtvedt, and D. Vokrouhlický. Improved constraint on the $\alpha_{1}$ ppn parameter from lunar motion. Phys. Rev. D, 54:R5927-R5930, 1996.

[929] T. Multamäki and I. Vilja. Cosmological expansion and the uniqueness of the gravitational action. Phys. Rev. D, 73(2):024018, Jan. 2006, arXiv:astro-ph/0506692 
[930] T. Multamäki and I. Vilja. Constraining Newtonian stellar configurations in $f(R)$ theories of gravity. Physics Letters B, 659:843-846, Feb. 2008, 0709.3422.

[931] R. C. Myers. Higher derivative gravity, surface terms and string theory. Phys. Rev., D36:392, 1987.

[932] R. Myrzakulov. Accelerating universe from F(T) gravities. ArXiv e-prints, June 2010, 1006.1120.

[933] R. Myrzakulov. F(T) gravity and k-essence. ArXiv e-prints, Aug. 2010, 1008.4486.

[934] R. Nagata, T. Chiba, and N. Sugiyama. Observational consequences of the evolution of primordial fluctuations in scalar-tensor cosmology. Phys. Rev. D, 66(10):103510, Nov 2002.

[935] R. Nagata, T. Chiba, and N. Sugiyama. WMAP constraints on scalar-tensor cosmology and the variation of the gravitational constant. Phys. Rev. D, 69(8):083512, Apr. 2004, arXiv:astro$\mathrm{ph} / 0311274$

[936] M. Nakahara. Geometry, topology, and physics. Taylor \& Francis, 2003.

[937] S. Nakamura and A. Hosoya. Density Fluctuation in Extended Inflationary Universe. Progress of Theoretical Physics, 87:401-407, Feb. 1992.

[938] H. Nariai. On the Green's Function in an Expanding Universe and Its Role in the Problem of Mach's Principle. Progress of Theoretical Physics, 40:49-59, July 1968.

[939] H. Nariai. Gravitational Instability in the Brans-Dicke Cosmology. Progress of Theoretical Physics, 42:544-554, Sept. 1969.

[940] T. Narikawa and K. Yamamoto. Characterizing the linear growth rate of cosmological density perturbations in an $\mathrm{f}(\mathrm{R})$ model. Phys. Rev. D, 81(4):043528, Feb. 2010, 0912.1445.

[941] P. Nath and M. Yamaguchi. Effects of Extra Space-time Dimensions on the Fermi Constant. Phys. Rev., D60:116004, 1999, hep-ph/9902323

[942] A. Navarro, A. Serna, and J. M. Alimi. Asymptotic and exact solutions of perfect-fluid scalartensor cosmologies. Phys. Rev. D, 59(12):124015, May 1999.

[943] I. Navarro and K. van Acoleyen. On the Newtonian limit of generalized modified gravity models. Physics Letters B, 622:1-5, Aug. 2005, arXiv:gr-qc/0506096

[944] I. Navarro and K. Van Acoleyen. Consistent long distance modification of gravity from inverse powers of the curvature. J. Cosmology Astropart. Phys., 3:8, Mar. 2006, arXiv:gr-qc/0511045

[945] I. Navarro and K. Van Acoleyen. f(R) actions, cosmic acceleration and local tests of gravity. $J$. Cosmology Astropart. Phys., 2:22, Feb. 2007, arXiv:gr-qc/0611127.

[946] J. F. Navarro, C. S. Frenk, and S. D. White. The Structure of cold dark matter halos. Astrophys.J., 462:563-575, 1996, astro-ph/9508025

[947] B. Nayak, A. Majumdar, and L. Singh. Astrophysical constraints on primordial black holes in brans-dicke theory. Journal of Cosmology and Astroparticle Physics, 2010(08):039, 2010.

[948] B. Nayak, L. P. Singh, and A. S. Majumdar. Effect of accretion on primordial black holes in brans-dicke theory. Phys. Rev. D, 80(2):023529, Jul 2009.

[949] W. Nelson. Restricting fourth-order gravity via cosmology. Phys. Rev. D, 82(12):124044, Dec. 2010, 1012.3353

[950] W. Nelson. Static solutions for fourth order gravity. Phys. Rev. D, 82(10):104026, Nov. 2010, 1010.3986.

[951] W. Nelson, J. Ochoa, and M. Sakellariadou. Constraining the Noncommutative Spectral Action via Astrophysical Observations. Physical Review Letters, 105(10):101602, Sept. 2010, 1005.4279.

[952] W. Nelson, J. Ochoa, and M. Sakellariadou. Gravitational waves in the spectral action of noncommutative geometry. Phys. Rev. D, 82(8):085021, Oct. 2010, 1005.4276.

[953] W. Nelson and M. Sakellariadou. Inflation mechanism in asymptotic noncommutative geometry. Physics Letters B, 680:263-266, Sept. 2009, 0903.1520.

[954] W. Nelson and M. Sakellariadou. Cosmology and the noncommutative approach to the standard model. Phys. Rev. D, 81(8):085038, Apr. 2010, 0812.1657.

[955] A. Neronov and I. Sachs. On metric perturbations in brane-world scenarios. Phys. Lett., B513:173-178, 2001, hep-th/0011254

[956] S. Nesseris, A. De Felice, and S. Tsujikawa. Observational constraints on Galileon cosmology. Phys.Rev., D82:124054, 2010, 1010.0407.

[957] S. Nesseris and A. Mazumdar. Newton's constant in $f\left(R, R_{\mu \nu} R^{\mu \nu}, \square R\right)$ theories of gravity and constraints from BBN. Phys. Rev. D, 79(10):104006, May 2009, 0902.1185.

[958] S. Nesseris, F. Piazza, and S. Tsujikawa. The universe is accelerating. Do we need a new mass scale? Phys.Lett., B689:122-128, 2010, 0910.3949.

[959] I. P. Neupane and B. M. N. Carter. Dynamical relaxation of dark energy: A solution to early inflation, late-time acceleration and the cosmological constant problem. Physics Letters B, 638:94-99, July 2006, arXiv:hep-th/0510109 
[960] I. P. Neupane and B. M. N. Carter. Towards inflation and dark energy cosmologies from modified Gauss Bonnet theory. J. Cosmology Astropart. Phys., 6:4, June 2006, arXiv:hep-th/0512262

[961] P. Nicolini, Noncommutative Black Holes, The Final Appeal To Quantum Gravity: A Review Int. J. Mod. Phys. A24 (2009) 1229-1308. arXiv:0807.1939 [hep-th]].

[962] A. Nicolis and R. Rattazzi. Classical and quantum consistency of the DGP model. JHEP, 0406:059, 2004, hep-th/0404159

[963] A. Nicolis, R. Rattazzi, and E. Trincherini. The Galileon as a local modification of gravity. Phys.Rev., D79:064036, 2009, 0811.2197.

[964] A. Nicolis, R. Rattazzi, and E. Trincherini. Energy's and amplitudes' positivity. JHEP, 1005:095, 2010, 0912.4258.

[965] H.-P. Nilles, A. Papazoglou, and G. Tasinato. Selftuning and its footprints. Nucl.Phys., B677:405429, 2004, hep-th/0309042

[966] G. Niz, A. Padilla, and H. K. Kunduri. Braneworld Isotropization and Magnetic Fields. JCAP, 0804:012, 2008, 0801.3462

[967] D. R. Noakes. The initial value formulation of higher derivative gravity. Journal of Mathematical Physics, 24:1846-1850, July 1983

[968] S. Nojiri and S. D. Odintsov. Modified gravity with negative and positive powers of curvature: Unification of inflation and cosmic acceleration. Phys. Rev. D, 68(12):123512, Dec. 2003, arXiv:hep-th/0307288

[969] S. Nojiri and S. D. Odintsov. Introduction to Modified Gravity and Gravitational Alternative for Dark Energy. Int. J. Geom. Meth. Mod. Phys., 4:115, 2007, arXiv:hep-th/0601213

[970] S. Nojiri and S. D. Odintsov. Modified Gravity with ln R Terms and Cosmic Acceleration. General Relativity and Gravitation, 36:1765-1780, Aug. 2004, arXiv:hep-th/0308176

[971] S. Nojiri and S. D. Odintsov. Modified Gauss Bonnet theory as gravitational alternative for dark energy. Physics Letters B, 631:1-6, Dec. 2005, arXiv:hep-th/0508049

[972] S. Nojiri and S. D. Odintsov. Modified $\mathrm{f}(\mathrm{R})$ gravity consistent with realistic cosmology: From a matter dominated epoch to a dark energy universe. Phys. Rev. D, 74(8):086005, Oct. 2006, arXiv:hep-th/0608008

[973] S. Nojiri and S. D. Odintsov. Modified gravity as an alternative for $\Lambda$ CDM cosmology. Journal of Physics A Mathematical General, 40:6725-6732, June 2007, arXiv:hep-th/0610164

[974] S. Nojiri and S. D. Odintsov. Unifying inflation with $\Lambda$ CDM epoch in modified $\mathrm{f}(\mathrm{R})$ gravity consistent with Solar System tests. Physics Letters B, 657:238-245, Dec. 2007, 0707.1941.

[975] S. Nojiri and S. D. Odintsov. Future evolution and finite-time singularities in f(R) gravity unifying inflation and cosmic acceleration. Phys. Rev. D, 78(4):046006, Aug. 2008, 0804.3519.

[976] S. Nojiri and S. D. Odintsov. Modified $\mathrm{f}(\mathrm{R})$ gravity unifying $R^{m}$ inflation with the $\Lambda$ CDM epoch. Phys. Rev. D, 77(2):026007, Jan. 2008, 0710.1738

[977] S. Nojiri and S. D. Odintsov. Modified non-local-f(R) gravity as the key for the inflation and dark energy. Physics Letters B, 659:821-826, Jan. 2008, 0708.0924.

[978] S. Nojiri and S. D. Odintsov. Unified cosmic history in modified gravity: from $F(R)$ theory to Lorentz non-invariant models. Phys. Rep., 505:59-144, Aug. 2011, 1011.0544.

[979] S. Nojiri, S. D. Odintsov, and O. G. Gorbunova. Dark energy problem: from phantom theory to modified Gauss Bonnet gravity. Journal of Physics A Mathematical General, 39:6627-6633, May 2006, arXiv:hep-th/0510183

[980] S. Nojiri, S. D. Odintsov, and D. Sáez-Gómez. Cosmological reconstruction of realistic modified f(R) gravities. Physics Letters B, 681:74-80, Oct. 2009, 0908.1269.

[981] S. Nojiri, S. D. Odintsov, and M. Sami. Dark energy cosmology from higher-order, string-inspired gravity, and its reconstruction. Phys. Rev. D, 74(4):046004, Aug. 2006, arXiv:hep-th/0605039

[982] S. Nojiri, S. D. Odintsov, and M. Sasaki. Gauss-Bonnet dark energy. Phys. Rev. D, 71(12):123509, June 2005, arXiv:hep-th/0504052

[983] S. Nojiri, S. D. Odintsov, and P. V. Tretyakov. From Inflation to Dark Energy in the Non-Minimal Modified Gravity. Progress of Theoretical Physics Supplement, 172:81-89, 2008, 0710.5232.

[984] M. R. Nolta et al. Five-Year Wilkinson Microwave Anisotropy Probe (WMAP) Observations: Angular Power Spectra. Astrophys. J. Supp., 180:296-305, 2009, 0803.0593.

[985] G. Nordstrom. On the possibility of unifying the electromagnetic and the gravitational fields. Phys. Z., 15:504-506, 1914, physics/0702221.

[986] K. Nordtvedt. Equivalence Principle for Massive Bodies. I. Phenomenology. Physical Review, 169:1014-1016, May 1968.

[987] K. Nordtvedt. Probing gravity to the second post-Newtonian order and to one part in 10 to the 7 th using the spin axis of the sun. ApJ, 320:871-874, Sept. 1987. 
[988] K. Nordtvedt, Jr. PostNewtonian metric for a general class of scalar tensor gravitational theories and observational consequences. Astrophys. J., 161:1059-1067, 1970.

[989] K. Nordtvedt, Jr. and C. M. Will. Conservation Laws and Preferred Frames in Relativistic Gravity. II. Experimental Evidence to Rule Out Preferred-Frame Theories of Gravity. ApJ, 177:775, Nov. 1972.

[990] A. Núñez and S. Solganik. Ghost constraints on modified gravity. Physics Letters B, 608:189-193, Feb. 2005, arXiv:hep-th/0411102

[991] N. J. Nunes and E. J. Copeland. Tracking quintessential inflation from brane worlds. Phys. Rev., D66:043524, 2002, astro-ph/0204115

[992] A. M. Nzioki, S. Carloni, R. Goswami, and P. K. S. Dunsby. New framework for studying spherically symmetric static solutions in $\mathrm{f}(\mathrm{R})$ gravity. Phys. Rev. D, 81(8):084028, Apr. 2010, 0908.3333 .

[993] J. O' Hanlon and B. O. J. Tupper. Vacuum-field solutions in the Brans-Dicke theory. Nuovo Cim., B7:305-312, 1972 .

[994] N. Ohta, and T. Torii. Black Holes in the Dilatonic Einstein-Gauss-Bonnet Theory in Various Dimensions. III - Asymptotically AdS Black Holes with $\mathrm{k}=+/-1$. Prog. Theo. Phys., 121:959-981, May 2009, 0902.4072.

[995] N. Ohta, and T. Torii. Black Holes in the Dilatonic Einstein-Gauss-Bonnet Theory in Various Dimensions. IV - Topological Black Holes with and without Cosmological Term. Prog. Theo. Phys., 122:1477-1500, Dec. 2009, 0908.3918.

[996] N. Ohta, and T. Torii. Global Structure of Black Holes in String Theory with Gauss-Bonnet Correction in Various Dimensions. Prog. Theo. Phys., 124:207-225, Aug. 2010, 1004.2779.

[997] Y. Okada. Inflation in Kaluza-Klein cosmology. Phys. Lett., B150:103, 1985.

[998] G. J. Olmo. The gravity lagrangian according to solar system experiments. Phys. Rev. Lett., 95(26):261102, Dec 2005.

[999] G. J. Olmo. Post-Newtonian constraints on $\mathrm{f}(\mathrm{R})$ cosmologies in metric and Palatini formalism. Phys. Rev. D, 72(8):083505, Oct. 2005, arXiv:gr-qc/0505135

[1000] G. J. Olmo. The Gravity Lagrangian According to Solar System Experiments. Physical Review Letters, 95(26):261102, Dec. 2005, arXiv:gr-qc/0505101.

[1001] G. J. Olmo. Limit to general relativity in $\mathrm{f}(\mathrm{R})$ theories of gravity. Phys. Rev. D, 75(2):023511, Jan. 2007, arXiv:gr-qc/0612047

[1002] G. J. Olmo, H. Sanchis-Alepuz, and S. Tripathi. Dynamical aspects of generalized Palatini theories of gravity. Phys. Rev. D, 80(2):024013, July 2009, 0907.2787.

[1003] M. Ostrogradsky. Memoires de l'Academie Imperiale des Science de Saint-Petersbourg, 4:385, 1850 .

[1004] A. Oukouiss. Exact and asymptotic solutions to tensor-scalar cosmologies. Nuclear Physics B, 486:413-422, Feb. 1997.

[1005] J. M. Overduin and P. S. Wesson. Kaluza-Klein gravity. Phys. Rept., 283:303-380, 1997, grqc/9805018

[1006] H. Oyaizu. Nonlinear evolution of $\mathrm{f}(\mathrm{R})$ cosmologies. I. Methodology. Phys. Rev. D, 78(12):123523, Dec. 2008, 0807.2449

[1007] H. Oyaizu, M. Lima, and W. Hu. Nonlinear evolution of $f(R)$ cosmologies. II. Power spectrum. Phys. Rev. D, 78(12):123524, Dec. 2008, 0807.2462.

[1008] A. Padilla. Brane world cosmology and holography. 2002, hep-th/0210217

[1009] A. Padilla. CFTs on non-critical braneworlds. Phys. Lett., B528:274-282, 2002, hep-th/0111247

[1010] A. Padilla. Surface terms and the Gauss-Bonnet Hamiltonian. Class. Quant. Grav., 20:3129-3150, 2003, gr-qc/0303082

[1011] A. Padilla. Ghost-free braneworld bigravity. Class. Quant. Grav., 21:2899-2918, 2004, hepth/0402079

[1012] A. Padilla. Cosmic acceleration from asymmetric branes. Class. Quant. Grav., 22:681-694, 2005, hep-th/0406157

[1013] A. Padilla. Infra-red modification of gravity from asymmetric branes. Class. Quant. Grav., 22:1087-1104, 2005, hep-th/0410033

[1014] A. Padilla. A covariant approach to braneworld holography. Class. Quant. Grav., 23:3983-3992, 2006, hep-th/0512083

[1015] A. Padilla. A short review of 'DGP Specteroscopy'. J. Phys., A40:6827-6834, 2007, hepth/0610093

[1016] A. Padilla. The good, the bad and the ugly .... of Horava gravity. J.Phys.Conf.Ser., 259:012033, 2010, 1009.4074 
[1017] A. Padilla, P. M. Saffin, and S.-Y. Zhou. Bi-galileon theory I: motivation and formulation. 2010, 1007.5424

[1018] A. Padilla, P. M. Saffin, and S.-Y. Zhou. Bi-galileon theory II: Phenomenology. 2010, 1008.3312.

[1019] A. Padilla, P. M. Saffin, and S.-Y. Zhou. Multi-galileons, solitons and Derrick's theorem. 2010, 1008.0745 .

[1020] A. Palatini. Deduzione invariantiva deUe equazioni gravitazionali dal principio di Hamilton. Rend. Circ. Mat. Palermo, 43:203, 1919.

[1021] E. Papantonopoulos and A. Papazoglou. Cosmological evolution of a purely conical codimension-2 brane world. JHEP, 0509:012, 2005, hep-th/0507278

[1022] E. Papantonopoulos, A. Papazoglou, and V. Zamarias. Regularization of conical singularities in warped six- dimensional compactifications. JHEP, 03:002, 2007, hep-th/0611311

[1023] E. Papantonopoulos, A. Papazoglou, and V. Zamarias. Induced cosmology on a regularized brane in six-dimensional flux compactification. Nucl. Phys., B797:520-536, 2008, 0707.1396.

[1024] A. Papapetrou. Spinning test particles in general relativity. 1 Proc.Roy.Soc.Lond., A209: 248, 1951.

[1025] A. Papazoglou and T. P. Sotiriou. Strong coupling in extended Horava-Lifshitz gravity. Phys. Lett., B685:197-200, 2010, 0911.1299.

[1026] C. Park, J. Hwang, and H. Noh. Constraints on a $f(R)$ gravity dark energy model with early scaling evolution. ArXiv e-prints, Dec. 2010, 1012.1662.

[1027] B. C. Paul, S. Mukherjee, and R. Tavakol. Singular instantons in higher derivative theories. Phys. Rev. D, 65(6):064020, Mar. 2002, arXiv:hep-th/0201009.

[1028] J. A. Peacock. Cosmological Physics. Jan. 1999.

[1029] E. Pechlaner and R. Sexl. On quadratic lagrangians in General Relativity. Communications in Mathematical Physics, 2:165-175, Dec. 1966.

[1030] P. Peebles. The void phenomenon. Astrophys.J., 557:495-504, 2001, astro-ph/0101127

[1031] P. J. E. Peebles. The large-scale structure of the universe. 1980.

[1032] P. Peldan. Actions for gravity, with generalizations: A Review. Class. Quant. Grav., 11:10871132, 1994, gr-qc/9305011

[1033] S. E. Perez Bergliaffa Constraining $\mathrm{f}(\mathrm{R})$ theories with the energy conditions. Phys. Lett. B, 642:311-314, 2006, gr-qc/0608072

[1034] S. Perlmutter et al. Measurements of Omega and Lambda from 42 high redshift supernovae. Astrophys.J., 517:565-586, 1999, astro-ph/9812133 The Supernova Cosmology Project.

[1035] F. Perrotta, C. Baccigalupi, and S. Matarrese. Extended quintessence. Phys. Rev. D, 61(2):023507, Dec 1999.

[1036] F. Piazza. Modifying Gravity in the Infra-Red by imposing an 'Ultra-Strong' Equivalence Principle. 2009, 0904.4299.

[1037] F. Piazza. The IR-Completion of Gravity: What happens at Hubble Scales? New J.Phys., 11:113050, 2009, 0907.0765.

[1038] L. Pilo, R. Rattazzi, and A. Zaffaroni. The fate of the radion in models with metastable graviton. JHEP, 07:056, 2000, hep-th/0004028

[1039] S. Pireaux and J. Rozelot. Solar quadrupole moment and purely relativistic gravitation contributions to Mercury's perihelion advance. Ap\&SS, 284:1159-1194, 2003, arXiv:astro-ph/0109032

[1040] E. V. Pitjeva. Experimental testing of relativistic effects, variability of the gravitational constant and topography of Mercury surface from radar observations 1964-1989. Celestial Mechanics and Dynamical Astronomy, 55:313-321, Apr. 1993.

[1041] E. V. Pitjeva. Modern Numerical Ephemerides of Planets and the Importance of Ranging Observations for Their Creation. Celestial Mechanics and Dynamical Astronomy, 80:249-271, July 2001.

[1042] J. F. Plebański. On the separation of Einsteinian substructures. Journal of Mathematical Physics, 18:2511-2520, Dec. 1977.

[1043] L. Pogosian and A. Silvestri. Pattern of growth in viable $\mathrm{f}(\mathrm{R})$ cosmologies. Phys. Rev. D $77(2): 023503$, Jan. 2008, 0709.0296

[1044] J. Polchinski. Dirichlet-Branes and Ramond-Ramond Charges. Phys. Rev. Lett., 75:4724-4727, 1995, hep-th/9510017

[1045] M. Pospelov and Y. Shang. On Lorentz violation in Horava-Lifshitz type theories. 2010, 1010.5249

[1046] O. Pujolas, I. Sawicki, A. Vikman, The Imperfect Fluid behind Kinetic Gravity Braiding arXiv:1103.5360 [hep-th]].

[1047] T. Qiu and K. C. Yang. Non-Gaussianities of single field inflation with nonminimal coupling. Phys. Rev. D, 83(8):084022, 2011, 1012.1697. 
[1048] F. Quevedo. Lectures on string/brane cosmology. Class. Quant. Grav., 19:5721-5779, 2002, hep-th/0210292

[1049] I. Quiros, Y. Leyva, and Y. Napoles. Note on stability of de Sitter solutions of $f(R)$ theories. Phys. Rev. D, 80(2):024022, July 2009, 0906.1190.

[1050] A. Rajaraman. Newtonian Gravity in Theories with Inverse Powers of R. ArXiv Astrophysics e-prints, Nov. 2003, arXiv:astro-ph/0311160

[1051] L. Randall and R. Sundrum. A large mass hierarchy from a small extra dimension. Phys. Rev. Lett., 83:3370-3373, 1999, hep-ph/9905221

[1052] L. Randall and R. Sundrum. An alternative to compactification. Phys. Rev. Lett., 83:4690-4693, 1999, hep-th/9906064

[1053] R. H. Rapp. An estimate of equatorial gravity from terrestrial and satellite data. Geophys. Res. Lett., 14:730-732, July 1987.

[1054] S. M. M. Rasouli, M. Farhoudi, and H. R. Sepangi. An anisotropic cosmological model in a modified Brans-Dicke theory. Classical and Quantum Gravity, 28(15):155004, 2011, 1105.5086.

[1055] P. Rastall. A theory of gravity. Canadian Journal of Physics, 54:66-75, Jan. 1976.

[1056] P. Rastall. A note on a theory of gravity. Canadian Journal of Physics, 55:38-42, Jan. 1977.

[1057] M. J. Rebouças and J. Santos. Gödel-type universes in $\mathrm{f}(\mathrm{R})$ gravity. Phys. Rev. D, 80(6):063009, Sept. 2009, 0906.5354.

[1058] B. A. Reid, W. J. Percival, D. J. Eisenstein, L. Verde, D. N. Spergel, R. A. Skibba, N. A. Bahcall, T. Budavari, J. A. Frieman, M. Fukugita, J. R. Gott, J. E. Gunn, Ž. Ivezić, G. R. Knapp, R. G. Kron, R. H. Lupton, T. A. McKay, A. Meiksin, R. C. Nichol, A. C. Pope, D. J. Schlegel, D. P. Schneider, C. Stoughton, M. A. Strauss, A. S. Szalay, M. Tegmark, M. S. Vogeley, D. H. Weinberg, D. G. York, and I. Zehavi. Cosmological constraints from the clustering of the Sloan Digital Sky Survey DR7 luminous red galaxies. MNRAS, 404:60-85, May 2010, 0907.1659.

[1059] S. Renaux-Petel, Orthogonal non-Gaussianities from Dirac-Born-Infeld Galileon inflation Class. Quant. Grav. 28 (2011) 182001. arXiv:1105.6366 [astro-ph.CO]].

[1060] R. Reyes, R. Mandelbaum, U. Seljak, T. Baldauf, J. E. Gunn, L. Lombriser, and R. E. Smith. Confirmation of general relativity on large scales from weak lensing and galaxy velocities. Nature, 464:256-258, Mar. 2010, 1003.2185.

[1061] A. Riazuelo and J.-P. Uzan. Cosmological observations in scalar-tensor quintessence. Phys. Rev. $D, 66(2): 023525$, Jul 2002.

[1062] A. Riazuelo, F. Vernizzi, D. A. Steer, and R. Durrer. Gauge invariant cosmological perturbation theory for braneworlds. 2002, hep-th/0205220

[1063] A. G. Riess et al. Observational Evidence from Supernovae for an Accelerating Universe and a Cosmological Constant. Astron. J., 116:1009-1038, 1998, astro-ph/9805201

[1064] A. G. Riess et al. Type Ia supernova discoveries at z \&gt; 1 from the Hubble Space Telescope: Evidence for past deceleration and constraints on dark energy evolution. Astrophys.J., 607:665687, 2004, astro-ph/0402512

[1065] S. Rippl, H. van Elst, R. Tavakol, and D. Taylor. Kinematics and dynamics of $f(R)$ theories of gravity. General Relativity and Gravitation, 28:193-205, Feb. 1996, arXiv:gr-qc/9511010

[1066] D. C. Rodrigues. Evolution of Anisotropies in Eddington-Born-Infeld Cosmology. Phys.Rev., D78:063013, 2008, 0806.3613.

[1067] N. Rosen. General Relativity and Flat Space. II. Physical Review, 57:150-153, Jan. 1940.

[1068] N. Rosen. A bi-metric theory of gravitation. General Relativity and Gravitation, 4:435-447, Dec. 1973.

[1069] N. Rosen. Bimetric gravitation theory on a cosmological basis. General Relativity and Gravitation, 9:339-351, Apr. 1978.

[1070] E. Rosenthal. Extended Palatini action for general relativity. Phys. Rev. D, 80(8):084029, Oct. 2009, 0809.2053.

[1071] N. P. Ross, J. da Angela, T. Shanks, D. A. Wake, R. D. Cannon, et al. The 2dF-SDSS LRG and QSO Survey: The 2-Point Correlation Function and Redshift-Space Distortions. Mon.Not.Roy.Astron.Soc., 2006, astro-ph/0612400

[1072] V. A. Rubakov. Large and infinite extra dimensions: An introduction. Phys. Usp., 44:871-893, 2001, hep-ph/0104152

[1073] V. A. Rubakov. Strong coupling in brane-induced gravity in five dimensions. 2003, hepth/0303125

[1074] V. A. Rubakov and M. E. Shaposhnikov. Do We Live Inside a Domain Wall? Phys. Lett., B125:136-138, 1983.

[1075] V. A. Ruban and A. M. Finkel'Shtejn. Generalization of the Taub-Kazner cosmological metric 
in the scalar-tensor gravitation theory. Nuovo Cimento Lettere, 5:289-293, 1972.

[1076] H. Ruegg and M. Ruiz-Altaba. The Stuckelberg field. Int.J.Mod.Phys., A19:3265-3348, 2004, hep-th/0304245

[1077] M. L. Ruggiero. Gravitational lensing and $\mathrm{f}(\mathrm{R})$ theories in the Palatini approach. General Relativity and Gravitation, 41:1497-1509, July 2009, 0712.3218.

[1078] M. L. Ruggiero and L. Iorio. Solar System planetary orbital motions and f(R) theories of gravity. J. Cosmology Astropart. Phys., 1:10, Jan. 2007, arXiv:gr-qc/0607093

[1079] G. Rybka, M. Hotz, L. J. Rosenberg, S. J. Asztalos, G. Carosi, C. Hagmann, D. Kinion, K. van Bibber, J. Hoskins, C. Martin, P. Sikivie, D. B. Tanner, R. Bradley, and J. Clarke. A Search for Scalar Chameleons with ADMX. ArXiv e-prints, Apr. 2010, 1004.5160.

[1080] S. Rydbeck, M. Fairbairn, and A. Goobar. Testing the DGP model with ESSENCE. JCAP, 0705:003, 2007, astro-ph/0701495

[1081] E. Sagi. Preferred frame parameters in the tensor-vector-scalar theory of gravity and its generalization. Phys. Rev., D80:044032, 2009, 0905.4001.

[1082] E. Sagi and J. D. Bekenstein. Black holes in the TeVeS theory of gravity and their thermodynamics. Phys. Rev., D77:024010, 2008, 0708.2639.

[1083] D. Sahdev. Perfect fluid higher dimensional cosmologies. Phys. Rev., D30:2495-2507, 1984.

[1084] D. Sahdev. Towards a Realistic Kaluza-Klein Cosmology. Phys. Lett., B137:155-159, 1984.

[1085] D. Sahdev. Perfect fluid higher dimensional cosmologies. 2. Phys. Rev., D39:3155, 1989.

[1086] V. Sahni, Y. Shtanov, Brane world models of dark energy JCAP 0311 (2003) 014. astro$\mathrm{ph} / 0202346$

[1087] V. Sahni, M. Sami, and T. Souradeep. Relic gravity waves from brane world inflation. Phys. Rev., D65:023518, 2002, gr-qc/0105121

[1088] N. Sakai and K. Maeda. Bubble dynamics and space-time structure in extended inflation. Phys. Rev. D, 48:5570-5575, Dec. 1993.

[1089] N. Sakai, J. Yokoyama, and K. Maeda. Monopole inflation in Brans-Dicke theory. Phys. Rev. D 59(10):103504, May 1999, arXiv:gr-qc/9811024

[1090] M. Sami, A. Toporensky, P. Tretjakov, and S. Tsujikawa. The fate of (phantom) dark energy universe with string curvature corrections. Physics Letters B, 619:193-200, July 2005, arXiv:hepth/0504154

[1091] H. Sanctuary and R. Sturani. Effective field theory analysis of the self-interacting chameleon. General Relativity and Gravitation, 42:1953-1967, Aug. 2010, 0809.3156

[1092] R. Sanders. The Universal Faber-Jackson Relation. 2010, 1002.2765.

[1093] R. H. Sanders. A stratified framework for scalar-tensor theories of Modified Dynamics. Astrophys. J., 480:492-502, 1997, astro-ph/9612099

[1094] R. H. Sanders and S. S. McGaugh. Modified Newtonian dynamics as an alternative to dark matter. Ann.Rev.Astron.Astrophys., 40:263-317, 2002, astro-ph/0204521

[1095] D. I. Santiago, D. Kalligas, and R. V. Wagoner. Scalar-tensor cosmologies and their late time evolution. Phys. Rev. D, 58(12):124005, Nov 1998.

[1096] C. Santos and R. Gregory. Cosmology in Brans-Dicke Theory with a Scalar Potential. Annals of Physics, 258:111-134, July 1997, arXiv:gr-qc/9611065

[1097] A. K. Sanyal. If Gauss Bonnet interaction plays the role of dark energy. Physics Letters B, 645:1-5, Feb. 2007, arXiv:astro-ph/0608104

[1098] E. N. Saridakis. Aspects of Horava-Lifshitz cosmology. 2011, 1101.0300.

[1099] A. Savas Arapoglu, C. Deliduman, and K. Yavuz Eksi. Constraints on Perturbative f(R) Gravity via Neutron Stars. ArXiv e-prints, Mar. 2010, 1003.3179.

[1100] I. Savonije and E. P. Verlinde. CFT and entropy on the brane. Phys. Lett., B507:305-311, 2001, hep-th/0102042

[1101] I. Sawicki and S. M. Carroll. Cosmological structure evolution and cmb anisotropies in DGP braneworlds. 2005, astro-ph/0510364

[1102] I. Sawicki and W. Hu. Stability of cosmological solutions in $\mathrm{f}(\mathrm{R})$ models of gravity. Phys. Rev. D, 75(12):127502, June 2007, arXiv:astro-ph/0702278

[1103] I. Sawicki, Y.-S. Song, and W. Hu. Near-horizon solution for DGP perturbations. Phys. Rev., D75:064002, 2007, astro-ph/0606285

[1104] D. W. Sciama. On the analogy between charge and spin in General Relativity. (in Recent developments in General Relativity), Pergamon + PWD (Oxford), 415, 1962.

[1105] D. W. Sciama. The physical structure of General Relativity. Rev. Mod. Phys., 36, 463, 1962.

[1106] B. E. Schaefer. The Hubble Diagram to Redshift greater than 6 from 69 Gamma-Ray Bursts. Astrophys.J., 660:16-46, 2007, astro-ph/0612285. 
[1107] M. A. Scheel, S. L. Shapiro, and S. A. Teukolsky. Collapse to black holes in brans-dicke theory. ii. comparison with general relativity. Phys. Rev. D, 51(8):4236-4249, Apr 1995.

[1108] C. A. O. Schelpe. Chameleon-photon mixing in a primordial magnetic field. Phys. Rev. D, 82(4):044033, Aug. 2010, 1003.0232.

[1109] C. Schimd, J. Uzan, and A. Riazuelo. Weak lensing in scalar-tensor theories of gravity. Phys. Rev. D, 71(8):083512, Apr. 2005, arXiv:astro-ph/0412120

[1110] S. Schlamminger, K. Choi, T. A. Wagner, J. H. Gundlach, and E. G. Adelberger. Test of the Equivalence Principle Using a Rotating Torsion Balance. Physical Review Letters, 100(4):041101, Feb. 2008, 0712.0607

[1111] F. Schmidt. Cosmological Simulations of Normal-Branch Braneworld Gravity. Phys.Rev., D80:123003, 2009, 0910.0235.

[1112] F. Schmidt. Self-consistent cosmological simulations of dgp braneworld gravity. Phys. Rev., D80:043001, 2009

[1113] F. Schmidt, W. Hu, and M. Lima. Spherical Collapse and the Halo Model in Braneworld Gravity. Phys.Rev., D81:063005, 2010, 0911.5178.

[1114] F. Schmidt, M. Lima, H. Oyaizu, and W. Hu. Nonlinear evolution of $\mathrm{f}(\mathrm{R})$ cosmologies. III. Halo statistics. Phys. Rev. D, 79(8):083518, Apr. 2009, 0812.0545.

[1115] F. Schmidt, A. Vikhlinin, and W. Hu. Cluster constraints on $\mathrm{f}(\mathrm{R})$ gravity. Phys. Rev. D, 80(8):083505, Oct. 2009, 0908.2457.

[1116] H. Schmidt. On static spherically symmetric solutions of the Bach-Einstein gravitational field equations. Astronomische Nachrichten, 306:67-70, 1985.

[1117] H. Schmidt. Variational derivatives of arbitrarily high order and multi-inflation cosmological models. Classical and Quantum Gravity, 7:1023-1031, June 1990.

[1118] H. Schmidt. Fourth Order Gravity: Equations, History, and Applications to Cosmology. International Journal of Geometric Methods in Modern Physics, 4:209, 2007, arXiv:gr-qc/0602017

[1119] B. F. Schutz. Perfect Fluids in General Relativity: Velocity Potentials and a Variational Principle. Phys. Rev. D, 2:2762-2773, Dec. 1970.

[1120] M. D. Schwartz. The emergence of localized gravity. Phys. Lett., B502:223-228, 2001, hepth/0011177

[1121] R. Scoccimarro. Large-Scale Structure in Brane-Induced Gravity I. Perturbation Theory. Phys.Rev., D80:104006, 2009, 0906.4545.

[1122] S. S. Seahra and C. G. Böhmer. Einstein static universes are unstable in generic $f(R)$ models Phys. Rev. D, 79(6):064009, Mar. 2009, 0901.0892.

[1123] S. S. Seahra and W. Hu. Analytic Description of DGP Perturbations on All Scales. Phys.Rev. D82:124015, 2010, 1007.4242.

[1124] M. D. Seifert. Stability of spherically symmetric solutions in modified theories of gravity. Phys. Rev., D76:064002, 2007, gr-qc/0703060

[1125] A. Serna and J. M. Alimi. Scalar-tensor cosmological models. Phys. Rev. D, 53(6):3074-3086, Mar 1996.

[1126] A. Serna, R. Dominguez-Tenreiro, and G. Yepes. Primordial nucleosynthesis bounds on the Brans-Dicke theory. ApJ, 391:433-442, June 1992.

[1127] T. R. Seshadri. Generation of density perturbations by inflation in scalar-tensor gravity theories. Physics Letters B, 277:67-72, Feb. 1992.

[1128] H. Shan, M. Feix, B. Famaey, and H. Zhao. An analytic model for non-spherical lenses in covariant MOdified Newtonian Dynamics. Mon. Not. Roy. Astron. Soc., 387:1303-1312, 2008, 0804.2668.

[1129] C. Shao, R. Cai, B. Wang, and R. Su. An alternative explanation of the conflict between 1/R gravity and solar system tests. Physics Letters B, 633:164-166, Feb. 2006, arXiv:gr-qc/0511034

[1130] I. I. Shapiro. Fourth Test of General Relativity. Physical Review Letters, 13:789-791, Dec. 1964.

[1131] S. S. Shapiro, J. L. Davis, D. E. Lebach, and J. S. Gregory. Measurement of the Solar Gravitational Deflection of Radio Waves using Geodetic Very-Long-Baseline Interferometry Data, 1979 1999. Physical Review Letters, 92(12):121101, Mar. 2004.

[1132] M. Sharif and M. Farasat Shamir. Exact solutions of Bianchi-type I and V spacetimes in the f(R) theory of gravity. Classical and Quantum Gravity, 26(23):235020, Dec. 2009, 0910.5787.

[1133] M. Sharif and M. F. Shamir. Non-vacuum Bianchi types I and V in f(R) gravity. General Relativity and Gravitation, 42:2643-2655, Nov. 2010, 1005.2798.

[1134] D. J. Shaw and J. D. Barrow. Local experiments see cosmologically varying constants. Phys. Lett., B639:596-599, 2006, gr-qc/0512117.

[1135] D. J. Shaw and J. D. Barrow. The local effects of cosmological variations in physical 'constants' and scalar fields. I: Spherically symmetric spacetimes. Phys. Rev., D73:123505, 2006, 
gr-qc/0512022

[1136] D. J. Shaw and J. D. Barrow. The local effects of cosmological variations in physical 'constants' and scalar fields. II: Quasi-spherical spacetimes. Phys. Rev., D73:123506, 2006, gr-qc/0601056.

[1137] R. K. Sheth and G. Tormen. Large scale bias and the peak background split. Mon.Not.Roy.Astron.Soc., 308:119, 1999, astro-ph/9901122

[1138] T. Shiromizu and D. Ida. Anti-de Sitter no hair, AdS/CFT and the brane-world. Phys. Rev., D64:044015, 2001, hep-th/0102035

[1139] T. Shiromizu and K. Koyama. Low energy effective theory for two brane systems: Covariant curvature formulation. Phys. Rev., D67:084022, 2003, hep-th/0210066

[1140] T. Shiromizu, K.-i. Maeda, and M. Sasaki. The Einstein equation on the 3-brane world. Phys. Rev. D62:024012, 2000, gr-qc/9910076

[1141] Y. Shtanov, V. Sahni, Bouncing brane worlds. Phys. Lett. B557 (2003) 1-6. gr-qc/0208047.

[1142] Y. Shtanov, V. Sahni, Unusual cosmological singularities in brane world models. Class. Quant. Grav. 19 (2002) L101-L107. gr-qc/0204040.

[1143] Y. Shtanov, V. Sahni, A. Shafieloo, A. Toporensky, Induced cosmological constant and other features of asymmetric brane embedding. JCAP 0904 (2009) 023. arXiv:0901.3074 [gr-qc]].

[1144] F.-W. Shu. Extended Hořava Gravity with Physical Ground-State Wavefunction. 2010, 1009.3677.

[1145] F. P. Silva and K. Koyama. Self-Accelerating Universe in Galileon Cosmology. Phys.Rev., D80:121301, 2009, 0909.4538.

[1146] J. D. Simon and M. Geha. The Kinematics of the Ultra-Faint Milky Way Satellites: Solving the Missing Satellite Problem. Astrophys.J., 670:313-331, 2007, 0706.0516.

[1147] C. Skordis. TeVeS cosmology: Covariant formalism for the background evolution and linear perturbation theory. Phys. Rev., D74:103513, 2006, astro-ph/0511591

[1148] C. Skordis. Generalizing TeVeS Cosmology. Phys. Rev., D77:123502, 2008, 0801.1985.

[1149] C. Skordis. Consistent cosmological modifications to the Einstein equations. Phys. Rev. D, 79(12):123527, June 2009, 0806.1238.

[1150] C. Skordis. Eddington-Born-Infeld theory and the dark sector. Nucl.Phys.Proc.Suppl., 194:338343,2009

[1151] C. Skordis. Modifications of gravity. Nucl.Phys.Proc.Suppl., 194:332-337, 2009.

[1152] C. Skordis. The Tensor-Vector-Scalar theory and its cosmology. Class. Quant. Grav., 26:143001, 2009, 0903.3602

[1153] C. Skordis, D. F. Mota, P. G. Ferreira, and C. Boehm. Large scale structure in Bekenstein's theory of relativistic MOND. Phys. Rev. Lett., 96:011301, 2006, astro-ph/0505519

[1154] C. Skordis and T. Zlosnik. The Geometry Of Modified Newtonian Dynamics. 2011, 1101.6019.

[1155] A. Slosar, A. Melchiorri, and J. Silk. Did Boomerang hit MOND? Phys. Rev., D72:101301, 2005, astro-ph/0508048

[1156] R. Smith et al. Stable clustering, the halo model and nonlinear cosmological power spectra. Mon.Not.Roy.Astron.Soc., 341:1311, 2003, astro-ph/0207664

[1157] Y. Song, W. Hu, and I. Sawicki. Large scale structure of $\mathrm{f}(\mathrm{R})$ gravity. Phys. Rev. D, 75(4):044004 Feb. 2007, arXiv:astro-ph/0610532

[1158] Y. Song, H. Peiris, and W. Hu. Cosmological constraints on $\mathrm{f}(\mathrm{R})$ acceleration models. Phys. Rev. D, 76(6):063517, Sept. 2007, 0706.2399.

[1159] Y. Song, G. Zhao, D. Bacon, K. Koyama, R. C. Nichol, and L. Pogosian. Complementarity of Weak Lensing and Peculiar Velocity Measurements in Testing General Relativity. ArXiv e-prints, Nov. 2010, 1011.2106.

[1160] Y.-S. Song. Large Scale Structure Formation of normal branch in DGP brane world model. Phys.Rev., D77:124031, 2008, 0711.2513.

[1161] Y.-S. Song, I. Sawicki, and W. Hu. Large-scale tests of the DGP model. Phys. Rev., D75:064003, 2007, astro-ph/0606286

[1162] T. P. Sotiriou. Constraining $\mathrm{f}(\mathrm{R})$ gravity in the Palatini formalism. Classical and Quantum Gravity, 23:1253-1267, Feb. 2006, arXiv:gr-qc/0512017

[1163] T. P. Sotiriou. The nearly Newtonian regime in non-linear theories of gravity. General Relativity and Gravitation, 38:1407-1417, Sept. 2006, arXiv:gr-qc/0507027.

[1164] T. P. Sotiriou. Unification of inflation and cosmic acceleration in the Palatini formalism. Phys. Rev. D, 73(6):063515, Mar. 2006, arXiv:gr-qc/0509029.

[1165] T. P. Sotiriou. Curvature scalar instability in $\mathrm{f}(\mathrm{R})$ gravity. Physics Letters B, 645:389-392, Feb. 2007, arXiv:gr-qc/0611107

[1166] T. P. Sotiriou. Horava-Lifshitz gravity: a status report. 2010, 1010.3218

[1167] T. P. Sotiriou and V. Faraoni. f(R) theories of gravity. Reviews of Modern Physics, 82:451-497, 
Jan. 2010, 0805.1726.

[1168] T. P. Sotiriou and S. Liberati. Metric-affine $\mathrm{f}(\mathrm{R})$ theories of gravity. Annals of Physics, 322:935966, Apr. 2007, arXiv:gr-qc/0604006

[1169] T. P. Sotiriou, M. Visser, and S. Weinfurtner. Phenomenologically viable Lorentz-violating quantum gravity. Phys.Rev.Lett., 102:251601, 2009, 0904.4464.

[1170] T. P. Sotiriou, M. Visser, and S. Weinfurtner. Quantum gravity without Lorentz invariance. JHEP, 0910:033, 2009, 0905.2798.

[1171] M. E. Soussa and R. P. Woodard. Letter: The Force of Gravity from a Lagrangian Containing Inverse Powers of the Ricci Scalar. General Relativity and Gravitation, 36:855-862, Apr. 2004, arXiv:astro-ph/0308114

[1172] D. Spergel et al. First year Wilkinson Microwave Anisotropy Probe (WMAP) observations: Determination of cosmological parameters. Astrophys.J.Suppl., 148:175-194, 2003, astro-ph/0302209

[1173] D. N. Spergel et al. Wilkinson Microwave Anisotropy Probe (WMAP) three year results: Implications for cosmology. Astrophys. J. Suppl., 170:377, 2007, astro-ph/0603449

[1174] D. N. Spergel and P. J. Steinhardt. Observational evidence for selfinteracting cold dark matter. Phys.Rev.Lett., 84:3760-3763, 2000, astro-ph/9909386

[1175] S. Speziale. Bi-metric theory of gravity from the non-chiral Plebanski action. Phys.Rev., D82:064003, 2010, 1003.4701.

[1176] I. Stairs, A. Faulkner, A. Lyne, M. Kramer, D. Lorimer, M. McLaughlin, R. Manchester, G. Hobbs, F. Camilo, A. Possenti, M. Burgay, N. D’Amico, P. Freire, and P. Gregory. Discovery of three wide-orbit binary pulsars: Implications for binary evolution and equivalence principles. Astrophys. J., 632:1060-1068, 2005.

[1177] I. H. Stairs. Testing General Relativity with Pulsar Timing. Living Reviews in Relativity, 6:5, Sept. 2003, arXiv:astro-ph/0307536

[1178] A. A. Starobinskii. The Perturbation Spectrum Evolving from a Nonsingular Initially De-Sitter Cosmology and the Microwave Background Anisotropy. Soviet Astronomy Letters, 9:302, June 1983.

[1179] A. A. Starobinsky. A new type of isotropic cosmological models without singularity. Physics Letters B, 91(1):99 - 102, 1980.

[1180] A. A. Starobinsky. Disappearing cosmological constant in $\mathrm{f}(\mathrm{R})$ gravity. JETP Lett., 86:157-163, 2007, 0706.2041

[1181] A. A. Starobinsky and J. Yokoyama. Density fluctuations in Brans-Dicke inflation. Feb. 1995, arXiv:gr-qc/9502002

[1182] J. H. Steffen and Gammev Collaboration. Constraints on chameleons and axions-like particles from the GammeV experiment. In Identification of Dark Matter 2008, 2008, 0810.5070.

[1183] J. H. Steffen, A. Upadhye, A. Baumbaugh, A. S. Chou, P. O. Mazur, R. Tomlin, A. Weltman, and W. Wester. Laboratory constraints on chameleon dark energy and power-law fields. ArXiv e-prints, Oct. 2010, 1010.0988.

[1184] P. J. Steinhardt. Recent advances in extended inflationary cosmology. Classical and Quantum Gravity, 10:33, Dec. 1993, arXiv:astro-ph/9212004

[1185] P. J. Steinhardt and F. S. Accetta. Hyperextended inflation. Physical Review Letters, 64:27402743, June 1990.

[1186] K. S. Stelle. Renormalization of higher-derivative quantum gravity. Phys. Rev. D, 16(4):953-969, Aug 1977.

[1187] K. S. Stelle. Classical gravity with higher derivatives. General Relativity and Gravitation, 9:353371, Apr. 1978.

[1188] J. M. Stewart and M. Walker. Perturbations of spacetimes in general relativity. Proc. Roy. Soc. Lond., A341:49-74, 1974.

[1189] H. Stoica, S. H. H. Tye, and I. Wasserman. Cosmology in the Randall-Sundrum brane world scenario. Phys. Lett., B482:205-212, 2000, hep-th/0004126

[1190] E. e. a. Sullivan. SNLS3: Constraints on Dark Energy Combining the Supernova Legacy Survey Third Year Data with Other Probes. Astrophys. J., 737:102, 2011, 1104.1444.

[1191] M. Susperregi. How typical is General Relativity in Brans-Dicke chaotic inflation? Dec. 1997, arXiv:gr-qc/9712031

[1192] M. Susperregi and A. Mazumdar. Extended inflation with an exponential potential. Phys. Rev. D, 58(8):083512, Oct. 1998, arXiv:gr-qc/9804081

[1193] M. P. K. Susperregi. Spectrum of density fluctuations in Brans-Dicke chaotic inflation. Phys. Rev. D, 55:560-572, Jan. 1997, arXiv:astro-ph/9606018

[1194] L. Susskind. The anthropic landscape of string theory. 2003, hep-th/0302219 
[1195] J. Tafel. A non-singular homogeneous universe with torsion. Phys. Lett., A45: 341, 1975.

[1196] T. Takahashi, J. Soda, Chiral Primordial Gravitational Waves from a Lifshitz Point Phys. Rev. Lett. 102 (2009) 231301. arXiv:0904.0554 [hep-th]].

[1197] C. Talmadge, J. Berthias, R. W. Hellings, and E. M. Standish. Model-independent constraints on possible modifications of Newtonian gravity. Physical Review Letters, 61:1159-1162, Sept. 1988

[1198] T. Tamaki. Post-Newtonian parameters in the tensor-vector-scalar theory. Phys. Rev. D77:124020, 2008, 0803.4309.

[1199] T. Tamaki and S. Tsujikawa. Revisiting chameleon gravity: Thin-shell and no-shell fields with appropriate boundary conditions. Phys. Rev. D, 78(8):084028, Oct. 2008, 0808.2284.

[1200] N. Tanahashi and T. Tanaka. Black holes in braneworld models. 2011, 1105.2997.

[1201] T. Tanaka. Classical black hole evaporation in Randall-Sundrum infinite braneworld. Prog. Theor. Phys. Suppl., 148:307-316, 2003, gr-qc/0203082.

[1202] F. Tangherlini. Schwarzschild field in $\mathrm{n}$ dimensions and the dimensionality of space problem. $I l$ Nuovo Cimento (1955-1965), 27(3):636-651, 1963.

[1203] M. Tegmark et al. Cosmological Constraints from the SDSS Luminous Red Galaxies. Phys.Rev., D74:123507, 2006, astro-ph/0608632

[1204] H. Thirring. Über die Wirkung rotierender ferner Massen in der Einsteinschen Gravitationstheorie. Physikalische Zeitschrift, 19:33, 1918.

[1205] S. Thomas. Baryons and dark matter from the late decay of a supersymmetric condensate. Physics Letters B, 356(2-3):256 - 263, 1995.

[1206] I. Thongkool, M. Sami, and S. R. Choudhury. How delicate are the f(R) gravity models with a disappearing cosmological constant? Phys. Rev. D, 80(12):127501, Dec. 2009, 0908.1693.

[1207] K. S. Thorne and C. M. Will. Theoretical Frameworks for Testing Relativistic Gravity. I. Foundations. ApJ, 163:595, Feb. 1971.

[1208] A. J. Tolley, C. Burgess, D. Hoover, and Y. Aghababaie. Bulk singularities and the effective cosmological constant for higher co-dimension branes. JHEP, 0603:091, 2006, hep-th/0512218

[1209] T. Torii, H. Yajima, and K. Maeda. Dilatonic black holes with a Gauss-Bonnet term. Phys. Rev. D, 55:739-753, Jan. 1997, arXiv:gr-qc/9606034

[1210] D. F. Torres. Nucleosynthesis bounds on scalar-tensor gravity: power-law couplings. Physics Letters B, 359:249-253, Feb. 1995.

[1211] D. F. Torres. Boson stars in general scalar-tensor gravitation: Equilibrium configurations. Phys. Rev. D, 56(6):3478-3484, Sep 1997.

[1212] D. F. Torres. Slow roll inflation in nonminimally coupled theories: hyperextended gravity approach. Physics Letters A, 225:13-17, Feb. 1997, arXiv:gr-qc/9610021

[1213] D. F. Torres, A. R. Liddle, and F. E. Schunck. Gravitational memory of boson stars. Phys. Rev. D, 57(8):4821-4825, Apr 1998 .

[1214] A. Trautman. On the Einstein-Cartan equations III Bull. Acad. Pol. Sci., Ser. Sci. Math. Astron. Phys., 20: 895, 1972.

[1215] A. Trautman. Einstein-Cartan theory. gr-qc/0606062, 2006.

[1216] A. A. Tseytlin and C. Vafa. Elements of string cosmology. Nucl. Phys., B372:443-466, 1992, hep-th/9109048

[1217] S. Tsujikawa. Density perturbations in the ekpyrotic Universe and string-inspired generalizations Physics Letters B, 526:179-185, Feb. 2002, arXiv:gr-qc/0110124

[1218] S. Tsujikawa. Cosmologies from higher-order string corrections. Annalen der Physik, 518:302-315, Apr. 2006, arXiv:hep-th/0606040

[1219] S. Tsujikawa. Matter density perturbations and effective gravitational constant in modified gravity models of dark energy. Phys. Rev. D, 76(2):023514, July 2007, 0705.1032.

[1220] S. Tsujikawa. Observational signatures of $f(R)$ dark energy models that satisfy cosmological and local gravity constraints. Phys. Rev. D, 77(2):023507, Jan. 2008, 0709.1391.

[1221] S. Tsujikawa, R. Gannouji, B. Moraes, and D. Polarski. Dispersion of growth of matter perturbations in $\mathrm{f}(\mathrm{R})$ gravity. Phys. Rev. D, 80(8):084044, Oct. 2009, 0908.2669

[1222] S. Tsujikawa, K. Maeda, and T. Torii. Preheating with nonminimally coupled scalar fields in higher-curvature inflation models. Phys. Rev. D, 60(12):123505, Dec. 1999, arXiv:hep$\mathrm{ph} / 9906501$

[1223] S. Tsujikawa and M. Sami. String-inspired cosmology: a late time transition from a scaling matter era to a dark energy universe caused by a Gauss Bonnet coupling. J. Cosmology Astropart. Phys., 1:6, Jan. 2007, arXiv:hep-th/0608178

[1224] S. Tsujikawa, M. Sami, and R. Maartens. Observational constraints on braneworld inflation: The Effect of a Gauss-Bonnet term. Phys.Rev., D70:063525, 2004, astro-ph/0406078 
[1225] S. Tsujikawa, T. Tamaki, and R. Tavakol. Chameleon scalar fields in relativistic gravitational backgrounds. J. Cosmology Astropart. Phys., 5:20, May 2009, 0901.3226.

[1226] S. Tsujikawa, K. Uddin, and R. Tavakol. Density perturbations in $\mathrm{f}(\mathrm{R})$ gravity theories in metric and Palatini formalisms. Phys. Rev. D, 77(4):043007, Feb. 2008, 0712.0082.

[1227] P. Y. Tsyba, I. I. Kulnazarov, K. K. Yerzhanov, and R. Myrzakulov. Pure kinetic k-essence as the cosmic speed-up and $\mathrm{f}(\mathrm{T})$ - gravity. ArXiv e-prints, Aug. 2010, 1008.0779

[1228] M. S. Turner, G. Steigman, and L. M. Krauss. Flatness of the universe - Reconciling theoretical prejudices with observational data. Physical Review Letters, 52:2090-2093, June 1984.

[1229] M. S. Turner and F. Wilczek. Relic gravitational waves and extended inflation. Physical Review Letters, 65:3080-3083, Dec. 1990.

[1230] S. G. Turyshev, M. Shao, and K. Nordtvedt. The laser astrometric test of relativity mission. Classical and Quantum Gravity, 21:2773-2799, June 2004, arXiv:gr-qc/0311020

[1231] S. G. Turyshev, J. G. Williams, M. Shao, J. D. Anderson, K. L. Nordtvedt, Jr, and T. W. Murphy, Jr. Laser Ranging to the Moon, Mars and Beyond. ArXiv General Relativity and Quantum Cosmology e-prints, Nov. 2004, arXiv:gr-qc/0411082

[1232] K. Uddin, J. E. Lidsey, and R. Tavakol. Cosmological perturbations in Palatini modified gravity. Class. Quant. Grav., 24:3951-3962, 2007, 0705.0232.

[1233] K. Uddin, J. E. Lidsey, and R. Tavakol. Cosmological scaling solutions in generalised GaussBonnet gravity theories. General Relativity and Gravitation, 41:2725-2736, Dec. 2009, 0903.0270.

[1234] K. -i. Umezu, K. Ichiki, T. Kajino, G. J. Mathews, R. Nakamura, M. Yahiro, Observational constraints on accelerating brane cosmology with exchange between the bulk and brane. Phys. Rev. D73 (2006) 063527. astro-ph/0507227.

[1235] A. Unzicker and T. Case. Translation of Einstein's Attempt of a Unified Field Theory with Teleparallelism. ArXiv Physics e-prints, Mar. 2005, arXiv:physics/0503046

[1236] A. Upadhye, S. S. Gubser, and J. Khoury. Unveiling chameleon fields in tests of the gravitational inverse-square law. Phys. Rev. D, 74(10):104024, Nov. 2006, arXiv:hep-ph/0608186

[1237] A. Upadhye and W. Hu. Existence of relativistic stars in $\mathrm{f}(\mathrm{R})$ gravity. Phys. Rev. D, 80(6):064002, Sept. 2009, 0905.4055.

[1238] A. Upadhye, J. H. Steffen, and A. Weltman. Constraining chameleon field theories using the GammeV afterglow experiments. Phys. Rev. D, 81(1):015013, Jan. 2010, 0911.3906.

[1239] R. Utiyama and B. S. Dewitt. Renormalization of a Classical Gravitational Field Interacting with Quantized Matter Fields. Journal of Mathematical Physics, 3:608-618, July 1962.

[1240] J. Uzan. The fundamental constants and their variation: observational and theoretical status. Reviews of Modern Physics, 75:403-455, Apr. 2003, arXiv:hep-ph/0205340.

[1241] A. I. Vainshtein. To the problem of nonvanishing gravitation mass. Phys. Lett., B39:393-394, 1972 .

[1242] K. Van Acoleyen and J. Van Doorsselaere. Galileons from Lovelock actions. 2011, 1102.0487.

[1243] H. van Dam and M. J. G. Veltman. Massive and massless Yang-Mills and gravitational fields. Nucl. Phys., B22:397-411, 1970.

[1244] C. van de Bruck, M. Dorca, R. H. Brandenberger, and A. Lukas. Cosmological perturbations in brane-world theories: Formalism. Phys. Rev., D62:123515, 2000, hep-th/0005032

[1245] C. van de Bruck, M. Dorca, C. J. A. P. Martins, and M. Parry. Cosmological consequences of the brane/bulk interaction. Phys. Lett., B495:183-192, 2000, hep-th/0009056

[1246] H. van Elst, J. E. Lidsey, and R. Tavakol. Quantum cosmology and higher-order Lagrangian theories. Classical and Quantum Gravity, 11:2483-2497, Oct. 1994, arXiv:gr-qc/9404044

[1247] M. A. Vasiliev. Consistent equation for interacting gauge fields of all spins in (3+1)-dimensions. Phys.Lett., B243:378-382, 1990.

[1248] L. Verde, A. F. Heavens, W. J. Percival, S. Matarrese, C. M. Baugh, et al. The 2dF Galaxy Redshift Survey: The Bias of galaxies and the density of the Universe. Mon.Not.Roy.Astron.Soc., 335:432, 2002, astro-ph/0112161

[1249] A. Vilenkin. Classical and quantum cosmology of the Starobinsky inflationary model. Phys. Rev. D, 32:2511-2521, Nov. 1985.

[1250] J. Vinet and J. M. Cline. Can codimension-two branes solve the cosmological constant problem? Phys. Rev., D70:083514, 2004, hep-th/0406141

[1251] D. N. Vollick. $1 / R$ curvature corrections as the source of the cosmological acceleration. Phys. Rev. D, 68(6):063510, Sept. 2003, arXiv:astro-ph/0306630

[1252] H. von Borzeszkowski. On singularities of general relativity and gravitational equations of fourth order. Annalen der Physik, 493:239-248, 1981

[1253] R. V. Wagoner. Scalar tensor theory and gravitational waves. Phys. Rev., D1:3209-3216, 1970. 
[1254] R. M. Wald. Final states of gravitational collapse. Phys. Rev. Lett., 26(26):1653-1655, Jun 1971.

[1255] R. M. Wald. Asymptotic behavior of homogeneous cosmological models in the presence of a positive cosmological constant. Phys. Rev. D, 28(8):2118-2120, Oct 1983.

[1256] A. Wang. Vector and tensor perturbations in Horava-Lifshitz cosmology. Phys.Rev., D82:124063, 2010, 1008.3637.

[1257] A. Wang and R. Maartens. Linear perturbations of cosmological models in the Horava- Lifshitz theory of gravity without detailed balance. Phys. Rev., D81:024009, 2010, 0907.1748.

[1258] A. Wang, D. Wands, and R. Maartens. Scalar field perturbations in Horava-Lifshitz cosmology. JCAP, 1003:013, 2010, 0909.5167.

[1259] A. Wang and Y. Wu. Cosmology in nonrelativistic general covariant theory of gravity. 2010, 1009.2089.

[1260] Y. Wang. Constraints on generalized extended inflationary models. Phys. Rev. D, 44:991-998, Aug. 1991.

[1261] Y. Wang and P. Mukherjee. Robust dark energy constraints from supernovae, galaxy clustering, and three-year wilkinson microwave anisotropy probe observations. Astrophys.J., 650:1-6, 2006, astro-ph/0604051

[1262] J. Weenink and T. Prokopec. Gauge invariant cosmological perturbations for the nonminimally coupled inflaton field. Phys. Rev. D, 82(12):123510, Dec. 2010, 1007.2133.

[1263] H. Wei. Growth Index of DGP Model and Current Growth Rate Data. Phys.Lett., B664:1-6, 2008, 0802.4122.

[1264] E. J. Weinberg. Some problems with extended inflation. Phys. Rev. D, 40:3950-3959, Dec. 1989.

[1265] S. Weinberg. The Cosmological Constant Problem. Rev.Mod.Phys., 61:1-23, 1989. Morris Loeb Lectures in Physics, Harvard University, May 2, 3, 5, and 10, 1988.

[1266] S. Weinfurtner, T. P. Sotiriou, and M. Visser. Projectable Horava-Lifshitz gravity in a nutshell. J.Phys.Conf.Ser., 222:012054, 2010, 1002.0308.

[1267] J. Weisberg, D. J. Nice and J. H. Taylor. Timing Measurements of the Relativistic Binary Pulsar PSR B1913+16. Astrophys. J., 722:1030-1034, 2010.

[1268] J. Weisberg and J. H. Taylor. General relativistic geodetic spin precession in binary pulsar b1913+16: Mapping the emission beam in two dimensions. Astrophys. J., 576:942-949, 2002.

[1269] H. Weyl. Gravitation und Elektrizität. Sitzungsber. Preuss. Akad. d. Wiss. Teil, 1(465), 1918.

[1270] B. Whitt. Fourth-order gravity as general relativity plus matter. Physics Letters B, 145:176-178, Sept. 1984.

[1271] B. Whitt. Stability of Schwarzschild black holes in fourth-order gravity. Phys. Rev. D, 32:379-388, July 1985.

[1272] C. Will. The confrontation between general relativity and experiment. Living Rev. Rel., 9:3, 2006 .

[1273] C. M. Will. in General relativity. An Einstein centenary survey. 1979.

[1274] C. M. Will. Theory and Experiment in Gravitational Physics. Mar. 1993.

[1275] C. M. Will and D. M. Eardley. Dipole gravitational radiation in Rosen's theory of gravity Observable effects in the binary system PSR 1913+16. ApJ, 212:L91-L94, Mar. 1977.

[1276] C. M. Will and K. Nordtvedt, Jr. Conservation Laws and Preferred Frames in Relativistic Gravity. I. Preferred-Frame Theories and an Extended PPN Formalism. ApJ, 177:757, Nov. 1972

[1277] J. G. Williams, S. G. Turyshev, and D. H. Boggs. Progress in Lunar Laser Ranging Tests of Relativistic Gravity. Physical Review Letters, 93(26):261101, Dec. 2004, arXiv:gr-qc/0411113

[1278] T. Wiseman. Relativistic stars in Randall-Sundrum gravity. Phys. Rev., D65:124007, 2002, hep-th/0111057

[1279] B. Withers. Einstein-aether as a quantum effective field theory. Classical and Quantum Gravity, 26(22):225009, Nov. 2009, 0905.2446.

[1280] W. Wood-Vasey et al. Observational Constraints on the Nature of the Dark Energy: First Cosmological Results from the ESSENCE Supernova Survey. Astrophys.J., 666:694-715, 2007, astro-ph/0701041

[1281] R. Woodard. Avoiding Dark Energy with 1/R Modifications of Gravity. In L. Papantonopoulos, editor, The Invisible Universe: Dark Matter and Dark Energy, volume 720 of Lecture Notes in Physics, Berlin Springer Verlag, page 403, 2007, arXiv:astro-ph/0601672

[1282] P. Worden, R. Torii, J. C. Mester, and C. W. F. Everitt. The STEP Payload and Experiment. Advances in Space Research, 25:1205-1208, 2000.

[1283] A. Wu. Constraints on the nucleation rate in extended inflation. Phys. Rev. D, 45:2653-2664, Apr. 1992.

[1284] F. Wu and X. Chen. Cosmic microwave background with Brans-Dicke gravity II: constraints with 
the WMAP and SDSS data. 2009, 0903.0385.

[1285] F. Wu, L.-e. Qiang, X. Wang, and X. Chen. Cosmic microwave background with Brans-Dicke Gravity: I. Covariant Formulation. 2009, 0903.0384.

[1286] P. Wu and H. Yu. $\mathrm{f}(\mathrm{T})$ models with phantom divide line crossing. ArXiv e-prints, Aug. 2010, 1008.3669 .

[1287] P. Wu and H. Yu. Observational constraints on $\mathrm{f}(\mathrm{T})$ theory. Physics Letters B, 693:415-420, Oct. 2010, 1006.0674.

[1288] P. Wu and H. Yu. The dynamical behavior of $\mathrm{f}(\mathrm{T})$ theory. Physics Letters B, 692:176-179, Aug. 2010, 1007.2348.

[1289] M. Wyman. Static spherically symmetric scalar fields in general relativity. Phys. Rev., D24:839$841,1981$.

[1290] M. Wyman and J. Khoury. Enhanced Peculiar Velocities in Brane-Induced Gravity. Phys.Rev., D82:044032, 2010, 1004.2046.

[1291] D. Xu et al. Is Gravitational Lensing by Intercluster Filaments Always Negligible? Astrophys. J., 682:711-720, 2008, 0710.4935.

[1292] J. Yang, D. N. Schramm, G. Steigman, and R. T. Rood. Constraints on cosmology and neutrino physics from big bang nucleosynthesis. ApJ, 227:697-704, Jan. 1979.

[1293] R. Yang. Conformal transformation in $\mathrm{f}(\mathrm{T})$ theories. ArXiv e-prints, Oct. 2010, 1010.1376.

[1294] A. Yoho, F. Ferrer, and G. D. Starkman. Degree-scale anomalies in the CMB: localizing the first peak dip to a small patch of the north ecliptic sky. 2010, 1005.5389.

[1295] M. Yoshimura. Extended inflation, Brans-Dicke dilaton, and baryon asymmetry. Physical Review Letters, 66:1559-1562, Mar. 1991.

[1296] V. I. Zakharov. Linearized gravitation theory and the graviton mass. JETP Lett., 12:312, 1970.

[1297] J. Zanelli. Lecture notes on Chern-Simons (super-)gravities. Second edition (February 2008). 2005, hep-th/0502193

[1298] Y. Zeldovich and A. A. Starobinsky. Particle production and vacuum polarization in an anisotropic gravitational field. Sov.Phys.JETP, 34:1159-1166, 1972.

[1299] P. Zhang. Testing gravity against the early time integrated Sachs-Wolfe effect. Phys. Rev. D, 73(12):123504, June 2006, arXiv:astro-ph/0511218.

[1300] P. Zhang. Behavior of $\mathrm{f}(\mathrm{R})$ gravity in the solar system, galaxies, and clusters. Phys. Rev. D, 76(2):024007, July 2007, arXiv:astro-ph/0701662

[1301] P. Zhang, M. Liguori, R. Bean, and S. Dodelson. Probing Gravity at Cosmological Scales by Measurements which Test the Relationship between Gravitational Lensing and Matter Overdensity. Physical Review Letters, 99(14):141302, Oct. 2007, 0704.1932.

[1302] G. Zhao, T. Giannantonio, L. Pogosian, A. Silvestri, D. J. Bacon, K. Koyama, R. C. Nichol, and Y. Song. Probing modifications of general relativity using current cosmological observations. Phys. Rev. D, 81(10):103510, May 2010, 1003.0001.

[1303] G. Zhao, B. Li, and K. Koyama. N-body Simulations for $f(R)$ Gravity using a Self-adaptive Particle-Mesh Code. ArXiv e-prints, Nov. 2010, 1011.1257.

[1304] G. Zhao, L. Pogosian, A. Silvestri, and J. Zylberberg. Cosmological Tests of General Relativity with Future Tomographic Surveys. Physical Review Letters, 103(24):241301, Dec. 2009, 0905.1326.

[1305] H. Zhao. Constraining TeVeS gravity as effective dark matter and dark energy. Int. J. Mod. Phys., D16:2055-2063, 2008, astro-ph/0610056

[1306] H.-S. Zhao. An introduction to gravitational lensing in TeVeS gravity. 2006, astro-ph/0611777

[1307] H.-S. Zhao, D. J. Bacon, A. N. Taylor, and K. Horne. Testing Bekenstein's Relativistic MOND gravity with Gravitational Lensing. Mon. Not. Roy. Astron. Soc., 368:171-186, 2006, astro$\mathrm{ph} / 0509590$

[1308] R. Zheng and Q. Huang. Growth factor in $\mathrm{f}(\mathrm{T})$ gravity. ArXiv e-prints, Oct. 2010, 1010.3512.

[1309] S. Zhou, E. J. Copeland, and P. M. Saffin. Cosmological constraints on $\mathrm{f}(\mathrm{G})$ dark energy models. J. Cosmology Astropart. Phys., 7:9, July 2009, 0903.4610.

[1310] S.-Y. Zhou. Goldstone's Theorem and Hamiltonian of Multi-galileon Modified Gravity. 2010, 1011.0863.

[1311] T. G. Zlosnik, P. G. Ferreira, and G. D. Starkman. Growth of structure in theories with a dynamical preferred frame. Phys. Rev. D, 77(8):084010, Apr. 2008, 0711.0520.

[1312] M. A. Zumberge, J. A. Hildebrand, J. M. Stevenson, R. L. Parker, F. N. Spiess, A. D. Chave, and M. E. Ander. Submarine measurement of the Newtonian gravitational constant. Physical Review Letters, 67:3051-3054, Nov. 1991.

[1313] B. Zumino. Gravity theories in more than four dimensions 1. Physics Reports, 137(1):109-114, 
1986.

[1314] J. Zuntz, T. G. Zlosnik, F. Bourliot, P. G. Ferreira, and G. D. Starkman. Vector field models of modified gravity and the dark sector. Phys. Rev. D, 81(10):104015, May 2010, 1002.0849.

[1315] J. A. Zuntz, P. G. Ferreira, and T. G. Zlosnik. Constraining Lorentz Violation with Cosmology. Physical Review Letters, 101(26):261102, Dec. 2008, 0808.1824.

[1316] B. Zwiebach. Curvature Squared Terms and String Theories. Phys. Lett., B156:315, 1985. 